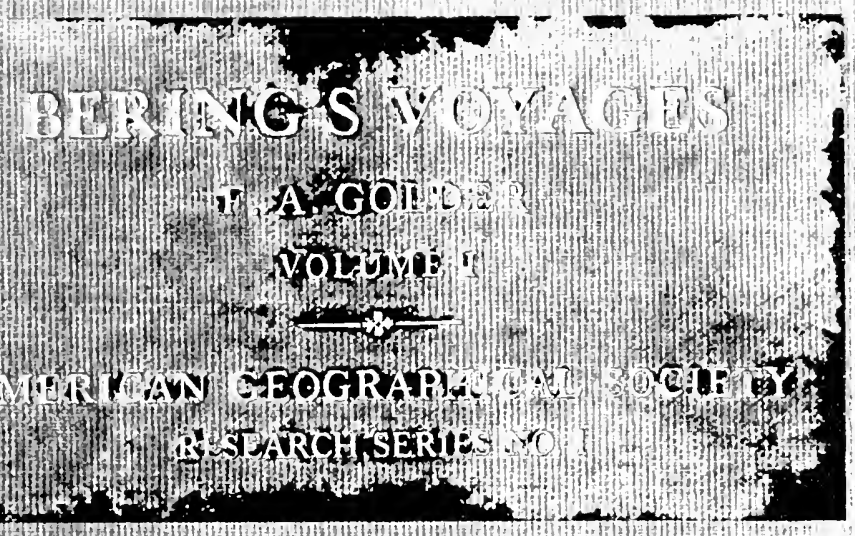


$\therefore$

$$
-
$$




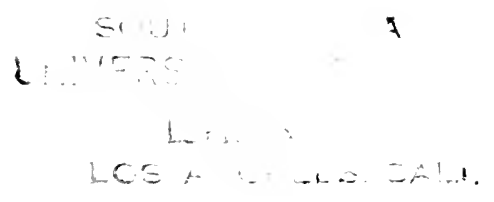



BERING'S VOYAGES

VOLUME I 

AMERICAN GEOGRAPHICAL SOCIETY RESEARCH SERIES NO. I W. L. G. JOERG, Editor

\title{
BERING'S VOYAGES
}

An Account of the Efforts of the Russians to

Determine the Relation of Asia and America

\author{
BY \\ F. A. GOLDER \\ IN TWO VOLUMES \\ VOLUN1E I:
}

The Log Books and Official Reports of the First and Second Expeditions

$1725-1730$ and $1733^{-174^{2}}$

WITH A CHART OF THE SECOND VOYAGE

BY

ELLSWORTH P. BERTHOLF

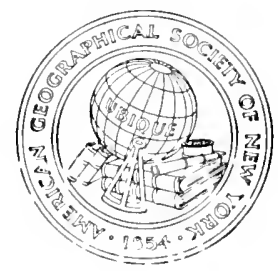

AMERICAN GEOGRAPHICAL SOCIETY

BROADWAY AT I 56 TH STREET

NEW YORK

I 922 
COPYRIGHT, I 922

BY

THE AMERICAN GEOGRAPHICAL SOCIETY OF NEW YORK 


\section{TO}

PROFESSOR EDWARD CHANNING

$$
\mathrm{OF}
$$

HARVARD UNIVERSITY 


\section{CONTENTS}

CHAPTER

PAGE

Preface. . . . . . . . . . . . . . . . . . $\mathrm{ix}$

\section{PART I}

The First Expedition, 1725-1730, and Its Setting

I The Geographical KNowledge of the North

Pacific Ocean at the BeginNing of the Eighteenth Century . . . . . . . . . . . . I I

II Bering's First Expedition, i725-1730 . . . . . 6

III Grozdev's Voyage to AMerica in I732 . . . . 2 I

\section{PART II}

The Second Expedition, 1733-1742

IV Bering's Second Expedition, From Its Inception to the Beginning of the Sea Voyage . . . . 25

$V$ The Lor BOOK OF Bering's Vessel, the "St.

Peter," and of Her Successor, the HoOker "St. Peter" . . . . . . . . . . . . . . . . 36

VI Lieutenant Waxel's Report on the Voyage of THE "ST. Peter" . . . . . . . . . . . . . . 270

VII The Journal of Chirikov's Vessel, the "St. Paul". . . . . . . . . . . . . . . . 283

VIII Chirikov's Report on the Voyage of the "ST. PAUl". . . . . . . . . . . . . . . . . . $3 \mathrm{I} 2$

IX The End of the Expedition . . . . . . . . . 328

Note to Accompany the Chart of the Voyage OF Bering AND ChIRIKOV From KaMCHATKA TO the Alaskan Coast and Return, 174I, BY the late Ellsworth P. Bertholf . . . . . . 330 Bibliographical Note . . . . . . . . . . . 349

(Index to both volumes in Vol. II.) 


\section{LIST OF ILLUSTRATIONS}

Fig.

I Section of a map by Guillaume Delisle, I 74 , to illustrate contemporary knowledge of the North Pacific . facing

2 Facsimile of part of Peter the Great's orders for the first expedition, with comments in his own handwriting

facing 7

3 Map showing route of the first expedition from St. Petersburg to Bering Strait

+ Facsimile of a manuscript map showing the route of the first expedition from Yudoma Cross to Urak near Okhotsk. (Drawing by Spanberg in the Russian archives.) . . . . . . . . . . . . . . . facing

5 Copy of the eastern section of the map accompanying Bering's report on his first expedition. (After Dall's facsimile.) . . . . . . . . . . . . . . I4-I5

6 Facsimile of Berkh's map, I823, showing route of the first expedition from Okhotsk to Bering Strait and return. . . . . . . . . . . . . . . . facing

7 Facsimile of a manuscript map of the Harbor of St. Peter and St. Paul (Petropavlovsk), I 740. (Drawing by Elagin in the Russian archives.) . . . . . . . . . . facing

8 The Harbor of St. Peter and St. Paul. (From an old engraving). . . . . . . . . . . . . . facing

9 Facsimile of signatures of Bering and his officers. (From the log book of the St. Peter: entry of May $4, \mathrm{I} 7+\mathrm{I}$.$) . .$

Io Facsimile of a page of the $\log$ book of the St. Peter kept by Yushin: entry of June $7,177^{I}$. . . . . . . . facing

I I Facsimile of a page of the log book of the St. Peter kept by Khitrov: end of entry of September 5, I 7+1, with map of the Shumagin Islands . . . . . . . . . facing 
Fig.

12 Upper: An Aleut in his baidarka, or skin boat. (Drawing on the chart of the voyage of the St. Peter by Waxel and Khitrov in the Russian archives.)

Lower: A native of Unalaska in a baidarka. (Drawing by Levashev in the Russian archives.) . . . . . facing 149

13 Facsimile of a page of the journal of the St. Paul: entries of July $10-15,1741$. . . . . . . . . . facing 290

14 A man of Unalaska. (Drawing by Levashev.) . . facing 304

15 Left: Inside and outside views of native hut, Unalaska. Right: A native woman of Unalaska. (Drawing by Levashev.). . . . . . . . . facing 305

PI.

I Chart of the voyage of Bering and Chirikov in the St. Peter and the St. Paul from Kamchatka to the Alaskan coast and return, 174I, based on the log books and other original records and adjusted to known physical conditions. Mean meridional scale, I:5,500,000. By Ellsworth P. Bertholf . . . . . . . . . . . facing 


\section{PREFACE}

THE discovery of the northwest coast of America by the Russians deserves far more consideration than it has yet received. Our information on the subject is very fragmentary-a chapter here and a chapter there in the histories of the Russian Far East and the American Far West. This neglect is due not so much to lack of interest as to lack of material and to the peculiar problems which the handling of the material presents. But these difficulties are gradually disappearing, and it is now possible to undertake a task that twenty years ago seemed out of the question. In this work the author has made the actors tell their own story of the discovery of the northwest coast of America. It would have been worth while to bring together all the interesting material relating to the subsidiary voyages of Bering's second expedition - the voyages of Spanberg to Japan and the explorations along the Arctic coast-but neither time nor space permitted it.

Volume $x$ includes an account of the log books and reports of the navigators, and Volume 2 deals with the journal of Steller, which throws much light on the second expedition and furnishes valuable scientific data. In the translation the terminology of the navigators has been retained and their ideas faithfully rendered in so far as it was possible, considering that their penmanship was not always legible, nor their language grammatical, nor their expressions intelligible. With these original data before him the reader is in a position to form an independent conclusion respecting the controversies raging around this voyage. This book is not the last word on the subject, and it is hoped that other investigators will use its material to make further studies in the field.

The late Captain E. P. Bertholf, until within two years of his death in I92I Commandant of the U.S. Coast Guard, and an authority on the waters and shores of Bering Sea and the North Pacific Ocean, has made a distinct contribution to science by plotting out scientifically the tracks and landfalls of the 
St. Peter and the St. Paul. In addition he read the manuscript and suggested improvements. Lieutenant Raymond Burhen, U. S. N., and Captain Sam Hoyt, of Seattle, have helped in the matter of nautical expressions. To all these gentlemen the author is sincerely grateful. He takes this occasion also to testify publicly to the courtesy and genuine good will of the Russian archivists and scholars of the old régime with whom he had contact in Petrograd and Moscow in his search for material. They were ever ready to inconvenience themselves to help him in his work. Some of these men have been forced from their position and their country, and others, like Professor Lappo-Danilerski, have died of starvation. They were real scholars and true gentlemen, and it is with a feeling of reverence and gratitude that this tribute is paid to them. 


\section{CHAPTER I}

\section{THE GEOGRAPHICAL KNOWLEDGE OF THE NORTH PACIFIC OCEAN AT THE BEGINNING OF THE EIGHTEENTH CENTURY}

THE numerous naval expeditions of the sixteenth century in search of a short passage to Asia gave the geographers a fairly good idea of the waters and shores of the Atlantic and of those parts of the Arctic that were nearest to Europe and European settlements. Equally helpful in making the Indian and the South Pacific waters familiar to the educated world were the voyages via the Cape of Good Hope route to the Indies, China, and Japan, the annual voyages of the Spanish naval officers between Mexico and the Philippines, and the occasional raids of the English freebooters along the Spanish-American coast. From the early years of the seventeenth century onward more or less reliable maps existed for the Pacific Ocean south of the parallel which runs through Cape Mendocino and the northern part of the main island of Japan, but for the vast region north of that line not a single map that could in any way lay claim to accuracy was to be found before the time of the Bering voyages. It was not even known whether the North Pacific area was all land or all water, whether Asia and America were separated or united (Fig. I).

There were many reasons for believing that islands or a continent were to be found in that northern region. When the Jesuits came to Japan in the middle of the sixteenth century they learned that north of Japan proper there was a body of land called Yezo, but they could not fully inform themselves as to its shape and size. A somewhat similar report reached Europe from another quarter. Richard Cocks, an English merchant in Japan, in a letter written in 1611 , made mention of "an island called Yedzo, which is thought to be rather some part of the continent Tartaria." 
But this was not the only vague information the cartographers had to go on. The credulous were as eager as ever to hear of isl xnds with golden streets, and they accepted as a fact every baseless rumor which helped to confirm them in their belief. One

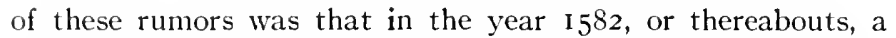
Spanish vessel in going from Manila eastward ran into a storm which drove her to an island situated in latitude $37^{\circ} 30^{\prime} \mathrm{N}$. and some 400 miles east of Japan. The inhabitants of this island were hospitable and rich to such an extent that even the pots and pans were made of gold and silver.

\section{VIZCAÍNO AND VRIES}

The Spanish government attached enough importance to current gossip to send Sebastian Vizcaíno from Mexico in search of the rumored El Dorado. He spent the autumns of 16 i I and 1612 in cruising north, south, and east of Japan without, however, being able to locate the prize. In the meantime the Dutch traders heard the story, and they in turn became interested. In I639 the Dutch East India Company instructed Mathijs Quast and Abel Janszoon Tasman to find the gold island, but all their efforts were in vain. Four years later the company sent another expedition in command of Maerten Gerritszoon Vries. He sailed north and east of Japan, sighting the island of Yezo without, howcame ever, being able to determine either its shape or its size, and among (what are now known as) the Kurile Islands. One of these he named State Island and the other (which he thought to be part of the American continent) Company Land. But neither gold nor silver did Vries find, though he sailed north to Sakhalin, south to Formosa, and east 460 miles from Japan. The Vries discoveries were put on the map by Jansson in $165^{\circ}$. The preceding year (1649) another cartographer, Texeira by name, published a map on which he marked the discoveries (in the early part of the seventeenth century) of a certain Juan de Gama just about where Vries placed his Company Land, that is to say between latitude $40^{\circ}$ and $45^{\circ} \mathrm{N}$. (Fig. I). 


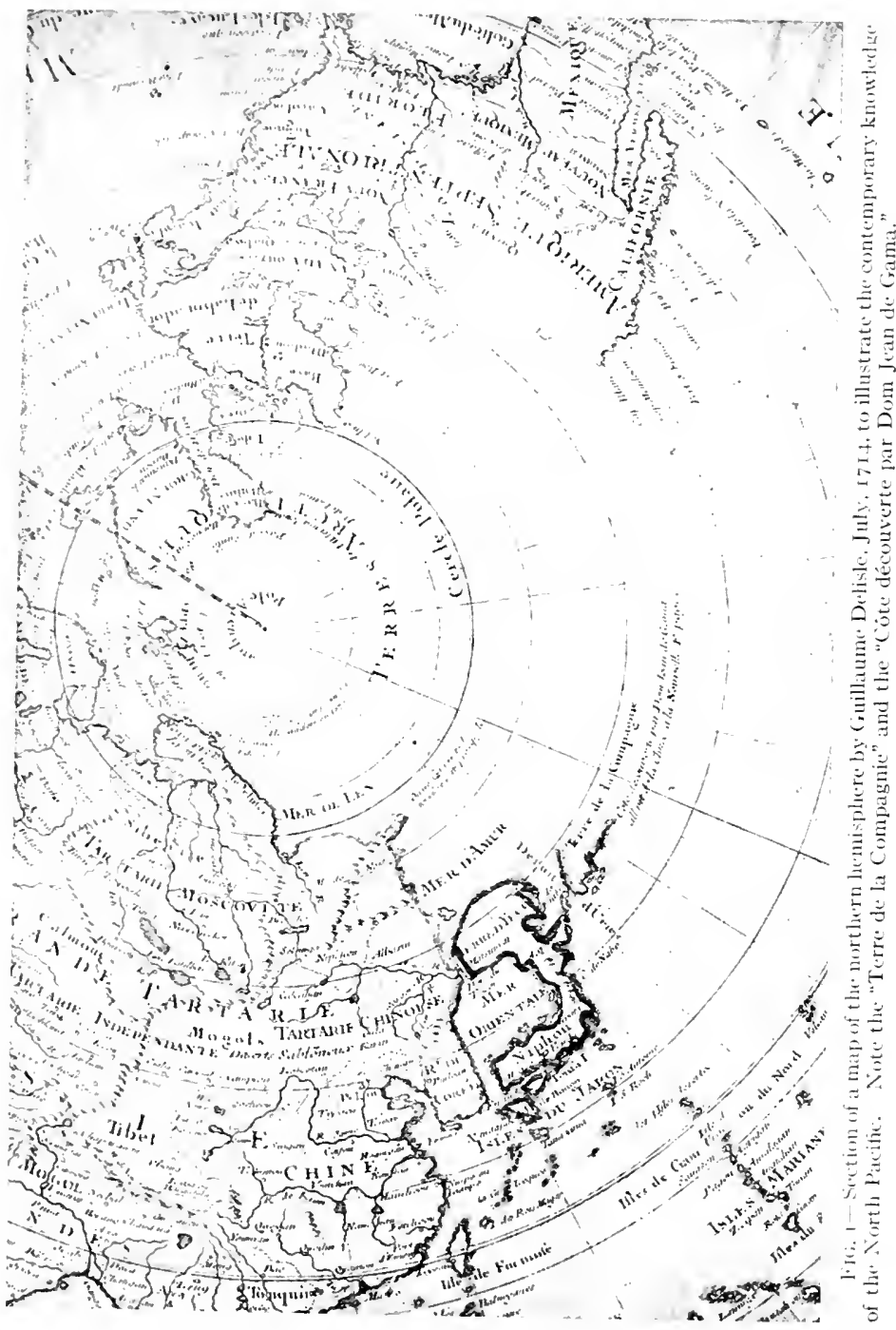





\section{Company Land and Gama Land}

It is no wonder that these three reports, that of the Jesuits, Vries, and Texeira, of the three poorly described territories, Yezo, Company Land, and Gama Land, bewildered the cartographers. They were at a loss to know whether the lands in question were islands or continents, whether they were parts of America or of Asia, whether they were three distinct lands or one and the same. On the maps of the second half of the seventeenth and the early part of the eighteenth century all these views, and many others besides, found expression. Johann Baptist Homann of Nuremberg, on his map of about 1712, suggested that Terra Esonis was probably the northwest coast of America and the same land that Gama discovered; on the other hand Guillaume Delisle, in a memoir which he read before the Paris Academy of Sciences in 1720 , made the statement that Yezo was a part of Asia and that Japan was a peninsula of it. The difference in the point of view of these two men gives an idea of the confusion on the subject that existed at the beginning of the eighteenth century. There was only one way of clearing up these difficulties, and that was by actual investigation.

\section{The Russian Advance in Siberia}

During the century and a half that the cartographers were losing themselves in their speculations and paper explorations of the North Pacific the Russian trappers, traders, tribute gatherers, and missionaries were wandering over the length and breadth of Siberia and pushing their discoveries to the "Eastern" Ocean. They had not, however, a clear idea of what they were doing; they had not even a very good mental picture of the land they discovered.

By actual navigation they had learned that the Ob, Yenisei, and Lena emptied into the "Icy" Sea, that the Amur, Ud, and Okhota flowed into the Eastern Sea; but they were ignorant as to whether there existed a passage between these two seas. They 
knew that one could go by water from the mouth of the $\mathrm{Ob}$ to the mouth of the Lena, from the mouth of the Amur to the mouth of the Okhota, but they did not know whether one could navigate from the mouth of the Lena to the mouth of the Okhota. They were not agreed among themselves whether there was land to the north and east of the two last-named rivers. Many of these Siberian hunters believed that not far from the mouth of the Kolyma River a large continent (bolshaya zemlya) extended northward and that the Asiatic mainland stretched out indefinitely to the eastward. Something was also said and known of the Anadyr and Kamchatka Rivers, but they were supposed to discharge their waters into the Arctic. According to some curious Siberian maps of the late seventeenth and early eighteenth centuries eastern Asia ended somewhere near the mouth of the Okhota River and northern Asia close to the Yana River, and the two parts together, where they joined, formed a right angle. The areas which now go by the name of Kamchatka and the Chukchi Peninsula were then unnoticed. This may have been due to the Siberians' ignorance of the country or to their ignorance of the science of map making.

\section{Peter the Great's Interest}

When Peter the Great came into power he took deep interest in the activities of his energetic Cossacks and tried to give them intelligent guidance. He sent many of his Swedish prisoners of war into Siberian ports to teach his subjects to build sea-going vessels, to use nautical instruments, and to construct modern maps. Between 1700 and 17 I5 Kamchatka and the Kurile Islands were discovered and explored, and the terra incognita in Asia was pushed still farther eastward. Reports of these discoveries reached the scientific men of Western Europe, and when Peter came among them in 1716 and 1717 they discussed with him the question of the North Pacific and urged him to settle once for all the problem whether Asia and America were united. Peter accepted for himself and for his country the honor and the 
responsibility, and carried the undertaking through successfully, although at the time his success was somewhat doubted. When Bering's second expedition came to an end in 1749 , Bering Strait had been discovered, the Arctic coast of Asia from the IWhite Sea to the Kolyma River had been charted, and the North Pacific coast of America from Cape Addington to Bering Island had been placed on the map. This was Russia's share in the work of discovery and exploration, and a very important contribution to geographical knowledge it was. 


\section{CHAPTER II}

\section{BERING'S FIRST EXPEDITION, 1725-1730}

ON his return to his capital in 1718 Peter the Great went to work at once on the problem of the relation of Asia to America. He ordered two of his officers, Fedor Luzhin and Ivan Evreinov, to proceed to Asia to make certain investigations for him and "to go to Kamchatka and farther, as you have been ordered, and determine whether Asia and America are united; and go not only north and south but east and west, and put on a chart all that you see." 1 They left Russia in 1719, reached Kamchatka in 1720 , cruised among and explored the Kurile Islands in 1720 or 1721 , returned to St. Petersburg in 1722 or 1723 , and made a verbal report to the emperor. Just what they reported is not known, but it is certain that they did not "determine whether Asia and America are united."

\section{Peter the Great's Orders}

This failure did not discourage Peter; it probably convinced him that in order to be successful the undertaking would have to be maintained on a larger scale than he had contemplated in I7 8 . The time was more favorable now than before for such work; the Peace of Nystad gave him the leisure and the peace of mind for planning great things. Unfortunately his health began to fail, and, as he realized that he had not long to live, he made more haste. On December $23,1724,{ }^{2}$ he set matters in motion by drawing up the following orders:

1 Polnoe Sobranic Zakonov Rossiiskoi Imperii, Vol. 4. Doc. 3266.

${ }^{2}$ In the present work the dates are according to the old style, at that time eleven day's behind the new style. 


$$
\text { . }
$$


3.

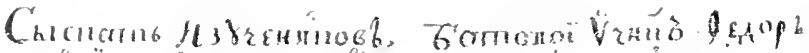

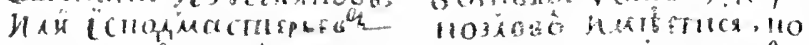
llomopot dhe Mofó niamo.

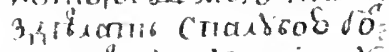

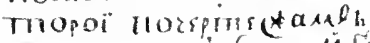

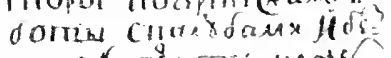
$110{ }^{2}$ '

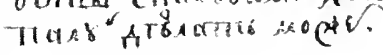

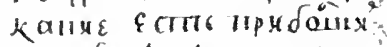
liaparax : neper ntozo:

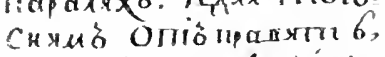

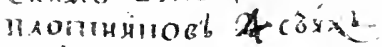

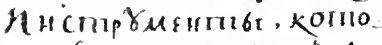

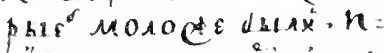
O"dHozo napmaris sonte. pa $i$. 8. inatu Marmo?

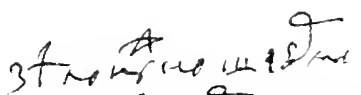

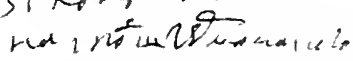

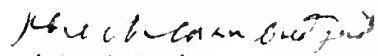
desegut

$-4$

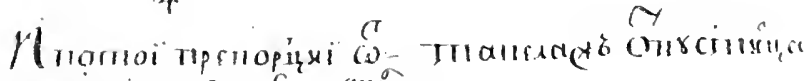

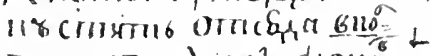
mopa: Trapdian?. diolio.

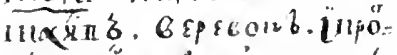

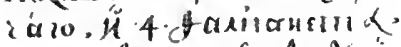
+ Eoror

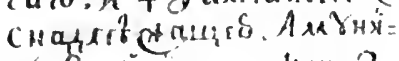

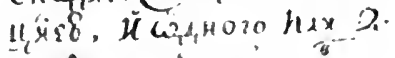
Trap 
. Orders of Peter the Great for the Selection of Officers for the FIRST EXPEDITION ${ }^{3}$

Peter's Orders

I. To find geodesists who have been in Siberia and have returned.

2. To find among the deserving naval lieutenants or sub-lieutenants such as could be sent to $\mathrm{Si}$ beria and Kamchatka.
3. To find among the apprentices or assistant master builders one who could build there a deck ship along the lines of the big ships here. For that purpose there should be sent with him young ship carpenters, such instruments as may be needed, one quartermaster, and eight sailors.

4. Likewise to forward from here one and a half* sets of sails; blocks, cables, and such like; four falconets with the necessary ammunition; and one or two sailmakers.

\section{Reports}

The Senate learns that the following named geodesists have been in Siberia: Ivan Zakharov, Peter Chichagov, Ivan Evreinov, who died, Fedor Luzhin, Peter Skobeltsyn, Ivan Svistunov, Dmitri Baskakov, Vasili Shetilov, Grigori Putilov.

Admiral Sievers and Rear Admiral Senyavin recommend among the naval lieutenants Stanberg, 5 Zverev, or Posenkov; among the sub-lieutenants Chirikov or Laptev. It would be advisable to have over them as commander Captain Bering or Fonverd. Bering has been in the East Indies and knows conditions, and Fonverd has had experience as navigator.

The student of shipbuilding. Fedor Koslov, is able to build either decked or open ships if he is furnished with a plan.

It is very necessary to have as nazigator or assistant navigator one who has been in North America.

Rigging is being sent.

*Two sets.

The rest is all right.

${ }^{3}$ Archives of the Ministry of Marine: Papers of the Admiralty Council, r 724 . No. 29, pp. I20-130. In the left-hand column are Peter's instructions, in the right is the report of the Senate or the Admiralty Council. The lines in italics are comments on the report by Peter made in his own handwriting (see Fig. 2).

- In 1720 or I 72 I Evreinov and Luzhin, as stated above, went to the Kurile Islands at the order of Peter. Soon after their return Evreinov died.

${ }^{5}$ I.e. Spanberg. 
5. If there are no such navigators in our navy, a letter should be dispatched at once to Holland that two men be sent who know the sea in the north and as far as Japan. These men should come by the admiralty post.

December 23, 1724 .
Vice-Admiral Sievers has written that he has among our men navigators who know the sea and that he will send them without delay.

\section{Ukase to the Governor of Siberia, Prince Dolgorukov ${ }^{6}$}

We are sending to Siberia Fleet-Captain Vitus Bering with assistants to undertake a naval expedition and to carry out such instructions as he has from us. When he comes to you and asks help of one kind and another for the expedition you are to give it to him.

January, I 725 .

\section{Account of The Expedition}

On the strength of the recommendations of the Senate and the Admiralty Council the tsar selected Vitus Bering ${ }^{7}$ to lead the expedition and gave him as lieutenants Martin Spanberg ${ }^{8}$ and Alexei Chirikov. In the early part of January, 1725 , he also drew up and signed the instructions; 9 but, owing to his failing health, he had to leave the execution of them to his friend Count Aprak$\sin ^{10}$ The death of Peter on January $28, \mathbf{1} 725$, did not in the least

${ }^{6}$ Archives of the Ministry of Marine: Papers of the Admiralty Council, 1724. No. 29.

${ }^{7}$ Vitus Bering was born at Horsens, Denmark, in the year 1681. As soon as he was old enough he went to sea and in 1703 made a voyage to the East Indies. In 1704 he joined the Russian navy with the rank of sub-lieutenant. He rose gradually in the service, being made lieutenant in 1707 , lieutenant-captain in 1710 , captain of the fourth rank in 1715 , captain of the third rank in 1717 , captain of the second rank in 1720 , and captain of the first rank in 1724 , when he was put in charge of the expedition. For a fuller account of Bering's tife the reader is referred to Peter Lauridsen's "Vitus Bering," transl. by J. E. Olson. Chicago, I 889.

${ }^{8}$ Spanberg was a Dane and Chirikov a Russian.

9 Printed below, pp. 10-II, in Bering's report.

${ }_{10}$ Nartov's account of the last day's of the monarch (Razskazi Nartova o Petre Vetikom, edited by L. N. Maikov, St.l'etersburg, 1891, p. 99) shows how important the latter regarded the expedition:

"In the beginning of January, 1725. Peter was realizing that he had not long to tive, yet his unconquerable spirit was busily at work for the good of the country. With his own hand be drew up the instructions relative to the Kamchatka expedition, which should determine the rolation between Asia and America. 
disturb the plans of the undertaking. Catherine was determined to carry out all the policies and ambitions of her husband. Already, on January 24 , the vanguard of the party had left the capital, but Bering and several others did not leave until February 5 , the day the instructions were handed to him, as may be read in the report of Bering which follows.

\section{Report of Fleet-Captain Bering on His Expedition to the Eastern COAST OF SIBERIA ${ }^{11}$}

To the Most Enlightened, August, and Great Empress of All the Russias [is submitted] a short account of the Siberian Expedition undertaken by Her Imperial Majesty's humble servant, Fleet-Captain V. I. Bering. ${ }^{12}$

On February 5. I 725. I received from Her Imperial Majesty the Empress Catherine Alexeyevna, ${ }^{13}$ of illustrious and immortal memory, the instructions drawn up by the hand of His Imperial Majesty Peter the Great, of deserving and eternal fame, a copy of which follows:

1. Build in Kamchatka or in some other place in that region one or two decked boats.

\section{(Footnote ro, continued.)}

He also selected the officers for this work-Vitus Bering, Martin Spangenberg ithis was the usual way of writing it at this period], and Alexei Chirikov.

"I was then almost constantly with the Emperor and saw with my own eyes how eager His Majesty" was to get the expedition under way, being, as it were, conscious that his end was near. When all had been arranged he seemed pleased and content. Ca!ling the general-admiral [Count Apraksin] to him he said: 'Bad health has obliged me to remain at home. Recently I have been thinking over a matter which has been on my mind for many' years, but other affairs have prevented me from carrying it out. I have reference to the finding of a passage to China and India through the Arctic Sea. On the map before me there is indicated sucl a passage bearing the name of Anian. There must be some reason for that. In my last travels I discussed the subject witl learned men, and they were of the opinion that such a passage could be found. Now that the country is in no danger from enemies we should strive to win for her glory along the lines of the arts and sciences. In seeking such a passage who knows but perhaps we may be more successful than the Dutch and English, who have made many such attempts along the American coast. I have written out these instructions and, on account of my health, I entrust the execution of them, point by point, to you, Feodor Matveyevich.' ".

11 Zapiski Voenno-Topogr. Depo, Vol. 1o, pp. 69-75, St. Petersburg, I 847. The original report in manuscript is in the Archives of the General Staff, Section $\mathrm{X}$. No, 566. [A translation of the published version appeared (pp. 135-143) in W. H. Dall: A Critical Review of Bering's First Expedition, 1725-30, Nall. Geogr. Mag., Vol. 2, 1890, pp. 111-I69.-EDir. Note.]

12 'itus Ivanovich Bering. Bering's father's name was Jonas.

${ }^{13}$ Catherine I, second wife of Peter, who succeeded him to the throne on February 8,1725 , and reigned until her death on May 6,1727 


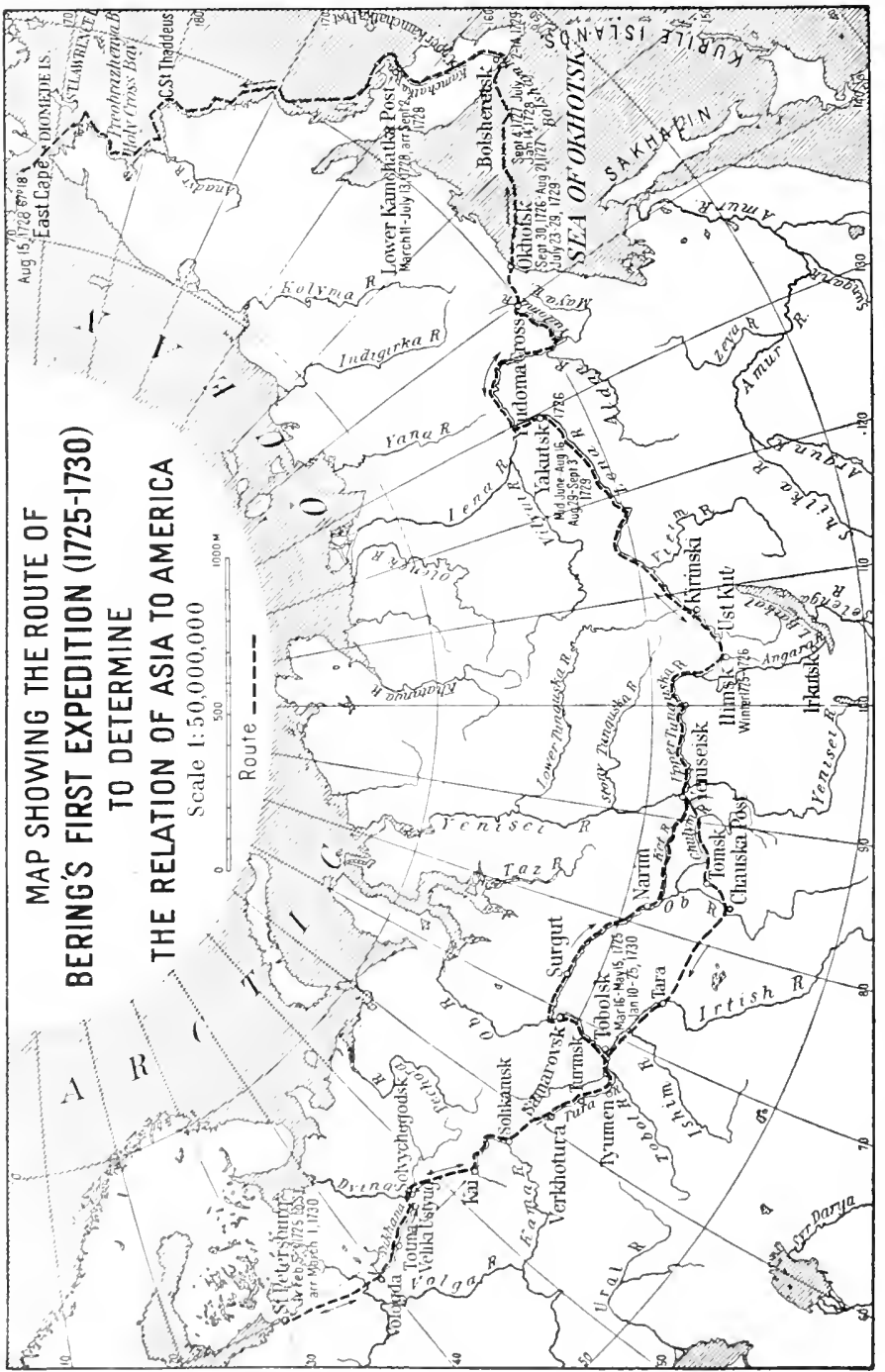

몰 竞苛

क⿺辶

辽速京

范告实

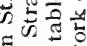

車了

足

ㄷำ

跣要

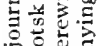

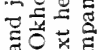

흘

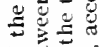

0 웅

$\dot{8} 8$

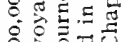

$\stackrel{\circ}{\circ}$

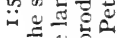

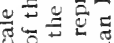

ऊ. 눙

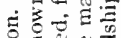

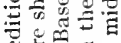

元气

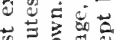

点过宫些

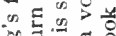

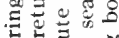

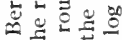

ㄷㄷㄴ동

$\stackrel{\text { व }}{2}$

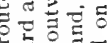

$\cong \cong$

-

E.

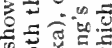

की

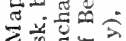

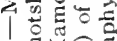

的全

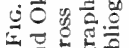

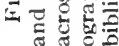


2. Sail on these boats along the shore which bears northerly and which (since its limits are unknown) seems to be a part of America.

3. Determine where it joins with America, go to some settlement under European jurisdiction; if you meet a European ship learn from it the name of the coast and put it down in writing, make a landing to obtain more detailed information, draw up a chart and come back here. ${ }^{14}$

In the orders given me by General-Admiral Count Apraksin ${ }^{15}$ it was stated that I was to ask for mechanies and supplies from the provincial government at Tobolsk and that I was to make monthly reports to the Admiralty College. ${ }^{16}$

On January 24 , before I received the instructions, there was dispatehed by the Admiralty College in the interest of the expedition one of my lieutenants with 26 men and 25 wagonloads of materials. Altogether there were under my command 33 men, and they were sent by way of Vologda. From St. Petersburg to Tobolsk we passed en route the following eities: Vologda, Totma, Veliki Ustyug, Solvychegodsk, Kai, Solikamsk, Verkhotura, Turinsk (also ealled Epanchin), and Tyumen. ${ }^{27}$

On March I 6 Tobolsk was reached and there we remained until May 15 because of the cold weather. During the stay I requisitioned the necessary material for the expedition. On May is we started down the Irtish to Samarovsk in four boats, known in Siberia as doshcheniki. ${ }^{18}$ They were loaded with supplies brought from St. Petersburg as well as other things gathered at Tobolsk. In the last-named city there were added to my company, at my request, a monk, a commissar, petty officers, and soldiers to the number of 34 .

From Samarovsk I sent ahead my marine guard, ${ }^{19}$ who earried with him orders from the provincial government of Tobolsk to the towns along the way to have boats prepared at Yeniseisk and Ust-Kut, ${ }^{20}$ but

14 Polnoe Sobranie Zakonov Rossiiskoi Imperii, Vol. 7, Doc. 4649.

is Feodor Matveyevich Apraksin (1671-1728), one of Peter's right-hand men. See also footnote 10.

16 The Admiralty College was created in 1718 and became a Ministry in 1802 .

17 For the route of the expedition, see map. Fig. 3. Most of the towns named, from Kai eastwards, were posts and stopping places for Siberian hunters and traders. Verkhotura (Upper Tura) was founded in 1598 , Tyumen in 1586, and Tobolsk in 1587. Turinsk was also called Epanchin because a Tatar chief of that name lived here before the Russians came.

$18 \mathrm{~A}$ doshchenik "is built of boards without a keel, flat-bottomed, about 35 to to feet long; rows and steers with long sweeps, two men to each; is furnished with a mast, and one square sail, and named from dosok, a board." (Martin Sauer: An Account of a Geographical and Astronomical Expedition to the Northern Parts of Russia, . . by Commodore Joseph Billings . . . . London, 1802, p. 20, footnote.) 19 Peter Chaplin.

${ }^{20}$ Ust-Kut (Mouth of the Kut), where the Kut falls into the Lena and marks the head of navigation. 
he himself was to proceed to Yakutsk. After leaving Samarovsk we went up the Ob to Surgut and to Narim, thence up the river Ket to Makovska Post. ${ }^{21}$ Along the course that we followed from Tobolsk to Makovska live the Ostyaks, ${ }^{22}$ who were formerly idolaters but through the labors of Metropolitan Philophei ${ }^{23}$ of Tobolsk were converted to Christianity in I 7 5. We proceeded overland from Makovska to Yeniseisk and there went aboard four boats and worked our way up the rivers Yenisei and [Upper] Tunguska to the mouth of the Ilim River. On the Tunguska there are three rapids (poroga) and several rocky shoals (shkver). In the rapids there are large submerged rocks across the whole width of the stream so that a boat can get through in only one or two places; the shoals likewise have rocks above and below the surface but they [the shoals] are not very large. The shoals differ from the rapids in that the former have little water and continue for a verst or two at a stretch, which causes some trouble in getting over them. At Yeniseisk 1 took for my service, in accordance with instructions from Tobolsk, 3o carpenters and blacksmiths. The rapids and shoals made it impossible to go up the llim to llimsk, and on that account there were sent out from that town smaller craft. On these the heavier materials were taken to Hlimsk, but the lighter were transported to the same place in the course of the winter.

Lieutenant Spanberg with 39 men, carpenters and laborers, was sent overland to Ust-Kut, where, during the winter, they constructed I 5 barges for taking the men and supplies down the Lena to Yakutsk. With the other members of the party I wintered at llimsk because there were not enough accomodations for all at Ust-Kut and we could not go through to Yakutsk owing to the snow and cold, the lack of teams, and the uninhabited country. According to orders from Tobolsk we were to receive our food supplies from lrkutsk and llimsk, as no grain grows at Yakutsk. During the winter I went from Ilimsk to Irkutsk to consult with the voivode ${ }^{24}$ of that city, who formerly held a similar position at Yakutsk, about that country, of which we knew very little, and to find out the best way of going from there to Okhotsk and Kamchatka. Towards the end of the winter I took over to Ust-Kut

${ }^{21}$ Built as a palisaded fort (ostrog) in 16 ro.

22 In the sixteenth and seventeenth centuries they made trouble for the Russians, but they are of no importance now. In 1911 the total number of Ostyaks in Siberia was 20,000 .

${ }^{3}$ Philophei (Leszczynski) $(1650-1727)$. In 1702 Peter put him in charge of the Siberian missions, and it is recorded that through his efforts 40,000 natives were converted to Christianity.

24 Mikhaelo Izmailov, voivode from October, 1724, to April, r 731 . In the Annals of Irkutsk (V. P. Sukachev: Pervoe stolyetie Irkutska, St. Pettersburg, 1902, pp. $1.3 .3-13.1)$ it is recorded that Spanberg and "Ivan Bering" were thete in the course of this winter. 



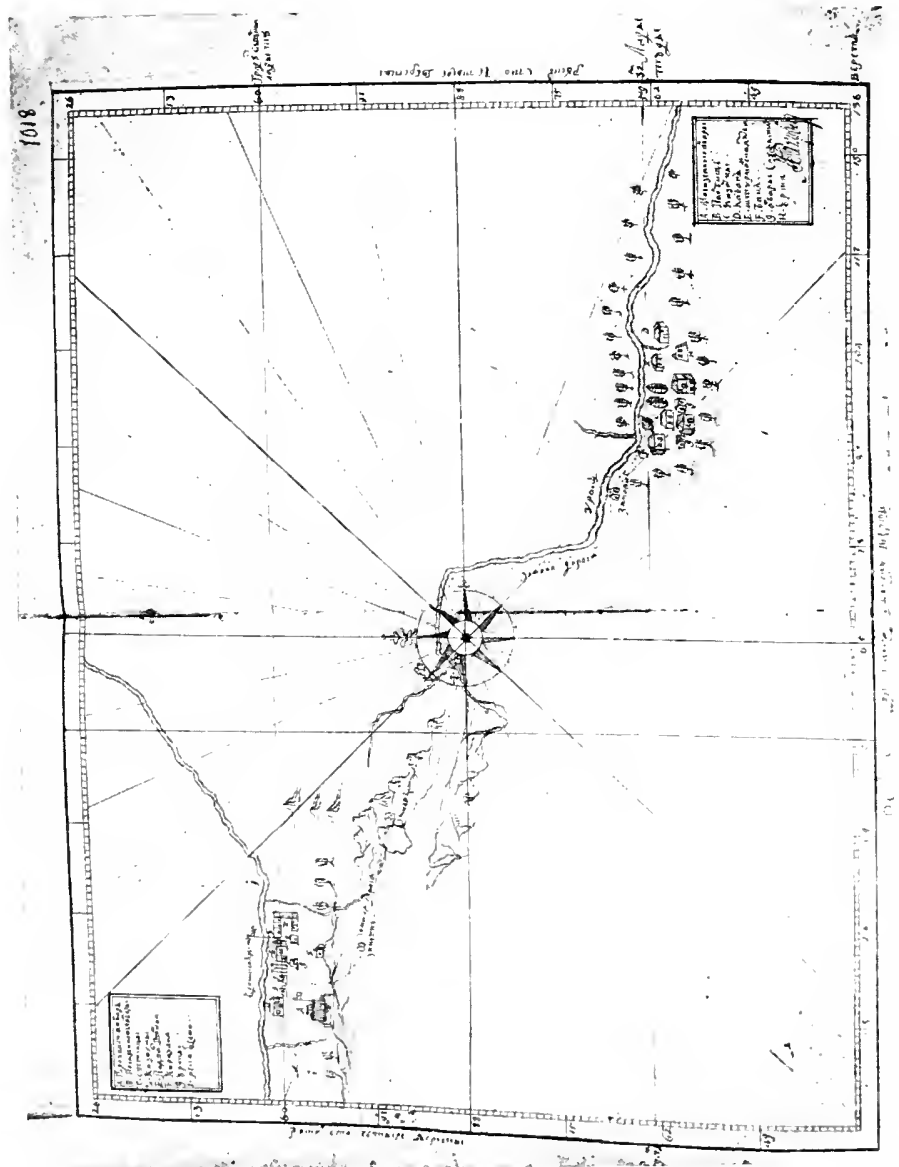

$\stackrel{\ddot{Z}}{ٍ}$

苛

2

$\ddot{E}$

ฮ

i

is

过

$\Xi$

U

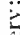

逆

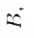

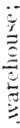

$=$

$\because \Xi$

है

$-3$

$=$

$\equiv$

告

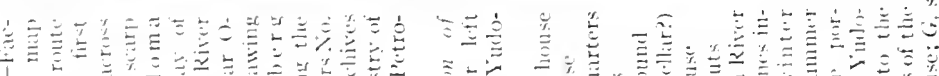
I

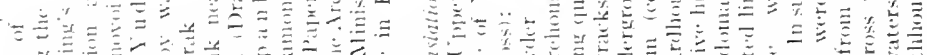

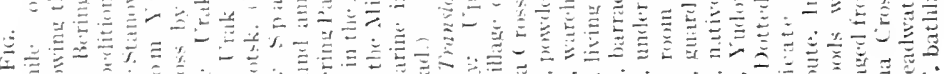

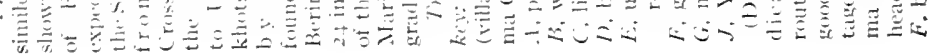


my whole company, which included 20 carpenters and blacksmiths from Irkutsk and two coopers from Ilimsk.

Along the Tunguska, Ilim, and Lena Rivers, as far as the Vitim, ${ }^{25}$ live the idolatrous Tungus who have deer for riding, but those who have no deer make their homes along the banks of streams an dsubsist on fish and get about in birch canoes.

In the spring of 1726 we left Ust-Kut in 15 barges and went down the Lena to Yakutsk. From the Vitim down along the Lena, on both banks, live the Yakuts and a small number of Tungus. The Yakuts have many horses and cattle which supply them with food and clothing, but those that have but few of these animals live on fish. They are idolaters; they worslip the sun and the moon and, of the birds, the swan, the eagle, and the raven. They hold in high respect their priests, whoin they call shamans, who keep in their possession small idols (bolvanov) known to them as shaitany. They claim to be of Tatar origin.

On arriving at Yakutsk I called for help to assist my men on the barges, and, as soon as they came, I dispatched Lieutenant Spanberg in I3 of the boats that were built at Ust-Kut and drew but little water down the Lena to the Aldan and from there up the Maya and the Yudoma. These barges were loaded with materials which could not be transported overland, besides other naval and land stores. I had hoped that this freight could be taken by water as far as Yudoma Cross, ${ }^{26}$ a much cheaper way than sending it by horses. That same summer I, with a small number of my men, went from Yakutsk to Okhotsk on horseback, taking with us a pack train, each horse carrying only a five$\operatorname{pood}^{27}$ load, and in this manner we brought over sixteen hundred poods of provisions. It was impossible to make use of wagons owing to the mud and the hills. At Okhotsk Post there are only ten Russian dwellings. Lieutenant Chirikov was left behind to winter at Yakutsk with the understanding that he was to come overland to Okhotsk in the spring.

Towards the end of December, I726, I received word from Lieutenant Spanberg that his boats were ice-bound in the Gorbea River, ${ }^{28}$ about $45^{\circ}$ versts from Yudoma Cross, and that he had made sleds and was hauling on them the most needed material. I made up a party from my own men and those of the ostrog and gave them dog teams and supplies, and sent them to the relief of the lieutenant. He arrived with his company about the first part of January, I 727, without, however, bringing

${ }^{25}$ The Vitim is a right branch of the Lena flowing into it in $113^{\circ} \mathrm{E}$. (sce Fig. 3 ) and is known for its fine sables. In 1911 the Tungus, including the Lamuts, numbered 75.204; the Yakuts 245,406.

${ }^{35}$ At the headwaters of the Yudoma, where navigation ceases, there was erected a cross, hence the name Yudoma Cross. For this part of the route, see map, Fig. 4.

${ }^{27} \mathrm{~A}$ pood is about 36 pounds.

${ }^{27}$ A left tributary of the Yudoma near its mouth. Probably the Derbi River of today. 


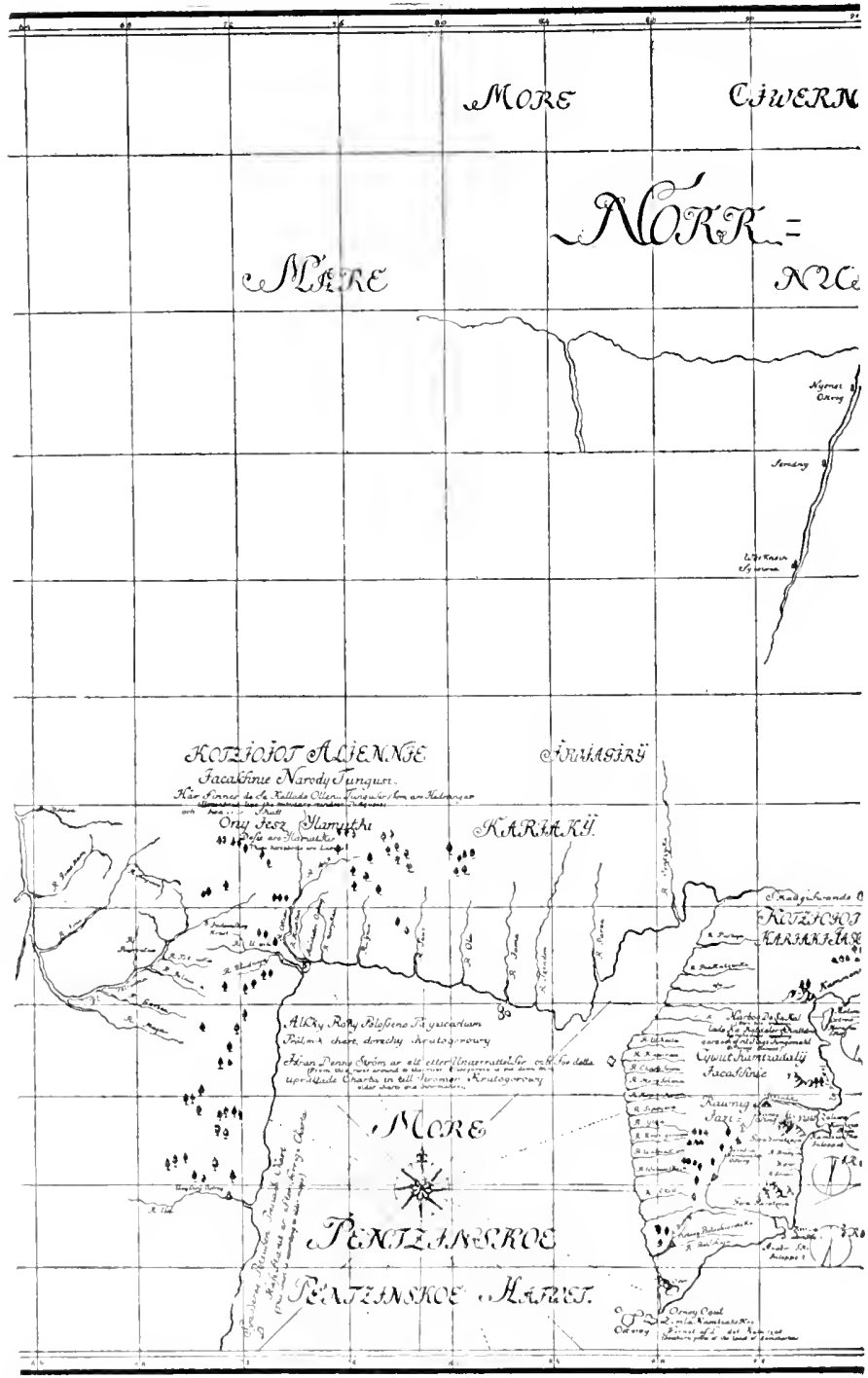

FIC: 5-The eastern section of the map accompanying Bering's report of his first expedition. Reproduced from W. II, Dall's facsimile (Appendix No. 19, U.S. Coast and Geodetic Survey Rept. for 1800, Pl. 69) of a copy of the map pre- 
BERING'S REPORT

I

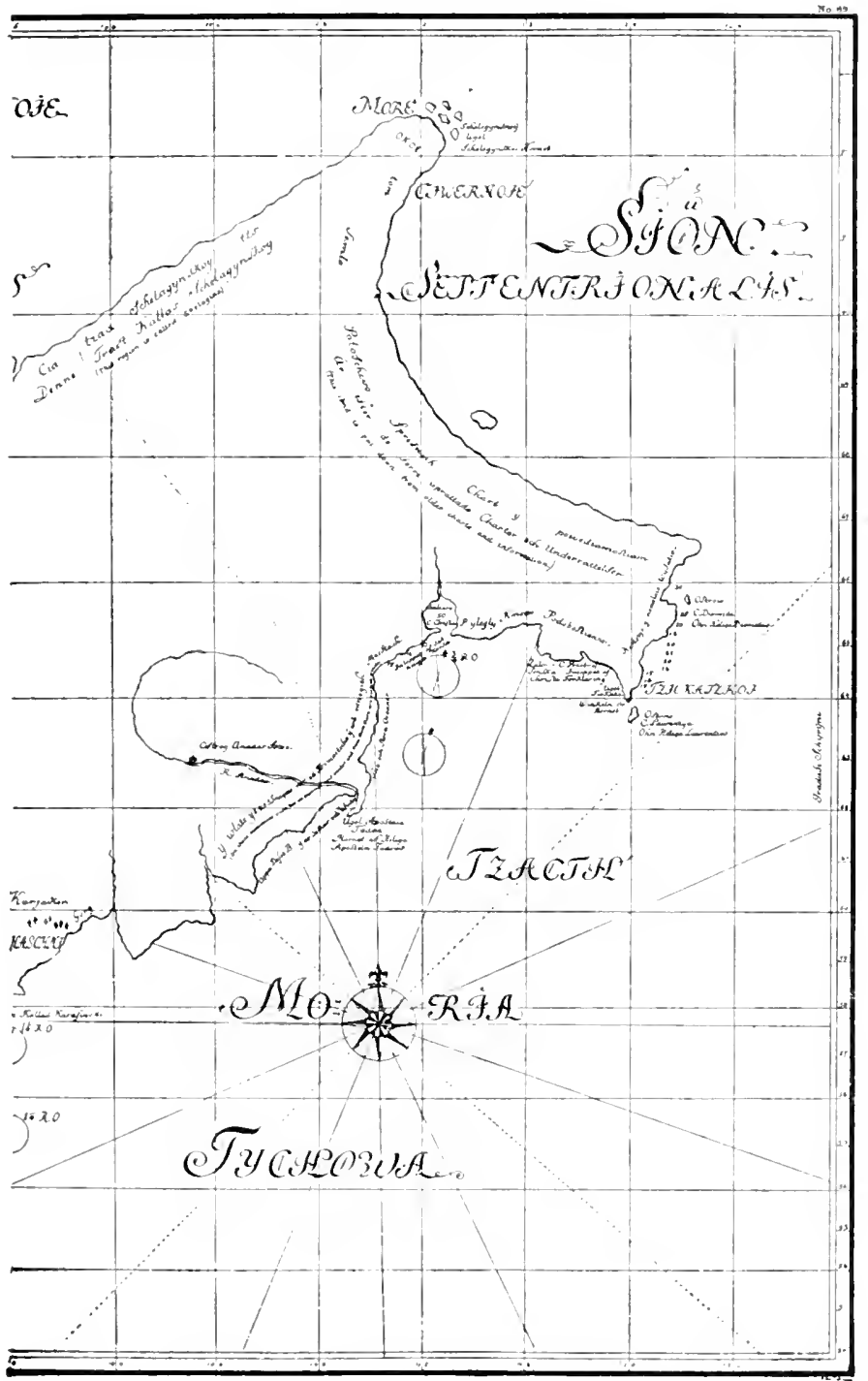

pared for use in Sweden, with transliteration of the Russian names, in 1880 in the collection of Baron Klinckofstrom. Compare with Bering's route and the coast outlines on Figs. 3 and 6. 
in any of the material, which he left in four different places along the uninhabited trail. They had been on the road ever since November 4 and during that time had suffered greatly from hunger, having been compelled to eat the dead horses that had dropped by the wayside, the harness, their leather elothing, and boots. Fortunately they found at Yudoma Cross the I 50 poods of flour which we had left behind when some of our liorses gave out.

Along the Aldan and Maya live the same kind of Yakuts as those on the Lena; but on the Yudoma, that part which is near Okhotsk Post, are the wandering coast Tungus, or Lamuts, as they call themselves. They have many deer, on the backs of which they ride summer and winter and which, as well as the wild deer, supply them with their food and clothing. Here, too, are to be seen the pedestrian Tungus who live near the sea and along the banks of the streams and depend on fish for their sustenance. They have the same religion as the Yakuts.

About the first part of February 1 gathered 90 men and several dog teams and gave them to Lieutenant Spanberg with orders to bring in whatever was left on the Yudoma. He himself returned during the first days of April, but some of his men did not come in until the middle of the month. Even then they failed to bring in everything, and it was necessary to send another party of 27 men to Yudoma Cross, which stayed away until May but brought in all the material on pack horses. In these regions people never go in winter from Yakutsk to Okhotsk or other faroff places on horseback; they go on foot, dragging behind them sleds-the kind we used between the Gorbea and Okhotsk-with such things as are needed, each sled carrying from Io to I 5 poods. A trip like this takes from eight to ten weeks. The snow here is sometimes seven feet deep, and in places even deeper, and people who travel in winter dig their way through the snow to the ground and there pass the night.

On June 3o, Lieutenant Spanberg sailed for the mouth of the Bolshaya River on his newly built ship ${ }^{29}$ which was loaded with all the materials. Ife had orders to discharge the cargo, send one of the petty officers with carpenters to Kanchatka to prepare ship timber, and return hinself to Okhotsk.

Lientenant Chirikov came from Yakutsk July 3, bringing with him in accordance with 1 y instruction 2,300 poods of flour. We loaded the flour on Spanberg's ship, which had returnc'd, and on an old boat, ${ }^{30}$ which had come in from the Bolshaya River, and sailed with my company for Bolsheretsk Post on August 2I. The supplies that were still in the boats on the Gorbea 1 sent back to Yakutsk in charge of the pilot and the men who had been detailed to watch over them, with orders to got a reesipt for them and then hasten hack with some part of the

${ }^{29}$ The Forlune.

30 The Lerliya. 
supply of iron and tar and join me in Kamchatka in 1728. At the mouth of the Bolshaya River we had to discharge the cargo into small boats and take it to Bolsheretsk. This post had only I 4 Russian houses. The heavier materials and part of the supplies were loaded into small boats and sent up the Bystra River, ${ }^{31}$ a stream with a swift current, to within 120 versts of the Upper Kamchatka Post. That same winter we left Bolsheretsk on dog teams for the Upper and Lower Kamchatka Posts. Each evening we made a camp in the snow and covered over the opening. This country has some bad blizzards, called purgas, ${ }^{32}$ and if a person is hit by one of them in an open place without having time to construct a slelter he is in danger of being snowed over and freezing to death. In the upper ostrog there are 17 houses; in the lower, 50 ; and in the other place, where the church is, there are 15 more. In all three places there lived, during otır stay, not more than I 50 persons [Russian]; their chief occupation is collecting tribute. The natives who drove the teams that brought us over from Bolsheretsk prepared 300 poods of whale blubber from a whale that had been washed ashore in the fall. In place of money they [the natives] were given Chinese tobacco. In southern Kamchatka live the Kurils, in the northern the Kamchadals. They have a common language, but there are a few minor differences. A small number of these people are idolaters. The others do not believe in anything and are strangers to all good customs ( $i$ chushdi vsiakykh dobrykh obychaev). Neither the natives of Kamchatka nor the Russians who live there sow any grain. Of domestic animals they have only the dog, which is used for driving, hauling, and for furnishing clothing. The food of the natives consists of fish, roots, berries, wild birds, and sea animals in season. In the neighborhood of the Yakutsk monastery, which is about a verst from the Kamchatka cluurch, there are raised at the present time oats, hemp, and radishes. Turnips are cultivated in all three ostrogs, and they reach such a size as is seldom seen in Russia, four of them making a pood. I took over to the monastery above mentioned some rye and barley and had it sown, but whether it matured or not I cannot tell, for sometimes early frosts kill the crop. In cultivating the soil the people are handicapped by lack of draft animals.

From all aborigines under Russian jurisdiction tribute in fur is collected. The natives have many evil practices. If a woman or a beast gives birth to twins one of them must be killed at once. Not to do so

${ }^{31}$ The Bystra (Swift), a branch of the Bolshaya, is full of rapids and shoals and is difficult to navigate. From Bolsheretsk to Lower Kamchatka Post the distance is 883 versts.

${ }_{32}$ "The poorga raged with redoubled fury; the clouds of sleet rolled like a dark smoke over the moor, and we wore all so benumbed with cold that our teeth chattered in our heads. The sleet, driven with such violence, had got into our clothes and penetrated even under our parkas, and into our baggage." (Peter Dobell: Travels in Kamchatka and Siberia, 2 vols., London, 1830; reference in Vol. I, p. 102.) 
is a great sin. It is a practice among the Kamchadals, if one of them is very sick, even a father or a mother, to take him out into the forest and leave him there, be it summer or winter, with just enough food to last a week, and as a result many die. The homes of the dead are not again occupied, and the dead themselves are not buried but are thrown into the woods, where the dogs devour them. ${ }^{33}$ The Koryaks burn their dead; this custom is now forbidden, but the law is not strictly enforced.

When I reached Lower Kamchatka Post the timber for our ship was in large part ready. We went to work on her April 4, I 728, and with the help of God had her completed by July $10 .{ }^{34}$ The timber we hauled on dog teams. Tar, since we had not brought any with us and the natives had none on hand as they did not know how to make it, we manufactured out of a tree known there as listvennik. ${ }^{35}$ For lack of anything better to take along on the sea voyage, we distilled liquor from grass by a process known in that country; $;^{36}$ salt we boiled out of sea water; butter we made from fish oil; and in place of meat we took salt fish. We had on board enough provisions to last 40 men a year. On July I 4 we sailed from the mouth of the Kamchatka River out to sea and followed the course laid down by the instructions of His Imperial Majesty Peter the Great and here marked on the chart.

On August 8, when we were in latitude $64^{\circ} 30^{\prime} \mathrm{N}$, eight men who claimed to be Chukchi (a people known for a long time to the Russians of the country) rowed to us from the shore in a leathern boat and, when near, asked who we were and why we came. On being invited on board, they put one man over, who, with the help of large inflated seal bladders, swam over to have a talk with us. A little later the boat moved up to us and the men in it told us that large numbers of Chukchi live along the shore, that a short distance from here the coast turns to the west, and that not far ahead of us is an island. We located this island, which we named St. Lawrence, in honor of the day, and found on it a few huts but no people, although I twice sent the midshipman to look for them.

By August I 5 we came to latitude $67^{\circ}$ I $8^{\prime} \mathrm{N}$. and turned back because

${ }^{33} \mathrm{~A}$ fuller and better account of the natives is to be found in S. P. Krasheninnikov's "History of Kamchatka," St. Petersburg, 1755, and G. WV. Steller's "Beschreibung von dem Lande Kamtschatka," Frankfort and Leipzig, 1774.

34 She was christened St. Gabriel. ${ }^{3}$ Larch.

${ }^{30}$ According to Steller the liquor is made in the following manner:

"Der Brandtewein wird aber folgendermassen angesetzt. Man gieset auf 2 Pud süsses Kraut 4 Eimer warmes und laues Wasser, leget zum Ferment entweder was von der Destillation übrig geblieben hinein, wovon er aber einen üblen Geruch oder Geschmack bekommt, oder Beeren von Schimalost ["Shimalost" is the Kamchatka honey'suekle bush (Lonicera caerulea) which bears delicious blue berries.-L. Stejneger.] wovon er sehr stark, angenelımer wird, und mehr Brandtewein giebet, oder man fermentiret ihn mit eingesauertem Mehl; nach Verlauf 2.4 Stunden wird er destilliret, und bekommt man einen Eimer Brandewein." ("Beschreibung von dem Lande Kamtschatka," p. 86.) 
the coast did not extend farther north and no land was near the Chukchi or East Cape and therefore it seemed to me that the instructions of His Imperial Majesty of illustrious and immortal memory had been carried out. Had we gone on and met with unfavorable winds we might have been prevented from returning to Kamchatka that season, and to have wintered where we were would not have been wise because there was no wood of any kind and the native population does as it pleases, is not under Russian control, and has nothing to do with the Russian tribute collectors. ${ }^{37}$

From the mouth of the Kamchatka River to the point from which we turned back the coast is mountainous, almost as straight as a wall, and covered with snow even in summer.

On August 20 there came to our ship four boats containing 40 Chukchi like those who had visited us before. They offered for sale meat, fish,

37 On August 13, having reached $65^{\circ} 30^{\prime}$, Bering called his officers together to discuss the question whether to go on or turn back. He said to them:

"Since we have come to latitude $65^{\circ} 30^{\prime}$ N. and according to my opinion and the statements of the Chukchi, we have reached and passed the most easterly point of their land, the question is now, shall we go farther north? If so, how far? When should we begin to look for harbors? Where does it seem bestlooking at it from the point of view of best serving our country-to go for the winter in order to protect men and ship?" The officers were divided in opinion. Spanberg, the senior officer, said: "Having come as far north as we have, and since on the Chukchi coast there are no harbors nor wood . . . so that we could preserve ourselves in such winter weather as we have in this region; and since these natives are not peaceful . . . I suggest that after we have gone on the course we are on until the sixteenth of this month, and if by that time we are not able to reach sixty-six degrees, we should then in God's name turn about and betimes seek shelter and harbor on the Kamchatka River, whence we came, in order to save men and ship." Chirikov made this argument: "As we have no positive information as to the degree of north latitude Europeans have ever reached in the Arctic Ocean on the Asiatic side we can not know with certainty whether America is really separated from Asia unless we touch at the mouth of the Kolyma, or at least the ice, because it is well known that there is alway's drift ice in the Arctic Ocean. Therefore it seems to me that according to your instructions we ought to sail without questioning-unless we are hindered by the ice, or the coast turns to the west-to the mouth of the Kolyma, as your instructions demand la place under European jurisdiction?] But should the land continue still farther to the north, it would be necessary on the twent $y$-fifth of this month to look for winter quarters in this neighborhood, and above all opposite Chukotski Cape, where, according to the accounts of the Chukchi through Peter Tatarinov, there is a forest. And if up to that time winds are contrary, then look there by all means for a place to winter." (Zapiski Hydrogr. Depart., Vol. 8, pp. 550-552, St. Petersburg, i850.)

[For details on the sea voyage, the most important part of the expedition, which Bering in this report deals with only' bricfly, the main source of information is the midshipman Peter Chaplin's log book, an abridged version of which, with map (cf. Fig. 6), was published by Berkh in 1823 and translated by Dall in 1891 . Polonskii's discussion of the first expedition, from which the above quotation is taken, is likewise of value. A narrative based on Berkh and Polonskii will be found in F. A. Golder's "Russian Expansion on the Pacific," pp. 140-147. For the references, see the bibliography.-Edir. Note.] 
water, about 5 red and white fox skins, four walrus tusks-all of which they disposed of to the crew for needles and such like articles. They told us that their relatives go to the Kolyma on deer and not by boat. that farther along the coast live some of their people, that they had known the Russians for a long time, and that one of their number had been at Anadyrsk Post to trade. The rest of their conversation did not differ greatly from what was said by those who had been to see us before. On September 2 we sailed into the mouth of the Kamchatka River and passed the winter in the Lower Kamchatka Post.

Having repaired the ship, which had been laid up, we left the mouth of the Kamchatka River on June 5, I729, and set an easterly course. because the inhabitants of Kamchatka said that in clear weather land could be seen across the sea. We made a careful search for it over a distance of 200 versts but could not find it. We circumnavigated and charted the southern part of Kamchatka, which up to this time had not been surveyed, and then sailed to the mouth of the Bolshaya River and from there to Okhostk Post. At the request of the authorities at Yakutsk I left with the government officials in charge of the posts of Lower Kamchatka and Bolsheretsk 800 poods of flour, dried meat, salt, and groats.

It was July 23 when we reached the mouth of the Okhota River, where I handed over the ship and all that went with it to the officer in command. We hired horses and went to Yudoma Cross, and from there we proceeded by boats and rafts down the Aldan to the portage (belskoi perepravy) and lower, where we again took horses and rode on to Yakutsk. From Okhotsk to Yakutsk it took us from July 29 to August 29 and to September $3 .{ }^{38}$ On September Io we went in two boats up the Lena and proceeded until October 1 , when we were blocked by ice and therefore spent a part of the autumn in the village of Peleduye. ${ }^{39}$ By October 29 there was sufficient snow on the ground, and the banks of the Lena were firm enough on account of the ice to permit us to go on. We followed the [Upper] Tunguska and the Yenisei to Ilimsk and Yeniseisk, passing Russian settlements on the way. From Yeniseisk to Tomsk we continued along the river Chulym, ${ }^{40}$ meeting with more Russian settlements and villages of newly converted Tatars. Between Tomsk and Chauska Post ${ }^{41}$ we passed through other Russian settlements. From Chauska to Tara we crossed the Barabinsk Steppe, from Tara to Tobolsk we followed the river Irtish and met with Tatar villages. At Tobolsk we arrived January IO, 1730 , and left there on the 25 th of the same month for St. Petersburg, going over the same country as on the outward journey to Tobolsk. On March 1 we reached St. Petersburg.

${ }^{38}$ Not clear. Possibly August 20 represents the break in the journey at the portage.

39 Peleluye is at the mouth of the river of the same name, a branch of the Lena.

4) A tributary of the $\mathrm{Ob}$ (see Fig. 3), lined with villages of Chulym Tatars.

4 On the Challs River, founded in 1713 . It is 223 versts from Tomsk. 
!

1 


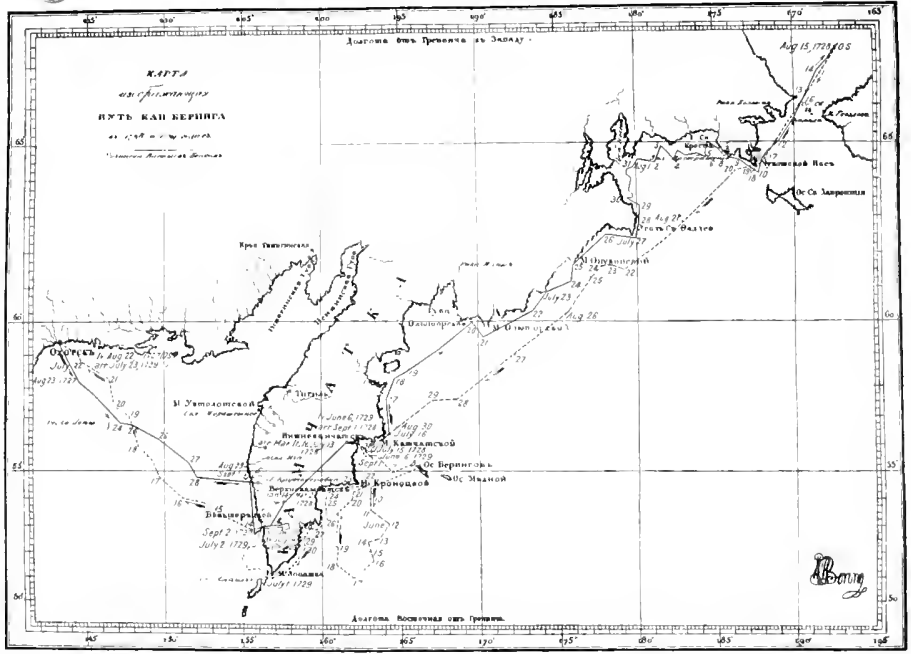

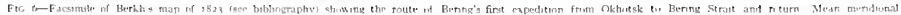

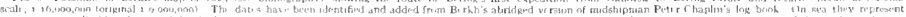

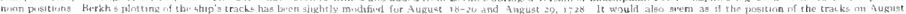

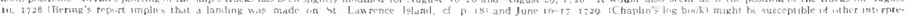

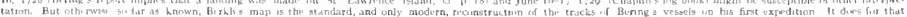

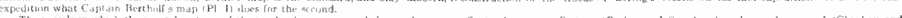

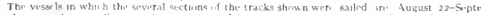

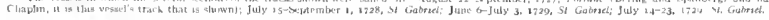




\section{CHAPTER III}

\section{GVOZDEY'S Y'OYAGE TO AMERICA IN 1732}

\section{Shestakov's Earlier Expedition}

After the Russians had conquered Kamchatka they pushed northward and established trading relations with the natives of East Cape. While they were in that neighborhood the traders heard that across from the cape there was a large country (bolshaya zemlya), and occasionally they saw the inhabitants of that country who were held captive by the Chukchi. The adventurous Cossacks decided to subdue these people, as well as the Chukchi, and force them to pay tribute. One of their leaders, Afanasi Shestakov, went to St. Petersburg to persuade the government to let him undertake this task. On March 23, 1727, the Senate gave him the necessary authority ${ }^{1}$ and force for the work in hand. It was Shestakov's plan to attack by land and sea the Chukchi and other hostile natives on the mainland and when they were conquered to send a force to the land beyond East Cape. With that in view the Cossack leader took possession in 1729 of all the seagoing vessels Bering had left behind hin at Okhotsk.

The campaign of 1730 ended disastrously for the Russians. Some of the boats were wrecked, the land forces were either waylaid ordefeated in open fight, and in one of these Shestakovlost his life. Out of all this great undertaking only one important thing was accomplished-the sighting of new land opposite East Cape.

\section{GVozdev's Expedition}

Shestakov was succeeded in command by Dmitri Pavlutski, captain of dragoons and a well-known Chukchi fighter. In addition to the other information about the "large country," Pavlutski had received in 1730 the report of one Melnikov on

1 Polnoe Sobranie Zakonov Rossiiskoi Imperii, Vol. 7, Doc. 5049. 
that subject which tended more and more to confirm him in the belief that such a bolshaya zemlya really existed. During the winter of 1731-1732 Pavlutski sent word to the officers of the St. Gabriel who were with the ship at Lower Kamchatka Post to bring him supplies to the Anadyr in the spring and after that go in search of the land opposite East Cape. ${ }^{2}$ When the time came for sailing only one of the officers, Michael Spiridonovich Grozdev was physically fit for duty, and he took command of the ship and set the course for the bolshaya zemlya. His achievement did not impress Pavlutski very much, and it was not until several years later that he was made to write out his report, which, with unimportant sections omitted, runs as follows: ${ }^{3}$

In May, 1732, we received orders from Major Pavlutski, who was at the time at the Anadyr fort, to go aboard the St. Gabriel with the pilot and underpilot and sail around Kamchatka Cape to the mouth of the Anadyr and opposite Anadyrski Cape to what is known as the "large country," examine and count the islands there, and gather tribute from the inhabitants. On July 23 we left Kamchatka River, and four days later Kamchatka Cape was doubled. We came to Anadyrski Cape August 3 and from there went to the islands to collect tribute. Moshkov told us of an island Bering had discovered, and we sailed about in order to find it. By this maneuvering we reached the southern part of Chukotski Cape, where, on August 5, we anchored three versts from shore. It was calm, and I went on land to examine the coast and fetch drinking water. Close to the shore we observed a small fresh stream into which we pulled. The country seemed uninhabited; but not far from where we stood was a herd of deer, numbering about 150 or more, guarded by two men, who ran away on seeing us. I killed two of the deer, filled two barrels with water, and went on board.

The next day two Chukchi came toward the ship in two baidaras but would not approach near enougl so that we could enter into conversation with them. When they had looked at us for a time they pulled away. On the norrow I, with nine men, went to the spot from which I had seen the natives issue the day before, but all that we found there were two huts of earth and whalebone. As we started back we caught

2 In the "Lettre d'un officier de la marine russienne," I 753 (see bibliography), the statement is made that Pavlutski ordered Grozdev to bring the provisions left by Bering to the country of the Chukchi, whom Pavlutski was fighting. Grozdev could not find Pavlutski and ther fore started back and accidentally. ran into the American coast.

3 Archives of the Ministry of Marine: Papers of Count Chernisliev, ${ }_{762-1768,}$ No. 367. See also Zapiski Hydrogr. Deparl. Vol. 9, pp. 88-103. St. Petcrsburg, 1851. 
a glimpse of two men who ran away on seeing us. We got under sail on the 8 th, steering for an island on the course suggested by Moshkov. On the following day Fedorov sent me a note saying that in his opinion we had not yet reached the place in question [large country] since we were still south of Chukotski Cape, and asked for my opinion. On the Ioth we sailed back to the spot where we had been a few days before and took on fresh water. Two days tater we ran into a calm and anchored. On going ashore we saw two huts and people, who, on noticing us, pulled away from the land in three baidaras. We managed to get into conversation with them and asked them for tribute, which they refused to give. Having a fair wind on the I5th we went on our way and on the 17 th sighted an island, but on account of the head wind we could not approach it but had to keep close to Chukotski Cape.

Here we saw many Chukchi, with whom we tried to enter into conversation but without much success. When the wind shifted once more to fair we steered again for the northern end of the island [one of the Diomedes]. Our attempt to land was resisted by a shower of arrows, to which we replied with muskets. After a great deal of difficulty the natives told us that they were Chukchi and that some of their people had fonght with the other Chukchi against Pavlutski. In cruising about the island, which is about two and a half versts long and a verst wide, we eame across other natives, but all refused to pay tribute. We made a landing and examined their homes, and from the island we saw the "large country". It was near one o'clock of the morning of August 20 when we left the first island, and six hours later we anchored off the second, which is smaller than the first, and about half a mile distant. A ship's boat and a baidara were sent to the shore, but meeting with an unfriendly reception they returned.

About three o'elock of the afternoon of August 2 I we sailed for the "large country" and anchored about four versts from its shore. It was now Fedorov's watch, and he, without consulting any one, gave orders to weigh anchor and approach the southern point of the shore. From there we could see huts, but in spite of our best efforts we did not come as close to them as we wished on account of the head wind and the shallow water. The breeze veering to north-northwest, we were obliged to stand out to sea on a southwest course and by doing so came to the fourth island on the 22nd. A strong wind was blowing, and when we tried to approach the shore the sails gave way. The sailors then came to me and asked that we return to Kamchatka because of the lateness of the season and the stormy weather. I referred them to the underpilot without whose consent I could not order such a move. In the meantime there came to us from the island a Chukchi in a leather boat which had room for but one man. He was dressed in a shirt of whale intestines which was fastened about the opening of the boat in such a manner that no water 
could enter even if a big wave should strike it. He told us that Chukchi lived in the "large country," where there were forests, streams, and animals. We had no opportunity of going ashore, and from the distance we could not tell whether all that he told us of the "large country" was true or not. When he was gone the sailors spoke to me again about returning to Kamchatka, and I answered them as before. Then they held a council and drew up a petition addressed to me and the underpilot, enumerating many reasons why we should go back. Taking these arguments into consideration we decided to return and entered the mouth of the Kamchatka River September 28.

Outside of the islands enumerated we saw no others, and the reason for not indicating their exact position is that the log book Fedorov and I kept was sent to Okhotsk in I 733. Another reason is that Fedorov when on watch often failed to make any observations in the journal. On returning to Kamchatka $I$ asked his aid in drawing up a map, but he refused to join me; and it was impossible for me to undertake it alone, for the reasons just enumerated.

From his own account it is quite evident that Gvodzer was not aware that he had sighted the American coast. He thought he had discovered an island. He says that after anchoring near the first and second islands he steered for the "large country" [third island] and from there to the "fourth island." This is all that is known of Grozder's voyage. 


\section{CHAPTER IV}

BERING'S SECOND EXPEDITION, FROM ITS INCEPTION TO THE BEGINNING OF THE SEA VOYAGE

When Bering returned to Russia he made his report to the Empress and to the officers of the Admiralty and the Senate and tried to persuade them that "the instructions of His Imperial Majesty . . . had been carried out." Some clapped their hands while others shrugged their shoulders. The doubters maintained that as long as the coast and waters between the Kolyma River and East Cape were unexamined the problem of the relation of Asia to America was unsolved. They were not without arguments. They called attention to the numerous Siberian rumors that a large body of land (bolshaya scmlya) existed north of the Kolyma River and another east of East Cape. Were these two or more distinct continents or islands or were they one? Were they part of Asia or part of America? These and such like questions were asked. They were fair questions. Bering was expected to answer them but could not answer them satisfactorily.

\section{Bering's Proposal of a Second Expedition}

He was not altogether discredited; for it was realized that he had done a good piece of work, even if he had not done it as well as he should. That he had ability no one doubted, and it was believed that with his experience and the lesson taught him he would do much better if he were given another chance. Bering. no doubt, desired another chance, and he submitted to the Empress the following propositions which would tend toencourage her to send another expedition.

1 According to Lauridsen's "Vitus Bering," Chicago, I889 (note 40, p. 208), first published in V. Berkh: Zhizneopisaniya Pervykh Rossiiskikh Admiralov, 4 vols., St. Petersburg, 1831-36; later reprinted by Sokolov in Zapiski Ilydrogr. Depart., Vol. 9, pp. 435-436, St. Petersburg, 1851.-Edit. Note. 
I. According to my observation the waves of eastern Kamchatka are smaller than in other seas, and I found on Karaginski Island large fir trees that do not grow on Kamchatka. These signs indicate that America, or some other land on this side of it, is not far from Kamchatka-perhaps from I50 to 200 miles. This could easily be ascertained by building a vessel of about $5^{\circ}$ tons and sending it to investigagate. If this be so [the existence of such a country], a trade might be established between the empire and the inhabitants of those regions.

2. Such a ship should be built in Kamchatka, because the necessary timber could be obtained more easily. The same holds true in the matter of food - fish and game are especially cheap there. Then, again, more help may be had from the natives of Kamchatka than from those of Okhotsk. One other reason should not be overlooked; the mouth of the Kamchatka River is deeper and offers a better shelter for boats.

3. It would not be without advantage to find a sea route from the Kamchatka or Okhota River to the Amur River or Japan, since it is known that these regions are inhabited. It would be very profitable to open trade relations with these people, particularly the Japanese. And as we have no ships there [in the Okhotsk Sea], we might arrange it with the Japanese that they meet us halfway in their boats. For such an expedition a ship about the size of the one mentioned would be needed, or one somewhat smaller might serve the purpose.

4. The cost of such an expedition-not including salaries, provisions, and materials for both boats, which can not be had there and would have to be taken from here and Siberia-would be from 10,000 to 12,000 ribles.

5. If it should be considered wise to map the northern regions of the coast of Siberia - from the $\mathrm{Ob}$ to the Yenisei and from there to the Lenathis could be done by boats or by land, since these regions are under Russian jurisdiction.

The question of a second voyage must have been raised even in 1730 , for early in 1731 arrangements for the undertaking were already made. The desire to determine the relation of Asia and America was not the only argument advanced in favor of the expedition. Some of the reports on that subject submitted to the Empress pointed out the benefits to be derived from territorial and commercial expansion, additional naval bases, and the discovery of precious metals. No doubt all these reasons had their weight with those in authority. ${ }^{2}$

2 Archives of State, XXIV, No. 8, I732; No. 9, 1732-1743. 


\section{LAND Versus SEA Expedition}

Another question that came up was how this expedition should be sent-by land or by water. There seems to have been a difference of opinion on that subject. Count Nikolai Golovin ${ }^{3}$ and Admiral Sanders submitted separate memoirs to Her Majesty recommending that two ships with naval and other supplies be chartered to go from Russia to Kamchatka by way of Cape Horn and Japan. After discharging their cargoes and resting the crews the ships could proceed on their voyage of discovery in the North Pacific. Count Golovin even offered to lead the expedition in person if the proper support were assured him.

A number of reasons were given why the sea route was preferable to the overland route. In the first place there would be a saving of time. It would take from ten months to a year for the sea voyage from St. Petersburg to Kamchatka and a year or a little more for the work of exploration and the return to Russia; on the other hand it would require two years to transport naval and other stores overland to Kamchatka, two more years to build sea-going vessels, and two more years to make the voyage and bring back the results to St. Petersburg.

Why the propositions of these two prominent men were not accepted is not clear. Perhaps because by 1731 and 1732 the expedition had ceased to be regarded in the light of purely maritime and geographic discovery and had come to be looked upon as one of scientific investigation in the larger sense, both by land and sea, in the Arctic Ocean as well as the Pacific. It had been decided to send along scientists of the newly founded Imperial Academy of Sciences to make a study of the people and the resources and to secure other scientific data about Siberia and the lands to be discovered. Then, too, in order to settle beyond dispute the much-debated question of the relation of Asia to America, it had been determined to survey and chart the Arctic coast from the White Sea to the mouth of the Kamclatka River. It was assumed as a matter of course that the leader of the expe-

3 Archives of State, XXIV, No. 8, 1732. 
dition could supervise its various activities, and therefore it would be necessary for him to be within land reach of the different parties. These may or may not have been the reasons, but the fact remains that Bering and his parties were ordered to proceed overland.

\section{Naval Preparations}

Because of the great distance to the Pacific, the difficulty of transporting material, and the lack of laborers, skilled and unskilled, for the building of sea-going vessels, the naval part of the expedition. needed most attention. On July 30, $173 \mathbf{1},{ }^{4}$ Grigori Pisarev (who had been appointed commander of the port of Okhotsk on May I O, I73I) ${ }^{5}$ was ordered to proceed to his post and make ready for Bering's coming. Pisarev was told to take, from different regions of Siberia, Russians and Tungus and settle them in the neighborhood of Okhotsk and Yudoma Crossthe Russians to be put to cultivating the soil and the Tungus to watch the flocks of sheep and the herds of horses and cattle which Pisarev would introduce. For the peopling of Okhotsk Pisarev had instructions to pick up at Yakutsk 300 young and strong men from those who were in prison for debt or for other crimes. In addition he was to take from Russia 20 ship carpenters to build four or six ships, under the supervision of naval architects who were to be sent by the Admiralty College, and from Ekaterinburg a number of iron workers to smelt iron and forge anchors and such things.

\section{Official Order for the Expedition}

The official order for the expedition, in which Bering is mentioned as commander, was announced by the Senate on April $\mathbf{1 7}_{7}$, $1732,{ }^{6}$ and this was followed up on May 2 of the same year by a general outline of the undertaking. ${ }^{7}$ On December 28, I732, the

1 Polnoe Sobranie Zakonov Rossiiskoi Imperii, Vol. 8, Doc. 5813, St. Petersburg.

G Ibid., Vol. 8, Doc. 5753.

${ }^{6}$ Ibid. Vol. 8. Doc. 602.3.

Ibid., Vol. 8, Docs. 604t, 6042. 
Senate issued the principal instructions, ${ }^{8}$ and a summary of the main articles that relate directly to the voyage to America is here given.

I. The Senate approves of the expedition in the hope that it will really be for the benefit and glory of Russia. It has given orders to the governor of Siberia, to the vice governor at Irkutsk, and to Pisarev to assist Bering. It is sending members from the Academy of Sciences, ${ }^{9}$ students from Moscow, ${ }^{10}$ assayers from Ekaterinburg, mechanics, and others. It confirms the project to examine not only the waters between Kamchatka and Japan, Kamchatka and America, but also the waters along the Arctic coast.

2. In the instructions which Peter gave to Bering it was evident that Peter desired to determine whether Asia and America were united. Bering says that he went as far as $67^{\circ} \mathrm{N}$. and found no connection between these two continents. It may be that north and west of the mouth of the Kolyma the two continents do not join, but no one knows whether this is so or not. In order to settle the relation between Asia and America the Admiralty proposes to send exploring expeditions along the Arctic shores from Archangel to the mouth of the Kamchatka River.

4. It has been reported that opposite the mouth of the Kolyma River there is a large land (bolshaya zemlya), that Siberians have been on it, and have seen the inhabitants. It is ordered that Bering should investigate this matter very thoroughly when he is at Yakutsk. If this is a true report, he should send a sloop to investigate. If it is found that Siberia really joins America so that it is impossible to proceed to Kamchatka. then the investigating party should follow the newly found coast as far as it can to learn in which direction it runs and return to Yakutsk.

If people are found there, they are to be treated kindly; they are to be given presents; they are to be asked the extent of their country and its resources, and they are to be invited to become our subjects and to pay tribute. If they are unwilling to do so, they are to be let alone; and no time slrould be wasted in arguing with them.

It is not at all likely, but it is possible, that by following the Arctic coast our explorers may come to some European settlement. In that case they should act according to the instructions given to Bering and Chirikov. If the explorers ascertain that Siberia and America are not connected, they should proceed to Kamchatka.

${ }^{8}$ Polnoe Sobranie Zakonov Rossiiskoi Imperii, Vol. 8, Doc. 629I.

${ }^{9}$ G. F. Müller, J. G. Gmelin, J. E. Fischer, Louis Delisle de la Croyère, and a little later G. W. Steller.

${ }^{10} \mathrm{~S}$. P. Krasheninnikov, author of the "History of Kamchatka," was among the number. 
5. In regard to going to America, it was ordered in I73 I that ships for this voyage should be built at Okhotsk. If they are ready, Bering should take two of them and proceed; if they are not finished, he should finish them. If they have not yet been started on, or if they are not seaworthy, the Admiralty College is of the opinion that the ships should not be built at Okhotsk. Bering recommends that Kamchatka, because it has more timber and a better harbor, should be selected as the place for shipbuilding. Bering desires to have two ships for the voyage so that in case of a misfortune to one of them the other would stand by. If one ship is completed at Okhotsk, it would be a good plan to take that and go to the Kamchatka River and there build the other one. Bering is to be in command of one of these vessels and Chirikov of the other. On the voyage they are to keep together, work together, and do all that is in their power to advance naval science. To help them a member of the Academy of Sciences [Louis Delisle de la Croyère] is sent along.

6. A late report of Captain Pavlutski, which was sent from Kamchatka, stated that recently Afanasi Melnikov with a small party returned from Chukchi Cape. This Melnikov was sent from Yakutsk in 1725 to bring the natives [Chukchi] under subjection and make them pay tribute. Melnikov says that in April, I730, while he was on Chukchi Cape, there came over from an island in the sea two men who had walrus teeth fastened to their own [pieces of walrus ivory in their lips]. These men told Melnikov that it takes a day to go from Chukchi Cape to their island, and another day from there to another island ahead of them. which island is called bolshaya zemlya. On this bolshaya zemlya all kinds of animals are to be had-sables, beavers, land otters, and wild deer. All kinds of green trees grow there. There are many natives on bolshaya zemlya; some of them have deer, and others have not. Although such reports cannot be trusted, yet they should be followed up and a voyage should be made in the direction of the islands. If they are located and people found on them, they should be treated as the instructions in Article 4 indicate. Go on [from there] to America and learn whether there is any continent, or islands, between Kamchatka and America; for, aside from the information furnished by Pavlutski, little is known on that subject. On the map of Professor Delisle a sea is located between Kamchatka and the Spanish province of Mexico in latitude $45^{\circ} \mathrm{N}$. If the American coast is discovered, Bering should carry out the instructions given him by Peter in 1725 , that is to say, to go to some European settlement. If a European ship is met with, he should learn from it the name of the coast, write it down, make a landing, obtain some definite information, draw a map, and return to Kamchatka. Be always on your guard not to fall into a trap and not to show the people you meet with the way to [Kamchatka]. 
9. Bering is to take with him 2,00o rubles' worth of presents to be distributed among the natives. Chinese tobacco, known as "shar log," is especially worth while because the natives are eager for it.

II. In these voyages search should be made for good harbors and for forests where timber for shipbuilding is to be had. Let mineralogists with a guard go ashore and prospect. If precious minerals are found in some place under Russian jurisdiction, the commander of Okhotsk and the principal officers elsewhere should be notified, and they shall send ships, miners, workmen, instruments, machinery, and provisions and begin working the mines.

12. Geodesists should be sent to examine all the rivers that fall into Lake Baikal from the east in order to find a nearer way to Kamchatka than by Yakutsk.

13. Captain Bering and all the officers in command of ships at sea should keep secret the instructions from the Admiralty College. For Bering, Chirikov, Spanberg, and the officers in command of the sloop which is to go east of the Lena to Kamchatka, special instructions are issued, and these may be made public. These public instructions are to state that at the request of the St. Petersburg, Paris, and other Academies the Emperor Peter the Great, of deserving and eternal fame, sent, out of curiosity, an expedition along his own shores to determine whether Asia and America are united. But the expedition did not settle that point. Now Your Imperial Majesty, influenced by the same reasons, is ordering a similar expedition and for a similar purpose. If you should come to settlements under European or Asiatic jurisdiction or if you should meet with ships of European or Asiatic governments, and are asked the object of your voyage, you may tell them what has just been said. If they demand to see your instructions, show them. This will allay their suspicions, because it is well known that European Powers have sent out expeditions and that the question whether Asia and America are united is still unanswered.

I4. In order that the expedition may not be retarded on account of delay in getting provisions and supplies of one kind and another, the Admiralty College should send at once special officers to Yakutsk to build boats and expedite the transportation of materials.

15. Because this expedition is harder and farther than any that have ever preceded it, will not Your Majesty reward with money all those who participate in it and give them double pay during the time that they are engaged, promote to the rank of sub-lieutenant the geodesists who have formerly been in Siberia, and to the rank of ensign ${ }^{11}$ those who go there for the first time? All officers should receive, either

"Bering was made Captain Commander and Chirikov Captain Lieutenant. 
here or in Moscow, a year's pay in advance and, if they desire it, another year's pay in advance either at Tobolsk or Yakutsk, so that they may get their outfits and depart in a contented frame of mind. Bering should arrange with the governor of Siberia and the vice governor at Yakutsk how the men are to be paid after that.

I6. Louis Delisle de la Croyère, ${ }^{12}$ at the recommendation of the Academy, is to have charge of the astronomical, physical, and other scientific observations of that nature. Instructions for that purpose have been given him. ${ }^{13}$ He is to have two geodesists to help him. Simeon Popov and Andrei Krasilnikov, who have been studying in the St. Petersburg Observatory. Professor de la Croyère requests that he have an interpreter who knows French or Latin and a mechanic who understands repairing his instruments. These two men are to be furnished him.

\section{Advance Parties}

Detachments of the expedition began leaving St. Petersburg in February, 1733, but Bering, who brought up the rear, did not get away until April, waiting apparently for supplementary instructions, which were issued in March, ${ }^{14}$ permitting him to build the ships at Okhotsk or in Kamchatka. The route followed was the same as the one taken at the time of the first expedition. Spanberg was in the lead and had orders to hasten to Okhotsk to supervise the completion of the ships which Pisarev was supposed to have on the ways or perhaps launched. When Spanberg reached his destination early in $\mathrm{I} 735$ he found no Pizarer, no ships, no quarters, no food, no Russian agriculturalists with full granaries, no Tungus with herds of fat cattle, nothing but the old cheerless and bare village that he had left behind him five years before. He was probably not surprised, for he must have heard on the way of the worthlessness and the evil deeds of Pisarev.

${ }^{12}$ Louis Delisle de la Croyère was the half brother of Guillaume Delisle and Joseph Nicolas Delisle, two well-known gengraphers of the first half of the eighteenth century. Joserh Nicolas was a member of the Russian Academy and was instrumental in securing this position for louis.

${ }_{13}$ A copy of these instructions, drawn up by Joseph Nicolas Delisle, is in the Library of Congress.

"I 'olnoe Sobranie Zakonov Rossiiskoi Imperii, Vol. 9. No. 6351. 


\section{Difficulties}

Not only at Okhotsk but elsewhere in Siberia the well-laid plans of the Senate and the Admiralty College miscarried. Bering, in place of being left free to look after the important work of leader, had to tarry at Yakutsk and other such places to do the work of a petty officer. He had to recruit men, requisition horses and supplies, and start them towards Okhotsk. Either the local authorities in Siberia could not or would not help him. One blamed another; Bering's own officers sided sometimes with one and sometimes with another; and as a result the mail carriers, especially provided for the expedition, were loaded down with charges and countercharges instead of reports of progress. The authorities at the capital were naturally greatly displeased. They tried to help Bering by relieving him of the control of the scientists and of the work of supervising the Arctic explorations. Notwithstanding this, month after month passed with comparatively little headway made. When the undertaking was planned it was assumed that in four years Bering would be ready for sea. According to Bering's own estimates the cost would be 10,000 to I2,000 rubles; but at the end of that period he was no farther than Yakutsk and the cost had run up to 300,000 rubles. At the capital there was considerable dissatisfaction, which the Admiralty College was made to feel. In 1738 the Imperial Cabinet, after taking into consideration the cost up to that time, the burden it threw on the Siberian people, the little progress made, asked the Admiralty College "to look into the Kamchatka Expedition and see if it can be brought to a head, so that from now on the treasury should not be emptied in vain."15

The Admiralty had to throw the blame on somebody and, justly or unjustly, blamed Bering and as leader lield him responsible and even went so far as to cut his pay in half from the beginning of the year 1738 until July, I7 fo. Bering was caught between two millstones which slowly but surely crushed him.

\footnotetext{
1s Opisanie Dyel Arkhiva Morskago Ministerstva, 10 vols., St. Petersburg, I877I905; reference in Vol. 7, p. 237.
} 
When the time came for his sea voyage he was pretty much discouraged and worn out, his physical strength was gone, and with it went the initiative and daring so necessary for a work of this kind. On his death bed Bering complained ${ }^{16}$ that too much had been expected from him - a man already advanced in years-and that those who should have stood by him failed to do so. In that great Russian world he stood alone, the target of the malcontents and the envious. That he was not wholly to blame may be seen from the orders of the Admiralty College to the local authorities in Siberia threatening them with torture unless they gave Bering more help. Finally, in 1739 , the Admiralty commissioned two officers on special duty to go to Siberia to take charge of the transportation of the supplies, and with their assistance most of the things needed for the voyage were deposited at Okhotsk in the autumn of 1740 .

\section{Preparation for the Sea Voyage}

Since coming to Okhotsk in 1737 Bering had been very busy supervising the building of the two ships for his voyage to America. In June, I740, they were launched and named the St. Peter and the St. Paul. Each measured 80 by 20 by 9 feet, had two masts and was brig-rigged, and carried fourteen guns, two and three pounders. All the needed supplies were loaded on these two vessels and on two freight boats, and on September 4 this small squadron left Okhotsk for the Bolshaya River (Fig. 3) in Kamchatka, where they anchored on September 20. Here Bering left the two freight boats because they were not strong enough to weather the autumn storms, and with the St. Peter and the St. Paul he sailed away for Petropavlovsk harbor in Avacha Bay (inset on Pl. I) and made that port on October 6. Petropavlovsk was a new harbor, having been surveyed and charted in 1740 by one of Bering's officers; and, judging from the sketch which he made (Fig. 7), Bering and his company constituted the whole population of that village. At this uninhabited spot the winter was spent in preparation for the great voyage.

${ }^{18}$ See Steller's account in Volume 2 of the present work 


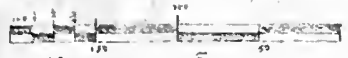

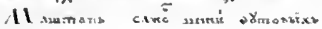

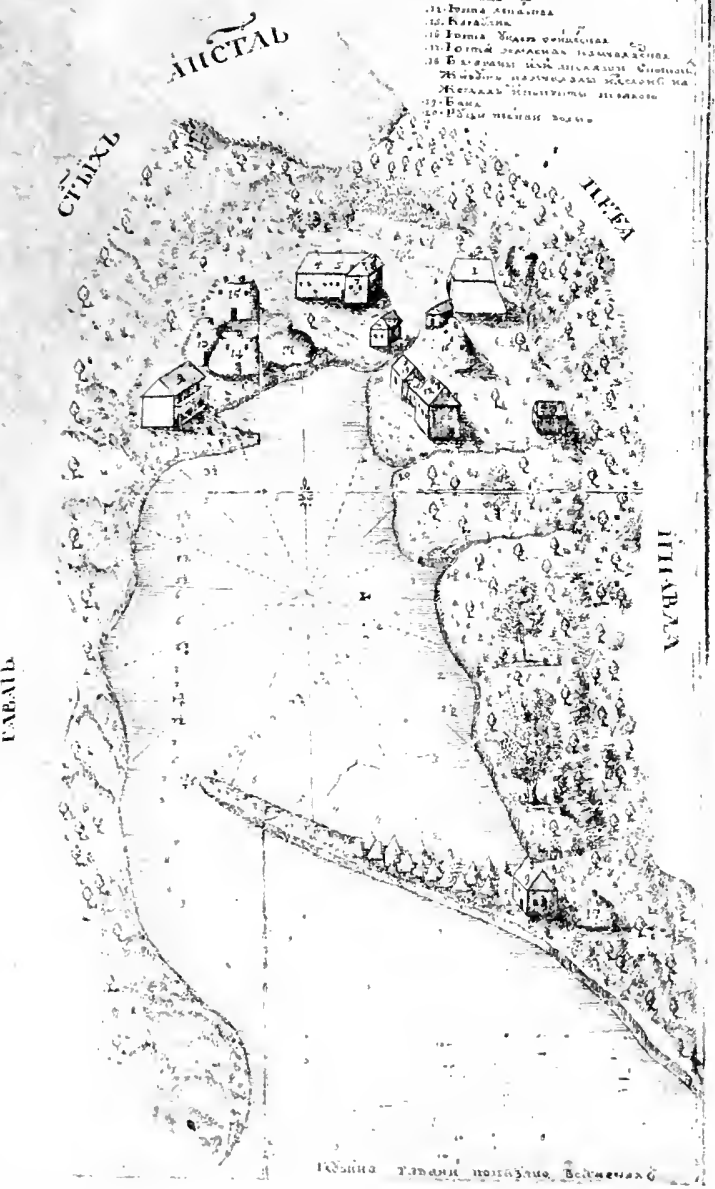

Translution of key:

I, traveling ethurch

2. warehouse

3. poweter house

4. Calotalis (onllmancle $r$

5. Captain ('hirikus and litutomant Chiklatches

7, jurefesert of alstrone my and andjunet

S. higher and lawer efticers

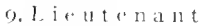
II axid

IG, L i c n t 11 at $n t$ Plautin

II, flevt-ma<ters and navigaters

12, quarters for the crew and workmen

13. blacksmith slopp

1. 4. medical quarters

1.5. guard house.

10, hower officess

17. native Kamchadal carthen hut

I8, native summori lomes raised on at folatform and (1) vered with grias

(1), bath liruse

20, It 5 . $\mathrm{h}-\mathrm{wat} \cdot \mathrm{r}$ spring.

In lower right arner:l 1).petle is ind is at + d i in fathems.

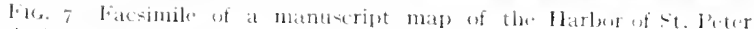

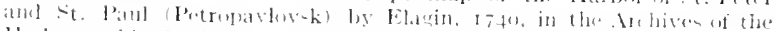

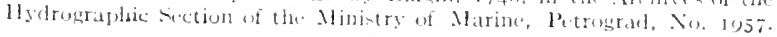




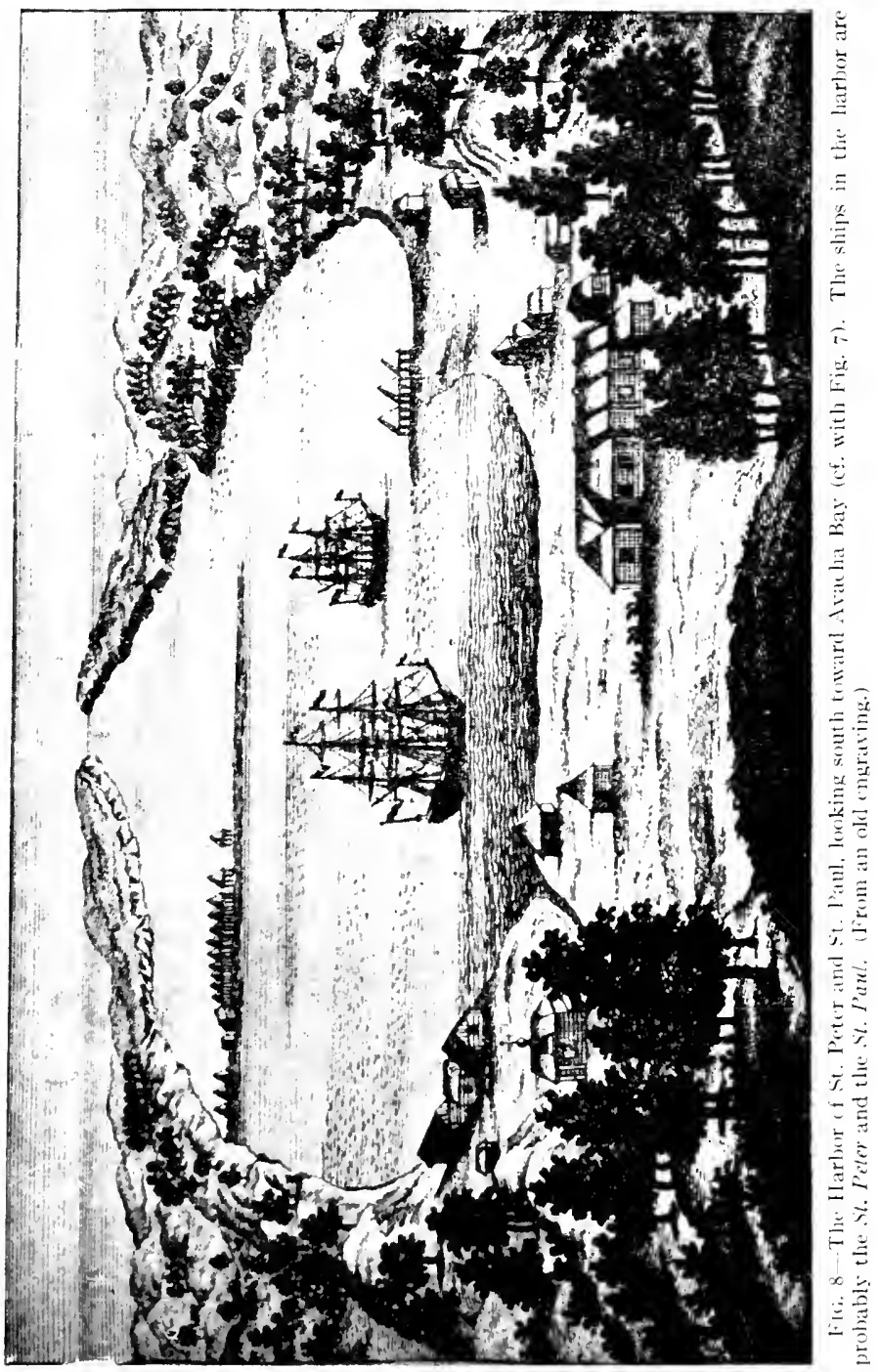


According to Steller, one of the scientists who went along, it had been Bering's original plan to depart from Kamchatka for America early in May, $174 \mathrm{r}$, and, after discovering that continent, to winter on it and return to Asia in 1742 ; but owing to a number of unforeseen accidents he was unable to bring this about. While at Okhotsk he had prepared the sea biscuit for the voyage, and these were lost at the mouth of the Okhota River in 1740 as they were being taken to Kamchatka. Then, again, because the two freight boats were unseaworthy he had to leave them behind at the Bolshaya River and have the supplies transported by the natives in the course of the winter. This was such a huge task that even the much-abused natives revolted, and it was some time before they were humbled and forced to do the bidding of the officers. As a result of all these misfortunes Bering was late in starting and not oversupplied on his departure.

On April 23, 1741, the navigation officers began to keep their records, and from this date on we will let them tell their own story. 


\section{CHAPTER V}

\section{THE LOG BOOK OF BERING'S VESSEL, THE "ST. PETER," AND OF HER SUCCESSOR, THE HOOKER "ST. PETER"}

The following is a translation, from the hitherto unpublished originals in the Russian archives, ${ }^{1}$ (I) of the log book of Bering's vessel, the St. Peter, covering the period from April 23, I74I, to August 9, 1742 , and describing the preparation of the ship for sailing, the voyage from Kamchatka to the Alaskan coast and return to Bering Island (June 4-November 6, I74I), the wreck of the St. Peter and the death of Bering, the life of the survivors on that island, and the building of a replacing vessel, the hooker St. Peter; and (2) of the log book of the hooker St. Peter from August io to September 7, 1742, describing the return voyage to Petropavlovsk, the home por in Kamchatka (August $\mathrm{I}_{3}$ to 27 ), and the attempted continuation of the voyage to Okhotsk and final return to Petropavlovsk (September i to 6).

\section{The Log BoOk of the "St. Peter"}

With the help of God this journal was begun this April 23, I 741, on the ship St. Peter by Fleet Master Safron Khitrov, under the command of Captain Commander Bering, in the Harbor of St. Peter and St. Paul, which is in Avacha Bay, latitude $53^{\circ} \mathrm{I}^{\prime} \mathrm{N}$; longitude, reckoned from St. Petersburg, according to the observation of the professor of astronomy.

1 There are two log books, one kept by the assistant navigator Kharlam Yushin (Arehives of the Hydrographic Section of the Ministry of Marine, Petrograd: 1741-42, Nos. $642,643,641$ ) and the other by the mate Sof ron Khitrov. Of Khitrov's there are two neat copies (Archives of State, Petrograd: $1732-43, \mathrm{XXIV}$, No.9, and Archives of the Imperial Academy of Sciences, Jetrograd: No. I20, 32:16:19), and it is almost certain that they were worked out from a third copy, whether Yushin's or Khitrov's is not clear. The Kintrov copies differ from one another in places, owing probably to the fault of the copyists; and they are not as rehible as Yushin's, which has alt the earmarks of an original. For that reason Yushin's is here used and is supplemented by Khitrov's wherever the latter contains additional material. Differences of more or less importance between the two logs are pointed out in the footnotes.

from April 23 to June 1 , inclusive, Khitrov's joirmal is Hsid, however. "Jotrnal" is used throughout in this clapter the same sense as "log book." 
Delisle de la Croyère, $127^{\circ} 3 \mathbf{I}^{\prime}$; variation of the compass $1 / 2$ rlumb easterly.2

In the Harbor of the Holy A postles Peter and Paul

\section{April 23, I74I}

Today with the help of God we began to load the ship St. Peter, on which worked eighteen men. The main shrouds were made fast to the mainmast, the preventer stay was also loosely secured; calked inside the ship, and near the mainmast hole nailed blocks for opening the cannon portholes; cleaned the hold, and in the afternoon took on ballast.

\section{April 24, I74I}

This day there were eighteen men at work, and they brought aboard the ship various rigging, also topmasts and yards; the cannons were secured on the port side while the starboard side was being calked. In addition we took on ballast.

$$
\text { A pril 25, I74I }
$$

Sixteen men were at work on board the ship. They rigged the mainsail and fore-topsail yards, worked about the rigging, and did some carpenter work.

$$
\text { April 26, 174I }
$$

This being Sunday there was no work.

$$
\text { A pril 27, } 1741
$$

There were seventeen men at work. Water and ballast were put into the hold. We rigged the fore and main yards; and other things were also done.

$$
\text { April } 28,1741
$$

This being the coronation day of Her Imperial Majesty there was no work.

$$
\text { A pril 29, } 1741
$$

Twenty-one men at work, rigging shrouds, backstays, and stays to the main-topmast and fore-topmast and stowing casks of water in the hold.

$$
\text { April 30, I74I }
$$

A strong unsteady wind from the NE accompanied by rain. We put barrels of water into the hold. raised the best bower anchor clear off the ice and secured it to the port side of the ship, and made fast the shore anchor line to the anchor on sliore.

\footnotetext{
2 "Rhumb" is used in two senses in the log book: ( $I$ ) in statements of the variation of the compass, as here and in the 2.-hour summary at the end of each day's log. in the sense of "point". or $11^{1}{ }^{\circ}$ of angular measure: (2) in the 2.4 -hour summaries and elsewhere, in the sense of "course,"i.e. the angle formed by the ship's path and the magnetic neridian.
} 
May $I, I 74 I$

Put casks of water in the hold, broke the ice around the small bower anchor, and let go into the water. Took on wood.

\section{May 2, I74I}

Eleven men worked today. In the hold wood was stowed between the casks. To the mainsail and fore-topsail yards were secured ties and blocks. We hoisted on board the spritsail yards from the ice, put the jib boom in the hoop, hauled aboard the small anchor from the ice, secured the hawser on the port side, did carpenter work in the cabin. Four inches of water in the hold.

\section{May 3, I74I}

Sunday and no work.

\section{May $4, I 74 I$}

A light wind from SW. In the morning there was frost and snow. Sixteen men were at work in the boatswain's storeroom, on the port side of the caboose; doors were made; and on the starboard side beds for the lower officers were furnished. Other work was done also.

This day Captain Commander Bering with Captain Chirikov and all the higher officers and navigators held a council, to which was invited the astronomer, Professor de la Croyère, to decide what course to sail first after leaving Avacha Bay in order to locate the Terra de Gama, which is indicated on the chart of the above-named professor and which extends northward to latitude $47^{\circ}$. [The following decision was made.]

\section{Decision on First Course to Sail}

On May 4, 1741, there was a council made up of Captain Commander Bering, officers, the professor of astronomy, and navigators. After listening to the instructions given to Captain Commander Bering by the lmperial Admiralty College, especially the nineth and seventeenth articles and the sixth article of the general instructions of the Senate, it was determined in order to carry out the instructions to find the American coast, to sail first after leaving Avacha SE by E, true, and to continue on that rhumb. If no land is found by the time latitude $46^{\circ}$ is reached to change the course to $\mathrm{E}$ by $\mathrm{N}$ steadily until land is discovered. If land is found either on the SE by $\mathbf{E}$ course or on the $\mathrm{E}$ by $\mathrm{N}$ course to coast alongside of it, depending on its extension from east to north or from north to west; if it should stretch between south and east, to leave the land and go east until we discovered more land and likewise to follow that land in a northerly direction, to latitude $65^{\circ}$, or as far as, with God's help, time may permit. If we should reach latitude $65^{\circ}$ in good time, then we should go west to the Chukchi Cape so that the distance between the American and Chukchi shores may be known, and from there we should return to this harbor. If on the above-mentioned rhumb the winds should be contrary we should keep as close to it as may be possible, and if with God's 
help we should discover land then we should make explorations as the instructions to the Captain Commander recommend.

In making the voyage it should be planned to return to this harbor during the last day's of September.

[Signed on the original:] BERING

Captain Alexei ChuRIKov

Lieutenant IVAN CHIKHACHEV

Lieutenant WAXEL

Lieutenant Plautin

Fleet Master SOFRon KHItrov

Louis Delisle de la Croyère

For Fleet Master AvraAu Dementiev

Navigator ANDREYAN ESELBERG

Navigator IVAN ELAGIN

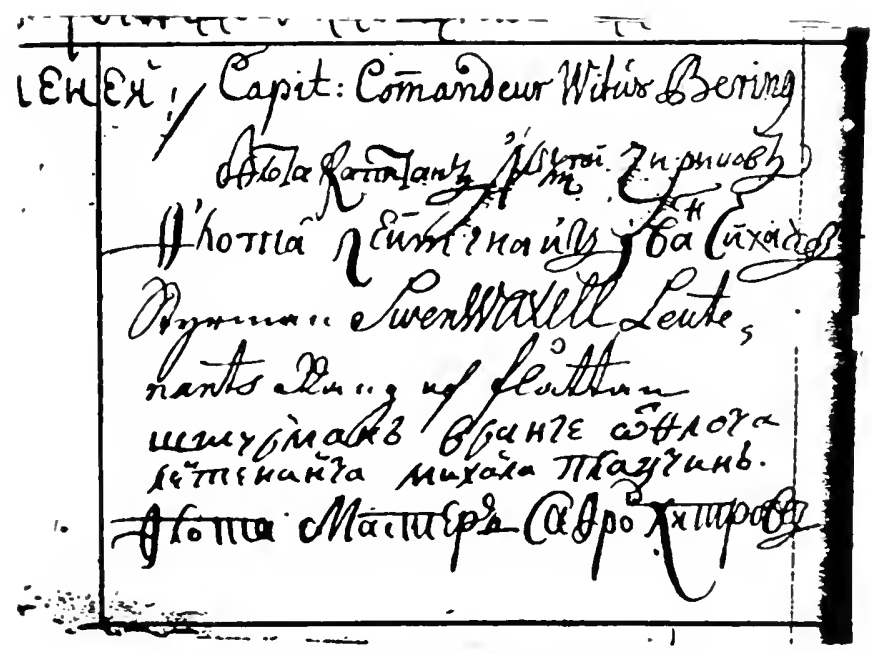

FIG. 9-Facsimile of signatures of Bering and his officers, probably from the "Decision on First Course to Sail" of May 4, 174I, which see for names.

\section{May 5, 1741}

Wind from SW. Seventeen men at work. On the main and fore shrouds the catharpings were fastened. Planed on the inside of the ship. Strengthened the foremast and nailed planks near the windlass. 


$$
\text { May 6, I74I }
$$

Wind from S. Gloomy weather. Seventeen men at work. On the fore-topsail the hoop was repaired, on both masts the chain plates were secured, and to the fore-topsail yards the gear was fitted. Water was brought from shore and stowed away in its place.

\section{May 7,1741}

Variable and uncomfortable SW wind. From shore there were brought two cannon, two falconets, and three gun carriages. We did also other kinds of work.

$$
\text { May } 8, i 74^{I}
$$

Wind from the NE and unsteady. Seventeen men were at work; rigged the fore and main-topsail yards and topsail sheets and clew lines. Stowed barrels of water in the hold, made fast two cannon, stowed 90 poods ${ }^{3}$ of strip iron under the commander's cabin.

\section{May $0, I 7+I$}

Unsteady wind from the $\mathrm{E}$ and gloomy weather. This being the day of Nikolai the Wonder-worker there was no work.

$$
\text { May Io, I74I }
$$

Wind from the S. Snowy weather. We hauled the ship farther from shore and let out the spare anchor from the port side of the stern and did other work about the ship.

$$
\text { May II, I74I }
$$

Wind from the E. The sky was overcast, and it rained and snowed. Nincteen men were at work; they brought casks of water and ten barrels of salt meat which they stowed aft in the hold, and they did other work as well.

$$
\text { May } 12, I 7+1
$$

Easterly wind with gloomy damp weather. Nineteen men were working. They brought aboard ship's stores. Scraped the foremast and workerl on the rigging. There was in the hold $7 \frac{3}{4}$ inches of water, and after pumping out there was $2 \frac{1}{2}$ inches left.

$$
\text { Niay } 13, I 7+1
$$

Wind from the S and unsteady. Seventeen men were at work bringing casks of water and stores from shore, polishing the foremast, putting in place the running rigging. Today we corrected the compasses with the meridian line-six of these compasses had a variation of $1 / 2$ rhumb east. and one ordinary stecring compass in a copper case had $3 / 4$ thumb east.

3.1 pood is equat to 40 Russian pounds, or $36 \mathrm{lbs}$. avoirdupois. 
May $1+17+1$

Wind from the E. Seventeen men at work, putting the rigging in order, stowing away the stores in the hold, greasing both topmasts with fat, and fastening bull's-eyes.

May $15,174 I$

Wind from S. Eighteen men at work. The anchor which was down forward on the port side was hoisted on board and put in the hold, and in its place was secured the spare anchor. Spare yards and topmasts were brought aboard and made fast. Ship stores were brought from shore.

$$
\operatorname{May} 16,17+1
$$

Light wind from SSE. There were eleven men at work who bent on the mainsail and foresail, both topsails, and the skyscraper. Dried the spare sails in the wind.

$$
\text { May } 17,1741
$$

Sunday and no work.

$$
\text { May } I 8, I 74 I
$$

Wind from E. Twenty-one men are at work. The port side of the ship from the upper wales was calked and pitched. Stores were brought on board. Water in the hold $5 \% 2$ inches.

\section{May 19,1741}

Unsteady wind from ENE. Cloudy, but occasionally it lightens in the E. Twenty-two men working, putting chocks under the longboat, calking and pitching the starboard side. bringing aboard provisions and boatswain's stores as well as the articles to be given as presents.

$$
\text { May } 20,1741
$$

No wind, wet snow. Twenty-three men were working. At the order of the Captain Commander, Ensign Lagunov took out from one of the casks on board a bucket of vodka and gave it to Adjunct Steller. We floored the powder room, made a bed for Adjunct Steller in the Commander's cabin. Examined the leak at the bow near the foremast and made revairs in the Captain Commander's cabin.

$$
\text { May } 2 I, I 74 I
$$

Heeled the ship on both sides and greased the under side. Gloomy weather and still. Twenty-two men were working today. 


$$
\text { May 22, } 174 I
$$

No wind, cloudy weather. This day the crew with its bags came aboard the St. Peter and did different kinds of work. Forward there was 8 feet 10 inches of drait. and aft 9 feet 4 inches, a difference of six inches.

\section{May $23,17+1$}

No wind. The crew has divided itself into two watches. Jib, mainsail, fore-topsail, staysail, and main-staysail were bent on. The deck over the crew's quarters was calked and pitched from below. Six inches of water in the hold.

$$
\text { May 24, I74I }
$$

This being Sunday there was no work. We hoisted the flag and the jack and dressed ship.

\section{May 25, I74I}

No wind, cloudy weather with rain. At ten o'clock in the morning Captain Commander Bering inspected the crews on board the St. Peter and the St. Paul, and as he left the last-named ship, of which Captain Chirikov was in command, the crew gave five hurrahs for the Captain Commander. From his boat the same number of hurrahs were given in return, which was answered by one more hurrah from the ship. The Captain Commander has moved his quarters to the ship. Powder, lead. and shells were brought aboard. This day Captain Commander Bering gave Captain Chirikov the signal code which is to be used on the voyage. What follows is an exact copy:

\section{SIGNALS FOR USE IN DAY'TIME}

I. When we shall wish to speak to you. Captain, we will hoist an ordinary pennant from the main-topmast and crosstrees and fire one gun. If your lieutenant should be needed the same pennant will fly from the same place and in addition the ensign from the ensign staff, but no gun will be fired.

2. If we should desire that you should take the lead, then the ordinary pennant will fly from the fore-topmast crosstrees, and it will be accompanied by one gun.

3. If it should be necessary to anchor, there will fly from the flagstaff a tricolor flag of long strips of red, white, and blue, and one gun will be fired.

4. When religious service is to be held, a white flag with a blue cross will fly from the gaff and one gun will be fired.

5. If the Captain Commander should wish to talk to you so that you will have to bring your ship near his, then, in addition to the signal for calling you, there will fly a blue flag from the main yard, accompanied by a gun.

6. If we should sail close-hauled and you in our wake, and if you should see the jack flying from the gaff and hear a shot, that is a signal for you to come about first.

7 . If in stormy weather it should be impossible to come about against the wind, we will signal to you to wear in succession, by hoisting a pennant at the stern and firing a gun. 
8. If in sailing close-hauled or free it should be necessary to sail with the wind then a blue flag will be hoisted on the flagstaff and one gun will be fired.

9. If on going with the wind it should be necessary to come about close-hauled on the starboard tack, having the sails on the port side, a red flag will fly from the gaff and one gun will be fired; if it slould be necessary to come about on the port tack with the sails on the starboard side, a blue flag will fly from the same place and one gun will be fired.

10. If a boat with men should be sent ashore for the purpose of exploring or taking on water a wide pennar: will fly from the gaff and one gun will be fired.

ii. If on the way we should become separated, we are to look for one another and cruise for three days near the spot where we lost sight of each other; the latitude of the place shall be determined and, by calculations, the distance and the rhumb from the harbor or from whatever spot the reckoning is made. If during that time we should not find one another, from which misfortune may God preserve us, then we are to sail on the rhumb agreed upon with you and all the officers, a copy of which agreement, with instructions signed by me, was handed to you. If three days have passed without our finding one another and if, going on the course agreed, we discover land on the rhumb where we expect, we are to beat up and down the coast for a day in order to wait for one another. If [land should be found] on the northern rhumb, coast along it in a southerly direction for a day as long as it is on the rhumb agreed upon, but do not spend more than a day in going south. But if [the land comes to an end before the day is out] keep on the course until the end of the day and after that continue on your course. If it should be found farther south than on the rhumb expected, coast along in a northerly direction until the course agreed upon is reached and there wait a day, and, if we should not find one another there, then go your own way and act according to your instructions.

12. If after separating we do not soon meet again and we should be in a region where foreign vessels might be expected, in order to recognize one another you should fly a blue flag from the main-topmast crosstrees and fire one gun and we will fly a red flag with a straight white cross from the same place and also fire one gun.

13. Signals which you, Captain Chirikov, while at anchor should give in order to keep the Captain Commander posted:

14. If you should see ships or sea vessels of any kind, you should fly your flag from the main-topmast and keep it there until it is acknowledged by one gun from the Captain Commander's ship. You should then dip the flag as many times as there are ships in sight.

\section{Signals While UNder Sail}

How to identify one another is indicated above in the 14th [1 2 th?] article.

If you see ships, you are to make the same signal as when at anchor and fire one gun. If the distance between us is so great that the flag cannot be seen, lower and hoist the topsail as many times as there are ships.

If the ship should spring a leak, from which misfortune God preserve us, or for some other reason it should not be possible to follow us, then stop and fire two guns.

Should you in the daytime desire to speak to the Captain Commander, put up the ordinary flag at the main-topmast or fore-topmast near the truck, hoist and lower the mainsail or foresail, according as to where the flag is flying, and continue to fire until you hear a gun in return. 
If you should see land fly both the flag and the jack and keep them flying until the Captain sees them and hoists his flag, and then lower yours. If, at the time, you have up the flag and the jack, lower them at once and fire from time to time unti] the Captain Commander hoists the flag and jack and fires one gun.

\section{Signals for Daytime in Foggy Weather}

If any one should in the daytime see breakers or reefs, the ship should put about, the jack should be hoisted at the main-topmast crosstrees, and the gun should be fired twice. If, may God preserve us, the ship should run aground, lower all sails, make the signal with the jack, and keep on firing until you make sure that the others are aware of the danger and are out of it.

\section{When SaIling in Foggy Weather}

If it should be decided to sail in foggy weather as before the fog set in, a gun will be fired every hour, and you are to keep for that purpose a half-hour and a minute glass, and as soon as you hear a shot turn your glass upside down and count the time between the shots and then do likewise [fire].

If it is desired that you should put on more sail, a gun will be fired every half hour, if to take off, every quarter of an hour, and you reply in like manner. During this time there should be ringing of bells and beating of drums on both ships.

If it should seem best to heave to on the starboard tack, three guns will be fired, if on the port tack, five; the beating of the drums and the ringing of the bells should go on.

If in foggy weather it should be necessary to lower the yards, four guns will be fired and the ship that is astern will in the meantime lower the yards.

If after drifting for a time we decide to make sail again, we should proceed as we did before we drifted: if we sailed with the wind before, we should do the same afterwards, each ship keeping in her position, so as not to become separated in the fog, and following the course agreed upon. If in the meantime the wind has shifted, keep on the course just the same, or if the wind is against you keep as close to the course as you can. Fire two guns, one after the other every six minutes.

If it is decided to anchor during the fog, the signal will be two guns, one after the oiher. Then after we have been at anchor a half hour we will fire two guns and, if you are far from us, will keep this up until you reply by a gun.

If in foggy weather it should seem best to get under way again, continue on the course held to the time of anchoring, that is. if we sailed close-hauled, then keep on the same tack on which we were until we anchored; if we sailed with fair wind, then continue on that course so that the two ships may keep on the same tack and not become separated in the fog.

Keep the course we sailed if the wind is favorable, if not, as near to that course as possible. Fire two guns one after the other every six minutes.

To fall off from close-hauled to with the wind, the signal is three guns, one after another every six minutes.

To head up from with the wind to close-hauled: if to the starboard tack, seven guns, if to the port tack, eight.

If we should be sailing with the wind aft or on the quarter and should decide to change course a few points, the following are the signals: for one point two guns, two weints three guns, four points five guns, five points six guns, and so on; in each case 
the number of guns will exceed by one the number of points. If we are to go to the starboard the gun will be every twenty-one [two?] minutes, if to the port every three minutes. If, in thick weather, land or a reef should be sighted, make the same signals which the Captain Commander would make in such a case, that is, lower the yards and fire four guns. If, may God guard us from such a misfortune, you should run aground, fire one gun after another so that we may put about or anchor, and from the other ship acknowledgment should be made by four guns.

\section{Signals To Be Made at Night While Under Sall}

If it should be necessary to back the yards against the wind, then two lanterns will be hung on the flagstaff, one under the other, and one gun will be fired. If with the wind, one lantern will be hung on the gaff and the other on the flagstaff, and one gun will be fired. You should also have lanterns at these places and keep them there until we take ours down. That ship should put about first which is under the wind, and care should be taken that at nighttime we do not run into each other.

If in bad weather it should be necessary to shorten sail and lay to, one lantern will be hung at the mainmast and another at the foreshrouds at the same height and on the same side, and two guns will be given. At the same time you should hang out lights from the same places.

If after drifting for a time we decide to make sail again, three lights will be hung from the mainshrouds at the same height, followed by two guns. You should hang your lanterns in the same place, and do not take them down until ours are down.

If in sailing with a fair wind it is necessary to sail close-hauled: if on starboard tack, that is to say the sails on the port side, two lanterns will be hung from the foreshrouds accompanied by four guns; if on port tack, that is to say sails on the starboard side, six guns will be fired. You should put out two lanterns at the same place.

If with a good wind at night we should be sailing free or with the wind on the quarter and it should be decided to change the course and if we are in the lead, we will hang out from the flagstaff as many lanterns, one under the other, as the number of points in the course to be changed. If two guns are fired, change the course as many points to starboard as there are lanterns, if three guns are fired change the same number of points to port. Should you be in the lead the signals as above will be made from the jack staff, accompanied by guns.

\section{Signals To Be Made at Night While Mooring Ship, Weighing, oR COMING to ANCHOR}

If at night it should seem best to anchor, three guns will be fired at short intervals apart, and two lanterns will be hung in the mainshrouds. You should then hang out one lantern from the same place. If the stream anchor is to go down, one light will hang from the jack staff while the guns are being fired. In that case you are to signal with a light from the foreshrouds and not from the mainshrouds.

To moor, or stand on two anchors, one light will be displayed from the mainmast and another from the foremast, and one gun will be fired. You are to show a light from the mainshrouds.

If at night it is necessary to raise one anchor, three lights will be shown from the main-topmast shrouds, higher than the light in the main-topsail, accompanied by two guns. You should hang out a light on the gaff. 
If at night the anchors are to be weighed, one light will be displayed from the mainshrouds and another from the main-topmast shrouds, followed by one gun. In this case you should show a light from the gaff.

If at night the yards should be lowered, a light will hang from each end of the yards and so long as they are down. You should show a light at the stern during this time.

If the topmasts are to be lowered, a light will be placed at the very top of each, and the topmasts with the lanterns will be let down at once. You are then to show a light halfway down the mainshrouds and keep it there so long as the topmasts are down.

When the topmasts and yards are to be put up again, two lanterns, one above the other, will be hung from the gaff, followed by two guns. You are to show one lantern at the same place. When our lights are taken down then yours are to come down too.

\section{Signals at Night While at Anchor}

If at night the anchor should not hold or the cable should part, two lanterns are to be lighted on the flagstaff and as many others as you have on the mainshrouds, and these are to be kept until the situation is righted.

If at night there should come near you a strange suspicious craft and if it should be impossible to notify us by the speaking trumpet or by sending the yawl, hang out all the lights that you have and fire, gun after gun, until the Commander replies with one gun.

\section{Signals at Night While Under Sail}

If you find it necessary to drop behind us, firc once and put up three lights on the foreshrouds.

If at night a ship should be scen, put up one lantern on the mainshroud and two on the stern and explode some powder or light a fuse and while heading for that ship continue to fire your gun, one shot after another. If we shonld also steer for this vessel and should fire one, two, or three guns, you are to follow us.

In case one of our ships meets with misfortune, from which may God preserve us, forcing her to return to the harbor, and conditions are such that it is impossible to talk over the situation, if she can make her way back without help of the other ship, a red flag should fly from the fore-topmast, followed by one gun. If, however, the situation is such that it is necessary that the other should follow to be of help in case of need, the same red flag should fly from the main-topmast crosstrees, and one gun after another should be fired until the other does follow. Should the misfortune happen at night or in foggy weather, eleven guns should be fired, and the other ship must approach until she is in sight.

If one of the ships should spring a leak or neet with some other harm so that she cannot continue the voyage, she should display two lights on the main-tonsail yards at equal height and continue to fire until the other ship comes to help.

Whichever ship sees land or a shoal at night should hang out as many lights as she can, fire once, and steer clear.

[The original signal code was signed:] BERING 


\section{May $26,174 I$}

No wind. We dried the upper and lower sails. At three o'clock we took in the sails. At six o'clock the wind was SSE. The Captain Commander went in the small boat to look over Rakovaya Bay.

$$
\text { May 27, I74I }
$$

No wind, cloudy and sunshiny. Pitched the boat on both sides.

$$
\text { May } 28, I 74 I
$$

No wind, cloudy, thick weather. Members of the crew went ashore by watches to have a bath. At ten o'clock the wind was SSE. At two o'clock in the afternoon we hauled on deck the spare anchor, with the cable, which had been down at the stern on the port side, and put in its place the stream anchor with the hawser made fast at the stern. The best bower anchor was lowered in the water to be cleaned and, after that, was stowed away in the hold, and to it was bent a cable I 50 fathoms long, which had been unwound and spliced.

\section{May 29, $I 74 I$}

At four in the morning a shot was fired to signal the St. Paul for religious service. Half an hour later another shot as a signal to get under way. (Main-topsail loose.) At six o'clock the small bower anchor and the stream anchor were weighed, and we were towed out of the Harbor of the Holy Apostles Peter and Paul into the roadstead of Avacha Bay. After passing the leading buoy, leaving it to starboard, which was in 15 feet of water and had a white flag at the top, we entered the roadstead and let go the small bower anchor and played out 20 fathoms of cable. Took soundings and got $7,8,9,10,5,3,4,9,12,13$ fathoms and blue slimy mud. When the gun was fired we hoisted the anchor flag.

\section{May 30,1741}

At one o'clock in the afternoon the wind was S by E; at four o'clock SE. Cleaned the small bower anchor cable. The Captain Commander issued a supplementary signal order for Captain Chirikov. In case it should be necessary to be towed, the ordinary jack should fly from the main-topmast-standing-backstays opposite the top. At four in the morning the signal was made for the lieutenant of the St. Paul to come to us, and in response navigator Elagin came and received the above signal order and returned to his ship. From six to nine in the morning we warped between $\mathrm{S}$ and W. During that time we made three tows of 390 fathoms each. From nine to ten we made two tows of the same length and then anchored in $91 / 2$ fathoms of water. The lighthouse at the entrance bore $\mathrm{SE}_{1}^{\prime} / 2 \mathrm{E}$ from us, and the native summer homes at the mouth of the Harbor of St. Peter and St. Paul NE by E. The winds were unsteady and shifted. 


\section{May $3 I, I 7+I$}

Topsail SSE wind, cloudy weather, and occasional sunshine. Eight o'clock in the evening the wind was from the SE but changeable. The longboat, in charge of the quartermaster and six men, was sent ashore for water and returned at two o'clock in the night with the water. At six in the morning the wind was from the NE; we hoisted the prayer flag when the gun was fired, let out a quarter of a cable on the small bower anchor.

While the ship was in the harbor it was loaded with all kinds of supplies, materials, and provisions which were needed for our sea voyage. The table below gives the kind and quantity of the cargo which we took aboard the St. Peter. At the stern the ship drew 9 feet 5 inches, at the stem 9 feet 4 inches of water, difference of one inch. In addition there is the small bower anchor with a half cable, the longboat, and the small yawl, all of which are in the water.

Table of the Materials, Supplies, and Provisions Takenon Board the "St. Peter" for the Sea Voyage

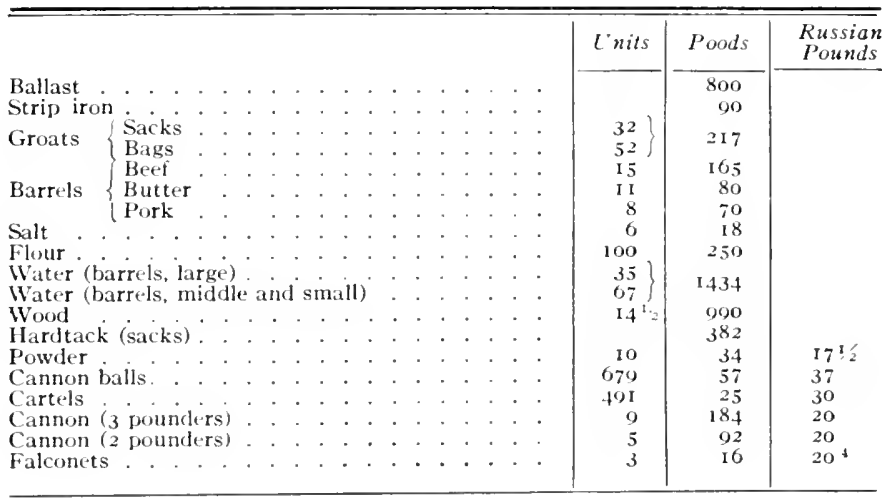

\section{June $I, I 7+I$}

At one o'clock topsail SSE wind, sun is shining. At five o'clock a very light wind from the E, which shifted to ESE by eight o'clock. We raised the small bower anchor in order to look at it and then let it go again. The lightlouse bears SE, the native summer luts in the harbor $\mathrm{N}_{2}^{1} \mathrm{E}$. During the whole twenty-four hours the winds veered back and forth between $S$ and $E$. In the hold there are four inclees of water.

\footnotetext{
4 total of 4.907 poods and $2.4^{\prime} ;$ Russian pounds (49 pounds to the pood), or about 88 :' tons.
} 
June 3, I7+I

At one o'clock the wind is SSE. At two o'clock we sent our boat ashore for water. At six o'clock we saw a ship under sail in the $S$ heading for Avacha Bay, which proved to be the double sloop Nadeshda coming from the mouth of the Bolshaya River. At nine o'clock the wind was SE; our boat returned with water. At eleven o'clock we put out a lighted lantern at the gaff for the benefit of the above-mentioned ship. At two $o$ 'clock we took down the lantern. At eleven in the morning the Nadezhda entered Avacha Bay and fired five guns as a salute, we replied with three; when she had come abreast, those on board gave three cheers for the Captain Commander, and we returned the compliment by three cheers, and then they gave three more. At twelve o'clock the double sloop came to anchor astern of us, and the navigator Ptichev came aboard our ship to make a report to the Captain Commander, after which he left. At one $o^{\prime}$ clock topgallantsail $\mathrm{NE}$ by $\mathrm{S}$ wind.

\section{June 4, I74I}

At seven o'clock the double sloop weighed anchor and sailed into the Harbor of St. Peter and St. Paul. At one o'clock calm. At two o'clock we had the anchor apeak and beat nearer towards the mouth; at four o'clock a gentle NW wind; we put up the fore-topsail and fore-topmaststaysail and steered for the mouth. St. Paul did likewise. At five o'clock the wind is WNIV; we hauled up the two topsails and were being towed $\mathrm{SE}$ by $\mathrm{S}$. The strong tide was the only obstacle to our going out, and we were, therefore, compelled to warp $\mathrm{SE}$ by $\mathrm{E}$ with the anchor in 13 fathoms. At eight o'clock set the sails and were towed $\mathrm{SE}$ by $\mathrm{E}$ into 8 , 9, IO, I2, I 4 fathoms. Lighthouse Vaua bore $\mathrm{N} 1 / 2 \mathrm{E}$, Vilyuchensk Volcano [SW3/4W?, depth of water 30 fathoms. At twelve o'clock Vaua Lighthouse bore NW by $\mathrm{N} 1 / 2 \mathrm{~W}, 2 \frac{1}{2}$ miles, Vilyuchensk Volcano WSW, Burning Volcano $\mathrm{N} 1 / 2 \mathrm{~W}$, Isopa Point $\mathrm{S}$ by $\mathrm{IV}$; carrying all sails except the spritsail..$^{5}$

5 Yutshin's journal gives these bearings: Vaua Lighthouse NW ís lthe same as Khitrov's NW by N/2W]. distant about $21 / 2$ German miles [1 5 German miles to a degree]. Vilyuchensk Volcano WSW, Burning Volcano N $1 / 2 W$, Isopa Cape S by W', about 9 German miles.

Vaua Lighthouse was located on Lighthouse (Mayachni) Cape at the entrance of Avacha Bay. Vilyuchensk Volcano is south of Avacha Bay and about three or four miles from the shore. North of Avacha are three volcanoes close together. According to Steller ("Beschreilung von dem Lande Kamtschatka," Frankfort and Leipzig, I 774. p. 44) the most northwesterly of the three was called Strelechnaya, the one next to it Corelaya (Burning), and the third had no name. After reading the various descriptions of Kanchatka and the log books of the navigators one is forced to conclude that there was a great deal of confusion on the subject. Strelechnaya Volcano was sometimes called Koryatskaya, Burning Voleano was also known as Avacha Volcano, and occasionally one of the last two names was given to the third volcano. which was not supposed to have a name. At the present time the names given to these mountains are: Koryatskaya, Avacha, and Kozelskaya.

The term lsopa disappeared from the maps and the books soon after Bering's time. Isopa Cape, or Ilook, judging from Steller's description "Beschreibung." etc., D. 18) and from early chats, is no other than Povorotni Cape of tnodem maps. (Mayachni Cane and the Harbor of St. Peter and St. Paul, or, in its modern form. Petroparfov'sk, may be located on 1 l. J in the inset of Avacha Bay; Vilyuchensk [Vilyuchin] and Koryatskaya Volcanoes, on the main map of Pl. 1.) 
I June 5, 1741. After Midday6

\begin{tabular}{|c|c|c|c|c|c|}
\hline$\stackrel{5}{0}$ & 营 & 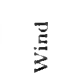 & $\begin{array}{l}\mathscr{0} \\
\stackrel{0}{0} \\
0 \\
0\end{array}$ & 离 & \\
\hline 1 & & $\mathrm{~s}$ & ESE & & Light wind and clear. \\
\hline 2 & & & $\bar{E}$ & & $\begin{array}{l}\text { Heavy fog. We beat the drum and rang the bell but } \\
\text { received no response from the St. Paul. }\end{array}$ \\
\hline 3 & & SE,S & $\mathrm{E} / \frac{1}{2} \mathrm{~N}$ & & \\
\hline 4 & & & $\mathrm{E} / \mathrm{N}$ & & \\
\hline 5 & & & NNE & & $\begin{array}{l}\text { Took aboard the longboat and yawl and lashed } \\
\text { them secure. }\end{array}$ \\
\hline 6 & & & & $\rightarrow$ & Fog cleared a bit. \\
\hline 7 & & & & & $\begin{array}{l}\text { Lowered topsails on caps, wind went down. } S t . \\
P \text { aul N by E, about }\left[7^{3} 4\right] \text { German miles. }\end{array}$ \\
\hline 8 & & $\stackrel{\Xi}{3}$ & & & $\begin{array}{l}\text { Lighthouse at the mouth of Avacha Bay WNWI/3W, } \\
4 \text { German miles, Isopa Point SSW. }\end{array}$ \\
\hline 9 & & & & & \\
\hline Io & & & & & Cloudy, stars out, St. Paul not in sight. \\
\hline II & - & & & & \\
\hline 12 & & WSW & SE E & & Hung out a lighted lantern over the stern. \\
\hline 1 & $\ldots$ & & & - & \\
\hline 2 & & & & & \\
\hline 3 & & & & _. & St. Paul NE by N I mile. \\
\hline 4 & - & & & & \\
\hline 5 & & & & & \\
\hline 6 & & WSW & & & Light wind, drizzly, cloudy. \\
\hline 7 & & & ESE & & \\
\hline 8 & & SW & & & Clear with passing clouds. \\
\hline 9 & & & & & \\
\hline 10 & & & & & $\begin{array}{l}\text { One gun was fired from the } S t \text {. Paul; the ordinary } \\
\text { flag was hoisted from the fore-topmast crosstrees, } \\
\text { and the fore-topsail was lowered. which was the } \\
\text { signal that the officers wished to speak to us. Whe } \\
\text { frred one gun in acknowledgment and started for } \\
\text { the St. Paul about eleven o'clock. }\end{array}$ \\
\hline if & & & $\mathrm{E}$ & & \\
\hline 12 & & & & & $\begin{array}{l}\text { Shipunski Cape NNE } 34 \mathrm{E}, \text { Burning Volcano } \mathrm{NW} \\
\text { by W, Isopa I'oint SW } 1 / 2 \mathrm{~S} \text {. }\end{array}$ \\
\hline
\end{tabular}

${ }^{6}$ Unless otherwise indicated Yushin's $\log$ book is used on and after this date. The $\log$ books were kept according to the astronomical day, which extends from noon of the previous civil date to the following noon, i.e. June 5 of the log began at noon, June 4 , civil time, and ended at noon. June 5, civil time. In timing the events of the voyage this should be borne in mind; also that the converted civil dates will be those of the Russian calendar, at that time eleven days behind the Gregorian calendar. See also footnote 3. p. 332. The noon positions on the chart. PI. I, are, as in the log, those of the end of the astronomical day, and the dates therefore coincide with the civil dates,

The symbols preceding the date indicate the day of the week, as follows:

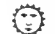
(3)
Sunday:
IMonday
$\delta$
Tuesday
$\gamma$
24
?
b)
Wednesday
Thursday
Friday
Saturday 


\section{h June 6, I77I. After Midday}

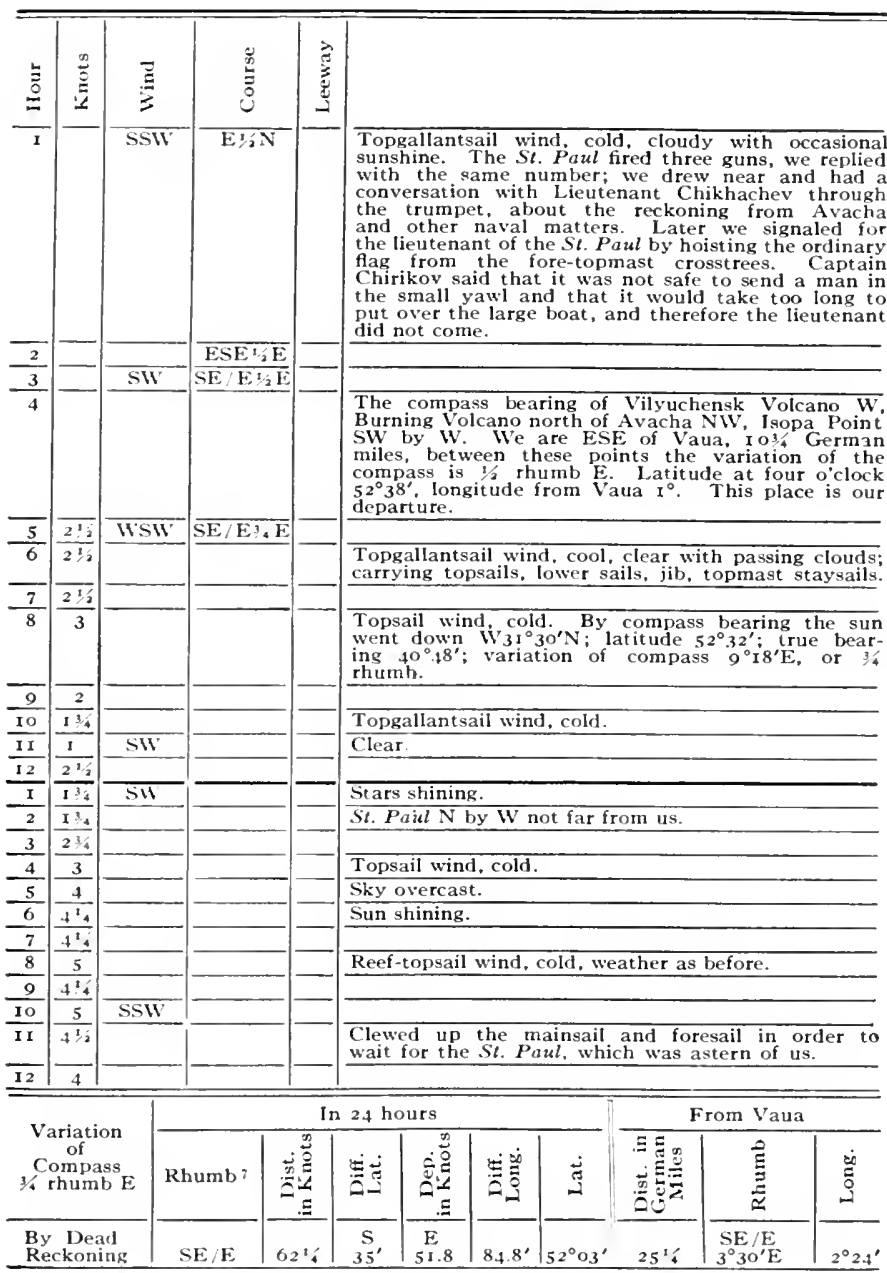

7 For different significance of "rhumb" in the $\log$, see footnote 2, above. 


\section{June 7, I741. After Midday}

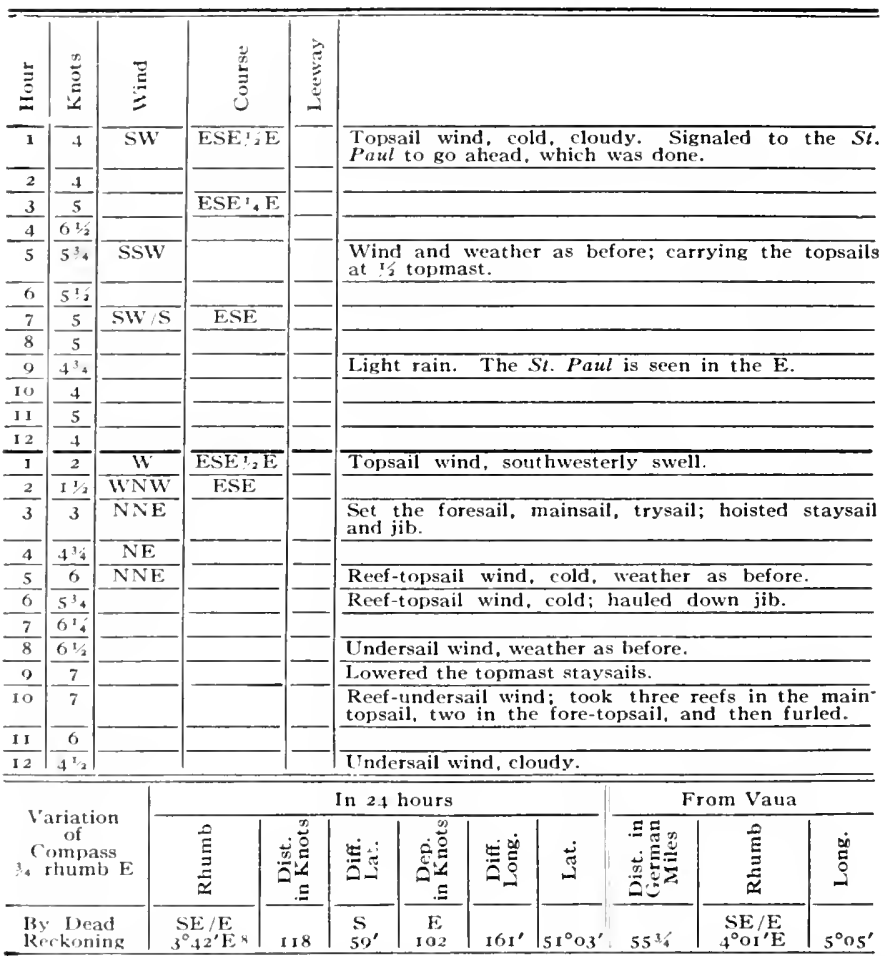

8 Throughout the log of the St. l'eter, the course from one noon position to the next and the rhumb from vaua are expressed, as here, in whole points of the conpass modified by that portion of a point $\left(\mathrm{II}^{\circ} \mathrm{I}^{\prime}\right)$ needed to define the course exactly to the minute. Thus, SE $/ \mathrm{I}^{\prime} 3^{\prime} 42^{\prime} \mathrm{E}$, expressed in azinuth, would be $123^{\circ} 45^{\circ}-3^{\circ} 42^{\prime}=120^{\circ} 03^{\prime}$. 


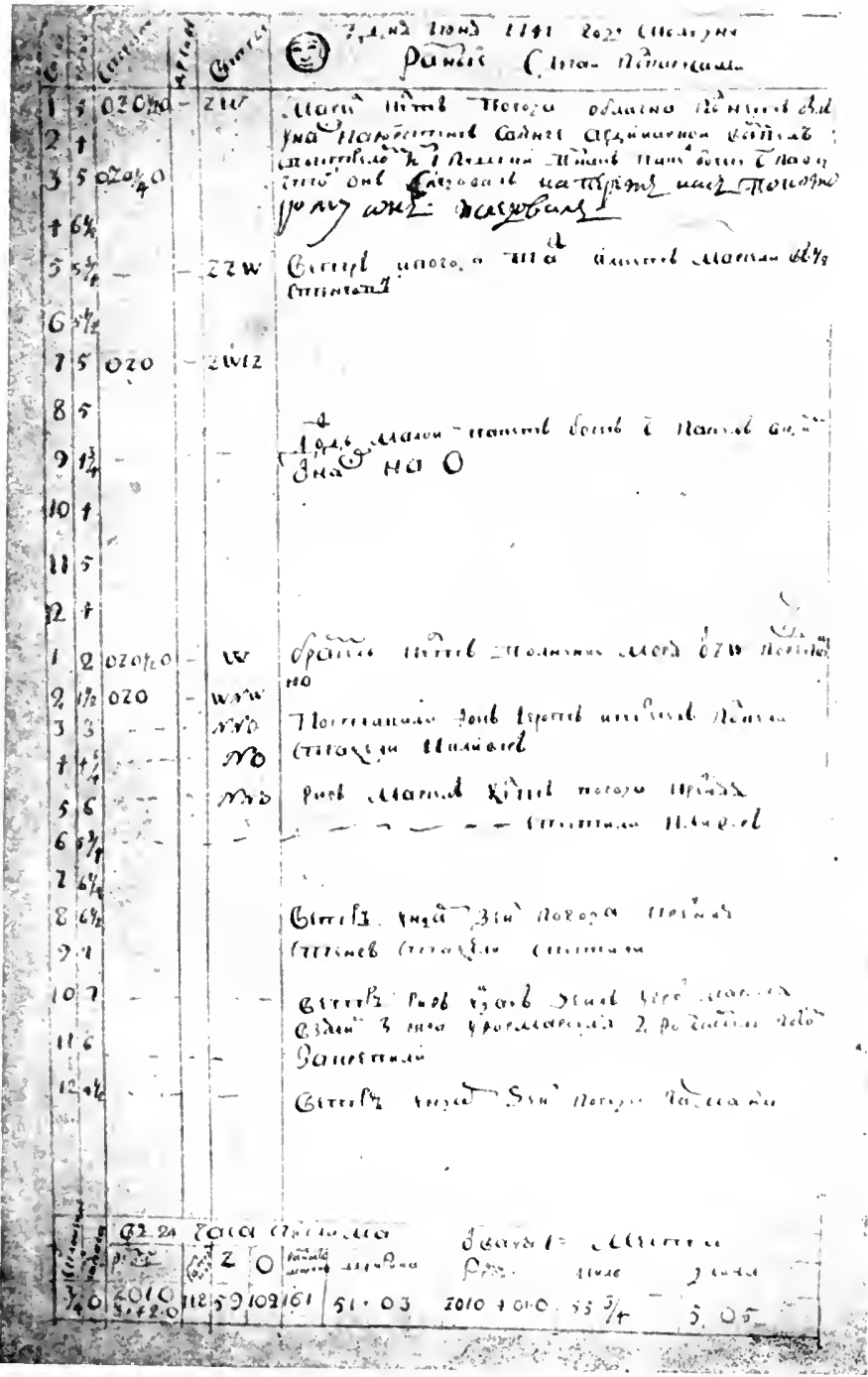

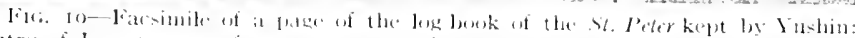

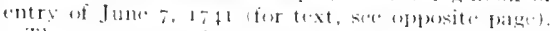

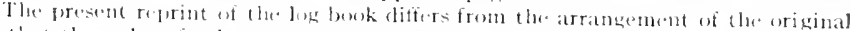

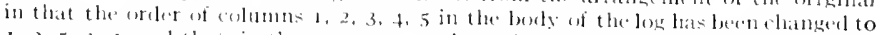
1. 2, 5. 3. - I, atel that, in the stmmary at the end, boxe's 1, 2. 3 mnder "From Vaua" lave beren transusedel to $2,1,3$. 

(3) June 8, I74I. Afler Midday

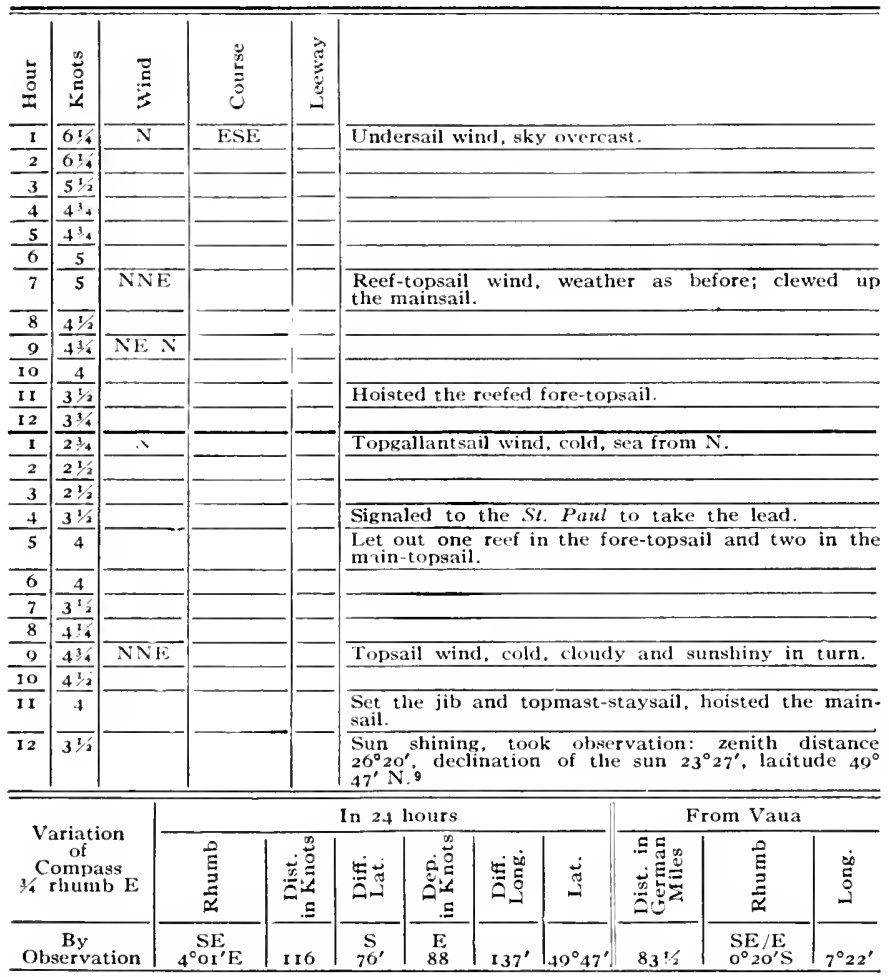

9 Khitrov gives zenith distance $26^{\circ} 59^{\prime}$, declination $23^{\circ} 27^{\prime}$, latitude $49^{\circ} 55^{\prime}$. This figure for latitude has been used on the chart (PI. I). 


\section{of June 9, I74I. After Midday}

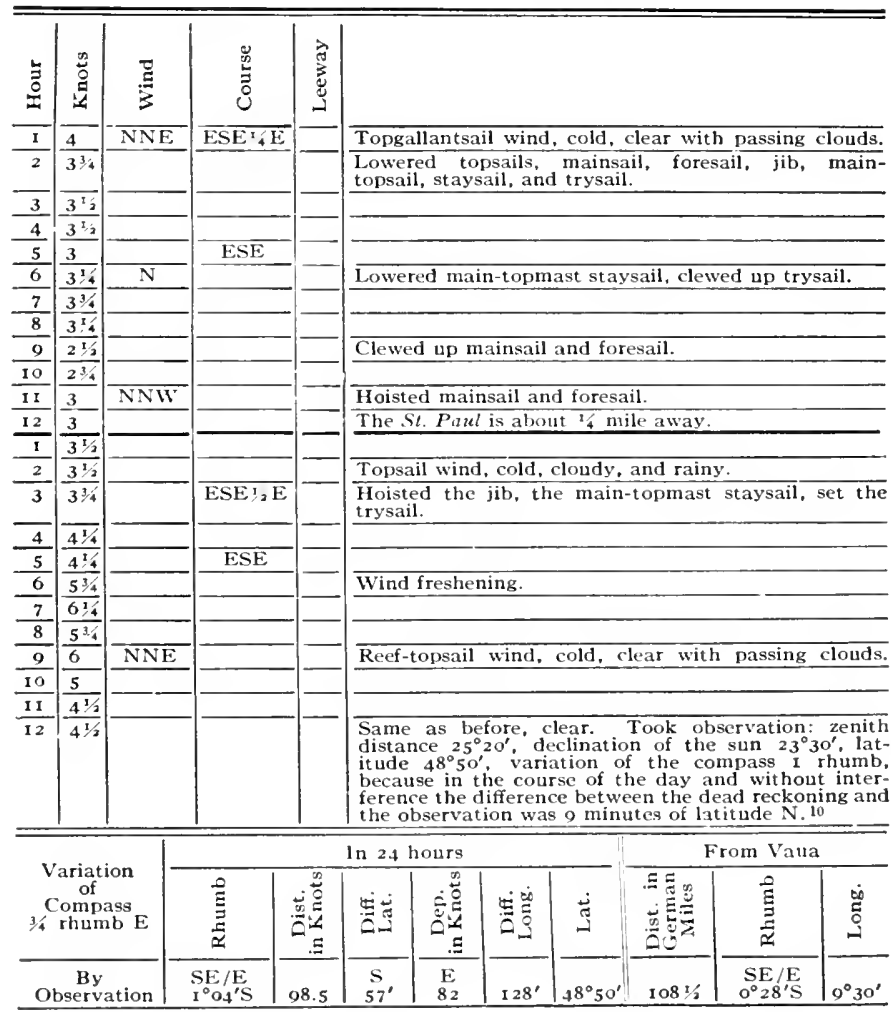

10 Khitrov gives the latitude as $48^{\circ} 48^{\prime}$. 
ఫ June 10, I741. After Midday

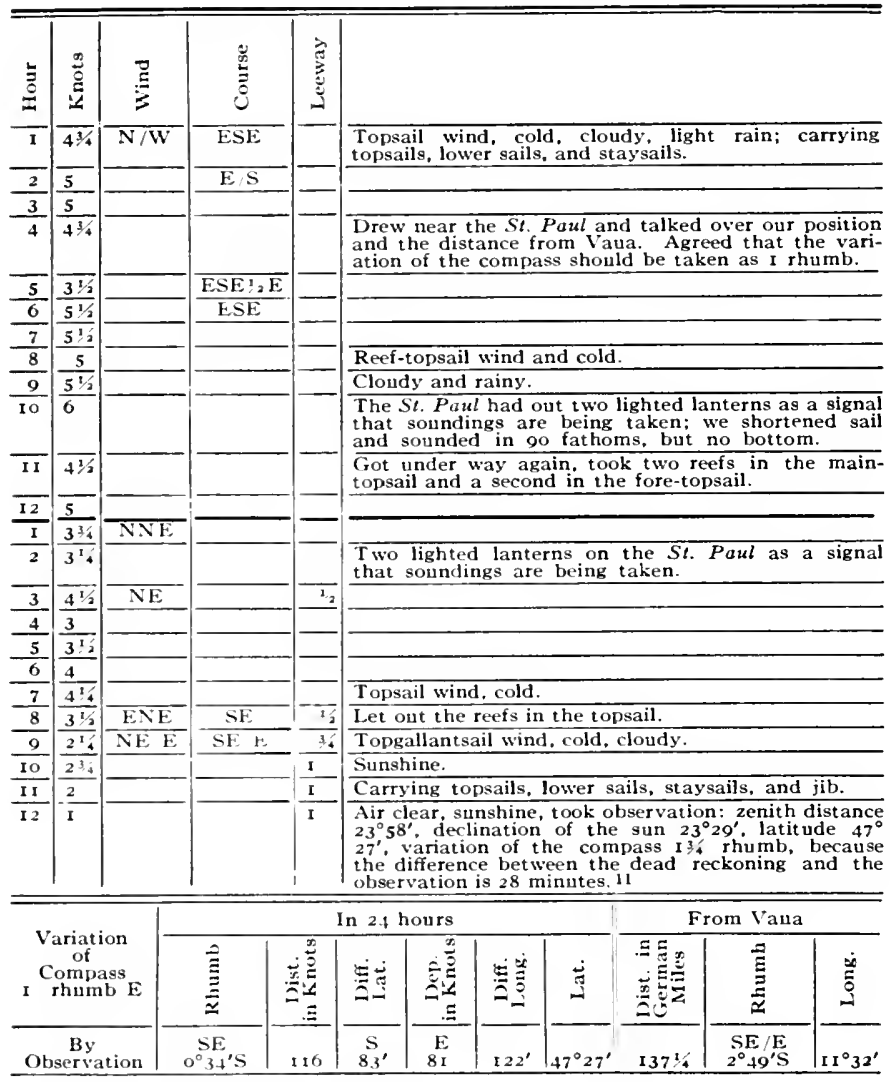

11 Khitrov gives the latitude as $47^{\circ} 29^{\prime}$. 


\section{2f June 11, I771. After Midday}

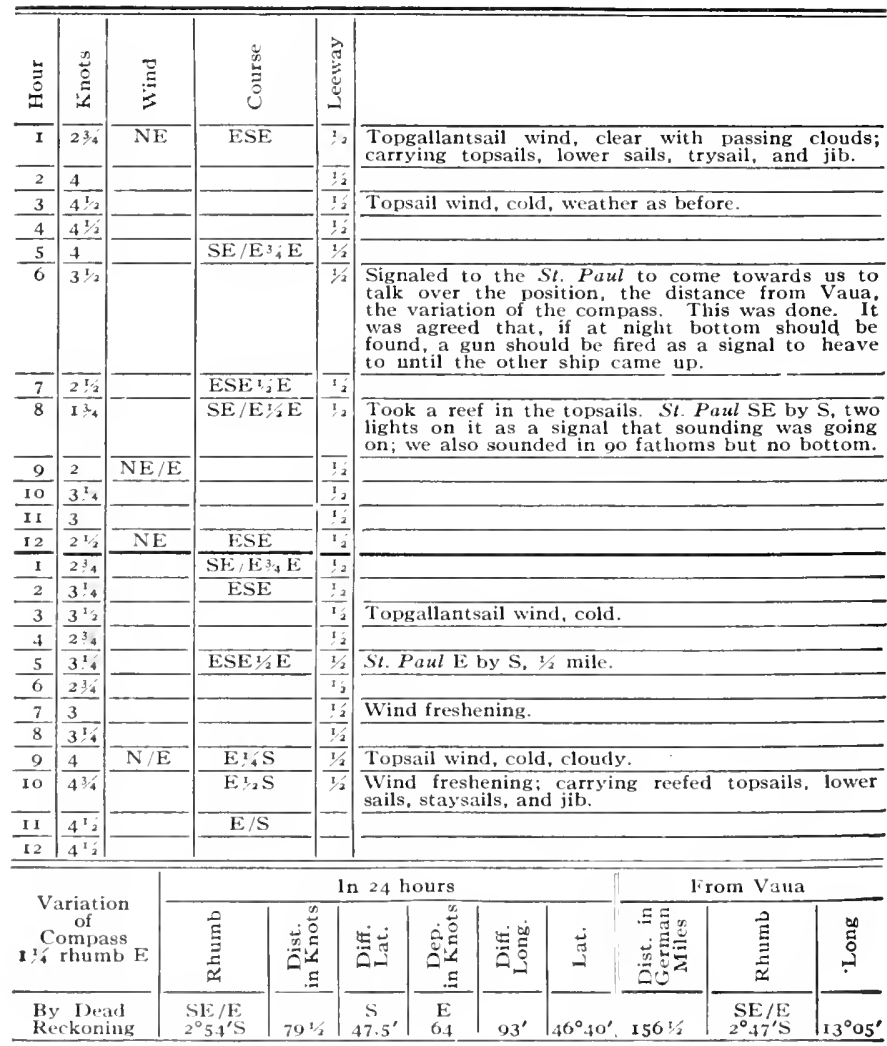


\& June 12, I74I. After Midday

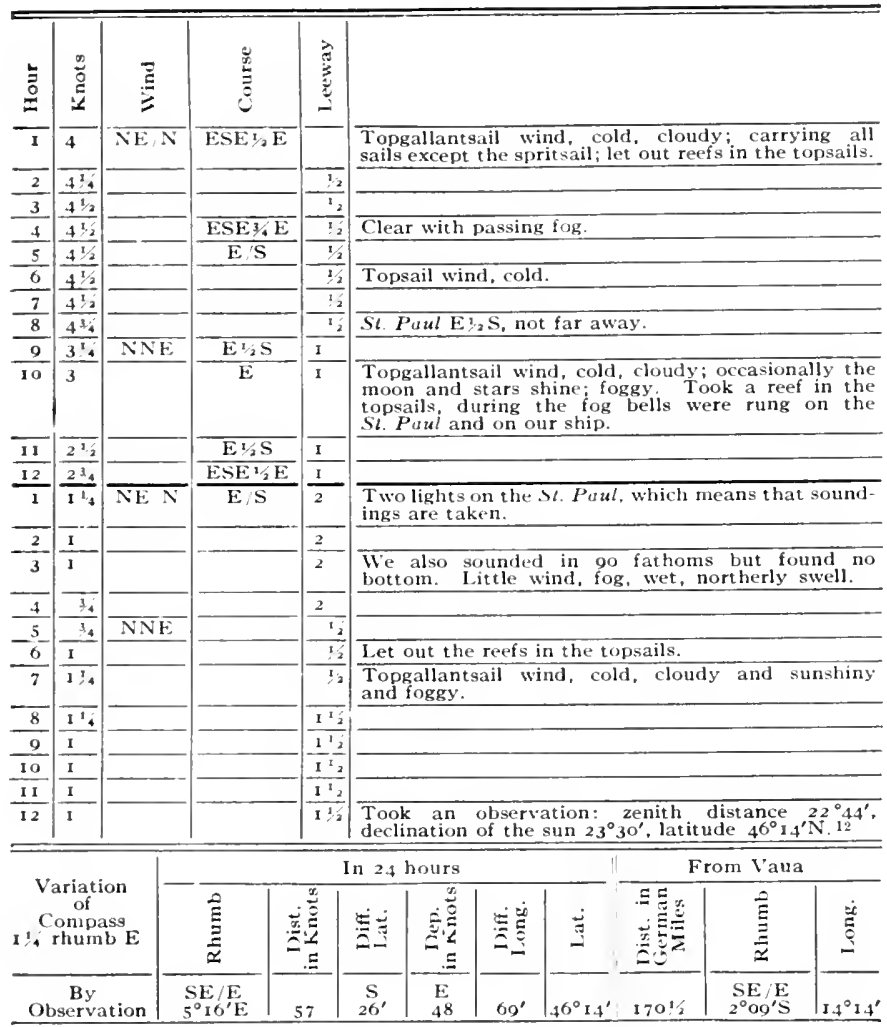

12 Khitrov gives the latitude as $46^{\circ} 09^{\prime}$. 


\section{b June 13, I74I. After Midday}

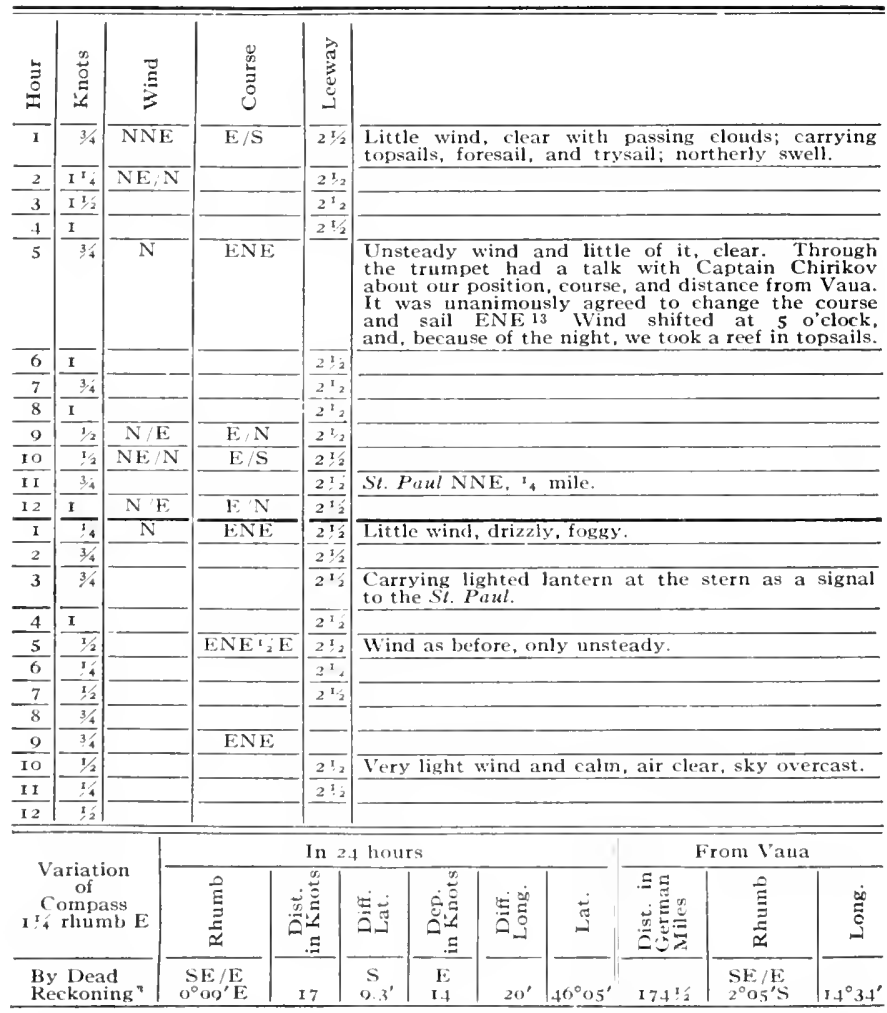

$13 \mathrm{E}$ by $\mathrm{N}$ according to Khitrov. This is the change in course agreed upon on May 4 (see 0.38 ) in case Juan de Gama liand were not found by the time tatitude $46^{\circ}$ lad been reached on the SE by E course from l'aua. See also the journal of the $\$ \%$. Paul, under June 13 (p. 286) and Chirikov's report, D. 313. 
June I4, I7tI. After Midday

\begin{tabular}{|c|c|c|c|c|c|c|c|c|c|c|c|}
\hline$\stackrel{5}{\stackrel{5}{3}}$ & 范 & $\underline{Z}$ & $\stackrel{3}{3}$ & 空 & & & & & & & \\
\hline 1 & 34 & $\mathrm{~N}$ & ENE & $21 / 3$ & $\begin{array}{l}\text { Very } \\
\text { swel }\end{array}$ & $\begin{array}{l}\text { light } \\
\text { carr }\end{array}$ & $\begin{array}{l}\text { ind a } \\
\text { ing to }\end{array}$ & $\begin{array}{l}\text { id unste } \\
\text { dsatls a }\end{array}$ & $\begin{array}{l}\text { ady, a } \\
\text { ad lowe }\end{array}$ & $\begin{array}{l}\text { thick, no } \\
\text { sails. }\end{array}$ & therly \\
\hline 2 & 3 & & & $21 / 2$ & & & & & & & \\
\hline 3 & $1 / 2$ & & & $2 x_{2}$ & & & & & & & \\
\hline+ & $1 / 2$ & & & $2 \frac{21}{2}$ & & & & & & & \\
\hline 5 & 34 & & & $2 ! / 2$ & & & & & & & \\
\hline 6 & 3 & & & $2 ! 2$ & & & & & & & \\
\hline 7 & 24 & $\mathrm{NE}$ & ESE & & $\begin{array}{l}\text { Ligh } \\
\text { and }\end{array}$ & $\begin{array}{l}\text { air } \\
\text { Irizzly }\end{array}$ & $\begin{array}{l}\text { calmi; } \\
\text { St. } P \text {; }\end{array}$ & $\begin{array}{l}\text { took a } \\
\text { ul N W }\end{array}$ & $\begin{array}{l}\text { reef in } \\
\text { by } w \text {. }\end{array}$ & topsails; & loudy \\
\hline 8 & 2 & & & $\overline{2^{12}}$ & & & & & & & \\
\hline 9 & -4 & & & $22_{2}$ & & & & & & & \\
\hline 10 & $1_{4}$ & NE H & $\mathrm{SE} \cdot \mathrm{E}$ & $\frac{2^{T_{2}}}{2}$ & & & & & & & \\
\hline 11 & $\mathrm{I}_{2}$ & ENE & $\mathrm{SE}$ & $21 / 2$ & & & & & & & \\
\hline 12 & $3 / 4$ & $E / S$ & $S E$ & $2 ! 3$ & $\begin{array}{l}\text { Caln } \\
\text { sail } \\
\text { runn }\end{array}$ & $\begin{array}{l}\text { i lowe } \\
\text { ind fo } \\
\text { ng fro }\end{array}$ & $\begin{array}{l}\text { red to } \\
\text { esail, } \\
\text { n N to }\end{array}$ & $\begin{array}{l}\text { sails or } \\
\text { llowed } \\
\text { S. St. }\end{array}$ & $\begin{array}{l}\text { caps, } \\
\text { for the } \\
\text { Paul }\end{array}$ & $\begin{array}{l}\text { lewed up } \\
\text { well whi } \\
\text { by N, }\end{array}$ & $\begin{array}{l}\text { main- } \\
\text { h was } \\
\text { mile. }\end{array}$ \\
\hline 1 & 5 & $\mathrm{ENE}$ & $\mathrm{SE}$ & $\sqrt{25}$ & & & & & & & \\
\hline 2 & 24 & $E / N$ & $\mathrm{SE}, \mathrm{S}$ & $\overline{2 ! 2}$ & $\begin{array}{l}\text { At t } \\
\text { fores }\end{array}$ & $\begin{array}{l}\text { mes li } \\
\text { iil and }\end{array}$ & $\begin{array}{l}\text { ht wi } \\
\text { mains }\end{array}$ & id and & fog; se & opsails, & oisted \\
\hline 3 & $\therefore 4$ & & & $22^{12}$ & & & & & & & \\
\hline 4 & $\overline{1}$ & $\mathrm{E} / \mathrm{N}$ & $\mathrm{NE}$ & I & $\begin{array}{l}\text { Ton } \\
\text { let } \\
\text { jib. }\end{array}$ & $\begin{array}{l}\text { allant } \\
\text { it the } \\
\end{array}$ & $\begin{array}{l}\text { ail wi } \\
\text { reefs }\end{array}$ & $\begin{array}{l}\text { ad. col } \\
\text { n topsa }\end{array}$ & $\begin{array}{l}\text { is tack } \\
\text { is, hoi }\end{array}$ & do staysa & 8 and \\
\hline 5 & $21 / 2$ & & $N^{1} / 2 E$ & & & & & & & & \\
\hline 6 & 3 & & & $I$ & Clon: & 1y. dri & zly, fi & ggy. & & & \\
\hline 7 & 23 & & & I & & & & & & & \\
\hline 8 & $2 ! 2$ & & & $I$ & & & & & & & \\
\hline 9 & 3 & & & & Tops & ail win & 1. cold & gloomy & & & \\
\hline 10 & $21 / 2$ & $\mathrm{E}$ & $\mathrm{NE} \mathrm{E}_{2} \mathrm{H}$ & & & & & & & & \\
\hline II & $2 ! 4$ & $E N$ & $\mathrm{NE}$ & $I$ & Top & allant & ail wi & d, clo & $\mathrm{ddy}, \mathrm{fc}$ & y, drizz & \\
\hline & $2 ! 4$ & & & 1 & & & & & & 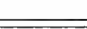 & \\
\hline \multirow{2}{*}{\multicolumn{3}{|c|}{$\begin{array}{c}\text { Variation } \\
\text { of } \\
\text { Compass } \\
\text { I } 4 \text { rhumb E }\end{array}$}} & \multicolumn{9}{|c|}{ In 24 hours } \\
\hline & & & 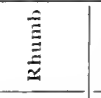 & 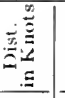 & 芫 & 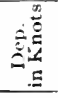 & 至 & ت્่ & 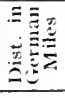 & $\stackrel{\vec{z}}{\underline{z}}$ & $\stackrel{0}{\Xi}$ \\
\hline $\begin{array}{l}\mathrm{By} \\
\mathrm{Re} \\
\end{array}$ & $\begin{array}{l}\text { Dea } \\
\text { ckon }\end{array}$ & & $\begin{array}{c}\text { NNE } \\
2^{\circ}{ }^{4} 6^{\prime} N \\
\end{array}$ & 20 & $\begin{array}{r}\mathrm{N} \\
18.5^{\circ} \\
\end{array}$ & $\begin{array}{c}E \\
6.5 \\
\end{array}$ & $9^{\prime}$ & $46^{\circ} 23^{\prime}$ & $1723 / 4$ & $\begin{array}{l}\mathrm{SE} / \mathrm{E} \\
0^{\circ}+0^{\prime} \mathrm{S}\end{array}$ & $14^{\circ} 43^{\prime}$ \\
\hline
\end{tabular}


(3) June 15, I74I. After Midday

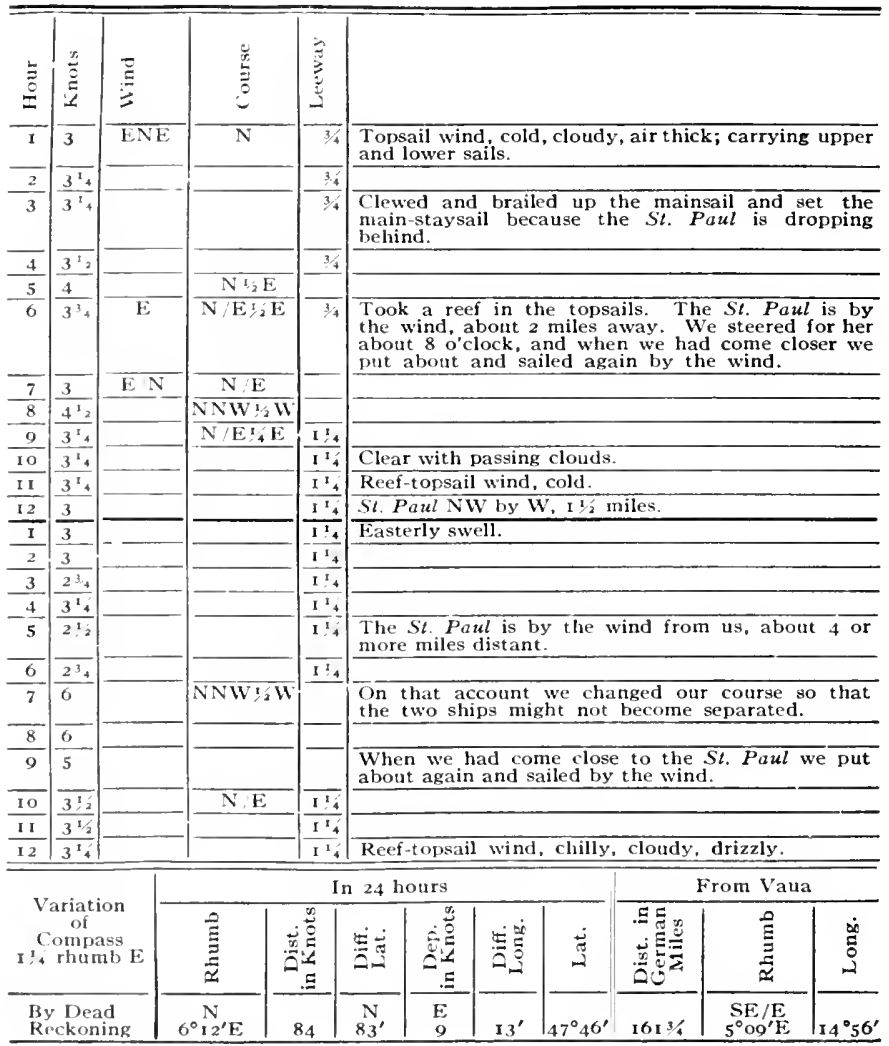


of June 16, 1741. Afler Midday

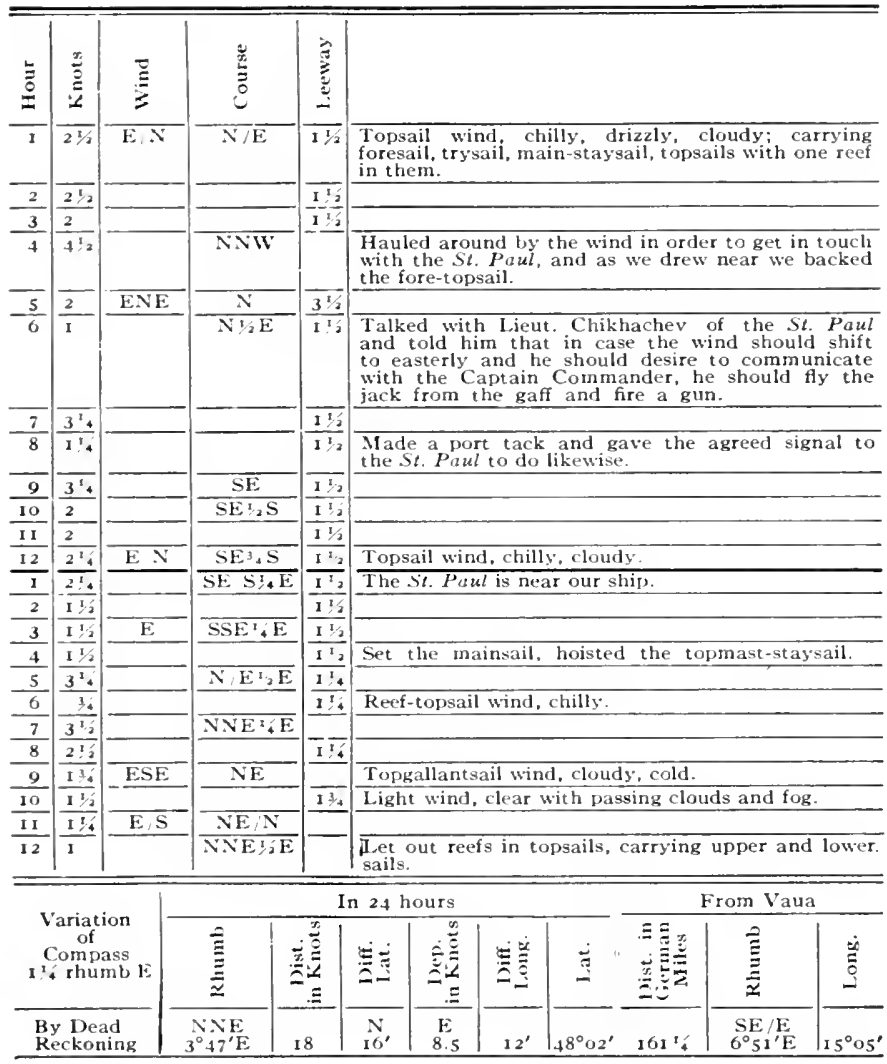


§ June 17, 1741. After Midday

\begin{tabular}{|c|c|c|c|c|c|c|c|c|c|c|c|c|}
\hline 产 & 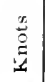 & $\stackrel{\Xi}{E}$ & 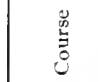 & & 崖 & & & & & & & \\
\hline 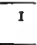 & $\overline{134}$ & $\mathrm{E}$ & NNE, 4 & & 2 & $\begin{array}{l}\text { Top } \\
\text { wet; }\end{array}$ & $\begin{array}{l}\text { lllants } \\
\text { arryir }\end{array}$ & $\begin{array}{l}\text { ail wi } \\
\text { g tops: }\end{array}$ & $\begin{array}{l}\text { ind, chi } \\
\text { ails, forc }\end{array}$ & $\begin{array}{l}\text { illy, clo } \\
\text { esail, try }\end{array}$ & $\begin{array}{l}\text { foggy } \\
\text { il, and sta }\end{array}$ & $\begin{array}{l}\text { and } \\
\text { iysails. }\end{array}$ \\
\hline 2 & $2, \frac{19}{4}$ & ESE & $\mathrm{NE}$ & & 2 & & & & & & & \\
\hline 3 & 18 & & & & 2 & & & & & & & \\
\hline 4 & $2^{1} 4$ & & $N E_{1}, N$ & & 2 & Clev & ed and & braile & d up the & foresail & & \\
\hline 5 & 2 & & $\mathrm{NE} / 2 \mathrm{E}$ & & 2 & $\begin{array}{l}\text { Laid } \\
\text { stay }\end{array}$ & $\begin{array}{l}\text { the } \mathrm{m} \\
\text { ails an }\end{array}$ & $\begin{array}{l}\text { ain-tol } \\
\text { d jib i }\end{array}$ & $\begin{array}{l}\text { sail on } \\
\text { n order }\end{array}$ & $\begin{array}{l}\text { the topl } \\
\text { to wait }\end{array}$ & $\begin{array}{l}\text { ist, lower } \\
\text { the St. }\end{array}$ & $\begin{array}{l}\text { ed the } \\
\text { Paul. }\end{array}$ \\
\hline 6 & I & & $\mathrm{NE}_{4} / \mathrm{H}$ & & 2 & & & & & & & \\
\hline 7 & $\mathrm{I}_{2}$ & & & & 2 & & & & & & & \\
\hline 8 & 34 & & & & 2 & $\begin{array}{l}\text { Too } \\
\text { topr } \\
\end{array}$ & $\begin{array}{l}\text { a reef } \\
\text { ast-st: }\end{array}$ & $\begin{array}{l}\text { in the } \\
\text { ysail. }\end{array}$ & topsails & , set for & ail, hoiste & d fore- \\
\hline 9 & $1 \frac{12}{2}$ & & & & 2 & & & & & & & \\
\hline 10 & 2 & $\mathrm{SE} \mathrm{H}$ & $\overline{\mathrm{NE} / \mathrm{E}^{1 / 2}}$ & & $11 / 2$ & Set & $\mathrm{ib}$ and & main-t & opmast & -staysail & & \\
\hline II & 3 & $\mathrm{SE}$ & ENE & & $11 / 2$ & foi & ted ma & nsail. & & & & \\
\hline 12 & $\overline{2^{34}}$ & & & & & $\begin{array}{l}\text { Low } \\
\text { crea } \\
\text { fore } \\
\text { in } 9 \\
\text { St } 9 \\
\end{array}$ & $\begin{array}{l}\text { ered ji } \\
\text { red, cle } \\
\text { topsail } \\
\text { fathor } \\
\text { aul be } \\
\end{array}$ & $\begin{array}{l}\text { and } \\
\text { wed u } \\
\text { heave } \\
\text { s, fille } \\
\text { ins NN }\end{array}$ & $\begin{array}{l}\text { staysai } \\
\text { p main } \\
\text { d the le. } \\
\text { d fore-t } \\
\text { W. }\end{array}$ & $\begin{array}{l}\text { is becal } \\
\text { sail and } \\
\text { ad witho } \\
\text { opsail. a }\end{array}$ & $\begin{array}{l}\text { the wi } \\
\text { oresail, } \\
\text { finding } \\
\text { set the f }\end{array}$ & $\begin{array}{l}\text { nd in- } \\
\text { owered } \\
\text { ottom } \\
\text { oresail. } \\
\end{array}$ \\
\hline $\bar{I}$ & $\overline{23,4}$ & & $\mathrm{ENE}_{2}$ & & $\overline{1 ! 2}$ & & & & & & & \\
\hline 2 & $3 / 2$ & $\mathrm{SE} / \mathrm{I}$ & NE,E & & & & & & & & & \\
\hline 3 & $21 / 2$ & SE & & & $1 \%$ & & & & & & & \\
\hline 4 & $2 \frac{114}{4}$ & & & & $\mathrm{I}^{1}$ & & & & & & & \\
\hline 5 & $21 / 3$ & & & & $\overline{I ! 2}$ & Set & he mai & nsail. & & & & \\
\hline 6 & 3 & & & & & & & & & & & \\
\hline 7 & $21 / 2$ & $\mathrm{SE} / \mathrm{S}$ & $\mathrm{ENE}^{3}$ & & $1 \frac{1}{2}$ & Top & Gallant & ail win & 1. chilly & y, cloudy & damp. & \\
\hline 8 & $2 T_{4}$ & & & & $r^{2}$ & & & & & & & \\
\hline 9 & $1 \div$ & & $\mathrm{E} / \mathrm{N}$ & & 2 & & & & & & & \\
\hline 10 & 154 & & & & & igh & $t$ wind & weath & er as be & fore. & & \\
\hline II & $I$ & SE & ENE $1 / 3$ & & & $\begin{array}{l}\text { Car } \\
\text { The }\end{array}$ & $\begin{array}{l}\text { ying u } \\
\text { topsail }\end{array}$ & $\begin{array}{l}\text { per an } \\
\text { have }\end{array}$ & $\begin{array}{l}\text { d lower } \\
\text { a reef in }\end{array}$ & $\begin{array}{l}\text { sails ex } \\
\text { nthem. }\end{array}$ & pt the sp & ritsail. \\
\hline 12 & {$[3 / 4$} & $\mathrm{SE} / \mathrm{F}$ & $\mathrm{NE} / \mathrm{H}$ & & 2 & & & & & & & \\
\hline \multirow{2}{*}{\multicolumn{3}{|c|}{$\begin{array}{c}\text { Variation } \\
\text { of } \\
\text { Compass } \\
\text { I t rhumb E }\end{array}$}} & \multicolumn{7}{|c|}{$\ln 2.4$ hours } & \multicolumn{3}{|c|}{ From Vaua } \\
\hline & & & 总 & 然 & 垔 & 曹示 & 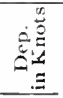 & 方气 & $\stackrel{5}{5}$ & 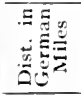 & 总 & $\stackrel{\infty}{\Xi}$ \\
\hline \multicolumn{3}{|c|}{$\begin{array}{l}\text { By Dead } \\
\text { Reckoning }\end{array}$} & $\begin{array}{l}\text { NEE } \\
I^{\circ} 27^{\prime} N \\
\end{array}$ & 44 & & $\begin{array}{r}\mathrm{N} \\
25.4^{\prime}\end{array}$ & $\begin{array}{r}E \\
36 \\
\end{array}$ & $54^{\prime}$ & $48^{\circ} 27^{\prime}$ & $165^{3 / 4}$ & $\begin{array}{c}\mathrm{ESE} \\
1^{\circ} 25^{\prime} \mathrm{S} \\
\end{array}$ & $15^{\circ} 39$ \\
\hline
\end{tabular}




\section{2f June 18, 1741. After Midday}

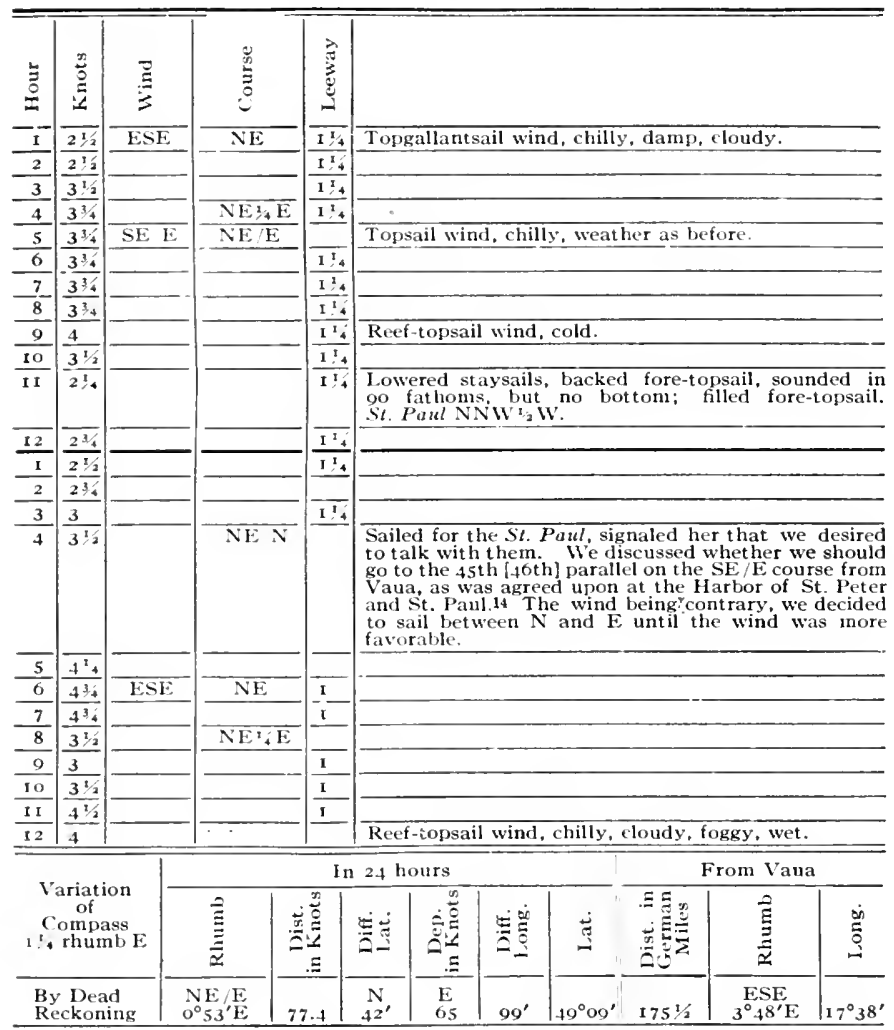

14 Khitrov"s journat: "When we drew nearer we inguired whether they still agreed to go on the northerly course since we had not yet gone in that direction as far as we had planned. They were of the opinion that this course should be followed when the wind was fair and, when it was not, we should keep more easterly." See also the journal of the St. Paul under June 18 (p. 287). 


\section{June I9, I741. After Midday}

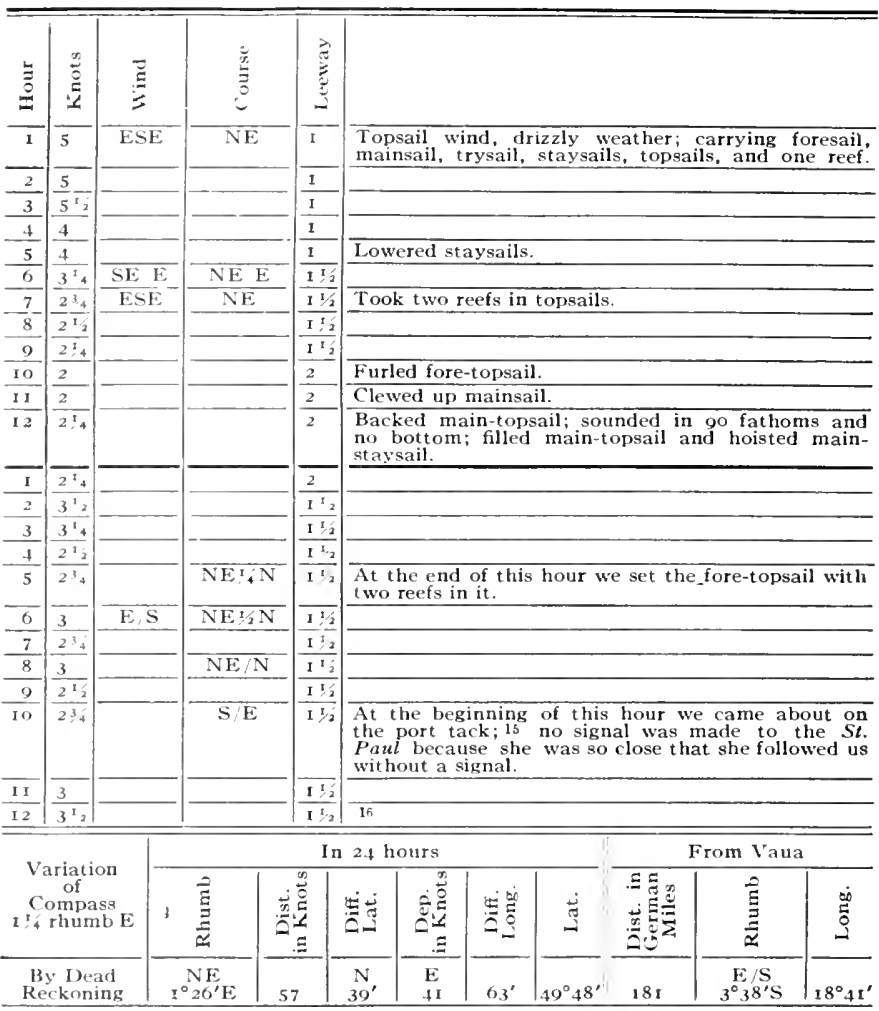

15 Called "backboard" tack in the original.

16 According to Khitrov an observation taken at noon gave latitude $50^{\circ} 13^{\prime}$, but owing to the haze on the horizon it was not used. 


\section{Ђ June 20, 1741. After Midday}

\begin{tabular}{|c|c|c|c|c|c|c|c|c|c|c|c|}
\hline$\stackrel{5}{3}$ & 总 & 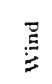 & 窇 & 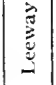 & & & & & & & \\
\hline I & 3 & $\mathrm{E} / \mathrm{S}$ & S/E & 2 & $\begin{array}{l}\text { Stro } \\
\text { lowe } \\
\text { in m }\end{array}$ & $\begin{array}{l}\text { g reef } \\
\text { ed ma } \\
\text { ain-tor }\end{array}$ & $\begin{array}{l}\text { opsai } \\
\text {-stay } \\
\text { ail. }\end{array}$ & $\begin{array}{l}\text { wind, } \\
\text { ail, set }\end{array}$ & $\begin{array}{c}\text { wet; f } \\
\text { mainsai }\end{array}$ & $\begin{array}{l}\text { ed fore- } \\
\text { took thr }\end{array}$ & $\begin{array}{l}\text { psail, } \\
\text { reefs }\end{array}$ \\
\hline 2 & 234 & & & 2 & & & & & & & \\
\hline 3 & $21 / 2$ & & & 2 & & & & & & & \\
\hline 4 & $21 / 2$ & & & 2 & & & & & & & \\
\hline 5 & 231 & & & 2 & Und & rsail $w$ & nd, ga & & & & \\
\hline 6 & 2 & & & $2 \%$ & $\begin{array}{l}\text { Furl } \\
\text { tryse } \\
\text { tanc } \\
\text { mair }\end{array}$ & $\begin{array}{l}\mathrm{d} \\
\mathrm{ma} \\
\text { from } \\
\text { frays }\end{array}$ & $\begin{array}{l}\text {-tops } \\
\text { ause } \\
\text { is, we } \\
\text { l. }\end{array}$ & $\begin{array}{l}\text { il, car } \\
\text { he St. } \\
\text { clewed }\end{array}$ & $\begin{array}{l}\text { rying f } \\
\text { Paul is } \\
\text { up ma }\end{array}$ & $\begin{array}{l}\text { esail, m } \\
\text { onsideral } \\
\text { ail and }\end{array}$ & $\begin{array}{l}\text { insail, } \\
\text { e dis- } \\
\text { oisted }\end{array}$ \\
\hline 7 & 2 & & & $2 T_{2}^{\prime}$ & & & & & & & \\
\hline 8 & 2 & $E$ & $\mathrm{~S} \mathrm{E}_{2}=\mathrm{E}$ & 3 & & & & & & & \\
\hline 9 & 234 & & & 3 & & & & & & & \\
\hline IO & $11 / 2$ & & & $41 / 2$ & $\begin{array}{l}S t .1 \\
\text { with } \\
\text { of } t h\end{array}$ & $\begin{array}{l}\text { aul N } \\
\text { passin } \\
\text { e fores }\end{array}$ & $\begin{array}{l}2 \mathrm{~m} \\
\text { cloll }\end{array}$ & $\begin{array}{l}\text { iles; hov } \\
\text { ds; hat }\end{array}$ & $\begin{array}{l}\text { vered } m \\
\text { led up }\end{array}$ & $\begin{array}{l}\text { n-staysai } \\
\text { e weath }\end{array}$ & $\begin{array}{l}\text { clear } \\
\text { clew }\end{array}$ \\
\hline II & $1 \%$ & & & 42 & & & & & & & \\
\hline 12 & 114 & & & $4^{x_{2}^{\prime}}$ & & & & & & & \\
\hline$x$ & I & & $\mathrm{SE}, \mathrm{S}$ & $5^{12}$ & $\begin{array}{l}S t . I \\
\text { stay }\end{array}$ & $\begin{array}{l}\text { ul no } \\
\text { iil, ho }\end{array}$ & $\begin{array}{l}\text { in sig } \\
\text { eto; }\end{array}$ & $\begin{array}{l}\text { at. Cle } \\
\text { ale blow }\end{array}$ & $\begin{array}{l}\text { wed } u p \\
\text { ing. } 17\end{array}$ & resail, s & main- \\
\hline 2 & I & & & $55^{2}$ & & & & & & & \\
\hline 3 & $11 / 2$ & & & $5 / 2$ & & & & & & & \\
\hline 4 & 5 & & NW & & $\begin{array}{l}\text { Got } \\
\text { of ff } \\
\text { o'clo } \\
\text { and } \\
\end{array}$ & $\begin{array}{l}\text { under } \\
\text { ading } \\
\text { k; fa } \\
\text { nain-s }\end{array}$ & $\begin{array}{l}\text { lay, h } \\
\text { he St } \\
\text { ed to } \\
\text { aysail }\end{array}$ & $\begin{array}{l}\text { isted fo } \\
\text { Paul } \\
\text { sight h }\end{array}$ & $\begin{array}{l}\text { resail, } \\
\text { which d } \\
\text { er; hov }\end{array}$ & $\begin{array}{l}\text { led with } \\
\text { appeared } \\
\text { to under }\end{array}$ & $\begin{array}{l}\text { view } \\
\text { at io } \\
\text { rysail }\end{array}$ \\
\hline 5 & $11 / 2$ & & $\mathrm{SE}: 2 \mathrm{~S}$ & $51 / 2$ & & & & & & & \\
\hline 6 & I & & & 513 & & & & & & & \\
\hline 7 & 1 & & & $51 / 2$ & & & & & & & \\
\hline 8 & $I$ & & & $5 \frac{1}{2}$ & & & & & & & \\
\hline 9 & $\mathrm{I}$ & & & $51 / 2$ & Stro & g reef & inder: & ail wind & & & \\
\hline 10 & $I$ & & & $5 \%$ & & & & & & & \\
\hline II & $I$ & & & $5 \%$ & Clea & with & assing & clouds. & & & \\
\hline 12 & $\mathrm{I}$ & & & $53 / 2$ & & & & & & & \\
\hline \multirow{2}{*}{\multicolumn{3}{|c|}{$\begin{array}{c}\text { Variation } \\
\text { of } \\
\text { Compass } \\
\text { I r r tumb E }\end{array}$}} & \multicolumn{6}{|c|}{ In 24 hours } & & om Vauz & \\
\hline & & & $\underset{\Xi}{\tilde{\Xi}}$ & 点 & 电泀 & $\begin{array}{r}\stackrel{n}{0} \\
\vdots \\
0\end{array}$ & 象晃 & షే & 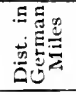 & E & 봉 \\
\hline $\begin{array}{l}\mathrm{B} \\
\mathrm{R} \\
\end{array}$ & $\begin{array}{l}\text { De } \\
\text { kor }\end{array}$ & & $\begin{array}{l}S W / \mathrm{S} \\
3^{\circ} 29^{\prime} \mathrm{V}\end{array}$ & 34 & $\begin{array}{c}\mathrm{S} \\
26.4^{\prime} \\
\end{array}$ & $\begin{array}{c}\text { WV } \\
22.5 \\
\end{array}$ & $35^{\prime}$ & $49^{\circ} 22^{\prime}$ & $178 \div$ & $\begin{array}{c}\mathrm{ESE} \\
5^{\circ} 12^{\prime} \mathrm{E}\end{array}$ & $18^{\circ} 06^{\prime}$ \\
\hline
\end{tabular}

17 Khitrov's journal: "At the beginning [of ro o'clock at night] we lowered the mainstaysail, made fast the fore-sheet [in order to wait] for the $S t$. Paul because she is from us [?] and not near. At I o'clock she disappeared from sight. We carried the foresail with the fore-sheet fast; mizzen sail against the mast. At 1 o'clock at the change of the watch I learned that the St. Paul was out of sight and therefore clewed up the foresail and laid to under the mizzen- and main-staysails. At 3:30 we hoisted the foresail and sailed in the direction [?] in search of the $S t$. Paul, because she had been seen on that course; at $4: 30$ we furled the foresail and laid to under the main-staysail and mizzensail. Undersail wind."

On the separation of the two vessels sce also Waxel's report, p. 271; the journal of the

St. Paul under June 20 (p. 287); and Chirikov's report. p. $3 \pi_{3}$. 
June 21, I74I. After Midday

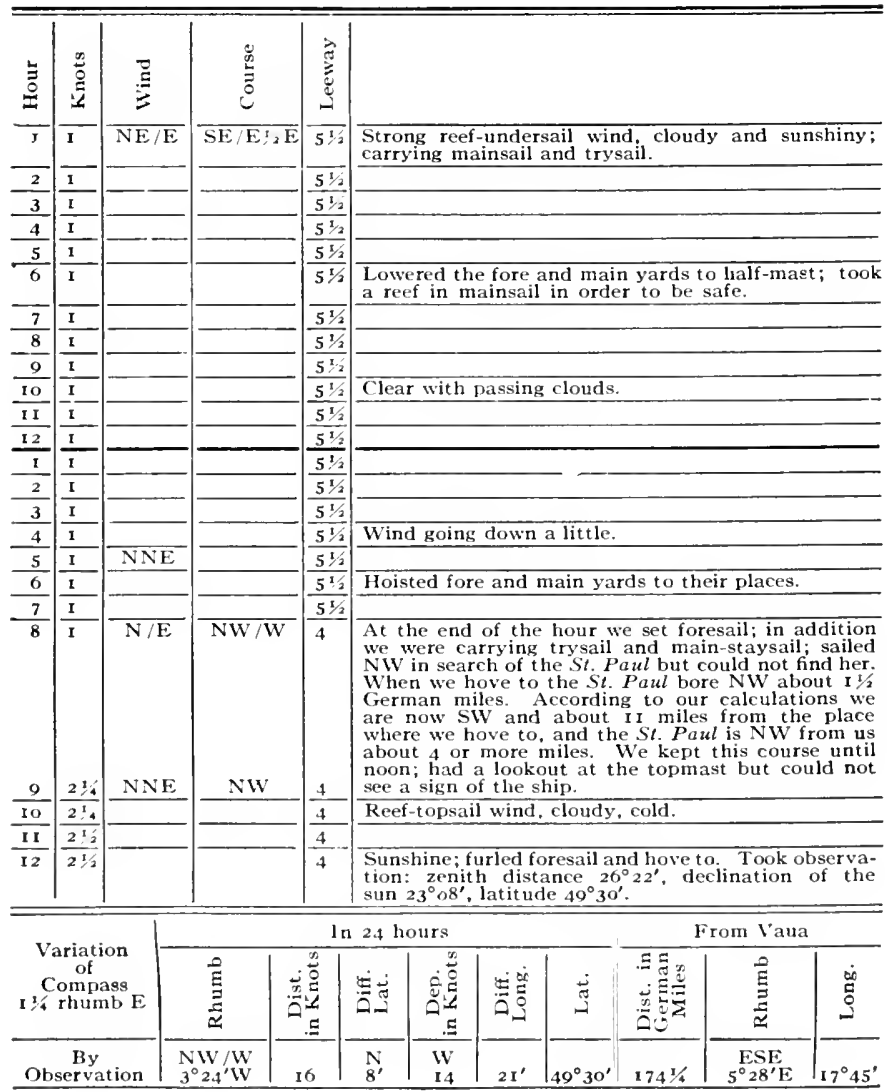




\section{(3) June 22,174I. After Midday}

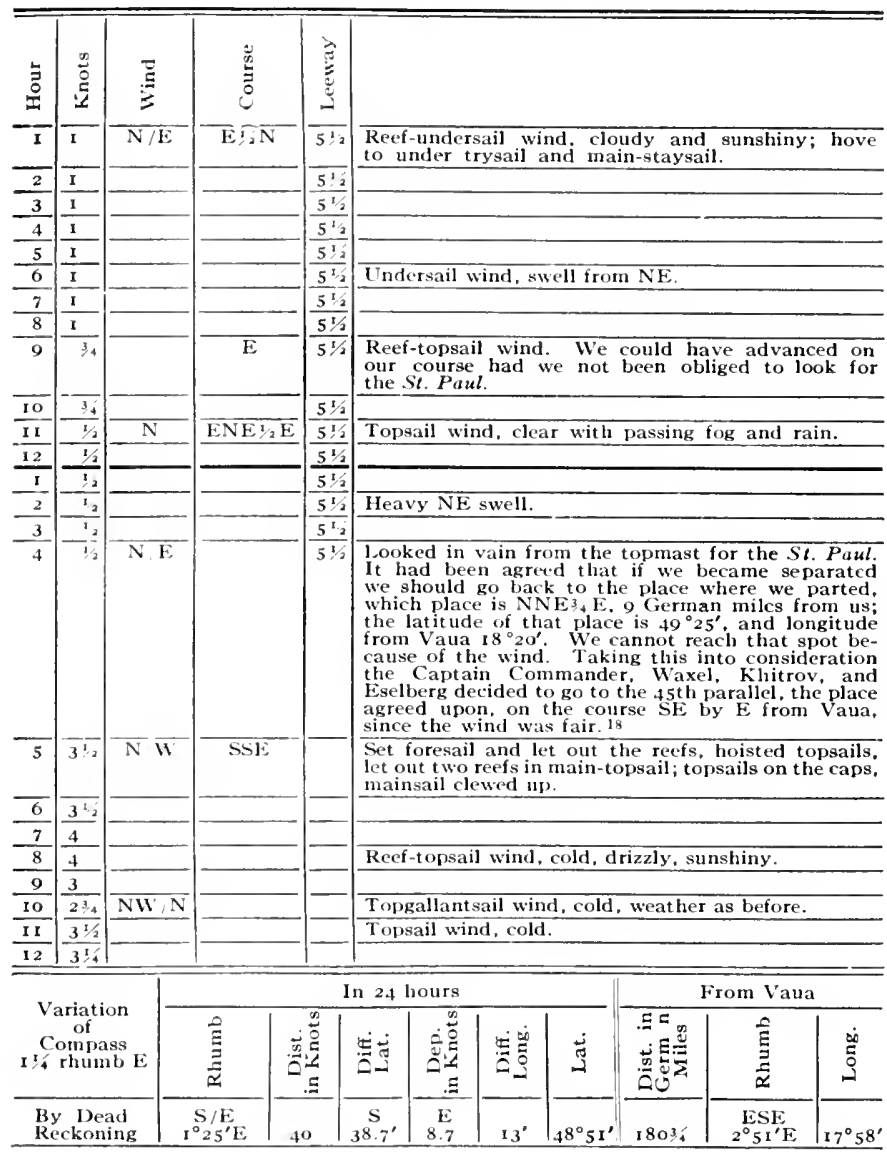

18 Khitrov's journal: A A. M. "We looked in vain from the crosstrees for the $S t$. Paul. According to the understanding we were to go to the place where she was last seen by us, which place was by our reckoning NNE3 E. 9 German miles distant, but head winds prevented our doing so. On that account the Captain Commander decided to go to latitude $46^{\circ} \mathrm{N}$, on the rhumb from Vaua SE by E. We cleared the foresail and stcered SSE, lowered the foreyards, set both topsails reefed." 
\& June 23, I7t1. After Midday

\begin{tabular}{|c|c|c|c|c|c|c|c|c|c|c|c|}
\hline$\stackrel{5}{3}$ & 苟 & $\stackrel{\vec{E}}{3}$ & 峁 & 峞 & & & & & & & \\
\hline $\bar{I}$ & $3 x^{x}$ & $\mathrm{~N} / \mathrm{W}$ & SSE & & $\begin{array}{l}\text { Tops } \\
\text { fores }\end{array}$ & $\begin{array}{l}\text { ail win } \\
\text { ail and }\end{array}$ & $\begin{array}{l}\text { id, clea } \\
\text { mains }\end{array}$ & $\begin{array}{l}\text { ir with } \\
\text { iil; } \mathrm{NE}\end{array}$ & $\begin{array}{l}\text { passing } \\
\text { swell. }\end{array}$ & louds; ca & rrying \\
\hline 2 & 234 & & & & & & & & & & \\
\hline 3 & $23 / 4$ & & & & & & & & & & \\
\hline 4 & $3 \underline{1}$ & & & & & & & & & & \\
\hline 5 & $3 \sqrt{3 / 4}$ & & $\mathrm{~s}$ & & $\begin{array}{l}\text { At tl } \\
\text { Khit }\end{array}$ & $\begin{array}{l}\text { lis hot } \\
\text { rov agr }\end{array}$ & $\begin{array}{l}r \text { the } C \\
\text { eed to }\end{array}$ & $\begin{array}{l}\text { aptain } \\
\text { sail the }\end{array}$ & $\begin{array}{l}\text { Comman } \\
\text { course in }\end{array}$ & $\begin{array}{l}\text { der, Waxe } \\
\text { dicated. }\end{array}$ & , and \\
\hline 6 & $4 \frac{1 / 4}{4}$ & & & & & & & & & & \\
\hline 7 & $3 \frac{21 / 2}{4}$ & & & & & & & & & & \\
\hline 8 & $23 \frac{14}{4}$ & $\mathrm{NW}$ & & & Topg & rallants & ail win & d. cold & 1 ; hoisted & topsails. & \\
\hline 9 & 35 & & & & Set $I$ & nainsai & and $f c$ & resail. & & & \\
\hline 10 & $2 ! 2$ & & & & & & & & & & \\
\hline 11 & 4 & & & & Hois & ted sta & ysail an & id jib. & & & \\
\hline 12 & $\sqrt{3+4}$ & & & & $\begin{array}{l}\text { Soun } \\
\text { in m }\end{array}$ & $\begin{array}{l}\text { ded in } \\
\text { ain-top }\end{array}$ & $\begin{array}{l}90 \text { fath } \\
\text { sail. }\end{array}$ & oms, b & ut no bot & om; let o & it reef \\
\hline $\mathrm{I}$ & 33 & $\mathrm{M} / \mathrm{N}$ & & & & & & & & & \\
\hline 2 & $3 \%$ & & & & Drizz & zly, we & t. clear & with $p$ & assing $\mathrm{clo}$ & ids. & \\
\hline 3 & 4 & & & & & & & & & & \\
\hline 4 & $4^{5 / 2}$ & & & & $\begin{array}{l}\text { Tops } \\
\text { light }\end{array}$ & $\begin{array}{l}\text { ail wir } \\
\text { ed lant }\end{array}$ & $\begin{array}{l}\text { d and } \\
\text { erns. }\end{array}$ & cold; & all sails & eefed; ca & rrying \\
\hline 5 & $4 \overline{4^{7 / 2}}$ & & $\mathrm{~S} / \mathrm{WV}$ & & Let 0 & out the & spritsa & & & & \\
\hline 6 & 5 & & & & & & & & & & \\
\hline 7 & $\overline{43 / 4}$ & IV & & & $\begin{array}{l}\text { Stror } \\
\text { topsa }\end{array}$ & $\begin{array}{l}\text { ig tops } \\
\text { iils. }\end{array}$ & ail win & d, cold & ; let out & the last $r$ & efs in \\
\hline 8 & 5 & & & & & & & & & & \\
\hline 9 & 4 4, & & & & Clou & $\mathrm{ds}$, fog & gy, wet & & & & \\
\hline 10 & $4^{1 / 2}$ & & & & & & & & & & \\
\hline 11 & 35 & & & & Occa & sional & unshin & & & & \\
\hline 12 & 5 & & & & Reef & topsai & wind, & cold, w & veather as & before. & \\
\hline \multirow{2}{*}{\multicolumn{3}{|c|}{$\begin{array}{c}\text { Variation } \\
\text { of } \\
\text { Compass } \\
\text { I, rhumb E }\end{array}$}} & \multicolumn{6}{|c|}{ In 24 hours } & \multicolumn{3}{|c|}{ From Vaua } \\
\hline & & & $\underset{\underline{E}}{\stackrel{E}{E}}$ & 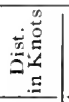 & 它票 & 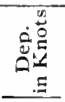 & 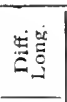 & 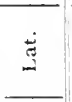 & 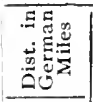 & E & है \\
\hline \multicolumn{3}{|c|}{$\begin{array}{l}\text { By Dead } \\
\text { Reckoning }\end{array}$} & $\begin{array}{l}\mathrm{S} / \mathrm{W} \\
4^{\circ} 0^{\prime} \mathrm{W}\end{array}$ & 91 & $\begin{array}{c}5 \\
87.5^{\prime}\end{array}$ & $\begin{array}{c}\mathrm{IV} \\
24.5\end{array}$ & $36^{\prime}$ & $47^{\circ} 24^{\prime}$ & $185^{3 / 4}$ & $\begin{array}{c}\mathrm{ESE} \\
3^{\circ} 5 I^{\prime} S \\
\end{array}$ & $17^{\circ} 22^{\prime}$ \\
\hline
\end{tabular}


ఫ June 24, I74I. After Midday

\begin{tabular}{|c|c|c|c|c|c|c|c|c|c|c|c|}
\hline 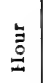 & $\stackrel{\infty}{0}$ & $\stackrel{0}{E}$ & 号 & 忢 & & & & & & & \\
\hline$I$ & $\overline{5 / 4}$ & W/S & SW W & $T / 2$ & $\begin{array}{l}\text { Top: } \\
\text { ing } \\
\text { swel }\end{array}$ & $\begin{array}{l}\text { ail wi } \\
\text { opsail } \\
\end{array}$ & $\begin{array}{l}\mathrm{d}_{y_{3}} \mathrm{col} \\
\text { fores: }\end{array}$ & ii, mair & $\begin{array}{l}\text { yi furle } \\
\text { sail, ano }\end{array}$ & $\begin{array}{l}\text { Spritgail } \\
\text { trysail; }\end{array}$ & $\begin{array}{l}\text { carry- } \\
\text { esterly }\end{array}$ \\
\hline 2 & 6 & & & $1 / 3$ & & & & & & & \\
\hline 3 & $51 / 2$ & & & $1 / 3$ & & & & & & & \\
\hline 4 & 5 & & $S / W 1 / 2 W$ & $\bar{v} \quad 1 / 2$ & Reef & topsai & wind, & cold. & & & \\
\hline 5 & $4 \frac{1}{4}$ & W & & $1 / 2$ & Tool & a ree & in top & sails; $d r$ & izzly, da & & \\
\hline 6 & $41 / 2$ & & & $1 / 2$ & & & & & & & \\
\hline 7 & $21 / 2$ & & SSW & $\bar{v} \quad \frac{1}{12}$ & Tool & two 1 & efs in & topsails & & & \\
\hline 8 & $21 / 2$ & & SSW & $T_{1 / 2}$ & & & & & & & \\
\hline 9 & $4^{1 / 4}$ & & & $1 / 2$ & & & & & & & \\
\hline 10 & $33 / 4$ & & & $\frac{1 / 2}{1 / 2}$ & Soun & ded in & 90 fat & oms, at & ad no bo & om. & \\
\hline II & $3 \frac{1}{2}$ & & & $1 / 2$ & & & & & & & \\
\hline 12 & 4 & & & $1 / 2$ & Win & mode & ating. & & & & \\
\hline 1 & $3^{3 / 4}$ & & & $3 / 2$ & & & & & & & \\
\hline 2 & $31 / 2$ & & & $3 / 2$ & Hea & ed the & lead $t$ & wice, an & id no bot & om. & \\
\hline 3 & 4 & & & $\frac{13}{12}$ & & & & & & & \\
\hline 4 & $4 \%$ & & & $1 / 2$ & Hois & ted sta & sail. & & & & \\
\hline 5 & $4 \%$ & $W / N$ & & $\sqrt{1 / 2}$ & & & & & & & \\
\hline 6 & $4 \frac{3}{4}$ & & $\mathrm{SW}^{2} \mathrm{WH}$ & & Let & ut ree & in $\mathrm{ma}$ & insail. & & & \\
\hline 7 & 43 & & & & & & & & & & \\
\hline 8 & $41 / 4$ & & & & & & & & & & \\
\hline 9 & $41 / 2$ & & & & & & & & & & \\
\hline 10 & 4 & $\mathrm{WW}$ & & & Tops & ail win & 1. cold & , drizzl & & & \\
\hline II & $4^{1 / 4}$ & $W N$ & & & Let & ut the & last re & efs in tl & he topsai & & \\
\hline & 5 & & $\mathrm{SE} \mathrm{E}_{4} \mathrm{E}$ & & Sky & verca & & & & & \\
\hline \multirow{2}{*}{\multicolumn{3}{|c|}{$\begin{array}{c}\text { Variation } \\
\text { of } \\
\text { Compass } \\
\text { I } / 4 \text { rhumb E }\end{array}$}} & \multicolumn{6}{|c|}{ In 24 hours } & \multicolumn{3}{|c|}{ From Vaua } \\
\hline & & & $\stackrel{\hat{\Xi}}{\underline{\Xi}}$ & . & 苞 & & 至总 & $\stackrel{\pi}{9}$ & 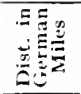 & 泀 & 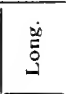 \\
\hline \multicolumn{3}{|c|}{$\begin{array}{l}\text { By Dead } \\
\text { Reckoning }\end{array}$} & $\underset{3^{\circ} \mathrm{O}^{\prime} \mathrm{SSW}}{\mathrm{SSW}}$ & 102 & $\underset{92^{\prime}}{S}$ & IV & $64^{\prime}$ & $45^{\circ} 52^{\prime}$ & $190 \%$ & $\underset{O^{\circ} O g^{\prime} \mathrm{E}}{\mathrm{SE} / \mathrm{E}}$ & $16^{\circ} 18^{\prime}$ \\
\hline
\end{tabular}




\section{2f June 25, I74I. After Midday}

\begin{tabular}{|c|c|c|c|c|c|c|c|c|c|c|c|}
\hline$\stackrel{0}{0}$ & 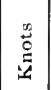 & $\stackrel{\Xi}{=}$ & 苫 & 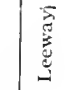 & & & & & & & \\
\hline $\bar{I}$ & $\overline{4: 4}$ & $\mathrm{~W} / \mathrm{N}$ & $\mathrm{SE} / \mathrm{E}$ & & $\begin{array}{l}\text { Tops } \\
\text { fore }\end{array}$ & $\begin{array}{l}\text { ail wi } \\
\text { and } m\end{array}$ & $\begin{array}{l}\text { 1. } \mathrm{col} \\
\mathrm{n}-\mathrm{top}\end{array}$ & $\begin{array}{l}\text { 1, cloudy } \\
\text { sails. }\end{array}$ & y. suns & $y$; carry & ng the \\
\hline 2 & 4 & & & & & & & & & & \\
\hline 3 & $23 / 4$ & & & & Saw & many & ucks. & & & & \\
\hline 4 & $21 / 2$ & & & & 19 & & & & & & \\
\hline 5 & $21 / 2$ & & $\mathrm{SE} / \mathrm{S}$ & & Topg & allant & il wi & ad, chil & ly, we & er as b & fore. \\
\hline 6 & 2 & & & & & & & & & & \\
\hline 7 & 2 & W & & & & & & & & & \\
\hline 8 & 214 & & & & & & & & & & \\
\hline 9 & $2 \%$ & & & & Litt) & e wind & & & & & \\
\hline 10 & $I^{1}+4$ & & & & $\begin{array}{l}\text { Clew } \\
90 \mathrm{fa}\end{array}$ & $\begin{array}{l}\text { ed an } \\
\text { thoms }\end{array}$ & $\begin{array}{l}\text { brail } \\
\text { and } n\end{array}$ & $\begin{array}{l}\mathrm{dup} t \\
\text { o botton }\end{array}$ & $\begin{array}{l}\text { he ma } \\
\text { In. }\end{array}$ & ail; soun & led in \\
\hline II & 1 & & & & & & & & & & \\
\hline 12 & 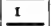 & & & & & & & & & & \\
\hline$I$ & $1: 4$ & & & & War & m, dri & y, we & & & & \\
\hline 2 & $I$ & & & & Now & and $t$ & en the & moon a & nd stal & re seen. & \\
\hline 3 & $3 / 4$ & & & & & & & & & & \\
\hline 4 & $\mathrm{I}$ & & & & & & & & & & \\
\hline 5 & $I$ & & & & Hois & ted th & jib at & d topma & st-stay & & \\
\hline 6 & 3,4 & & & & & & & & & & \\
\hline 7 & $1 \mathrm{I} / 2$ & & & & & & & & & & \\
\hline 8 & 3 & W'SWV & 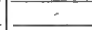 & & & & & & & & \\
\hline 9 & & calm & & & $\begin{array}{l}\text { Clou } \\
\text { up t } \\
\text { caps }\end{array}$ & $\begin{array}{l}\text { dy, su } \\
\text { he for } \\
\text { No }\end{array}$ & $\begin{array}{l}\text { shiny } \\
\text { and } \\
\text { ind. }\end{array}$ & $\begin{array}{l}\text { lowered } \\
\text { nainsail, }\end{array}$ & $\begin{array}{l}1 \text { jib a } \\
\text { lower }\end{array}$ & $\begin{array}{l}\text { staysail, } \\
\text { topsails }\end{array}$ & $\begin{array}{l}\text { clewed } \\
\text { on the }\end{array}$ \\
\hline Io & & calm & & & & & & & & & \\
\hline II & & calm & & & & & & & & & \\
\hline 12 & & calm & & & Wea & ther a & befort & atmose & here $t$ & & \\
\hline \multirow{2}{*}{\multicolumn{3}{|c|}{$\begin{array}{c}\text { Variation } \\
\text { of } \\
\text { Compass } \\
\text { I thumb E }\end{array}$}} & \multicolumn{9}{|c|}{ In 24 hours } \\
\hline & & & $\vec{\Xi}$ & 莙 & 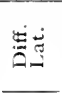 & 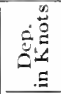 & 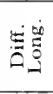 & $\stackrel{\leftrightarrow}{\stackrel{0}{\pi}}$ & 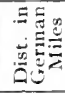 & 总 & ఏే \\
\hline \multicolumn{3}{|c|}{$\begin{array}{l}\text { By Dead } \\
\text { Reekoning }\end{array}$} & $\underset{2^{\circ} 25^{\prime} \mathbf{E}}{S}$ & 37 & $\begin{array}{c}\mathrm{S} \\
35.8^{\prime} \\
\end{array}$ & $\begin{array}{c}\mathrm{E} \\
8.7\end{array}$ & $12^{\prime}$ & $45^{\circ} 16^{\prime}$ & $198^{2} 4$ & $\begin{array}{l}\mathrm{SE} / \mathrm{E} \\
I^{\circ} 33^{\prime} \mathrm{S}\end{array}$ & $16^{\circ} 30^{\prime}$ \\
\hline
\end{tabular}

19 In Khitrov's journal it is stated that at 4 o'clock in the afternoon Bering called Waxel, Khitrov, and Eselberg into consultation about the course to sail. At the Harbor of St. Peter and St. Paul it had been agreed to sail to the $46 \mathrm{th}$ parallel in search of land; but this parallel had already been passed, and, though birds were seen, indieating that land was not far off land itself was not sighted. Taking all these points into consideration the officers decided to continue on the southerly course until noon of the $26 t h$, and accordingly the course steered was $\mathrm{SE}$ by $\mathrm{S}$. 
T June 26, I77I. After Midday

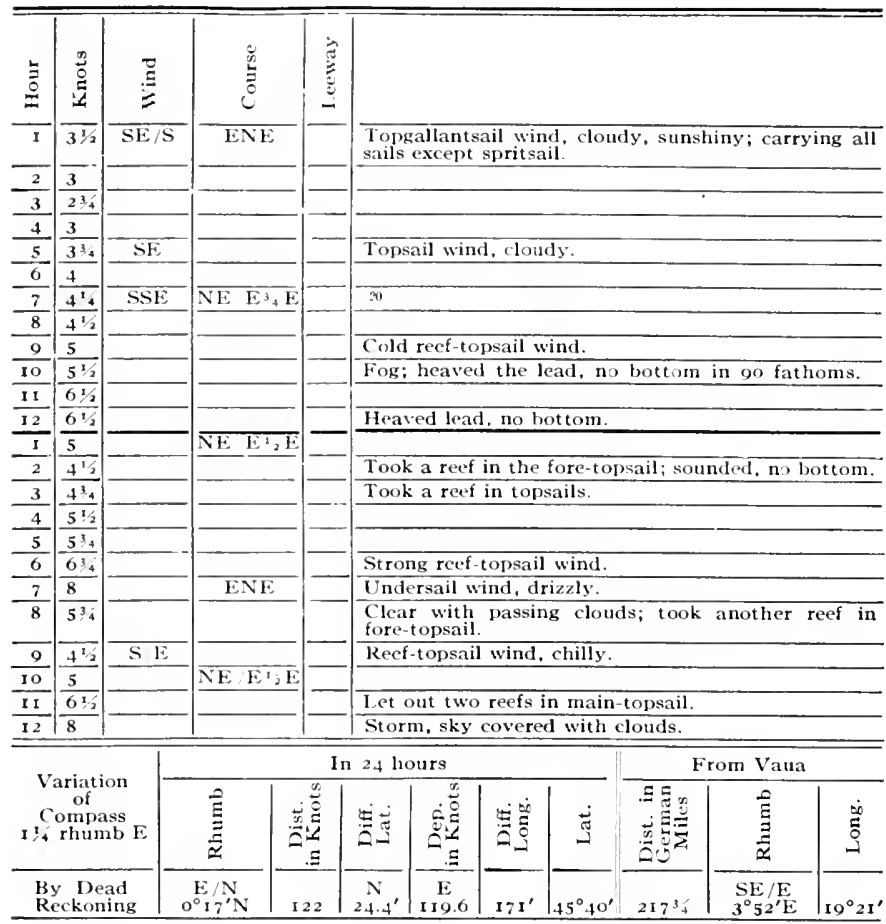

30 Khitrov"s journal: "Although on the 25 th we agreed to sail south, yet we found no land, which proves that the map of Delisle de la Croyere, which locates Juan de Gama Land as far as the 24 th $\mid 4, t h$ ? $]$ degrce is not accurate. Under the circumstances we concluded not to go farther soutli but instead to carry out the original plan made at the Harbor of St. Peter and St. Paul and sail between north and east." 
b) June 27, 1741. After Midday

\begin{tabular}{|c|c|c|c|c|c|c|c|c|c|c|c|c|}
\hline$\stackrel{\Xi}{\Xi}$ & 龸 & $\bar{\Xi}$ & 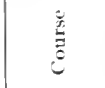 & & 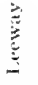 & & & & & & & \\
\hline $\bar{I}$ & $\overline{4^{2}}$ & $\mathrm{SE}, \mathrm{S}$ & ENE & & & $\begin{array}{l}\text { Tops } \\
\text { in tol }\end{array}$ & $\begin{array}{l}\text { ail win } \\
\text { psails: }\end{array}$ & $\begin{array}{l}\text { d, clon } \\
\text { arryin }\end{array}$ & $\begin{array}{l}\text { idy, chil } \\
\mathrm{g} \text { all the }\end{array}$ & $\begin{array}{l}11 \mathrm{y} ; \text { let } \\
\text { esails ex }\end{array}$ & $\begin{array}{l}\text { ut all th } \\
\text { pt the sI }\end{array}$ & $\begin{array}{l}\text { reefs } \\
\text { itsail. }\end{array}$ \\
\hline 2 & 4 & & & & & & & & & & & \\
\hline 3 & $5 ! 4$ & & & & & & & & & & & \\
\hline 4 & $5: 2$ & & & & & Stror & ig reef- & opsail & wind. & & & \\
\hline 5 & $5 \frac{1}{2}$ & & & & & & & & & & & \\
\hline 6 & $5 ? 2$ & & & & & & & & & & & \\
\hline 7 & $5 \div 3$ & & $\overline{N E} \mathrm{E}_{2}$ & & & & & & & & & \\
\hline 8 & $\overline{6^{2} / 2}$ & & & & & Reef & -unders & il win & d; took & a reef ir & opsails. & \\
\hline 9 & $5^{3 / 4}$ & SSE & & & & Haul & ed dow & $\mathrm{njib}$ a & nd main & 1-topma & staysail. & \\
\hline 20 & $51_{2}$ & & & & & Soun & ded in & 0 fath & loms, bu & it no bo & om. & \\
\hline II & $\overline{5}$ & & & & & Cold & reef-to & psail w & ind. & & & \\
\hline 12 & 5 & & & & & Heav & ed the & kead. & & & & \\
\hline$\overline{1}$ & 5 & SE? & ENL & & & & & & & & & \\
\hline 2 & 414 & & & & & Heav & ed the & lead, $n$ & 10 bottor & & & \\
\hline$\overline{3}$ & 4 & & & & & $\begin{array}{l}\text { Cold } \\
\text { sail; }\end{array}$ & $\begin{array}{l}\text { topsail } \\
\text { let out }\end{array}$ & $\begin{array}{l}\text { wind; } \\
\text { all the }\end{array}$ & $\begin{array}{l}\text { set jib } \\
\text { e reefs is }\end{array}$ & $\begin{array}{l}\text { and } m \\
\mathrm{n} \text { topsa }\end{array}$ & n-topmas & -stay- \\
\hline 4 & $4^{3} 4$ & & & & & & & & & & & \\
\hline 5 & $44^{2}$ & & $\mathrm{NE} \mathrm{E}^{\prime}$ & & & & & & & & & \\
\hline 6 & $4^{34}$ & & & & & Winc & 1 as bef & ore, $\mathrm{dr}$ & izzly we & ather. & & \\
\hline 7 & $41 / 2$ & & & & 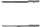 & & & & & & & \\
\hline 8 & $4 ! 4$ & & & & & & & & & & & \\
\hline 9 & 434 & & & & & Took & a reef & in tops & sails. & & & \\
\hline 10 & $3 ! 4$ & & & & & Topg & rallants & ail win & d, weath & her as b & & \\
\hline 11 & 3 & & & & & & & & & & & \\
\hline 12 & $\mid 3 \div 4$ & & & & & Carr & ying all & sails e & xcept th & 1e sprits & & \\
\hline \multirow{2}{*}{\multicolumn{3}{|c|}{$\begin{array}{c}\text { Variation } \\
\text { of } \\
\text { Compass } \\
1, \text { rhumb } \mathrm{E}\end{array}$}} & \multicolumn{7}{|c|}{ In 2.4 hours } & \multicolumn{3}{|c|}{ From Vaua } \\
\hline & & & $\stackrel{\vec{E}}{\underline{\Xi}}$ & $\frac{.00}{3}$ & $\begin{array}{l}\frac{\pi}{3} \\
\vdots \\
\vdots \\
\end{array}$ & 运 & 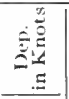 & 象至 & $\stackrel{5}{ت}$ & 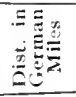 & 产 & 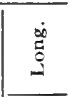 \\
\hline \multicolumn{3}{|c|}{$\begin{array}{l}\text { By Dead } \\
\text { Reckoning }\end{array}$} & $\underset{0^{\circ} 16^{\prime} E}{N}$ & I1 3 & & $\begin{array}{c}\mathrm{N} \\
21.6^{\prime}\end{array}$ & $\begin{array}{ccc}E \\
1 & 1 & 1\end{array} \mid$ & $163^{\prime}$ & $46^{\circ} \mathrm{O}^{\prime}$ & $238 \%$ & $\begin{array}{c}\text { ESE } \\
3^{\circ} 08^{\prime} S \\
\end{array}$ & $122^{\circ} 04^{\prime}$ \\
\hline
\end{tabular}


June 28,1741. Afler Midday

\begin{tabular}{|c|c|c|c|c|c|c|c|c|c|c|c|}
\hline \multirow{2}{*}{$\frac{\stackrel{5}{\vdots}}{1}$} & 营 & $\bar{z}$ & 㫄 & 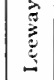 & & & & & & & \\
\hline & $\overline{43 / 4}$ & $\mathrm{SE} / \mathrm{S}$ & $\overline{N E !_{2} E}$ & & \multicolumn{7}{|c|}{$\begin{array}{l}\text { Topsail wind, chilly, drizzly; carrying all sails except } \\
\text { the spritsail. The topsails have a reef in them. }\end{array}$} \\
\hline \multirow{2}{*}{$\frac{2}{3}$} & $43_{4}$ & & & & & & & & & & \\
\hline & 5 & & & & \multirow{2}{*}{\multicolumn{7}{|c|}{ Thick fog, wet, heavy SW swell. }} \\
\hline 4 & $41 / 2$ & & & & & & & & & & \\
\hline 5 & $4 \div 4$ & & & & \multicolumn{7}{|c|}{ 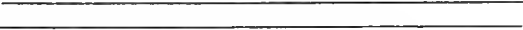 } \\
\hline$\frac{6}{6}$ & 3 & & & & \multicolumn{7}{|c|}{ Fog; sounded in I 80 fathoms, no bottom. } \\
\hline$\overline{7}$ & 4 & & ENE & & \\
\hline 8 & 4 & & & & \multicolumn{7}{|c|}{ Took two reefs in topsails. } \\
\hline 9 & $41 / 3$ & & \multirow{2}{*}{$\longrightarrow$} & & \\
\hline 10 & $31 / 2$ & & & & \multicolumn{7}{|c|}{ Sounded in 90 tathoms, no bottom. } \\
\hline II & 3 & & - & & \multicolumn{7}{|c|}{ Topgallantsail wind, chilly, wet, foggy. } \\
\hline 12 & $2 x_{4}$ & & \multirow{2}{*}{\multicolumn{2}{|c|}{$\overline{\overline{\mathrm{NE} / \mathrm{E} \tau_{2} \mathrm{E}}}$}} & \multirow{2}{*}{\multicolumn{7}{|c|}{ 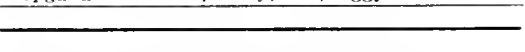 }} \\
\hline $\mathbf{I}$ & $23 / 4$ & SSE & & & & & & & & & \\
\hline 2 & 2 & & & & \multicolumn{7}{|c|}{ Fog; sounded in 90 fathoms, no bottom. } \\
\hline 3 & $\overline{21 / 2}$ & & & & \multicolumn{7}{|c|}{$\begin{array}{l}\text { Let out two reefs in fore-topsail and the second reef } \\
\text { in main-topsail. }\end{array}$} \\
\hline 4 & 3.4 & & \multirow{2}{*}{ 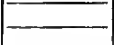 } & & \multirow{2}{*}{\multicolumn{7}{|c|}{ 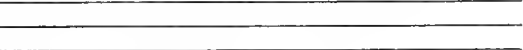 }} \\
\hline 5 & $4 \div 4$ & & & & & & & & & & \\
\hline 6 & $4 \frac{14}{4}$ & & & - & \multicolumn{7}{|c|}{ Let out a third reef in the main-topsail. } \\
\hline 7 & 4 & & & & \multirow{2}{*}{\multicolumn{7}{|c|}{ Set the spritsail. }} \\
\hline 8 & $3 \frac{3}{4}$ & & & & \\
\hline 9 & 3.4 & & & & & & & & & & \\
\hline 10 & $21 / 2$ & & & & $\mathrm{Cle}$ & with & passing & fogs. & & & \\
\hline II & $33^{2}$ & & & & & & & & & & \\
\hline 12 & $31 / 2$ & & & & Car & ying al & 1 sails $e$ & xcept t & he main & aysail. & \\
\hline & & & & & 241 & urs & & & & m Vaua & \\
\hline & $\begin{array}{l}\text { ariati } \\
\text { of } \\
\text { ompa } \\
\text { rhum }\end{array}$ & $\begin{array}{l}\text { on } \\
\text { iss } \\
\text { ab E E }\end{array}$ & $\stackrel{0}{\Xi}$ & 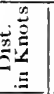 & نَّة & 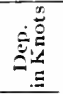 & 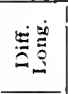 & 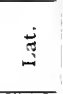 & 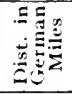 & 总 & 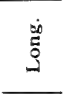 \\
\hline & $\begin{array}{l}y \text { De } \\
\text { eckon }\end{array}$ & & $\underset{I^{\circ} 2 I^{\prime} N}{E / N}$ & 87 & in & $\begin{array}{c}\mathrm{E} \\
85\end{array}$ & $129.6^{\prime}$ & $46^{\circ} 2 \mathrm{I}^{\prime}$ & $253 \frac{3 / 4}{4}$ & $\begin{array}{c}\mathrm{ESE} \\
0^{\circ} \mathrm{I}^{\prime} \mathrm{S}\end{array}$ & $24^{\circ} \mathrm{O}^{\prime}$ \\
\hline
\end{tabular}


(1) June 29, I7fI. After Midday

\begin{tabular}{|c|c|c|c|c|c|c|c|c|c|c|c|c|}
\hline$\stackrel{\Xi}{\Xi}$ & $\stackrel{n}{\varrho}$ & $\stackrel{\Xi}{\Xi}$ & 范 & & 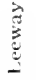 & & & & & & & \\
\hline $\bar{I}$ & $\overline{4 \pi / 4}$ & SSIV & $N E, E$ & & & $\begin{array}{l}\text { Top } \\
\text { exce }\end{array}$ & $\begin{array}{l}\text { sail wi } \\
\text { pt the }\end{array}$ & $\begin{array}{l}\text { nd, hes } \\
\text { main-st }\end{array}$ & $\begin{array}{l}\text { avy fog. } \\
\text { tay'sail. } 21\end{array}$ & chilly; & arrying a & all sails \\
\hline$\frac{2}{2}$ & $4+2$ & & & & & & & & & & & \\
\hline 3 & 4 & & & & & Clea & r with & passing & $y$ clouds. & & & \\
\hline 4 & 4 & & & & & Low & ered $m$ & tainsails & $\mathrm{s}$ and jib. & & & \\
\hline 5 & $3 \frac{3}{3 / 4}$ & & & & & Top & gallant & sail wit & id, weath & ner as b & ore. & \\
\hline 6 & $3^{1 / 2}$ & & & & & & & & & & & \\
\hline 7 & 35 & & $N E: 2$ & & & & & & & & & \\
\hline 8 & $3^{1 / 2}$ & & & & & Hea & $y$ and & damp & fog. & & & \\
\hline 9 & $3^{\pi / 2}$ & & & & & Too & k reef & in topsa & ails. & & & \\
\hline ro & $3^{3 / 4}$ & & & & & & & & & & & \\
\hline II & $3 \div 4$ & & & & & Sou & ided in & 190 fat & homs, no & bottor & & \\
\hline 12 & $3^{1 / 2}$ & & & & & & & & & & & \\
\hline I & $31 / 2$ & & NE E & & & & & & & & & \\
\hline 2 & $3^{3} 4$ & & & & & Sour & ided as & gain, no & a bottom & & & \\
\hline 3 & 4 & & & & 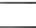 & & & & & & & \\
\hline 4 & $\sqrt{4^{2}}$ & & & & & Top & sail wir & nd, chil & $1 y$, foggy & damp & & \\
\hline 5 & $44_{4}^{3}$ & & $\mathrm{NE}_{2} \mathrm{H}$ & & & & & & & & & \\
\hline 6 & $4^{1 / 2}$ & & & & & $\overline{\mathrm{Clev}}$ & ved up & the ma & insail. & & & \\
\hline 7 & $\overline{4 ! 4}$ & & & & & Occe & isional & sunshit & & & & \\
\hline 8 & 4 & & & & & & & & & & & \\
\hline 9 & $43^{3}$ & & & & & Furl & ed spri & it sail. & & & & \\
\hline 10 & $4^{r_{2}}$ & & & & & Ree & -topsa & il wind, & , chilly, " & weather & before. & \\
\hline II & $44^{1 ! 2}$ & & NE E & & 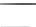 & & & & & & & \\
\hline 12 & $+4^{3}$ & & & & & Car & ying t & opsails & ; clewed & up fore & $\mathrm{il}$ and $\mathrm{m}$ & ainsail. \\
\hline \multirow{2}{*}{\multicolumn{3}{|c|}{$\begin{array}{c}\text { Variation } \\
\text { of } \\
\text { Compass } \\
\mathrm{I}: 4 \text { rhumb E }\end{array}$}} & \multicolumn{7}{|c|}{ In 24 hours } & \multicolumn{3}{|c|}{ From Vaua } \\
\hline & & & 竭 & 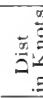 & 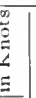 & 氙 & 告 & 窇 & हुं। & 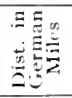 & $\stackrel{\vec{E}}{\underline{\underline{E}}}$ & $\stackrel{\dot{0}}{\stackrel{0}{0}}$ \\
\hline \multicolumn{3}{|c|}{$\begin{array}{l}\text { By Dead } \\
\text { Reckoning }\end{array}$} & $\begin{array}{c}\mathrm{ENE} \\
\mathrm{O}^{\circ} \mathrm{O}^{\prime} \mathrm{E}\end{array}$ & 94.5 & & $\begin{array}{c}N \\
36^{\prime}\end{array}$ & \begin{tabular}{|c}
$E$ \\
87.4 \\
\end{tabular} & $127^{\prime}$ & $46^{\circ} 57^{\circ}$ & $268 \%$ & $\begin{array}{c}\text { ESE } \\
3^{\circ} \mathrm{C}^{\prime} \mathrm{E}\end{array}$ & $26^{\circ} 14^{\prime}$ \\
\hline
\end{tabular}

21 Khitrov's journal: "Because we had gone too far southward we agreed with the "aptain Commander to sail true ENE in order to strike the E by $N$ course, as planned." 
d June 30, I7tI. Afler Midday

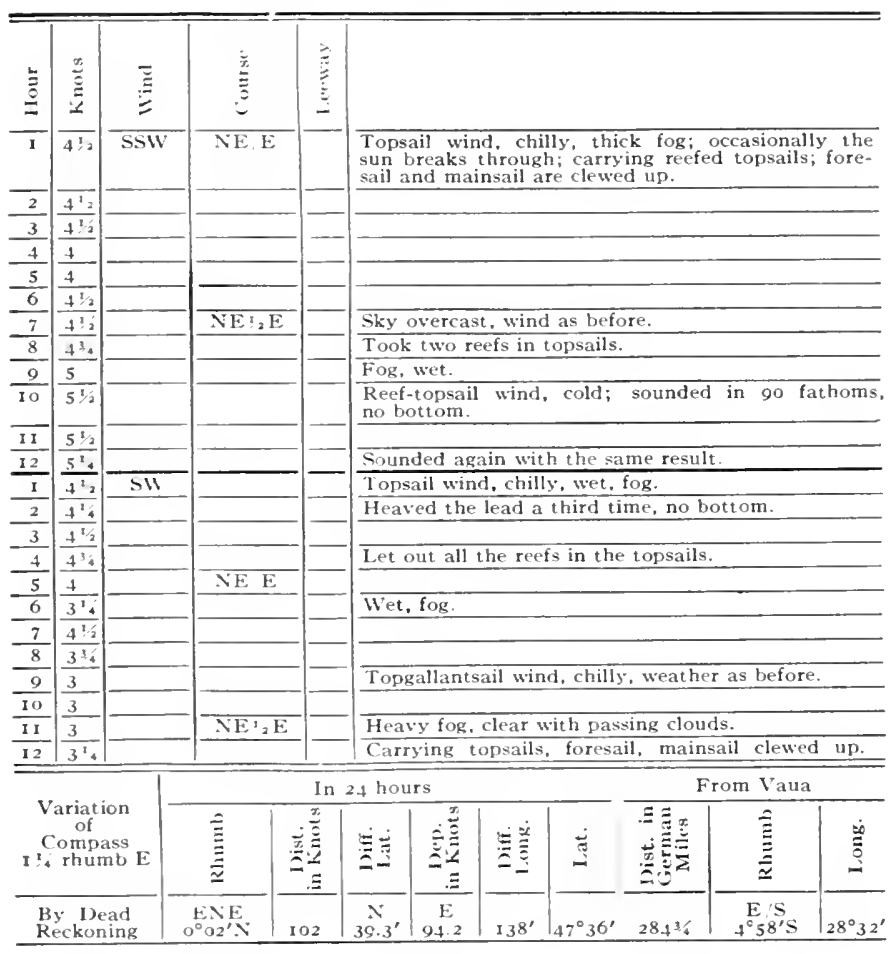




\begin{tabular}{|c|c|c|c|c|c|c|c|c|c|c|c|}
\hline$\stackrel{\Xi}{\Xi}$ & 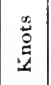 & $\frac{9}{3}$ & $\stackrel{\mathscr{U}}{\rightleftarrows}$ & $\sum_{i}^{2}$ & & & & & & & \\
\hline I & $\overline{33 / 4}$ & $\overline{\mathrm{SW} / \mathrm{W}}$ & $\mathrm{NE}_{1 / 2 \mathrm{E}}$ & & $\begin{array}{l}\text { Topg } \\
\text { carry }\end{array}$ & $\begin{array}{l}\text { allant } \\
\text { ing to }\end{array}$ & $\begin{array}{l}\text { il win win } \\
\text { sails, } f\end{array}$ & $\begin{array}{l}\text { d, wet, } \\
\text { oresail; }\end{array}$ & $\begin{array}{l}\text { fog, oce } \\
\text { mainsai }\end{array}$ & $\begin{array}{l}\text { ional sur } \\
\text { clewed } 1\end{array}$ & $\begin{array}{l}\text { shine; } \\
\text { p. }\end{array}$ \\
\hline 2 & $3^{2}+$ & & & & & & & & & & \\
\hline 3 & $3 / 2$ & & & & $\begin{array}{l}\text { It w } \\
\text { have } \\
\text { mus! } \\
\text { was } \\
\text { was } \\
\end{array}$ & $\begin{array}{l}\text { soci } \\
\text { fordin } \\
\text { and } \\
\text { o have } \\
\text { omma }\end{array}$ & $\begin{array}{l}\text { ed by } \\
\text { ler wa } \\
\text { ecause } \\
\text { limit } \\
\text { ded te }\end{array}$ & $\begin{array}{l}\text { the offi } \\
\text { ery mus } \\
\text { of the s } \\
\text { d amou } \\
\text { see tha }\end{array}$ & $\begin{array}{l}\text { cers tha } \\
\text { h and in } \\
\text { carcity } \\
\text { int of it. } \\
\text { it the or }\end{array}$ & $\begin{array}{l}\text { he crew } \\
\text { le evenin } \\
\text { vater eac } \\
\text { nsign La } \\
\text { rs were o }\end{array}$ & $\begin{array}{l}\text { hould } \\
\text { thick } \\
\text { h man } \\
\text { gunov } \\
\text { beyed. }\end{array}$ \\
\hline 4 & 3 & & & & & & & & & & \\
\hline 5 & 23 & & & & & & & & & & \\
\hline 6 & 214 & & & & & & & & & & \\
\hline 7 & 13 & & $\mathrm{NE} / \mathrm{E}$ & & & & & & & & \\
\hline 8 & $23 / 4$ & & & & & & & & & & \\
\hline 9 & 4 & & & & Rain & & & & & & \\
\hline 10 & 43 & & & & Set t & rysail. & & & & & \\
\hline II & 43 & & & & No t & ottom & at $90 \mathrm{f}$ & athoms. & & & \\
\hline 12 & 4 & & & & & & & & & & \\
\hline$I$ & 4 & & $\mathrm{NE}: \mathrm{E}$ & & Tops & ail wir & 1, fog, & heavy I & ain, hig & ea. & \\
\hline 2 & 4 & & & & & & & & & & \\
\hline 3 & $41 / 4$ & & & & Hea & ed lea & . no b & ttom. & & & \\
\hline 4 & $4 \frac{1}{2}$ & & & & Hois & ed $\mathrm{ma}$ & nsail. & & & & \\
\hline 5 & $51 / 2$ & & $\mathrm{NE} / \mathrm{E}$ & & & & & & & & \\
\hline 6 & 5 & & & & Reef & topsai & wind. & & & & \\
\hline 7 & 4 & & & & & & & & & & \\
\hline 8 & $31 / 2$ & & & & Clew & ed up & rysail. & & & & \\
\hline 9 & $4^{1} 4$ & & & & Did & the sat & e to $n$ & ainsail. & & & \\
\hline 10 & $4^{1}$ & & $\mathrm{NE} 1 / 2 \mathrm{E}$ & & Win & and & eather & as befo & re. light & in. & \\
\hline II & $\overline{4^{3}}$ & & & & $\begin{array}{l}\text { Sigh } \\
\text { lengt } \\
\text { near }\end{array}$ & $\begin{array}{l}\text { ed a } \\
h \text {, and }\end{array}$ & $\begin{array}{l}\text { iece o } \\
\text { this le }\end{array}$ & $\begin{array}{l}\text { drift } \\
\text { ds us } t\end{array}$ & $\begin{array}{l}\text { od abo } \\
\text { o believe }\end{array}$ & $\begin{array}{l}\text { two fath } \\
\text { hat the } s\end{array}$ & $\begin{array}{l}\text { oms in } \\
\text { hore is }\end{array}$ \\
\hline 12 & $31 / 2$ & & & & $\begin{array}{l}\text { Soun } \\
\text { did }\end{array}$ & $\begin{array}{l}\text { ded w } \\
\text { ot tou }\end{array}$ & $\begin{array}{l}\text { h tw } \\
\text { li bot }\end{array}$ & $\begin{array}{l}\text { lead } 1 \\
\text { om. }\end{array}$ & ines in & o fathor & ns but \\
\hline \multirow{2}{*}{\multicolumn{3}{|c|}{$\begin{array}{c}\text { Variation } \\
\text { of } \\
\text { Compass } \\
\text { I rhumb } \mathrm{E}\end{array}$}} & \multicolumn{6}{|c|}{ In 2.4 hours } & \multicolumn{3}{|c|}{ From Vaua } \\
\hline & & & 点 & 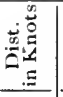 & 氙苟 & 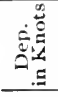 & 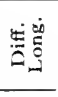 & 苟 & 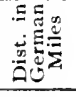 & है & 兽 \\
\hline \multicolumn{3}{|c|}{$\begin{array}{l}\text { By Dead } \\
\text { Reckoning }\end{array}$} & $\begin{array}{l}\text { ENE } \\
0^{\circ} 0^{\prime} N\end{array}$ & 90.3 & $\begin{array}{c}\mathrm{N}^{\prime} \\
34.8^{\prime}\end{array}$ & $\begin{array}{c}\mathrm{E} \\
83.4 \\
\end{array}$ & $125^{\prime}$ & $48^{\circ} 11^{\prime}$ & $2901 / 4$ & $\begin{array}{c}E / S \\
2^{\circ} 24^{\prime} S\end{array}$ & $30^{\circ} 37^{\prime}$ \\
\hline
\end{tabular}




\section{2f July 2, 174I. After Midday}

\begin{tabular}{|c|c|c|c|c|c|c|c|c|c|c|c|}
\hline 녹 & 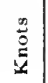 & $\frac{\vec{E}}{3}$ & 旁 & 突 & & & & & & & \\
\hline$I$ & 3 & $\mathrm{~S}$ & $\mathrm{NE} / \mathrm{E}$ & & $\begin{array}{l}\text { Top } \\
\text { and }\end{array}$ & $\begin{array}{l}\text { allant: } \\
\text { rysail. }\end{array}$ & ai) win & 1, rain; & carryin & psails, f & $\overrightarrow{\text { resail, }}$ \\
\hline 2 & 3 & & & & & & & & & & \\
\hline 3 & 23 & & & & & & & & & & \\
\hline 4 & 3 & & & & & & & & & & \\
\hline 5 & 4 & & & & Set & b and & opmas & t-staysa & il. hoist & mainsail & \\
\hline 6 & $31 / 2$ & & & & & & & & & & \\
\hline 7 & 234 & & $\mathrm{NE}, \mathrm{E}$ & & Top & allant & ail wir & d, fogg & y, dam & & \\
\hline 8 & 2 & & & & Too & reef $\mathrm{i}$ & topsa & ils, low & red sta & ils and & \\
\hline 9 & I & & & & & & & & & & \\
\hline 10 & 134 & & & & Soul & ded in & 90 fatl & loms, n & bottor & & \\
\hline 11 & $\mathbf{I}$ & & & & & & & & & & \\
\hline 12 & $I^{3} / 2$ & & & & Sour & ded ag & in, no & bottom & & & \\
\hline$I$ & 3.4 & & NlE E & & Litt & wind & SW s & vell, fog & and $w$ & & \\
\hline 2 & 3 & & & & & & & & & & \\
\hline 3 & $1 / 2$ & & & & $\begin{array}{l}\text { Cley } \\
\text { mai }\end{array}$ & $\begin{array}{l}\text { ed and } \\
\text { sail; ] }\end{array}$ & $\begin{array}{l}\text { braile } \\
\text { t out }\end{array}$ & $\begin{array}{l}\text { up thie } \\
80 \text { fath }\end{array}$ & $\begin{array}{l}\text { foresai } \\
\text { ms of }\end{array}$ & $\begin{array}{l}\text { did the } \\
\text { no bot }\end{array}$ & $\begin{array}{l}\text { ime to } \\
\text { om. }\end{array}$ \\
\hline 4 & $1 / 2$ & & & & & & & & & & \\
\hline 5 & & calm & & & Calr & ; lowe & red tor & sails on & the cal & & \\
\hline 6 & $1 / 2$ & $N$ & ENE & I & $\begin{array}{l}\text { Ligl } \\
\text { then }\end{array}$ & $\begin{array}{l}\text { wind } \\
\text { set } \mathrm{ji}\end{array}$ & $\begin{array}{l}\text { hoist } \\
\text { and }\end{array}$ & $\begin{array}{l}\text { d topsa } \\
\text { opmast }\end{array}$ & $\begin{array}{l}18 \text { and } \\
\text { staysai }\end{array}$ & out the & eefs in \\
\hline 7 & 112 & & & I & & & & & & & \\
\hline 8 & 214 & & & $\mathbf{I}$ & & & & & & & \\
\hline 9 & 4 & $\mathrm{~N} E$ & $\mathrm{E} N$ & $\mathbf{I}$ & Top & ail wir & d, driz & zly. & & & \\
\hline 10 & 3 & & & $\mathbf{I}$ & Too & two $r$ & $\mathrm{efs}$ in $f$ & ore-tops & iil and c & in main & topsail. \\
\hline II & 4 & & & 1 & Low & red jil & took & another & reef in & in-topsa & \\
\hline 12 & 3 & & & 1 & Win & 1 and & eather & as befo & & & \\
\hline \multirow{2}{*}{\multicolumn{3}{|c|}{$\begin{array}{c}\text { Variation } \\
\text { of } \\
\text { Compass } \\
1 / 4 \text { rhumb E }\end{array}$}} & \multicolumn{6}{|c|}{ In 24 hours } & \multicolumn{3}{|c|}{ From Vaua } \\
\hline & & & $\underset{\Xi}{\stackrel{\Xi}{\Xi}}$ & 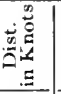 & 突望 & 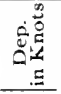 & 象蹗 & 苞 & 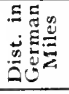 & 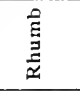 & 䠄 \\
\hline \multicolumn{3}{|c|}{$\begin{array}{l}\text { By Dead } \\
\text { Reckoning }\end{array}$} & $\begin{array}{l}\mathrm{E} / \mathrm{N} \\
I^{\circ} 36^{\prime} \mathrm{E} \\
\end{array}$ & 48 & $\begin{array}{c}\mathrm{N} \\
7.8^{\prime}\end{array}$ & $\begin{array}{c}E \\
46.9 \\
\end{array}$ & $70^{\prime}$ & $48^{\circ} 19^{\prime}$ & $309: 2$ & $\begin{array}{c}\mathrm{E} / \mathrm{S} \\
\mathbf{1}^{\circ} 35^{\prime} \mathrm{S}\end{array}$ & $31^{\circ} 47^{\prime}$ \\
\hline
\end{tabular}


q July 3, I74I. After Midday

\begin{tabular}{|c|c|c|c|c|c|c|c|c|c|c|c|c|}
\hline$\stackrel{\Xi}{3}$ & 总 & 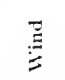 & 递 & & 范 & & & & & & & \\
\hline $\mathrm{I}$ & $3 ! 2$ & $\mathrm{NE}$ & ENE $/ 2 / 2$ & & $1 \%$ & Reef & topsail & wind, & cloudy. & & & \\
\hline 2 & $3 \div 4$ & & & & $11 / 2$ & $\begin{array}{l}\text { Tool } \\
\text { and }\end{array}$ & $\begin{array}{l}\text { third } \\
\text { nain-to }\end{array}$ & $\begin{array}{l}\text { eef in } \\
\text { mast- }\end{array}$ & $\begin{array}{l}\text { main-t } \\
\text { staysait }\end{array}$ & opsail. f & led fore- & opsail \\
\hline 3 & $3: 4$ & & & & 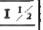 & & & & & & & \\
\hline 4 & 3 & & & & $I^{1 / 2}$ & & & & & & & \\
\hline 5 & $3 x_{4}$ & $N$ & ENE & & $\mathrm{I}^{\frac{1}{2}}$ & Wint & increa & & & & & \\
\hline 6 & $33_{4}$ & & & & $\mathrm{I}^{1}$ & & & & & & & \\
\hline 7 & 25 & $\mathrm{~N} \cdot \mathrm{W}$ & $\mathrm{NE} \mathrm{E}^{\mathrm{I}}$ & & $\mathrm{I}^{12}$ & llea & y sea f & om N. & & & & \\
\hline 8 & $2 \sqrt{2,4}$ & N. W & & & 2 & & & & & & & \\
\hline 9 & $1 \div 4$ & & & & $2 ! 2$ & Furl & d mair & topsai & & & & \\
\hline 10 & $1^{1} 4$ & & & & $2^{11}$ & & & & & & & \\
\hline $1 \mathrm{I}$ & 114 & & & & $2 ! 2$ & & & & & & & \\
\hline$\sqrt{2}$ & I!4 & & & & 2 & & & & & & & \\
\hline $\mathrm{I}$ & $1 \%$ & & $\mathrm{NE} / \mathrm{E}$ & & 2 & $\begin{array}{l}\text { Hois } \\
\text { hois }\end{array}$ & $\begin{array}{l}\text { ted } \\
\text { ed mai } \\
\end{array}$ & $\begin{array}{l}\text { in-tops } \\
\text { i-topm }\end{array}$ & $\begin{array}{l}\text { ail and } \\
\text { ast-stay }\end{array}$ & $\begin{array}{l}1 \text { put } \\
\text { tsail. } \\
\end{array}$ & ee reefs & in it, \\
\hline 2 & $1 ! 4$ & & & & 2 & & & & & & & \\
\hline 3 & $13 \cdot 4$ & & & & 2 & $\begin{array}{l}\text { lyois } \\
\text { two }\end{array}$ & $\begin{array}{l}\text { ed for } \\
\text { eeefs in }\end{array}$ & $\begin{array}{l}\text {-topsa } \\
\text { main- }\end{array}$ & $\begin{array}{l}1 \text { and } 1 \mathrm{e} \\
\text { copsail. }\end{array}$ & out a & ef in it. & out \\
\hline 4 & $2^{2}$ & & & & 1 & & & & & & & \\
\hline 5 & 2 & & & & $1^{52}$ & & & & & & & \\
\hline 6 & $\mathrm{I}^{3} 4$ & & & & 1.2 & Top: & allant: & il win & 1, sky o & vercast. & & \\
\hline 7 & 2 & & & & $1 \div 2$ & & & & & & & \\
\hline 8 & $2 \%$ & NNW & $\mathrm{NE} / 2 \mathrm{E}$ & & $x \frac{1 / 2}{1 / 2}$ & Let & ut a re & $\mathrm{finth}$ & e topsai & & & \\
\hline 9 & $23 / 4$ & & $\mathrm{NE}$ & & $\overline{\mathrm{I}_{2}}$ & Nor & herlys & vell. & & & & \\
\hline ro & $1^{3} 4$ & & & & $\overline{\mathrm{X}^{1}+2}$ & & & & & & & \\
\hline$x 1$ & $2 \%$ & NW & $\mathrm{NNE}_{2}$ & & $\mathrm{I}_{2}$ & Top: & ail win & 1. clou & & & & \\
\hline & 213 & $\mathrm{NW} / \mathrm{I}$ & $\mathrm{NE} / \mathrm{N}$ & & $\mathrm{I}_{2}$ & & & & & & & \\
\hline \multirow{2}{*}{\multicolumn{3}{|c|}{$\begin{array}{c}\text { Variation } \\
\text { of } \\
\text { Compass } \\
I 1 / 4 \text { rhumb E }\end{array}$}} & \multicolumn{7}{|c|}{ In 24 hours } & \multicolumn{3}{|c|}{ From Vaua } \\
\hline & & & 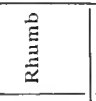 & 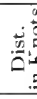 & $\Xi$ & 葛 & 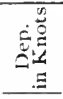 & 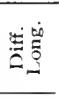 & 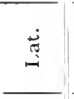 & 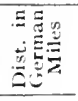 & 泀 & 㫣 \\
\hline \multicolumn{3}{|c|}{$\begin{array}{l}\text { By Dead } \\
\text { Reckoning }\end{array}$} & $\begin{array}{c}\text { E/S } \\
I^{\circ} 37^{\prime} S \\
\end{array}$ & 52. & & $\underset{\mathrm{I} .4^{\prime}}{\mathrm{S}}$ & $\begin{array}{l}\mathrm{E} \\
52 \\
\end{array}$ & $76^{\prime}$ & $4^{\circ} \times 8^{\prime}$ & 32134 & $\begin{array}{c}\mathrm{E} / \mathrm{S} \\
\mathrm{I}^{\circ} \mathrm{I} 6^{\prime} \mathrm{S}\end{array}$ & $3.3^{\circ} 03^{\circ}$ \\
\hline
\end{tabular}




\section{h July 4, 174I. After Midday}

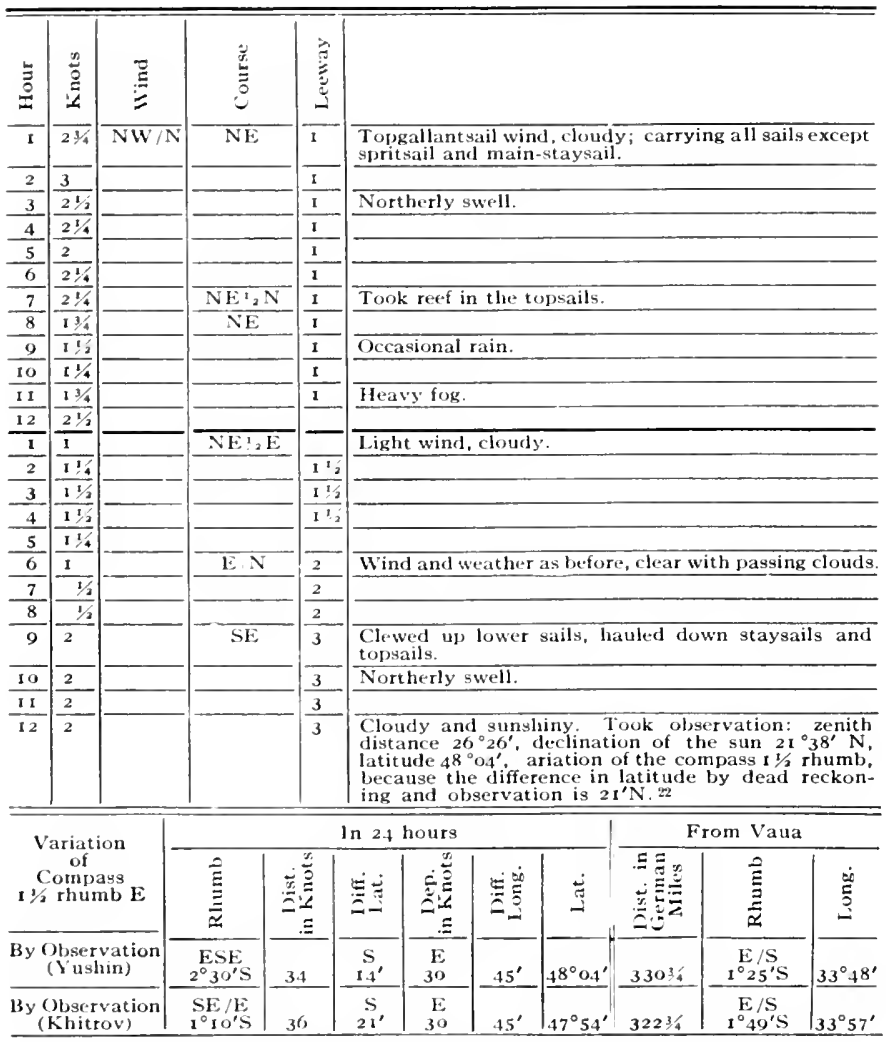

22 As indicated in the 24 -hour summary, Khitrov's journal gives latitude $47^{\circ} 54^{\prime}$. This value has been used on the chart (Pl. 1). 
July 5, 174I. After Midday

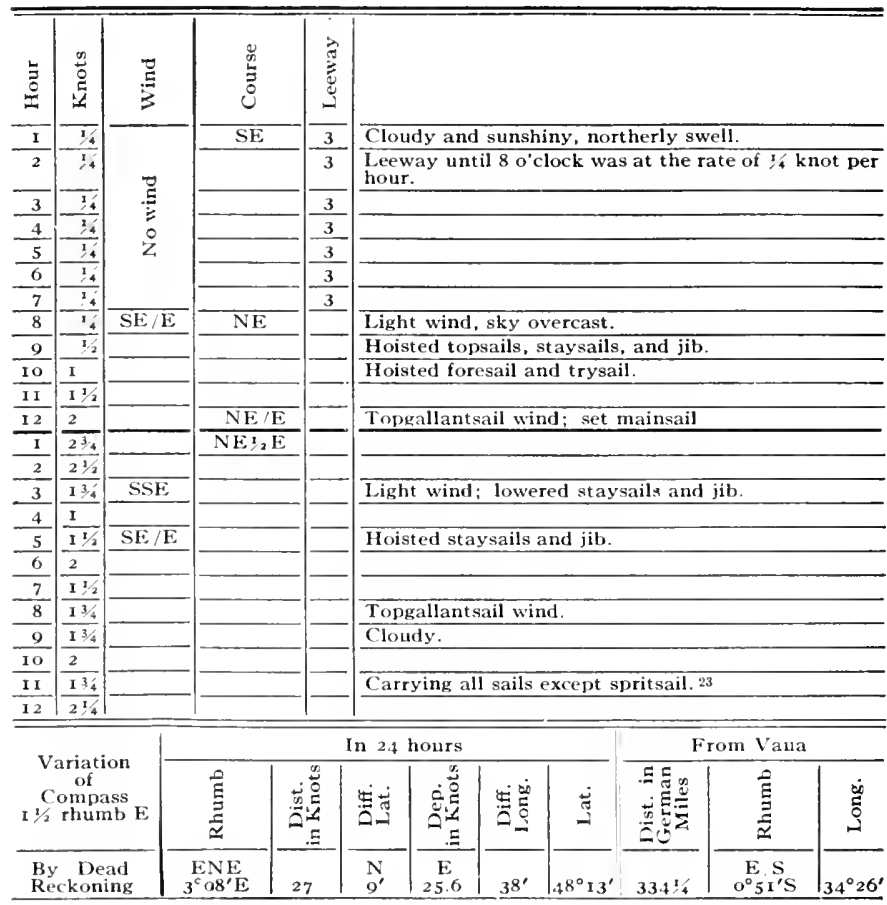

23 Khitrov's journal: "Filled 39 barrels with salt water for ballast." 
(3 July 6, I74I. After Midday

\begin{tabular}{|c|c|c|c|c|c|c|c|c|c|c|c|c|}
\hline 岂 & $\stackrel{\infty}{\circ}$ & $\stackrel{\square}{E}$ & 递 & & 育 & & & & & & & \\
\hline$I$ & 3 & $\mathrm{SE} / \mathrm{S}$ & $\mathrm{NE}: 2$ & & & $\begin{array}{l}\text { Top } \\
\text { spri }\end{array}$ & $\begin{array}{l}\text { allant } \\
\text { ail. }\end{array}$ & ail wi & d. cloud & y; carry & gall sails & except \\
\hline 2 & $31 / 2$ & & & & & & & & & & & \\
\hline 3 & $3 \pi$ & & & & & & & & & & & \\
\hline 4 & $3 \frac{1}{4}$ & & & & & Let & but the & sprits & & & & \\
\hline 5 & $3 \overline{3}$ & & & & & $\begin{array}{l}\text { Unh } \\
\text { befo }\end{array}$ & $\begin{array}{l}\text { ent the } \\
\text { re and }\end{array}$ & $\begin{array}{l}\text { fore-t } \\
\text { oook } t\end{array}$ & $\begin{array}{l}\text { psail at } \\
\text { ree reef }\end{array}$ & $\begin{array}{l}\text { Id bent } \\
s \text { in it. }\end{array}$ & the one & ve had \\
\hline 6 & 3 & & & & & & & & & & & \\
\hline 7 & $32 / 4$ & $\mathrm{SE} / \mathrm{H}$ & & & & & & & & & & \\
\hline 8 & 3 & & & & & Fur & ed the & pritsa & & & & \\
\hline 9 & $21 / 3$ & & & & & & & & & & & \\
\hline 10 & $2 \%$ & & & & & & & & & & & \\
\hline II & 234 & & & & & & & & & & & \\
\hline 12 & $2 \div 6$ & & & & & Top & Gallant & ail wi & d. clots & & & \\
\hline $\mathrm{I}$ & $2 \frac{213}{3}$ & & & & & & & & & & & \\
\hline 2 & $21 / 2$ & & & & & & & & & & & \\
\hline 3 & 4 & & & & & Top & ail wir & d, we: & ther as & before. & & \\
\hline 4 & 23 & & & & & & & & & & & \\
\hline 5 & $23 / 4$ & & & & & $\overline{T O P}$ & sallant & ail wi & & & & \\
\hline 6 & $2 \%$ & & & & & & & & & & & \\
\hline 7 & 5 & & & & & Top & ail wir & d. chi & y, clous & & & \\
\hline 8 & $31 / 2$ & & & & & Clea & r with & oassin & clouds & & & \\
\hline 9 & 3 & & & & & & & & & & & \\
\hline 10 & $3 \frac{1}{4}$ & ESE & $\mathrm{NE}$ & & 82 & $\begin{array}{l}\text { Top } \\
\text { alls }\end{array}$ & $\begin{array}{l}\text { Gallant } \\
\text { ills exc }\end{array}$ & $\begin{array}{l}\text { ail w } \\
\text { pt sp }\end{array}$ & $\begin{array}{l}\text { nd, we: } \\
\text { itsail an }\end{array}$ & $\begin{array}{l}\text { ther as } \\
\text { d main-s }\end{array}$ & $\begin{array}{l}\text { efore; } \\
\text { aysail: }\end{array}$ & rrying \\
\hline$I I$ & 3 & & & & $\frac{T}{12}$ & & & & & & & \\
\hline 12 & $2 \pi / 2$ & & & & 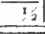 & & & & & & & \\
\hline \multirow{2}{*}{\multicolumn{3}{|c|}{$\begin{array}{c}\text { Variation } \\
\text { of } \\
\text { Compass } \\
1 / 2 \text { rhumb E }\end{array}$}} & \multicolumn{7}{|c|}{ In 24 hours } & \multicolumn{3}{|c|}{ From Vaua } \\
\hline & & & 胥 & $\dot{m}$ & 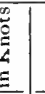 & 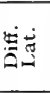 & 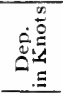 & 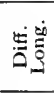 & 岇 & 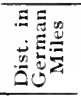 & $\underset{\Xi}{E}$ & : \\
\hline \multicolumn{3}{|c|}{$\begin{array}{l}\text { By Dead } \\
\text { Reckoning }\end{array}$} & ENE & 72 & & $\underset{29^{\prime}}{N}$ & $\begin{array}{r}E \\
66\end{array}$ & $99^{\prime}$ & $.4^{\circ} 42^{\prime}$ & $347 \%$ & $\begin{array}{c}\mathbf{E} / \mathrm{S} \\
0^{\circ} 4^{\prime} \mathrm{E}\end{array}$ & $36^{\circ} \mathrm{o}$ \\
\hline
\end{tabular}




\section{o July 7, I74I. After Midday}

\begin{tabular}{|c|c|c|c|c|c|c|c|c|c|c|c|}
\hline$\stackrel{2}{3}$ & 营 & $\stackrel{\Xi}{\Xi}$ & 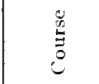 & 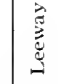 & & & & & & & \\
\hline$\overline{1}$ & $\overline{23 / 4}$ & ESE & $\mathrm{NE}$ & $3 / 2$ & $\begin{array}{l}\text { Tops } \\
\text { excel }\end{array}$ & $\begin{array}{l}\text { allant } \\
\text { t spri }\end{array}$ & $\begin{array}{l}\text { iil } w \\
\text { ail an }\end{array}$ & $\begin{array}{l}\text { ad, clo } \\
1 \text { main-s }\end{array}$ & $\begin{array}{l}\text { Iudy; } \\
\text { staysail. }\end{array}$ & rying al & sails \\
\hline 2 & $3^{x}$ & & $\mathrm{NE}_{2} \mathrm{~N}$ & $3 / 2$ & & & & & & & \\
\hline 3 & 3 & & & $\frac{1 / 2}{2}$ & & & & & & & \\
\hline 4 & $23 / 4$ & & & $1 / 2$ & & & & & & & \\
\hline 5 & 3 & & & $1 / 2$ & Tops & ail wi & at $\mathrm{ti}$ & nes. & & & \\
\hline 6 & 3 & & & $i_{2}$ & Wea & ther a: & before & & & & \\
\hline 7 & $23 / 4$ & & & $\frac{11}{1}$ & & & & & & & \\
\hline 8 & 3 & & & 12 & & & & & & & \\
\hline 9 & 23,4 & $\mathrm{E} / \mathrm{S}$ & NE N & 3 & Tool & reef & the $t$ & psails. & & & \\
\hline IO & 3 & & & $i_{2}$ & Win & fresh & ing ; & ounded & in $90 \mathrm{fa}$ & oms, no b & ottom. \\
\hline II & $21 / 2$ & & & 3 & & & & & & & \\
\hline 12 & $3^{2} 4$ & & & $\mathrm{I}_{2}$ & & & & & & & \\
\hline$I$ & $31 / 2$ & $\mathrm{ESE}$ & $\mathrm{NE} 1 / 2 \mathrm{~N}$ & $\overline{T / 2}$ & & & & & & & \\
\hline 2 & 3 & & & $y_{2}$ & Let & out rec & in to & osail. & & & \\
\hline 3 & $2 \div 4$ & E S S & $\mathrm{NE} / \mathrm{N}$ & $\mathbf{I}$ & & & & & & & \\
\hline 4 & 2 & $\mathrm{E}$ & NNE & $I$ & Top & allant & iil wir & & & & \\
\hline 5 & $\mathbf{I}$ & & & 1 & Ligh & $t$ wind & & & & & \\
\hline 6 & 274 & $\mathrm{E}, \mathrm{S}$ & $\mathrm{NE} / \mathrm{N}$ & $I$ & & & & & & & \\
\hline 7 & 3 & $\mathbf{E}$ & NNE & 1 & Top & ail wi & & & & & \\
\hline 8 & $3 \pi 4$ & & & I & $\begin{array}{l}\text { Tet } \\
\text { that } \\
\text { mov }\end{array}$ & $\begin{array}{l}\text { out } 4 \\
\text { when } \\
\text { ment. }\end{array}$ & $\begin{array}{l}\text { whes } \\
\text { was }\end{array}$ & $\begin{array}{l}\text { n the m } \\
\text { ight the }\end{array}$ & $\begin{array}{l}\text { lainstay } \\
\text { e vessel }\end{array}$ & $\begin{array}{l}\text { ecause we } \\
\text { d not hav }\end{array}$ & $\begin{array}{l}\text { notice } \\
\text { e a free }\end{array}$ \\
\hline 9 & 3 & & & $I$ & & & & & & & \\
\hline 10 & 3 & & & $I^{2} 4$ & & & & & & & \\
\hline 11 & $3 \%$ & & & $\mathrm{I}^{\mathrm{I}}$ & Tool & reef i & topsa & & & & \\
\hline 12 & $\underline{3^{x}}{ }^{x}$ & & & $I^{1} \cdot 4$ & Top: & ail wir & 1, clou & y and & rainy. & & \\
\hline \multirow{2}{*}{\multicolumn{3}{|c|}{$\begin{array}{c}\text { Variation } \\
\text { of } \\
\text { Compass } \\
1 / 2 \text { rhumb } E\end{array}$}} & \multicolumn{6}{|c|}{ In 24 hours } & \multicolumn{3}{|c|}{ From Vaua } \\
\hline & & & 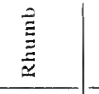 & 无 & 岕芯 & ¿. & 氙 & 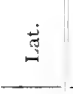 & 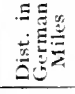 & 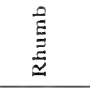 & $\stackrel{\dot{H}}{\dot{0}}$ \\
\hline \multicolumn{3}{|c|}{$\begin{array}{l}\text { Ry Dead } \\
\text { Reckcning }\end{array}$} & $\begin{array}{l}\mathrm{NE} \\
3^{\circ}+8^{\prime} \mathrm{N}\end{array}$ & 67 & $\begin{array}{c}\mathrm{N} \\
50.7^{\prime}\end{array}$ & $\begin{array}{c}E \\
44.4 \\
\end{array}$ & $68^{\prime}$ & $40^{\circ} 33^{\prime}$ & 353 & $\begin{array}{c}\text { E } \\
3^{\circ} \mathrm{O}_{4}^{\prime} \mathrm{E}\end{array}$ & $37^{\circ} 13^{\prime}$ \\
\hline
\end{tabular}


$\Varangle$ July 8, 1741. After Midday

\begin{tabular}{|c|c|c|c|c|c|c|c|c|c|c|c|c|}
\hline 苛 & 总 & 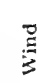 & 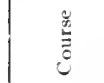 & & לें & & & & & & & \\
\hline I & $\overline{43 / 4}$ & $\mathrm{E}$ & NNE & & $I$ & $\begin{array}{l}\text { Top } \\
\text { sails }\end{array}$ & $\begin{array}{l}\text { sail win } \\
\text { stays }\end{array}$ & $\begin{array}{l}\text { d, clou } \\
\text { ils, jib. }\end{array}$ & $\begin{array}{l}\mathrm{dy} \text { and } \\
\text { mainsa }\end{array}$ & $\begin{array}{l}\text { loggy, es } \\
\text { il, and }\end{array}$ & $\begin{array}{l}\text { ying ree } \\
\text { sail. }\end{array}$ & d top- \\
\hline 2 & $4 ! 4$ & & & & $I$ & & & & & & & \\
\hline 3 & 4 & & & & 1 & & & & & & & \\
\hline 4 & $41 / 2$ & & & & 1 & Reef & -topsai & wind. & & & & \\
\hline 5 & 4 & & & & I & Tool & twor & cefs in & topsails. & & & \\
\hline 6 & $23 / 4$ & & & & $11 / 2$ & 1.ow & ered jil & & & & & \\
\hline 7 & 3 & & & & $11 / 2$ & & & & & & & \\
\hline 8 & 3 & & & & $11 / 2$ & & & & & & & \\
\hline 9 & $21 / 2$ & & & & $21 / 2$ & Tool & k three & reefs in & main-t & opsail. & & \\
\hline 10 & $23 / 4$ & & & & $21 / 2$ & Furl & ed mai & 1-topsai & & & & \\
\hline II & $2 \pi$ & & & & $21 / 2$ & & & & & & & \\
\hline 12 & 234 & & & & $21 / 2$ & Top & sail wir & & & & & \\
\hline 1 & 2 & & & & 3 & Fog & $y$ and & wet. & & & & \\
\hline 2 & 13.4 & & & & 3 & & & & & & & \\
\hline 3 & $21 / 2$ & $\mathrm{E}, \mathrm{N}$ & $N E$ & & $11 / 3$ & $\begin{array}{l}\text { Hois } \\
\text { reef: }\end{array}$ & $\begin{array}{l}\text { ted for } \\
\text { in ma }\end{array}$ & e-topsa & $\begin{array}{l}\text { il with th } \\
\text { iil. }\end{array}$ & wo reefs & it, let o & it three \\
\hline 4 & $3 ! 4$ & & & & $11 / 2$ & & & & & & & \\
\hline 5 & $23 / 4$ & & & & $11 / 2$ & Sigh & ted a $g$ & ood-siz: & ed piece & of drift & ood. & \\
\hline 6 & $23 / 4$ & & & & $11 \frac{12}{2}$ & & & & & & & \\
\hline 7 & 3 & & & & 12 & Let & out tw & reefs i & $n$ main & topsail. & & \\
\hline 8 & $23 / 4$ & & & & $11 / 2$ & & & & & & & \\
\hline 9 & 3 & ENF & $\mathbf{N}$ & & $1 \frac{1 / 2}{4}$ & & & & & & & \\
\hline 10 & 234 & & & & $1+4$ & Sour & ided in & $180 \mathrm{fat}$ & homs, & io botto & & \\
\hline II & $23_{4}$ & & & & $\mathrm{I}_{4}$ & & & & & & & \\
\hline 12 & $23 / 4$ & & $\mathrm{~N}^{1 / 2} \mathrm{E}$ & & $\overline{I ' *}$ & Top & sail wit & d, driz & $2 \mathrm{Iy}, \mathrm{SE}$ & swell. & & \\
\hline \multirow{2}{*}{\multicolumn{3}{|c|}{$\begin{array}{l}\text { Variation } \\
\text { of } \\
\text { Compass } \\
1 / 2 \text { rliumb E }\end{array}$}} & \multicolumn{7}{|c|}{ ln 24 hours } & \multicolumn{3}{|c|}{ From Vaua } \\
\hline & & & 言 & 葴造 & 离 & 岛 & 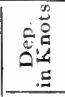 & 造莒 & ஹூ & 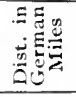 & $\stackrel{\vec{E}}{\underline{\Xi}}$ & $\stackrel{\infty}{\stackrel{0}{0}}$ \\
\hline \multicolumn{3}{|c|}{$\begin{array}{l}\text { By loead } \\
\text { Reckoning }\end{array}$} & $\begin{array}{c}\mathrm{N} / \mathrm{E} \\
5^{\circ} / 2^{\prime} \mathrm{E}\end{array}$ & 68.8 & & N & $\begin{array}{c}\mathrm{E} \\
19.4 \\
\end{array}$ & $30.4^{\prime}$ & $50^{\circ} 39^{\prime}$ & $3.51 \%$ & $\begin{array}{c}\mathrm{E} \\
6^{\circ} 3 I^{\prime} \mathrm{S} \\
\end{array}$ & $37^{\circ} 43$ \\
\hline
\end{tabular}


2 July 9, I741. After Midday

\begin{tabular}{|c|c|c|c|c|c|c|c|c|c|c|c|c|}
\hline 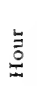 & 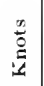 & $\stackrel{\Xi}{\Xi}$ & $\stackrel{\mathscr{n}}{3}$ & & 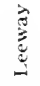 & & & & & & & \\
\hline$I$ & $\overline{21 / 2}$ & ENE & $\mathrm{NI}^{1 / 2} \mathrm{~V}$ & & 2 & $\begin{array}{l}\text { Top } \\
\text { stay }\end{array}$ & $\begin{array}{l}\text { ail wir } \\
\text { sails, } f\end{array}$ & $\begin{array}{l}\text {, cloud } \\
\text { esail, }\end{array}$ & $\begin{array}{l}\text { y, wet; } \\
\text { nainsai }\end{array}$ & $\begin{array}{l}\text { carryin } \\
\text { l, and } t\end{array}$ & $\begin{array}{l}\text { eef-tops } \\
\text { iil. }\end{array}$ & Is and \\
\hline 2 & $23 / 4$ & & & & 2 & & & & & & & \\
\hline 3 & $21 / 2$ & & & & 2 & & & & & & & \\
\hline 4 & 13 & $\mathrm{NE} /$ & $\mathrm{N} / \mathrm{H}$ & & 2 & Top & gallant & ail win & & & & \\
\hline 5 & 2 & & & & 2 & Wea & ther a & before. & & & & \\
\hline 6 & 2 & & $\mathrm{~N} / \mathrm{W}$ & $\mathrm{W}$ & 2 & & & & & & & \\
\hline$\frac{7}{7}$ & I & $\mathrm{NE}$ & NNI & & 2 & Litt & e wing & & & & & \\
\hline$\frac{1}{8}$ & $1_{2}$ & $\tau$ & & & 2 & Sou & ided ir & $180 \mathrm{fat}$ & homs, & no botte & & \\
\hline 9 & 34 & 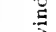 & & & & Low & ered st & ysails, & clewed & up main & and $\mathrm{tr}$ & ail. \\
\hline 10 & 14 & 3 & & & & $\begin{array}{l}\text { Fro } \\
\text { to }\end{array}$ & Niw. & $2 \mathrm{o}^{\prime} \mathrm{clo}$ & ck we & Irifted & ut $3 / 4$ o & knot \\
\hline$\overline{\text { I I }}$ & 7. & & & & & & & & & & & \\
\hline 12 & $1 / 2$ & SSW & $\mathrm{NE}$ & & & Ligl & t wine & drizzl & & & & \\
\hline 1 & 1 & & & & & Clo & $\mathrm{dy}, \mathrm{st}$ & s out, & topgall & antsail & & \\
\hline 2 & 134 & SW & & & & Fog & wet. & & & & & \\
\hline 3 & $11 / 2$ & & & & & & & & & & & \\
\hline 4 & 214 & & & & & & & & & & & \\
\hline 5 & $\mathrm{I}$ & & & & & Let & out th & e reefs & in $\mathrm{mai}$ & a-topsai & & \\
\hline 6 & $\begin{array}{ll}14 \\
\end{array}$ & & & & & & & & & & & \\
\hline 7 & $11 / 2$ & & & & & Hea & y fog & clear 4 & ith pas & sing clo & & \\
\hline 8 & 2 & & & & & & & & & & & \\
\hline 9 & $21 / 2$ & SW & & & & Top & sail wi & d, wea & her as & before. & & \\
\hline Io & 3 & & & & & & & & & & & \\
\hline II & $21 / 2$ & & & & & & & & & & & \\
\hline 12 & $\overline{21 / 2}$ & & & & & $\begin{array}{l}\text { Cle: } \\
\text { obse } \\
\text { of } 5\end{array}$ & $\begin{array}{l}\text { r with } \\
\text { rvatio } \\
\text { in } 20^{\circ} \\
\end{array}$ & $\begin{array}{l}\text { passin } \\
6^{\prime} \text {, lati } \\
\end{array}$ & $\begin{array}{l}\text { cloud } \\
\text { th dis } \\
\text { ude } 5 i\end{array}$ & $\begin{array}{l}\text { S, sun } \\
\text { ance } \\
0_{20} .24\end{array}$ & $4^{\prime}$. dec & $\begin{array}{l}\text { ok an } \\
\text { nation } \\
\end{array}$ \\
\hline \multirow{2}{*}{\multicolumn{3}{|c|}{$\begin{array}{c}\text { Variation } \\
\text { of } \\
\text { Compass } \\
1 \% \text { rhumb E }\end{array}$}} & \multicolumn{7}{|c|}{ In 24 hours } & \multicolumn{3}{|c|}{ From Vaua } \\
\hline & & & $\stackrel{\vec{\Xi}}{\stackrel{\Xi}{\Xi}}$ & a & 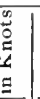 & 岂䓵 & م. & 电完 & 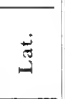 & 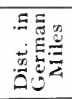 & है & $\stackrel{\leftrightarrow}{\check{a}}$ \\
\hline \multicolumn{3}{|c|}{$\begin{array}{c}\text { By } \\
\text { Observation }\end{array}$} & $\begin{array}{l}\text { NNE } \\
2^{\circ} \mathrm{O} 2^{\prime} \mathrm{N}\end{array}$ & 44 & & $\underset{4}{N}$ & $\underset{15.3}{E}$ & $24.3^{\prime}$ & $5 \mathrm{I}^{\circ} 2 \mathrm{O}^{\prime}$ & $35 \mathrm{I}^{3} \frac{1}{4}$ & $\underset{3^{\circ}}{\mathrm{E}} \frac{0^{\prime} \mathrm{S}}{}$ & $38^{\circ} 07^{\prime}$ \\
\hline
\end{tabular}

24 Khitrov's journal gives latitude $5 \mathrm{I}^{\circ} 27^{\prime}$, 
\& July IO, I7tI. After Midday

\begin{tabular}{|c|c|c|c|c|c|c|c|c|c|c|c|}
\hline$\stackrel{5}{0}$ & 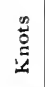 & $\stackrel{\square}{\Xi}$ & 荿 & 高 & & & & & & & \\
\hline$I$ & $\overline{23 / 4}$ & $\mathrm{~S} / \mathrm{W}$ & $\mathrm{NE}$ & & $\begin{array}{l}\text { Top } \\
\text { exce }\end{array}$ & $\begin{array}{l}\text { sail wi } \\
\text { pt spri }\end{array}$ & $\begin{array}{l}\text { d, clo } \\
\text { sail an }\end{array}$ & $\begin{array}{l}\text { udy, st } \\
\text { d trysa }\end{array}$ & $\begin{array}{l}\text { inshiny, } \\
\text { i. }\end{array}$ & rrying : & 11 sails \\
\hline 2 & 3 & & & & & & & & & & \\
\hline 3 & 23 & & & & $\overline{\text { At t }}$ & imes tl & ck fog & & & & \\
\hline 4 & $2 \frac{1 / 2}{2}$ & & & - & Thic & $k$, wet & og. & & & & \\
\hline 5 & 2 & & & & & & & & & & \\
\hline 6 & $11 / 2$ & & & & & & & & & & \\
\hline 7 & 3 & & & & $\mathrm{Clos}$ & $\mathrm{dy}, \mathrm{fo}$ & 'ifting & & & & \\
\hline 8 & 3 & & & & & & & & & & \\
\hline 9 & $3^{3 / 4}$ & & & & Top & sail wi & & & & & \\
\hline 10 & $3^{3 / 2}$ & & & & Star & s out. & & & & & \\
\hline II & 3 & & & & Hea & ved lea & 1. no b & ottom & it 90 fat & mis. & \\
\hline 12 & $21 / 2$ & & & & Low & ered st & ysails. & & & & \\
\hline $\mathrm{I}$ & $23 / 4$ & SIV & & & & & & & & & \\
\hline 2 & $1 \overline{4}$ & & & & Top & gallant & ail win & & & & \\
\hline 3 & $\mathrm{I}$ & & & & & & & & & & \\
\hline 4 & $3 / 4$ & & & & & & & & & & \\
\hline 5 & 1 & SE S & & & & & & & & & \\
\hline 6 & $I 1 / 2$ & & & & & & & & & & \\
\hline 7 & $22_{4}$ & & & 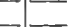 & Let & out thr & reef: & in the & fore-tops & & \\
\hline 8 & $2 \frac{1}{2}$ & & & & Hoi & ited st & sail a & nd jib. & & & \\
\hline 9 & $2 \pi$ & & & & & & & & & & \\
\hline 10 & $21 / 2$ & $\mathrm{SE} \mathrm{H}$ & & & Clor & dy an & sunsh & & & & \\
\hline II & 23 & ESE & & & & & & & & & \\
\hline & 3 & & & & Thi & , wet & & & & & \\
\hline \multirow{2}{*}{\multicolumn{3}{|c|}{$\begin{array}{c}\text { Variation } \\
\text { of } \\
\text { Compass } \\
1 \% \text { rhumb E }\end{array}$}} & \multicolumn{6}{|c|}{ In 2.4 hours } & \multicolumn{3}{|c|}{ From Vaua } \\
\hline & & & 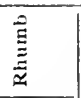 & 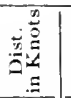 & 象焉 & 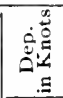 & 象葟 & 苞 & 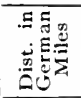 & $\frac{\hat{E}}{\underline{\Xi}}$ & $\stackrel{\infty}{\stackrel{\infty}{0}}$ \\
\hline \multicolumn{3}{|c|}{$\begin{array}{l}\text { By Dead } \\
\text { Reckoning }\end{array}$} & $\mathrm{E} / \mathrm{E} / \mathrm{E}$ & $57 ! 4$ & $\begin{array}{c}\mathrm{N} \\
27^{\prime}\end{array}$ & $\begin{array}{c}E \\
50.5 \\
\end{array}$ & $8 \mathrm{I}^{\prime}$ & $51^{\circ} 47^{\prime}$ & $362 \%$ & $2^{\circ} \frac{E}{39^{\prime} S}$ & $39^{\circ} 28^{\prime}$ \\
\hline
\end{tabular}


h July I1, I741. After Midday

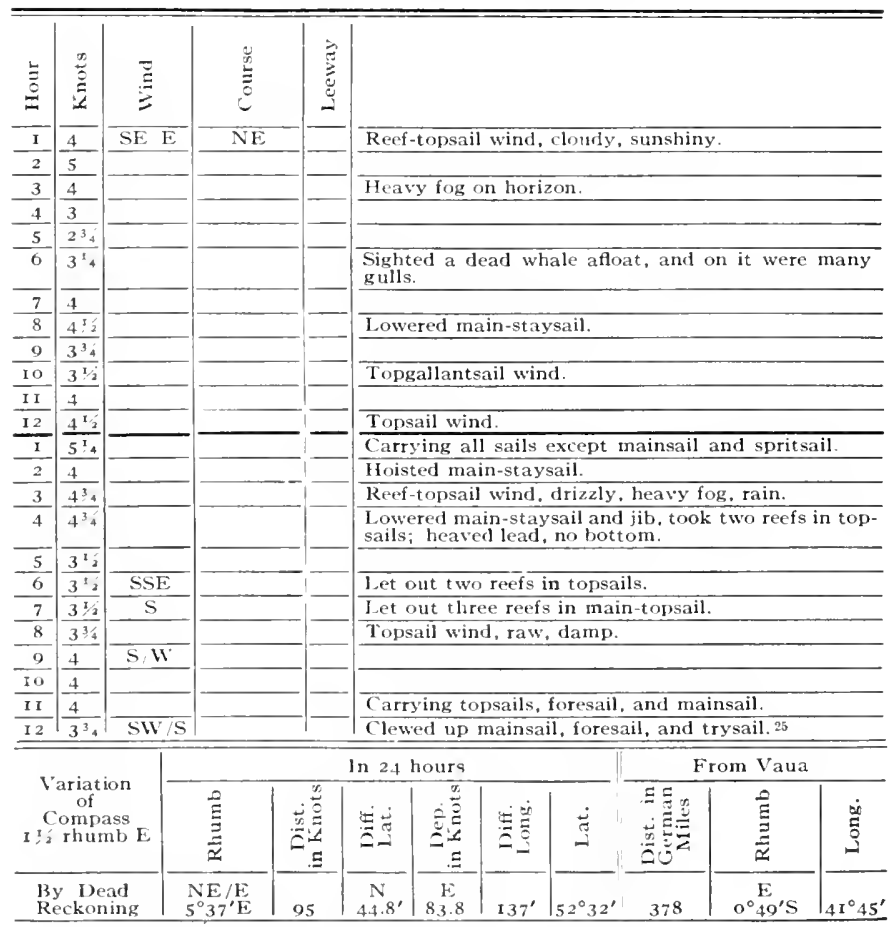

25 Khitrov's journal gives latitude by observation $52^{\circ} 40^{\prime}$. 
July I2, I74I. After Midday

\begin{tabular}{|c|c|c|c|c|c|c|c|c|c|c|c|}
\hline$\stackrel{\underline{z}}{0}$ & 足 & $\stackrel{\vec{g}}{=}$ & 总 & 空 & & & & & & & \\
\hline$I$ & $41 / 2$ & SW & $\mathrm{NE}$ & & Reef & -topsail & wind, & cloudy. & & & \\
\hline 2 & $41 / 2$ & & & & & & & & & & \\
\hline 3 & $4 \frac{1 / 2}{4}$ & & & & & & & & & & \\
\hline 4 & 5 & & & & Pass & ed muc & drift & vood. & & & \\
\hline 5 & 5 & & & & & & & & & & \\
\hline 6 & 5 & & & & Win & d fresh & ning: & took $\mathrm{tw}$ & o reefs is & topsails. & \\
\hline 7 & $43 / 4$ & & & & & & & & & & \\
\hline 8 & $4 \frac{13}{4}$ & & & & & & & & & & \\
\hline 9 & $41 / 2$ & & & & Too & three & reefs ir & main-t & opsail. & & \\
\hline I0 & 334 & & & & & & & & & & \\
\hline II & $3^{3 / 4}$ & & & & & & & & & & \\
\hline 12 & $3 \frac{13}{3}$ & $\mathrm{SW} / \mathrm{S}$ & & & Clev & ed up & oresail & & & & \\
\hline $\mathrm{I}$ & $\overline{1}$ & & & & Furl & ed fore & & & & & \\
\hline 2 & 2 & & $\mathrm{SE} \div / \mathrm{E}$ & 6 & & & & & & & \\
\hline 3 & $41 / 2$ & & $\mathrm{NE}$ & & At 3 & :30 we & Tewed & up trys & ail. & & \\
\hline 4 & 5 & & & & Set & nain ax & d fore- & topsails & with ret & Sin them & \\
\hline 5 & $41 / 2$ & & & & Rair & , reef-t & psail & wind, $\mathrm{el}$ & illy. & & \\
\hline 6 & 5 & & & & & & & & & & \\
\hline 7 & $4 \frac{3 / 4}{4}$ & & & & Let & out a $r$ & ef in $n$ & tain-top & sail. & & \\
\hline 8 & 5 & & & & Let & out a $t$ & ef in $f$ & oresail. & & & \\
\hline 9 & 5 & & & & Let & out $t w$ & reefs & $n$ main & topsail. & & \\
\hline 10 & $5 \%$ & & & & Und & ersail y & ind. & & & & \\
\hline II & 6 & & & & Set & fore-tof & sail ree & fed. & & & \\
\hline & 3 & & & & Ree & topsai & wind, & cloudy & and sur & hiny. & \\
\hline \multirow{2}{*}{\multicolumn{3}{|c|}{$\begin{array}{l}\text { Variation } \\
\text { of } \\
\text { Compass } \\
1 / 2 \text { rhumb E }\end{array}$}} & \multicolumn{6}{|c|}{ In 2.4 hours } & \multicolumn{3}{|c|}{ From Vaua } \\
\hline & & & 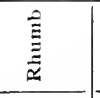 & $\begin{array}{r}\stackrel{\infty}{0} \\
0 \\
0 \\
0 \\
0\end{array}$ & 蛆苛 & 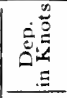 & 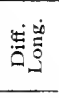 & $\stackrel{\dot{\pi}}{\leftrightarrows}$ & 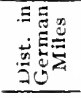 & 急 & 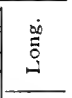 \\
\hline \multicolumn{3}{|c|}{$\begin{array}{l}\text { B:- Dead } \\
\text { Reckoning }\end{array}$} & $\begin{array}{l}N \mathrm{E} / \mathrm{E} \\
5^{\circ} 56^{\prime} \mathrm{E}\end{array}$ & 104 & ${ }_{48.7^{\prime}}^{N}$ & $\begin{array}{c}E \\
92.3\end{array}$ & $154^{\prime}$ & $53^{\circ} 2 I^{\prime}$ & $394 \div 4$ & $0^{\circ} \mathrm{E}^{\prime} \mathrm{N}$ & $44^{\circ} \mathrm{J} 3^{\prime}$ \\
\hline
\end{tabular}


(1) July I3, I77I. After Midday

\begin{tabular}{|c|c|c|c|c|c|c|c|c|c|c|c|c|}
\hline 总 & $\stackrel{\substack{0 \\
:}}{:}$ & $\frac{E}{E}$ & 总 & & 密 & & & & & & & \\
\hline 1 & 5 & W/S & $\mathrm{NE}$ & & & $\begin{array}{l}\text { Strol } \\
\text { and }\end{array}$ & $\begin{array}{l}\text { hunde } \\
\text { hird } \mathrm{re}\end{array}$ & $\begin{array}{l}\text { reail wi } \\
\text { ef in m }\end{array}$ & $\begin{array}{l}\text { nd, clou } \\
\text { ain-tops }\end{array}$ & $\begin{array}{l}\text { dy, sun } \\
\text { sail. }\end{array}$ & iny; took & $\overline{\text { second }}$ \\
\hline 2 & 5 & & & & & & & & & & & \\
\hline 3 & 5 & W & & & & & & & & & & \\
\hline 4 & 5 & & & & & Sigh & ed a p & ece of & irif twod & od three & thons lo & \\
\hline 5 & $4^{34}$ & WNW & & & & & & & & & & \\
\hline 6 & 43 & & & & & Hea & y sea & unning & & & & \\
\hline 7 & $41 / 2$ & & & & & & & & & & & \\
\hline 8 & $\overline{.3 / 4}$ & & & & & $\begin{array}{l}\text { Suns } \\
\text { W2.: } \\
\text { vari: }\end{array}$ & $\begin{array}{l}\text { hine. } \\
\text { oN; } \\
\text { tion } \\
\text { tion }\end{array}$ & $\begin{array}{l}\text { Took } \\
\text { titude } \\
\text { comp }\end{array}$ & $\begin{array}{l}\text { the ant } \\
53^{\circ} 39^{\prime} \\
\text { ass } 11^{\circ}\end{array}$ & $\begin{array}{l}\text { iplitude } \\
\text { true } \\
38^{\prime} \mathrm{E}, \mathrm{o}\end{array}$ & $\begin{array}{l}\text { of settin } \\
\text { earing } \\
\text { one rhur }\end{array}$ & $\begin{array}{l}5^{\circ} 3^{\text {sun }} \\
b^{\prime}\end{array}$ \\
\hline 9 & $41 / 2$ & WV & & & & & & & & & & \\
\hline 10 & 43 & & & & & Sky & lear, 1 & toon at & dd stars & shining & & \\
\hline 11 & $3^{34}$ & & & & & Furl & d mair & topsa & & . & & \\
\hline$\overline{12}$ & 3 & & & & & Furl & d fore & ail bec & ause of & darknes & & \\
\hline$\overline{1}$ & $\overline{1}$ & Wh/S & $\overline{\mathrm{N}} \mathrm{W \textrm {V } _ { 1 / 2 }}$ & & $\overline{6 ! 2}$ & Hov & to, le & out tr & ysail. & & & \\
\hline 2 & $\frac{13}{12}$ & & & & & Sour & ded, $n$ & botto & & & & \\
\hline 3 & 3 & & & & & $\begin{array}{l}\text { Got } \\
\text { out }\end{array}$ & $\begin{array}{l}\text { under } \\
\text { wo ree }\end{array}$ & $\begin{array}{l}\text { ay, set } \\
\text { s, clew }\end{array}$ & $\begin{array}{l}\text { foresai } \\
\text { ed up t }\end{array}$ & $\begin{array}{l}\text { and } m \\
\text { rysail. }\end{array}$ & -topsail : & nd let \\
\hline 4 & $4^{\frac{1}{2}}$ & & & & & Set & nainsai & and $\mathrm{f}$ & re-tops & & & \\
\hline 5 & $41 / 2$ & & & & 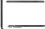 & & & & & & & \\
\hline 6 & $4 / 2$ & & & & & Und & rsail y & ind, $w$ & & & & \\
\hline 7 & 412 & & & & & Let & sut ree & in $\mathrm{ma}$ & insail, c & lewed it & $p$ and bra & iled it. \\
\hline 8 & 42 & & & & & Sun: & hine. & & & & & \\
\hline 9 & 5 & $\mathrm{~N} / \mathrm{W}$ & & & & Ree & -under & ail win & & & & \\
\hline 10 & 6 & & & & & Clea & with & assing & clouds: & set ma & sail. & \\
\hline 11 & $41 / 2$ & & & & & & & & & & & \\
\hline 12 & $|43|$ & & & & & Win & going & down. & & & & \\
\hline \multirow{2}{*}{\multicolumn{3}{|c|}{$\begin{array}{l}\text { Variation } \\
\text { of } \\
\text { Compass } \\
\text { I rhumb E }\end{array}$}} & \multicolumn{7}{|c|}{ In 24 hours } & \multicolumn{3}{|c|}{ From Vaua } \\
\hline & & & 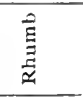 & 它: & 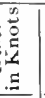 & 我薄 & 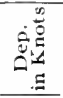 & 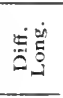 & $\stackrel{\dot{B}}{\mathfrak{I}}$ & 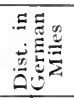 & $\frac{\hat{E}}{E}$ & ¿ \\
\hline \multicolumn{3}{|c|}{$\begin{array}{l}\text { By Dead } \\
\text { Reckoning }\end{array}$} & $\mathrm{NE} / \mathrm{E}$ & Itot & & $\begin{array}{c}\mathrm{N} \\
56^{\prime}\end{array}$ & $\begin{array}{l}E \\
84\end{array}$ & I.1.2 & $54^{\circ} 17^{\prime}$ & 415 & $\begin{array}{c}E \\
2^{\circ} 52^{\prime} \mathrm{N}\end{array}$ & $46^{\circ} 41^{\prime}$ \\
\hline
\end{tabular}


S July It, I7+t. After Midday

\begin{tabular}{|c|c|c|c|c|c|c|c|c|c|c|c|}
\hline 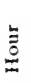 & $\begin{array}{l}\stackrel{n}{0} \\
\stackrel{4}{4}\end{array}$ & $\stackrel{\Xi}{\underline{E}}$ & 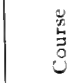 & 䒿 & & & & & & & \\
\hline$I$ & 43 & WSW & $\mathrm{NE}$ & & $\begin{array}{l}\text { Und } \\
\text { reef }\end{array}$ & $\begin{array}{l}\text { ersail } \\
\text { d, for }\end{array}$ & $\begin{array}{l}\text { nnd, d } \\
\text { ail, a }\end{array}$ & $\begin{array}{l}\text { izzly s } \\
\text { d main }\end{array}$ & $\begin{array}{l}\text { Inshiny } \\
\text { ail. }\end{array}$ & carrying & opsails \\
\hline 2 & $41 / 2$ & & & & & & & & & & \\
\hline 3 & 43.4 & & & & & & & & & & \\
\hline 4 & $4 \sqrt{3 / 2}$ & & & & $\begin{array}{l}\text { The } \\
\text { Khi } \\
\text { and }\end{array}$ & $\begin{array}{l}\text { Capta } \\
\text { rov, a } \\
\text { decide }\end{array}$ & $\begin{array}{l}\text { Com } \\
\text { Nay } \\
\text { to ste }\end{array}$ & $\begin{array}{l}\text { nander, } \\
\text { gator } \mathrm{E} \\
\mathrm{r} \mathrm{N} \text { by }\end{array}$ & $\begin{array}{l}\text { Lieuten } \\
\text { selberg } \\
\text { E to the }\end{array}$ & $\begin{array}{l}\text { nt Waxel, } \\
\text { ad a cons } \\
\text { and sight }\end{array}$ & $\begin{array}{l}\text { Master } \\
\text { ltation } \\
\text { d. } 26\end{array}$ \\
\hline 5 & $5 \div 4$ & & $\mathrm{~N}, \mathrm{H}$ & & & & & & & & \\
\hline 6 & 5 & & & & Too & two & efs in & topsails & & & \\
\hline 7 & $5+4$ & & & & Ree & -unde & ail wi & & & & \\
\hline 8 & 512 & & & & & & & & & & \\
\hline 9 & $51 / 2$ & & & & & & & & & & \\
\hline 10 & 53 & & & & & & & & & & \\
\hline II & 514 & & & & & & & & & & \\
\hline 12 & 534 & & & & & & & & & & \\
\hline $\bar{I}$ & 43 & $W$ & & & $\begin{array}{l}\text { Furl } \\
\text { beca } \\
\text { the }\end{array}$ & $\begin{array}{l}\text { ed to } \\
\text { use it } \\
\text { og. }\end{array}$ & $\begin{array}{l}\text { ails, } \\
\text { as da }\end{array}$ & $\begin{array}{l}\text { lowered } \\
\text { gerous }\end{array}$ & $\begin{array}{r}\text { staysa } \\
\text { o navig }\end{array}$ & $\begin{array}{l}s \text { on th } \\
\text { te at nigh }\end{array}$ & $\begin{array}{r}\text { yards } \\
\text { and in }\end{array}$ \\
\hline 2 & 4 & & & & & & & & & & \\
\hline 3 & $5 \%$ & & & & $\begin{array}{l}\text { Hoi } \\
\text { stay }\end{array}$ & $\begin{array}{l}\text { ted to } \\
\text { sails a }\end{array}$ & $\begin{array}{l}\text { sails } \\
\text { a trys }\end{array}$ & $\begin{array}{l}\text { vith thr } \\
\text { ii. }\end{array}$ & se reef & in each, & hoisted \\
\hline 4 & $5 \frac{3}{4}$ & & & & & & & & & & \\
\hline 5 & 612 & II: & & & & & & & & & \\
\hline 6 & 6 & & & & Let & out a & ef in & main-to & psail, c & wed up t & ysail. \\
\hline 7 & $5 \%$ & & & & $\begin{array}{l}\text { Sun } \\
\text { let } 0\end{array}$ & $\begin{array}{l}\text { shinin } \\
\text { ut two }\end{array}$ & $\begin{array}{l}\text { let } 0 \\
\text { reefs }\end{array}$ & $\begin{array}{l}\text { t a reef } \\
\text { a main- }\end{array}$ & $\begin{array}{l}\text { in fore-t } \\
\text { opsail. }\end{array}$ & psail, hoi & ted jib. \\
\hline 8 & 5 & & & & & & & & & & \\
\hline 9 & $41 / 2$ & & & & & & & & & & \\
\hline 10 & 45 & & & & & & & & & & \\
\hline$I I$ & 43 & & & & Let & out tw & reefs & in fore-t & opsail. & & \\
\hline 12 & $41 / 3$ & & & & $\begin{array}{l}\text { Reef } \\
\text { dist } \\
\text { but } \\
\text { reck }\end{array}$ & $\begin{array}{l}\text { - topsa } \\
\text { nce } 36 \\
\text { no co } \\
\text { oningl }\end{array}$ & $\begin{array}{l}\text { wind } \\
\text { I s' } \\
\text { ectio } \\
\text { Vari }\end{array}$ & $\begin{array}{l}\text { clear. } \\
\text { clinatio } \\
\text { was } n \\
\text { tion of }\end{array}$ & $\begin{array}{l}\text { Took o } \\
\text { n I } 9^{\circ} 50 \\
\text { ade [in } \\
\text { he com }\end{array}$ & $\begin{array}{l}\text { servation } \\
\text { latitude } \\
\text { latitude } \\
\text { ass } 1.4 \mathrm{rh}\end{array}$ & $\begin{array}{l}\text { zenith } \\
56^{\circ} \text { oI'; } \\
\text { y dead } \\
\text { mb E. }\end{array}$ \\
\hline \multirow{2}{*}{\multicolumn{3}{|c|}{$\begin{array}{c}\text { Variation } \\
\text { of } \\
\text { Compass } \\
\text { I rhumb E }\end{array}$}} & \multicolumn{9}{|c|}{ In 24 hours } \\
\hline & & & $\stackrel{\stackrel{E}{E}}{\stackrel{E}{\Xi}}$ & $\stackrel{5}{2}$ & 苟 & 递苛 & 乐离 & 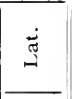 & 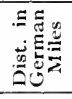 & 尽 & 㑒 \\
\hline \multicolumn{3}{|c|}{$\begin{array}{l}\text { By Dead } \\
\text { Reckoning }\end{array}$} & $\begin{array}{l}\text { NNE } \\
4^{\circ} 50^{\prime} E\end{array}$ & 119 & $\underset{106^{\prime}}{\mathrm{N}}$ & $\begin{array}{c}\mathrm{E} \\
54.8\end{array}$ & $96^{\prime}$ & $56^{\circ} \mathrm{o} 3^{\prime} \mid$ & $4231 / 2$ & $\begin{array}{c}\mathrm{E} \\
6^{\circ} 20^{\prime} \mathrm{N}\end{array}$ & $48^{\circ} 17^{\prime}$ \\
\hline
\end{tabular}

26 Khitrov does not mention sighting land on this day. Steller says that he saw land on the 15 th, but the officers paid no attention to him. It is possible that without Steller's being told about it orders were given to steer in the direction he indicated. 


\author{
July $14,174 I$. \\ Decision to Change Course ${ }^{27}$
}

Captain Commander Bering, Lieutenant Waxel, Fleet Master Khitrov, and Navigator Andreyan Eselberg had a mceting to discuss the decision reached on May $4, I_{4} \mathbf{I}$, in the Harbor of St. Peter and St. Paul. At that time and place it had been agreed after leaving the said harbor to sail $\mathrm{SE}$ by $\mathrm{E}$, true, to latitude $46^{\circ} \mathrm{N}$, unless land was found before; but if no land was sighted to sail from that parallel $\mathrm{E}$ by $\mathrm{N}$ steadily until land was discovered. If land should be found either on a SE by $\mathrm{E}$ or $\mathrm{E}$ by $\mathrm{N}$ course we were to coast alongside of it for as long a time as possible so that we might return to the Harbor of St. Peter and St. Paul by the end of September. After we left the said harbor we kept the $\mathrm{SE}$ by $\mathrm{E}$ course not only to $46^{\circ}$ but even to $45^{\circ}$ without seeing any land. We then sailed $\mathrm{E}$ by $\mathrm{N}$ close to $9^{\circ}$ of latitude and $35^{\circ}$ of longitude, but on this course we equally failed to discover land. On that account we concluded to change our course one point and keep closer to $\mathrm{N}$, that is to go ENE, which course we are now holding as far as the wind permits. By dead reckoning, we find ourselves at present in latitude $54^{\circ} 34^{\prime} \mathrm{N}$. and longitude from Vaua near $48^{\circ}$, a distance of more than 400 German miles. We had on board 102 barrels of water of different sizes, and by today, July $\mathrm{I}_{4}$, half of it has been used up by the crew. What is left, even if the allowance were reduced, would not last longer than August 25 or at the most until September, for we do not know whether the barrels of water in the lower hold are full or whether some of the water has leaked out. Taking these points into consideration we have decided to sail northward by true compass on the rhumb ENE so that we might in good time examine those northern regions and see whether there is not some land, as we suppose there is, judging by various reports. If we were to continue on easterly courses we should be sailing farther and farther from Kamchatka and, not being able to replenish our supply of water, we should suffer great hardships and, may God preserve us, extreme misfortune. But in going northerly we stand a better chance of finding water for our needs.

\author{
BERING \\ Lieutenant WAXEL \\ Fleet Master Sofron Kuitrov \\ Navigator ANDREYAN ESELBERG
}

27 From Khitrov's journal, "The reasons for our action will be found in the exact copy of the minutes given on the next page." (Entry under July 14,4 P. M.) 
$\Varangle$ July $15,17+1$. After Midday

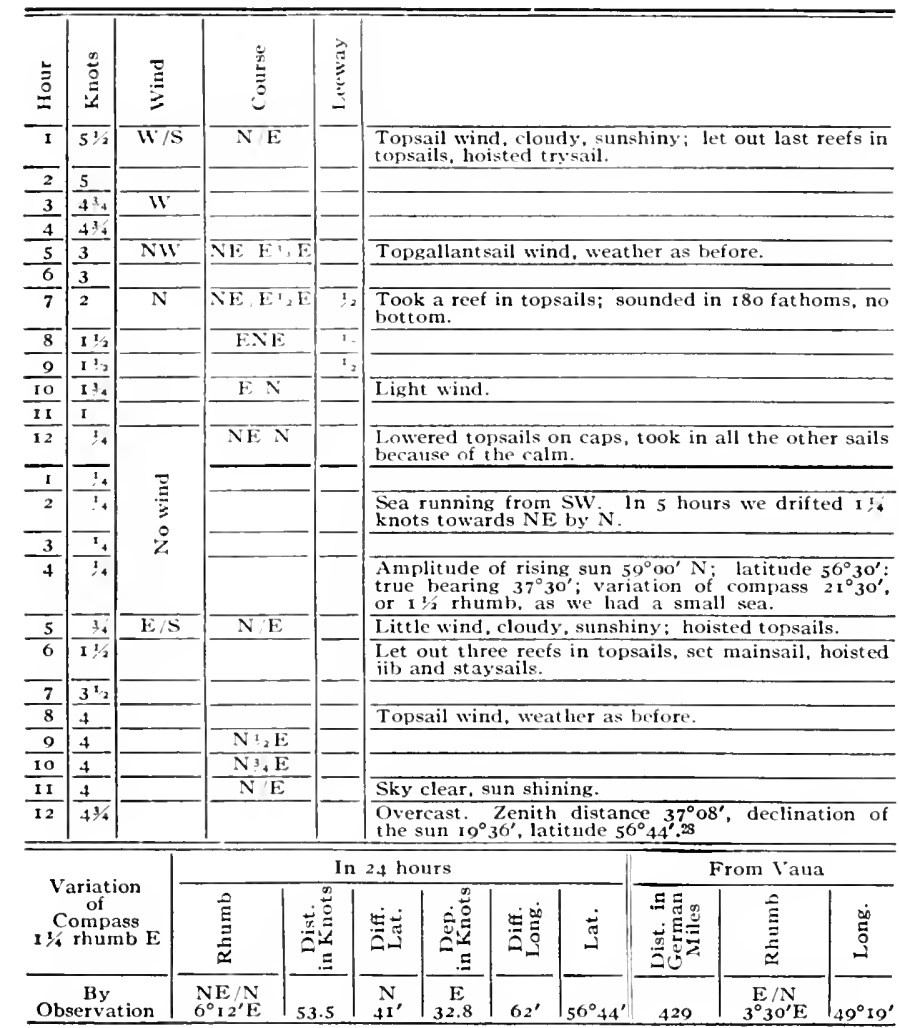

28 Khitrov's journal gives latitude by observation $56^{\circ} 5 I^{\prime}$. 


\section{$2\left(\right.$ July $_{16} 6,1741$. After Midday}

\begin{tabular}{|c|c|c|c|c|c|c|c|c|c|c|c|}
\hline 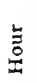 & 总 & $\stackrel{\Xi}{\vdots}$ & 岂 & 总 & & & & & & & \\
\hline I & 5 & $\mathrm{E}$ & $\mathrm{N} / 2 \mathrm{E}$ & & $\begin{array}{l}\text { Top } \\
\text { exce }\end{array}$ & $\begin{array}{l}\text { ail wi } \\
\text { te mai }\end{array}$ & $\begin{array}{l}\text { d. clor } \\
\text {-stayse }\end{array}$ & $\begin{array}{l}\text { idy, su } \\
\text { ail. }\end{array}$ & shiny; & arrying & II sails \\
\hline 2 & $4^{34}$ & & & & & & & & & & \\
\hline 3 & $\overline{412}$ & & & & & & & & & & \\
\hline 4 & $44^{3}$ & & & & Fur & ed spr & sail. & & & & \\
\hline 5 & 5 & & & & & & & & & & \\
\hline 6 & 514 & & & & Ree & -topsa & wind. & & & & \\
\hline 7 & $53 / 4$ & & & & Clo & dy. & & & & & \\
\hline 8 & $4 \frac{1 / 2}{4}$ & & & & Too & two & eefs in & topsails & & & \\
\hline 9 & $4^{3} 4$ & & & & & & & & & & \\
\hline 10 & $4 \pi / 4$ & $\mathrm{E} / \mathrm{N}$ & & $3 / 2$ & Stro & ig reef & topsail & wind. & & & \\
\hline I I & $4 \%$ & & & $3 / 2$ & Sou & ded in & 90 fat & loms, n & bottor & & \\
\hline 12 & 4 & & & $1 / 2$ & & & & & & & \\
\hline$I$ & 3 & ENE & $\mathrm{N}$ & I & & & & & & & \\
\hline 2 & $23 / 4$ & $\mathrm{NE} / \mathrm{I}$ & $\mathrm{N} 3 / 2 \mathrm{~W}$ & I & Top & ail wi & & & & & \\
\hline 3 & 3 & & $\mathrm{~N} / \mathrm{W}$ & $I$ & & & & & & & \\
\hline 4 & 314 & & $\mathrm{~N}_{2} \mathrm{~W}$ & I & Let & out tw & reefs & in topsa & is. & & \\
\hline 5 & $31 / 2$ & & $\mathrm{~N}$ & I & & & & & & & \\
\hline 6 & $31 / 2$ & & $\mathrm{~N}$ & $I$ & & & & & & & \\
\hline 7 & 3 & & $\mathrm{~N}_{4} \mathrm{~W}$ & $I$ & & & & & & & \\
\hline 8 & 4 & & $\mathrm{~N}_{3}{ }_{4} \mathrm{VV}$ & $\mathbf{I}$ & Ree & topsa & wind. & & & & \\
\hline 9 & $3 \%$ & & $\mathrm{~N} / \mathrm{W}$ & $11 / 2$ & Clor & dy, su & shiny. & & & & \\
\hline 10 & $31 / 2$ & & & $I 1 / 2$ & Too & two & efs in & topsails & & & \\
\hline II & $3^{1} 4$ & & & $11 / 2$ & & & & & & & \\
\hline 12 & $23 / 4$ & & & $11 / 2$ & \begin{tabular}{|c} 
Dri \\
tanc \\
58 \\
\end{tabular} & $\begin{array}{l}38^{\circ} 4 \\
22^{\circ}\end{array}$ & $\begin{array}{l}\text { shiny } \\
\text { decli } \\
\text { o corre }\end{array}$ & $\begin{array}{l}\text { Took } \\
\text { nation } \\
\text { ction } \mathrm{m}\end{array}$ & $\begin{array}{l}\text { observa } \\
\text { f the } \mathrm{S} \\
\text { ade. } 9\end{array}$ & $\begin{array}{l}\text { on: zen } \\
19^{\circ} 23^{\prime} \text {. }\end{array}$ & $\begin{array}{l}\text { th dis- } \\
\text { atitude }\end{array}$ \\
\hline \multirow{2}{*}{\multicolumn{3}{|c|}{$\begin{array}{c}\text { Vatiation } \\
\text { of } \\
\text { Compass } \\
1 / 2 \text { rhumb E }\end{array}$}} & \multicolumn{6}{|c|}{ In 24 hours } & \multicolumn{3}{|c|}{ From V'aua } \\
\hline & & & $\stackrel{\mathscr{E}}{\Xi}$ & 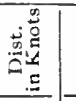 & 芯芯 & 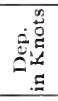 & 至莣 & $\stackrel{\leftrightarrow}{\sim}$ & 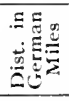 & $\stackrel{\Xi}{E}$ & ¿ \\
\hline \multicolumn{3}{|c|}{$\begin{array}{l}\text { By Dead } \\
\text { Reckoning }\end{array}$} & $\begin{array}{c}N / E \\
0^{\circ} 8^{\prime} N\end{array}$ & 91.6 & $\begin{array}{c}N \\
90^{\prime}\end{array}$ & $\begin{array}{c}\mathrm{E} \\
\mathrm{I} 7.4 \\
\end{array}$ & $32.4^{\prime}$ & $58^{\circ} 14^{\prime}$ & $429^{3 / 4}$ & $\begin{array}{c}\mathrm{E} / \mathrm{N} \\
0^{\circ} 3 \mathrm{I}^{\prime} \mathrm{E}\end{array}$ & $49^{\circ} 51^{\prime}$ \\
\hline
\end{tabular}

29 Khitrov's journal gives latitude by observation $58^{\circ} 17^{\prime}$.

Footnotes to Log of July 17 .

30 Mt. St. Elias. (Identifications in this and the next chapter by E. P. B.)

31 The bluffs just west of the entrance of Vakutat Bay.

$32 \mathrm{By}$, the calendar St. Aphinogena day is July 16, and St. Mariny day is July I7. St. Mariny Point is Cape Suckling of today.

${ }_{33}$ Khitrov's jolurnal: "Took observation of setting sun, W $15^{\circ} \mathrm{N}$, latitude $58^{\circ} 30$; true bearing $39^{\circ} 30^{\prime}$; variation of compass $24^{\circ} 30^{\prime}$." 
o Inly I7, I74I. Afler Midday

\begin{tabular}{|c|c|c|c|c|c|c|c|c|c|c|c|}
\hline 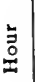 & 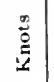 & $\stackrel{\bar{\Xi}}{\Xi}$ & 产 & 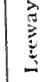 & & & & & & & \\
\hline 1 & 3 & $\mathrm{NE}$ & NNW & $I$ & $\begin{array}{l}\text { At } 1 \\
\text { and } \\
\text { wind } \\
\text { stay } \\
\text { fath } \\
\end{array}$ & $\begin{array}{l}2: 30 \text { w } \\
\text { mong } \\
\text { clear } \\
\text { als, fo } \\
\text { ms, no }\end{array}$ & $\begin{array}{l}\text { sight } \\
\text { hem a } \\
\text { with } \\
\text { resail. } \\
\text { botton }\end{array}$ & $\begin{array}{l}\text { h ligh } \\
\text { high vo } \\
\text { assing } \\
\text { and m } \\
\end{array}$ & $\begin{array}{l}\text { snow-ce } \\
\text { lcano N } \\
\text { clouds; } \\
\text { lainsail; }\end{array}$ & $\begin{array}{l}\text { ered mol } \\
\text { ay W. } 30 \\
\text { arrying to } \\
\text { sounded }\end{array}$ & $\begin{array}{l}\text { ntains } \\
\text { opsail } \\
\text { psails, } \\
\text { n 190 }\end{array}$ \\
\hline$\sqrt{2}$ & $\overline{4^{3} 4}$ & NE N & $\mathrm{NW} \mathrm{N}$ & 1 & & & & & & & \\
\hline 3 & 3 & & & 1 & Let & ut thrt & e reefs & in tops: & ails. & & \\
\hline$\frac{4}{4}$ & $21 / 2$ & NE & $\mathrm{NW} / \mathrm{W}$ & $\mathrm{I}$ & Top & allants & ail win & & & & \\
\hline 5 & 2 & & & 1 & Clou & & & & & & \\
\hline 6 & $1^{1}$ & $\mathrm{~N}$ & WNW & 1 & Ligh & wind. & & & & & \\
\hline 7 & $\overline{I^{1 / 2}}$ & $\mathrm{~N} / \mathrm{W}$ & $\mathrm{W} / \mathrm{N}$ & 1 & $\begin{array}{l}8 \text { o' } \\
\text { bear } \\
\text { rhun } \\
\text { nam } \\
\text { Gern } \\
\text { thos } \\
\text { Nob } \\
\text { hour } \\
\text { shor } \\
\text { we c } \\
\text { is a }\end{array}$ & $\begin{array}{l}\text { ock to } \\
\text { ng } 39^{\circ} \\
\text { bs } \mathrm{E} . \\
\text { d } \mathrm{St} . \\
\text { an } \mathrm{mi} \\
\text { we ha } \\
\text { wet } 18 \\
\text { of } 8 \\
\text { in Ny } \\
\text { lled } \mathrm{St} \\
\text { igh eley }\end{array}$ & $\begin{array}{l}\text { ok am } \\
\text { ok, vo } \\
\text { A poi } \\
\text { phino } \\
\text { es th th } \\
\text { isight } \\
\text { niles, a } \\
\text { we sir } \\
\text { inabo } \\
\text { irarin } \\
\text { ation }\end{array}$ & $\begin{array}{l}\text { plitude } \\
\text { riation } \\
\text { it of th } \\
\text { gena } 31 \\
\text { e moun } \\
\text { ed befor } \\
\text { nd } 5 \mathrm{mi} \\
\text { hted a } \\
\text { it } 12 \mathrm{mi} \\
\text { y.32 Fr } \\
\text { bearng }\end{array}$ & $\begin{array}{l}\text { of sun } \\
\text { of comi } \\
\text { e sighte } \\
\text { bore N } \\
\text { tains on } \\
\text { e. The } \\
\text { les insho } \\
\text { nother p } \\
\text { les from } \\
\text { om this } \\
\text { NNW } 1 / 2\end{array}$ & $\begin{array}{l}15^{\circ} \mathrm{Oo}^{\prime} \mathrm{I} \\
\text { sh } 24^{\circ} 30 \\
\text { shore wh } \\
\text { by E ab } \\
\text { t are low } \\
\text { lcano bea } \\
\text { e. At thi } \\
\text { int of th } \\
\text { is, and th } \\
\text { int insho } \\
V \text {. }\end{array}$ & $\begin{array}{l}\text { true } \\
\text { or } 2 \\
\text { ich we } \\
\text { ut } 10 \\
\text { r than } \\
\text { rs now } \\
\text { same } \\
\text { same } \\
\text { s point } \\
\text { e there }\end{array}$ \\
\hline 8 & 2 & NNW & IV & $I$ & & & & & & & \\
\hline 9 & $I$ & NW & NNE $1 / 2 \mathrm{E}$ & 1 & & & & & & & \\
\hline 10 & I & & & $\mathrm{I}$ & Sour & ded in & $180 \mathrm{fa}$ & homs, n & ao botto & & \\
\hline 11 & $3 / 4$ & & $\overline{\mathrm{NNE}}$ & 1 & $\begin{array}{l}\text { Sour } \\
\text { trys }\end{array}$ & $\begin{array}{l}\text { ded ag } \\
\text { ii. }\end{array}$ & in, no & bottom. & Clewe & up mains & iil and \\
\hline 12 & $7 / 2$ & NNW & $\mathrm{NE}$ & 1 & Sour & ded, $n$ & botto & & & & \\
\hline $\bar{I}$ & $?_{4}$ & & & & $\begin{array}{l}\text { Low } \\
\text { ther }\end{array}$ & $\begin{array}{l}\text { red to } \\
\text { is no }\end{array}$ & $\begin{array}{l}\text { osails } \\
\text { sind. }\end{array}$ & n caps, & lowered & taysails & ecause \\
\hline 2 & 14 & $\Xi$ & & & & & & & & & \\
\hline 3 & $3 / 4$ & 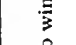 & & & $\begin{array}{l}\text { Eas } \\
\text { in } \mathrm{s}\end{array}$ & $\begin{array}{l}\text { erly sw } \\
\text { hours }\end{array}$ & 11. for & ng us N & SEat th & rate of $I^{3}$ & knots \\
\hline 4 & 214 & $z$ & & & & & & & & & \\
\hline 5 & $\begin{array}{ll}1 \\
1 \\
\end{array}$ & & & & & & & & & & \\
\hline 6 & $\therefore$ & & & & & & & & & & \\
\hline 7 & $13 / 4$ & $\bar{E} / \mathrm{S}$ & NVE:E & I & $\begin{array}{l}\text { Top } \\
\text { tops } \\
\text { jib. }\end{array}$ & $\begin{array}{l}\text { allants } \\
\text { iils anc }\end{array}$ & $\begin{array}{l}\text { ail wir } \\
\text { main }\end{array}$ & $\begin{array}{l}\text { d, clear } \\
\text { ail, hois }\end{array}$ & $\begin{array}{l}\text { with p } \\
\text { sted to }\end{array}$ & $\begin{array}{l}\text { sing clou } \\
\text { ast-stays }\end{array}$ & $\begin{array}{l}\text { is; set } \\
\text { iil and }\end{array}$ \\
\hline 8 & $\overline{I ! 4}$ & $\mathrm{E}$ & NNE & $\mathrm{I}$ & St. I & Iariny & ears $\mathrm{N}$ & W by 1 & $\frac{V \text {, about }}{\text {. }}$ & o Germa & miles. \\
\hline 9 & 2 & & & $I$ & & & & & & & \\
\hline 10 & 4 & ESE & $\mathrm{NE}$ & & & & & & & & \\
\hline 11 & $31 / 2$ & & $\mathrm{~N}$ & & Top & ail win & d; let & out reef: & $\mathrm{s}$ in tops & & \\
\hline 12 & 2 & & & & $\begin{array}{l}\text { Clea } \\
\text { get } \\
\text { clou } \\
\text { the } \\
\text { of } t\end{array}$ & $\begin{array}{l}\text { with } \\
\text { beari } \\
\text { s. In } \\
\text { liart } \\
\text { e comp } \\
\end{array}$ & $\begin{array}{l}\text { passin } \\
\text { g beca } \\
\text { marki } \\
\text { e took } \\
\text { ass as } \\
\end{array}$ & $\begin{array}{l}\text { clouds } \\
\text { use the } \\
\text { in the at } \\
\text { into co } \\
3 / 4 \text { rhun } \\
\end{array}$ & $\begin{array}{l}\text { sir th } \\
\text { shore is } \\
\text { bove-me } \\
\text { onsidera } \\
\text { mb easte }\end{array}$ & $\begin{array}{l}\mathrm{k} \text {. impos } \\
\text { idden by } \\
\text { tioned po } \\
\text { on the v } \\
y .33\end{array}$ & $\begin{array}{l}\text { ible to } \\
\text { heavy } \\
\text { ints on } \\
\text { riation }\end{array}$ \\
\hline \multirow{2}{*}{\multicolumn{3}{|c|}{$\begin{array}{c}\text { Variation } \\
\text { of } \\
\text { Compass } \\
1 \% \text { rhumb E }\end{array}$}} & \multicolumn{6}{|c|}{ In 24 hours } & & om Vaua & \\
\hline & & & $\stackrel{\vec{E}}{\underline{\underline{E}}}$ & 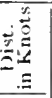 & 晃 & 竞: & 题总 & ت & 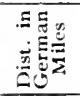 & 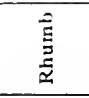 & 离 \\
\hline & $\begin{array}{l}\text { y De: } \\
\text { eckon }\end{array}$ & & $\begin{array}{c}N \\
0^{\circ} 0^{\prime} \mathrm{N}\end{array}$ & 30 & $\begin{array}{c}\mathrm{N} \\
29^{\prime}\end{array}$ & $\begin{array}{l}11 \\
5.7 \\
\end{array}$ & $10.9^{\prime}$ & $58^{\circ} 43^{\prime}$ & 42614 & $\begin{array}{c}\mathrm{E} / \mathrm{N} \\
\mathrm{O}^{\circ} 33^{\prime} \mathrm{N}\end{array}$ & $49^{\circ} 40^{\prime}$ \\
\hline
\end{tabular}

(Footncit 30-33 at bottom of opposite page.) 


\section{Ћ July 18, I74I. After Midday}

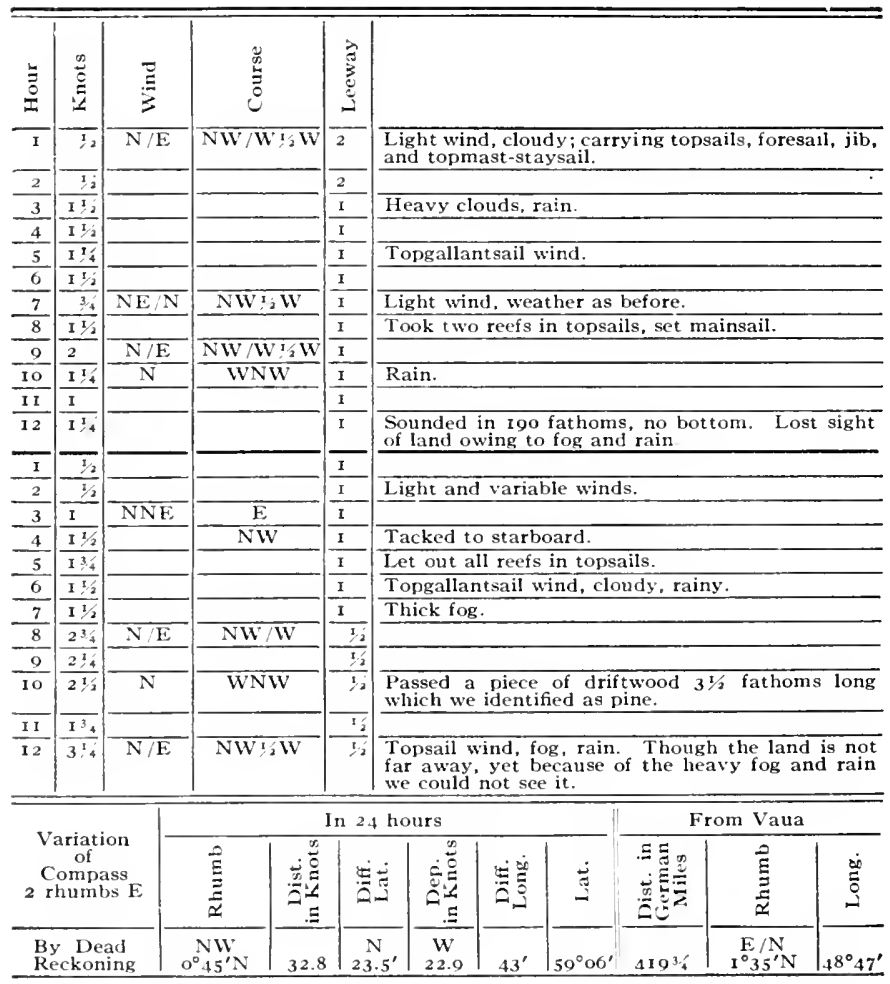


July I9, I7fI. After Midday

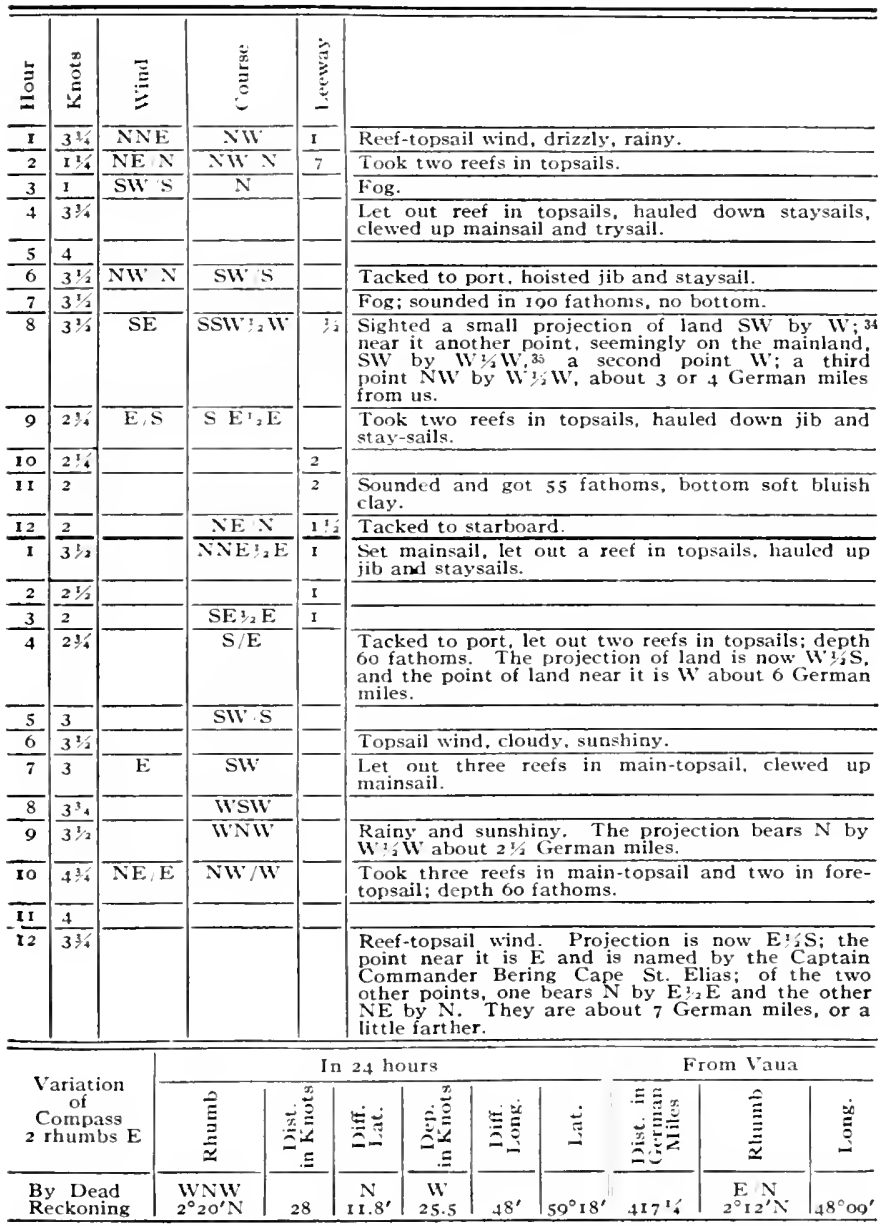

34 Pinnacle Rock off Cape St. Elias,

js Cape St. Elias. 


\section{(3) July 20, I74T. After Midday}

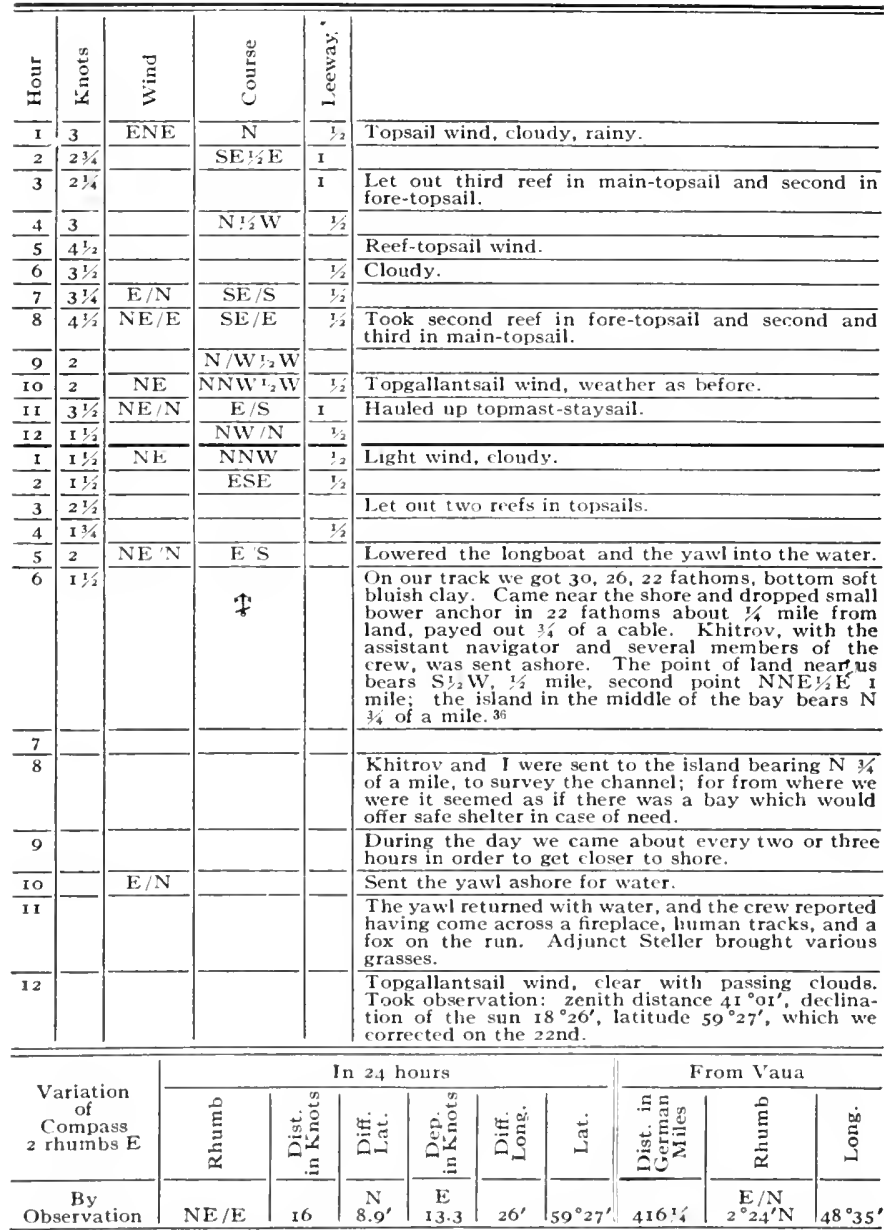

35 Khitrov's journal: "At six o'clock we came within $1 / 2$ mile of the island and dropped the small bower anchor in 22 fathoms of water. We called that island St. Elias. - The Captain Commander sent me in the longboat to examine the strait and to find an anchorage among the islands seen by us in the N."July 20 is St. Elias day. "The island in the middle of the bay [which] bears $\mathrm{N} 3 / 4$ of a mile" is Wingham Island. 
of July $21, I 74 I$. After Midday

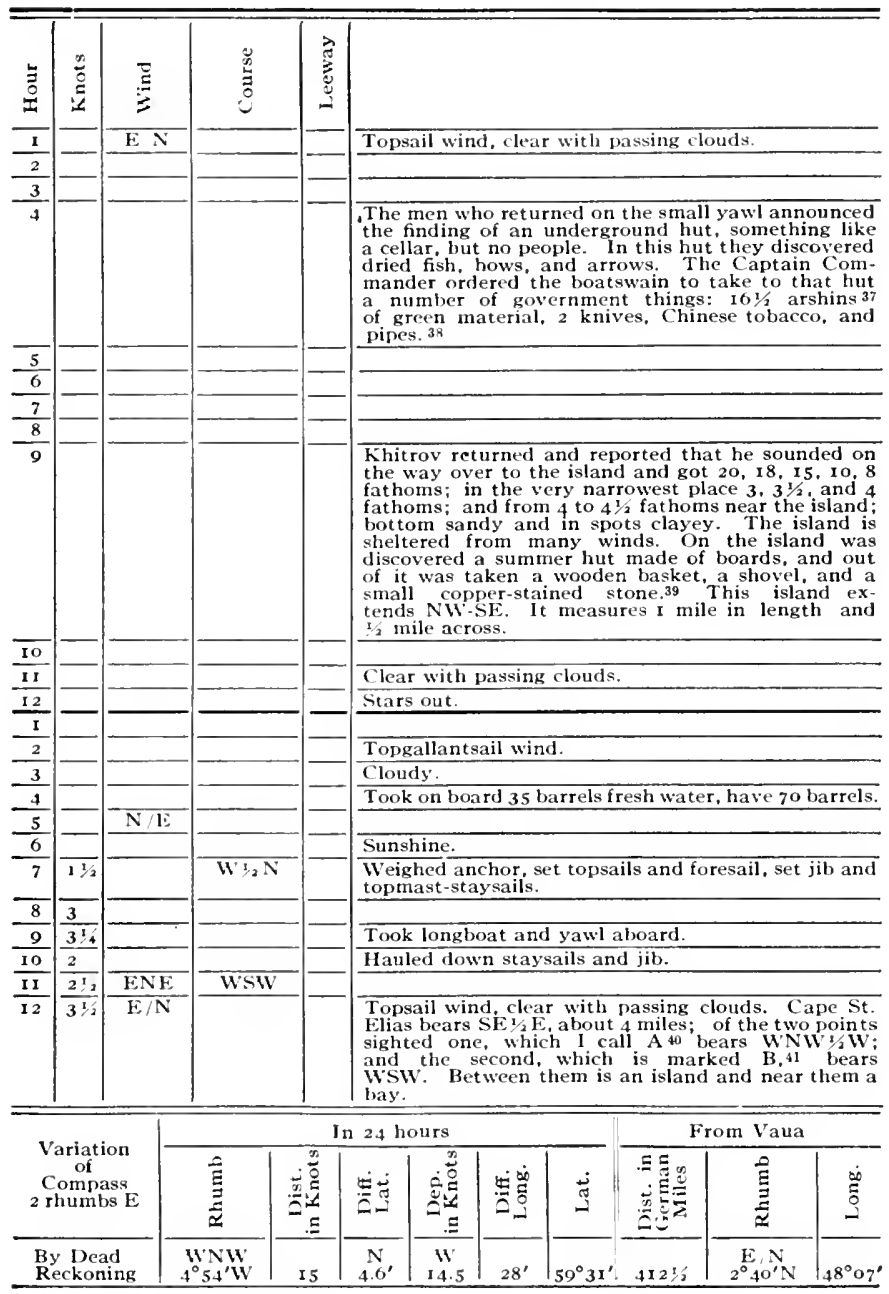

(Foolnotes 37-4I on nexl page.) 
Footnotes to Log of July $2 I$ on page 97.

37 An arshin is equal to 28 inches.

$3 s$ In 1790 , when the Billings expedition visited this island one of the old natives said that he remernbered that when he was a boy a ship had been close into the bay on the west side of the island and had sent a boat on shore; but on its approaching land the natives all ran away. When the ship sailed, they returned to their hut and found in their subterraneous storeroom some glass beads, leaves (tobacco), an iron kettle, and something else" (Martin Sauer: An Account of a Geographical and Astronomical Expedition to the Northern Larts of Russia. . . by Commodore Joseph Billings, Londcn, I 802 , p. 194.)

Bering's coming must have made quite a stir among the natives of Alaska. The in. habitants of one of the islands in Prince William Sound told Sarychev (one of Billings' officers) that a ship came to Kayak lsland and that the people came ashore and left some knives (Sarychev: Account of a loyage of Discovery, London, I806, Part II, pp. $52-53$ )

39 Khitrov's journal: "I came aboard from shore in the longboat and reported to the Captain Commander that the channel to the island has a depth of $3,31 / 2$, and 4 fathoms. that the bottom is sandy and in places slimy, and that it is sheltered from many winds. I reported also that I found on that island a hut made of hewn boards, the floor of the hut being also of boards. In place of an oven it had a kind of fireplace. From this hut I brought along to show Captain Commander Bering a wooden basket, a shovel, a small stone which had copper stains on it, and one common round stone with a hole. All of these things I gave to the Captain Commander. During the whole time that we remained at anchor we took in 35 barrels of water. At seven in the morning we weighed anchor. set the topsails, foresail, mainsail, jib, and staysails, took on board the small and large boats, and sailed away. That which happened to me on that island is noted down in the report which I made on the island of St. Elias."

40 A point on the mainland.

41 Cape Hinchinbrook. 
July $2 I, I 74 I$

\section{RePORt ON St. Elias [KaYAK] ISLAND42}

The island of St. Elias lies $\mathrm{NE}$ and SW. It is about $3 \frac{1}{2}$ miles from the mainland to the bay. It is about $3 \frac{1}{4}$ German miles long and not more than $31 / 2$ versts across. According to my calculation it is in latitude $59^{\circ} 39^{\prime}$ [or $49^{\prime}$-not clear] and $48^{\circ} 42^{\prime}$ of longitude from Vaua on the course $\mathrm{E}$ by $\mathrm{N} 1 / 4 \mathrm{~N}$, distant $4161 / 2$ miles from the northerly point. This island stands out alone in the sea like a stone column; extending from it a submerged reef of rocks may be seen in low water. From its northerly point about $1 / 4$ of a mile there lie, in the bay between the island and the mainland, other islands which offer shelter from winds. To one of these islands the Captain Commander sent me with several men in the longboat to examine the strait and to find a good anchorage. In going there between $\mathrm{N}$ and $\mathrm{E}$, the depth of the channel was $25,22,18,10,7,6,4$, and $31 / 2$ fathoms where it is possible to anchor; and in going to it one should keep in the middle between St. Elias and the island and watch out for the projecting cape of the island, because running out from the middle of the island of St. Elias and this island are submerged reefs. On this island there are many trees, namely, fir, larch, cedar, and other kinds, but little of it is good for shipbuilding because we had oecasion to look for a piece of timber for the topsail yards but did not find it. We came across (on the island) a hut which was made of hewn boards; the floor was also made of these boards. In place of an oven there was in one corner of the hut a fireplace. Near the hut was found a wooden basket in which were shell fish, which showed that the inhabitants here used them for food. We did not see any people, but it was quite evident that they had been here shortly before our arrival. I brought from this island, in order to show our Captain Commander, one basket, a shovel, a small stone with copper stains on it. It was not possible to tell whether there was or was not suitable timber for shipbuilding on the mainland, which land is two miles from this island. The mainlan 1 has high snow-covered mountains and volcanoes, and, from this as well as from the fact that the material for the hut and floor was of good wide boards, we may assume that there may be good timber there.

On St. Elias Island there is also plenty of wood. On that island there was discovered an earthen hut containing dried fish which were cured this summer. On the beach were seen human tracks in the sand, a fireplace, and running foxes. From all this it may be reasoned that the natives, on seeing us, ran away and hid or that they have their habitations on the mainland and come to the island in summer to cateh fish and other sea animals. The Captain Commander deeided to send to that hut, in order to please the people, several of the things which we had to give as presents: namely, $16 \frac{1}{2}$ arshins of green material, 2 iron knives, 20 Clinese strings of beads, 2 iron pipes for smoking Chinese tobacco, called "sliar." All these things were left there. The attached sketch map gives the position of the bay and the islands and their relation to the mainland.

42 From Khitrov's journal. Khitroy's sketch map, mentioned in the last sentence of this report, was published by Sokolov in Zapisk: Hydrogr. Depart... Tol. 9. St. Petersburg. 1851, and reproduced by Lauridsen in his "Vitus Bering," Chicago, 1889 . In style of execution it resembles his hitherto unpublished map of the Shumagin Islands, our Fig. II. 


\section{$\Varangle$ July 22, I74I. After Midday}

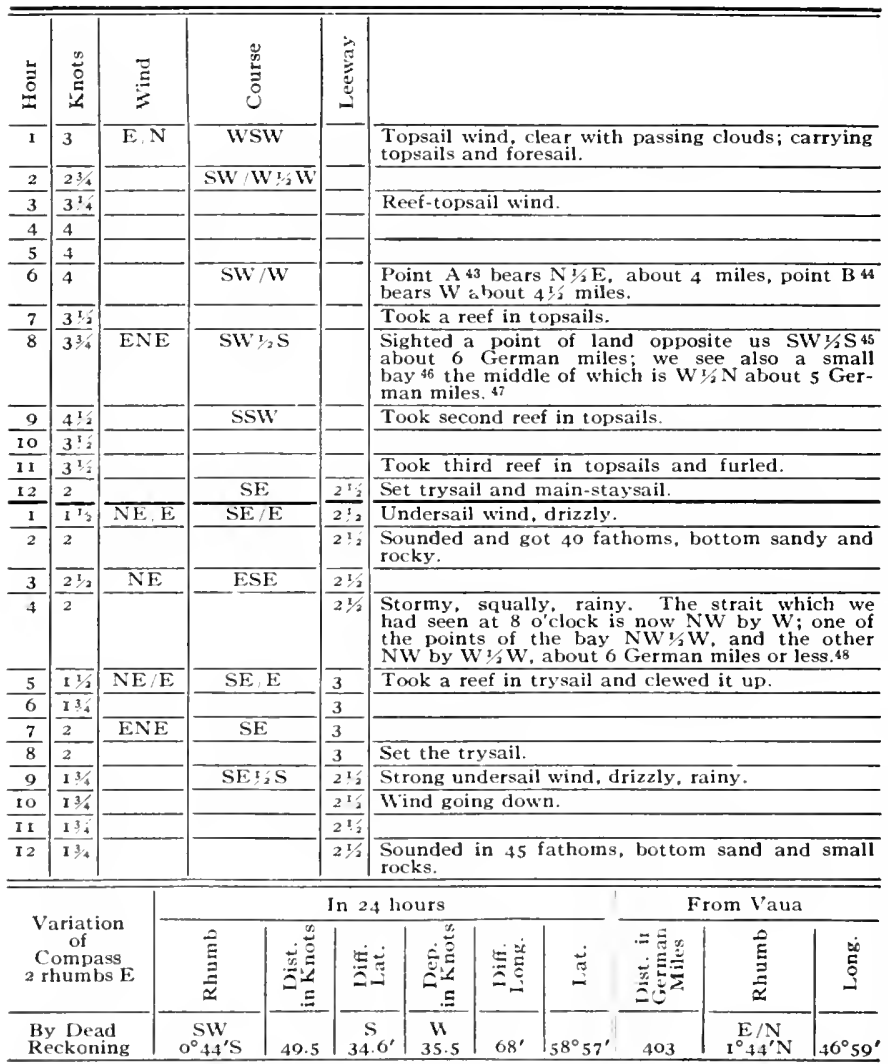

43 Some point on the mainland. See log of July 21. last liour.

4t Cave IIinchinbrook.

45. SE point of Montague Island.

46 The strait between Cape Hinchinbrook and Montague Island.

47 Klitrov's journal: "Point $\mathrm{E}$ [easterly point] bears $\mathrm{SW} / \mathrm{s} \mathrm{S}, 61 / 2$ [miles]; the strait $W^{1 / 3} \mathrm{~N}$,

$\$$ Khitrov's journal: "The strait bears NW/W, Point D NW'; W, 6 miles." 
2f July 23, 1741. After Midday

\begin{tabular}{|c|c|c|c|c|c|c|c|c|c|c|c|c|}
\hline$\stackrel{5}{\Xi}$ & $\stackrel{\infty}{0}$ & 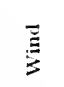 & \multicolumn{2}{|c|}{ 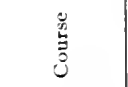 } & $\hat{\ddot{Z}}$ & & & & & & & \\
\hline 1 & 23 & ENE & \multicolumn{2}{|c|}{$\mathrm{SE}_{1 / 2 \mathrm{~S}}$} & $21 / 2$ & \multicolumn{7}{|c|}{$\begin{array}{l}\text { Undersail wind, drizzly; set topsail reefs; depth } \\
\text { so and } 60 \text { fathoms, sandy botcom. }\end{array}$} \\
\hline 2 & 2 & & & & $\frac{T / 2}{1 / 2}$ & & & & & & & \\
\hline 3 & 2 & $\mathrm{E} / \mathrm{N}$ & \multicolumn{2}{|c|}{$\mathrm{SE} / \mathrm{S}$} & $21 / 2$ & & & & & & & \\
\hline 4 & 2 & & & & $2 \%$ & & & & & & & \\
\hline 5 & $I 3 / 4$ & & & & $2 \%$ & \\
\hline 6 & $11 / 2$ & \multirow[b]{2}{*}{$\mathrm{E}$} & & & $25 / 2$ & \multicolumn{7}{|c|}{ Topsail wind, heavy swell. } \\
\hline 7 & $\overline{13 / 4}$ & & \multicolumn{2}{|c|}{$\mathrm{S} E I_{2} \mathrm{E}$} & $2 \%$ & & & & & & & \\
\hline 8 & 2 & & & & $2 \%$ & \multicolumn{4}{|c|}{ Let out reef in trysail. } & & & \\
\hline 9 & 2 & & & & $2 \%$ & \multicolumn{7}{|c|}{ Hauled up staysails. } \\
\hline 10 & 25 & & & & $2 \pm / 2$ & & & & & & & \\
\hline II & $21 / 2$ & & & & $21 / 2$ & \multicolumn{7}{|c|}{ Hauled down staysails, furled fore-topsail. } \\
\hline 12 & 214 & & & & $2 \frac{1 / 2}{2}$ & & & & & & & \\
\hline $\mathrm{I}$ & $21 / 2$ & & & & $21 / 2$ & & & & & & & \\
\hline 2 & $\overline{3}$ & & & & $21 / 2$ & \multicolumn{7}{|c|}{$\begin{array}{l}\text { Set the fore-topsail reefed, hauled up fore-top- } \\
\text { mast-staysail. }\end{array}$} \\
\hline 3 & 2 & $\mathrm{EN}$ & $\mathrm{SSE}$ & $\mathrm{E}$ & 2 & \multicolumn{7}{|c|}{ Topsail wind. } \\
\hline 4 & 2 & & & & 2 & \multicolumn{7}{|c|}{ Drizzly, rainy. } \\
\hline 5 & $2 \frac{2}{4}$ & & & & 2 & & & & & & & \\
\hline 6 & $2 \%$ & & & & 2 & \multicolumn{7}{|c|}{ Hauled down fore-topmast-staysail. } \\
\hline 7 & $2 \%$ & & & & 2 & \multicolumn{7}{|c|}{ (2) } \\
\hline 8 & $2 \pi / 2$ & & & & 2 & \multirow{2}{*}{\multicolumn{7}{|c|}{ Reef-topsail wind. }} \\
\hline 9 & $2 \%$ & & & & 2 & & & & & & & \\
\hline Io & $23 / 4$ & & & & 2 & \multicolumn{7}{|c|}{ Drizzly, rainy. } \\
\hline I I & $3 \frac{1}{4}$ & & & & 2 & \multicolumn{7}{|c|}{ Hauled up fore-topmast-staysail. } \\
\hline 12 & 3 & & & & 2 & & & & & & & \\
\hline \multirow{2}{*}{\multicolumn{3}{|c|}{$\begin{array}{l}\text { Variation } \\
\text { of } \\
\text { Compass } \\
2 \text { rhumbs E. }\end{array}$}} & \multicolumn{7}{|c|}{ In 24 hours } & \multicolumn{3}{|c|}{ From Vaua } \\
\hline & & & $\frac{\vec{E}}{\underline{E}}$ & 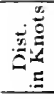 & 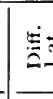 & & 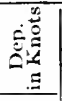 & $\underset{0}{0}$ & 殅 & 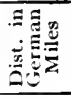 & 章 & 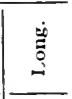 \\
\hline & $\begin{array}{l}\text { Dea } \\
\text { eckon }\end{array}$ & & $\begin{array}{l}\mathrm{SSWV} \\
0^{\circ} 35^{\prime} \mathrm{S} \\
\end{array}$ & 54.6 & $\begin{array}{r}\mathrm{S} \\
50 . \\
\end{array}$ & & $\begin{array}{c}\text { W } \\
20.4\end{array}$ & $39^{\prime}$ & $58^{\circ} \mathrm{o} 6^{\prime}$ & 400 & $\begin{array}{r}\mathrm{E} / \mathrm{N} \\
0^{\circ} \mathrm{O} 2^{\prime} \mathrm{N}\end{array}$ & $46^{\circ} 20^{\prime}$ \\
\hline
\end{tabular}


ㅇ July 24, I74I. After Midday

\begin{tabular}{|c|c|c|c|c|c|c|c|c|c|c|c|}
\hline 苛 & 点 & 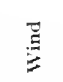 & 总 & 离 & & & & & & & \\
\hline 1 & 2 & $\mathrm{E}$ & SSE & $21 / 2$ & \begin{tabular}{|l} 
Reef \\
fore \\
topn
\end{tabular} & $\begin{array}{l}\text { topsail } \\
\text { and } n \\
\text { ast-sta }\end{array}$ & $\begin{array}{l}\text { wind, } \\
\text { ain-t, } \\
\text { sail. }\end{array}$ & $\begin{array}{l}\text { sky ov } \\
\text { psail re }\end{array}$ & $\begin{array}{l}\text { ercast, } r \\
\text { efed, tr }\end{array}$ & $\begin{array}{l}\text { n; carryi } \\
\text { sail, and }\end{array}$ & $\begin{array}{l}\text { fore- } \\
\text { fore }\end{array}$ \\
\hline 2 & 2 & & & $\overline{21_{2}}$ & & & & & & & \\
\hline 3 & 2 & & & $2 ! 2$ & & & & & & & \\
\hline 4 & $1 \%$ & & & $21 / 2$ & Ligh & wind, & easter & y swell. & & & \\
\hline 5 & $1: 3$ & & & $2+\frac{1}{2}$ & & & & & & & \\
\hline 6 & 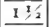 & & & $2^{2 / 2}$ & & & & & & & \\
\hline 7 & 212 & $\mathrm{E} / \mathrm{S}$ & $\mathrm{S} / \mathrm{E}$ & $2 ! 2$ & & & & & & & \\
\hline 8 & $\mathrm{I}^{34_{4}}$ & & & $2 \frac{11 / 2}{2}$ & & & & & & & \\
\hline 9 & 3 & $E$ & $\mathrm{~S} \mathrm{E}^{I_{z} \mathrm{E}}$ & 2 & Stro & g wine & and $r$ & in. & & & \\
\hline 10 & 3 & $\mathrm{E} / \mathrm{N}$ & $\mathrm{SSE}, 2 \mathrm{E}$ & 2 & & & & & & & \\
\hline 11 & 3 & $\mathrm{NE} / \mathrm{I}$ & $\mathrm{SE}^{2} \mathrm{E}$ & 2 & & & & & & & \\
\hline 12 & 3 & & & 2 & & & & & & & \\
\hline 1 & 2 & & & 2 & $\overline{\text { Furl }}$ & $d \operatorname{tons}$ & & & & & \\
\hline 2 & $\mathrm{I}_{4}$ & & $\mathrm{SE}$ & 3 & & & & & & & \\
\hline 3 & $\mathrm{I}^{2} 4$ & & & 3 & & & & & & & \\
\hline 4 & $11 / 2$ & & & 3 & Hea & ed the & lead a & I night & without & nding bot & tom. \\
\hline 5 & 112 & & & 3 & Top & ail win & 1, swe & & & & \\
\hline 6 & 2 & & & 3 & & & & & & & \\
\hline 7 & $2 ! 4$ & $\mathrm{E} / \mathrm{N}$ & $\mathrm{SE} / \mathrm{S}$ & 3 & & & & & & & \\
\hline 8 & $1^{3 / 4}$ & & & 3 & Ree & topsai & wind & & & & \\
\hline 9 & $1 ! 2$ & ENE & $\mathrm{SSE}^{2}=\mathrm{E}$ & 3 & Glor & my. & & & & & \\
\hline 10 & $\overline{15 / 2}$ & & & 3 & Top & ail win & & & & & \\
\hline $1 \mathrm{I}$ & $\mathrm{I}_{2}$ & & & 3 & East & erly sw & & & & & \\
\hline 12 & $1 \%$ & & & 3 & $\begin{array}{l}\text { Furl } \\
\text { and } \\
\text { wing } \\
\mathrm{N} \text { a } \\
\text { the } \\
\text { we } \\
\text { by } 1\end{array}$ & $\begin{array}{l}\text { d fore } \\
\text { trysail } \\
\text { it } \\
\text { id } w \\
\text { fog } n \\
\text { aw lon } \\
\text { a dista } \\
\end{array}$ & $\begin{array}{l}\text { ail an } \\
\text { on ac } \\
\text { tad b } \\
\text { o obs } \\
\text { vente } \\
\text { the } \\
t \quad 27 \\
\end{array}$ & $\begin{array}{l}\text { hove } \\
\text { count of } \\
\text { en our } \\
\text { rve mor } \\
\text { us f } \\
\text { 2nd? b } \\
\text { erman }\end{array}$ & $\begin{array}{l}\text { o under } \\
\text { the dri } \\
\text { purposs } \\
\text { e carefu } \\
\text { om doin } \\
\text { ears by } \\
\text { miles. }\end{array}$ & $\begin{array}{l}\text { he main- } \\
\text { ly weath } \\
\text { to sail b } \\
\text { y the coa } \\
\text { so. Th } \\
\text { rue com! }\end{array}$ & $\begin{array}{l}\text { taysail } \\
\text { er and } \\
\text { etween } \\
\text { t, but } \\
\mathrm{e} \text { land } \\
\text { ass } \mathrm{N} \\
\end{array}$ \\
\hline \multirow{2}{*}{\multicolumn{3}{|c|}{$\begin{array}{l}\text { Variation } \\
\text { of } \\
\text { Compass } \\
2 \text { rhumbs E }\end{array}$}} & \multicolumn{6}{|c|}{$\ln 24$ hours } & \multicolumn{3}{|c|}{ From Vaua } \\
\hline & & & $\frac{\hat{E}}{E}$ & 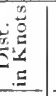 & 氙苞 & 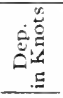 & 逗总 & 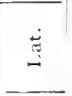 & 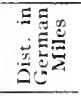 & $\underset{\underline{E}}{\bar{E}}$ & 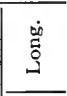 \\
\hline \multicolumn{3}{|c|}{$\begin{array}{l}\text { By Dead } \\
\text { Reckoning }\end{array}$} & $\begin{array}{l}\mathrm{SSW} \\
3^{\circ} \mathrm{OI} \text { 'S }\end{array}$ & 45 & $\begin{array}{c}\mathrm{S} \\
4^{2} 2.3^{\prime} \\
\end{array}$ & $\begin{array}{l}W \\
15 \\
\end{array}$ & $28^{\prime}$ & $57^{\circ} 24^{\prime}$ & $397^{3 / 4}$ & $\begin{array}{r}\mathrm{E} / \mathrm{N} \\
1^{\circ} 29^{\prime} \mathrm{E}\end{array}$ & $45^{\circ} 59^{\prime}$ \\
\hline
\end{tabular}


h) July 25, I74I. Afler Midday

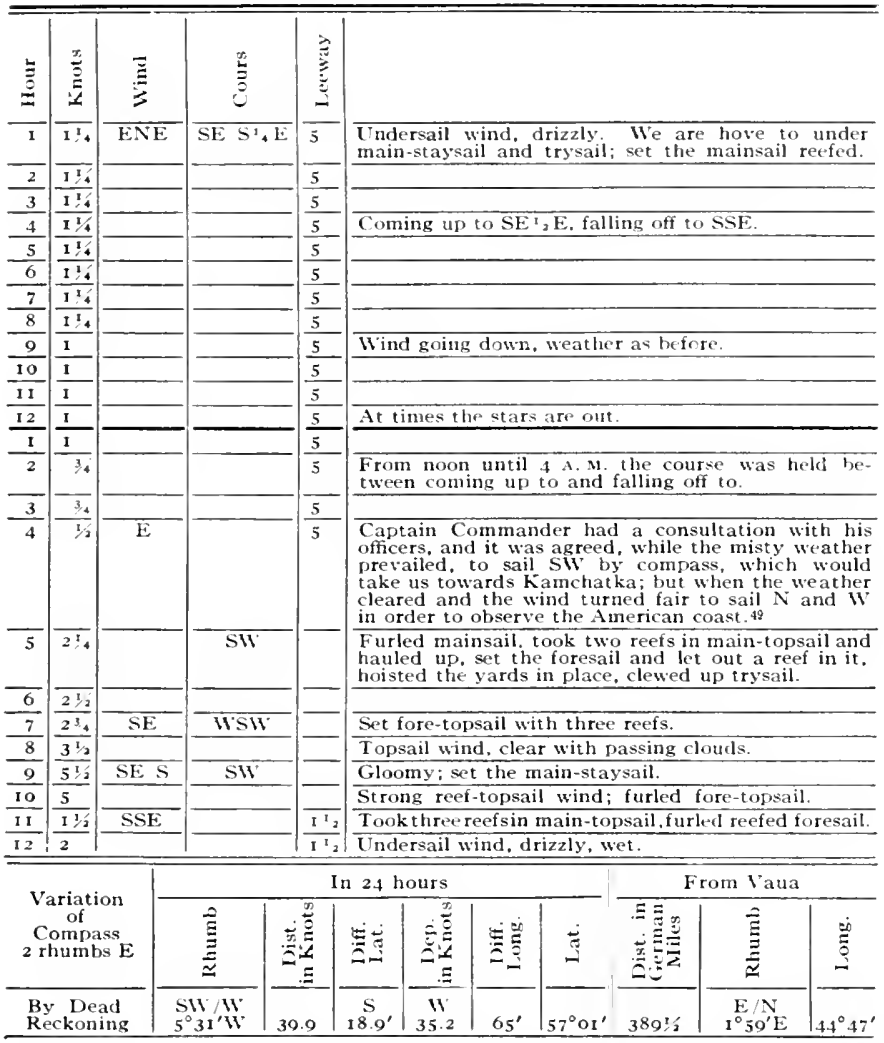

19 Klitrov's journal: "Got under way again. With the Captain Commander it was agreed while this gloomy weather lasted to hold the course SW in order to keep off the land about which we knew nothing, but in clear weather to hold a iv by $N$ course in order to see the mainland." 


\section{4 \\ LOG BOOK OF THE "ST. PETER"}

July 26, I74I. After Midday

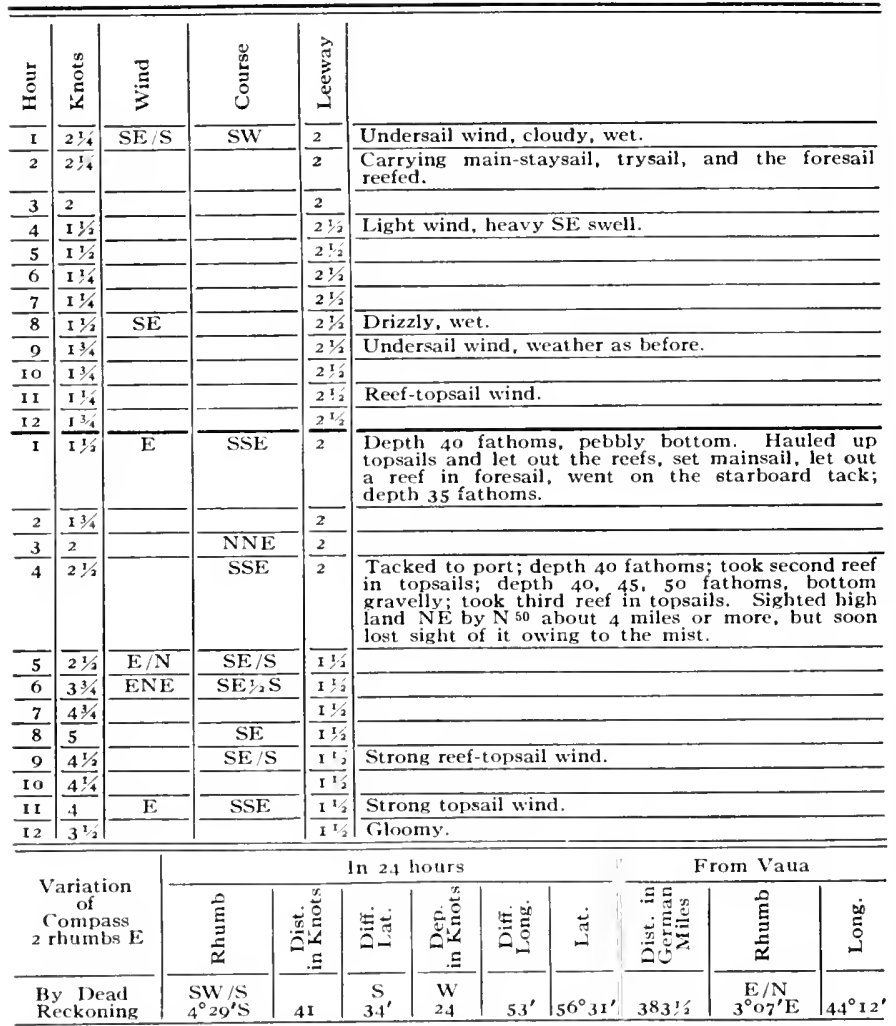

50 Black Point, the southern extremity of Sitkalidak Island. Khitrov's journal: "Sighted land $\mathrm{NE}$ by $\mathrm{N}$, about 8 miles away." 


\section{(3) July 27, 17.71. After Midday}

\begin{tabular}{|c|c|c|c|c|c|c|c|c|c|c|c|c|}
\hline$\stackrel{5}{3}$ & 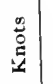 & $\stackrel{D}{3}$ & 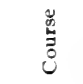 & & 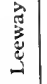 & & & & & & & \\
\hline$I$ & 3 & $\mathrm{E} / \mathrm{S}$ & $\mathrm{S} / 2 \mathrm{E}$ & & $\overline{12 / 2}$ & $\begin{array}{l}\text { Ree } \\
\text { tops }\end{array}$ & $\begin{array}{l}\text { opsai } \\
\text { Is. }\end{array}$ & wind, & cloudy & , wet: I & out a $r$ & eef in \\
\hline 2 & $2 \%$ & $\mathrm{SE} / \mathrm{S}$ & $\mathrm{S} / \mathrm{W}$ & & $\overline{15}$ & & & & & & & \\
\hline 3 & 314 & $\mathrm{SE} / \mathrm{S}$ & SSW $1 / 2$ & & $11 / 2$ & Let & ut two & reefs in & n topsal & ils. & & \\
\hline 4 & $23 / 4$ & & $\mathrm{SW} / \mathrm{S}$ & & $11 / 2$ & Set & b and & fore-top & mast-st & taysail. & & \\
\hline 5 & $31 / 2$ & & & & $1 \%$ & & & & & & & \\
\hline 6 & $31 / 2$ & & & & $11 / 2$ & Sou & ded an & d got 7 & 0 and 6 & o fathon & & \\
\hline 7 & $3 \%$ & & & & $1 \%$ & & & & & & & \\
\hline 8 & $31 / 2$ & & & & $I 1 / 2$ & Top & ail win & d, clouc & dy. & & & \\
\hline 9 & 334 & & & & $12 / 3$ & Too & two $t$ & eefs in & topsail & Is. 51 & & \\
\hline IO & $\overline{21 / 2}$ & $\mathrm{SE}$ & SSW & & 2 & $\begin{array}{l}\text { Dep } \\
\text { hau }\end{array}$ & h dow & $\begin{array}{l}35.40 \\
\text { n stays }\end{array}$ & $\begin{array}{l}45 \text { fa } \\
\text { ail, too }\end{array}$ & $\begin{array}{l}\text { thoms, } \\
k \text { three }\end{array}$ & $\begin{array}{l}\text { ravelly b } \\
\text { eefs in top }\end{array}$ & $\begin{array}{l}\text { ot tom; } \\
\text { sails. }\end{array}$ \\
\hline 11 & $2 \%$ & & & & 2 & & & & & & & \\
\hline 12 & 2 & ESE & $\mathrm{S}$ & & 2 & Hea & ed leas & 1. no bo & ottom. & & & \\
\hline 1 & 2 & & $5: 2$ & & 2 & & & & & & & \\
\hline 2 & $31 / 2$ & & & & 2 & $\begin{array}{l}\text { Hat } \\
\text { fore } \\
\text { stay }\end{array}$ & $\begin{array}{l}\text { led up } \\
\text { topsail } \\
\text { sail, fur }\end{array}$ & $\begin{array}{l}\text { fore-to } \\
\text { und } \\
\text { led mai }\end{array}$ & $\begin{array}{l}\text { psail; d } \\
\text { ersail } \\
\text { in-topsa }\end{array}$ & $\begin{array}{l}\text { depth } 40 \\
\text { wind; } \\
\text { ail. }\end{array}$ & $\begin{array}{l}\text { fathoms } \\
\text { pwered }\end{array}$ & $\begin{array}{l}\text { furled } \\
\text { retop- }\end{array}$ \\
\hline 3 & $31 / 2$ & $\mathrm{SE} / \mathrm{E}$ & S IV & & 2 & & & & & & & \\
\hline 4 & $23 / 4$ & ESE & $\mathrm{S} 1 / 2 \mathrm{~V}$ & & 3 & & & & & & & \\
\hline 5 & $21 / 2$ & $\mathrm{SE}$ & SSIV & & 3 & & & & & & & \\
\hline$\overline{6}$ & $2 \div 4$ & & & & 3 & $\begin{array}{l}\text { Set } \\
\text { pas }\end{array}$ & $\begin{array}{l}\text { main-t } \\
\text { ng clor }\end{array}$ & $\begin{array}{l}\text { opsail; } \\
\text { ids. }\end{array}$ & reef-to & opsail v & nd, clear & with \\
\hline 7 & 2 & $\mathrm{SE} / \mathrm{S}$ & $\mathrm{SW} / \mathrm{s}$ & & 215 & & & & & & & \\
\hline 8 & $2 \%$ & & & & $21 / 2$ & $\begin{array}{l}\text { Loo } \\
\text { but } \\
\text { seen } \\
\text { lead }\end{array}$ & $\begin{array}{l}\text { ed fro } \\
\text { saw n } \\
\text { about } \\
\text { line is }\end{array}$ & $\begin{array}{l}\text { m cross } \\
\text { one. I } \\
5 \text { or I } \\
\text { all we }\end{array}$ & $\begin{array}{l}\text { strees i } \\
\text { n clear } \\
\text { o Germ } \\
\text { have to }\end{array}$ & $\begin{array}{l}\text { in all di } \\
\text { weathe } \\
\text { nan mile } \\
\text { o go on. }\end{array}$ & $\begin{array}{l}\text { ections fo } \\
\text { land mis } \\
\text { from us. }\end{array}$ & $\begin{array}{c}\text { land } \\
\text { The be } \\
\text { The }\end{array}$ \\
\hline$\overline{9}$ & 2 & & & & $21 / 2$ & $\begin{array}{l}\text { Sou } \\
\text { sma }\end{array}$ & $\begin{array}{l}\text { ded an } \\
1 \text { rocks }\end{array}$ & $\begin{array}{l}\text { got } \\
\text { set for }\end{array}$ & $\begin{array}{l}50 \text { fathe } \\
\text { e-topsal }\end{array}$ & $\begin{array}{l}\text { oms, bot } \\
\text { iil. }\end{array}$ & om of sar & d and \\
\hline 10 & $31 / 2$ & & & & 2 & & & & & & & \\
\hline 11 & $31 / 2$ & & & & 2 & I.et & out twe & reefs is & n topsai & ils. & & \\
\hline 12 & 3 & & & & 2 & Ree & topsai & wind & and $a$ & head swe & 1. drizzly, & rainy. \\
\hline \multirow{2}{*}{\multicolumn{3}{|c|}{$\begin{array}{c}\text { Variation } \\
\text { of } \\
\text { Compass } \\
2 \text { rhumbs E }\end{array}$}} & \multicolumn{7}{|c|}{ In 24 hours } & & om Vaua & \\
\hline & & & 莺 & $\stackrel{\dot{\omega}}{\vec{\sigma}}$ & 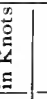 & 苛 & 造递 & 量 & 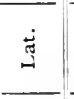 & 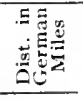 & 章 & ํํㄹ \\
\hline \multicolumn{3}{|c|}{$\begin{array}{l}\text { By Dead } \\
\text { Reckoning }\end{array}$} & $\begin{array}{l}\text { WSW } \\
5^{\circ} 05^{\prime} W\end{array}$ & 67 & & $\underset{20^{\prime}}{\mathrm{S}}$ & $\begin{array}{c}W^{\prime} \\
6.3 .8\end{array}$ & $114.7^{\prime}$ & $56^{\circ} 11^{\prime}$ & $370 \% / 4$ & $\begin{array}{l}\mathrm{E} / \mathrm{N} \\
33^{\circ} 7 \mathrm{E}\end{array}$ & $42^{\circ} \times 7^{\prime}$ \\
\hline
\end{tabular}

5! Khitrov's journal: "Sounded, but no bottom. We are in danger of running on sand banks and therefore cannot keep close to land, hence have kept off a few points." 
of July 28, 174I. After Midday

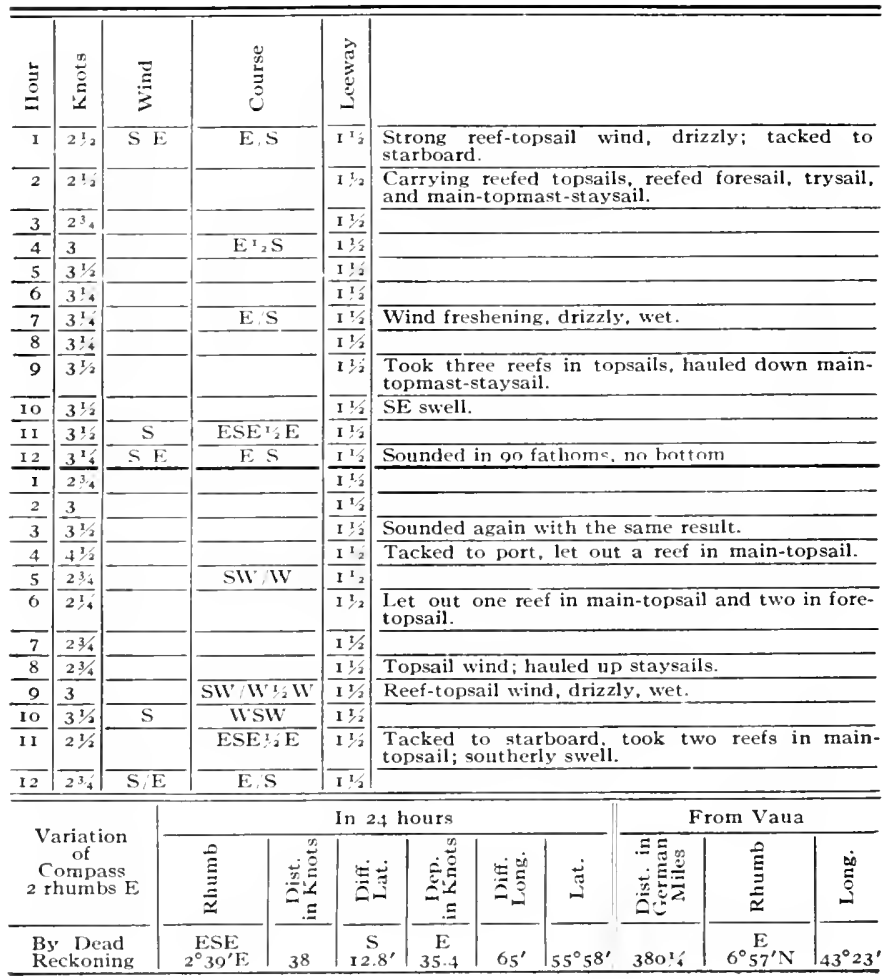




\section{§ July 29, 174I. After Midday}

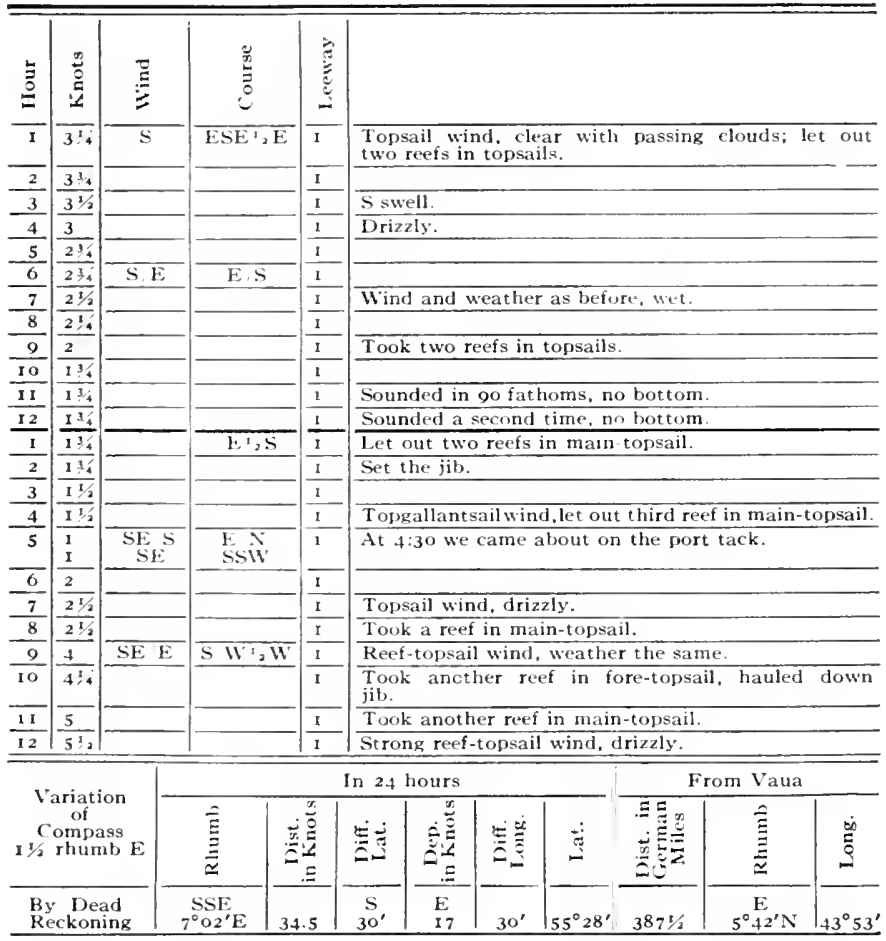


2f July 3o, 174I. After Midday

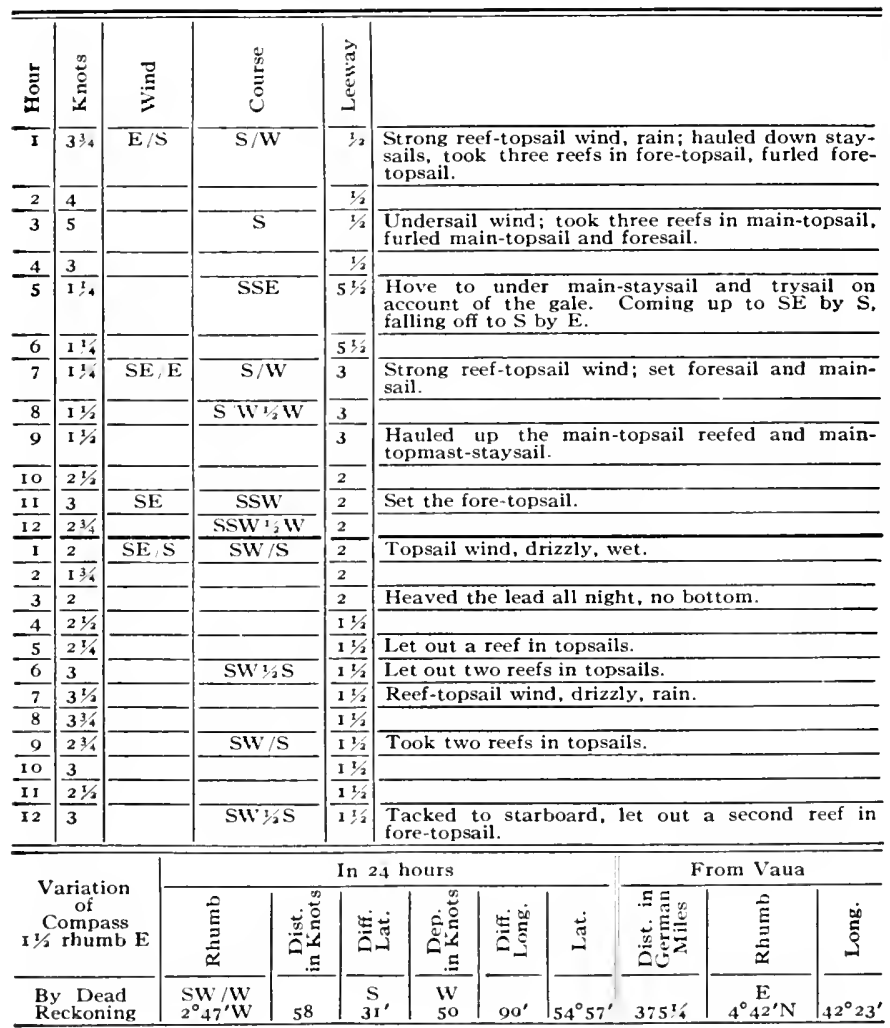




\section{ㅇ July 31, I741. After Midday}

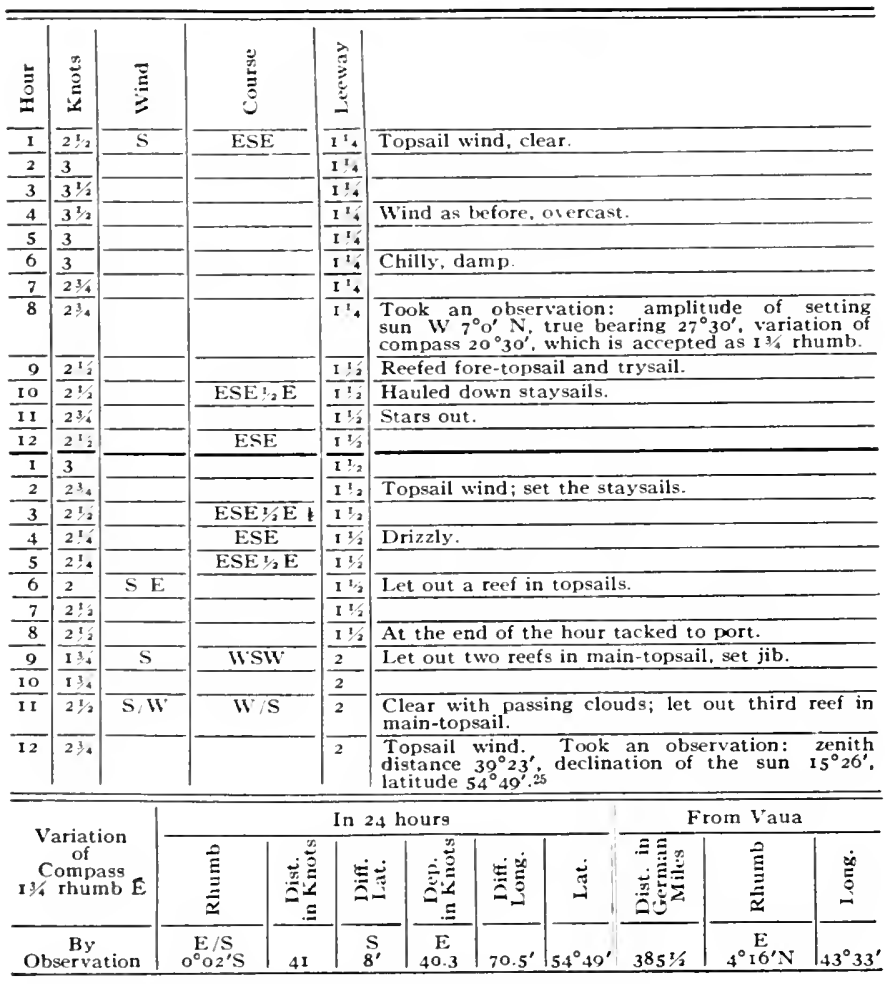

$\$ 2$ Khitrov's journal gives latitude by observation $54^{\circ} 46^{\prime}$. 
h August I, 17+1. After Midday

\begin{tabular}{|c|c|c|c|c|c|c|c|c|c|c|c|}
\hline$\stackrel{\Xi}{\Xi}$ & $\stackrel{\stackrel{2}{g}}{\stackrel{2}{g}}$ & $\stackrel{\Xi}{\Xi}$ & \multicolumn{2}{|l|}{ 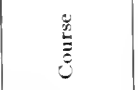 } & 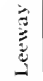 & & & & & & \\
\hline 1 & 3 & $\mathrm{SE}$ & \multicolumn{2}{|c|}{$\overline{\mathrm{W}_{4} \mathrm{~N}}$} & & \multicolumn{6}{|c|}{$\begin{array}{l}\text { Topsail wind, clear with passing clouds; carry- } \\
\text { ing all sails; the fore-topsail has one reef. }\end{array}$} \\
\hline 2 & 3 & & & & & \multirow{2}{*}{\multicolumn{6}{|c|}{$\begin{array}{l}\text { Lowered two cannons with their carriages into } \\
\text { the loold. }\end{array}$}} \\
\hline 3 & 3 & & & & & & & & & & \\
\hline 4 & 2 & & & & & & & & & & \\
\hline 5 & $I 1 / 2$ & & & & & \multicolumn{6}{|c|}{ Topgallantsail wind, weather as before. } \\
\hline 6 & $\overline{114}$ & & & & & \multicolumn{6}{|c|}{ 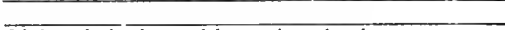 } \\
\hline 7 & 114 & & & & & \multicolumn{6}{|c|}{ Light wind, clear with passing clouds. } \\
\hline 8 & $I 1 / 4$ & & & & & \multicolumn{6}{|c|}{ Took two reefs in main-topsail. } \\
\hline 9 & $11^{2}$ & & II & & & \multicolumn{6}{|c|}{ Hauled down jib and topmast-staysail. } \\
\hline 10 & $I_{2}$ & & & & & \multicolumn{6}{|c|}{ Light wind, thick mist on the horizon. } \\
\hline ii & $1+$ & & & & & \multirow{2}{*}{\multicolumn{6}{|c|}{ Calm, stars out. }} \\
\hline 12 & & & & & & & & & & & \\
\hline $\mathrm{I}$ & & & & & & \multicolumn{6}{|c|}{ Clewed up trysail. } \\
\hline 2 & $I$ & ENE & WI & & & \multicolumn{6}{|c|}{ Light wind, cloudy. } \\
\hline$\frac{3}{3}$ & $\overline{\mathrm{I}^{\mathrm{I}}}$ & & & & & & & & & & \\
\hline$\frac{4}{4}$ & $\mathrm{I}^{34}$ & & IVN & & & \multirow{2}{*}{\multicolumn{6}{|c|}{ Topgallantsail wind; let out two reefs in topsails. }} \\
\hline 5 & $\overline{2^{3} 4}$ & NE & & & $\ldots$ & & & & & & \\
\hline 6 & 4 & & & & & \multirow{2}{*}{\multicolumn{6}{|c|}{$\begin{array}{l}\text { Topsail wind; let out third reef in main-topsail. } \\
\text { hauled up spritsail. }\end{array}$}} \\
\hline 7 & 3 & & $\mathrm{NW} / \mathrm{V}$ & & & & & & & & \\
\hline 8 & 5 & & & & & \multicolumn{6}{|c|}{ let out third reef in fore-topsail. } \\
\hline 9 & $44^{3}$ & & & & & \multicolumn{6}{|c|}{ Reef-topsail wind. } \\
\hline 10 & 5 & & & & & \multicolumn{6}{|c|}{ Let out reefs in foresail and mainsail. } \\
\hline II & $\overline{4^{3} 4}$ & & & & & \multicolumn{6}{|l|}{ Drizzly. } \\
\hline 12 & $\mid \overline{4^{1} 4}$ & & & & & \multicolumn{6}{|c|}{ Fog, damp. } \\
\hline \multirow{2}{*}{\multicolumn{3}{|c|}{$\begin{array}{l}\text { Variation } \\
\text { of } \\
\text { Compass } \\
13 \text { rhumb E }\end{array}$}} & \multicolumn{9}{|c|}{$\ln 24$ hours } \\
\hline & & & $\frac{\vec{E}}{\vec{E}}$ & 吾 & 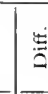 & تُ & 惡崫 & 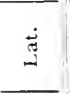 & 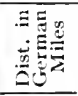 & 苂 & $\stackrel{\dot{\theta}}{\stackrel{0}{\oplus}}$ \\
\hline & $\begin{array}{l}\text { De } \\
\text { eckon }\end{array}$ & & $\underset{7^{\circ} 29^{\prime} \mathrm{W}}{\mathrm{N} / \mathrm{W}}$ & 54 & & $\begin{array}{l}W \\
43\end{array}$ & $75^{\prime}$ & $55^{\circ} 22^{\prime}$ & $373^{3 / 4}$ & $5^{\circ}+1^{\prime} N$ & $42^{\circ} 18^{\prime}$ \\
\hline
\end{tabular}




\section{August 2, I74I. After Midday}

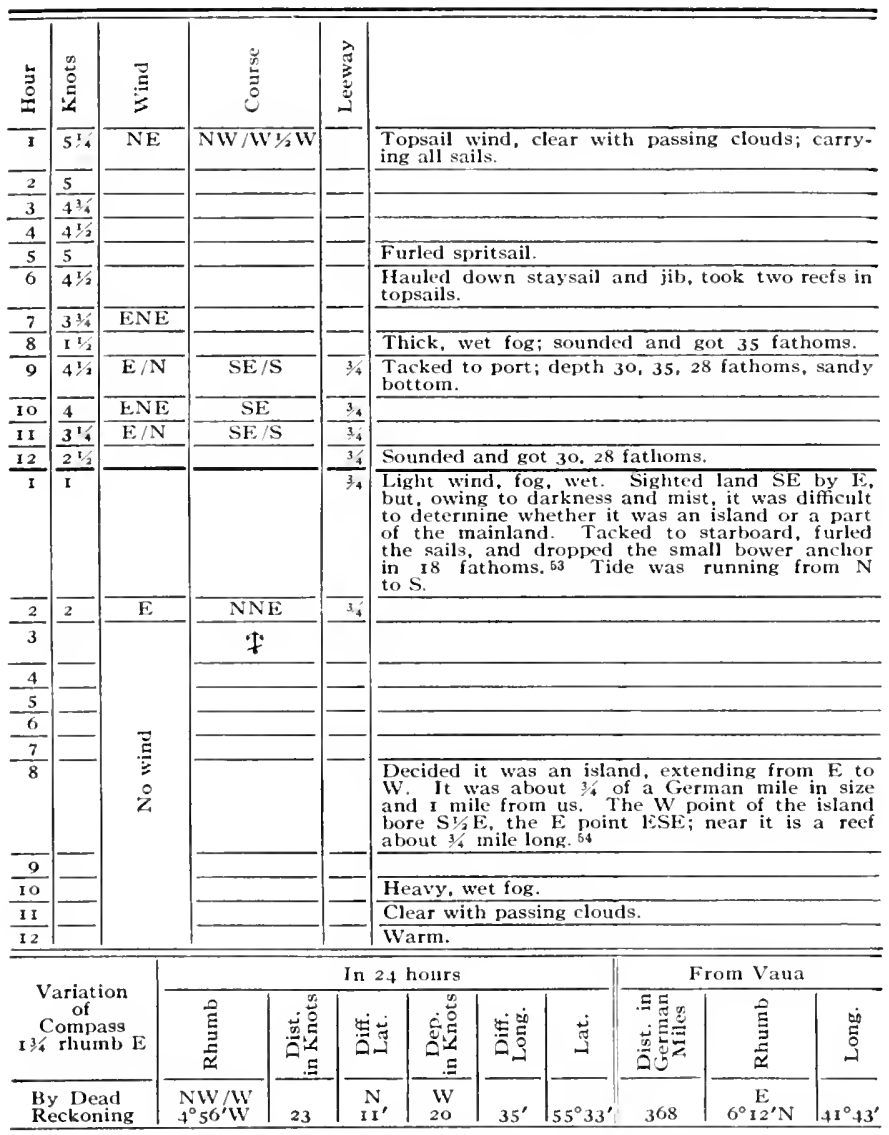

53 Northern end of Chirikov Island, so named by Vancouver on April 4, 1794, in honor of the commander of the St. Paul (George Vancouver: A Voyage of I)iscovery to the North Pacific Ocean... 3 vols. and atlas, 1,ondon, 1798 ; reference in Vol. 3, p. 87). For the names given by Bering and his officers, see foot note 56 , below.

$54 \mathrm{Kh}$ htrov's journal: "8 o"clock in the morning. One point of the island bore $S / 2 \mathrm{E}$, $11 / 2$ miles, another point ESE, 2 miles. From the first-mentioned point there is a rocky reef to the $\mathrm{N}, \mathrm{I}$ mile." 
(1) August 3, 1741. After Midday

\begin{tabular}{|c|c|c|c|c|c|c|c|c|c|c|c|}
\hline 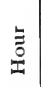 & 焉 & 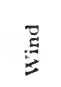 & \multicolumn{2}{|c|}{ 号 } & 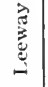 & & & & & & \\
\hline \multicolumn{2}{|l|}{ I } & \multirow{4}{*}{ 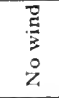 } & & & & \multicolumn{6}{|c|}{ Clear with passing fog. } \\
\hline 2 & & & & & & & & & & & \\
\hline \multicolumn{2}{|l|}{3} & & & & & \multicolumn{6}{|c|}{ Weather as above, wet. } \\
\hline 4 & & & & & & & & & & & \\
\hline \multirow{2}{*}{$\frac{5}{6}$} & & SW & & & & \multicolumn{6}{|c|}{ Light wind, weather as before. } \\
\hline & & No & & & & \\
\hline 7 & & wind & & & & & & & & & \\
\hline 8 & I & $\mathrm{S} / \mathrm{W}$ & \multicolumn{2}{|c|}{ NNE } & & \multicolumn{6}{|c|}{$\begin{array}{l}\text { Topgallantsail wind, cloudy, } 8: 30 \text {, weighed the } \\
\text { anclor, set topsails, foresail, and got under way, } \\
\text { having } 18,20 \text { fathoms of water on our track, } \\
\text { bottom sandy and shelly. }\end{array}$} \\
\hline 9 & 2 & & & & & \multirow{2}{*}{\multicolumn{6}{|c|}{ 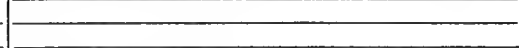 }} \\
\hline \multirow{2}{*}{$\frac{10}{11}$} & 2 & & \multirow{2}{*}{\multicolumn{2}{|c|}{$\mathrm{N} / \mathrm{E}$}} & & & & & & & \\
\hline & 2 & & & & & \\
\hline \multirow{2}{*}{$\frac{12}{1}$} & 2 & & \multirow{2}{*}{\multicolumn{2}{|c|}{ NNE }} & & \multicolumn{6}{|c|}{ Depth $20,24,26$ fathoms, bottom as above. } \\
\hline & $8 / 3$ & s & & & & \multicolumn{6}{|c|}{$\begin{array}{l}\text { light wind, } 25,22 \text { fathoms, bottom of small } \\
\text { rocks. Topgallantsail wind, cloudy. Depth } 20 \text {, } \\
21,19,18 \text {; bottom as before, in places shells. } 65\end{array}$} \\
\hline \multirow{2}{*}{$\frac{2}{3}$} & 2 & & \multicolumn{2}{|c|}{$\mathrm{N} / \mathrm{E}$} & & \multirow{2}{*}{\multicolumn{6}{|c|}{ 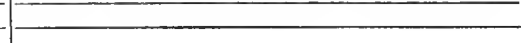 }} \\
\hline & 2 & & & & & & & & & \\
\hline$(4$. & 3 & SW & $\mathrm{NN}$ & & & & & & & & \\
\hline 5 & $3 \frac{1}{4}$ & & $\mathrm{WN}$ & & $\mathrm{x}^{\mathrm{x}} / 2$ & Fog, wet; & I 7 fatl & ioms of & water. & & \\
\hline 6 & $31 / 2$ & & & & 15 & $\begin{array}{l}20,23,2 \\
\text { rocks. }\end{array}$ & 5 fath & $\mathrm{ms}$ of & water. & ot tom of & small \\
\hline 7 & 33 & & WNW & $1 / 2 \mathrm{~W}$ & $5 / 2$ & $\begin{array}{l}\text { The isla } \\
\text { named } \\
\text { center of } \\
\text { the cross } \\
\text { cause the }\end{array}$ & $\begin{array}{l}\text { nd wh } \\
\text { rchdea } \\
\text { it, S } \\
\text { trees } \\
\text { air wa }\end{array}$ & $\begin{array}{l}\text { ere we } \\
\text { con St } \\
\text { about } \\
\text { e coulc } \\
\text { s not cl } \\
\end{array}$ & $\begin{array}{l}\text { stood, } \\
\text { ephen, } 56 \\
\text { Germ } \\
\text { see no } \\
\text { ear. } 28 \\
\end{array}$ & $\begin{array}{l}\text { and whi } \\
\text { bears no } \\
\text { miles. } \\
\text { other lar } \\
\text { nd } 30 \mathrm{fa}\end{array}$ & $\begin{array}{l}\text { ch we } \\
\text { w, the } \\
\text { From } \\
\text { d be- } \\
\text { homs. }\end{array}$ \\
\hline 8 & $31 / 2$ & & & & $1 / 2$ & & & & & & \\
\hline 9 & 3 & & $W N$ & & 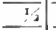 & Topsail u & ind, cl & sudy, fo & ggy. & & \\
\hline$\overline{10}$ & $21 / 2$ & & & & $3 / 2$ & $\begin{array}{l}35 \text { and } \\
\text { trysail, st }\end{array}$ & $\begin{array}{l}40 \text { fat } \\
\text { aysail. }\end{array}$ & hib, mai & $\begin{array}{ll}\text { sandy } \\
\text { nsail; }\end{array}$ & $\begin{array}{l}\text { ttom; ce } \\
\text { ded, no b }\end{array}$ & $\begin{array}{l}\text { rrying } \\
\text { rttom. }\end{array}$ \\
\hline 11 & $2 \pi / 2$ & & & & $5 / 2$ & & & & & & \\
\hline 12 & 23 & & & & $1 /\left.2\right|^{2}$ & & & & & & \\
\hline & & & & & $\operatorname{In} 2.4$ & thours & & & & $\mathrm{m}$ Vaua & \\
\hline $\begin{array}{r}C \\
13 / 4\end{array}$ & $\begin{array}{l}\text { ariati } \\
\text { of } \\
\text { ompa } \\
\text { rhum }\end{array}$ & on & 总 & 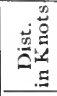 & 苔 & 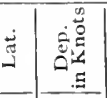 & 象莣 & 苞 & 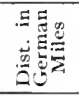 & 节 & $\stackrel{\leftrightarrow}{\stackrel{\leftrightarrow}{\circ}}$ \\
\hline $\begin{array}{l}\mathrm{B} \\
\mathrm{R} \\
\mathrm{R}\end{array}$ & $\begin{array}{l}\text { De } \\
\text { eckon }\end{array}$ & & $6^{\circ} \mathrm{N} / \mathrm{W}$ & 33.5 & $\begin{array}{r}\mathrm{N} \\
\mathbf{3 2}\end{array}$ & W & $17.8^{\prime}$ & $56^{\circ} 05^{\prime}$ & $364 \%$ & $\underset{3^{\circ} 43^{\prime} \mathrm{E}}{\mathrm{E} / \mathrm{N}}$ & $4 \mathrm{I}^{\circ} 25^{\prime}$ \\
\hline
\end{tabular}

G5 Khitrov'si journal: "The going out of the tide as well as the fog and rocky bottom made our situation critical. On that account we clianged our course to NNW."

56 Archdeacon Stephen day is August 2. This jsland, the modem Chirikov Island, would, according to $\mathrm{K}$ hitrov (see footnote 60 , below), seem to have been named Tumannoi (Foggy) Island. Cf, also Waxel's report, p. 272 . 


\section{of August 4, I74I. After Midday}

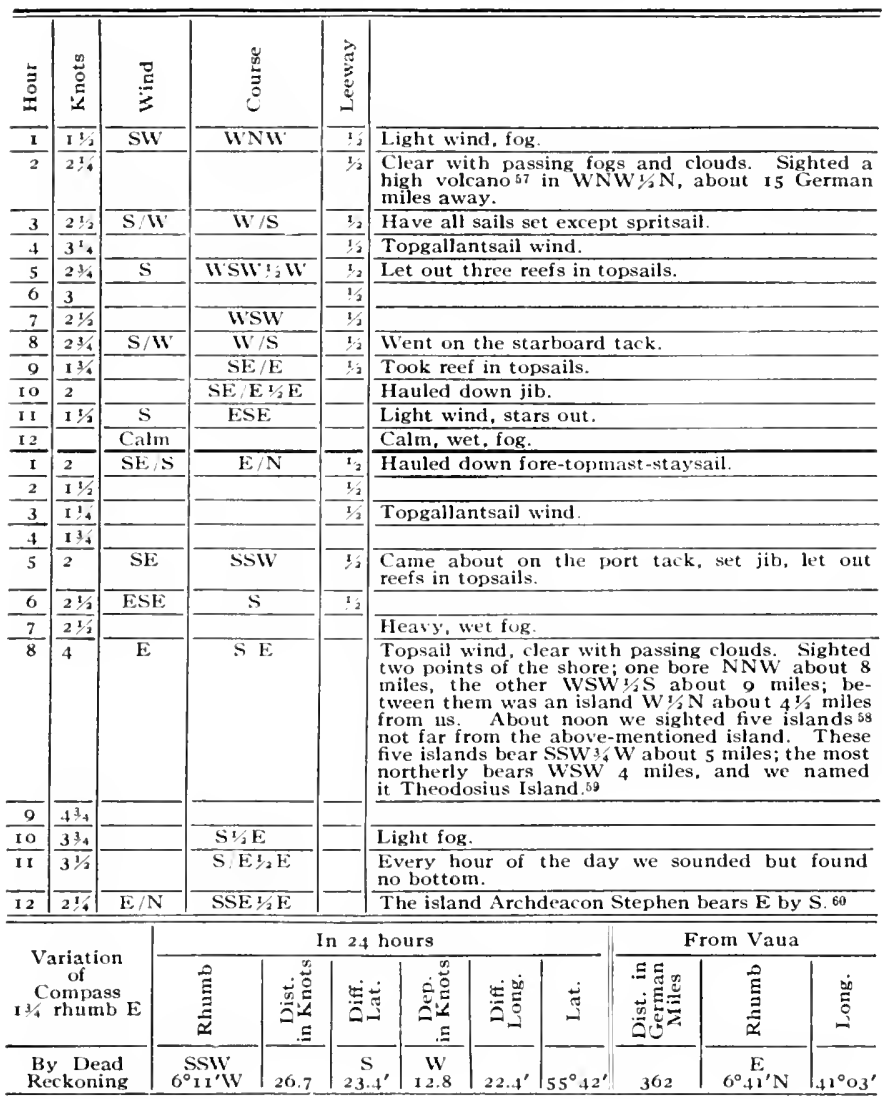

57 Mt. Chiginagak.

ss Semidi Islands. Named the Eudoxia 1slands according to Sokolov, Za pisk: IIydrogr. De part. Vol. 9. St. Petersburg, 185t, p. 388.

59 Khitrov's journal: "Sighted five islands; the southern one SSW ${ }_{3}, W_{5}$ miles, the northern WSW $1 / 2$ W 5 miles."

w Khitrov's journal: "Foggy Island where we stood at anchor bore $\mathrm{E}$ by S." 
广 August 5, I74I. After Midday

\begin{tabular}{|c|c|c|c|c|c|c|c|c|c|c|c|c|}
\hline$\stackrel{5}{\tilde{Z}}$ & $\stackrel{\substack{0 \\
\vdots}}{: 1}$ & $\underset{\Xi}{\Xi}$ & כִ & & $\hat{己}$ & & & & & & & \\
\hline I & 23.4 & $\mathrm{E}$ & SS & & $\mathrm{I}$ & To & psail v & ind, $f o$ & g, wet, & rain. & & \\
\hline 2 & $3{ }^{2} 2$ & & & & $\mathrm{I}$ & & & & & & & \\
\hline 3 & $4^{\mathrm{t}} 4$ & & & & $I$ & To & ok twr & reefs & n topsal & ails. & & \\
\hline 4 & 3 & & & & $I$ & $\mathrm{Re}$ & ef-top & ail wis & & & & \\
\hline 5 & $31 / 2$ & & $\mathrm{~S} / \mathrm{E}$ & ${ }_{2} \mathrm{E}$ & $\mathrm{I}$ & $\mathrm{Fo}_{\mathrm{O}}$ & g, rain & & & & & \\
\hline 6 & $\sqrt{3^{1}}$ & & & & I & & & & & & & \\
\hline 7 & 4 & E S S & $\mathrm{S}$ & & $I$ & $\mathrm{St}$ & ong re & f-tops & ail wind & & & \\
\hline 8 & 4 & ESE & $\mathrm{S}$ & & I & $\begin{array}{l}\mathrm{Ca} \\
\text { sai }\end{array}$ & rrying & topsa & ls, fore & esail, ma & ssail, and & stay- \\
\hline 9 & $3^{3 / 4}$ & & & & I & & & & & & & \\
\hline 10 & $I^{3} 4$ & SE & $\mathrm{NE}, \mathrm{I}$ & $5, \mathrm{E}$ & $\mathrm{I}$ & I 0 & 30. ta & ked to & starboa & ard, & & \\
\hline II & $33^{14}$ & & & & I & & & & & & & \\
\hline 12 & $\begin{array}{l}3^{1} 2 \\
\end{array}$ & & & & & & & & & & & \\
\hline$I$ & $\overline{23_{4}}$ & & & & I & To & psail 1 & ind. & & & & \\
\hline 2 & 23 & & & & I & WV & fog. & & & & & \\
\hline 3 & $23_{4}^{3}$ & & SS & & $\mathbf{I}$ & At & the er & $\mathrm{d}$ of $\mathrm{tl}$ & te hour & came al & ut on por & t tack. \\
\hline 4 & $2 \pm 2$ & & & & $\mathrm{I}$ & & & & & & & \\
\hline 5 & 4 & & & & $I$ & $\begin{array}{l}\text { Fr } \\
\text { no }\end{array}$ & $\begin{array}{l}\text { mo no } \\
\text { bottor }\end{array}$ & in to & $\begin{array}{l}\text { his hou } \\
\text { tit a ree }\end{array}$ & $\begin{array}{l}\text { ur we so } \\
\text { ef in maj }\end{array}$ & $\begin{array}{l}\text { nded but } \\
\text { topsail. }\end{array}$ & found \\
\hline 6 & 3 & $\mathrm{SE} / 1$ & $\mathrm{~S} / \mathrm{W}$ & $1 / 2 \mathrm{~W}$ & $\mathrm{I}$ & So & unded & and $\mathrm{g}$ & $40 \mathrm{fa}$ & athoms. & ottom of & gravel. \\
\hline 7 & $2 \div 4$ & $\mathrm{SE} / \mathrm{S}$ & E $/$ & & 2 & $\mathrm{Ca}$ & me ab & ut on & starboar & rd tack. & & \\
\hline 8 & $\overline{2^{I_{2}}}$ & & & & 2 & $\mathrm{He}$ & avy $S$ & swell. & & & & \\
\hline 9 & 2 & & ENE & $\angle \mathrm{E}$ & 2 & To & pgalla & tsail $v$ & ind, he & ary fog. & & \\
\hline IO & 2 & & & & 2 & & & & & & & \\
\hline II & 2 & & & & 2 & $\begin{array}{l}\mathrm{Cl} \\
\text { ha }\end{array}$ & $\begin{array}{l}\text { ar wi } \\
\text { ve soul }\end{array}$ & $\begin{array}{l}\text { ly pas } \\
\text { ded b }\end{array}$ & $\begin{array}{l}\text { sing for } \\
\text { it found }\end{array}$ & $\begin{array}{l}\text { Sin. Sin } \\
\text { d no bot }\end{array}$ & In. & ck we \\
\hline I 2 & 1 & $\mathrm{SE} /$ & \& 1 & & & & & & & & & \\
\hline \multirow{2}{*}{\multicolumn{3}{|c|}{$\begin{array}{c}\text { Variation } \\
\text { of } \\
\text { Compass } \\
\text { 13 rhumb E }\end{array}$}} & \multicolumn{7}{|c|}{$\ln 24$ hours } & \multicolumn{3}{|c|}{ From Vaua } \\
\hline & & & 劳 & 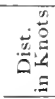 & & نَ & 递苋 & $\stackrel{\square}{2}$ & 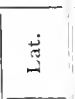 & 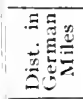 & 点 & $\stackrel{80}{\Xi}$ \\
\hline \multicolumn{3}{|c|}{$\begin{array}{l}\text { By Dead } \\
\text { Reckoning }\end{array}$} & $3^{\circ} 5_{5} / \mathrm{E}$ & $30^{1 / 2}$ & & $\begin{array}{l}S \\
9.4^{\prime}\end{array}$ & $\begin{array}{l}k \\
8\end{array}$ & $14^{\prime}$ & $55^{\circ} 13^{\prime}$ & $364^{3 / 4}$ & $\begin{array}{c}\mathrm{li} \\
5^{\circ} 28^{\prime} \mathrm{N}\end{array}$ & $41^{\circ} 17^{\prime}$ \\
\hline
\end{tabular}




\section{August 6, I7+1. After Midday}

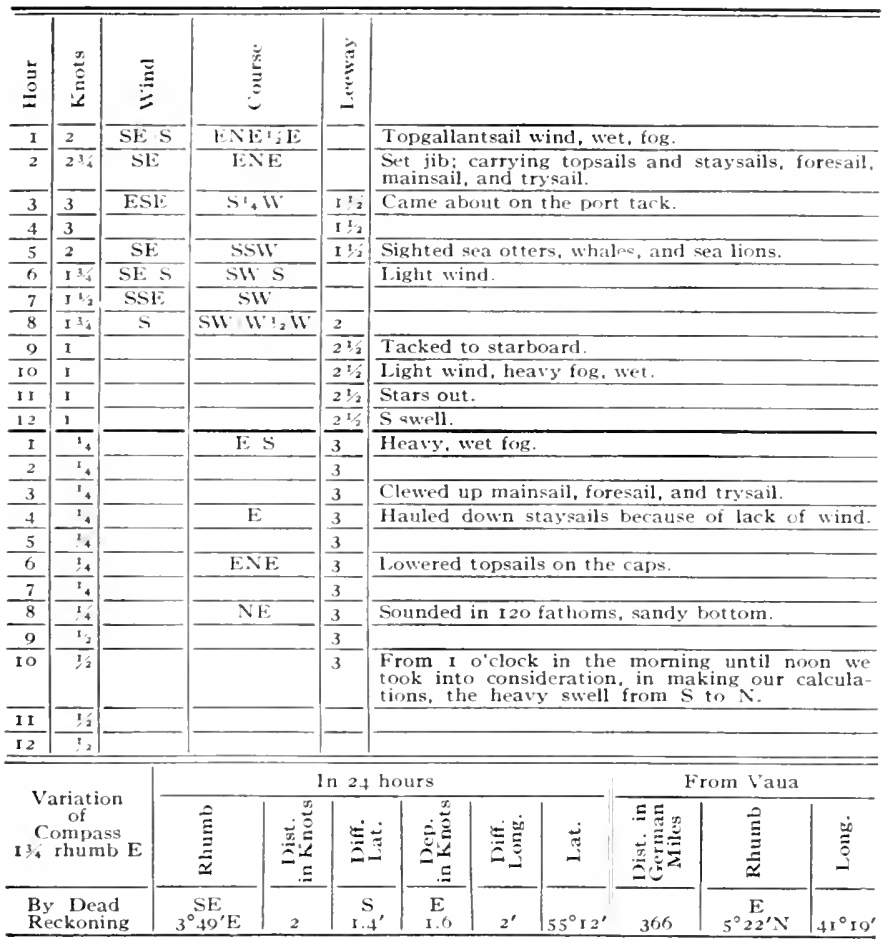




\section{if August 7, 174I. After Midday}

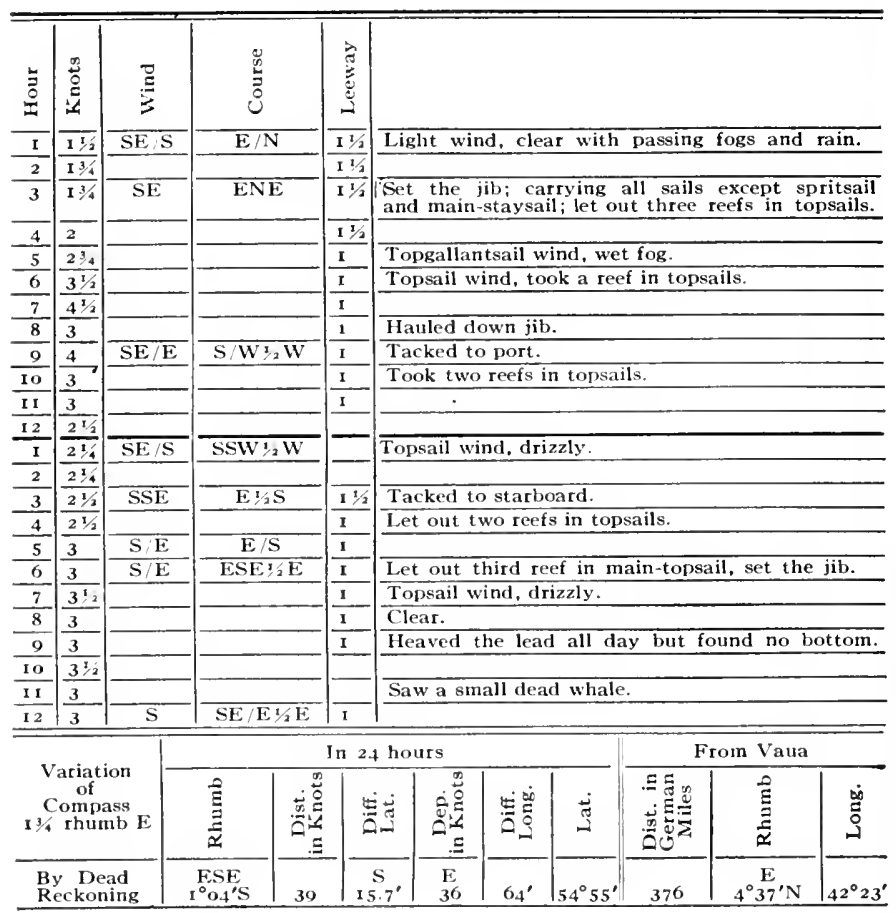


h) Augusl $8, I 7+I$. Afler Midday

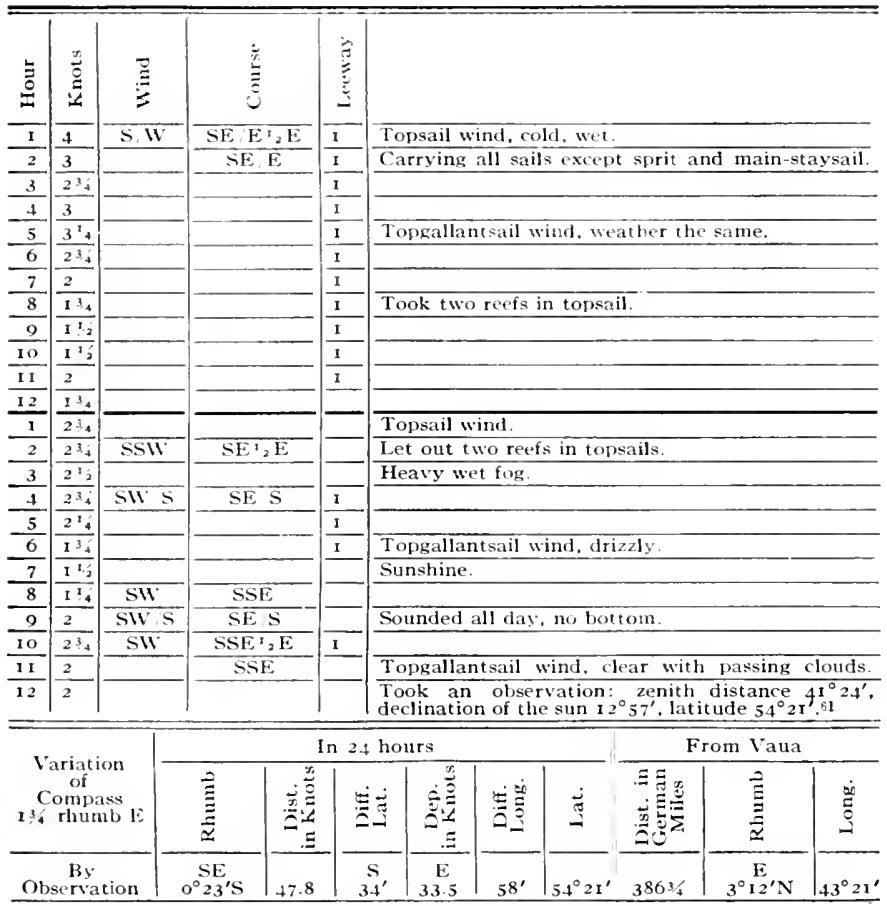

61 Khitrov's journal gives latitude by observation $54^{\circ} 1 \mathrm{I}^{\prime}$. 
August 9, 1741. After Midday

\begin{tabular}{|c|c|c|c|c|c|c|c|c|c|c|c|c|c|}
\hline 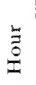 & $\begin{array}{l}0 \\
0 \\
0 \\
0\end{array}$ & $\stackrel{\Xi}{E}$ & & \multicolumn{2}{|c|}{ 泀 } & 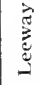 & & & & & & & \\
\hline $\bar{I}$ & 13 & sw/ & & \multicolumn{2}{|c|}{$\mathrm{S} 33_{4} \mathrm{E}$} & $I$ & \multicolumn{7}{|c|}{$\begin{array}{l}\text { Light wind, clear with passing clouds; carrying } \\
\text { all sails except spritsail and main-staysail. }\end{array}$} \\
\hline 2 & 13.4 & & & & & $I$ & \\
\hline 3 & $I^{1 / 2}$ & & & & & $I$ & & & & & & & \\
\hline 4 & $\overline{I^{2}+4}$ & & & & & $\mathrm{I}$ & \multicolumn{7}{|c|}{ Fog, wet. } \\
\hline 5 & $\mathrm{I}$ & & & & & $I$ & \multicolumn{7}{|c|}{ 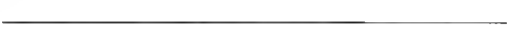 } \\
\hline$\frac{6}{6}$ & $\mathrm{I3}_{2}$ & & & & & 1 & \multirow{2}{*}{\multicolumn{7}{|c|}{ - }} \\
\hline$\overline{7}$ & $\mathrm{I}^{3 / 2}$ & WSW & & $\mathrm{S}^{1}=$ & & 1 & & & & & & & \\
\hline 8 & $1 \frac{1 / 2}{1 / 2}$ & SW I & & $\mathrm{s}$ & & $I$ & \multicolumn{7}{|c|}{ Took a reef in topsails. } \\
\hline 9 & 2 & & & $\mathrm{~S} \cdot \mathrm{E}$ & $\mathrm{E}$ & $\mathbf{I}$ & \\
\hline 10 & $I^{3} 4$ & & & $\mathrm{~S}$ & & $\mathrm{I}$ & \multicolumn{7}{|c|}{ Topgallantsail wind. } \\
\hline 11 & $13 / 4$ & & & & & 1 & \multicolumn{7}{|c|}{ Fog, stars out. } \\
\hline 12 & $\mathrm{I}^{\mathrm{1}_{2}}$ & & & & & $I$ & \\
\hline $\mathrm{I}$ & $I_{4}^{3}$ & WSV & & \multicolumn{2}{|c|}{$\mathrm{s}$} & $I$ & \multicolumn{6}{|c|}{ Light wind. } & \\
\hline 2 & 13 & & & & & 1 & \multirow{2}{*}{\multicolumn{7}{|c|}{$\ldots$}} \\
\hline 3 & $I \frac{1 / 2}{2}$ & & & & & $I$ & \multirow{2}{*}{\multicolumn{7}{|c|}{ Let out reefs in topsails. }} \\
\hline 4 & 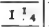 & $W / \mathrm{W}$ & & $\mathrm{S}$ & & $\mathrm{I}$ & & & & & & & \\
\hline 5 & $\overline{I_{4}}$ & & & & & $\mathrm{I}$ & \multicolumn{7}{|c|}{ Cloudy. } \\
\hline 6 & $\overline{I_{4}}$ & & & & & I & \\
\hline 7 & $\overline{1} i_{4}$ & WST & & & & I & \\
\hline 8 & $\overline{34}$ & & & $\$$ & & $I$ & & & & & & & \\
\hline$\frac{9}{9}$ & & Caln & & & & $I$ & \multicolumn{7}{|c|}{ Calm, foggy. } \\
\hline Io & $\frac{3}{2}$ & SW & & ss & & I & \multicolumn{7}{|c|}{ Light wind. } \\
\hline II & $\overline{\mathrm{I}_{3}}$ & iWsi & & $s$ & & 1 & \multicolumn{7}{|c|}{ Fog, wet. } \\
\hline 12 & 2 & IIS & & $s$ & & $I$ & \multicolumn{7}{|c|}{ Topgallantsail wind, weather as before. } \\
\hline \multirow{2}{*}{\multicolumn{4}{|c|}{$\begin{array}{l}\text { Variation } \\
\text { of } \\
\text { Compass } \\
13 \text { rhumb E }\end{array}$}} & \multicolumn{7}{|c|}{ In 21 hours } & \multicolumn{3}{|c|}{ From Vaua } \\
\hline & & & & $\frac{\stackrel{0}{\Xi}}{\underline{\Xi}}$ & 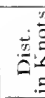 & & हु & 离总 & 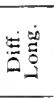 & $\stackrel{\text { ت̇ }}{\leftrightarrows}$ & 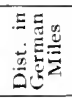 & $\stackrel{\vec{E}}{\underline{\underline{\Xi}}}$ & $\stackrel{\infty}{\check{0}}$ \\
\hline & De: & & & ${ }_{25} \mathrm{~S}$ & 30 & & \begin{tabular}{|c|}
$\mathrm{S}$ \\
.8
\end{tabular} & $\begin{array}{l}\text { WV } \\
2.3\end{array}$ & $3^{\prime}$ & $53^{\circ} 5 \mathrm{I}^{\prime}$ & $3863 / 4$ & $2^{\circ} \mathrm{O}_{4^{\prime}}^{\mathrm{E}} \mathrm{N}$ & $43^{\circ} 18^{\prime}$ \\
\hline
\end{tabular}


(S A ugust 10, I74I, After Midday

\begin{tabular}{|c|c|c|c|c|c|c|c|c|c|c|c|c|}
\hline$\stackrel{\Xi}{\Xi}$ & 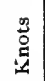 & $\stackrel{\vec{E}}{\stackrel{\vec{E}}{\xi}}$ & $\stackrel{\square}{0}$ & & 育 & & & & & & & \\
\hline I & 23 & iV N & SSW & $1 \mathrm{~W}$ & & & pgallas & tsail w & nd, clo & jy. & & \\
\hline 2 & 2 & & SW & & 1 & & rrying & topsail & stay & ails, fo & sail, and & main- \\
\hline 3 & $1 / 2$ & & & & & & & & & & & \\
\hline 4 & 1 & NW V & SW & & & & $t, f \circ g$ & $y$ on th & e horiz & & & \\
\hline 5 & $I$ & $\mathrm{~W} / \mathrm{N}$ & SSW & ${ }_{3} \mathrm{WW}$ & 1 & & hat win & 1. weat & her the & same. & & \\
\hline 6 & $23 / 4$ & & SW & & $T / 2$ & & pgallaı & tsail w & & & & \\
\hline 7 & 2 & & & & $1 / 2$ & & & & & & & \\
\hline 8 & $21 / 2$ & & & & $1 / 2$ & & ok a r & ef in to & psails. & & & \\
\hline 9 & 3 & WNW & SS & & $1 / 2$ & & oudy. & & & & & \\
\hline 10 & 4 & & & & $3 / 2$ & & psail $n$ & ind. & & & & \\
\hline 11 & $31 / 2$ & & & & & & on an & stars & & & & \\
\hline 12 & 4 & & & & & & & & & & & \\
\hline $\mathrm{I}$ & $\overline{4 ! 4}$ & $W / N$ & & & & & ef-tops & iil wint & & & & \\
\hline 2 & $3^{3 / 4}$ & & & & & & & & & & & \\
\hline 3 & 4 & & & & & & & & & & & \\
\hline 4 & $4^{14}$ & & & & & & & & & & & \\
\hline 5 & 3 & & $\mathrm{SW} / \mathrm{S}$ & $1 / 2 \mathrm{~W}$ & & & psail w & ind, ck & udy. & & & \\
\hline 6 & $23 / 4$ & WNW & SI & & $I_{2}$ & & out $r$ & efs in & opsails & & & \\
\hline 7 & 2 & & & & & & & & & & & \\
\hline 8 & $11 / 2$ & & & & & & pgallat & tsail w & & & & \\
\hline 9 & $I$ & NW/V & SW & $1 / 2 \mathrm{~W}$ & $\overline{112}$ & & & & & & & \\
\hline 10 & $3 / 4$ & & & & $\overline{1 \%}$ & & & & & & & \\
\hline II & $\overline{I^{2} 4}$ & IV $/ \mathrm{S}$ & $\mathrm{S} W$ & ${ }_{2} \mathrm{WW}$ & & & pgalla & tsail w & nd. clo & udy. & & \\
\hline 12 & $13^{3}$ & & $s$ & & $I$ & & riation & of com & pass $\mathrm{r}$ & rhuml & asterly.62 & \\
\hline \multirow{2}{*}{\multicolumn{3}{|c|}{$\begin{array}{c}\text { Variation } \\
\text { of } \\
\text { Compass } \\
1 / 2 \text { rhumb E }\end{array}$}} & \multicolumn{7}{|c|}{ In 24 hours } & \multicolumn{3}{|c|}{ From Vaua } \\
\hline & & & है & 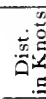 & & हैं & 要 & 幽是 & 㟧 & 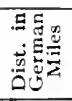 & $\frac{\hat{E}}{\underline{E}}$ & $\stackrel{\infty}{\stackrel{\infty}{ٍ}}$ \\
\hline \multicolumn{3}{|c|}{$\begin{array}{l}\text { By Dead } \\
\text { Reckoning }\end{array}$} & $\underset{3^{\circ} 12^{\prime} S}{S}$ & 58.3 & & $\begin{array}{l}S \\
3.5^{\prime}\end{array}$ & $\begin{array}{c}W \\
38.9\end{array}$ & $64.6^{\prime}$ & $53^{\circ} \circ 8^{\prime}$ & $38 \mathrm{r}^{3 / 4}$ & $0^{\circ} \underset{32^{\prime} \mathrm{N}}{\mathrm{E}}$ & $42^{\circ} \mathrm{I}$ \\
\hline
\end{tabular}

62 Khitrov's journal: "Captain Bering with the rest of us signed the decision we came to, an exact cupy of which is here attached." (See next page.) 


\section{August IO, I74I}

\section{Decision to HASTEN RETURN ${ }^{63}$}

Captain Commander Bering, Lieutenant Waxel, Fleet Master Khitrov, and Navigator Eselberg met to discuss the agreement reached by us, together with Captain Chirikov and the officers of the St. Paul, on May 4, I74I, in the Harbor of St. Peter and St. Paul. It was then agreed that while at sea we should keep in mind to return to the above-named port towards the end of September. Now it is August ro, which is regarded as already autumn, and according to all calculations we are not very far short of 400 German miles [from our destination]. Although, according to our former decision, we should still spend some time in examining the discovered American coast, yet we find this to be dangerous because of the violent autumn storms and continuous heavy fogs. It is not safe to approach the land, for we do not know the lay of it. There are many sand banks and islands, as may be seen from the chart which we have drawn up. We do not even know where to look for shelter. The assistant surgeon, Betge, has submitted a report in which he says that there are five men on the sick list, totally unfit for duty and that, of the others, sixteen are badly affected with scurvy and if we continue at sea until the late autumn these men too will be unfit for service.

On examining the log book we notice that up to now the winds have been easterly, and we fear lest the westerly winds may set in soon and prevent us from reaching port before the late autumn. We summoned before us Ensign Lagunov and all petty officers, namely, Assistant Navigator Yushin, Assistant Skipper Khatianintsov, Assistant Constable Roselius, Boatswain Nils Jansen, Boatswain's Mate Alexei Ivanov and read to them our arguments just mentioned. They agreed witl them; also in the idea that we should steer for the Harbor of St. Peter and St. Paul along the 53rd parallel of latitude, or as near to it as the winds will permit, because the last we saw of the American coast was on the 55th parallel and by going to the 53rd we may learn if the coast extends that far.

63 Khitrov's journal.

BERING

Lieutenant WAXEL

Fleet Master Sof Ron KHitrov

Navigator ANDREYAN ESELBERG 


\section{of August 11, 1741. After Midday}

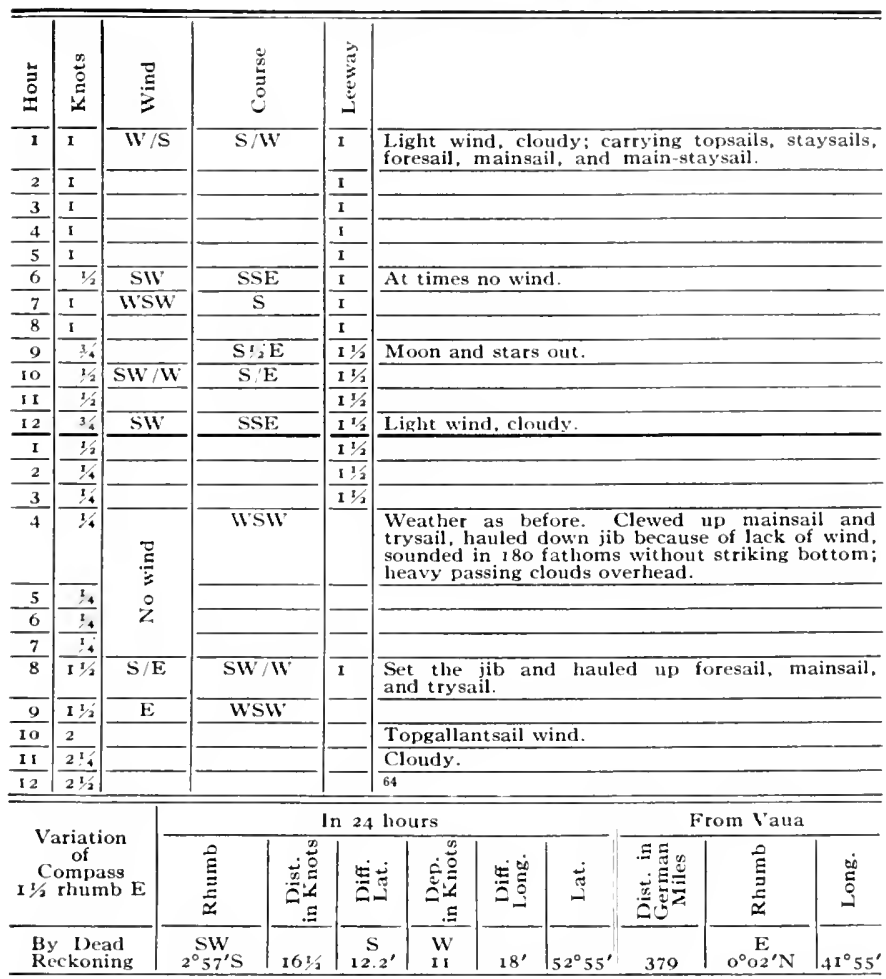

Ghitrov's journal gives latitude by observation $52^{\circ} 40^{\prime}$. This value "has been used on the chart (Pl. I). 
$\Varangle$ August 12, 17+1. After Midday

\begin{tabular}{|c|c|c|c|c|c|c|c|c|c|c|c|c|}
\hline 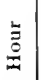 & 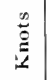 & \multirow{2}{*}{\multicolumn{2}{|c|}{$\frac{\vec{J}}{E}$}} & \multicolumn{2}{|c|}{ 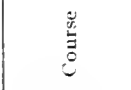 } & 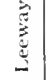 & & & & & & \\
\hline 1 & $2 ! 4$ & & & \multicolumn{2}{|c|}{ WSW } & & \multicolumn{6}{|c|}{$\begin{array}{l}\text { Topgallantsail wind, cloudy; carrying topsails } \\
\text { foresail, mainsail, trysail, and topmast-staysail }\end{array}$} \\
\hline 2 & $21_{4}$ & & & \multirow{2}{*}{\multicolumn{2}{|c|}{ 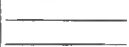 }} & & \multicolumn{6}{|c|}{ Clewed up mainsail and trysail. } \\
\hline 3 & 2 & & & & & & \\
\hline 4 & $13 / 4$ & \multirow{3}{*}{$\mathrm{SE}$} & & & & & & & & \\
\hline 5 & $13 / 2$ & & \multirow{2}{*}{\multicolumn{3}{|c|}{$\bar{\square}$}} & & \multicolumn{6}{|c|}{ 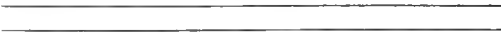 } \\
\hline 6 & $I^{x_{4}}$ & & & & & & \multicolumn{6}{|c|}{+2} \\
\hline 7 & $\mathrm{I}^{1}$ & \multirow{2}{*}{\multicolumn{2}{|c|}{-}} & \multirow{2}{*}{\multicolumn{2}{|c|}{ 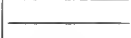 }} & & \\
\hline 8 & $\pi / 2$ & & & & & & \multicolumn{6}{|c|}{ Light wind, drizzly, wet. } \\
\hline 9 & $\pi_{4}$ & \multirow{6}{*}{$\begin{array}{l}\vec{E} \\
\ddot{E} \\
z\end{array}$} & \multicolumn{3}{|c|}{ SW } & 2 & \multicolumn{6}{|c|}{$\begin{array}{l}\text { Clewed up foresail, lowered topsail on caps be- } \\
\text { cause of little wind. }\end{array}$} \\
\hline 10 & $-x_{4}$ & & & 2 & \multirow{2}{*}{\multicolumn{6}{|c|}{$\begin{array}{l}\text { E swell. From } 9 \text { o'clock until } 3: 20 \text { we allowed } \\
x_{4} \text { knot to } \mathrm{S} \text {. }\end{array}$}} \\
\hline II & 14 & & & 2 & & & & & & \\
\hline 12 & $1 / 4$ & & \multirow{2}{*}{\multicolumn{3}{|c|}{ 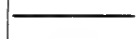 }} & 2 & \multirow{2}{*}{\multicolumn{6}{|c|}{ 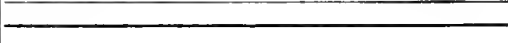 }} \\
\hline $\mathrm{I}$ & $\therefore$ & & & & & 2 & & & & & & \\
\hline 2 & r. & & & & & 2 & \multicolumn{6}{|c|}{ 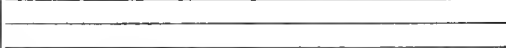 } \\
\hline 3 & $3 / 4$ & \multicolumn{2}{|c|}{ WSW } & \multicolumn{2}{|c|}{$\mathrm{S}, 2 \mathrm{E}$} & $\mathrm{II}^{1}$ & \multicolumn{6}{|c|}{ Light wind; set topsails. } \\
\hline 4 & $1 / 4$ & \multirow[b]{2}{*}{ SSW } & & & & $1 \div 2$ & Cloudy. & atmosp & lere he: & avy: & & \\
\hline 5 & $\frac{1}{12}$ & & & 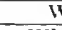 & & $I 1 / 2$ & & & & & & \\
\hline 6 & $3 / 4$ & SW & & $\mathrm{W}$ & & $I^{1 / 2}$ & Set forese & il and & rysail. & & & \\
\hline 7 & 1 & SW & & W & & $1 \frac{1 / 2}{2}$ & Hauled $u$ & p mair & ail, set & jib and & aysails. & \\
\hline 8 & $I t / 2$. & SSW & & i) & & $I$ & & & & & & \\
\hline 9 & 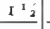 & & & $\mathrm{W}^{\mathrm{r}}$ & & $I$ & & & & & & \\
\hline 10 & 2 & SW & & Wर & & $I$ & Topgalla & atsail 3 & ind, cle & oudy. & & \\
\hline II & $I^{\frac{3}{4}}$ & & & & & $I$ & Clear wit & 12 pass & ig clou & & & \\
\hline 12 & $1 / 2$ & SW, & & $\mathrm{NW}$ & & I & Light wit & d, wea & her as & before. & & \\
\hline & & & & & & n 24 & hours & & & & om Vaua & \\
\hline $\mathrm{C}$ & $\begin{array}{l}\text { of } \\
\text { of } \\
\text { rhpa } \\
\text { rhum }\end{array}$ & & & $\stackrel{\Xi}{\Xi}$ & 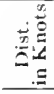 & 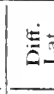 & 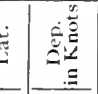 & 㔛泀 & $\stackrel{\stackrel{5}{5}}{-}$ & 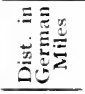 & 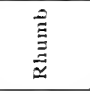 & $\stackrel{\infty}{E}$ \\
\hline & $\begin{array}{r}\text { Dea } \\
\text { ckoni }\end{array}$ & & & NW & 22 & $\begin{array}{r}\mathrm{N} \\
4.9 \\
\end{array}$ & $\begin{array}{c}\mathrm{WW} \\
2 \mathrm{I} .3 \\
\end{array}$ & $36^{\prime}$ & $53^{\circ} \mathrm{O}^{\prime}$ & $373 ! 2$ & $0_{0}^{\circ} I_{2}^{\prime} N$ & $41^{\circ} 19^{\prime}$ \\
\hline
\end{tabular}


24 August 13, 1741. After Midday

\begin{tabular}{|c|c|c|c|c|c|c|c|c|c|c|c|c|}
\hline \multirow{2}{*}{ 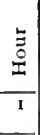 } & \multirow{2}{*}{$\frac{\stackrel{0}{0}}{\stackrel{0}{0}}$} & \multicolumn{2}{|c|}{$\frac{D}{3}$} & \multicolumn{2}{|c|}{ 造 } & 芳 & & & & & & \\
\hline & & \multicolumn{2}{|c|}{$\overline{\text { SW }}$} & \multicolumn{2}{|c|}{ WNW } & & \multicolumn{6}{|c|}{$\begin{array}{l}\text { Light wind, cloudy; carrying topsails, foresaits, } \\
\text { mainsail, trysail, jib, and topmast-staysail. }\end{array}$} \\
\hline 2 & $I$ & \multirow{2}{*}{\multicolumn{2}{|c|}{$\frac{\mathrm{SWI}^{\prime} \mathrm{S}}{\mathrm{SSW}}$}} & \multicolumn{2}{|c|}{$W^{+}: 2 \mathrm{~N}$} & & & & & & & \\
\hline 3 & 114 & & & \multicolumn{2}{|c|}{ IV } & $15 / 2$ & & & & & & \\
\hline 4 & $I$ & & & \multicolumn{2}{|c|}{$W: S$} & & & & & & & \\
\hline 5 & $I 3,4$ & \multicolumn{2}{|c|}{$\mathrm{SW} / \mathrm{S}$} & \multicolumn{2}{|c|}{$W^{1 / 2} \mathrm{~N}$} & & \multicolumn{6}{|c|}{ Topgallantsail wind, weather as before. } \\
\hline 6 & $21 / 2$ & \multirow{2}{*}{\multicolumn{2}{|c|}{ SSW }} & \multicolumn{2}{|c|}{$\mathrm{W} / \mathrm{N}$} & $1 \%$ & \multicolumn{6}{|c|}{ Topsail wind. } \\
\hline 7 & 3 & & & \multicolumn{2}{|c|}{$1 V_{2}^{2} \mathrm{~N}$} & & \multicolumn{6}{|c|}{ Took a reef in topsails. } \\
\hline$\overline{8}$ & 23,4 & \multirow{2}{*}{\multicolumn{2}{|c|}{ SW' }} & & & & & & & & & \\
\hline 9 & 3 & & & \multicolumn{2}{|c|}{$\mathrm{WNW}$} & & \multicolumn{6}{|c|}{ Sounded in I so fathoms, no bottom. } \\
\hline Io & $44^{2}$ & & & \multicolumn{2}{|c|}{ WNWt/2W } & 134 & \multicolumn{6}{|c|}{ Reef-topsail wind, wet. } \\
\hline$\overline{I I}$ & $\overline{23}$ & & \multicolumn{2}{|c|}{$\mathrm{S}_{2} \mathrm{E}$} & & \multicolumn{6}{|c|}{ Made a starboard tack at 11 3o. } \\
\hline 12 & 234 & & & & & & & & & & & \\
\hline 1 & 3 & SIV & & $\overline{S I}$ & & & Drizzly. & & & & & \\
\hline 2 & 23,4 & & & & & & Hauled $\mathrm{c}$ & wn jil & & & & \\
\hline 3 & $21 / 2$ & & & & & & Wind go & g dow & & & & \\
\hline 7 & $\overline{23}$ & & & $\mathrm{ST}_{2}$ & & $1 \pm 2$ & Set jib. & & & & & \\
\hline 5 & 4 & & & & & $\overline{1.2}$ & & & & & & \\
\hline 6 & 2 & & & WNW & W & & Came ab & ut on & he port & tack a & 30 & \\
\hline 7 & $21 / 2$ & & & & & & & & & & & \\
\hline 8 & $2: 4$ & & & & & & Clear wi & passi & $2 g$ fog. & & & \\
\hline 9 & $2 \%$ & & & & & & & & & & & \\
\hline 10 & 3 & WS & & NII & W & & At the en & 1 of $t h$ & hour $t$ & acked t & starboard & \\
\hline 11 & 3 & $w$ & & $\mathrm{NW}$ & W & $\sqrt{11_{2}}$ & Let out $r$ & ef in 1 & ain-top & sail. & & \\
\hline 12 & 24 & WS & & $S$ & & & & & & & & \\
\hline & & & & & & n 24 & hours & & & & m Vaua & \\
\hline $\begin{array}{l}\mathrm{V} \\
\mathrm{C} \\
1 \%\end{array}$ & $\begin{array}{l}\text { iriati } \\
\text { of } \\
\text { ompa } \\
\text { rhum }\end{array}$ & ss & & $\stackrel{\Xi}{\Xi}$ & 点 & 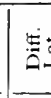 & 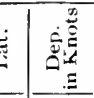 & 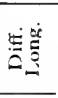 & है & 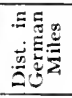 & $\underset{\Xi}{\tilde{E}}$ & $\dot{0}$ \\
\hline $\begin{array}{l}\mathrm{B}_{\mathrm{y}} \\
\mathrm{R}_{\mathrm{e}}\end{array}$ & $\begin{array}{l}\text { Dea } \\
\text { ckoni }\end{array}$ & & & $\begin{array}{l}\text { W/W } \\
56^{\prime} \mathrm{W}\end{array}$ & 19.5 & $3^{\prime}$ & $\begin{array}{c}\text { W } \\
17.7\end{array}$ & $28.7^{\prime}$ & $53^{\circ} \circ 8^{\prime}$ & $3721 / 2$ & $0^{\circ} 3^{\prime} \mathrm{N}$ & $40^{\circ} 50^{\prime}$ \\
\hline
\end{tabular}


ㅇ August 14, I741. After Midday

\begin{tabular}{|c|c|c|c|c|c|c|c|c|c|c|c|c|}
\hline$\stackrel{3}{\Xi}$ & 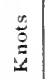 & $\sum^{\Xi}$ & : & & $\widehat{\overbrace{}}$ & & & & & & & \\
\hline$I$ & 2 & $11^{\circ} \mathrm{S}$ & $\mathrm{S}_{13}$ & & $11 / 2$ & & psail & ind. $c$ & oudy. & & & \\
\hline 2 & $\overline{2 ! 4}$ & & & & $11 / 2$ & & $\begin{array}{l}\text { rrying } \\
\text { d trys }\end{array}$ & il. $^{\text {topsa }}$ & ils, sta & sails, & resail, & ainsail, \\
\hline 3 & 2 & & & & $11 / 2$ & & & & & & & \\
\hline 4 & $2 \%$ & & & & 15 & & & & & & & \\
\hline 5 & $2 ! / 2$ & W & $\mathrm{SW}$ & $\mathrm{WV}$ & $11 / 2$ & & ggy ar & d wet & & & & \\
\hline 6 & $23 / 4$ & & & & $13 / 2$ & & $V$ swel & & & & & \\
\hline 7 & $21 / 2$ & WIS & & & $12 / 2$ & & & & & & & \\
\hline 8 & 2 & & & & & & ok a I & ef in & nain-tof & sail. & & \\
\hline 9 & 28 & & & & $I^{2} z^{2}$ & & & & & & & \\
\hline 10 & 2 & & $\mathrm{~S}$ & & $11 / 2$ & & & & & & & \\
\hline II & $21 / 2$ & & & & $11 / 2$ & & oudy. & & & & & \\
\hline 12 & $2 t / 2$ & & & & $\mathrm{I}^{\mathrm{I} / 2}$ & & ars out & & & & & \\
\hline I & $2 \%$ & & & & $\overline{1 ! 2}$ & & & & & & & \\
\hline 2 & 212 & & & & & & & & & & & \\
\hline 3 & $2 i 4$ & WW & $\mathrm{S} / \mathrm{W}$ & $\mathrm{W}$ & & & psail & ind, d & rizzly. & et. & & \\
\hline 4 & $2 ! 3$ & & & & $\begin{array}{ll}1 \\
\end{array}$ & & & & & & & \\
\hline 5 & 2 & & SS & & 13 & Le & tout & in & nain-to & sail. & & \\
\hline 6 & $\overline{11 / 2}$ & & & & 2 & & & & & & & \\
\hline 7 & $\begin{array}{l}1+4 \\
\end{array}$ & & $\mathrm{SW}$ & IV & & & wered & ib. & & & & \\
\hline 8 & 1 & IISI & 5 & & & & ick we & fog. & & & & \\
\hline 9 & $2{ }^{1}$ & W & $\mathrm{S} / \mathrm{WV}$ & $3 \mathrm{~W}$ & 2 & & ear wi & 1 pass & ing clou & & & \\
\hline 10 & $\begin{array}{l}2 ! 4 \\
\end{array}$ & & & & 2 & & & & & & & \\
\hline I I & $\overline{13 / 4}$ & & $\mathrm{SS}$ & & 2 & & $\begin{array}{l}\text { wered } \\
\text { e hold }\end{array}$ & $\begin{array}{l}\text { two } \\
\text { topga! }\end{array}$ & $\begin{array}{l}\text { cannon } \\
\text { lantsail }\end{array}$ & $\begin{array}{l}\text { with th } \\
\text { wind. }\end{array}$ & ir carria & es into \\
\hline 12 & 2 & & & & & & izzly. & & & & & \\
\hline \multirow{2}{*}{\multicolumn{3}{|c|}{$\begin{array}{c}\text { Variation } \\
\text { of } \\
\text { Compass } \\
11 / 2 \text { rhumb E }\end{array}$}} & \multicolumn{7}{|c|}{ In $2+$ hours } & \multicolumn{3}{|c|}{ From Vaua } \\
\hline & & & 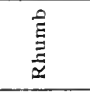 & 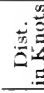 & 范 & & 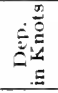 & 参曷 & 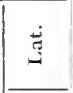 & 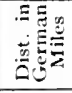 & है & $\stackrel{\dot{\theta}}{\tilde{g}}$ \\
\hline \multicolumn{3}{|c|}{$\begin{array}{l}\text { By Dead } \\
\text { Reckoning }\end{array}$} & $\underset{I_{13}^{\circ} \mathrm{W}}{\mathrm{S} / \mathrm{W}}$ & 51 & 49 & & $\begin{array}{l}\text { U } \\
\text { II }\end{array}$ & $18^{\prime}$ & $52^{\circ} 18^{\prime}$ & 37234 & $\mathrm{I}^{\circ} 23^{\prime} \mathrm{S}$ & $40^{\circ} 32^{\prime}$ \\
\hline
\end{tabular}


Ђ August 15, 174I. After Midday

\begin{tabular}{|c|c|c|c|c|c|c|c|c|c|c|c|c|}
\hline$\stackrel{\Xi}{\Xi}$ & $\underset{2}{\infty}$ & $\Xi$ & \multicolumn{2}{|c|}{ 茪 } & 产 & & & & & & & \\
\hline$I$ & $I 1 / 2$ & \multirow{2}{*}{$\frac{\text { WSW }}{\text { SW } 11}$} & \multicolumn{2}{|c|}{$\mathrm{S}$} & $11 / 2$ & \multicolumn{7}{|c|}{ Topsail wind, cloudy, wet. } \\
\hline 2 & $2 \frac{1}{4}$ & & \multirow{2}{*}{\multicolumn{2}{|c|}{$\mathrm{NW}, W \frac{1}{2} \mathrm{WV}$}} & & \multicolumn{7}{|c|}{ Tacked to port. } \\
\hline 3 & $\overline{234}$ & & & & $I$ & \multicolumn{7}{|c|}{$\begin{array}{l}\text { Carrying topsails, foresail, mainsail, trysail, } \\
\text { jib, and topmast-staysail. }\end{array}$} \\
\hline 4 & 28 & & \multicolumn{2}{|c|}{$\mathrm{NW} / \mathrm{W}$} & & \multicolumn{7}{|c|}{ jib, and topmast-staysail. } \\
\hline 5 & $2 \frac{14}{4}$ & WSW & \multicolumn{2}{|c|}{$\mathrm{NW}^{1 / 2 \mathrm{~W}}$} & & \multicolumn{7}{|c|}{ Fog, wet. } \\
\hline 6 & 3 & & \multicolumn{2}{|c|}{ NW } & 1 & \\
\hline 7 & $15 / 2$ & & \multicolumn{2}{|c|}{$\mathrm{NW} / 2 \mathrm{~N}$} & & \\
\hline 8 & $4^{1 / 2}$ & W & \multicolumn{2}{|c|}{$\mathrm{S} / \mathrm{W} 1 / 2 \mathrm{~W}$} & & & & & \multicolumn{4}{|c|}{ Starboard tack. } \\
\hline 9 & $2 \%$ & & & & $\mathrm{I}$ & & & & & & & \\
\hline 10 & 3 & & & & & \\
\hline II & $23 / 4$ & & \multicolumn{2}{|c|}{ SsW } & & & \multicolumn{6}{|c|}{ Light wind. } \\
\hline 12 & $23 / 4$ & & & & $t$ & \multicolumn{7}{|c|}{ Drizzly, wet. } \\
\hline 1 & $3 \%$ & & & & & \\
\hline 2 & $2 \%$ & & & & & & & & & & & \\
\hline 3 & $21 / 3$ & & & & & \multirow{2}{*}{\multicolumn{7}{|c|}{ Let out a reef in topsails. }} \\
\hline 4 & $2 \pi / 2$ & & & & $\sqrt{1 \div 2}$ & & & & & & & \\
\hline 5 & $2 \% / 2$ & & & & & & & & & & & \\
\hline 6 & $\overline{2^{2} 4}$ & & & & & & & & & & & \\
\hline 7 & $\overline{134}$ & & & & 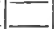 & & pgallai & itsail $n$ & ind. & & & \\
\hline 8 & I! & & & & 2 & & & & & & & \\
\hline 9 & $I 1 / 2$ & & & & & & & & & & & \\
\hline Io & 3 & W:S & $\mathrm{NW}$ & & & & psail w & ind, fo & g, wet. & & & \\
\hline II & $2 \sqrt{214}$ & & & & $\mathrm{I}$ & & & & & & & \\
\hline 12 & $2 \frac{13}{2 ! 3}$ & & & & & & swell & & & & & \\
\hline & & & & & In 2.4 & ho & urs & & & & m Vaua & \\
\hline 13 & $\begin{array}{l}\text { ariati } \\
\text { of } \\
\text { omp } \\
\text { rhun }\end{array}$ & $\begin{array}{l}\text { on } \\
\text { ass } \\
\text { nb E }\end{array}$ & $\stackrel{\hat{\Xi}}{\underline{\Xi}}$ & 竞: & $\Xi$ & & 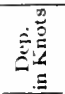 & 至递 & $\stackrel{\dot{\Xi}}{-}$ & 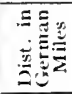 & $\stackrel{\vec{\Xi}}{\underline{\Xi}}$ & : \\
\hline$\stackrel{\mathrm{R}}{\mathrm{R}}$ & $\begin{array}{l}\text { De } \\
\text { eckon }\end{array}$ & & $\begin{array}{l}S W / N V \\
3^{\circ} 2 I^{\prime}\end{array}$ & 26 & 13 & & $\underset{22.5}{W}$ & $35.8^{\prime}$ & $52^{\circ} \mathrm{O}^{\prime}$ & $3663 / 4$ & $\underset{1^{\circ} 56^{\prime} S}{E}$ & $39^{\circ} 56^{\prime}$ \\
\hline
\end{tabular}


August 16, 1741. After Midday

\begin{tabular}{|c|c|c|c|c|c|c|c|c|c|c|c|c|}
\hline$\stackrel{气}{0}$ & $\underset{\stackrel{\infty}{0}}{\stackrel{\infty}{L}}$ & $\stackrel{5}{3}$ & 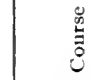 & & 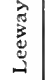 & & & & & & & \\
\hline 1 & $21 / 2$ & WSW & NV & & I & & $\begin{array}{l}\text { psail } \\
\text { ept } m\end{array}$ & $\begin{array}{l}\text { vind, } \\
\text { in-stay }\end{array}$ & $\begin{array}{l}\text { cloudy, } \\
\text { sail anc }\end{array}$ & $\begin{array}{l}\text { wet; } \\
\text { trysail }\end{array}$ & rying al & sails \\
\hline 2 & 3 & & & & $\mathrm{I}$ & & & & & & & \\
\hline 3 & 3 & SW $/ 1$ & NW & & $I$ & & & & & & & \\
\hline 4 & $33_{4}^{19}$ & & & & 1 & & & & & & & \\
\hline 5 & 3.4 & & WNW & $1 / 2 \mathrm{~N}$ & $I$ & & auled d & own jit & & & & \\
\hline 6 & $3 ! 4$ & & & & 1 & & & & & & & \\
\hline 7 & 2 & & & & $I$ & & & & & & & \\
\hline 8 & 3 & & & & $\mathrm{I}$ & & ef-top: & ail win & & & & \\
\hline 9 & $2 ! 2$ & & & & & & & & & & & \\
\hline I0 & $22^{x}$ & & & & $11 / 2$ & & & & & & & \\
\hline II & 2 & WSW & NI & & $\overline{15 / 2}$ & & & & & & & \\
\hline 12 & $2 \frac{13}{2}$ & & & & $\overline{15 / 2}$ & & unded & out did & not str & ike bott & & \\
\hline 1 & $\overline{2 ! 2}$ & & $\mathrm{NIV}$ & & $\overline{1^{5 / 2}}$ & & & & & & & \\
\hline 2 & $\overline{213}$ & & NV & & $11 / 2$ & & auled & jib. & & & & \\
\hline 3 & 24 & $\mathrm{SW} / \mathrm{V}$ & NW & & $11 / 2$ & & $t$ out a & reef in & main-t & opsail. & & \\
\hline 4 & 2 & Siv & $W N$ & & $\overline{1}^{1 / 2}$ & & & & & & & \\
\hline 5 & 2 & & WNW & $1 / 2 N$ & $11 / 2$ & & psail y & ind. & & & & \\
\hline 6 & $\overline{354}$ & & & & $\overline{I^{1}}$ & & $\begin{array}{l}\text { ef-tops } \\
\text { psail. }\end{array}$ & ail win & d, fog. & wet; to & a reef in & main- \\
\hline 7 & 3 & & & & 1 & & & & & & & \\
\hline 8 & 25 & & & & $I$ & & & & & & & \\
\hline 9 & 3 & & WN & & $\mathrm{I}$ & & auled $\mathrm{d}$ & own jit & & & & \\
\hline 10 & 3 & & & & $\mathrm{I}$ & & ear, ch & & & & & \\
\hline II & 213 & & $W^{1}$ & & I & & & & & & & \\
\hline 12 & $21 / 2$ & & W/ & & $\overline{132}$ & & unded & in $90 \mathrm{f}$ : & tnoms, & no bott & & \\
\hline \multirow{2}{*}{\multicolumn{3}{|c|}{$\begin{array}{c}\text { Variation } \\
\text { of } \\
\text { Compass } \\
x 1 / 2 \text { rhumb E }\end{array}$}} & \multicolumn{7}{|c|}{ In 2.4 bours } & \multicolumn{3}{|c|}{ From Vaua } \\
\hline & & & & 蒡苛 & $\mathscr{\Xi}$ & & 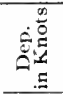 & 电它 & $\stackrel{ت}{ت}$ & 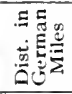 & $\stackrel{\vec{E}}{\underline{\underline{E}}}$ & 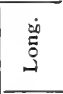 \\
\hline \multicolumn{3}{|c|}{$\begin{array}{l}\text { By Dead } \\
\text { Reckoning }\end{array}$} & $\begin{array}{l}\text { NNW } \\
7^{\circ} 06^{\prime} W\end{array}$ & 62.3 & 54 & $2^{\prime}$ & $\begin{array}{l}\text { W } \\
30.8\end{array}$ & $50.3^{\prime}$ & $552^{\circ} 59^{\prime}$ & $3531 / 2$ & $0^{\circ} \stackrel{\mathrm{E} 2^{\prime} \mathrm{N}}{\mathrm{E}}$ & $39^{\circ} 06^{\prime}$ \\
\hline
\end{tabular}


(2) August 17, 1741. After Midday

\begin{tabular}{|c|c|c|c|c|c|c|c|c|c|c|c|c|}
\hline$\stackrel{\mathscr{g}}{\Xi}$ & 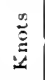 & $\vec{\Xi}$ & & & & $\stackrel{\vdots}{\vdots}$ & & & & & & \\
\hline$I$ & $3 ! 2$ & SII & & & & 114 & Reef-top & ail win & d. fog. & wet. & & \\
\hline 2 & $\overline{34}$ & $\mathrm{SH}$ & & $W N$ & $2 \mathrm{WV}$ & $1: 4$ & $\begin{array}{l}\text { Carrying } \\
\text { and stay }\end{array}$ & $\begin{array}{l}\text { reef-tc } \\
\text { sails. }\end{array}$ & psails. & foresail. & mainsail. & trysail. \\
\hline 3 & 352 & & & & & $I^{1} 4$ & & & & & & \\
\hline 4 & $3^{5} 4$ & SW & & & & $1 ! 4$ & & & & & & \\
\hline 5 & 234 & & & & & $\overline{1^{2} 4}$ & & & & & & \\
\hline 6 & 25 & sw & & IVN & $516^{\circ}$ & $1 \%$ & Took tw & reefs & n topsa & iils. & & \\
\hline 7 & 2 & & & & & $1 \div 3$ & Hauled & own st & aysails. & & & \\
\hline 8 & 2 & & & & & $11 / 2$ & Furled to & psails, & strong & gale. & & \\
\hline 9 & I & $\mathrm{SW}$ & & $\mathrm{S} / \mathrm{I}$ & & 5 & $\begin{array}{l}\text { Reefed ff } \\
\text { mainsail. }\end{array}$ & $\begin{array}{l}\text { oresail } \\
\text { trysail }\end{array}$ & $\begin{array}{c}\text { and } m a \\
\text { furled }\end{array}$ & $\begin{array}{l}\text { insail at } \\
\text { foresail. }\end{array}$ & id hove to & under \\
\hline IO & $I$ & & & & & 5 & Coming & ip to S & falling & off to $\mathrm{S}$ & by $\mathrm{S}$ & \\
\hline II & I & & & & & 5 & & & & & & \\
\hline 12 & 1 & & & & & 5 & & & & & & \\
\hline$I$ & $\overline{114}$ & & & & & 5 & Real sto & & & & & \\
\hline 2 & I!4 & II & & & & 5 & Coming & $1 \mathrm{D}$ to $\mathrm{S}$ & Sw, fal & ling off $t$ & $5 \mathrm{Sby} \mathrm{E}$. & \\
\hline-3 & $\mathrm{I}^{1} 4$ & & & & & 5 & & & & & & \\
\hline 4 & 1.4 & & & & & 5 & Cloudy. & & & & & \\
\hline 5 & $\mathrm{I}^{1}$ & IV & & & & 5 & & & & & & \\
\hline 6 & $5 ! 4$ & & & & & 5 & Coming & $1 \mathrm{p}$ to $\mathrm{S}$ & 4 by $\mathrm{S}$ & falling & fff to $S$ by & W: \\
\hline 7 & $x^{14}$ & 11 & & SSI & $2 \mathrm{WV}$ & 5 & Coming & p to $\mathrm{S}$ & W, falli & ng off to & S by W. & \\
\hline 8 & $I^{1} 4$ & & & & & 5 & & & & & & \\
\hline 9 & $1 ! 3$ & WN & & & & 5 & Coming & ip to & IV by & T. fallin & off to $\mathrm{S}$ & W. \\
\hline 10 & $\mathrm{I}^{\mathrm{I}}$ & & & & & 5 & Gale, clo & ady. & & & & \\
\hline 11 & $I_{3}$ & & & & & 5 & Clear wi & h pass & ng clous & & & \\
\hline & $1 ! 2$ & & & & & 5 & & & & & & \\
\hline \multirow{2}{*}{\multicolumn{3}{|c|}{$\begin{array}{c}\text { Variation } \\
\text { of } \\
\text { Compass } \\
11 / 2 \text { rhumb E }\end{array}$}} & \multicolumn{7}{|c|}{ In 24 hours } & \multicolumn{3}{|c|}{ From Vaua } \\
\hline & & & & 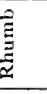 & 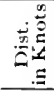 & 5 & 递离 & 象造 & $\stackrel{\overrightarrow{7}}{-}$ & 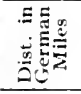 & $\stackrel{\text { E }}{\stackrel{\Xi}{\Xi}}$ & 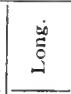 \\
\hline \multicolumn{3}{|c|}{$\begin{array}{l}\text { By Dead } \\
\text { Reckoning }\end{array}$} & & W & 7.0 & $o^{\prime}$ & $\begin{array}{l}11 \\
7.0 \\
\end{array}$ & II. $7^{\prime}$ & $52^{\circ} 59^{\prime}$ & $3513 \%$ & $0_{0}^{\circ}{ }_{12}^{\prime} \mathrm{N}$ & $38^{\circ} 54^{\prime}$ \\
\hline
\end{tabular}


of August I8, I74I. After Midday

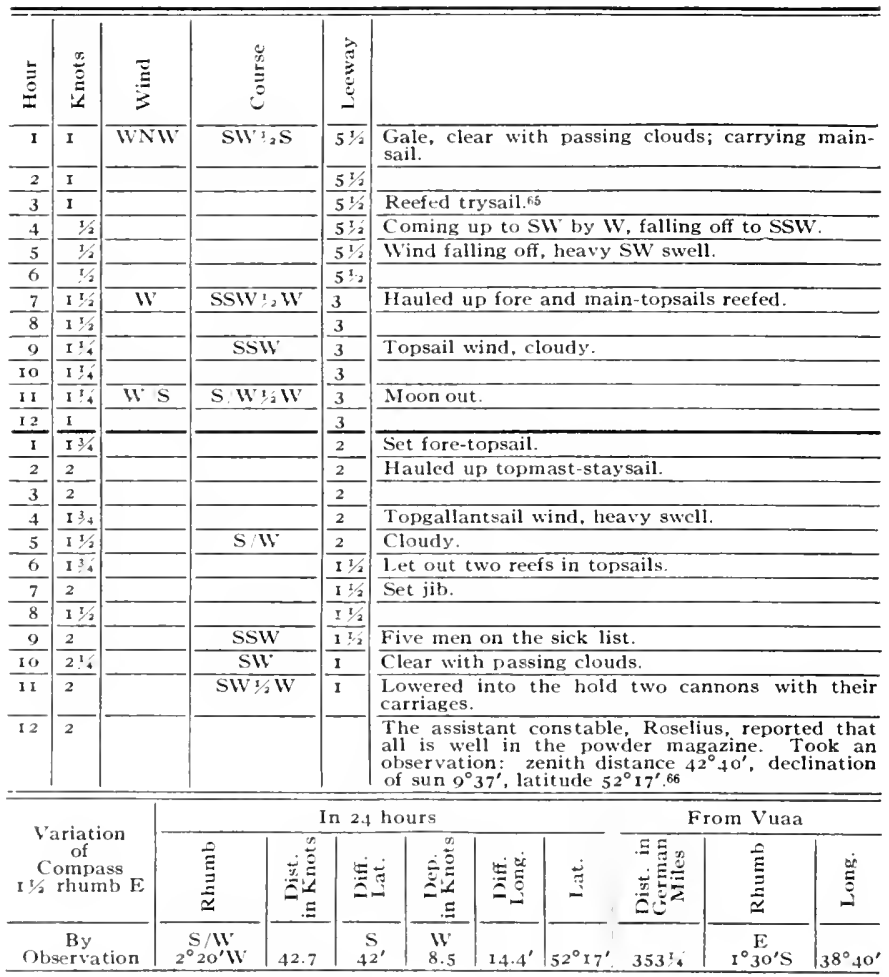

65 Khitrov's journal: "IIove to."

66 Khitrov's journal gives latitude by observation $52^{\circ} \mathrm{o} 7^{\prime}$. 
$\Varangle$ August 19, I7fI. After Midday

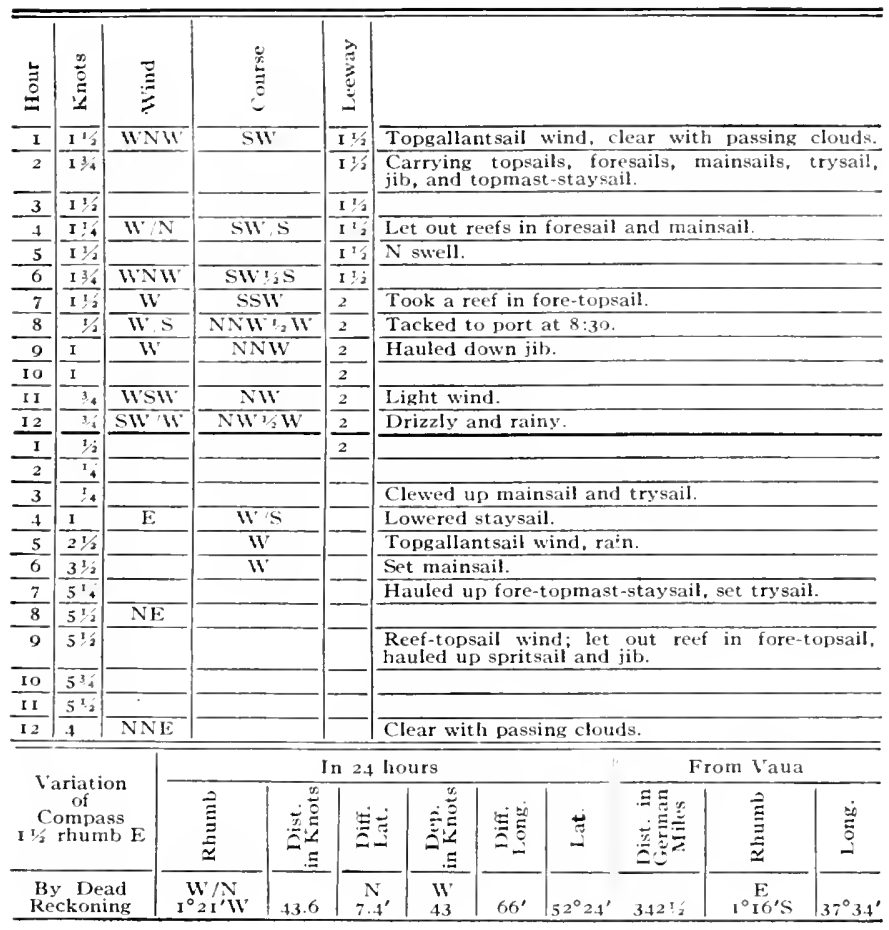


2f August 20, I74I. After Midday

\begin{tabular}{|c|c|c|c|c|c|c|c|c|c|c|c|}
\hline 冚 & 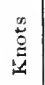 & $\vec{\Xi}$ & \multicolumn{2}{|c|}{ 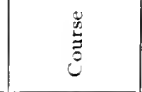 } & 莺 & & & & & & \\
\hline $\bar{I}$ & $\overline{31 / 2}$ & NNE & \multicolumn{2}{|c|}{ W } & & \multicolumn{5}{|c|}{$\begin{array}{l}\text { Topsail wind, clear with passing clouds; } \\
\text { ing all sails. }\end{array}$} & carry- \\
\hline 2 & $\mathrm{I} \div 2$ & 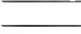 & \multirow{2}{*}{\multicolumn{2}{|c|}{$\ldots \ldots$}} & & \multirow{2}{*}{\multicolumn{6}{|c|}{ Topgallantsail wind. }} \\
\hline 3 & $\mathrm{I}^{34}$ & & & & 1 & & & & & & \\
\hline 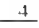 & $1^{3,4}$ & & \multicolumn{2}{|c|}{ W/S } & $\mathrm{I}$ & \\
\hline 5 & $\mathbf{1}^{3 / 4}$ & $\mathrm{NWV} / \mathrm{N}$ & & $\mathrm{I}$ & \multicolumn{6}{|c|}{ Furled spritsail. } \\
\hline 6 & $I^{5}+4$ & \multirow{5}{*}{ ¿ั } & & & $\mathrm{I}$ & \multicolumn{6}{|c|}{ Sounded in 180 fathoms, no bottom. } \\
\hline 7 & \multirow{2}{*}{$\frac{I}{r_{2}}$} & & \multicolumn{2}{|c|}{ Insw $2 \mathrm{~W}$} & $I^{1 / 2}$ & \multirow{2}{*}{\multicolumn{6}{|c|}{ lleavy SW swell. }} \\
\hline 8 & & & \multicolumn{2}{|c|}{ NNW } & 2 & & & & & & \\
\hline 9 & \multirow{2}{*}{$\begin{array}{l}1 / 4 \\
14 \\
\end{array}$} & & & & 2 & & & & & & \\
\hline Io & & & & & 2 & \multicolumn{6}{|c|}{ Sounded, no bottom. } \\
\hline II & 1 & \multirow[t]{2}{*}{$\mathrm{sW} / \mathrm{W}$} & \multicolumn{2}{|c|}{ NW/W } & $1 \frac{1}{2}$ & & & & & & \\
\hline 12 & $\overline{\mathrm{I}^{2}}$ & & & & $11 / 2$ & \multicolumn{6}{|c|}{ light wind, clear with passing clouds. } \\
\hline 1 & 2 & WSW & \multicolumn{2}{|c|}{ NW } & $\overline{1 / 2}$ & \multicolumn{6}{|c|}{ Topgallantsail wind and unsteady. } \\
\hline 2 & $2 i_{+}$ & IV & NN & & $\mathrm{t}$ & Passing c & ouds. & & & & \\
\hline 3 & $\overline{I^{3}+}$ & & & & $\mathrm{I}$ & & & & & & \\
\hline 4 & $\overline{1 ! 2}$ & $\mathrm{~W} / \mathrm{N}$ & $\mathrm{N} /$ & & $\mathrm{I}$ & & & & & & \\
\hline 5 & $\overline{1^{3} 4_{4}}$ & $\mathrm{~W}$ & NN & & $1 \% / 2$ & Wind an & weath & er as be & fore. & & \\
\hline 6 & $\overline{11 / 2}$ & NW & SI & & $I^{2} / 2$ & Tacked $\mathrm{t}$ & starb & ard. & & & \\
\hline 7 & 1 & $\mathrm{NW}$ & SW & & $11 / 2$ & & & & & & \\
\hline 8 & $\mathrm{I}^{\mathrm{T}}$ & & & & $I 1 / 2$ & & & & & & \\
\hline 9 & $13 / 2$ & WNV & SW 1 & $\mathrm{~W}$ & $I^{1 / 2}$ & Topgalla & itsail w & ind, cle & udy. & & \\
\hline 30 & $11^{2}$ & & & & $51 / 2$ & Passing $\mathrm{C}$ & louds. & & & & \\
\hline II & $I$ & & SI & & $11 / 2$ & SW swell & & & & & \\
\hline 12 & $13 \frac{14}{4}$ & $\mathrm{~W} / \mathrm{N}$ & $\mathrm{SW}$ & & $\mid 1,2$ & & & & & & \\
\hline & & & & & $\ln 24$ & hours & & & & m Vaua & \\
\hline$\underset{1 / 2}{C}$ & $\begin{array}{l}\text { arrats } \\
\text { of } \\
\text { ompa } \\
\text { rhum }\end{array}$ & $\begin{array}{l}\text { on } \\
\text { iss } \\
\text { b E E }\end{array}$ & & 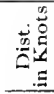 & 总 & 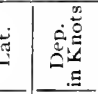 & 电它 & 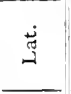 & 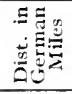 & $\underset{\underline{E}}{\underline{E}}$ & $\begin{array}{l}\dot{0} \\
\stackrel{0}{0} \\
\end{array}$ \\
\hline & $\begin{array}{l}\text { De } \\
\text { ckon }\end{array}$ & & $\begin{array}{l}W / N \\
2^{\circ} 23^{\prime} N\end{array}$ & 21 & $\begin{array}{r}N \\
4.9\end{array}$ & $\begin{array}{l}\mathrm{WV} \\
20.2\end{array}$ & $33.8^{\prime}$ & $52^{\circ} 29^{\prime}$ & $3371 / 4$ & $\mathrm{I}^{\circ} \mathrm{E} 4^{\prime} \mathrm{S}$ & $37^{\circ} \mathrm{O}^{\prime}$ \\
\hline
\end{tabular}


q August 21, 174I. After Midday

\begin{tabular}{|c|c|c|c|c|c|c|c|c|c|c|c|}
\hline$\stackrel{\underline{z}}{\underline{z}}$ & 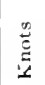 & $\stackrel{\bar{Z}}{Z}$ & & & 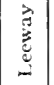 & & & & & & \\
\hline I & $I$ & W & & & $\overline{11 / 2}$ & $\begin{array}{l}\text { Light w } \\
\text { topsails. } \\
\text { staysail. }\end{array}$ & $\begin{array}{l}\text { id. } \\
\text { oresal }\end{array}$ & $\begin{array}{l}\text { udy, he } \\
\text { mains }\end{array}$ & $\begin{array}{l}\text { ayy } \mathrm{S} \\
\text { ail, try }\end{array}$ & $\begin{array}{l}\text { swell: } \\
\text { il, and }\end{array}$ & $\begin{array}{l}\text { arrying } \\
\text { opmast }\end{array}$ \\
\hline 2 & $\overline{I^{3 / 4}}$ & & & & $I 1 / 2$ & & & & & & \\
\hline 3 & 2 & & ssl & $\mathrm{WW}$ & $I 1 / 2$ & & & & & & \\
\hline 4 & $\overline{I^{3 / 4}}$ & $\mathrm{~W} / \mathrm{N}$ & & & $I 1 / 2$ & & & & & & \\
\hline 5 & $\overline{13}$ & 11 & & & $11 / 2$ & Topgalla & tsail & ind. & & & \\
\hline 6 & I & & 5,11 & / $3 \mathrm{~W}$ & $11 / 2$ & Drizzly. & & & & & \\
\hline 7 & $\overline{\mathrm{I}^{3}+4}$ & WNV & & & $I 1 / 2$ & Tacked & port. & & & & \\
\hline 8 & $2^{13}$ & NW & & & $I^{1 / 3}$ & Tacked & start & bard. & & & \\
\hline 9 & $\overline{2 ! 4}$ & & WS & $1 / 25$ & 13 & Took red & in tol & sails. & & & \\
\hline 10 & $\overline{1 ! 4}$ & & & & $I \frac{1 / 2}{4}$ & & & & & & \\
\hline II & $I$ & $\mathrm{~W} / \mathrm{N}$ & & & $1 / 2$ & Light an & shift & g wind & & & \\
\hline 12 & $8 / 2$ & WV & & & $11 / 3$ & Sounded & no be & tom. & & & \\
\hline I & $I$ & & 5.11 & $2 \mathrm{~W}$ & 13 & & & & & & \\
\hline 2 & $\overline{2 ! 3}$ & & $\mathrm{~N} / \mathrm{V}$ & $1 . \mathrm{W}$ & $11 / 2$ & Tacked & port. & & & & \\
\hline 3 & 4 & & & & $11 / 2$ & Hauled & wn ji & & & & \\
\hline 4 & $\mathrm{Ir}_{3}$ & & SSI & $2 \mathrm{~W}$ & $11 / 2$ & Starboar & tack & $4: 30$ & & & \\
\hline 5 & $\overline{I^{3}+4}$ & $\mathrm{~W} / \mathrm{N}$ & & & $I^{1 / 2}$ & & & & & & \\
\hline 6 & I & IV & S II & iW & $\overline{1 ! 2}$ & Light wi & $\mathrm{d}$ and & unstead & $y=$ drizz & wet. & \\
\hline 7 & $\overline{23,}$ & $w^{\prime} / \mathrm{s}$ & NNI & $1 / 3 \mathrm{WH}$ & $I$ & Port tac & & & & & \\
\hline 8 & $21 / 2$ & & NV & & I & Topsail & ind. & & & & \\
\hline 9 & 23 & IV & & & $I^{1 / 3}$ & Starboar & tack. & & & & \\
\hline IO & $21 / 2$ & & NNI & $1 / 2 W$ & $I$ & Port tac & & & & & \\
\hline II & $\overline{I^{3} 4}$ & WW/S & $\mathrm{Nu}$ & & I & & & & & & \\
\hline 12 & 2 & & & & $\mathrm{I}$ & Topsail & ind, $c$ & oucly, S & W'swell & & \\
\hline \multirow{2}{*}{\multicolumn{3}{|c|}{$\begin{array}{c}\text { Variation } \\
\text { of } \\
\text { Compass } \\
\text { I } 1 / 2 \text { rhumb E }\end{array}$}} & \multicolumn{6}{|c|}{ In 24 hours } & \multicolumn{3}{|c|}{ From Vaua } \\
\hline & & & 总 & 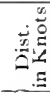 & 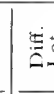 & 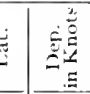 & 空 & : & 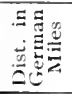 & 当 & 递 \\
\hline \multicolumn{3}{|c|}{$\begin{array}{l}\text { By Dead } \\
\text { Reckoning }\end{array}$} & $\begin{array}{c}\text { IV } \\
4^{\circ} \mathrm{I} 6^{\prime} \mathrm{S}\end{array}$ & I3.5 & S & $\begin{array}{c}W \\
13.4\end{array}$ & $2 \mathrm{I}^{\prime}$ & $52^{\circ} 28^{\prime}$ & $334 ! 2$ & $I^{\circ} \mathrm{E}$ & $36^{\circ} 39^{\prime}$ \\
\hline
\end{tabular}




\section{h August 22, 1741. After Midday}

\begin{tabular}{|c|c|c|c|c|c|c|c|c|c|c|c|c|}
\hline$\stackrel{5}{3}$ & 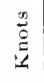 & $\underset{Z}{Z}$ & \multicolumn{3}{|c|}{ 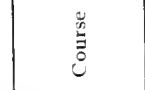 } & \multirow[t]{2}{*}{ 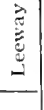 } & & & & & & \\
\hline$\underline{I}$ & $\overline{374}$ & \multicolumn{2}{|c|}{ WSII } & \multicolumn{2}{|c|}{ NW } & & \multicolumn{6}{|c|}{ Topsail wind, drizzly, wet. } \\
\hline 2 & $23 / 4$ & & & & & & \multicolumn{6}{|c|}{$\begin{array}{l}\text { Carrying topsails, foresail, mainsail, trysail, and } \\
\text { staysails. }\end{array}$} \\
\hline 3 & $2 \frac{1 / 2}{2}$ & \multicolumn{2}{|c|}{$511 / \mathrm{W}^{2}$} & \multicolumn{2}{|c|}{ NWI/2W } & I & & & & & & \\
\hline 4 & 314 & & & & & & \multicolumn{6}{|c|}{ SWW swell. } \\
\hline 5 & $\overline{23 / 4}$ & & & \multicolumn{2}{|c|}{ NIV $/ W$} & & & & & & & \\
\hline 6 & $\overline{23 / 4}$ & \multicolumn{2}{|c|}{ SW } & \multicolumn{2}{|c|}{$\mathrm{NW} / \mathrm{W} / 2 \mathrm{~W}$} & & & & & & & \\
\hline 7 & 3 & \multicolumn{2}{|c|}{$\$ 11.11$} & \multicolumn{2}{|c|}{$\mathrm{NIV} / 2 \mathrm{~W}$} & $\mathbf{I}$ & \multicolumn{6}{|c|}{ Reef-topsail wind, drizzly, wet. } \\
\hline 8 & 3 & & & & & $\mathrm{I}_{\mathrm{I} / 2}$ & & & & & & \\
\hline 9 & 4 & \multicolumn{2}{|c|}{ 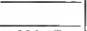 } & \multicolumn{2}{|c|}{-} & $\mathrm{I}^{\mathrm{T} / 2}$ & \multirow{2}{*}{\multicolumn{6}{|c|}{$\begin{array}{l}\text { Light wind, sounded but no bottom, starboard } \\
\text { tack. }\end{array}$}} \\
\hline 10 & $\mathrm{I}^{\mathrm{I}} \mathrm{2}_{2}$ & \multicolumn{2}{|c|}{$\mathrm{W} / \mathrm{S}$} & \multicolumn{2}{|c|}{$\mathrm{S}_{2} \mathrm{WT}^{\prime}$} & 1,2 & & & & & & \\
\hline I I & \multirow[t]{2}{*}{$\mathbf{I}$} & \multirow{2}{*}{\multicolumn{2}{|c|}{ 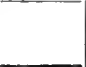 }} & \multirow{2}{*}{\multicolumn{2}{|c|}{ S W }} & \multirow[t]{2}{*}{5,2} & \multicolumn{6}{|c|}{$\begin{array}{l}\text { Furled sails and hove to under mainsail and } \\
\text { trysaul because of darkness and danger. }\end{array}$} \\
\hline 12 & & & & & & & \multicolumn{6}{|c|}{ Coming up to SSW, falling off to S. } \\
\hline 1 & $I$ & \multicolumn{2}{|c|}{$\mathrm{W}^{+}$} & \multirow{2}{*}{\multicolumn{2}{|c|}{$\mathrm{NW}_{2} \mathrm{~N}$}} & $5+2$ & & & & & & \\
\hline 2 & $\overline{12 / 2}$ & WS & & & & & $\begin{array}{l}\text { Set top } \\
\text { port } .67\end{array}$ & ils. & resail. & and st: & sails; tac & to \\
\hline 3 & 2 & & & $\mathrm{NV}$ & & I & & & & & & \\
\hline 4 & $21 / 4$ & & & NW & ${ }_{2} \mathrm{~N}$ & $\mathrm{I}$ & Sounded & no be & tom. & & & \\
\hline 5 & $21 / 2$ & & & & & I & & & & & & \\
\hline 6 & $13 / 4$ & W & & $\mathrm{NV}$ & & & Topgall & tsail & ind, do & udy. & & \\
\hline 7 & $\mathbf{1} 3 / 4$ & WN & & $\mathrm{N} / \mathrm{S}$ & $2 W$ & I $1 / 2$ & Came al & ut on & starboa & d tack & $7: 30$ & \\
\hline 8 & $\mathbf{I 3 / 4}$ & NW & & 511 & & $I_{2}$ & Let out & efs in & topsail. & & & \\
\hline 9 & $\mathrm{I}^{3} 4$ & WN & & & & $I^{1 / 2}$ & Topsail & ind, & ear witl & passin & clouds. & \\
\hline Jo & 2 & $\mathrm{NIV}$ & $\mathrm{IV}$ & $\mathrm{SII}$ & $W$ & $1 / 2$ & & & & & & \\
\hline II & $\mathrm{I}^{3} 4$ & & & & & $I_{12}$ & SWr swe & & & & & \\
\hline 12 & $2^{I_{2}}$ & $W N$ & & & & & & & & & & \\
\hline & & & & & & n 2.4 & hours & & & & om Vaua & \\
\hline $1 / 2$ & $\begin{array}{l}\text { of } \\
\text { ompa } \\
\text { rhun }\end{array}$ & 89 & & $\stackrel{\rho}{\stackrel{E}{\Xi}}$ & 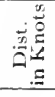 & $\stackrel{\leftrightarrows}{0}$ & 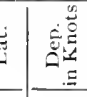 & 过 & 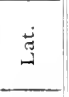 & 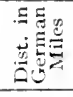 & $\underline{\Xi}$ & Е \\
\hline $\begin{array}{l}\text { By } \\
\text { Re }\end{array}$ & $\begin{array}{r}\text { De: } \\
\text { ckon }\end{array}$ & & & $\begin{array}{l}W / N \\
17 \% \mathrm{~W}\end{array}$ & 32.7 & 26. & $\begin{array}{l}\text { IV } \\
\text { I9 } \\
\end{array}$ & $3 \mathrm{I}^{\prime}$ & $52^{\circ} 55^{\prime}$ & $326^{3} 4$ & $\begin{array}{c}\mathrm{E} \\
0^{\circ} \mathrm{O} 3^{\prime} \mathrm{N}\end{array}$ & $36^{\circ} 08^{\prime}$ \\
\hline
\end{tabular}

67 Khitrov"s journal: "At the beginning of this hour we got under way again and tacked to port."

6s Flitrov's journal: "On sick list, two sailors, two grenadiers, two marines, one Siberian soldier." 
August 23. 1741. Afler Midday

\begin{tabular}{|c|c|c|c|c|c|c|c|c|c|c|c|c|c|}
\hline \multirow{2}{*}{$\frac{\stackrel{5}{\Xi}}{1}$} & \multirow{2}{*}{ 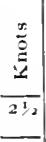 } & \multicolumn{2}{|l|}{$\bar{E}$} & \multicolumn{2}{|c|}{ 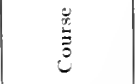 } & \multirow{2}{*}{$\frac{2}{13}$} & & & & & & & \\
\hline & & \multicolumn{2}{|c|}{ WNW } & \multicolumn{2}{|c|}{ SW } & & \multicolumn{7}{|c|}{$\begin{array}{l}\text { Reef-topsail wind, clear with passing clouds; } \\
\text { took a reef in topsails. }\end{array}$} \\
\hline 2 & $2+\frac{1}{2 !}$ & & & & \multicolumn{8}{|c|}{-7} \\
\hline 3 & \multicolumn{3}{|l|}{$21 / 2$} & & & \multicolumn{8}{|l|}{13} \\
\hline 4 & $2: 2$ & \multirow{2}{*}{\multicolumn{2}{|c|}{$\mathrm{NW} / \mathrm{WW}$}} & \multirow{2}{*}{\multicolumn{2}{|c|}{$S I V, W$}} & \multirow[t]{2}{*}{$\overline{I 3 / 4}$} & \\
\hline 5 & 21,2 & & & & & & \multicolumn{7}{|c|}{ W swell. } \\
\hline 6 & 23 & \multicolumn{2}{|c|}{-} & & \multicolumn{8}{|l|}{$I 3 / 4$} \\
\hline 7 & & \multicolumn{2}{|c|}{$\mathrm{SW} \mathrm{t}_{2}$} & 2 & \multicolumn{7}{|c|}{ Took two reefs in topsails. } \\
\hline 8 & 2 & \multirow{2}{*}{\multicolumn{2}{|c|}{$\ldots$}} & & & 2 & & & & & & & \\
\hline 9 & 13 & & & & & 2 & & pgalla & tsail v & ind anc & unstead & & \\
\hline Io & $I 1 / 2$ & WN & & SV & & 2 & & avy $\mathrm{S}$ & swel & & & & \\
\hline I I & $\overline{21 / 2}$ & IV & & SSII & IV & $\overline{13,4}$ & & & & & & & \\
\hline 12 & 2 & W & & SSI & & 2 & & ear wit & passi & ig clou & & & \\
\hline $\mathbf{I}$ & $11 / 2$ & $\pi$ & & $\mathrm{NNIV}$ & 211 & & & cked $t$ & port. & & & & \\
\hline 2 & 134 & IIS & & $\mathrm{NW}$ & $\mathrm{N}$ & $I I_{2}$ & & out & vo ree & in tor & sails. & & \\
\hline 3 & $13 / 4$ & & & $\mathrm{NI}$ & & & & & & & & & \\
\hline 4 & 2 & SV & & & & & & pgalla & tsail s & ind, $\mathrm{clc}$ & udy. & & \\
\hline 5 & 3 & SW & & NW & 216 & & & psail & ind, $d$ & izzly, & et. & & \\
\hline 6 & $2 ! 2$ & & & $\mathrm{NW}$ & & & & & & & & & \\
\hline 7 & $21 / 2$ & SSI & & & & & & $t$ out & ird re & $f$ in to & sails. & & \\
\hline 8 & $21 / 4$ & & & & & & & & & & & & \\
\hline 9 & 3 & & & WIN & & & & & & & & & \\
\hline I0 & $4^{x} 4$ & & & & & & & psail & ind, d & izzly, r & ainy. & & \\
\hline I I & 4 & $\mathrm{SIV}$ & & $11 \times 11$ & $2 \mathrm{IV}$ & 16 & & & & & & & \\
\hline 12 & 4 & $\mathrm{SW}$ & & $\mathrm{NIV}$ & W & $\therefore$ & & ok a $\mathrm{r}$ & ef in $t$ & psail, & veather & s before. & \\
\hline & & & & & & In & h & urs & & & & om Vaua & \\
\hline $\begin{array}{c}\mathrm{V} \\
\mathrm{I} / 2\end{array}$ & $\begin{array}{l}\text { ariati } \\
\text { of } \\
\text { ompa } \\
\text { thum }\end{array}$ & on & & $\stackrel{\bar{\Xi}}{\underline{\Xi}}$ & 泀苔 & $\stackrel{2}{2}$ & & $=\frac{\overbrace{}^{n}}{5}$ & 要离 & 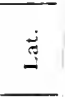 & 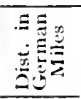 & $\underline{\vec{\Xi}}$ & $\stackrel{\dot{0}}{\tilde{C}}$ \\
\hline $\begin{array}{l}\text { By } \\
\text { Re }\end{array}$ & $\begin{array}{l}\text { I } \\
\text { ack or }\end{array}$ & & & $\begin{array}{l}\mathrm{IV} / \mathrm{N} \\
{ }^{\circ} 9^{\prime} \mathrm{V}\end{array}$ & .38 & 6. & & $\begin{array}{c}\text { W } \\
37.4\end{array}$ & $61^{\prime}$ & $53^{\circ} \mathrm{or}{ }^{\prime}$ & $317^{\circ} 4$ & $0^{\circ}{ }_{19^{\prime}}^{\mathrm{E}} \mathrm{N}$ & $35^{\circ} 07^{\prime}$ \\
\hline
\end{tabular}


(3) A ugust 24, I74I. After Midday

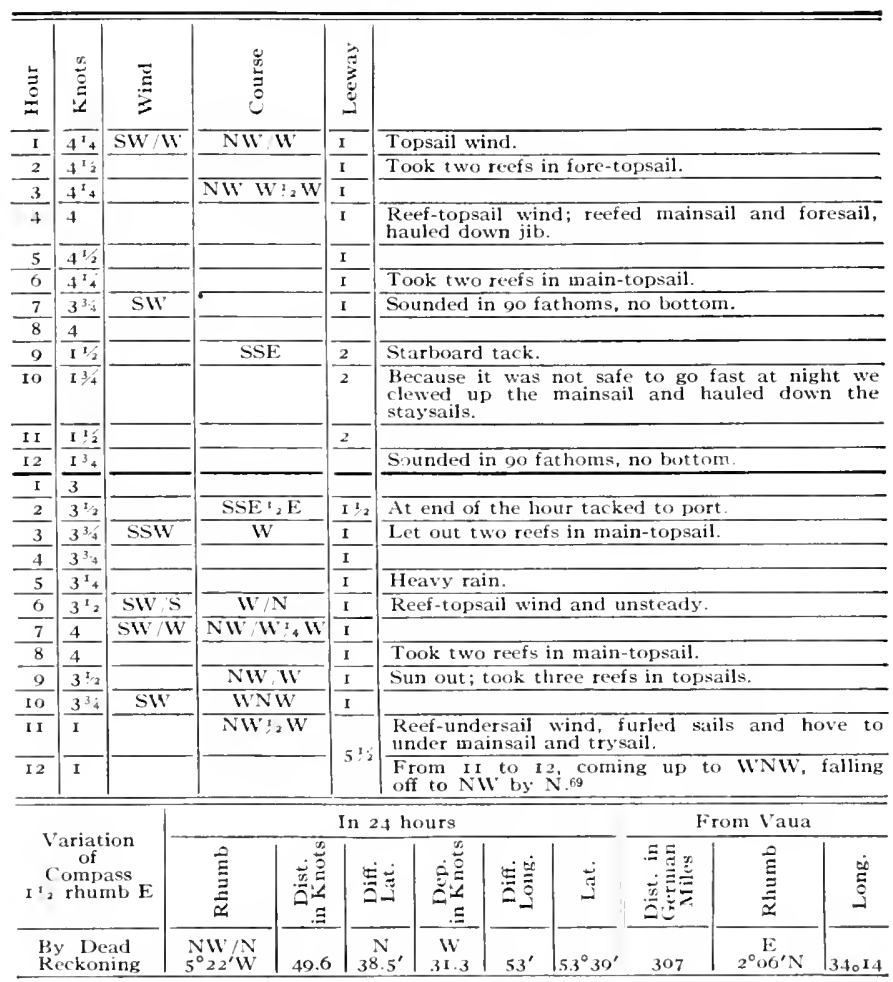

69 Khitrov's journal gives an observation for lutitude, $53^{\circ} 37^{\prime}$. 
\& August 25, 1741. After Midday

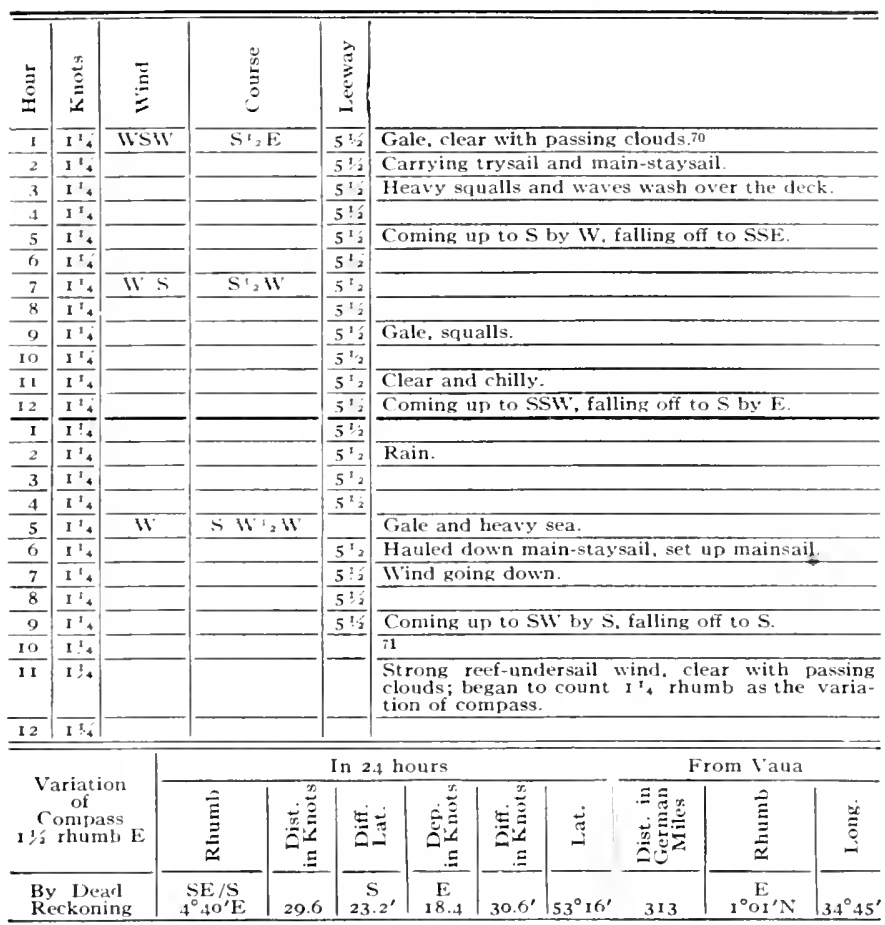

to Khitrov"s journal: "Jove to."

il Khitrov's journal states that nine were on the sick list. 


\section{$\Varangle$ August 26, 174t. After Midday}

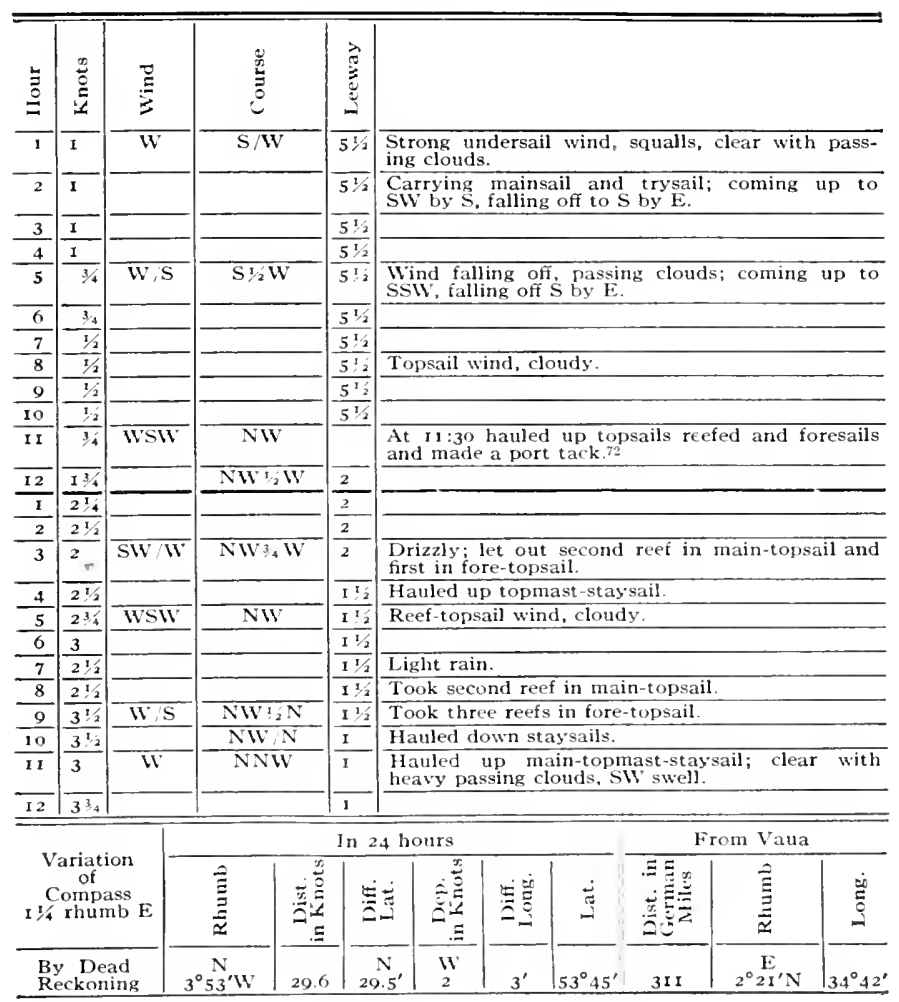

72 Khitrov's journal: "Got under way again," 


\section{A A}

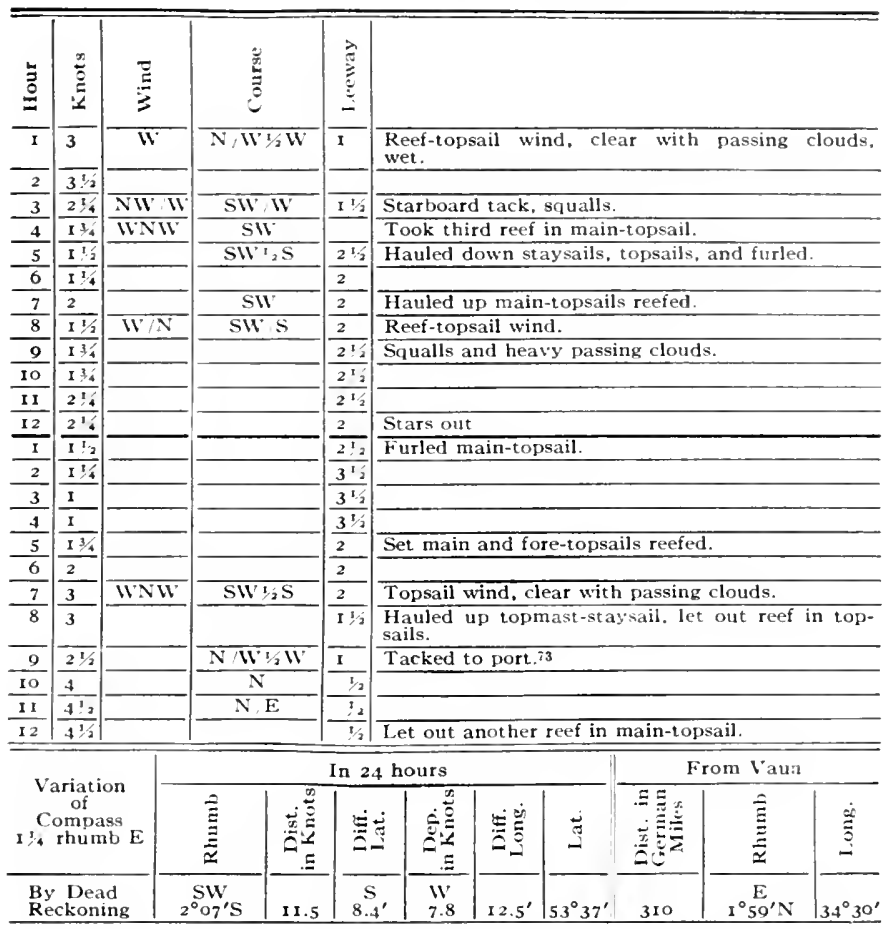

73 Khitrov's journal: "Together with the Captain Commander we signed today that which we agreed upon, a copy of which is here attached." (See next page.) 


\author{
A ugust 27, 1741
}

\title{
Decision To Land IN Order To Take ON Water 74
}

Captain Commander Bering, Lieutenant Waxel, Fleet Master Khitrov, and Navigator Eselberg met to talk over the decision, which we with our petty officers came to on August Io, relative to our returning to Avacha harbor. Our decision at the time to start back, though it was yet early, was based on the fear that west winds would begin to blow and hinder us. Since then this has happened, and now we have of the water taken in America only 25 barrels, which is not enough for returning to Avacha if the contrary west winds continue to blow.

The American coast which we last saw was between latitude $55^{\circ}$ and $56^{\circ} \mathrm{N}$, and according to our reckoning it is not more than 60 German miles from us. Therefore we, the undersigned, have unanimously agreed for safety's sake to go nearer the land with a view to finding good anchorage where we might take on water enough to last until our return so that in case of head winds we should not suffer extremely.

74 Khitrov's iournal.

BERING

Lieutenant WAXEL

Fleet Master Sofron KHItrov Navigator Andreyan Eselberg and petty officers. 


\section{ㅇ August 28, 17+1. After Midday}

\begin{tabular}{|c|c|c|c|c|c|c|c|c|c|c|}
\hline$\stackrel{2}{3}$ & $\underset{0}{2}$ & $\stackrel{\Xi}{\Xi}$ & $\stackrel{\frac{\mathscr{H}}{3}}{3}$ & 㐫 & & & & & & \\
\hline$I$ & $\sqrt[3]{4}$ & $\mathrm{~W} / \mathrm{N}$ & $\mathbf{N} \mathbf{E}$ & 12 & $\begin{array}{l}\text { Top } \\
\text { reef }\end{array}$ & $\begin{array}{l}\text { ail win } \\
\text { in fore }\end{array}$ & $\begin{array}{l}\text { 1, clear } \\
\text { topsail }\end{array}$ & with passing $c$ & uds; let o & ut two \\
\hline 2 & $2 t_{2}$ & IVNW & $5 W^{\prime} \cdot{ }_{2} S$ & $\begin{array}{r}1.2 \\
\end{array}$ & Tac & $\mathrm{ed}$ in $\mathrm{s}$ & Iccessio & & & \\
\hline 3 & 5 & $\mathrm{~W} / \mathrm{N}$ & $\mathrm{N} E$ & $\mathrm{I}^{2}$ & 1 ac & Ea in $\mathrm{s}$ & ICcessio & & & \\
\hline 4 & 5 & & & 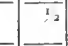 & $\begin{array}{l}\text { Cart } \\
\text { stay }\end{array}$ & $\begin{array}{l}\text { ying to } \\
\text { fail. }\end{array}$ & psails, & foresail, main & iil. trysai & I, and \\
\hline 5 & 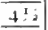 & & & $I_{2}$ & Too & all the & reefs is & n tousails. & & \\
\hline 6 & 4 & & & $\frac{12}{2}$ & Clea & with I & eavy $p$ & assing clouds, s & Lalls, rain & \\
\hline 7 & 4 & & & 12 & & & & & & \\
\hline 8 & $3^{3}$ & IV & & $T_{2}$ & & & & & & \\
\hline 9 & 2 & & NNW & $1 ! 2$ & Fur & d tops & iils. & & & \\
\hline 10 & $1: 4$ & & & 132 & $\begin{array}{l}\text { Stro } \\
\text { and }\end{array}$ & $\begin{array}{l}\text { ig and } \\
\text { rain. }\end{array}$ & old top & ssail wind, squa & s. passing & clouds \\
\hline II & 1 & & & 3 & & & & & & \\
\hline 12 & 1 & & & 3 & Hea & y pass & ng clou & ds and rain. & & \\
\hline 1 & $\overline{112}$ & & $N 15,211$ & 25 & & & & & & \\
\hline 2 & $\overline{134}$ & & & $2 \div 2$ & $\begin{array}{l}\text { Dur } \\
\text { bott }\end{array}$ & $\begin{array}{l}\text { ng the } \\
\text { im. }\end{array}$ & night w & ve heaved the I & d but str & uck no \\
\hline 3 & 134 & & & $21_{2}^{2}$ & & & & & & \\
\hline+4 & $x^{x}$ & $\mathrm{~W} / \mathrm{N}$ & $\mathrm{N}, \mathrm{W}$ & $21_{2}$ & Top & ail win & 1. cloud & dy. & & \\
\hline 5 & 3 & & $\mathrm{~N}$ & $21 / 2$ & Set & opsails, & hauled & 1 up staysails at & jib. & \\
\hline 6 & $3^{3} 4$ & & & $1 / 2$ & & & & & & \\
\hline 7 & $3{ }^{\prime}{ }_{2}$ & & & $3 / 2$ & Let & ut all & ceefs in & topsails. & & \\
\hline 8 & 4 & & & $\sqrt{1+1}$ & & & & & & \\
\hline 9 & + & WV & & 1 & Cle: & & & & & \\
\hline 10 & $\overline{3^{3}}$ & & & 12 & & & & & & \\
\hline 11 & 3 & & & $\mathrm{I}_{2}$ & Has & ed up & pritsail & & & \\
\hline 12 & 4 & & & $\pi$ & $\begin{array}{l}\text { Cle: } \\
\text { zeni } \\
\text { latit } \\
\text { east } \\
\text { E:2 }\end{array}$ & $\begin{array}{l}\text { with } \\
\text { h dista } \\
\text { ade } 54^{\circ} \\
\text { rly bec } \\
4,75\end{array}$ & $\begin{array}{l}\text { passing } \\
\text { nce } 48^{\circ} \\
\text { o5'. var } \\
\text { ause at } \\
\end{array}$ & $\begin{array}{l}\text { clonds Toot } \\
14 \text {, declination } \\
\text { riation of the co } \\
\text { t noon the obse }\end{array}$ & $\begin{array}{l}\text { an obser } \\
\text { f the sun } \\
\text { apass } 1 \text { ! } \\
\text { vation wa }\end{array}$ & $\begin{array}{l}\text { 'ation: } \\
5^{\circ} 51^{\prime} \\
\text { rhumb } \\
\text { is } S \text { by }\end{array}$ \\
\hline \multirow{2}{*}{\multicolumn{3}{|c|}{$\begin{array}{c}\text { Variation } \\
\text { of } \\
\text { Compass } \\
1,2 \text { rhumb E }\end{array}$}} & \multicolumn{8}{|c|}{ In 2.4 hours } \\
\hline & & & $\stackrel{\vec{E}}{\underline{\Xi}}$ & $\begin{array}{l}5 \\
\end{array}$ & 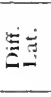 & 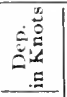 & $\stackrel{i=}{2}$ & 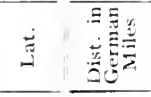 & 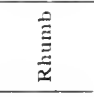 & 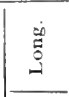 \\
\hline \multicolumn{3}{|c|}{$\begin{array}{c}\text { By } \\
\text { ()bservation }\end{array}$} & $\begin{array}{r}\mathrm{NE} \\
255^{\prime} \mathrm{E} \\
\end{array}$ & 41.7 & $\begin{array}{l}N \\
28^{\prime} \\
\end{array}$ & $\begin{array}{l}\mathrm{E} \\
.32 \\
\end{array}$ & $52.7^{\prime}$ & $54^{\circ} 05^{\prime}, \quad 31.4 \%$ & $\begin{array}{c}\mathrm{E} \\
3^{\circ}+4^{\prime} \mathrm{N}^{\top}\end{array}$ & $35^{\circ} 23^{\prime}$ \\
\hline
\end{tabular}

is Khitrov's journal gives latitude by observation $54^{\circ} 07^{\prime}$. 


\section{I) August 29, I74I. After Midday}

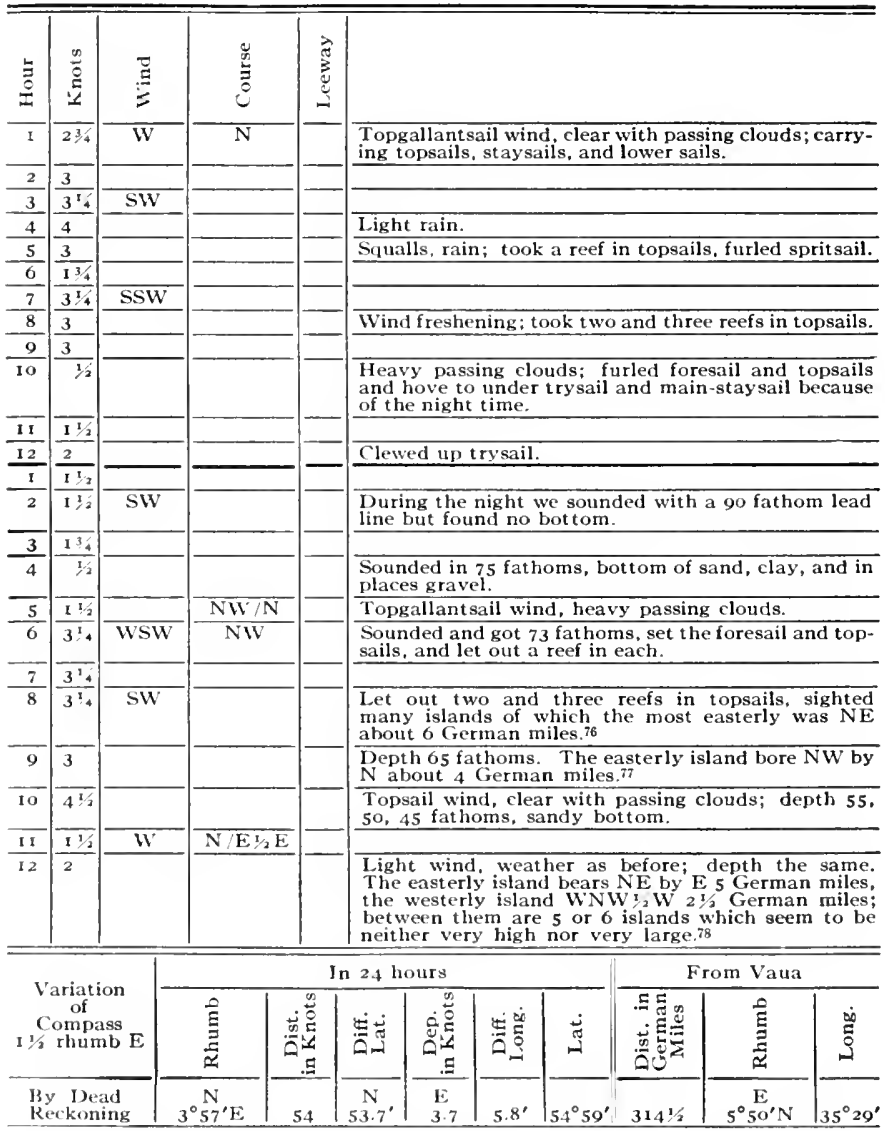

76 Shumagin Islands. Khitrov's journal: "Siaw many islands of which the southernmast bore NE",

7 Khitrov's journal: "looked fur islands toward the $\mathrm{N}$; the last bore NW by N."

is Khitrov's journal: "Southernmost island NE by E. Northernmost WNW'/4W." 
August 30, 1741. After Midday

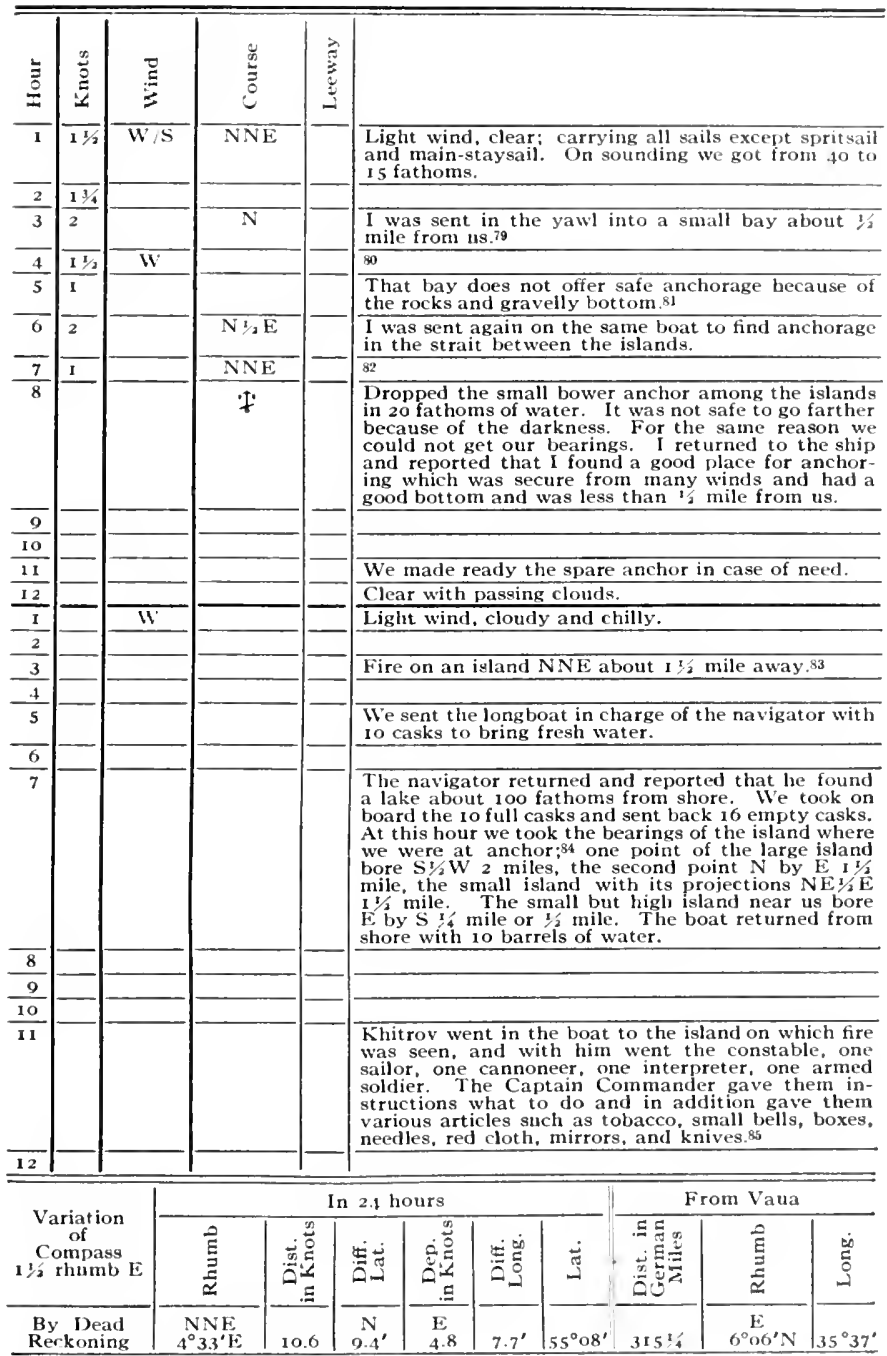

(Footnotes 79-85 at bottom of next page.) 


\begin{tabular}{|c|c|c|c|c|c|}
\hline 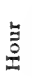 & 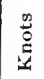 & $\stackrel{\vec{D}}{\partial}$ & . & 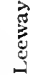 & \\
\hline $\mathbf{I}$ & & $\mathrm{S}, \mathrm{IV}$ & & & $\begin{array}{l}\text { Light wind, clear with passing clouds; } 10 \text { more casks } \\
\text { of water brought on board; the sick were taken } \\
\text { ashore. }\end{array}$ \\
\hline 2 & & & & & \\
\hline 3 & & & & & \\
\hline 4 & & $\mathrm{SE} \mathrm{S}$ & & & Light rain. \\
\hline 5 & & & & & The boat returned with 10 more casks of water. \\
\hline 6 & & & & & \\
\hline 7 & & $\mathrm{SE}$ & & & Io empty casks sent ashore. \\
\hline 8 & & & & & \\
\hline 9 & & & & & \\
\hline 10 & & $S$ & & & $\begin{array}{l}\text { The quartermaster returned to the ship with io more } \\
\text { casks and reported that the sailor Nikita Shumagin } \\
\text { died on shore. He was buried on the island, which } \\
\text { was named Shumagin after him. } 36 \text { The small boat } \\
\text { was tied astern. }\end{array}$ \\
\hline I I & & & & & \\
\hline 12 & & & & & \\
\hline $\mathrm{I}$ & & & & & \\
\hline 2 & & & & & \\
\hline 3 & & & & & 31 casks were filled. \\
\hline 4 & & & & & $\begin{array}{l}\text { During the night we had a lantern on the gaff so } \\
\text { Khitrov could sce it. }\end{array}$ \\
\hline 5 & & $\mathrm{SE}$ & & & Sent the boat for more water. \\
\hline h & & $\mathrm{E}$ & & & \\
\hline $\bar{i}$ & & & & & I I more casks were brought from shore. \\
\hline 8 & & & & & \\
\hline 9 & & & & & Sent ro empty casks ashore. \\
\hline Io & & & & & Wind freshening, payed out $3 / 4$ of a cable. \\
\hline I I & & & & & $\begin{array}{l}\text { Io more casks taken on board with which we filled } \\
\text { the casks in the hold. }\end{array}$ \\
\hline 12 & & & & & \\
\hline
\end{tabular}

56 Khitrov's journal: "He was buried on the island, and over his grave a wooden cross was erected." The name Shumagin is now given to the whole group of islands, and the island which used to be called Stumagin is now known as Nagai.

\section{Footnoles to Log of August 30}

79 Khitrov's journal: "Lowered the small yawl and sent the assistant navigator in search of good anchorage."

so Khitrov's journal: "Took in all sail, dropped the anchor in 24 fathoms, and payed ont cable. The assistant navigator returned and reported that he failed to find a good place to anchor. Put over the longboat into the water."

si Khitrov's journal: "Weighed anchor. set sails, and steered between the islands into the bay, getting as we went along 24, 25, 20, 15 fathoms, bottom gravelly and shelly."

th: Khitrov's journal: "Went in tow; and, when we came within $1 / 2$ mile of one of the islands, we anchored in 20 fathoms and payed out $3 / 2$ cable. Because of the fog we could not get our bearings."

$k 3$ Khitrov's journal: "Saw a fire on one of the islands about $2 \frac{1}{2}$ miles away on the rhumb NNE."

netween Near fsland and Nagai 1sland. This anchorage is matked on Khitrov's map, reproduced in Fig. II, by an inverted anchor at the 10 and 20 fathom soundings Khitrov's journal: "When fog cleared it was possible to get bearings: the southern point of the large island bore $\mathrm{S} \mathrm{s} / \mathrm{W}$; the northern point of the same island bore $\mathrm{N}$ by $\mathrm{E}$; the small island with its projections $N$ by $\mathrm{E}, \mathrm{E} E$; the southern point of the island where we are at anchor bore" $\mathrm{E}$ by $\mathrm{C}^{\circ}$

ss Khitrov"s journal: "Captain Commander sent me to the island on which fire was secn and which is from us NNE. In case 1 found human beings I was told to be kind to them and was guren for fistribution various presents, namely 1, pound Chinesc tobacco, 5 copper bells, 1,0 beads, 20 needles, 2 arshins red material, 5 small mirrors, and 5 knives. I went in the small yawl and took with the I assistant constable, I sailor, I cannoneer, I soldier, I Chukchi, and I Koriak interpreter." 
8 September I, I74I. After Midday

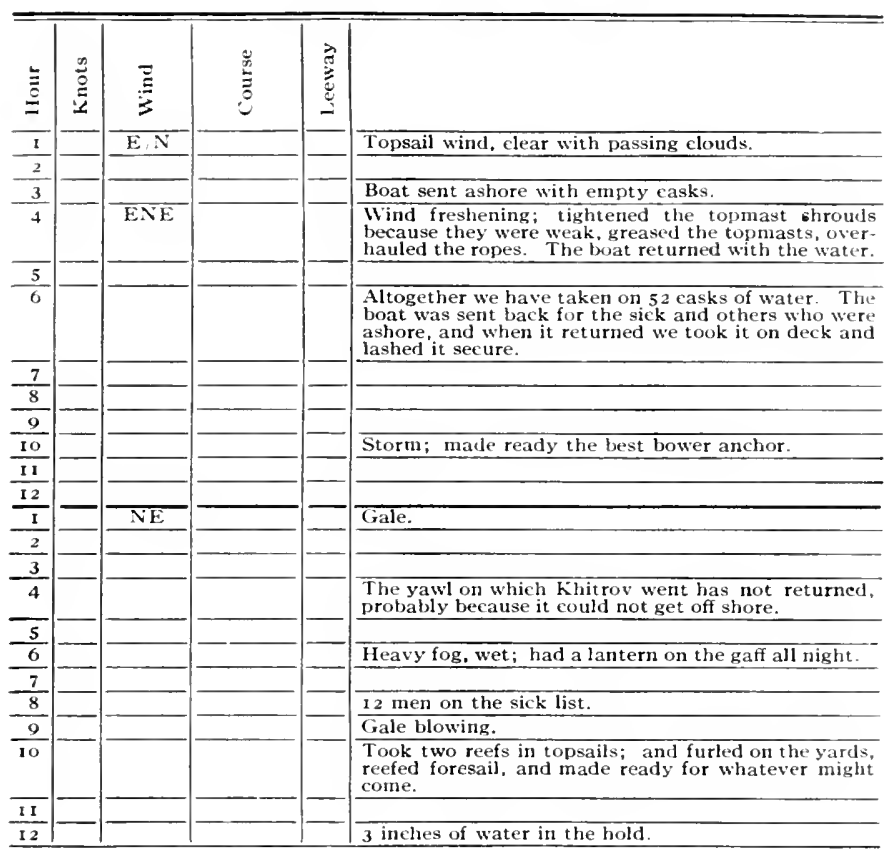


I44 LOG BOOK OF THE "ST. PETER"

$\Varangle$ September 2, I74I. After Midday

\begin{tabular}{|c|c|c|c|c|c|}
\hline$\stackrel{2}{2}$ & $\stackrel{\infty}{\stackrel{\infty}{0}}$ & $\stackrel{\vec{z}}{z}$ & $\stackrel{\mathscr{D}}{ٍ}$ & $\vec{己}$ & \\
\hline $\mathrm{I}$ & & NE & & & Reef-topsail wind, cloudy and rainy. \\
\hline 2 & & & & & \\
\hline 3 & & & & & \\
\hline 4 & & & & & Wind going down. \\
\hline 5 & & & & & 4 inches of water in the hold. \\
\hline 6 & & & & & \\
\hline 7 & & & & & \\
\hline$\overline{8}$ & & & & & Wind comes in gusts. \\
\hline 9 & & & & & \\
\hline 10 & & & & & Squally. \\
\hline 11 & & & & & \\
\hline 12 & & & & & \\
\hline $\mathbf{I}$ & & & & & \\
\hline 2 & & & & & \\
\hline 3 & & $\mathrm{E}$ & & & Unsteady wind. \\
\hline 4 & & & & & $\begin{array}{l}\text { Began to warp the anchor because it was dangerous } \\
\text { to remain where we were. }\end{array}$ \\
\hline 5 & & $\mathrm{SE}$ & & & \\
\hline 6 & & & & & $\begin{array}{l}\text { Weighed anchor, set the sails, and started for the } \\
\text { island in order to protect oursclves from the wind. } \\
\text { From } 6 \text { o'clock until } 8: 20 \text { we sounded as we went } \\
\text { along and got } 20,17,15,13 \text { fathoms; rhumb } E \text { by } \\
N_{1} / 2 \text {; advanced } 34 \text { of a German mile. }\end{array}$ \\
\hline 7 & & & & & Thick wet fog. \\
\hline 8 & & & & & $\begin{array}{l}\text { Dropped anchor in } 16 \text { fathoms, } 87 \text { took in the sails, } \\
\text { payed out } 3 / 4 \text { of a cable; bottom sandy. Because } \\
\text { of the fog could not get the bearings. }\end{array}$ \\
\hline 9 & & & & & \\
\hline 10 & & & & & Put over the boat into the water, sea going down. \\
\hline 11 & & & & & \\
\hline 12 & & SSE & & & Sent the boat ashore for Khitrov 88 \\
\hline
\end{tabular}

87 This second anchorage is the one marked on Khitrov's map (Fig. II) by an anchor lying on its side at the 16 fathom sounding.

88 Khitrov's journal: "The Jongboat was put into the water and sent for me on Shumagin Island because 1 could not get on board in the small boat, owing to the gale. Iligh sea going down. Bearings from anchor: S point Shumagin Island $S$ by $W, N$ point of the same island $\mathrm{Nt}_{3} \mathrm{E}$, the $\mathrm{N}$ point of the two islands from Shumagin $\mathrm{NNE}$." 
2 September 3, I74I, After Midday

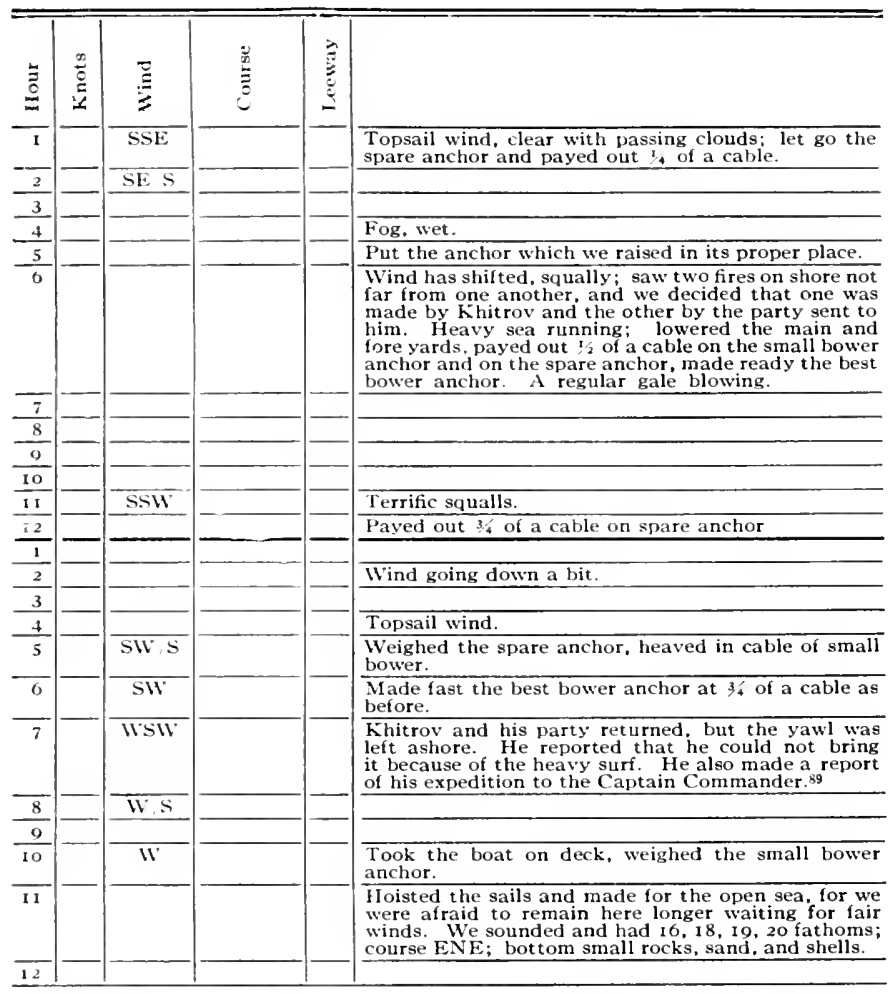

89 Khitrov's journal: "I reported to the Captain Commander that while I was on the island to which 1 was sent 1 saw a fireplace and many other signs of human beings but no human beings thenselves. I sighted the mainland back of the islands about 12 miles away." 
I46 LOG BOOK OF THE "ST. PETER"

Q September 4. I7fI. After Midday

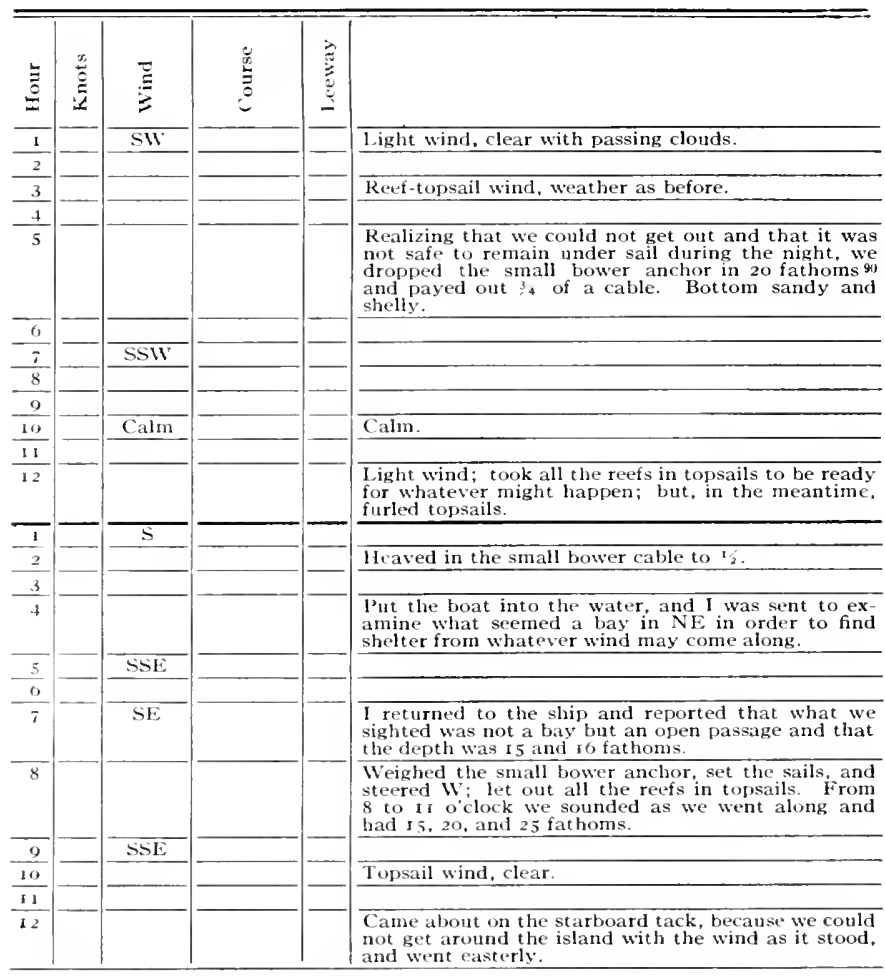

91 Off Bird Island. This thirt anchorage is marked on Khitrov's map (Fig. 11) by an anchor at the end of the arrow pointing north. 


\section{h September 5, 1741. Afler Midday}

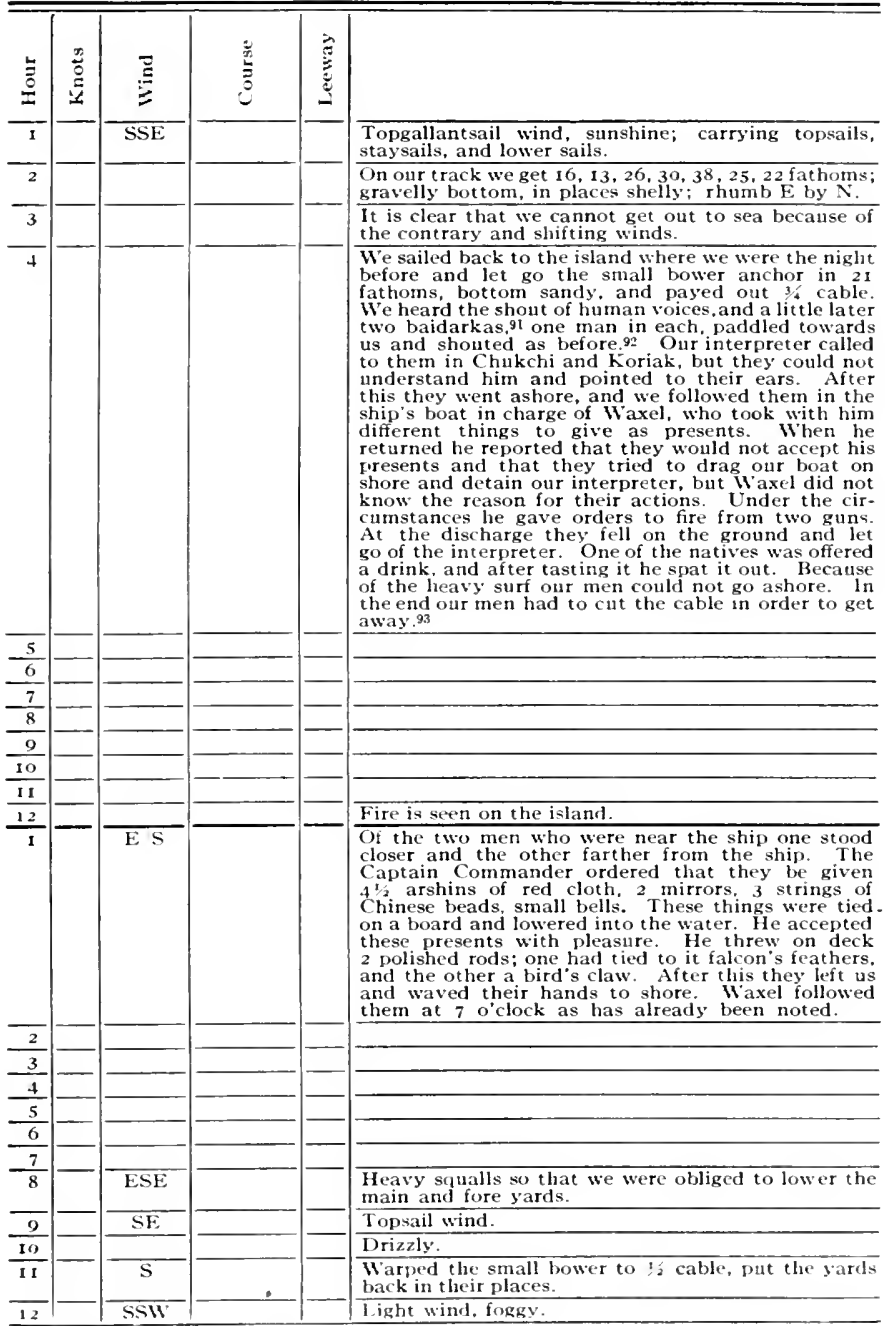

(Footnotes or-03 on next page.) 


\section{Footnotes to Log of September 5}

91 A baidar (Russ. baidára) is a large open skin boat able to accommodate a number of passengers and a considerable amount of freight. A baidarka (Russ. baidárka) is a small skin boat covered all over, except the round openings at the top to admit the paddler or paddlers (ef. Fig. II). The baidar is related to the Eskimo $1 \mathrm{miak}$, the baidarka to the kayak. Baidars may be seen today in the Bering Sea and baidarkas in the Aleutian Islands. When the Russians first came in contact with the Aleuts the baidarkas were all of the "one-hateh" type, that is, large enough for one person. This type has almost entirely disappeared and has been succeeded by the "two-hatch" baidarka, and occasionally" one may even see a "three-hatch" baidarka. These little boats are very light and strong, and the Aleuts are very skillul in handling them.

Samer. who visited Unalaska in 1790 . has leit a full description of these skin boats: "The baidars, or boats, of Oonalashka are infinitely superior to those of any other island. If perfect symmetry, smoothness, and proportion constitute beauty, they are beautiful: to me they appeared so beyond anything that 1 ever beheld. I have seen some of them as transparent as oiled paper, through which you conld trace every formation of the inside, and the manner of the native's sitting in it; whose light dress, painted and plumed bonnet. together with his perfeet ease and aetivity, added infinitely to its elegance. Their first appearance struck me with amazement beyond expression." (Martin Sauer An Account of a Geographical and Astronomical Expedition to the Northern Parts of Russia . . by Commodore Joseph Billings, London, 1802, p. 157.)

${ }_{92}$ This was a form of greeting more or less common to all the natives of the northwest coast of America. Cook met with it in Nootka Sound. "On their first coming. they generally went through a singular mode of introdueing themselves. They wonld paddle, with all their strength, quite round both ships. a Chief, or other principal person on the canoe, standing up with a spear or some other weapon in his hand and speaking. or rather hollowing, all the time." (James Cook: A Voyage to the Paeific Ocean, London, 1 78., Vol. 2, p. 273.)

93 Khitrov's journal: "At 4:30 we heard shouts of human voices from the island alongside of which we were at anchor.

"At 6 o'clock two baidarkas were seen coming towards 118 , one man in each boat, and when they were within so fathoms of the ship they stopped and ealled in their own tongue, but our Chukehi and Koriak interpreters could not make out what they said nor could they make themselves understood when they shouted to them ithe islanders]. because they pointed to their ears and to the island. On the island people were also calling. One of the two men just mentioned paddled up close to the ship but not quite alongside. At the order of Captain Commander Bering we threw overboard a piece of board on which were tied a number of presents, namely, $5 \frac{1 / 2}{2}$ arshins of red material, 2 small mirrors, 2 strings of Chinese iron beads, 20 smali copper bells and 5 [knives?]. The American received these things with pleasure and in return threw to us as a present two thinly shaped rods to one of which were tied bird's feathers and to the other bird's claws with feathers on them. The feathers we identified as those of the falcon. When we had taken these things they paddled away for the shore and called to us and pointed to the land. We lowered the longboat into the water lor the purpose of going ashore. In the boat was sent, by the order of the Captain, Lieutenant Waxel, who took with him several members of the crew, (who were armed), a lew presents, and Russian liquor. He was gone about two hours, returning at 8 o'clock and reporting to the Captain Commander that when he came to the island on which were the Americans he offered them presents but they would not take them. He offered one of the islanders a glass of liquor, but as soon as he tasted it he spat it out and returned the glass. The lientenant allowed several of the men, among them being the interpreter, to land; but he himself remained in the boat which he anchored out a little distance from shore, for owing to the strong wind and submerged rocks near the beach it was dangerous to approach it. The Americans led the interpreter to their camp and gave him whale blubber, but when he attempted to leave them to return to the boat, nine of the Americans seized him and would not let him go, which shows that they regarded him as one of their own people even though he is a Kamchadal by origin. In order to free the interpreter an order was given to fire off several guns in the air, and when that was done the Americans fell down on the ground, letting go of the interpreter, who hurried to the boat. When our party was ready to leave the Americans seized the painter and started to haul the boat ashore. Seeing this, the order was given to cut the painter and leave the anchor and pull for the ship. In the drawing attached is given the position of the bay and the island [H.g. II], and a sketch of the Americans and their lair-seal skin boats." (Sce Fig. 12, upper.) 


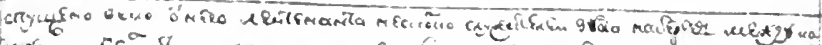

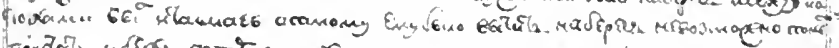

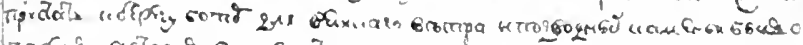

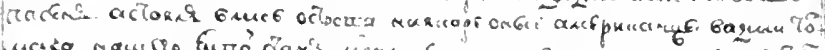

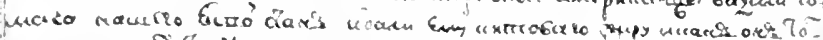

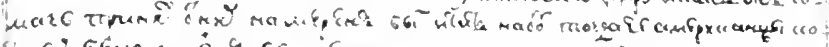

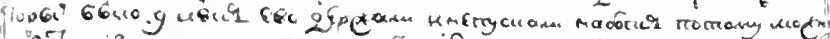

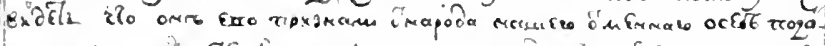

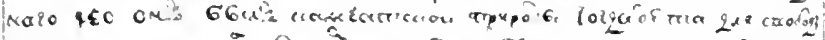

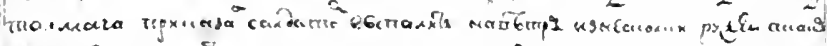

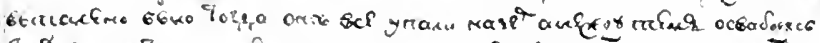

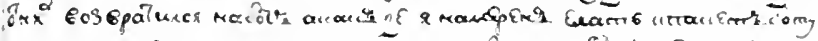

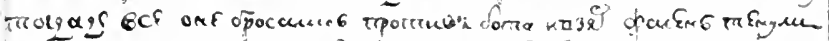

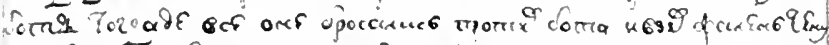

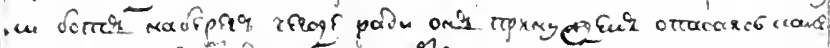

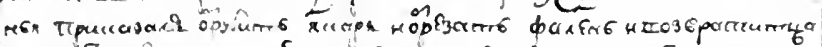

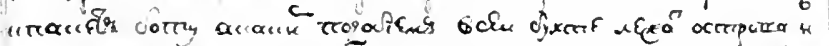

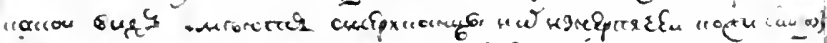

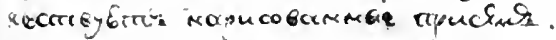

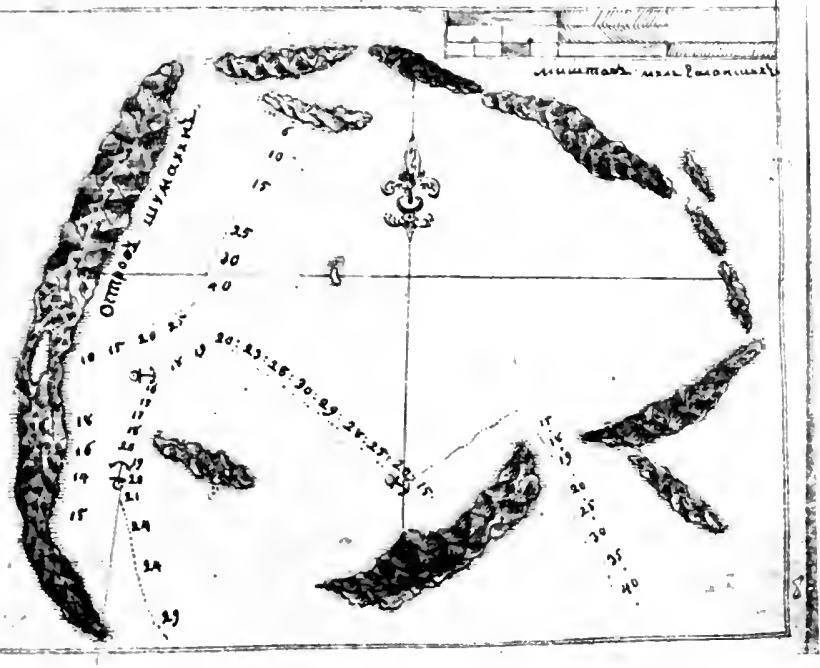

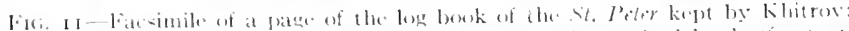

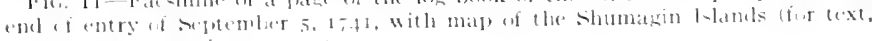

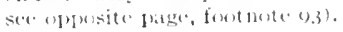



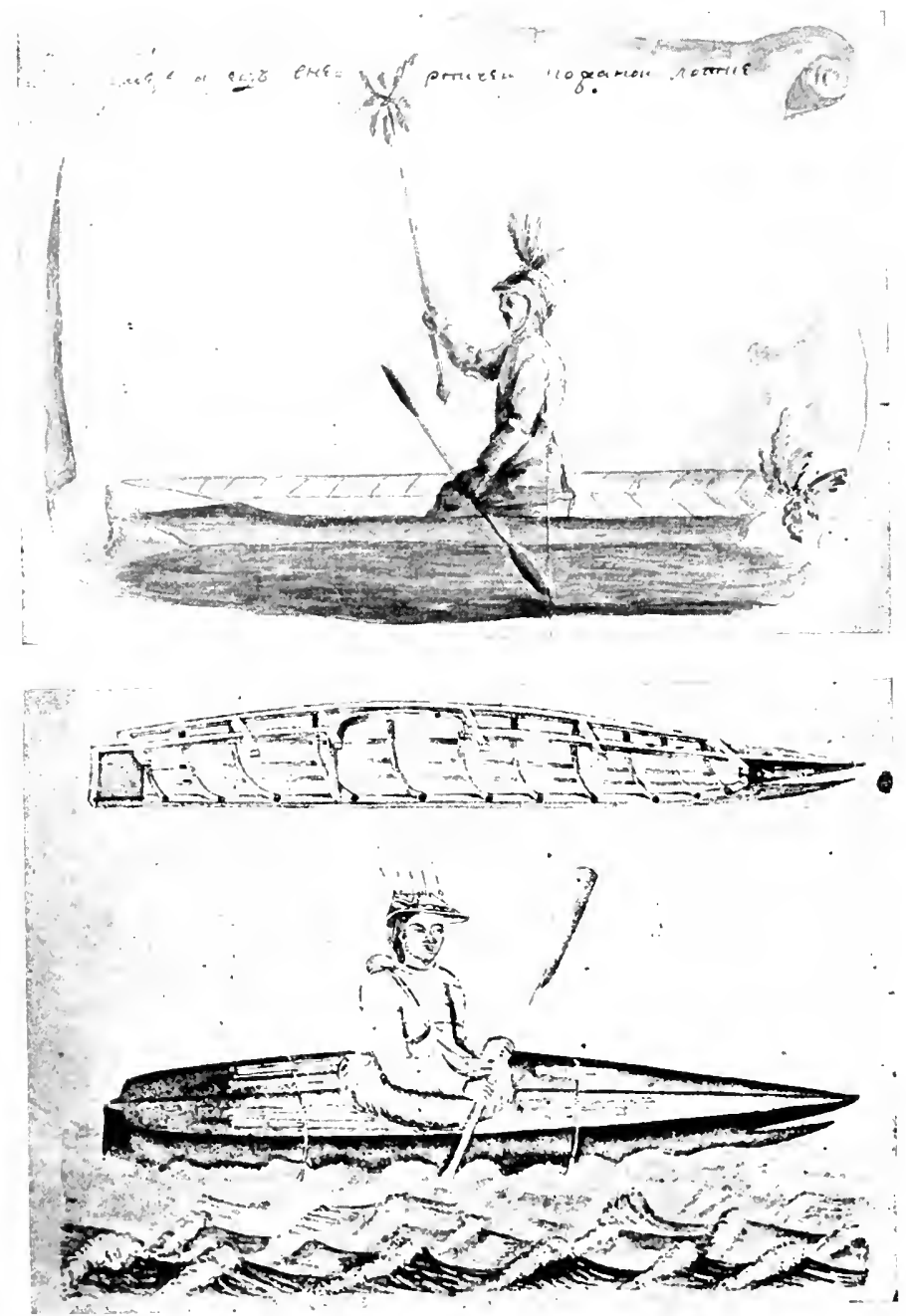

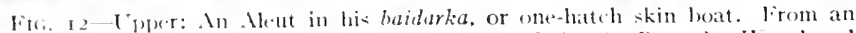

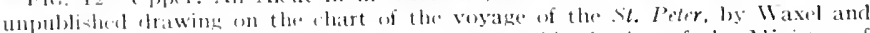

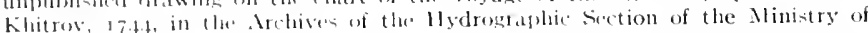
Matrime. Pettograll. No, 10) 70 .

Luwer: a from a book of unpublished draw-

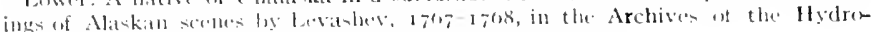

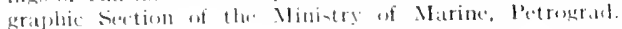




\section{September 6, 1741. After Midday}

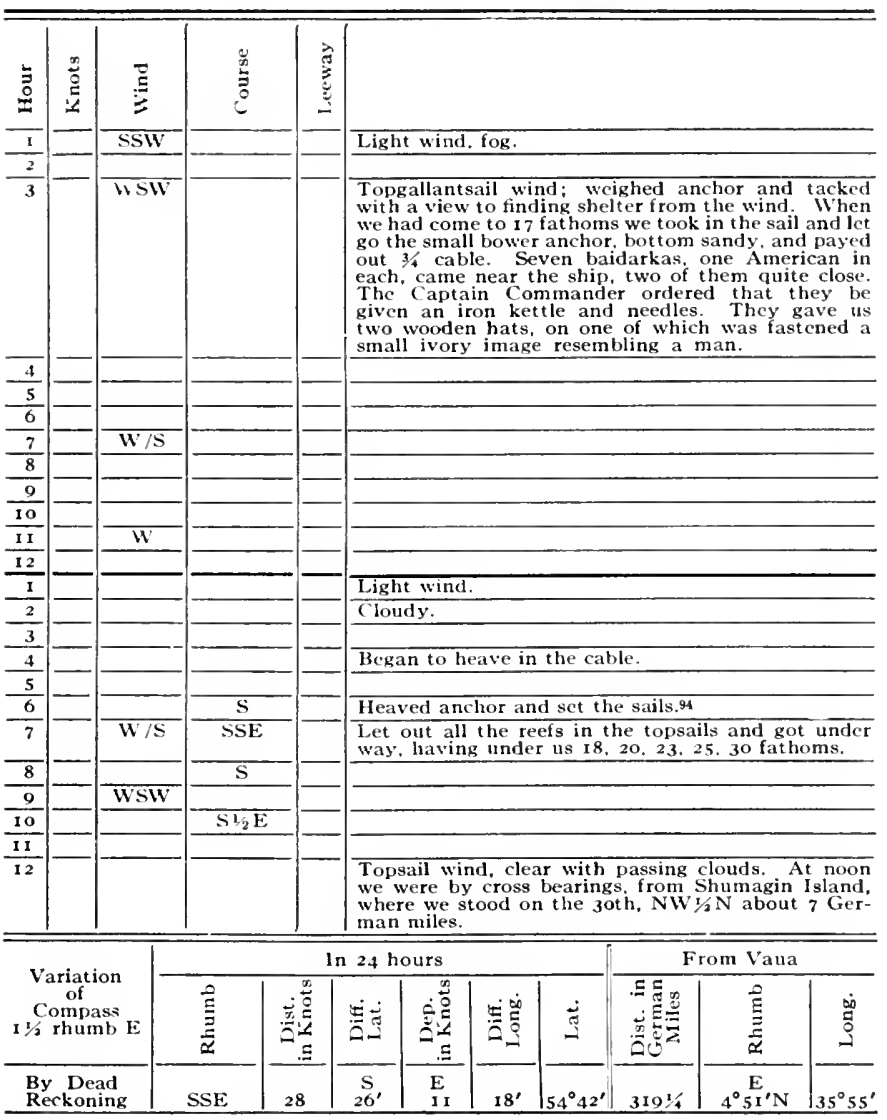

9 Khitrov's journal: "Got under way." 
(3eptember 7, 1741. After Midday

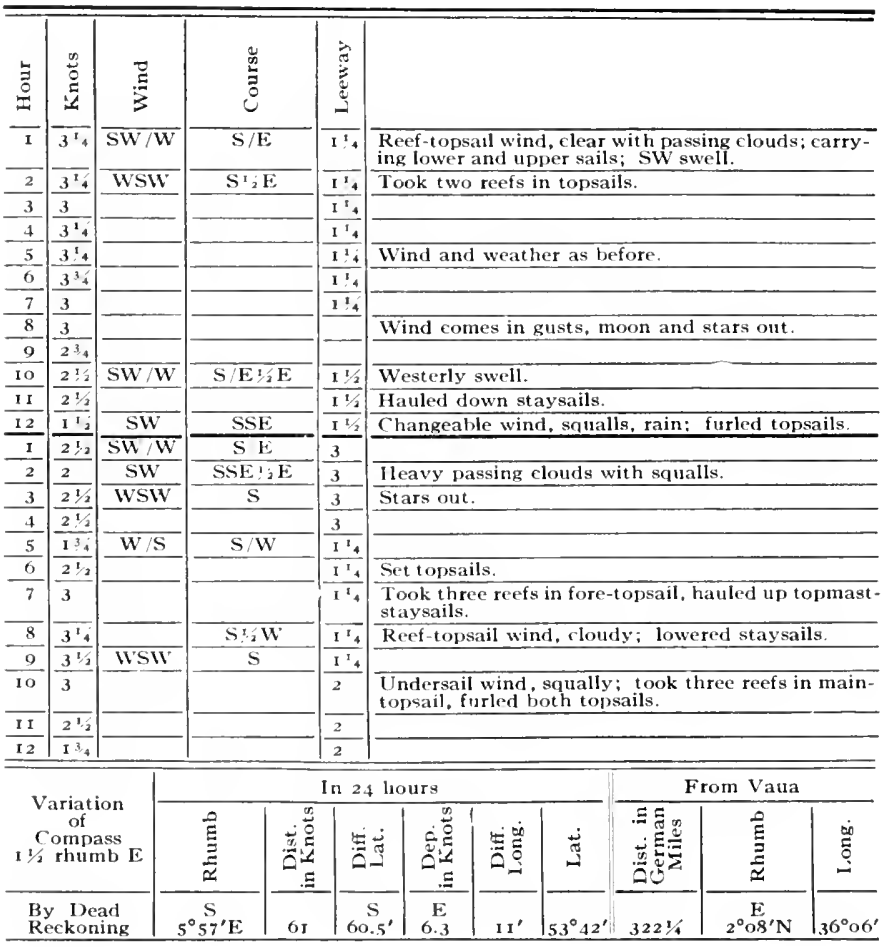


万S September 8, I74I. After Midday

\begin{tabular}{|c|c|c|c|c|c|c|c|c|c|c|c|c|}
\hline$\stackrel{\Xi}{\Xi}$ & 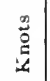 & 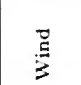 & \multicolumn{2}{|c|}{ 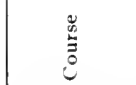 } & \multicolumn{2}{|l|}{ 育 } & & & & & & \\
\hline$I$ & $\overline{134}$ & WV & $\mathrm{S} / \mathrm{W}$ & W & $\overline{21 / 2}$ & & $\begin{array}{l}\text { ef-und } \\
\text { sterly. } \\
\text { insail. } \\
\text { sail. }\end{array}$ & $\begin{array}{l}\text { rsail } \\
\text { vell; } \\
\text { and }\end{array}$ & $\begin{array}{l}\text { ind, } \\
\text { eefed f } \\
\text { ove to }\end{array}$ & $\begin{array}{l}\text { ear with } \\
\text { oresail, } \\
\text { under }\end{array}$ & $\begin{array}{l}\text { passing } \\
\text { rled fores } \\
\text { ain-stay'sa }\end{array}$ & $\begin{array}{l}\text { louds } \\
\text { ii and } \\
\text { il and }\end{array}$ \\
\hline 2 & 2 & & & & 23 & & $\operatorname{ming} \mathrm{u}$ & to S & by W & falling & ff to SSIV & \\
\hline 3 & $7 ! 2$ & IV $/ \mathrm{N}$ & SSW & $\mathrm{W}$ & & & & & & & & \\
\hline 4 & $11_{2}$ & & & & $\overline{5^{1 / 2}}$ & & & & & & & \\
\hline 5 & I15 & WNW & SW" & & $51 / 2$ & & avy st & $\mathrm{rm}$. & & & & \\
\hline 6 & $1 \%$ & & & & $51 / 2$ & & & & & & & \\
\hline 7 & $1 ! 2$ & & & & $5 \frac{1}{2}$ & & oudy, : & r thic & & & & \\
\hline 8 & $11 / 2$ & & & & 5 & & ars out & & & & & \\
\hline 9 & $\overline{114}$ & & & & & & & & & & & \\
\hline 10 & $1^{2}+4$ & & & & & & ind dec & easin & & & & \\
\hline II & $1 !$ & W/N & SSW & $2 W$ & & & ming & to S & $V$, falli & ig off to & by $\mathrm{W}$ ? & \\
\hline 12 & 74 & & & & $\overline{5^{1 / 2}}$ & & & & & & & \\
\hline$I$ & 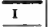 & & & & 6 & & dersail & wind. & Neathe & as befor & & \\
\hline 2 & $I$ & & & & 6 & & & & & & & \\
\hline 3 & $\mathrm{I}$ & & & & 6 & & & & & & & \\
\hline 4 & $I$ & & & & & & psail y & nd. & & & & \\
\hline 5 & 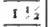 & & SW & & $\overline{I_{2}}$ & & isted t & prails & let ou & a reef ir & topsails. & \\
\hline 6 & 23 & WNW & SW & & $1 ! 4$ & & isted & pmas & staysa & is, set f & resail and & main- \\
\hline 7 & 3 & $W / N$ & SW & & $I^{1} 4_{4}$ & & ind un & eady & ind in & usts. & & \\
\hline 8 & $2 t_{2}$ & & & & $I^{\prime} 4$ & & & & & & & \\
\hline 9 & 23 & & & & $1^{2} 4$ & & & & & & & \\
\hline 10 & 3 & & & & $1 \frac{14}{4}$ & & ear wit & pass & g clou & & & \\
\hline II & 23 & & & & & & & & & & & \\
\hline 12 & 23 & & SW & & $1^{1} 4$ & & esterly & well. & & & & \\
\hline & & & & & In & 24 & ours & & & & om Vaua & \\
\hline $1 \%$ & $\begin{array}{l}\text { arjati } \\
\text { of } \\
\text { ompa } \\
\text { rhun }\end{array}$ & $\begin{array}{l}\text { on } \\
\text { lass } \\
\text { ib E }\end{array}$ & $\frac{\hat{E}}{\stackrel{\Xi}{\Xi}}$ & 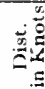 & & - & 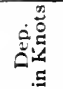 & 象葛 & $\stackrel{\text { Ij }}{-}$ & 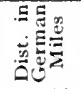 & 胥 & 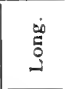 \\
\hline & De & & $\underset{2^{\circ}}{\stackrel{s}{3} / \mathrm{W}}$ & 39 & 37 & $7^{\prime}$ & $\begin{array}{c}W \\
9\end{array}$ & $15^{\prime}$ & $53^{\circ} 0.4^{\prime}$ & $323 \%$ & $0^{\circ} \mathbf{2} 4^{\prime} \mathrm{N}$ & $35^{\circ} 51^{\prime}$ \\
\hline
\end{tabular}


$\Varangle$ September 9, I74I. After Midday

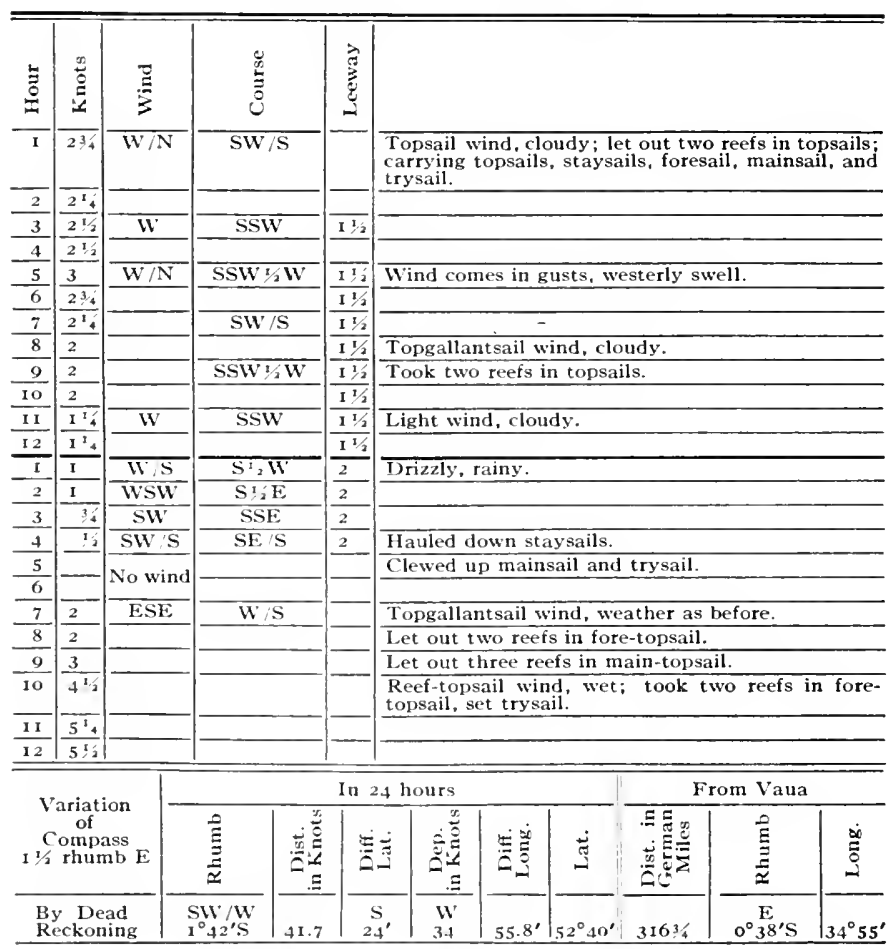


2 September 10, 174I. After Midday

\begin{tabular}{|c|c|c|c|c|c|c|c|c|c|c|c|c|}
\hline$\stackrel{\Xi}{\Xi}$ & $\stackrel{n}{\vdots}$ & $\stackrel{\Xi}{\Xi}$ & \multicolumn{2}{|c|}{$\stackrel{0}{0}$} & 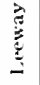 & & & & & & & \\
\hline $\bar{I}$ & $\overline{5 \% 3}$ & ESE & \multicolumn{2}{|c|}{ WSW } & & \multicolumn{7}{|c|}{$\begin{array}{l}\text { Undersail wind, drizzly, rainy; carrying reefed } \\
\text { topsails. foresail, and trysail. }\end{array}$} \\
\hline \multicolumn{13}{|c|}{ (2) } \\
\hline 3 & 5 & $\mathrm{SE}$ & & & & & & & & & & \\
\hline 4 & 5 & $\mathrm{SE} / \mathrm{S}$ & \multicolumn{2}{|c|}{$\mathrm{SW} / \mathrm{JV}$} & $11 / 2$ & \multicolumn{7}{|c|}{ Set mainsail and topmast-staysails. } \\
\hline 5 & 2 & $\mathrm{~S} / \mathrm{E}$ & \multicolumn{2}{|c|}{$\mathrm{s}$} & $1: 2$ & & & & & & & \\
\hline 6 & $\overline{13.4}$ & $S$ & \multicolumn{2}{|c|}{ WSW } & $11 / 2$ & \multicolumn{7}{|c|}{$\begin{array}{l}\text { Topgallantsail wind, weather as before, damp, } \\
\text { heavy swell from SE. }\end{array}$} \\
\hline 7 & $2+4$ & & & & $1 \%$ & & & & & & & \\
\hline 8 & 214 & $\mathrm{~S} E$ & \multicolumn{2}{|c|}{ SW IV } & $11 / 2$ & & & & & & & \\
\hline 9 & 24 & & & $11 / 2$ & \multicolumn{7}{|c|}{ Drizzly, wet. } \\
\hline 10 & 2 & & & & $1 \mathrm{I} / 2$ & \multicolumn{7}{|c|}{ 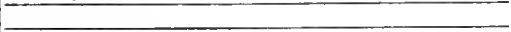 } \\
\hline II & $11 / 2$ & $\mathrm{~s}$ & \multicolumn{2}{|c|}{ WSW } & $1 \%$ & \multicolumn{7}{|c|}{ Southerly swell. } \\
\hline 12 & $11 / 4$ & & & & 2 & & 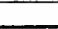 & +2 & & & & \\
\hline$I$ & 132 & & & & 2 & \multicolumn{7}{|c|}{ Light wind, weather as before. } \\
\hline 2 & $1 \%$ & & & & 2 & & & & & & & \\
\hline 3 & $\mathbf{I}$ & $\mathrm{S} / \mathrm{V}$ & \multicolumn{2}{|c|}{ W $\mathrm{S}$} & $\mathbf{I}$ & & & & & & & \\
\hline 4 & $2 \%$ & SSW & \multicolumn{2}{|c|}{11} & $\mathrm{I}$ & \multicolumn{7}{|c|}{ Topgallantsail wind. } \\
\hline 5 & 2 & & & & $\mathbf{I}$ & \multicolumn{7}{|c|}{ Let out two reefs in fore-topsail. } \\
\hline 6 & 234 & & & & $\mathbf{I}$ & \multicolumn{7}{|c|}{ Set the jib. } \\
\hline 7 & 234 & & & & $\mathbf{I}$ & \multicolumn{7}{|c|}{ Topsail wind, clear with passing clouds. } \\
\hline 8 & $32 / 2$ & & & & $\mathbf{I}$ & \\
\hline 9 & $33^{2}$ & $\mathrm{~S}$ & \multicolumn{2}{|c|}{ WSI" } & $\overline{15}$ & \multicolumn{7}{|c|}{ Southerly swell. } \\
\hline 10 & $33^{*}$ & & & & $\overline{I^{5} 4}$ & \multicolumn{7}{|c|}{+2} \\
\hline 11 & $2^{2 / 2}$ & & & & 15 & \multicolumn{7}{|c|}{ Wind decreasing. } \\
\hline 12 & $\overline{23}+1$ & & WSW & ${ }_{2} 11$ & $\overline{124}$ & & s. wet & & & & & \\
\hline & & & & & In & $2+1$ & urs & & & & m Vaua & \\
\hline $\begin{array}{r}\mathrm{V} \\
\mathrm{C}\end{array}$ & $\begin{array}{l}\text { ariati } \\
\text { of } \\
\text { ompa } \\
\text { rhum }\end{array}$ & $\begin{array}{l}\mathrm{On} \\
\mathrm{Ss} \\
\mathrm{b} \mathrm{E}\end{array}$ & $\underset{\Xi}{\Xi}$ & 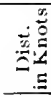 & 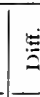 & & 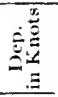 & 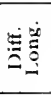 & $\stackrel{ت}{ت}$ & 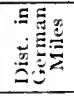 & $\frac{\vec{E}}{\underline{E}}$ & $\stackrel{\leftrightarrow}{\stackrel{0}{2}}$ \\
\hline $\begin{array}{l}\mathrm{B} y \\
\mathrm{R}\end{array}$ & $\begin{array}{c}\text { Dea } \\
\text { ckon }\end{array}$ & & $\begin{array}{l}1 \mathrm{~W} / \mathrm{N} \\
3^{\circ} \mathrm{O} / \mathrm{W}\end{array}$ & 6.1 & & 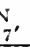 & Wo & $98^{\prime}$ & $52^{\circ} 49^{\prime}$ & $3011 / 2$ & $0^{\circ}{ }_{14}{ }^{\prime} S$ & $33^{\circ} 17^{\prime}$ \\
\hline
\end{tabular}


q September 11, 174I. After Midday

\begin{tabular}{|c|c|c|c|c|c|c|c|c|c|c|c|c|}
\hline$\stackrel{5}{\Xi}$ & 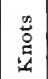 & $\frac{\pi}{3}$ & & 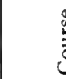 & & 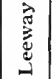 & & & & & & \\
\hline I & 3 & $\mathrm{~S} / \mathrm{I}$ & & $\mathrm{W}$ & & & $\begin{array}{l}\text { Topsail } \\
\text { carrying } \\
\text { staysails }\end{array}$ & $\begin{array}{l}\text { ind, } f \\
\text { eefed }\end{array}$ & $\begin{array}{l}\text { ggy, cl } \\
\text { topsail }\end{array}$ & $\begin{array}{l}\text { ear wit } \\
\text { fores }\end{array}$ & $\begin{array}{l}\text { passing } \\
\text { mains }\end{array}$ & $\begin{array}{l}\text { louds; } \\
1 \text {, and }\end{array}$ \\
\hline 2 & $21 / 2$ & & & & & & & & & & & \\
\hline 3 & 3 & SSI & & W & & & Wind un & teady & and in $g$ & usts. & & \\
\hline 4 & 3 & & & & & & & & & & & \\
\hline 5 & $31 / 2$ & & & & & & Reef-top & ail wir & & & & \\
\hline 6 & $33 / 4$ & & & $\mathrm{~V}$ & & & & & & & & \\
\hline 7 & 3 & $\mathrm{~S} / \mathrm{I}$ & & SW & W & I & & & & & & \\
\hline 8 & $21 / 2$ & $\mathrm{~S}$ & & W & & & Topgalla & tsail & ind, we & & & \\
\hline 9 & $\mathrm{I}^{\mathrm{T}}$ & $\mathrm{S} / \mathrm{H}$ & & SW & W & $\mathrm{I}$ & & & & & & \\
\hline 10 & I & $\mathrm{E} N \mathrm{~N}$ & & $\mathrm{~S}$ & & & Light wi & d, we: & ther as & before. & & \\
\hline II & 2 & $\mathrm{NN}$ & & SW & $\mathrm{W}$ & & Sounded & but $n$ & botton & & & \\
\hline 12 & $21 / 2$ & & & & & & Topsail & ind, $\mathrm{cl}$ & oudy. & & & \\
\hline$I$ & 3 & & & & & & Occasion & $11 y \mathrm{th}$ & stars a & re seten. & & \\
\hline 2 & $31 / 2$ & $\bar{N}$ & & & & & Let out $t$ & vo ree & $\mathrm{s}$ in $\mathrm{ma}$ & n-topsa & & \\
\hline 3 & $3^{3 / 4}$ & & & & & & Drizzly, & et. & & & & \\
\hline 4 & 4 & NW & & & & & & & & & & \\
\hline 5 & 5 & $\mathrm{NN}$ & & W: & & & Wind un & eady. & & & & \\
\hline 6 & $5 \%$ & $\mathrm{~N}$ & & & & & & & & & & \\
\hline 7 & 5 & & & & & & Let out $\mathrm{t}$ & vo ree & $\mathrm{s}$ in for & -topsai & & \\
\hline 8 & 5 & & & & & & & & & & & \\
\hline 9 & 5 & & & & & & Strong re & f-tops & iil wind & & & \\
\hline 10 & 5 & & & & & & & & & & & \\
\hline II & 5 & NN & & & & & Drizzly. & & & & & \\
\hline 12 & 5 & & & & & & Wet. & & & & & \\
\hline \multirow{2}{*}{\multicolumn{3}{|c|}{$\begin{array}{c}\text { Variation } \\
\text { of } \\
\text { Compass } \\
\text { I } 4 \text { rlumb E }\end{array}$}} & \multicolumn{7}{|c|}{ In 24 hours } & \multicolumn{3}{|c|}{ From Vaua } \\
\hline & & & & 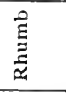 & 苔范 & i & 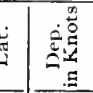 & 両号 & 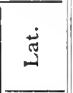 & 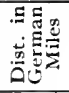 & $\frac{\vec{E}}{\underline{E}}$ & $\stackrel{0}{\stackrel{0}{0}}$ \\
\hline \multicolumn{3}{|c|}{$\begin{array}{l}\text { By Dead } \\
\text { Reckoning }\end{array}$} & & $\begin{array}{l}\mathrm{W} / \mathrm{S} \\
13^{\prime} \mathrm{W}\end{array}$ & 83.7 & $\begin{array}{r}S \\
8.8\end{array}$ & $\begin{array}{r}W \\
83\end{array}$ & $140^{\prime}$ & $\mid 52^{\circ}{ }_{4} 0^{\prime}$ & $283 \%$ & $0^{\circ} \mathrm{E}$ & $30^{\circ} 57^{\prime}$ \\
\hline
\end{tabular}


Ђ September 12, 1741. After Midday

\begin{tabular}{|c|c|c|c|c|c|c|c|c|c|c|c|c|}
\hline$\underset{3}{3}$ & 号 & $\stackrel{0}{3}$ & & 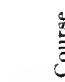 & & 彥 & & & & & & \\
\hline 1 & $\overline{34}$ & $\mathbf{N}$ & & WS & & I & $\begin{array}{l}\text { Topsail } \\
\text { staysails }\end{array}$ & ind, & rizzly, & $\begin{array}{l}\text { wet; c } \\
\text { sail, an }\end{array}$ & $\begin{array}{l}\text { rying to } \\
\text { trysail. }\end{array}$ & sails, \\
\hline 2 & $\overline{35}$ & & & & & I & & & & & & \\
\hline 3 & 3 & NNI & & & & 1 & & & & & & \\
\hline 4 & $\begin{array}{ll}132 \\
\end{array}$ & $\mathrm{NW}$ & & SW & $\mathrm{W}$ & 1 & Northea & erly : & vel1. & & & \\
\hline 5 & 3 & NNV & & WS & & 1 & & & & & & \\
\hline 6 & $2 \%$ & & & & & 1 & Topgalla & tsail & ind, elo & udy. & & \\
\hline 7 & 136 & & & & & 1 & & & & & & \\
\hline 8 & $I$ & & & & & & Light wi & 1, we & her as & before. & & \\
\hline 9 & $\sqrt{3}$ & & & & & & Northerl & swell & & & & \\
\hline IO & & Caln & & & & & Cloudy. & & & & & \\
\hline 11 & $71^{12}$ & IV : & & $\mathrm{S}^{1}$ & & 1 & Took tw & reef $\mathrm{s}$ & n topsa & ils. & & \\
\hline 12 & 2 & & & & & I & & & & & & \\
\hline$I$ & $21 / 2$ & & & $S$ & & 1 & & & & & & \\
\hline 2 & 2 & & & & & $I$ & Weather & $s$ bef & re, moo & $\mathrm{n}$ and $\mathrm{s}$ & s out. & \\
\hline 3 & $13 / 2$ & WSI & & 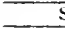 & & 1 & & & & & & \\
\hline 4 & 2 & W & & SS & & $x$ & Heavy $\mathrm{cl}$ & uds f & m the & west. & & \\
\hline 5 & 2 & & & & & 1 & & & & & & \\
\hline 6 & 2 & $\mathbf{W} / \mathrm{s}$ & & $\mathrm{S}$ & & $\mathrm{I}$ & Topgalla & tsail & ind. & & & \\
\hline 7 & $I^{3}+4$ & & & $\mathrm{~S}^{\mathrm{I}}$ & & I & Changea & e win & 1. cloud & & & \\
\hline 8 & 23 & WSI & & Si & & $\mathrm{I}$ & Clear wi & pass & ig clou & & & \\
\hline 9 & $11 / 2$ & $\mathrm{SIV} /$ & & NW & $2 \mathrm{~W}$ & 1 & let out & cond & reef in & topsails & tacked $t$ & port. \\
\hline Io & 2 & & & & & 1 & & & & & & \\
\hline I I & 2 & & & & & 1 & Topgalla & tsail & ind. & & & \\
\hline 12 & 2 & IVSV & & $\mathrm{N}$ & & 1 & Cloudy, & & & & & \\
\hline \multirow{2}{*}{\multicolumn{3}{|c|}{$\begin{array}{l}\text { Variation } \\
\text { of } \\
\text { Compass } \\
13 \text { rhumb E }\end{array}$}} & \multicolumn{7}{|c|}{ In 2.4 hours } & \multicolumn{3}{|c|}{ From Vaua } \\
\hline & & & & $\underset{\Xi}{\vec{\Xi}}$ & 总兽 & 幽 & 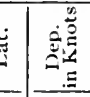 & 需总 & ঙํ & 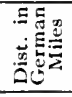 & 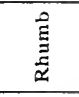 & 离 \\
\hline \multicolumn{3}{|c|}{$\begin{array}{l}\text { By Dead } \\
\text { Reckoning }\end{array}$} & & $\begin{array}{l}\mathrm{w} / \mathrm{w} \\
06^{\prime} \mathrm{W}\end{array}$ & $31 \%$ & $\underset{16}{S}$ & $\begin{array}{l}W \\
27\end{array}$ & $.44^{\prime}$ & $52^{\circ} 24^{\prime}$ & $276 \frac{1}{4}$ & $1^{\circ} \frac{\mathrm{E}}{34^{\prime} \mathrm{S}}$ & $30^{\circ} 13^{\prime}$ \\
\hline
\end{tabular}


September 13, I74I. After Midday

\begin{tabular}{|c|c|c|c|c|c|c|c|c|c|c|c|c|}
\hline 号 & 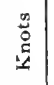 & \multicolumn{2}{|l|}{$\stackrel{\Xi}{E}$} & \multicolumn{2}{|c|}{$\stackrel{\mathscr{n}}{\stackrel{\mathscr{n}}{5}}$} & لِّ & & & & & & \\
\hline $\mathrm{I}$ & I & \multicolumn{2}{|c|}{$\mathrm{SW} / \mathrm{W}$} & \multicolumn{2}{|c|}{ NW $1 / 2 W$} & $11 / 2$ & \multicolumn{6}{|c|}{$\begin{array}{l}\text { Topgallantsail wind, cloudy, wet; came about } \\
\text { on the starboard tack. }\end{array}$} \\
\hline 2 & $I$ & \multicolumn{2}{|l|}{$\mathrm{W}$} & \multicolumn{2}{|c|}{ SSW } & $11 / 2$ & \\
\hline 3 & 1.4 & \multirow{2}{*}{\multicolumn{2}{|c|}{ 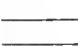 }} & & & 3 & & & & & & \\
\hline 4 & 14 & & & & & 3 & \multicolumn{6}{|c|}{ Drizzly, wet. } \\
\hline 5 & 1 & \multirow{2}{*}{\multicolumn{2}{|c|}{$\mathrm{SW} / \mathrm{W}$}} & \multicolumn{2}{|c|}{$\mathrm{S} / \mathrm{E}$} & $I 1 / 2$ & \multicolumn{6}{|c|}{ Light wind. } \\
\hline 6 & $23 \frac{4}{4}$ & & & \multicolumn{2}{|c|}{$\mathrm{S} / \mathrm{E} / 2 / 2 \mathrm{E}$} & I & & & & & & \\
\hline 7 & $\mathrm{I}$ & \multicolumn{2}{|c|}{ WSW } & \multirow{2}{*}{\multicolumn{2}{|c|}{$N W / W 1 / 2 W$}} & $11 / 29$ & \multicolumn{6}{|c|}{ Came about on the port tack. } \\
\hline 8 & $1 / 2$ & \multicolumn{2}{|c|}{$\mathrm{SW} / \mathrm{W}$} & & & & \multicolumn{6}{|c|}{ Cloudy. } \\
\hline 9 & $1 ! 2$ & & & & & $\mathrm{I}$ & \multirow{2}{*}{\multicolumn{6}{|c|}{$\frac{\text { Moon out; took two reefs in topsails. }}{\text { Topgallantsail wind. }}$}} \\
\hline Io & 2 & \multirow{2}{*}{\multicolumn{2}{|c|}{ 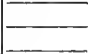 }} & \multirow{2}{*}{\multicolumn{2}{|c|}{$\bar{\square}$}} & $\mathrm{I}$ & \multirow{2}{*}{\multicolumn{5}{|c|}{ Topgallantsail wind. }} & \\
\hline II & $2 \pi / 4$ & & & & & 1 & \\
\hline $\mathrm{I} 2$ & 2 & \multirow{2}{*}{\multicolumn{2}{|c|}{ 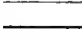 }} & \multicolumn{2}{|c|}{ NW/W } & & & & & & & \\
\hline $\mathrm{I}$ & $11 / 2$ & & & \multicolumn{2}{|c|}{$\mathrm{S} / \mathrm{E}$} & $r 1 / 2 \pi$ & \multicolumn{6}{|c|}{ Made the starboard tack. } \\
\hline 2 & 114 & 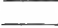 & & $\mathrm{S} / \mathrm{F}$ & $1 / 2 \mathrm{E}$ & $\mathrm{I}$ & & & & & & \\
\hline 3 & $I$ & & & & & 2 & & & & & & \\
\hline 4 & 1 & & & & & 2 & & & & & & \\
\hline 5 & $1 / 2$ & & & WI & & 3 & & & & & & \\
\hline 6 & $1 / 2$ & & & & & 3 & Light wi & & & & & \\
\hline 7 & $3 / 4$ & $\mathrm{~s}$ & & WSV & $\frac{1}{2} \mathrm{~W}$ & I!21 1 & Let out : & cond: & and thir & d reefs & topsails. & \\
\hline 8 & $3 / 4$ & & & & & $I ! / 2$ & & & & & & \\
\hline 9 & $23 / 4$ & $\mathrm{~S} / \mathrm{E}$ & & $\mathrm{W}$ & & & Topgalla & tsail n & vind. & & & \\
\hline 10 & $3 \% 4$ & & & & & & Clear wi & h passi & ing cloud & & & \\
\hline II & 314 & & & & & & & & & & & \\
\hline I2 & $3 \frac{3}{4}$ & & & & & & Topsail & ind, $w$ & eather a & as before & & \\
\hline & & & & & & In 2.4 & 1 hours & & & & om Vaus & \\
\hline $\begin{array}{r}V_{i} \\
\mathrm{C} 1 / 4\end{array}$ & $\begin{array}{l}\text { ariatio } \\
\text { of } \\
\text { ompa } \\
\text { rhum }\end{array}$ & $\begin{array}{l}\text { on } \\
\text { iss } \\
\text { ab E E }\end{array}$ & & 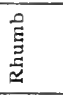 & 焉 & 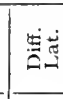 & 离莒 & 电苞 & 苞 & 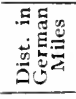 & $\stackrel{0}{E}$ & ف \\
\hline & $\begin{array}{l}\text { y Dea } \\
\text { ckoni } \\
\text { Y ushir } \\
\end{array}$ & & & $\begin{array}{ll}W / S \\
{ }^{2}\end{array}$ & 18.3 & $\begin{array}{l}\mathrm{S} \\
1.5^{\prime}\end{array}$ & $\begin{array}{c}W \\
18\end{array}$ & $28.8^{\prime}$ & $52^{\circ} 23^{\prime}$ & $2721 / 4$ & $\begin{aligned} E \\
1^{\circ} 3^{\prime} S \\
\end{aligned}$ & $29^{\circ} 44^{\prime}$ \\
\hline Obs & $\begin{array}{l}\text { By } \\
\text { ervat } \\
\text { Ehitro }\end{array}$ & ion & & W/S & 20.8 & $\underset{24^{\prime}}{S}$ & $\begin{array}{l}W \\
17.7\end{array}$ & $28.8^{\prime}$ & {$\left[52^{\circ} \mathrm{Or}^{\prime}\right]$} & $271 \frac{14}{4}$ & $2^{\circ} .48^{\prime} \mathrm{S}$ & $20^{\circ} 35^{\prime}$ \\
\hline
\end{tabular}

95 As indicated in the 2.4 -hour summary, Khitrov's journal gives latitude by observa tion $52^{\circ} \mathrm{or}^{\prime}$. 'This value has been used on the chart (Pl. I). 
(1) September I.4, I74I. After Midday

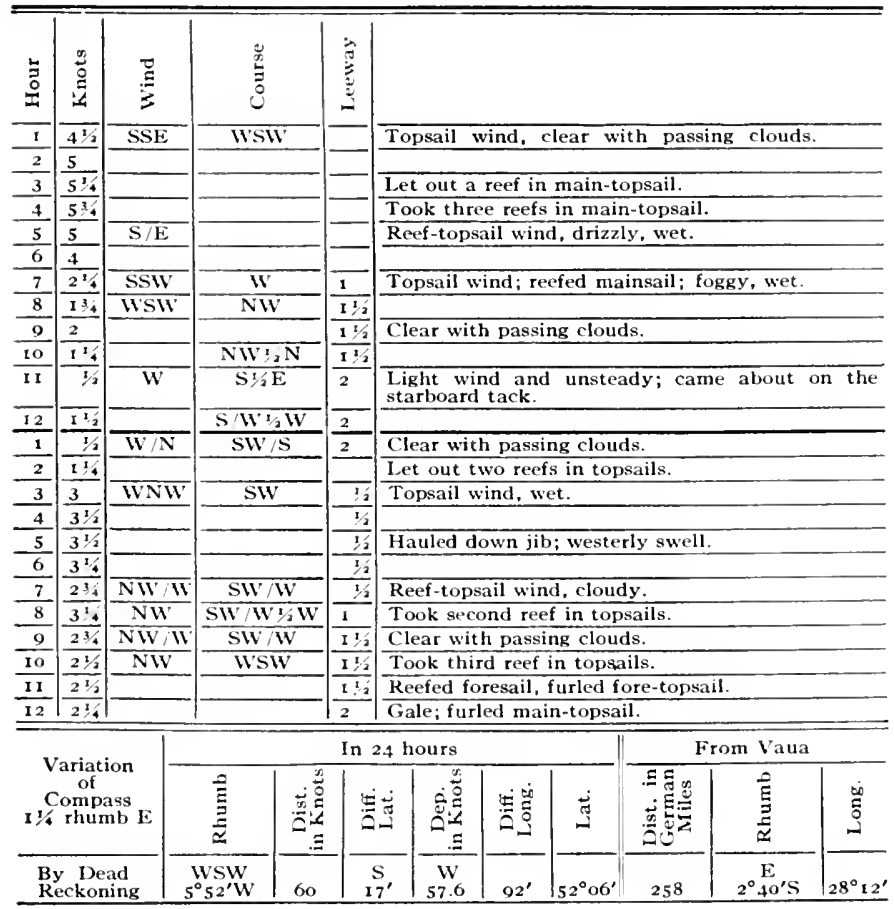




\section{\& September 15, 1741. After Midday}

\begin{tabular}{|c|c|c|c|c|c|c|c|c|c|c|c|c|}
\hline 冚 & 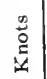 & $\underset{\Xi}{3}$ & \multicolumn{2}{|c|}{ 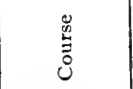 } & 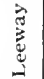 & & & & & & & \\
\hline $\bar{I}$ & 2 & NW & \multicolumn{2}{|c|}{ WSWI/2S } & $\overline{21 / 3}$ & \multicolumn{7}{|c|}{$\begin{array}{l}\text { Reef-undersail wind, clear witl passing clouds; } \\
\text { hauled down staysails; squally; furled foresail, } \\
\text { hove to under mainsail and trysail; coming up } \\
\text { to } W \text {, falling off to SW by W. }\end{array}$} \\
\hline 2 & $2 \frac{x}{2}$ & & & & $\overline{21 / 2}$ & & & & & & & \\
\hline 3 & $\mathrm{II/2}$ & NW/N & \multicolumn{2}{|c|}{ WSW $t / 2 W$} & $21 / 2$ & & & & & & & \\
\hline 4 & $1 \mathrm{I} / 2$ & & & & 5 & & & & & & & \\
\hline 5 & $13 \frac{1}{2}$ & & & & 5 & & & & & & & \\
\hline 6 & $11 / 2$ & & & & 5 & \multicolumn{7}{|c|}{ Lowered the foreyard. } \\
\hline 7 & $1 \mathrm{x} / 4$ & & & & & \multicolumn{7}{|c|}{ Stormy. } \\
\hline 8 & $\overline{\mathrm{I}_{4}^{2}}$ & & & & $\overline{51 / 2}$ & \multicolumn{7}{|c|}{ Furled main-staysail. } \\
\hline 9 & $\overline{114}$ & & & & $51 / 2$ & & & & & & & \\
\hline I0 & $\overline{1+4}$ & & & & $51 / 2$ & \multicolumn{7}{|c|}{ Clear with passing clouds. } \\
\hline II & $\overline{114}$ & & & & $51 / 2$ & \multicolumn{7}{|c|}{ At times light rain. } \\
\hline 12 & $\overline{\mathrm{I}_{4}^{4}}$ & & & & & & & & & & & \\
\hline $\mathbf{I}$ & $\mathbf{I}$ & & & & & \multicolumn{7}{|c|}{ Wind decreasing. } \\
\hline 2 & 1 & & & & 6 & & & & & & & \\
\hline 3 & $\mathbf{I}$ & & & & 6 & \multicolumn{7}{|c|}{ Stars out. } \\
\hline 4 & $\mathrm{I}$ & & & & 6 & \multicolumn{7}{|c|}{ Chilly, damp. } \\
\hline 5 & $13 / 2$ & & & & & \multicolumn{7}{|c|}{ Undersail wind; hauled up foreyards. } \\
\hline 6 & $\overline{3 / 4}$ & NW & WS & & 3 & \multicolumn{7}{|c|}{ Set foresail and mainsail. } \\
\hline 7 & $11 / 2$ & & & & 3 & & & & & & & \\
\hline 8 & $\frac{1 / 2}{2}$ & & & & 3 & \multicolumn{7}{|c|}{ Sun shining. } \\
\hline 9 & 2 & & WSV & $1 / 2 \mathrm{~S}$ & $21 / 2$ & \multicolumn{7}{|c|}{$\begin{array}{l}\text { Topsail wind, clondy; set main-topsail and let } \\
\text { out two reefs. }\end{array}$} \\
\hline Io & $\overline{I T / 2}$ & & & & $21 / 2$ & \multicolumn{7}{|c|}{ Set fore-topsail reefed. } \\
\hline II & 2 & & Ws & & 2 & & & & & & & \\
\hline 12 & $2 ! / 2$ & & & & 2 & \multicolumn{7}{|c|}{$\begin{array}{l}\text { Northwesterly swell. Took an observation: zenith } \\
\text { distance } 52^{\circ} 20^{\prime} \text {. declination of the sun } 1^{\circ} 06^{\prime} \text {, } \\
\text { latitude } 51^{\circ} 14^{\prime} \text {. }\end{array}$} \\
\hline \multirow{2}{*}{\multicolumn{3}{|c|}{$\begin{array}{l}\text { Variation } \\
\text { of } \\
\text { Compass } \\
\text { I } 4 \text { rhumb E }\end{array}$}} & \multicolumn{10}{|c|}{ In 24 hours } \\
\hline & & & 胥 & $\stackrel{\infty}{\infty} \stackrel{\infty}{0}$ & $\dddot{g}$ & & 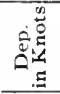 & 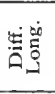 & 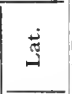 & 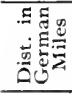 & $\stackrel{\rho}{\stackrel{E}{z}}$ & $\stackrel{\circ}{\stackrel{0}{0}}$ \\
\hline $\mathrm{Ob}$ & $\begin{array}{l}\text { By } \\
\text { serva }\end{array}$ & ation & $\operatorname{SSWV}_{28}$ & 56 & & & $\begin{array}{l}\text { WV } \\
20\end{array}$ & $32^{\prime}$ & $51^{\circ} 14^{\prime}$ & $2561 / 4$ & $\begin{array}{c}\mathrm{E} \\
5^{\circ} 36^{\prime} \mathrm{S}\end{array}$ & $27^{\circ} 40^{\prime}$ \\
\hline
\end{tabular}


\઼ September 16, 1741. After Midday

\begin{tabular}{|c|c|c|c|c|c|c|c|c|c|c|c|c|c|}
\hline \multirow{2}{*}{$\frac{\stackrel{\Xi}{\Xi}}{1}$} & \multirow{2}{*}{ 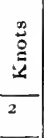 } & $\frac{\bar{E}}{3}$ & & \multicolumn{2}{|c|}{$\stackrel{\stackrel{D}{Z}}{3}$} & \multicolumn{2}{|l|}{ 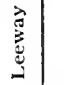 } & & & & & & \\
\hline & & WNV & & \multicolumn{2}{|c|}{$\mathrm{SW} / 3 \mathrm{~W}$} & $13 / 2$ & & \multicolumn{6}{|c|}{$\begin{array}{l}\text { Topsail wind, clear with passing clouds; let out } \\
\text { a reef in fore-topsail. }\end{array}$} \\
\hline 2 & $21 / 2$ & & & \multirow{2}{*}{\multicolumn{2}{|c|}{$\overline{\mathrm{SWV}}$}} & $11 / 2$ & & & & & & & \\
\hline 3 & 2 & & & & & $1 \mathrm{I} / 2$ & \multicolumn{7}{|c|}{$\begin{array}{l}\text { Sea running from NW; carrying topsails, stay- } \\
\text { sails, foresail, mainsail, and trysail. }\end{array}$} \\
\hline 4 & $21 / 2$ & & & & & $1 \frac{1 / 2}{2}$ & & & & & & & \\
\hline 5 & 134 & & & & & $13 / 2$ & \multicolumn{7}{|c|}{ Topgallantsail wind, weather as before. } \\
\hline 6 & 13 & & & & & $12 / 2$ & \\
\hline 7 & $13 / 2$ & W' & & \multicolumn{2}{|c|}{ SSW } & $18 / 2$ & \\
\hline 8 & $11 / 2$ & IVSW & & \multicolumn{2}{|c|}{$\mathbf{S}$} & $I \frac{1}{2}$ & \multicolumn{7}{|c|}{ Took three reefs in fore-topsail. } \\
\hline 9 & $I$ & & & \multicolumn{2}{|c|}{ NV } & & \multicolumn{7}{|c|}{ Light wind, made the port tack. } \\
\hline I0 & $11 / 2$ & SW & & \multirow{2}{*}{\multicolumn{2}{|c|}{$\frac{\text { WNW }}{\text { WI/2N }}$}} & 2 & \multicolumn{7}{|c|}{ Drizzly, clear. } \\
\hline II & 214 & $\mathrm{SW} / \mathrm{s}$ & & & & & & & & & & & \\
\hline 12 & $1 \mathrm{I}^{1}$ & & & & 11.2 & & & & & & & \\
\hline 1 & $I 1 / 2$ & SSE & & \multicolumn{2}{|c|}{$\mathrm{W} / \mathrm{S}$} & & \multicolumn{7}{|c|}{ Let out two reefs in iore-topsail. } \\
\hline 2 & 2 & & & & & & & & & & & & \\
\hline 3 & $3 ! / 3$ & ESE & & & & & \multicolumn{5}{|c|}{ Let out a reef in main-topsail. } & & \\
\hline 4 & $41 / 3$ & & & & & & \multicolumn{7}{|c|}{ Topsail wind. drizzly. } \\
\hline \multicolumn{13}{|c|}{$41 / 2$} & \\
\hline 6 & 434 & $\mathrm{SE}$ & & & & & & & & & & & \\
\hline 7 & $5: 4$ & & & & & & \multicolumn{7}{|c|}{ Reef-topsail wind, weather as before. ${ }^{96}$} \\
\hline 8 & 534 & $\mathrm{SE}:$ & & & & & & out : & reef is & foresai & set jil & & \\
\hline 9 & $5 \%$ & SSE & & & & & & & & & & & \\
\hline 10 & $3 \frac{2}{2}$ & SW & & & & & & psail y & ind. & & & & \\
\hline 11 & $3^{34}$ & $\mathrm{SW}$ & & & & $1 / 2$ & & ok ree & in $\mathrm{ma}$ & in-tops: & & & \\
\hline 12 & $4^{3 / 2}$ & SWI & & WN & $8 / 2 \mathrm{~N}$ & & & ef-top & ail wir & d, drizz & & & \\
\hline & & & & & & & 24 & nours & & & & m V'aua & \\
\hline C & $\begin{array}{l}\text { ariat } \\
\text { of } \\
\text { ompa } \\
\text { rhun }\end{array}$ & iss & & $\begin{array}{l}\vec{E} \\
\vec{\Xi}\end{array}$ & 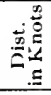 & בְ & & 窇: & 至莕 & تُ & 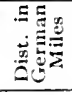 & $\stackrel{\varrho}{\Xi}$ & 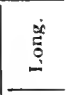 \\
\hline & $\begin{array}{l}\text { Dea } \\
\text { ckon }\end{array}$ & & & iv' & 60 & 0 & $a^{\prime}$ & WV & $96^{\prime}$ & $51^{\circ} 14^{\prime}$ & $2.411 / 4$ & $5^{\circ} 57^{\prime} \mathrm{S}$ & $26^{\circ} 04^{\prime}$ \\
\hline
\end{tabular}

96 Khitrov's journal: "On sick list: . . . 12 men." 


\section{September 17, I74I. After Midday}

\begin{tabular}{|c|c|c|c|c|c|c|c|c|c|c|c|c|}
\hline$\stackrel{\vdots}{\Xi}$ & 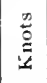 & $\ddot{\Xi}$ & \multicolumn{2}{|c|}{ 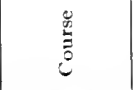 } & 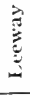 & & & & & & & \\
\hline 1 & 4 & SW & \multicolumn{2}{|c|}{ WNWI/2N } & I & \multicolumn{7}{|c|}{ Reef-topsail wind, drizzly, rain, } \\
\hline 2 & $33^{3}$ & $\mathrm{SW}, \mathrm{W}$ & \multicolumn{2}{|c|}{ NW/W } & $\mathrm{I}$ & \multicolumn{7}{|c|}{ Took second and third reef in topsails. } \\
\hline 3 & 3 & WSV & \multicolumn{2}{|c|}{ NW } & I & \multicolumn{7}{|c|}{ Hauled down jib. } \\
\hline 4 & $3 \frac{3}{4}$ & SW & \multicolumn{2}{|c|}{ WNW/2N } & 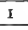 & \multicolumn{7}{|c|}{ Let out reef in topsails. } \\
\hline 5 & $3 ! 4$ & & \multicolumn{2}{|c|}{ WNW } & $I$ & \\
\hline 6 & 4 & SW $/ \mathrm{S}$ & \multicolumn{2}{|c|}{$\mathrm{W} / \mathrm{N}$} & I & \multicolumn{7}{|c|}{ 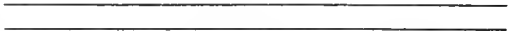 } \\
\hline 7 & $31 / 2$ & SIV & \multicolumn{2}{|c|}{ IVNW } & $\mathbf{I}$ & \multicolumn{7}{|c|}{ Took third reef in topsails and in foresail. } \\
\hline 8 & $33 / 4$ & & & & $\mathbf{I}$ & & & & & & & \\
\hline 9 & $31 / 2$ & $\mathrm{SW} / \mathrm{I}$ & \multicolumn{2}{|c|}{$\mathrm{NW} / \mathrm{W}$} & $I$ & \multicolumn{7}{|c|}{$\begin{array}{l}\text { Reef-topsail wind, drizzly, clear with passing } \\
\text { showers. }\end{array}$} \\
\hline Io & 4 & & & & $\mathbf{I}$ & & & & & & & \\
\hline II & $33^{3 \cdot 4}$ & & \multicolumn{2}{|c|}{ NW/W $/ 2 \mathrm{~W}$} & I & \multicolumn{7}{|c|}{ Wind comes in gusts. } \\
\hline 12 & $21 / 2$ & W'SI & \multicolumn{2}{|c|}{$\mathrm{NW}$} & I & \multicolumn{7}{|c|}{ Rain, southwesterly swell; tacked to starboard. } \\
\hline $\mathbf{r}$ & $23 / 4$ & & \multicolumn{2}{|c|}{$\mathrm{S}$} & 2 & \multicolumn{7}{|c|}{ 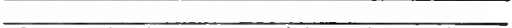 } \\
\hline 2 & $21 / 4$ & $W / \mathrm{S}$ & $\mathrm{Si}$ & & 2 & & avy $r$ & in: le & out a & reef in & main-tops & \\
\hline 3 & $11 / 2$ & W & SS & & 2 & & 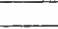 & (2) & 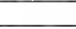 & 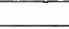 & +2 & \\
\hline 4 & 13/4 & $\mathrm{W} / \mathrm{N}$ & SSW & $\mathrm{W}$ & 2 & & psail & ind; 1 & $t$ out $t$ & so reef & in main-ts & psail. \\
\hline 5 & $11 / 2$ & & & & 2 & & & & & & & \\
\hline 6 & $I 1 / 2$ & & & & 2 & & out a & reef i & fore-to & psail. & & \\
\hline 7 & 2 & & SII & & 2 & & pgalla & tsail & rind, clo & udy. & & \\
\hline 8 & $I 2 / 2$ & & & & 2 & & avy sc & , ligh & rain. & & & \\
\hline 9 & 2 & W & SS & & 2 & & $\begin{array}{l}\text { tout } \\
\text { e-tops }\end{array}$ & $\begin{array}{l}\text { hird } \mathrm{r} \\
\text { il. }\end{array}$ & eef in $m$ & ain-to & ail and se & ond in \\
\hline Io & $\mathrm{I}^{1} 4$ & & & & 2 & & & & & & & \\
\hline II & $\overline{2: 4}$ & $W / N$ & SV & & 2 & & $\begin{array}{l}\text { ind } \mathrm{fr} \\
\text { in-tor } \\
\text { osail. }\end{array}$ & $\begin{array}{l}\text { shenir } \\
\text { ails }\end{array}$ & $\begin{array}{l}\text { g; took } \\
\text { nd seco }\end{array}$ & $\begin{array}{l}\text { first ar } \\
\text { ad and }\end{array}$ & $\begin{array}{l}\text { second } \\
\text { hird reefs }\end{array}$ & $\begin{array}{l}\text { eefs in } \\
n \text { fore- }\end{array}$ \\
\hline 12 & $I^{3 / 4}$ & W & SSW & $2 \mathrm{VV}$ & 2 & & & & & & & \\
\hline & & & & & & & ours & & & & rom Vaua & \\
\hline r & $\begin{array}{l}\text { ariat } \\
\text { of } \\
\text { ornp } \\
\text { rhur }\end{array}$ & ass & $\stackrel{0}{\Xi}$ & 놀 & & & 돌 & 进是 & $\stackrel{\check{3}}{-}$ & 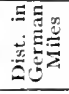 & $\underline{\underline{\Xi}}$ & 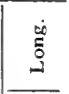 \\
\hline$\underset{\mathrm{B}}{\mathrm{B}}$ & $\begin{array}{l}\text { ye } \\
\text { eckon }\end{array}$ & & $\begin{array}{l}\text { WNW } \\
{ }^{\circ}{ }^{\prime} N\end{array}$ & 33 & & $\begin{array}{l}\text { N } \\
\text { i }\end{array}$ & $\begin{array}{l}\mathrm{W} \\
30\end{array}$ & $48^{\prime}$ & $51^{\circ} 27^{\prime}$ & $233: 4$ & $5^{\circ} \frac{\mathrm{E}}{2 I^{\prime} \mathrm{S}}$ & $25^{\circ} \times 6^{\prime}$ \\
\hline
\end{tabular}


O Seplember 18, 1741. Afler Midday

\begin{tabular}{|c|c|c|c|c|c|c|c|c|c|c|c|}
\hline$\stackrel{5}{\varrho}$ & $\frac{n}{0}$ & \multicolumn{2}{|c|}{$\underset{\Xi}{\Xi}$} & \multicolumn{2}{|c|}{ 苋 } & 离 & & & & & \\
\hline $\mathbf{I}$ & 2 & \multicolumn{2}{|c|}{ WNW } & \multicolumn{2}{|c|}{ SW } & $\sqrt{13} \cdot 4$ & \multicolumn{5}{|c|}{$\begin{array}{l}\text { Strong reef-topsail wind, westerly swell; carry- } \\
\text { ing reefed topsails, foresail, mainsaif, trysail, } \\
\text { and staysails. }\end{array}$} \\
\hline 2 & $13 / 4$ & & & & & $13 \%$ & & & & & \\
\hline 3 & $22^{2}$ & & & & & $13 / 4$ & & & & & \\
\hline 4 & $21 / 4$ & \multicolumn{2}{|c|}{$\mathrm{NW/W}$} & \multicolumn{2}{|c|}{$\mathrm{SW} / \mathrm{W}$} & $1 \%$ & \multicolumn{5}{|c|}{ Took three reefs in topsails. } \\
\hline 5 & 314 & \multirow{2}{*}{\multicolumn{2}{|c|}{$\mathrm{NW}$}} & \multirow{2}{*}{\multicolumn{2}{|c|}{ WSW }} & $11 / 2$ & & & & & \\
\hline 6 & $23 / 4$ & & & & & $13 / 2$ & \\
\hline 7 & 2 & & & \multicolumn{2}{|c|}{$11514: 2 \mathrm{~S}$} & 13 & \multicolumn{5}{|c|}{ Haufed down fore-topmast-stay'sail. } \\
\hline 8 & 2 & & & & & $2 \frac{1}{4 / 4}$ & \multicolumn{5}{|c|}{ Gale, heavy passing clouds. } \\
\hline 9 & $11 / 2$ & & & & & 23 & \multicolumn{5}{|c|}{ Wet; furled fore-topsail. } \\
\hline Io & $\mathrm{I3} / 4$ & & & & & $2 \$ 4$ & \multicolumn{5}{|c|}{ Wind decreasing, air chilly. } \\
\hline 11 & 13 & \multicolumn{2}{|c|}{$\mathrm{NW} / \mathrm{W}$} & \multicolumn{2}{|c|}{$S W W$} & $22^{2}$ & \multicolumn{5}{|c|}{ Hauled up fore-topmast-staysail. } \\
\hline 12 & $13 / 4$ & & & & & $22_{4}^{4}$ & & & & & \\
\hline $\mathbf{I}$ & $13 / 4$ & \multicolumn{2}{|c|}{ WNW } & \multicolumn{2}{|c|}{ SW } & 214 & \multicolumn{5}{|c|}{ Set fore-topsail. } \\
\hline 2 & $13 / 4$ & \multicolumn{2}{|c|}{$\mathrm{W} / \mathrm{N}$} & \multicolumn{2}{|c|}{$\mathrm{sW} s$} & I3:4 & & & & & \\
\hline 3 & 2 & \multirow{2}{*}{\multicolumn{2}{|c|}{ W }} & & & $1+3 / 4$ & \multicolumn{5}{|c|}{ Topsail wind. } \\
\hline 4 & 2 & & & \multicolumn{2}{|c|}{ SSW! W } & 13,4 & & & & & \\
\hline 5 & $21 / 2$ & & & SS & & $13 / 4$ & \multicolumn{5}{|c|}{ Let out a reef in main-topsail. } \\
\hline 6 & 2 & \multicolumn{2}{|c|}{$\mathrm{W} / \mathrm{S}$} & $\mathrm{S}$ & & 133 & At the en & of $t$ & hour we mad & he port & ck. \\
\hline 7 & 2 & WS & & $\mathrm{NW}$ & $2 \mathrm{~N}$ & $11 / 2$ & & & & & \\
\hline 8 & $22^{14}$ & $\mathrm{~W}^{*}$ & & $\mathrm{NW}$ & $\mathrm{N}$ & $11 / 2$ & Topsail & nd. & & & \\
\hline 9 & $31 / 2$ & & & & & $11 / 2$ & 97 & & & & \\
\hline ro & $31 / 2$ & WS & & $\mathrm{NW}$ & $2 \mathrm{~N}$ & $11 / 2$ & Reef-top & ill wi & & & \\
\hline I I & $31 / 2$ & & & & & I1/2 & Drizzly. & & & & \\
\hline 12 & 3 & & & NI & & $11 / 21$ & Wind co: & es in & usts. & & \\
\hline & & & & & & In 24 & hours & & & m Vau & \\
\hline C & $\begin{array}{l}\text { of } \\
\text { of } \\
\text { ompa } \\
\text { rhum }\end{array}$ & ss & & $\underset{\Xi}{\Xi}$ & . & 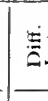 & . & 题 & ن & $\stackrel{\stackrel{\Xi}{\Xi}}{\Xi}$ & $\stackrel{\infty}{:}$ \\
\hline $\begin{array}{l}\mathrm{By} \\
\mathrm{R} \\
\end{array}$ & $\begin{array}{l}\text { Dea } \\
\text { ckoni }\end{array}$ & & & $\begin{array}{l}\text { iste } \\
\text { ig }\end{array}$ & 27 & Io & $\begin{array}{c}W \\
25.6\end{array}$ & $41^{\prime}$ & $51^{\circ} 17^{\prime} \quad 227 \%$ & $\begin{array}{c}E \\
6^{\circ} 07^{\prime} S\end{array}$ & $24^{\circ} 35^{\prime}$ \\
\hline
\end{tabular}

97 Khitrov's journal: "On sick list: I4 men". 
b) September 19, 174I. After Midday

\begin{tabular}{|c|c|c|c|c|c|c|c|c|c|c|c|}
\hline$\stackrel{气}{0}$ & 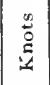 & $\stackrel{Z}{\Xi}$ & 崩 & & 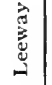 & & & & & & \\
\hline $\mathbf{I}$ & $\sqrt{3 \% 4}$ & WSW & NI & & 2 & $\begin{array}{l}\text { Recf-top } \\
\text { in main-t }\end{array}$ & $\begin{array}{l}\text { sail win } \\
\text { opsail. }\end{array}$ & d, squall & $y$, drizzl & took thr & reefs \\
\hline 2 & 2 & & NW & $12 \mathrm{~N}$ & 2 & & & & & & \\
\hline 3 & $21 / 3$ & & & & 2 & Squally; & furled & topsails & beavy & vesterly s & vell. \\
\hline 4 & $\mathrm{I}^{\mathrm{x}}$ & $\mathrm{W} / \mathrm{s}$ & NIV & & 3 & & & & & & \\
\hline 5 & $11 / 2$ & $W / N$ & SW & & 3 & $\begin{array}{l}\text { Topsail } \\
\text { board tac }\end{array}$ & $\begin{array}{l}\text { vind an } \\
\text { : } \text {, set }\end{array}$ & $\begin{array}{l}\text { d unstea } \\
\text { opsails }\end{array}$ & $\begin{array}{l}\text { ady: car } \\
\text { reefed. }\end{array}$ & e about o & star- \\
\hline 6 & $11 / 2$ & WNV & SV & & 3 & & & & & & \\
\hline 7 & $I \%$ & & SW & & 3 & Gale, clo & udy. & & & & \\
\hline 8 & $1 \%$ & $W / N$ & SW & & 3 & & & & & & \\
\hline 9 & $3 / 4$ & WV & SS & & 6 & $\begin{array}{l}\text { Furled to } \\
\text { trysail ar } \\
\text { falling of } \\
\end{array}$ & $\begin{array}{l}\text { Issails } \\
\text { id mais } \\
\text { to } 5\end{array}$ & $\begin{array}{l}\text { and low } \\
\text {-staysai } \\
\text { y W. }\end{array}$ & $\begin{array}{l}\text { ered sai } \\
1 \text {; comin }\end{array}$ & $\begin{array}{l}\text { hove to } \\
\text { up to SV }\end{array}$ & $\begin{array}{l}\text { under } \\
\text { by } \mathrm{S} \text {. }\end{array}$ \\
\hline Io & 1 & & & & 6 & & & & & & \\
\hline II & $\mathrm{I}$ & & & & 6 & & & & & & \\
\hline 12 & 1 & $\mathrm{~W} / \mathrm{N}$ & SW & & & Coming & ip to $S$ & W, fallir & ng ofi to & SIV. & \\
\hline $\mathrm{I}$ & $t$ & WNV & SW & & 6 & Coming 1 & $1 \mathrm{ptov}$ & $\mathrm{sW}, \mathrm{fal}$ & lling off & SW by & \\
\hline 2 & $\mathrm{I}$ & & & & & Clear wit & $\mathrm{h}$ pass & $\mathrm{ng}$ clond & & & \\
\hline 3 & $I$ & NW & SW/W & $13 \mathrm{~W}$ & 6 & & & & & & \\
\hline 4 & $\mathrm{I}$ & & & & 6 & Coming & p to $\mathrm{V}$ & by $\mathrm{S}, \mathrm{f}$ & falling of & to SW & \\
\hline 5 & $I$ & & & & 6 & Reef-tops & ail win & d. cloud & & & \\
\hline 6 & $1 \%$ & & WS & & 2 & Set fores: & iil and & mainsail & & & \\
\hline 7 & $2 \%$ & & & & $\mathrm{IT/2}$ & Set topsa & ils and & let out & a reef in & hein. & \\
\hline 8 & $21 / 2$ & & & & 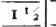 & & & & & & \\
\hline 9 & $21 / 2$ & NW/1 & W & & $11 / 2$ & Reef-tops & ail wir & d. cloud & & & \\
\hline Io & $3^{1 / 4}$ & NIV & WS & & $\overline{1 ! 4}$ & & & & & & \\
\hline II & $3 \frac{112}{2}$ & & & & $\overline{I_{4}}$ & Clear wit & $\mathrm{h}$ passi & ng clond & & & \\
\hline 12 & 3 & & & & 13 & & & & & & \\
\hline \multirow{2}{*}{\multicolumn{3}{|c|}{$\begin{array}{c}\text { Variation } \\
\text { of } \\
\text { Compass } \\
11 \text { rhumb E }\end{array}$}} & \multicolumn{6}{|c|}{ In 24 hours } & \multicolumn{3}{|c|}{ From Vaua } \\
\hline & & & है & 送 & 管: & 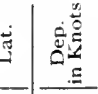 & 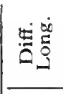 & $\stackrel{\text { 乌ึ }}{3}$ & 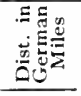 & है & $\stackrel{\infty}{0}$ \\
\hline \multicolumn{3}{|c|}{$\begin{array}{l}\text { By Dead } \\
\text { Reckoning }\end{array}$} & $\begin{array}{l}\mathrm{SW} / \mathrm{W} \\
\mathrm{O}^{\circ} \mathrm{O}^{\prime} \mathrm{W}\end{array}$ & 21.5 & $\begin{array}{r}\mathrm{S} \\
12\end{array}$ & $\begin{array}{l}w \\
18 \\
\end{array}$ & $28^{\prime}$ & $51^{\circ} \circ 5^{\prime \prime}$ & $2243 / 4$ & $6^{\circ}{ }_{58}^{\mathrm{E}} \mathrm{S}$ & $24^{\circ} 07^{\prime}$ \\
\hline
\end{tabular}


Seplember 20, I74I. Afler Midday

\begin{tabular}{|c|c|c|c|c|c|c|c|c|c|c|c|c|c|}
\hline$\stackrel{气}{=}$ & 离 & $\stackrel{\vec{E}}{E}$ & & 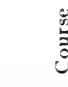 & & 产 & & & & & & & \\
\hline$I$ & 3 & NIV & & W's & & $11 / 2$ & & psail w & ind, $\mathrm{cl}$ & ondy. & & & \\
\hline 2 & $23_{4}$ & & & & & $11 / 2$ & & out $t$ & vo ree & $s$ in top & sails. & & \\
\hline 3 & 3 & NW & & sil & & $11 / 2$ & & $\begin{array}{l}\text { the o } \\
\text { re shif } \\
\text { provisi } \\
\text { ticient }\end{array}$ & $\begin{array}{l}\text { der o } \\
\text { ed fro } \\
\text { ons an } \\
y \text { dow }\end{array}$ & $\begin{array}{l}\text { the c } \\
n \text { the ho } \\
3 \text { jack } \\
n \text { in th }\end{array}$ & $\begin{array}{l}\text { aptain } \\
\text { ld aft to } \\
\text { s because } \\
\text { e water. }\end{array}$ & $\begin{array}{l}\text { mmander } \\
\text { the bow } 31 \\
\text { the bow w }\end{array}$ & $\begin{array}{l}\text { r there } \\
\text { I sacks } \\
\text { vas not } \\
\end{array}$ \\
\hline 4 & 3 & & & & & $11 / 2$ & & & & & & & \\
\hline 5 & 294 & & & & & $11 / 2$ & & & & & & & \\
\hline 6 & $3^{14}$ & & & & & $11 / 2$ & & & & & & & \\
\hline 7 & $21 / 2$ & NW & & IVSW & $1 / 2 \mathrm{~S}$ & $1 / 2$ & & ists of & vind a & nd rain. & & & \\
\hline 8 & 2 & & & & & $11 / 2$ & & $\begin{array}{l}\text { pgallar } \\
\text { west. }\end{array}$ & tsail & vind, $h$ & avy pas & ing clouds & 8 from \\
\hline 9 & 3 & NW/ & & SIV & & $\overline{11 / 2}$ & & ok two & reefs & in topsa & & & \\
\hline 10 & 3 & NIV & & WSW & $1 / 2 \mathrm{~S}$ & $I 1 / 3$ & & ght tof & sail w & nd. & & & \\
\hline II & $\overline{2^{3 / 2}}$ & NW & & SW & & $\overline{112}$ & & $\begin{array}{l}\text { the so } \\
\text { shes of }\end{array}$ & $\begin{array}{l}\text { th w: } \\
\text { lightn }\end{array}$ & $\begin{array}{l}\text { s seen s } \\
\text { ing. }\end{array}$ & omething & that looke & ed like \\
\hline 12 & $21 / 2$ & & & SW: & & $1^{1 / 2}$ & & & & & & & \\
\hline 1 & 234 & & & SW & & $1 \mathrm{I} / 2$ & & iilly. cl & ear. & & & & \\
\hline 2 & 214 & & & & & $\mathrm{I}_{2}^{\prime}$ & & ually a & nd rai & & & & \\
\hline 3 & 3 & NII & & WS & & $I^{1 / 2}$ & & & & & & & \\
\hline 4 & 3 & & & & & $1 \%$ & & & & & & & \\
\hline 5 & $3 \frac{x}{4}$ & & & WSW & $x_{2} \mathrm{~S}$ & $I: 2$ & & & & & & & \\
\hline 6 & 3 & & & WSW & $2 \mathrm{~W}$ & $\mathrm{II}^{\mathrm{I}_{2}}$ & & $t$ out $t$ & vo ree & $\mathrm{s}$ in $\mathrm{ma}$ & intopsail & & \\
\hline 7 & 3 & & & & & $I \frac{1 / 2}{2}$ & & & & & & & \\
\hline 8 & 3 & & & & & $11 / 2$ & & nshine & & & & & \\
\hline 9 & 28 & NW & & W & & $1^{3} 4$ & & men o & the & ick list. & & & \\
\hline to & 2 & NNI & & 11 & & $I^{3}$ & & & & & & & \\
\hline II & 2 & NW & & WI & & $\mathrm{I}^{1} 4_{4}$ & & psail w & ind, $c$ & oudy. & & & \\
\hline 12 & 2 & $\mathrm{NW}$ & & WSW & $2 \mathrm{~W}$ & $1^{3 / 4}$ & & ear wit & pass & ng clou & & & \\
\hline \multirow{2}{*}{\multicolumn{3}{|c|}{$\begin{array}{c}\text { Variation } \\
\text { of } \\
\text { Compass } \\
114 \text { rhumb E }\end{array}$}} & \multicolumn{8}{|c|}{ In 24 hours } & \multicolumn{3}{|c|}{ From Vaua } \\
\hline & & & & 音 & 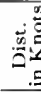 & & & 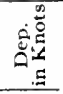 & 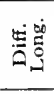 & 节 & 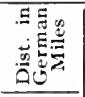 & 葛 & $\stackrel{\dot{0}}{\dot{0}}$ \\
\hline \multicolumn{3}{|c|}{$\begin{array}{l}\text { By Dead } \\
\text { Reckoning }\end{array}$} & & $\begin{array}{l}W / W \\
47^{\prime} W\end{array}$ & 62 & & & $\begin{array}{l}W \\
55\end{array}$ & $85^{\prime}$ & $150^{\circ} 35^{\prime}$ & $\left.213^{\prime \prime}\right)^{\prime}$ & $\begin{array}{c}E / S \\
1^{\circ} 53^{\prime} \mathrm{E}\end{array}$ & $122^{\circ} 42^{\prime}$ \\
\hline
\end{tabular}


(3eptember 21, 1741. After Midday

\begin{tabular}{|c|c|c|c|c|c|c|c|c|c|c|c|}
\hline$\stackrel{\Xi}{\Xi}$ & 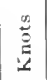 & $\bar{\Xi}$ & \multicolumn{2}{|c|}{ 巳ั } & 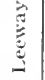 & & & & & & \\
\hline I & $\overline{132}$ & NW/N & \multicolumn{2}{|c|}{ W $/ \mathrm{s}$} & $\overline{132}$ & \multicolumn{6}{|c|}{$\begin{array}{l}\text { Topsail wind: let out three reefs in main-topsail: } \\
\text { air thick. }\end{array}$} \\
\hline 2 & $\sqrt{212}$ & & \multicolumn{2}{|c|}{ WSW:2W } & $\overline{x ! 2}$ & & & & & & \\
\hline 3 & $\overline{23_{4}}$ & NW & \multicolumn{2}{|c|}{ WSW } & $11_{2}$ & \multicolumn{6}{|c|}{ Wind in gusts. } \\
\hline 4 & $\overline{21 / 3}$ & & \multirow{2}{*}{\multicolumn{2}{|c|}{$\frac{S W W W W}{\text { WSIV }}$}} & $\overline{I_{1 / 2}}$ & \multicolumn{6}{|c|}{ Heavy westerly swell. } \\
\hline 5 & $\overline{25}$ & & & & $\overline{\mathrm{I}^{1} \overline{2}}$ & \multirow{2}{*}{\multicolumn{6}{|c|}{$\begin{array}{l}\text { Took one and two reefs in main-topsail, a second } \\
\text { reef in fore-topsail. }\end{array}$}} \\
\hline 6 & $\overline{231}$ & & & & $\overline{I^{1}{ }_{2}}$ & & & & & & \\
\hline 7 & 2 & $\mathrm{NW}$ & \multicolumn{2}{|c|}{$\mathrm{WSW}_{4}+\mathrm{W}$} & & \multicolumn{6}{|c|}{ 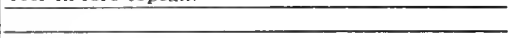 } \\
\hline 8 & $2 ! 4$ & & & & $13_{4}$ & \multirow{2}{*}{\multicolumn{6}{|c|}{ Light wind, clear with passing clouds. }} \\
\hline 9 & 2 & & & & $\overline{1^{3} 4}$ & \multirow{2}{*}{\multicolumn{6}{|c|}{ Light wind, clear with passing clouds. }} \\
\hline 10 & $I$ & & & & $\overline{I^{3}}$ & & & & & & \\
\hline 11 & $3_{4}$ & & & & $\sqrt{134}$ & \multirow{3}{*}{\multicolumn{6}{|c|}{$\begin{array}{l}\text { let ont the second and first reefs in main-topsail, } \\
\text { hoisted jib. }\end{array}$}} \\
\hline$\overline{12}$ & $3+$ & NNW & \multicolumn{2}{|c|}{ W } & 1. & & & & & & \\
\hline $\mathrm{I}$ & $\frac{T_{2}}{1 / 2}$ & NW & \multicolumn{2}{|c|}{ WSW } & 2 & & & & & & \\
\hline 2 & & \multirow{10}{*}{ 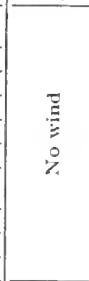 } & & & & \multicolumn{6}{|c|}{$\begin{array}{l}\text { Clewed up trysail, hauled down topsails and stay- } \\
\text { sails because there was no wind. }\end{array}$} \\
\hline 3 & & & & & & & & & & & \\
\hline 4 & & & & & & \multirow{2}{*}{\multicolumn{6}{|c|}{ Northwesterly swell. }} \\
\hline 5 & 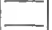 & & & & & & & & & & \\
\hline 6 & & & & & & \\
\hline 7 & - & & & & & \multirow{2}{*}{\multicolumn{6}{|c|}{$\begin{array}{l}\text { Unbent trysail because it needed repairing and in } \\
\text { its place bent on another. }\end{array}$}} \\
\hline 8 & & & & & & & & & & & \\
\hline 9 & 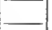 & & & & & \\
\hline 10 & ב & & & & & \multirow{2}{*}{\multicolumn{6}{|c|}{13 men on the sick list. }} \\
\hline II & & & & & & & & & & & \\
\hline 12 & 3 & SW & WV & & & \multicolumn{6}{|c|}{$\begin{array}{l}\text { Topsail wind; set topsails, staysails, and lower } \\
\text { sails. }\end{array}$} \\
\hline \multirow{2}{*}{\multicolumn{3}{|c|}{$\begin{array}{l}\text { Variation } \\
\text { of } \\
\text { Compass } \\
\text { I t rlutumb E }\end{array}$}} & \multicolumn{6}{|c|}{ In 2.4 hours } & \multicolumn{3}{|c|}{ From Vaua } \\
\hline & & & 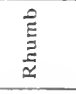 & 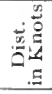 & 它? & 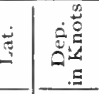 & 象战 & $\stackrel{\stackrel{\leftrightarrow}{ت}}{\sim}$ & 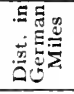 & 节 & 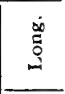 \\
\hline & $\begin{array}{l}\text { Dea } \\
\text { ckon }\end{array}$ & & $\begin{array}{l}\text { WI/S } \\
5^{\circ} \mathrm{O}^{\prime} \mathrm{S}\end{array}$ & 25 & S & $\begin{array}{l}W \\
24\end{array}$ & $37^{\prime}$ & $\left|50^{\circ} 28^{\prime}\right|$ & $2081 / 2$ & $\underset{1^{\circ}{ }_{1}^{\prime} / O^{\prime} E}{\mathrm{E} / \mathrm{E}}$ & $22^{\circ} 05^{\prime}$ \\
\hline
\end{tabular}




\section{d September 22, 1741. After Midday}

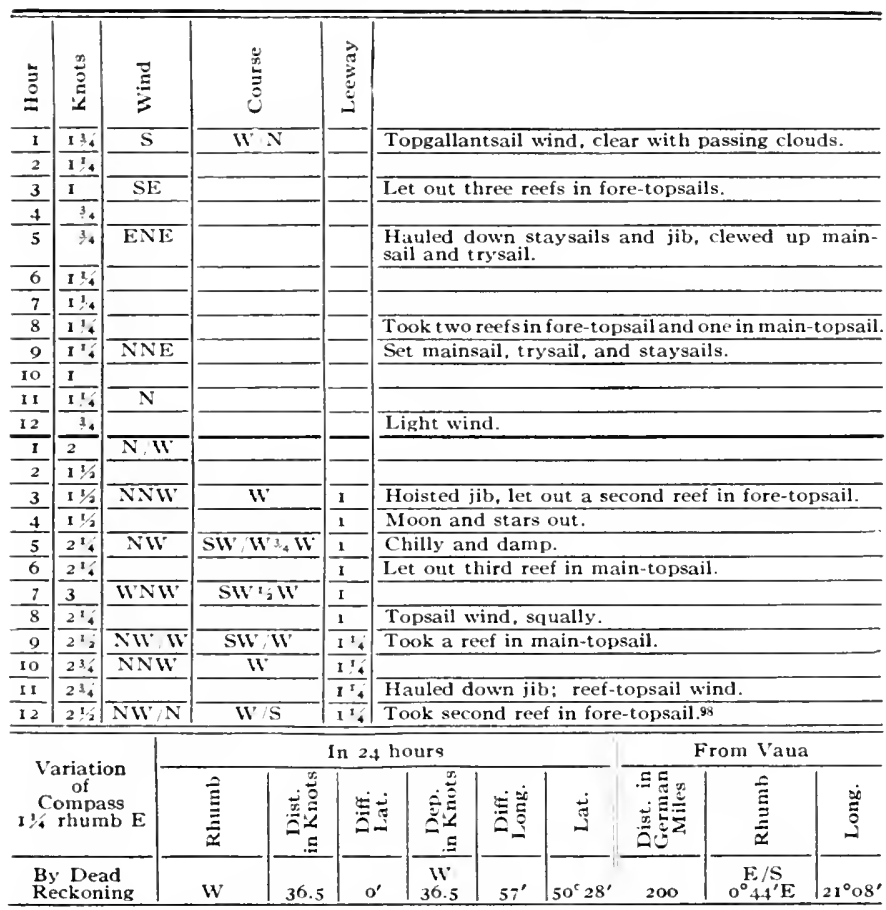

23 Khitrov's journal gives latitude by observation $50^{\circ} 27^{\prime}$. The noon position for this day is therefore indicated on the chart (Pl. I) as based on observation. 
$\Varangle$ September 23, 174I. After Midday

\begin{tabular}{|c|c|c|c|c|c|c|c|c|c|c|c|c|}
\hline$\stackrel{0}{=}$ & 焉 & 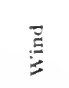 & 总 & & 总 & & & & & & & \\
\hline I & $\overline{23_{4}}$ & NW & $W$ & & $11 / 2$ & $\begin{array}{l}\text { To } \\
\text { sta }\end{array}$ & $\begin{array}{l}\text { psail y } \\
\text { ysails, }\end{array}$ & $\begin{array}{l}\text { ind, } \\
\text { and loy }\end{array}$ & $\begin{array}{l}\text { lear; c } \\
\text { ver sails }\end{array}$ & $\begin{array}{l}\text { carrying } \\
\text { s. }\end{array}$ & reefed to & psails, \\
\hline 2 & $\overline{134}$ & NW & ws & & 13 & & & & & & & \\
\hline 3 & 2 & & & & $\overline{1^{1}+2}$ & & esterly & well, r & eef-tops & sail wind & & \\
\hline 4 & $2 ! 4$ & & SW $/ 1$ & $3 \mathrm{WW}$ & $1^{1 / 2}$ & & & & & & & \\
\hline 5 & 3 & IVNW & SW! & & $\mathrm{II}^{\mathrm{I}}$ & & & & & & & \\
\hline 6 & $2 ! 4$ & & & & $\overline{I^{1}}$ & To & ok a se & ond $\mathrm{rc}$ & ef in $m$ c & ain-topsa & & \\
\hline 7 & 2 & & SVI & & $\overline{13 \cdot 6}$ & & & & & & & \\
\hline 8 & 2 & & & & $\mathrm{I}_{4}$ & & me abo & it with & the win & dd and $\mathrm{ma}$ & de the por & tack. \\
\hline 9 & $\overline{1^{3}}$ & W & NNW & $5 \mathrm{~W}$ & 2 & & & & & & & \\
\hline 10 & $\overline{I^{3} 4}$ & & & & 2 & & & & & & & \\
\hline 11 & 214 & WSW & $\mathrm{NW}$ & & $\mathrm{I}^{7 / 2}$ & & ok thre & reefs & in topsa & ails. & & \\
\hline 12 & $\overline{212}$ & & & & $I^{1 / 2}$ & & & & & & & \\
\hline$I$ & 2 & SW V & NIV & & & & rled tol & sails; & wind $\mathrm{fr}$ & reshening & & \\
\hline 2 & 2 & & & & $2 ! 2$ & $\mathrm{Sq}$ & ually. & & & & & \\
\hline 3 & $\overline{I^{2}}$ & & NIV & IV & 2 & & the $m$ & in-top & sail reef & fed; reef & topsail wi & \\
\hline 4 & $\overline{1^{3}}$ & SW & $\mathrm{NW}$ & LIV & 2 & & & & & & & \\
\hline 5 & $1^{3}$ & SW 1 & $\mathrm{NW}$ & & 2 & & fore-to & psail. & & & & \\
\hline 6 & $21 / 2$ & & & & $11^{3 \frac{3}{4}}$ & & & & & & & \\
\hline 7 & $I^{x} 4$ & & NWV W & $1,3 W$ & $2 \sqrt{3 / 4}$ & & psail w & nd. & & & & \\
\hline 8 & $22_{2}^{\prime}$ & & & & $13 / 4$ & & izzly. & & & & & \\
\hline 9 & $1^{3} 4$ & & NW & & 134 & & & & & & & \\
\hline 10 & 2 & & & & $13 \frac{3}{4}$ & & ind fres & nening & & & & \\
\hline 11 & $2 ! 2$ & & $\mathrm{NW}$ & $12 \mathrm{~W}$ & $\overline{1^{3 / 4}}$ & & dersail & wind. & & & & \\
\hline 12 & $2]^{12}$ & & & & & & avy' sw & fl fror & $\mathrm{nSW} ;$ & furled tof & sails. & \\
\hline \multirow{2}{*}{\multicolumn{3}{|c|}{$\begin{array}{l}\text { Variation } \\
\text { of } \\
\text { Compass } \\
\text { r : rliumb E }\end{array}$}} & \multicolumn{10}{|c|}{ In 24 hours } \\
\hline & & & $\stackrel{\vec{E}}{\underline{E}}$ & 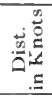 & 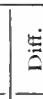 & 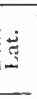 & 至 & 象葛 & 苞 & 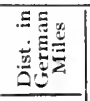 & 言 & $\stackrel{\dot{\phi}}{\dot{\omega}}$ \\
\hline \multicolumn{3}{|c|}{$\begin{array}{l}\text { By Dead } \\
\text { Reckoning }\end{array}$} & $6^{\circ}{ }^{N} I_{W}$ & 30 & & , & $\begin{array}{c}W \\
23.5\end{array}$ & $37^{\prime}$ & $50^{\circ} 47^{\prime} \mid$ & $1923 / 4$ & $\begin{array}{c}\mathrm{E} / \mathrm{S} \\
14^{\circ} 6^{\prime} \mathrm{E} \\
\end{array}$ & $20^{\circ} 31^{\prime}$ \\
\hline
\end{tabular}


2 September 24, 1741. After Midday

\begin{tabular}{|c|c|c|c|c|c|c|c|c|c|c|c|}
\hline$\stackrel{5}{E}$ & 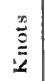 & $\stackrel{\bar{E}}{\bar{E}}$ & 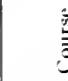 & & 章 & & & & & & \\
\hline$\overline{1}$ & $\overline{19 / 4}$ & SW & NW & $1 / 2 \mathrm{~W}$ & 23 & $\begin{array}{l}\text { Strong ur } \\
\text { and main } \\
\text { trysail; }\end{array}$ & $\begin{array}{l}\text { lersail } \\
\text { ail, ho } \\
\text { gming }\end{array}$ & $\begin{array}{l}\text { wind, } \\
\text { ve to ur } \\
\text { up to }\end{array}$ & $\begin{array}{l}\text { rizzly, } \\
\text { der the } \\
1 / 2 \mathrm{~N}, \mathrm{fa}\end{array}$ & $\begin{array}{l}\text { ti furled } \\
\text { ain-stay } \\
\text { ing off to }\end{array}$ & $\begin{array}{l}\text { resail } \\
\text { if and } \\
\text { viv. }\end{array}$ \\
\hline 2 & 18 & SW/S & NW & 3,4 & $5 / 2$ & & & & & & \\
\hline 3 & $11 / 2$ & & & & $51 / 2$ & & & & & & \\
\hline 4 & $11 / 2$ & & & & $5^{t / 2}$ & Gale. & & & & & \\
\hline 5 & $\overline{1 \% 3}$ & WSW & NW & $4 \mathrm{~N}$ & $\overline{51 / 2}$ & $\begin{array}{l}\text { By the } \\
\text { Andrei T }\end{array}$ & $\begin{array}{l}11 \text { of } \\
\text { etyaks }\end{array}$ & Fod die & of $\mathrm{scu}$ & $y$ the $g$ & nadier \\
\hline 6 & $1 / 2$ & & & & $\overline{5^{1}}$ & Coming & to $N$ & W by 1 & fallin & off to N. & \\
\hline 7 & $1 !$ & & NW & ${ }_{2} N$ & 6 & & & & & & \\
\hline 8 & $\overline{1+4}$ & & & & 6 & Wind goi & g dow & n a littl & & & \\
\hline 9 & 114 & & & & 6 & & & & & & \\
\hline 10 & $I^{1} 4$ & & & & 6 & Drizzly. & & & & & \\
\hline II & $1 ! 4$ & & & & 6 & & & & & & \\
\hline 12 & $\mathrm{I}^{14}$ & & & & 6 & & & & & & \\
\hline $\bar{I}$ & $\overline{I 1_{4}}$ & & & & 6 & Undersai & wind. & & & & \\
\hline 2 & 114 & & & & 6 & & & & & & \\
\hline 3 & 1 & W' & $\mathrm{NNW}$ & $1 / 3 \mathrm{~W}$ & $31 / 2$ & Set fores & $11, \mathrm{ma}$ & nsail. h & uled do & n stay'sal & \\
\hline 4 & 24 & & & & 13 & Set main & opsail & with th & ree reef & $n$ it. & \\
\hline 5 & 2 & & & & 134 & & & & & & \\
\hline 6 & 2 & & & & 136 & & & & & & \\
\hline 7 & 3 & & & & $I^{1 / 2}$ & Set fore-t & psail & vith thr & reefs. & & \\
\hline 8 & $21 / 2$ & & & & $11 / 2$ & Hauled u & topm & ast-stay & sails. & & \\
\hline 9 & 3 & & & & $1: 2$ & Reef-tops & iil win & i, cloud & & & \\
\hline 10 & $23 / 4$ & IV S & $\mathrm{NW}$ & & $\mathrm{I}^{3}$ & Let out $r$ & ef in $t$ & psails. & & & \\
\hline I I & 234 & & & & $\mathrm{I}^{1}$ & Lowered & he dea & d bod $y$ & nto the & & \\
\hline 12 & $31 / 2$ & WSW & $\bar{N}$ & & $\mid \overline{1}$ & Clear wit & passi & g cloud & 8.99 & & \\
\hline \multirow{2}{*}{\multicolumn{3}{|c|}{$\begin{array}{c}\text { Variation } \\
\text { of } \\
\text { Compass } \\
13 / 4 \text { rhumb E }\end{array}$}} & \multicolumn{6}{|c|}{ In 2.4 hours } & \multicolumn{3}{|c|}{ From Vaua } \\
\hline & & & $\stackrel{\vec{E}}{\underline{\Xi}}$ & 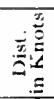 & $\Xi$ & 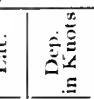 & 至苞 & 岕 & 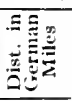 & 玄 & 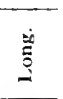 \\
\hline \multicolumn{3}{|c|}{$\begin{array}{l}\text { By Dead } \\
\text { Reckoning }\end{array}$} & $\frac{N / E}{0}$ & 40.7 & 39 & $\begin{array}{c}E \\
8.7\end{array}$ & $14^{\circ}$ & $51^{\circ} 27^{\prime}$ & $199^{3 / 4}$ & $6^{\circ}{ }_{30}^{\mathrm{E}} \mathrm{S}$ & $20^{\circ}+5^{\prime}$ \\
\hline
\end{tabular}

क Khitrov's journal gives latitude by observation $5 \mathrm{I}^{\circ} 30^{\prime}$. The noon position for this day is therefore indicated on the chart (PI. I) as based on observation. 


\section{q September 25, 1741. After Midday}

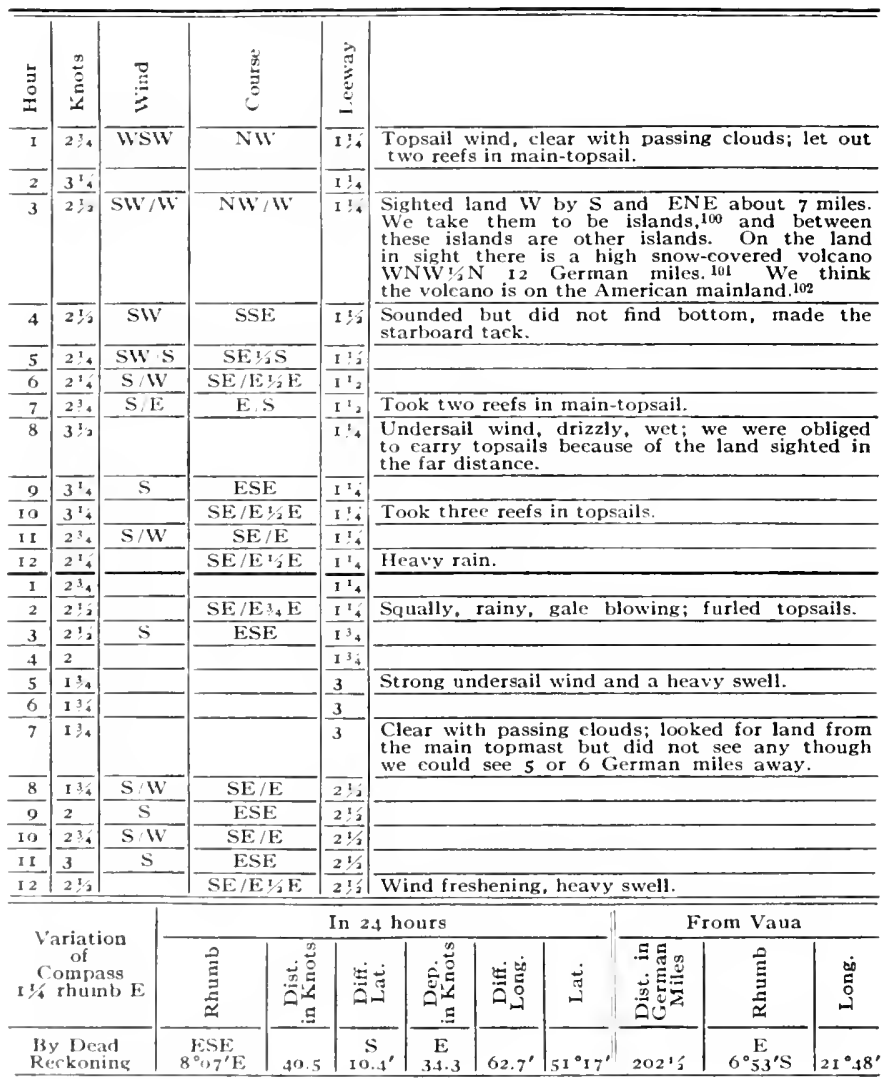

in Probably Adak and Atka Islands

101 ['rolbably the volean' on Great Sitkin Island.

102 Khitrov's journal: "Siglited land, W point iV by $S$, and $E$ point $E N E$ about 8 miles, which seems to be an island; a snow-covered mountain, which we think is on the mainland, WNW1/3N 12 German miles." 
h) September 26, I74I. After Midday

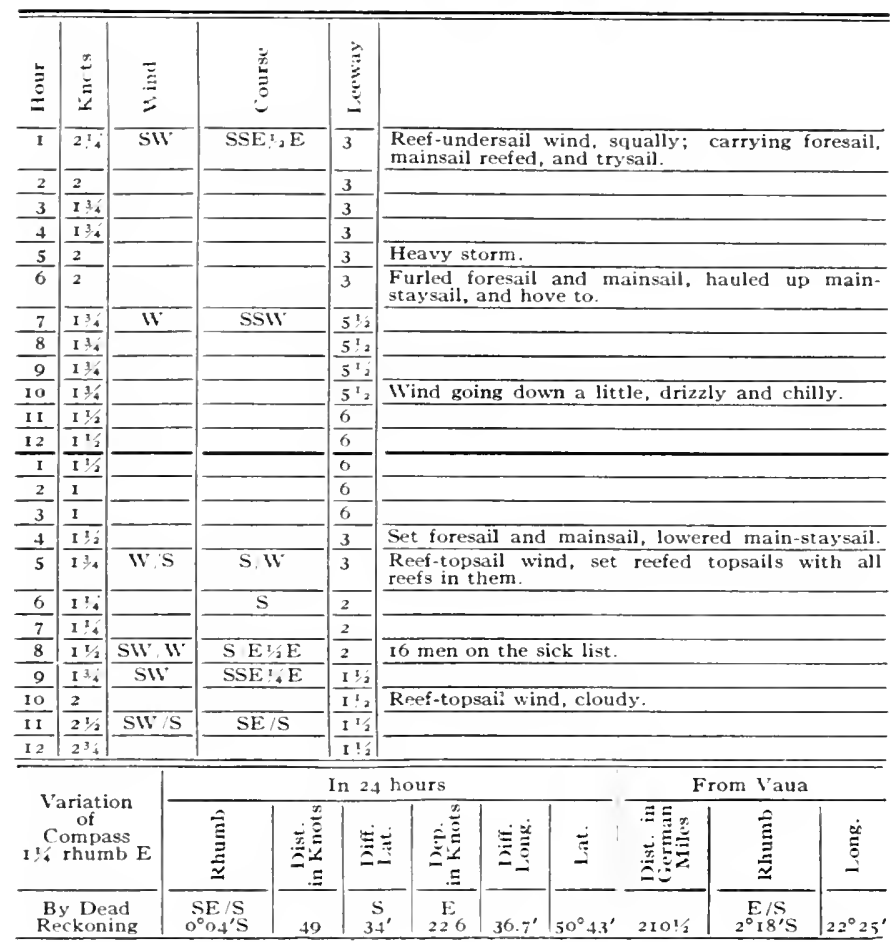


Seplember 27, I741. After Midday

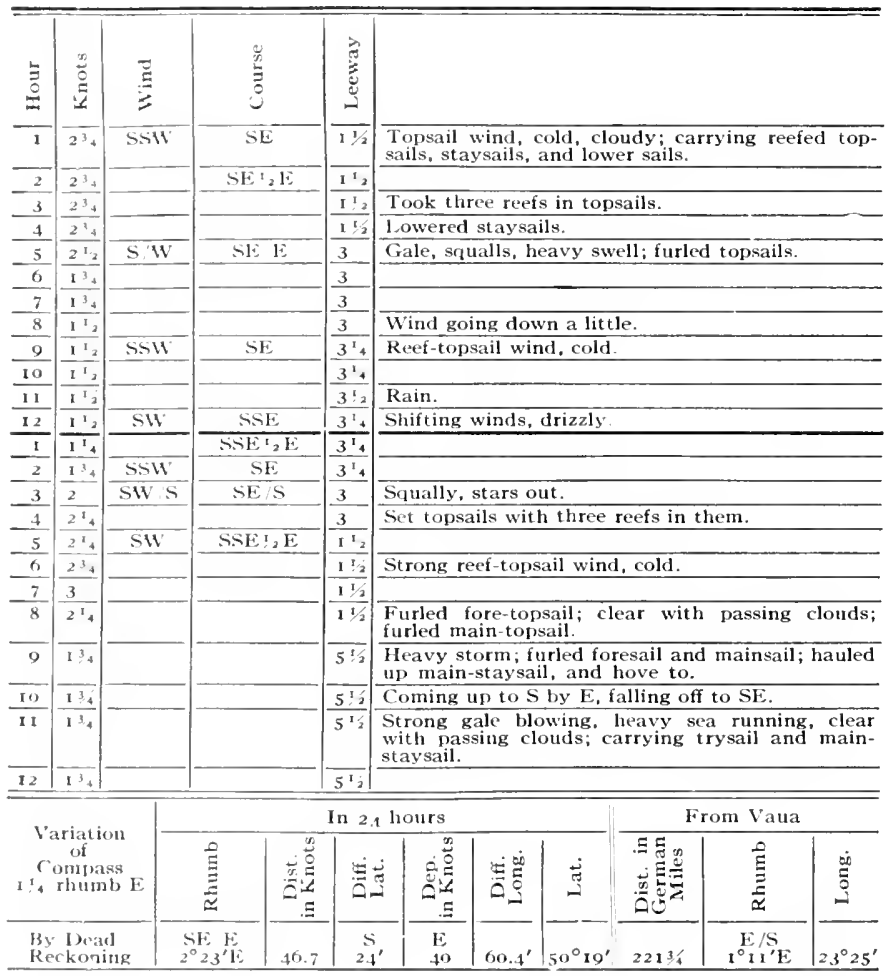


(3) September 28, I74I. Afler Midday

\begin{tabular}{|c|c|c|c|c|c|c|c|c|c|c|c|}
\hline$\stackrel{n}{3}$ & 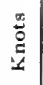 & $\overrightarrow{\mathrm{g}}$ & 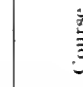 & & (ָ) & & & & & & \\
\hline I & 2 & WSN & $\$ ?$ & & $51 / 2$ & $\begin{array}{l}\text { Heavy s } \\
\text { ing main }\end{array}$ & $\begin{array}{l}\text { orm, } \\
\text { staysa }\end{array}$ & $\begin{array}{l}\text { lear wi } \\
1 \text { and } t r\end{array}$ & $\begin{array}{l}\mathrm{h} \text { passi } \\
\text { ysail. }\end{array}$ & 8 clouds; & carry- \\
\hline 2 & 2 & & & & $5 \%$ & & & & & & \\
\hline 3 & 2 & & & & 55 & & & & & & \\
\hline 4 & 2 & & & & $51 / 2$ & Coming & ptos & by $\mathrm{W}, \mathrm{f}$ & alling o & to SSE. & \\
\hline 5 & 2 & & & & $5 \frac{1 / 2}{4}$ & Wet. & & & & & \\
\hline 6 & 2 & & & & $51 / 2$ & & & & & & \\
\hline 7 & 2 & & $\mathrm{~s}$ & & $51 / 2$ & $\begin{array}{l}\begin{array}{l}\text { Lowered } \\
\text { storm. }\end{array} \\
\end{array}$ & ore at & d main & yards & account & of the \\
\hline 8 & 2 & & & & $5 \frac{1 / 2}{3}$ & & & & & & \\
\hline 9 & 2 & & & & $5 \%$ & Squalls, & in an & hail. & & & \\
\hline 10 & 2 & & & & 513 & Coming & p to $S$ & Wh, fall & ng off $t$ & SSE. & \\
\hline 11 & 2 & & & & $51 / 2$ & & & & & & \\
\hline 12 & 2 & & & & $5 \frac{112}{2}$ & Stars out & chilly & & & & \\
\hline $\mathrm{I}$ & 2 & II S & $51 / 2$ & & $5 ! 2$ & Coming & p to S & W, fall & $\mathrm{ng}$ off $\mathrm{t}$ & $S$ by $E$. & \\
\hline 2 & 2 & W & S W & $2 W$ & $51 / 2$ & Coming & $\mathrm{p}$ to $\mathrm{S}$ & $V$ by $s$, & falling & to $\mathrm{S}$. & \\
\hline 3 & 2 & & & & $51 / 2$ & & & & & & \\
\hline 4 & 2 & $w / N$ & SSW & W & $5 \%$ & Coming $\mathrm{v}$ & $p$ to $S$ & V, fallir & $g$ off to & by W. & \\
\hline 5 & 2 & & SSW & 41 & $51 / 2$ & Coming & p to $S$ & Dy $\mathrm{S}$, & falling & to $\mathrm{S} / 2 \mathrm{E}$ & \\
\hline 6 & 2 & & & & $5 \%$ & & & & & & \\
\hline 7 & 2 & & & & $51 / 2$ & & & & & & \\
\hline 8 & 2 & & & & $5 \frac{12}{2}$ & & & & & & \\
\hline 9 & 2 & & SS & & $5: 2$ & Coming 1 & $\mathrm{p}$ to $\mathrm{S}$ & $\therefore$, fallit & g off to & & \\
\hline 10 & 2 & & & & $51 / 2$ & Heavy st & $\mathrm{rm}, \mathrm{or}$ & casiona & rain. & & \\
\hline II & 2 & & & & $\overline{5^{12}}$ & & & & & & \\
\hline & 2 & & & & 5,2 & Carrying & main-s & aysail : & nd trys & & \\
\hline \multirow{2}{*}{\multicolumn{3}{|c|}{$\begin{array}{c}\text { Variation } \\
\text { of } \\
\text { Compass } \\
11 \text { rhumb E }\end{array}$}} & \multicolumn{6}{|c|}{ In 24 hours } & \multicolumn{3}{|c|}{ From Vaua } \\
\hline & & & $\hat{\underline{\Xi}}$ & 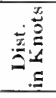 & ن & 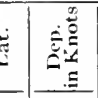 & 焉 & ज्ञ & 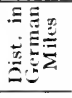 & $\overrightarrow{\underline{E}}$ & $\stackrel{\oplus}{\tilde{E}}$ \\
\hline \multicolumn{3}{|c|}{$\begin{array}{l}\text { By Dead } \\
\text { Reckoning }\end{array}$} & $\begin{array}{l}\mathrm{SE} / \mathrm{S} \\
\dot{1}^{\circ}+8^{\prime} \mathrm{E}\end{array}$ & 46.5 & $\begin{array}{r}\mathrm{S} \\
36.6 \\
\end{array}$ & $\begin{array}{r}E \\
29.2 \\
\end{array}$ & $46^{\prime}$ & $19^{\circ} 42^{\prime}$ & $2313 / 4$ & $\begin{array}{c}\text { E/S } \\
0^{\circ}+2^{\prime} S \\
\end{array}$ & $24^{\circ} 11^{\prime}$ \\
\hline
\end{tabular}


* Seplewber 20, 1741. After Midday

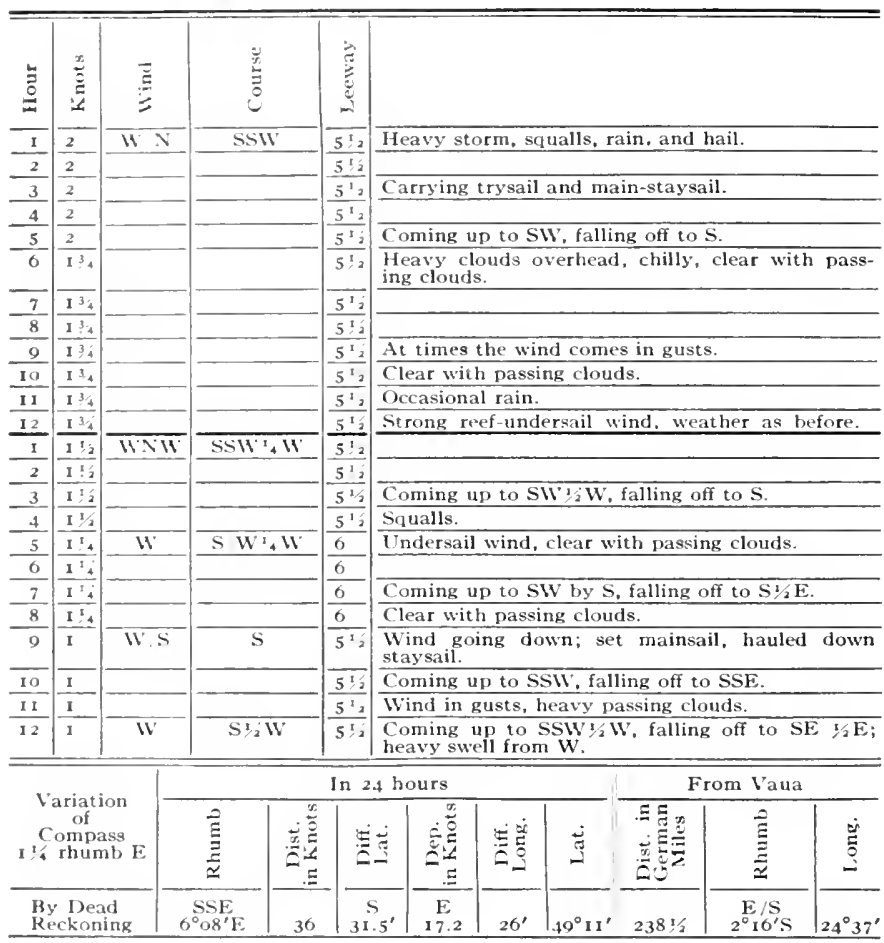


$\Varangle$ Seplember 30,1741 . After Midday

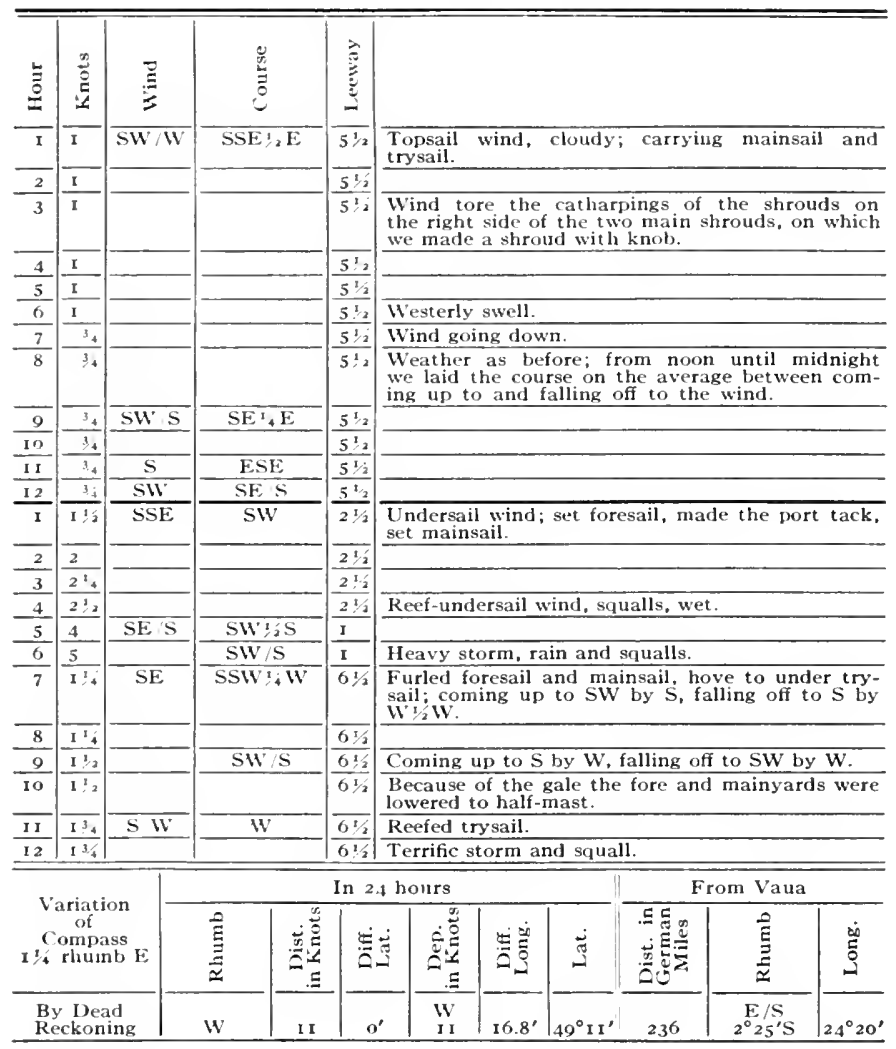


2 October I, I74I. After Midday

\begin{tabular}{|c|c|c|c|c|c|c|c|c|c|c|c|c|}
\hline$\stackrel{5}{3}$ & 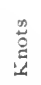 & 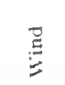 & 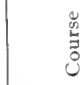 & & 党 & & & & & & & \\
\hline 1 & 2 & SSIV & II & & $\overline{0 ! 2}$ & $\begin{array}{l}\text { Fri } \\
\text { ove }\end{array}$ & $\begin{array}{l}\text { ghtful } \\
\text {-r the d }\end{array}$ & $\begin{array}{l}\text { storm } \\
\text { eck. }\end{array}$ & heavy & squalls, & waves $w$ & ashing \\
\hline 2 & 2 & & & & $\overline{6^{\prime}+2}$ & & & & & & & \\
\hline 3 & 2 & & & & $60^{2}$ & & & & & & & \\
\hline 4 & 2 & & & & $\overline{6^{12}}$ & & ar with & passi & ing cloud & & & \\
\hline 5 & 2 & SW S & IV 3 & & $\overline{6 \%}$ & $\mathrm{HC}$ & avy cio & tds.s & qualls. & & & \\
\hline 6 & 2 & & & & $\overline{6^{1}}$ & & & & & & & \\
\hline 7 & 2 & SW & WNW & $+W$ & $6: 2$ & & & & & & & \\
\hline 8 & 2 & & & & $6 ! 2$ & & rrific st & $\mathrm{rm}$ a & id great & waves. & & \\
\hline 9 & 2 & & & & $61 / 2$ & & & & & & & \\
\hline 10 & 2 & & & & $61 / 2$ & & & & & & & \\
\hline 11 & 2 & & & & $6 \overline{1 / 2}$ & & avy rai & & & & & \\
\hline 12 & 2 & & & & $\overline{6^{1 / 2}}$ & & izzly & & & & & \\
\hline 1 & 2 & & & & $61 / 2$ & & & & & & & \\
\hline 2 & 2 & & IWNII & $2 \mathrm{~W}$ & $\overline{6^{1}}$ & & & & & & & \\
\hline 3 & 2 & & & & $\overline{6_{1}^{2}}$ & & & & & & & \\
\hline 4 & 2 & & & & $6 ! 2$ & & $\begin{array}{l}\text { eavy clo } \\
\text { main } \\
\text { main-t }\end{array}$ & $\begin{array}{l}\text { uds, } \\
\text { shrouc } \\
\text { opma }\end{array}$ & $\begin{array}{l}\text { et, stor } \\
\text { on the } \\
\text { stay, }\end{array}$ & $\begin{array}{l}\text { Iny; one } \\
\text { left side } \\
\text { which we }\end{array}$ & $\begin{array}{l}\text { f the lany } \\
\text { tore loose } \\
\text { repaired. }\end{array}$ & $\begin{array}{l}\text { ards to } \\
; \quad \text { also } \\
\end{array}$ \\
\hline 5 & 2 & $S 11$ & NIV:II & ${ }_{2} \mathrm{~W}$ & $\overline{612}$ & & & & & & & \\
\hline 6 & 2 & & & & $\overline{612}$ & & & & & & & \\
\hline 7 & 2 & & & & $6 \div 2$ & & & & & & & \\
\hline 8 & 2 & & & & $6 !$ & & & & & & & \\
\hline 9 & 2 & $\mathrm{SW} / \mathrm{T}$ & NIV & & $6 ! 2$ & & $\begin{array}{l}\text { the rig } \\
\text { th to sea }\end{array}$ & $\begin{array}{l}\text { ht side } \\
\text {, and }\end{array}$ & $\begin{array}{l}\text { the gun } \\
\text { we naile }\end{array}$ & $\begin{array}{l}2 \text { port bu } \\
\text { ed board }\end{array}$ & $\begin{array}{l}\text { wark was } \\
\text { in its pla }\end{array}$ & $\begin{array}{l}\text { carried } \\
\text { ce. }\end{array}$ \\
\hline 10 & 2 & & & & $6^{1 / 2}$ & & & & & & & \\
\hline II & 2 & & & & $6 \div 2$ & & $\begin{array}{l}\text { avy gal } \\
\text { th side }\end{array}$ & $\begin{array}{l}\text { e. wet } \\
\text { wast }\end{array}$ & $\begin{array}{l}\text { squally } \\
\text { ed over }\end{array}$ & $\begin{array}{l}\text { y all da } \\
\text { the ded }\end{array}$ & the wave & es from \\
\hline$\overline{12}$ & 2 & SIV & N & & $\overline{652}$ & & & & & & & \\
\hline \multirow{2}{*}{\multicolumn{3}{|c|}{$\begin{array}{c}\text { Variation } \\
\text { of } \\
\text { Compass } \\
\text { 1 t rhumb E }\end{array}$}} & \multicolumn{7}{|c|}{ In 24 hours } & \multicolumn{3}{|c|}{ From Vaua } \\
\hline & & & है & 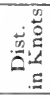 & 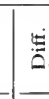 & 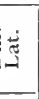 & 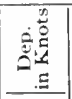 & 䓀高 & 泀 & 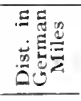 & है & $\stackrel{\infty}{\Xi}$ \\
\hline \multicolumn{3}{|c|}{$\begin{array}{l}\text { Isy Dead } \\
\text { Reckoning }\end{array}$} & $\begin{array}{c}N / \mathbb{E} \\
5^{\circ} 13^{\prime} \mathrm{E}\end{array}$ & 46 & +1 & $N$ & $\begin{array}{l}\mathrm{E} \\
13 \\
\end{array}$ & $20^{\prime}$ & $49^{\circ} 55^{\prime}$ & $230 \%$ & $\begin{array}{c}\mathrm{E} / \mathrm{S} \\
\mathrm{O}^{\circ} 16^{\prime} \mathrm{E} \\
\end{array}$ & $24^{\circ}+40^{\prime}$ \\
\hline
\end{tabular}


\& October 2, I74I. After Midday

\begin{tabular}{|c|c|c|c|c|c|c|c|c|c|c|c|c|}
\hline$\stackrel{\text { s. }}{\stackrel{5}{ \pm}}$ & $\stackrel{\substack{0 \\
:}}{:}$ & $\vec{E}$ & 苞 & & 窇 & & & & & & & \\
\hline I & 2 & WSW & NIV' & & $\overline{6^{12}}$ & & $\begin{array}{l}\text { rrific } \\
\text { izzly; }\end{array}$ & storm, & $\begin{array}{l}\text { heavy } \\
\text { trysail }\end{array}$ & squalls & and high & seas, \\
\hline 2 & 2 & & & & $6 \frac{1}{62}$ & & & & & & & \\
\hline 3 & 2 & & & & $\overline{6^{3,2}}$ & & $\begin{array}{l}\text { labent } t \\
\text { ady in } \\
\text { was not }\end{array}$ & $\begin{array}{l}\text { he spr } \\
\text { onace o } \\
\text { tlikely }\end{array}$ & $\begin{array}{l}\text { and to } \\
\text { the try } \\
\text { that thi }\end{array}$ & $\begin{array}{l}\text { ok a reef } \\
\text { sail, bece. } \\
\text { e trysail }\end{array}$ & $\begin{array}{l}\text { in it and } \\
\text { tse in this } \\
\text { could hold }\end{array}$ & $\begin{array}{l}\text { lade it } \\
\text { storm } \\
\text { out. } \\
\end{array}$ \\
\hline 4 & 2 & & & & $6 \%$ & & & & & & & \\
\hline 5 & 2 & & & & $6 \mathrm{x} / 2$ & & & & & & & \\
\hline 6 & 2 & & & & $\overline{6^{12}}$ & & & & & & & \\
\hline 7 & $I^{3} 4$ & $\mathrm{~W} / \mathrm{s}$ & NiV & & $\overline{6 r_{2}}$ & & a going & down & bit. & & & \\
\hline 8 & $I^{3} 4$ & & & & $61 / 2$ & & eather: & as befo & & & & \\
\hline 9 & 134 & & & & $66^{1}$ & & & & & & & \\
\hline 10 & 134 & & & & $\overline{6^{12}}$ & & eavy do & ouds, r & & & & \\
\hline II & $13 / 4$ & WSIV & NW & & 7 & & & & & & & \\
\hline 12 & $\overline{I^{3} 4}$ & & & & 7 & & rong ga & le, stat & ont. & & & \\
\hline$I$ & $I^{1 / 2}$ & & & & 7 & & oudy, c & hilly. & & & & \\
\hline 2 & $11 / 2$ & & & & 7 & & & & & & & \\
\hline 3 & $I^{\pi / 2}$ & & & & 7 & & regular & Storm & & & & \\
\hline 4 & $I^{x_{4}}$ & & & & 7 & & & & & & & \\
\hline 5 & $I^{2} 4$ & & & & 7 & & ondy. & & & & & \\
\hline 6 & $1^{x_{4}}$ & SW II & NW' & & 7 & & & & & & & \\
\hline 7 & $I$ & & & & 7 & & ear wit & 1 passi & $2 \mathrm{~g}$ clono & & & \\
\hline 8 & 1 & SW & $\mathrm{S}, \mathrm{E}$ & & 6 & & $\begin{array}{l}\text { le blo } \\
\text { out wi } \\
\text { ard tac } \\
\text { t side }\end{array}$ & $\begin{array}{l}\text { wing. } \\
\text { th the } \\
k \text {; exa } \\
\text { and res }\end{array}$ & $\begin{array}{l}\text { At } 8: 3 \\
\text { wind, } \\
\text { nined t } \\
\text { aired th }\end{array}$ & $\begin{array}{l}\text { o set fo } \\
\text { and hov } \\
\text { le damag } \\
\text { em. }\end{array}$ & $\begin{array}{l}\text { e-staysail. } \\
\text { to in the } \\
\text { d shrouds }\end{array}$ & $\begin{array}{l}\text { came } \\
\text { star- } \\
\text { on the } \\
\end{array}$ \\
\hline 9 & $I$ & & SS & & 6 & & & & & & & \\
\hline Io & $\mathrm{I}$ & & & & 6 & & & & & & & \\
\hline II & $I$ & & & & 6 & & & & & & & \\
\hline 12 & $I$ & & & & 6 & & ear wit & $h$ pass & ng clous & & & \\
\hline \multirow{2}{*}{\multicolumn{3}{|c|}{$\begin{array}{c}\text { Variation } \\
\text { of } \\
\text { Compass } \\
1 \% \text { rlumb E }\end{array}$}} & \multicolumn{7}{|c|}{ In 24 hours } & \multicolumn{3}{|c|}{ From Vaua } \\
\hline & & & 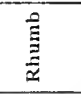 & 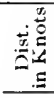 & $E$ & تี & 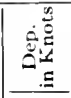 & 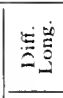 & ت્ & 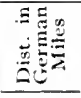 & 蔦 & $\stackrel{\mathscr{0}}{\stackrel{\leftrightarrow}{0}}$ \\
\hline \multicolumn{3}{|c|}{$\begin{array}{l}\text { By Dead } \\
\text { Reckoning }\end{array}$} & $\begin{array}{l}\mathrm{NE} / \mathrm{E} \\
\mathrm{O}^{\circ} 23^{\prime} \mathrm{E}\end{array}$ & $34^{1 / 2}$ & & $g^{\prime}$ & $\underset{28.7}{E}$ & $44^{\prime}$ & $50^{\circ} 14^{\prime}$ & $240^{1} 4$ & $\begin{array}{c}\mathrm{E} / \mathrm{S} \\
{ }_{1}^{\circ}+0^{\prime} \mathrm{E}\end{array}$ & $25^{\circ} 24^{\prime}$ \\
\hline
\end{tabular}


b October 3, I74I. After Midday

\begin{tabular}{|c|c|c|c|c|c|c|c|c|c|c|c|}
\hline$\stackrel{5}{E}$ & 苞 & $\ddot{E}$ & 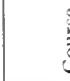 & & 毵 & & & & & & \\
\hline $\bar{I}$ & $\mathbf{I}$ & S.W & $\mathrm{SE}$ & & $\overline{5^{12}}$ & Undersai & wind, & heavy' & W swel & & \\
\hline 2 & $I$ & & & & $5^{1 / 2}$ & Drizzly, 1 & iny"; & arrying & trysail & d main-s & aysail. \\
\hline$\overline{3}$ & $\overline{3 i}$ & & & & $\overline{5 ! / 2}$ & $\begin{array}{l}\text { Wind goi } \\
\text { sail, patc }\end{array}$ & $\begin{array}{l}\text { g dow } \\
\text { ed the }\end{array}$ & $\begin{array}{l}\text { i: set } n \\
\text { trysail }\end{array}$ & $\begin{array}{l}\text { ainsail, } \\
\text { which h }\end{array}$ & $\begin{array}{l}\text { vered mai } \\
\text { been dan }\end{array}$ & $\begin{array}{l}\text { n-stay- } \\
\text { laged. }\end{array}$ \\
\hline 4 & 7 & & & & $\overline{5 \%}$ & & & & & & \\
\hline 5 & $\frac{1 / 2}{1 / 2}$ & & & & $5+2$ & & & & & & \\
\hline 6 & $1 / 3$ & & & & $5 \frac{12}{2}$ & Set trysa & & & & & \\
\hline 7 & $3_{4}$ & SE & $\mathrm{E}$ & & $5^{1 / 2}$ & & & & & & \\
\hline 8 & 34 & & & & $5 \%$ & & & & & & \\
\hline 9 & 34 & & & & $\overline{512}$ & Undersai & wind. & & & & \\
\hline I0 & $i$ & & & & $\overline{5^{2}}$ & Wind fre & henin & & & & \\
\hline It & 1 & & & & 512 & & & & & & \\
\hline 12 & 1 & & & & $5^{1 / 2}$ & & & & & & \\
\hline$\overline{\mathrm{I}}$ & $\sqrt{4^{x}}$ & ESE & $\$$ & & $5 \frac{1}{2}$ & $\begin{array}{l}\text { Set fores } \\
\text { tack, an }\end{array}$ & $\begin{array}{l}1, \text { cant } \\
\text { went }\end{array}$ & $\begin{array}{l}\text { e about } \\
\text { our wa }\end{array}$ & $\begin{array}{l}\text { with th } \\
\text { yi hea }\end{array}$ & $\begin{array}{l}\text { xind on } t \\
\text { rain. }\end{array}$ & ne port \\
\hline 2 & $3^{\frac{\pi}{2}}$ & & & & 7 & & & & & & \\
\hline 3 & I & SW & SSE & & 7 & $\begin{array}{l}\text { Undersa } \\
\text { trysail, } \mathrm{f}\end{array}$ & $\begin{array}{l}\text { wind } \\
\text { rled } \mathrm{f}\end{array}$ & $\begin{array}{c}\text { starb } \\
\text { resail. }\end{array}$ & ard tac & hove to & under \\
\hline 4 & $I$ & & & & 7 & & & & & & \\
\hline 5 & $\overline{I^{\prime} 2}$ & ssw & $\mathrm{SE}$ & & 6 & & & & & & \\
\hline 6 & $\overline{11 / 2}$ & & & & 6 & Heavy cl & uds, r & ain: se & main-s & sail. & \\
\hline 7 & $1 \frac{1}{2}$ & & & & 6 & & & & & & \\
\hline 8 & 13 & & & & 6 & Very chil & $y$ and & damp. & & & \\
\hline 9 & $\mathrm{I}^{1 / 3}$ & & & & 6 & & & & & & \\
\hline 10 & $1 ! 4$ & SW & & & 6 & Gale. & & & & & \\
\hline $1 \mathrm{I}$ & $\overline{153}$ & & & & 6 & $\begin{array}{l}\text { This day } \\
\text { on the a } \\
\text { off to the }\end{array}$ & $\begin{array}{l}\text { while } \\
\text { erage } \\
\text { wind. }\end{array}$ & $\begin{array}{l}\text { ve were } \\
\text { betweer }\end{array}$ & $\begin{array}{l}\text { hove to } \\
\text { coming }\end{array}$ & $\begin{array}{l}\text { e laid the } \\
\text { p to and }\end{array}$ & $\begin{array}{l}\text { course } \\
\text { falling } \\
\end{array}$ \\
\hline 12 & $I ! 2$ & & $\mathrm{~S} / \mathrm{F}$ & & 6 & & & & & & \\
\hline \multirow{2}{*}{\multicolumn{3}{|c|}{$\begin{array}{c}\text { Variation } \\
\text { of } \\
\text { Compass } \\
1 ! \text { rlumb E }\end{array}$}} & \multicolumn{6}{|c|}{ In 2.4 hours } & \multicolumn{3}{|c|}{ From Vaua } \\
\hline & & & $\underset{\Xi}{\stackrel{\Xi}{\Xi}}$ & 焉 & 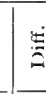 & 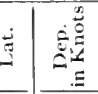 & 写苞 & $\stackrel{\text { ت̇ }}{\mathrm{i}}$ & 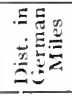 & 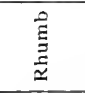 & $\stackrel{\infty}{0}$ \\
\hline \multicolumn{3}{|c|}{$\begin{array}{l}\text { By Dead } \\
\text { Reckoning }\end{array}$} & $\underset{7^{\circ} 21^{\prime} \mathrm{E}}{\mathrm{E} / \mathrm{s}}$ & I5 & & $\begin{array}{c}E \\
I .4 .7\end{array}$ & $23^{\prime}$ & $50^{\circ} \times 3^{\prime}$ & 24334 & $\begin{array}{c}\mathrm{E} / \mathrm{S} \\
{ }^{\circ}+5^{\prime} \mathrm{E} \\
\end{array}$ & $25^{\circ} 47^{\prime}$ \\
\hline
\end{tabular}


Ocioher 4. I74I. After Midday

\begin{tabular}{|c|c|c|c|c|c|c|c|c|c|c|c|c|c|}
\hline$\stackrel{5}{3}$ & 总 & $\bar{E}$ & & 葛 & & 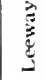 & & & & & & & \\
\hline $\bar{I}$ & $\overline{1 / 2}$ & $\overline{\mathrm{SW} / \mathrm{T}}$ & & $\mathrm{S}$ & & $61 / 2$ & & $\begin{array}{l}\text { rong u } \\
\text { ain-sta }\end{array}$ & $\begin{array}{l}\text { adersai } \\
\text { sail. }\end{array}$ & wind, & rain; ca & ying try & il and, \\
\hline 2 & $1 \% / 2$ & & & & & $61 / 2$ & & & & & & & \\
\hline 3 & $I \pi / 2$ & $W$ & & $S^{2} / 2$ & & $6 \%$ & & ualls. & & & & & \\
\hline 4 & $18 / 2$ & & & & & 6,2 & & orm; & auled & lown $\mathrm{m}$ & ain-stay & & \\
\hline 5 & $I^{2 / 2}$ & W & & SS & & $61 / 2$ & & & & & & & \\
\hline 6 & $11 / 3$ & & & & & $61 / 2$ & & ind as & before, & drizzly & and ver & chilly. & \\
\hline 7 & $11 / 2$ & & & & & $6 \%$ & & & & & & & \\
\hline 8 & $11 / 2$ & & & & & $\overline{6 \% 2}$ & & & & & & & \\
\hline 9 & $13 / 2$ & $W$ & & SSW & ${ }_{4} \mathrm{~W}$ & $6 \%$ & & & & & & & \\
\hline I0 & $11 / 2$ & & & & & $\overline{6 t_{2}}$ & & ght rai & & & & & \\
\hline $1 \mathrm{I}$ & $I 1 / 2$ & & & & & $6 \frac{12}{2}$ & & ear wit & h passi & $\mathrm{ig}$ clous & & & \\
\hline 12 & $\begin{array}{ll}135 \\
\end{array}$ & & & & & $\overline{611}$ & & & & & & & \\
\hline I & $I^{1 / 2}$ & & & & & 612 & & & & & & & \\
\hline$\sqrt{2}$ & $11 / 4$ & & & SW & & 7 & & ef-und & ersail w & ind, hea & avy sea. & izzly and & rainy. \\
\hline 3 & $I \mathrm{I} / 4$ & & & & & 7 & & & & & & & \\
\hline 4 & 15 & & & & & 7 & & ind cor & hes in & usts. & & & \\
\hline 5 & $I$ & & & SSW & $2 W$ & 6 & & adersai & wind, & chilly. & & & \\
\hline 6 & $\mathrm{I}$ & & & & & 6 & & isted & nain $n+s t$ & aysail. & & & \\
\hline 7 & $I$ & & & & & $5^{1 / 2}$ & & $t$ main & ail, ha & sled doy & wn main & aysail. & \\
\hline 8 & $\mathrm{I}$ & & & & & $5^{1 / 2}$ & & the er & $\mathrm{d}$ of $\mathrm{th}$ & hour & set the f & esail. & \\
\hline 9 & $\overline{1 \div 4}$ & W S & & 5 & & $\mathrm{I}^{1 / 2}$ & & oisted & opsails & with th & ree reef & n each. & \\
\hline 10 & $I 1 / 4$ & W'St & & $\mathrm{S}$ & & $11 / 2$ & & & & & & & \\
\hline II & $\begin{array}{l}15 / 4 \\
\end{array}$ & & & & & Is & & & & & & & \\
\hline & $\overline{13 / 2}$ & SW & & $\mathrm{S}$ & & $\mathrm{I}^{1 / 2}$ & & Tong $\mathrm{rt}$ & ef-tops & il wind & 1. drizzly & & \\
\hline \multirow{2}{*}{\multicolumn{3}{|c|}{$\begin{array}{c}\text { Variation } \\
\text { of } \\
\text { Compass } \\
1 / 4 \text { rhumb E }\end{array}$}} & \multicolumn{8}{|c|}{ In 24 hours } & \multicolumn{3}{|c|}{ From Vaua } \\
\hline & & & & $\stackrel{\hat{\Xi}}{\underline{\Xi}}$ & 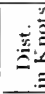 & 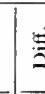 & & 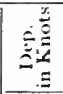 & 总 & ت & 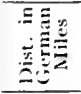 & $\frac{\vec{E}}{\underline{\underline{E}}}$ & 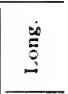 \\
\hline \multicolumn{3}{|c|}{$\begin{array}{l}\text { By Dead } \\
\text { Reckoning }\end{array}$} & & $S_{29^{\prime} E}$ & 30 & & $6^{\prime}$ & $\begin{array}{l}\mathrm{E} \\
\mathrm{I} 5\end{array}$ & $23.3^{\prime}$ & $49^{\circ} 47^{\prime}$ & $2493 / 4$ & ${ }_{0^{\circ} 28^{\prime} \mathrm{E}}^{\mathrm{E} / \mathrm{S}}$ & $26^{\circ} 10^{\prime}$ \\
\hline
\end{tabular}


(3) October 5, I74I. After Midday

\begin{tabular}{|c|c|c|c|c|c|c|c|c|c|c|c|}
\hline$\stackrel{\underline{\partial}}{=}$ & 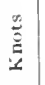 & 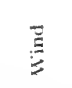 & 总 & & 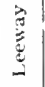 & & & & & & \\
\hline 1 & $2^{x_{2}}$ & SW & $S S E$ & & 3. & $\begin{array}{l}\text { Reef-tops: } \\
\text { sail, main } \\
\text { mast-stay }\end{array}$ & $\begin{array}{l}\text { iil win } \\
\text { sail, r } \\
\text { sails. }\end{array}$ & $\begin{array}{l}\text { d, west } \\
\text { eefed to }\end{array}$ & $\begin{array}{l}\text { rly swe } \\
\text { psails, }\end{array}$ & $\begin{array}{c}\text { carryin } \\
\text { ysail. an }\end{array}$ & $\begin{array}{l}\text { fore- } \\
\text { d top- }\end{array}$ \\
\hline 2 & 3 & SsW & S1 & & 34 & & & & & & \\
\hline 3 & 3 & & & & 34 & & & & & & \\
\hline 4 & $22^{3}$ & SW & $\mathrm{SE}$ & & $1 \frac{13}{2}$ & Lowered s & tay'sai & Is; rain & & & \\
\hline 5 & 3 & $\mathrm{~s}$ & WS & & $\overline{1^{1}}$ & At the be & inning & of the & hour $\mathrm{ma}$ & the port & tack. \\
\hline 6 & 3 & $\mathrm{SE}$ & WSII & WV & $\mathrm{I}^{\mathrm{I}_{1}}$ & & & & & & \\
\hline 7 & 312 & SSE & SWI & & 15 & Strong ret & f-tops & ail wind & and rai & & \\
\hline 8 & 3 & $S E$ & SW & & $11 / 3$ & Squalls. & & & & & \\
\hline 9 & 34 & & & & $1^{x_{2}^{2}}$ & Furled for & $e-t o p s$ & & & & \\
\hline 10 & 3 & SSE & SI & & $2 \%$ & $\begin{array}{l}\text { Furled m } \\
\text { downpour }\end{array}$ & ain-tor & psail ; re & ef-under & il wind, & heavy \\
\hline 11 & 2 & & & & 23 & & & & & & \\
\hline 12 & $\mathrm{I}_{2}$ & & & & $21 / 2$ & Furled $\mathrm{m}$ & insail & hauled & up mair & taysail. & \\
\hline $\mathrm{I}$ & $1 ! 2$ & $\mathrm{~s}$ & W: & & $2 ! 2$ & Light ratr & & & & & \\
\hline 2 & 2 & SSW & 11 & & $2 ! 2$ & Set main- & topsail & reefed. & & & \\
\hline 3 & 2 & SW & WNI & $2 \mathrm{~N}$ & $22^{12}$ & Chilly, cl & ar wit & h passin & g clouds & & \\
\hline 4 & 212 & & & & $21 / 2$ & & & & & & \\
\hline 5 & $22^{3} 4$ & SW 11 & $\mathrm{NI}$ & & $2 \frac{1 / 2}{2}$ & Storm, $f_{t}$ & rled $m$ & ain-tops & & & \\
\hline 6 & 1 & WSW & $s$ & & 4 & Made sta & rboard & tack. & & & \\
\hline 7 & $\mathrm{I}_{4}^{3}$ & & $\underline{s}$ & & 4 & & & & & & \\
\hline 8 & $\overline{I \cdot 3}$ & & & & 4 & Strong re & f-tops & ail wind & & & \\
\hline 9 & $1^{3} 4$ & SW W & $\mathrm{SE}$ & & 4 & & & & & & \\
\hline 10 & $1: 4$ & & & & 4 & & & & & & \\
\hline 11 & $1^{1} \cdot$ & & & & 4 & $\begin{array}{l}\text { Storm; } \\
\text { sail and }\end{array}$ & $\begin{array}{l}\text { rrled } \\
\text { rysail. }\end{array}$ & oresail, & hove to & nder mair & \\
\hline 12 & 1 & WSW & $\mathrm{S}^{\mathrm{s}}$ & & $\overline{5_{3}}$ & & & & & & \\
\hline \multirow{2}{*}{\multicolumn{3}{|c|}{$\begin{array}{c}\text { Variation } \\
\text { of } \\
\text { Compass } \\
\text { I rhumbl, E }\end{array}$}} & \multicolumn{6}{|c|}{ In 24 hours } & \multicolumn{3}{|c|}{ From Vaua } \\
\hline & & & $\stackrel{\vec{E}}{\underline{E}}$ & 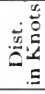 & 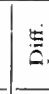 & 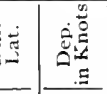 & 象是 & 苞 & 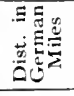 & 莫 & $\stackrel{\infty}{\circ}$ \\
\hline \multicolumn{3}{|c|}{$\begin{array}{l}\text { 13y l)ead } \\
\text { Reckoning }\end{array}$} & $\begin{array}{l}\text { WSW } \\
0^{\circ} 39^{\prime} \text { ' }\end{array}$ & 16 & & $\begin{array}{l}\text { IV } \\
1.4 .5\end{array}$ & $22^{\prime}$ & $49^{\circ} 41^{\prime}$ & 24613 & $\begin{array}{c}\mathrm{E} / \mathrm{S} \\
0^{\circ} \mathrm{O}^{\prime} \mathrm{S}\end{array}$ & $25^{\circ} .18^{\prime}$ \\
\hline
\end{tabular}


6 October 6, I741. After Midday

\begin{tabular}{|c|c|c|c|c|c|c|c|c|c|c|c|c|}
\hline 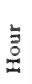 & 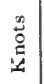 & $\underset{Z}{Z}$ & \multicolumn{2}{|c|}{ 㟧 } & 釆 & & & & & & & \\
\hline 1 & $11 / 2$ & WSW & \multicolumn{2}{|c|}{$\mathrm{S} / 2 \mathrm{E}$} & $5: 2$ & \multicolumn{7}{|c|}{$\begin{array}{l}\text { Stormy, clear with passing clouds, wet; carrying } \\
\text { trysail and main-staysail. }\end{array}$} \\
\hline 2 & $13 / 2$ & & & & $5 \div 2$ & & & & & & & \\
\hline 3 & $13 / 2$ & SIV II & \multicolumn{2}{|c|}{$\mathrm{S}, \mathrm{E}^{1} \mathrm{E}$} & $5 \frac{1 / 2}{\longrightarrow}$ & & & & & & & \\
\hline 4 & $11 / 2$ & & & & $51 / 2$ & \multicolumn{7}{|c|}{ Reefed trysail. } \\
\hline 5 & $1 \%$ & & & & 6 & \multicolumn{7}{|c|}{ Squalls. } \\
\hline 6 & $13 / 2$ & & & & 6 & \multicolumn{7}{|c|}{ Heavy clouds, cluilly. } \\
\hline 7 & $11 / 2$ & & & & 6 & \multicolumn{7}{|c|}{ Waves from both sides washing over the deck. } \\
\hline 8 & $1 \mathrm{~T} / 3$ & & & & 6 & \multicolumn{7}{|c|}{ 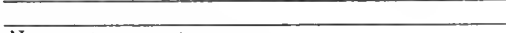 } \\
\hline 9 & $13 / 2$ & SW" & $\mathrm{SSE}$ & $2 \mathrm{E}$ & 6 & \multicolumn{7}{|c|}{ Heavy storm, wet. } \\
\hline 10 & 15 & & & & $6+$ & \multicolumn{7}{|c|}{ Hauled down main-staysail. } \\
\hline 11 & $11 / 2$ & & & & $6 \frac{1}{2}$ & & & & & & & \\
\hline 12 & $\mathrm{I} / 2$ & & & & $6^{1}$ & \multicolumn{7}{|c|}{ Clear with passing clonds. } \\
\hline 1 & 18 & & & & $61 / 2$ & \multirow{2}{*}{\multicolumn{7}{|c|}{ (2) }} \\
\hline 2 & $13 / 2$ & & & & $61 / 2$ & & & & & & & \\
\hline 3 & $1 \%$ & WSW & $\mathrm{S}$ & & 613 & \multicolumn{7}{|c|}{ W'ind going down a bit. } \\
\hline 4 & $11 / 3$ & & & & $61 / 2$ & & 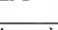 & $\ldots$ & 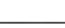 & & & \\
\hline 5 & 112 & & & & $61 / 2$ & \multicolumn{7}{|c|}{ Light rain. } \\
\hline 6 & 13 & & & & $61 / 2$ & \\
\hline 7 & $11 / 2$ & W/S & $\mathrm{St}$ & & 6 & \multicolumn{7}{|c|}{ Stormy, set main-staysail. } \\
\hline 8 & $18 / 3$ & & & & 6 & & & & & & & \\
\hline 9 & $1 \div 4$ & & & & 6 & \multicolumn{7}{|c|}{ Clear with passing clouds. } \\
\hline 10 & $1+4$ & & & & 6 & & & & & & & \\
\hline 11 & $11_{4}$ & & & & 6 & \multicolumn{4}{|c|}{ Weather as before, wet. } & & & \\
\hline & $1 \%$ & & & & 6 & \multicolumn{7}{|c|}{ Heavy westerly swell. } \\
\hline \multirow{2}{*}{\multicolumn{3}{|c|}{$\begin{array}{c}\text { Variation } \\
\text { of } \\
\text { Compass } \\
1 . \text { rhumb E }\end{array}$}} & \multicolumn{7}{|c|}{ In 24 hours } & \multicolumn{3}{|c|}{ From Vaua } \\
\hline & & & $\stackrel{\stackrel{\Xi}{\Xi}}{\Xi}$ & 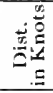 & 象 & & 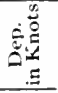 & 空苔 & ثึ & 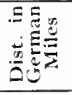 & $\stackrel{\hat{\Xi}}{a}$ & 范 \\
\hline $\begin{array}{l}\mathrm{B} \\
\mathrm{R} \\
\end{array}$ & $\begin{array}{l}\text { Der } \\
\text { ckon }\end{array}$ & & ESE & 33 & 12. & & $\begin{array}{c}\mathrm{E} \\
30.4 \\
\end{array}$ & $45.7^{\prime}$ & $49^{\circ} 29^{\prime}$ & $255^{1 / 4}$ & $\begin{array}{l}\mathrm{E} / \mathrm{S} \\
0^{\circ} 2 I^{\prime} \mathrm{S}\end{array}$ & $26^{\circ} 34^{\prime}$ \\
\hline
\end{tabular}




\section{ఫ October 7, I741. After Midday}

\begin{tabular}{|c|c|c|c|c|c|c|c|c|c|c|c|c|}
\hline$\stackrel{5}{3}$ & 常 & $\bar{\Xi}$ & 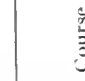 & & 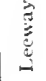 & & & & & & & \\
\hline$\overline{1}$ & $\overline{I_{32}}$ & W' & $\mathrm{Si}_{2}$ & & 6 & & $\begin{array}{l}\text { ayy s } \\
\text { trysaa }\end{array}$ & 1 and, & $\begin{array}{l}\text { lear wh } \\
\text { lain-sta }\end{array}$ & $\begin{array}{l}\text { th passi } \\
\text { ysail. }\end{array}$ & $\mathrm{g}$ cloud; & carry- \\
\hline 2 & $1 ! 2$ & & & & 6 & & & & & & & \\
\hline 3 & $1 \%$ & & & & 6 & & illy. & & & & & \\
\hline 4 & $\overline{1 \% 2}$ & & & & $5^{1 / 2}$ & & & & & & & \\
\hline 5 & $\overrightarrow{\mathrm{I} \cdot 4}$ & & & & $51 / 2$ & & rrific $s$ & orm. & & & & \\
\hline 6 & $\mathrm{I}^{3} 4$ & & & & 51 & & & & & & & \\
\hline 7 & $53_{4}$ & & & & $51 / 2$ & & ualls a & ad rain & & & & \\
\hline 8 & $I^{3} 4$ & & & & $51 / 2$ & & & & & & & \\
\hline 9 & $\overline{1 \%}$ & & & & 6 & & & & & & & \\
\hline 10 & $1 \frac{1 / 2}{4}$ & & & & 6 & & & & & & & \\
\hline 11 & $1 \div$ & & & & 6 & & $\mathrm{rm} \mathrm{go}$ & ng dov & n a bit & & & \\
\hline 12 & $\overline{\mathrm{I}^{1} 4}$ & IV'SI & $\mathrm{Si}$ & & 6 & & & & & & & \\
\hline$I$ & $I$ & WV & $S^{1}$ & & 6 & & idersail & wind. & & & & \\
\hline 2 & 1 & & & & 5 & & & & & & & \\
\hline 3 & $\overline{11 / 2}$ & WV & S II & $12 \mathrm{~W}$ & 5 & & main & ail, loy & ered $\mathrm{m}$ & ain-stays & & \\
\hline 4 & $\overline{\mathrm{I}^{1 / 2}}$ & & & & 5 & & & & & & & \\
\hline 5 & $\overline{1 \%}$ & $1 \mathrm{WN}$ & SW & & 5 & & ef-tops & ail win & d, cold. & & & \\
\hline 6 & $2^{\prime}$ & NW & SW & & $1^{3 / 4}$ & & fores: & il and & eefed $t$ & opsails. & & \\
\hline 7 & $2 !+4$ & & & & $\overline{13 / 4}$ & & & & & & & \\
\hline 8 & 2 & & SW & & $\overline{1^{3} 4}$ & & inled u & ptopm & ast-stay & sails. & & \\
\hline 9 & $21 / 4$ & & SW & & $13 / 4$ & & $\begin{array}{l}\text { in, he } \\
\text { inse , } \\
\text { ling of } \\
\text { ingude } \\
\text { tount }\end{array}$ & $\begin{array}{l}\text { avy we } \\
\text { as ke } \\
f^{4} \text { to. } \\
48^{\circ}+3^{\prime} \\
v^{2} 43^{2}\end{array}$ & $\begin{array}{l}\text { sterly } \\
\text { t bet } \\
\text { fook a } \\
\text { but on } \\
\text { en of it. }\end{array}$ & $\begin{array}{l}\text { well; du } \\
\text { een con } \\
\text { abserv } \\
\text { accoun }\end{array}$ & $\begin{array}{l}\text { ing the d } \\
\text { ing up } \\
\text { tion whic } \\
\text { of the s }\end{array}$ & $\begin{array}{l}\text { ay the } \\
\text { o and } \\
\text { gave } \\
\text { ell no }\end{array}$ \\
\hline 10 & $2 ! 3$ & & SW & W & $1^{3} 4$ & & & & & & & \\
\hline 11 & $2 ! 2$ & & & & $\mathrm{I}^{34}$ & & & & & & & \\
\hline 12 & 234 & & & & $\mathrm{I}^{3} 4_{4}$ & & & & & & & \\
\hline \multirow{2}{*}{\multicolumn{3}{|c|}{$\begin{array}{c}\text { Variation } \\
\text { of } \\
\text { Compass } \\
1^{1}+\text { rhumb E }\end{array}$}} & \multicolumn{7}{|c|}{ In 24 hours } & \multicolumn{3}{|c|}{ From Vaua } \\
\hline & & & Е & $\begin{array}{ll}0.5 \\
0\end{array}$ & & & 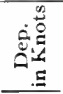 & 电曷 & 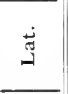 & 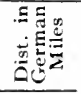 & $\begin{array}{l}\text { 咅 } \\
\text { 吾 }\end{array}$ & 离 \\
\hline \multicolumn{3}{|c|}{$\begin{array}{l}\text { Hy Dead } \\
\text { Reckoning }\end{array}$} & $8^{\circ} \mathrm{S}$ & 30 & & & $\begin{array}{c}E \\
4.5\end{array}$ & $6.8^{\prime}$ & $48^{\circ} 59^{\prime}$ & 259 & $\underset{1^{\circ}{ }_{5} / \mathrm{E} 2^{\prime} \mathrm{S}}{\mathrm{E} / \mathrm{S}}$ & $26^{\circ}{ }_{41}$ \\
\hline
\end{tabular}


2 October 8. I74I. After Midday

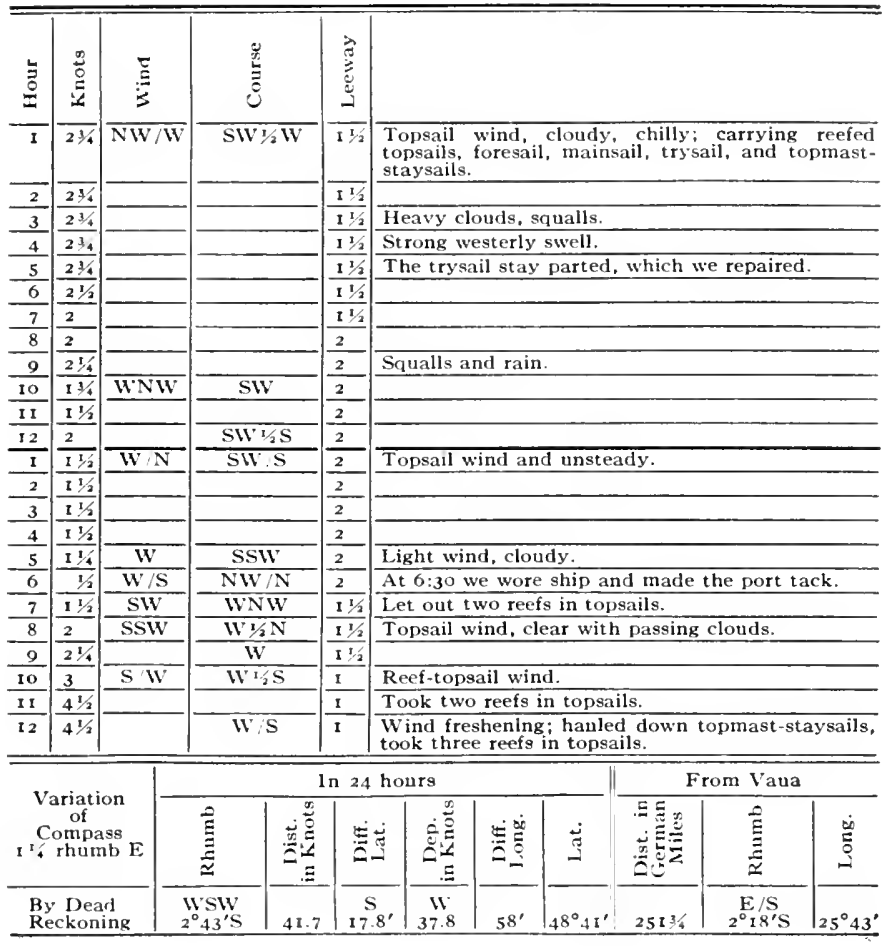


October 9, I74I. After Midday

\begin{tabular}{|c|c|c|c|c|c|c|c|c|c|c|c|c|}
\hline$\stackrel{\stackrel{s}{0}}{=}$ & $\stackrel{3}{0}$ & $\vec{E}$ & \multicolumn{2}{|c|}{ 总 } & 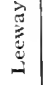 & & & & & & & \\
\hline$I$ & $\sqrt{42}$ & $\triangle E$ & \multicolumn{2}{|c|}{ WSW } & I & \multicolumn{7}{|c|}{$\begin{array}{l}\text { Topsail wind, cold, drizzly; carrying reefed top- } \\
\text { sails, foresail, mainsail, trysail. }\end{array}$} \\
\hline 2 & $\overline{3 x_{2}}$ & & & & $\mathrm{I}$ & \multicolumn{7}{|c|}{ Furled fore-topsail because of the gale. } \\
\hline 3 & $\overline{3^{2}}$ & & & & $\overline{\mathrm{I}^{\mathrm{I} / 2}}$ & \multicolumn{7}{|c|}{ Furled main-topsail. } \\
\hline+ & $\mathrm{I}_{4}$ & & & & $11 / 2$ & \multirow{2}{*}{\multicolumn{7}{|c|}{$\begin{array}{l}\text { At } 5: 30 \text { furled foresail and hove to under main- } \\
\text { staysail and trysail. }\end{array}$}} \\
\hline 3 & 2 & & \multicolumn{2}{|c|}{ SW Wt/2W } & 6 & & & & & & & \\
\hline 6 & $\overline{\mathrm{I}^{2}}$ & & & & 6 & & & & & & & \\
\hline 7 & $\overline{1_{2}}$ & SSW & \multicolumn{2}{|c|}{$\mathrm{W}^{2} \mathrm{~N}$} & 5 & \multicolumn{7}{|c|}{ Heavy swell from south. } \\
\hline 8 & $1^{1+2}$ & & & & 5 & \multicolumn{7}{|c|}{ Set mainsail, lowered main-staysail. } \\
\hline 9 & $\mathrm{I}^{3}$ & & & & 5 & \multicolumn{7}{|c|}{ Drizzly and rainy. } \\
\hline 10 & $\overline{1_{2}}$ & & & & 5 & \multicolumn{7}{|c|}{ Wind freshening. } \\
\hline II & $\overline{\mathrm{I}^{2}}$ & & & & 6 & \\
\hline 12 & $\sqrt{1^{1}}$ & SW S & \multicolumn{2}{|c|}{ W'NW!_W } & 6 & \multicolumn{7}{|c|}{ Furled mainsail, hauled up main-staysail. } \\
\hline $\mathrm{I}$ & $\overline{I_{2}}$ & & & & 6 & & & & & & & \\
\hline 2 & $\overline{I^{2}}$ & SW & \multicolumn{2}{|c|}{$\mathrm{NW} / \mathrm{W}_{2} \mathrm{~W}$} & 6 & \multicolumn{7}{|c|}{ Terrific storm. } \\
\hline 3 & $I^{12}$ & & & & 6 & \\
\hline 4 & $\sqrt{1^{2}}$ & & & & 6 & \multirow{2}{*}{\multicolumn{7}{|c|}{ Heavy squalls. }} \\
\hline 5 & $\overline{I^{2}}$ & WSW & \multicolumn{2}{|c|}{$\mathrm{NW}_{2} \mathrm{~N}$} & 6 & & & & & & & \\
\hline 6 & $\overline{\mathrm{I}^{2}}$ & & \multirow{2}{*}{\multicolumn{2}{|c|}{ NNW }} & 6 & \\
\hline 7 & $1 ! 2$ & II $/ \mathrm{S}$ & & & 6 & \multicolumn{7}{|c|}{$\begin{array}{l}\text { The lanyard of the main shroud on the left side } \\
\text { parted, which we repaired. }\end{array}$} \\
\hline 8 & $\overline{I^{12}}$ & IV & \multicolumn{2}{|c|}{$\mathrm{N} \mathrm{Wr} W^{\prime}$} & 6 & \multirow{3}{*}{\multicolumn{7}{|c|}{$\begin{array}{l}\text { Terrific gale blowing; hauled down main-staysail, } \\
\text { carrying only the reefed trysail. }\end{array}$}} \\
\hline 9 & $\overline{I^{3}+4}$ & $\mathrm{~W} / \mathrm{N}$ & \multicolumn{2}{|c|}{$\mathrm{N}+2 \mathrm{~W}$} & $6 / 2$ & & & & & & & \\
\hline Io & $1^{3}+4$ & & & & $65 / 2$ & & & & & & & \\
\hline 11 & $\overline{I^{2} 4}$ & & & & $61 / 2$ & \multirow{2}{*}{\multicolumn{7}{|c|}{$\begin{array}{l}\text { Heavy squalls, and the lee side of the ship is } \\
\text { under water. }\end{array}$}} \\
\hline 12 & $I^{3}+$ & & & & $\overline{65}$ & & & & & & & \\
\hline \multirow{2}{*}{\multicolumn{3}{|c|}{$\begin{array}{l}\text { Variation } \\
\text { of } \\
\text { Compass } \\
1+4 \text { rhumb } 1 \text { E }\end{array}$}} & & & $\ln 24$ & ho & & & & & om Vaua & \\
\hline & & & $\stackrel{\vec{E}}{\underline{\Xi}}$ & 这 & 结 & & 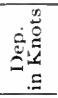 & 里突 & 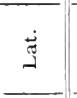 & 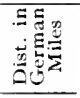 & 营 & $\stackrel{\dot{u}}{\Xi}$ \\
\hline & $\begin{array}{l}y \text { 1)e: } \\
\text { eckons }\end{array}$ & & $\mathrm{N}$ & 22 & 21 & & $\begin{array}{l}\text { W } \\
3.3\end{array}$ & $5^{\prime}$ & $49^{\circ} \mathrm{O}_{3}^{\prime}$ & 249 & $\begin{array}{c}\mathrm{E} / \mathrm{S} \\
{ }^{\circ}{ }^{\prime} 0^{\prime} \mathrm{S}\end{array}$ & $25^{\circ} 38^{\prime}$ \\
\hline
\end{tabular}


b) October IO, I74I. After Midday

\begin{tabular}{|c|c|c|c|c|c|c|c|c|c|c|c|c|}
\hline$\stackrel{\Xi}{\Xi}$ & 咅 & 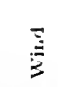 & $\stackrel{\mathscr{2}}{\mathscr{3}}$ & & 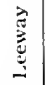 & & & & & & & \\
\hline$I$ & $\mathrm{IT}_{2}$ & WN1 & $\mathrm{N}_{1 / 2}$ & & 6 & & $\mathrm{rm}$ an & squa & s, clear & with pa & ing clou & \\
\hline 2 & 1.2 & & & & 6 & & & & & & & \\
\hline 3 & $I^{2}$ & & & & 6 & & rrying & eefed & rysail. & & & \\
\hline 4 & $\overline{I^{1 / 2}}$ & & & & 6 & & & & & & & \\
\hline 5 & $1: 2$ & & & & 6 & & ick clo & $\mathrm{ds}, \mathrm{ra}$ & & & & \\
\hline 6 & $\overline{\mathrm{I}_{2}}$ & & & & 6 & & & & & & & \\
\hline 7 & $11 / 2$ & $W / 2$ & $\mathrm{Nt}_{2}$ & & $5+2$ & & $\mathrm{rm}$. & & & & & \\
\hline 8 & 135 & & & & $5 \%$ & & uled u & main & staysail & & & \\
\hline 9 & $11_{2}$ & & & & 5.2 & & il, squ & IIs. & & & & \\
\hline 10 & 1,2 & & & & $5+2$ & & & & & & & \\
\hline 11 & $\overline{I^{x}}$ & & & & 6 & & rific $s$ & $\mathrm{rm} .1$ & wered I & ain-sta & ail. & \\
\hline$\sqrt{2}$ & $\mathrm{I}^{\mathrm{t}}$ & & & & 6 & & & & & & & \\
\hline $\mathbf{I}$ & $\overline{152}$ & & & & 6 & & & & & & & \\
\hline 2 & $\overline{I_{2}}$ & & & & 6 & & & & & & & \\
\hline$\sqrt{3}$ & $\overline{15 / 2}$ & & & & 6 & & $\begin{array}{l}\text { ve di } \\
\text { stron }\end{array}$ & $\begin{array}{l}\text { culty } \\
\text { wind }\end{array}$ & in car & ying tr & sail beca & se of \\
\hline 4 & $\overline{\mathrm{It}_{2}}$ & WNI & $\mathrm{NI}$ & & 6 & & & & & & & \\
\hline 5 & $\overline{1 \cdot 2}$ & $11 \%$ & $N$ & & 6 & & ghtful & quall & & & & \\
\hline 6 & 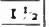 & & & & 6 & & & & & & & \\
\hline 7 & 13 & & & & 6 & & w. co & & & & & \\
\hline 8 & 19 & & & & 6 & & & & & & & \\
\hline 9 & 12 & & & & 6 & & ar wit & pass & gg clouc & & & \\
\hline 10 & $\begin{array}{ll}112 \\
\end{array}$ & & & & 6 & & & & & & & \\
\hline 11 & $I \div 2$ & & & & 6 & & $\begin{array}{l}\text { avyse } \\
\text { h side }\end{array}$ & runr & ing, wa & hing ox & the dec & from \\
\hline 12 & 13 & & & & & & & & & & & \\
\hline \multirow{2}{*}{\multicolumn{3}{|c|}{$\begin{array}{c}\text { Variation } \\
\text { of } \\
\text { Compass } \\
1 \text {, rhumb } \mathrm{E}\end{array}$}} & \multicolumn{7}{|c|}{ In 2.4 hours } & \multicolumn{3}{|c|}{ From Vaua } \\
\hline & & & 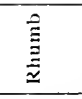 & 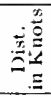 & $\underline{2}$ & 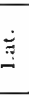 & 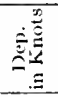 & 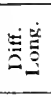 & క్ & 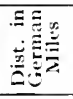 & $\underline{\Xi}$ & 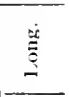 \\
\hline \multicolumn{3}{|c|}{$\begin{array}{l}\text { By Dead } \\
\text { Reckoning }\end{array}$} & $\underset{10}{\mathrm{E} / \mathcal{H}^{\prime} \mathrm{E}}$ & 34 & .6 & 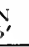 & $\begin{array}{c}\mathrm{E} \\
33.4\end{array}$ & $5 I^{\prime}$ & $49^{\circ} 00^{\prime}$ & 256 & $\underset{I^{\circ}}{E}, 7^{\prime} S$ & $26^{\circ} 29^{\prime}$ \\
\hline
\end{tabular}


October II, I741. After Midday

\begin{tabular}{|c|c|c|c|c|c|c|c|c|c|c|c|c|}
\hline$\stackrel{\bar{\Xi}}{\underline{3}}$ & 旁 & 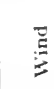 & & 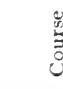 & & 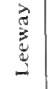 & & & & & & \\
\hline 1 & $I 1 / 2$ & W' & & $\mathrm{N}=$ & & 6 & $\begin{array}{l}\text { Storm, so } \\
\text { ing trysai }\end{array}$ & ualls, & clear w & th pass & g clouds; & carry- \\
\hline 2 & $11 / 2$ & & & & & 6 & & & & & & \\
\hline 3 & $I^{1 / 2}$ & & & & & 6 & & & & & & \\
\hline 4 & $\mathrm{I}^{\mathrm{I}}+$ & IV & & $\mathrm{NWW}$ & WW & 6 & Storm. & & & & & \\
\hline 5 & $\overline{I^{2}}$ & & & & & 6 & Hoisted I & ain-st: & aysail. & & & \\
\hline 6 & $\mathrm{I}^{\mathrm{I}}+$ & & & & & 6 & & & & & & \\
\hline 7 & $I^{2}+$ & & & & & 6 & & & & & & \\
\hline 8 & $I$ & & & & & 6 & Reef-und & rsail y & vind. & & & \\
\hline 9 & $\mathrm{I}$ & & & & & 6 & Cloudy. & & & & & \\
\hline 10 & $I$ & & & $\mathrm{~N}$ & & 6 & Gusts of & vind, 1 & reavy $\mathrm{cl}$ & ouds. & & \\
\hline 11 & $I$ & & & & & 6 & & & & & & \\
\hline 12 & $I$ & & & & & 6 & Stars out & chilly & & & & \\
\hline $\bar{I}$ & 3 & & & & & $6 ! 2$ & & & & & & \\
\hline 2 & $3 / 4$ & & & & & $61 / 2$ & Undersail & wind, & westerl & swell. & & \\
\hline 3 & $-1 / 2$ & & & $\mathrm{~N} / \mathrm{W}$ & $2 \mathrm{~W}$ & $5 . / 2$ & Set main & ail. & & & & \\
\hline 4 & $1 / 2$ & & & & & $5 \%$ & Hauled d & own $m$ & ain-stay & sail. & & \\
\hline 5 & $I ! / 2$ & $\mathrm{~W} /$ & & $\mathrm{SW}$ & & $1 y^{2}$ & Reef-tops & ail win & id, set fo & & & \\
\hline 6 & 234 & NW & & SW' & & $11 / 2$ & $\begin{array}{l}\text { Came ab } \\
\text { board tac }\end{array}$ & kut wit & the & ind an & lay on th & e star- \\
\hline 7 & 214 & W/ & & SW & & $x^{7}$ & Set topsa & 11 reef & ed, hau & led up & pmast-st & ysails. \\
\hline 8 & $23 / 4$ & WN & & Si & & $11 / 2$ & & & & & & \\
\hline 9 & $21 / 2$ & & & & & $1 \%$ & & & & & & \\
\hline 10 & $21 / 2$ & & & & & $15 / 2$ & Let out a & reef in & topsail & & & \\
\hline 11 & $2 ! 2$ & & & & & $11 / 2$ & & & & & & \\
\hline 12 & 234 & & & & & $1 / 2$ & $\begin{array}{l}\text { Reef-tops } \\
\text { from noo } \\
\text { the cour } \\
\text { to and fa } \\
\text { tion: ze } \\
\text { sun } 10^{\circ} 5 \\
\text { this obse }\end{array}$ & $\begin{array}{l}\text { ail wi } \\
\text { nil to } 5 \\
\text { e on } \\
\text { ling of } \\
\text { ith di } \\
\text {, latit } \\
\text { vation } \\
\end{array}$ & $\begin{array}{l}\text { ind, cle } \\
\text { o'clock } \\
\text { the ave } \\
\text { it to the } \\
\text { stance } \\
\text { ude } 48^{\circ} \\
\text { we kep } \\
\end{array}$ & $\begin{array}{l}\text { ar with } \\
\text { in the } \\
\text { rage be } \\
\text { wind, } \\
\text { ond } \\
9^{\circ} 4^{\prime} \text {. Fr } \\
\text { the co }\end{array}$ & $\begin{array}{l}\text { passing } \\
\text { orning } \\
\text { veen com } \\
\text { ook an o } \\
\text { climation } \\
\text { a the Iot } \\
\text { se by rec }\end{array}$ & $\begin{array}{l}\text { clouds: } \\
\text { e kept } \\
\text { ing up } \\
\text { bserva- } \\
\text { of the } \\
\text { h until } \\
\text { koning. }\end{array}$ \\
\hline \multirow{2}{*}{\multicolumn{3}{|c|}{$\begin{array}{c}\text { Variation } \\
\text { of } \\
\text { Compass } \\
I \text { r rlumb E }\end{array}$}} & \multicolumn{7}{|c|}{ In 24 hours } & \multicolumn{3}{|c|}{ From Vaua } \\
\hline & & & & $\underset{\underline{\underline{\Xi}}}{\underline{\underline{z}}}$ & 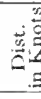 & $\underline{\Xi}$ & 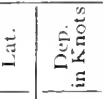 & 里 & تُ & 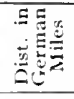 & $\begin{array}{l}\text { है } \\
\text { Е }\end{array}$ & 递 \\
\hline \multicolumn{3}{|c|}{$\begin{array}{l}\text { By Detall } \\
\text { Reckoning }\end{array}$} & & $\begin{array}{l}S S E \\
03^{\prime} \mathrm{E}\end{array}$ & 11 & io & $\begin{array}{r}\mathrm{E} \\
5 \\
\end{array}$ & $8^{\prime}$ & $48^{-5} 9^{\prime}$ & & & $26^{\circ} 31^{\prime}$ \\
\hline \multicolumn{3}{|c|}{$\begin{array}{c}\text { By } \\
\text { Observation }\end{array}$} & & $\begin{array}{l}S_{0} \\
0.3^{\prime} \mathrm{E}\end{array}$ & 01 & 54 & $\begin{array}{c}\mathrm{E} \\
27.5\end{array}$ & $41.7^{\prime}$ & $1.8^{\circ} 15^{\prime}$ & 268 & $\begin{array}{c}\mathrm{E} / \mathrm{S} \\
3^{\circ} 50^{\prime} \mathrm{S}\end{array}$ & $27^{\circ} 1^{\prime}$ \\
\hline
\end{tabular}


(3 October 12, 1741. After Midday

\begin{tabular}{|c|c|c|c|c|c|c|c|c|c|c|c|}
\hline$\stackrel{\frac{3}{3}}{2}$ & 总 & $\stackrel{\vec{g}}{\stackrel{\Xi}{3}}$ & \multicolumn{2}{|c|}{ 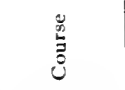 } & 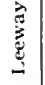 & \multicolumn{6}{|c|}{ - } \\
\hline 1 & $23 / 4$ & NW:W & \multicolumn{2}{|c|}{ SW $\% W$} & $1: 1 / 4$ & \multicolumn{6}{|c|}{$\begin{array}{l}\text { Topsail wind, cloudy; carrying topsails and lower } \\
\text { sails reefed and topmast-staysails. }\end{array}$} \\
\hline 2 & 2 & & \multirow{2}{*}{\multicolumn{2}{|c|}{$\square$}} & $1 \frac{13}{1 / 4}$ & \\
\hline 3 & 2 & & & & $I \frac{1 / 4}{1 / 4}$ & & & & & & \\
\hline 4 & 2 & & \multicolumn{2}{|c|}{ SW W } & $1 \%$ & \multirow{2}{*}{\multicolumn{6}{|c|}{ Topgallantsail wind; let out two reefs in topsails. }} \\
\hline 5 & I1/2 & WNW & & $1 \%$ & & & & & & \\
\hline 6 & $11 / 2$ & & & & I $1 / 2$ & & & & & & \\
\hline 7 & $1 \%$ & & \multicolumn{2}{|c|}{ SW/2S } & 2 & \multicolumn{6}{|c|}{ Took two reefs in topsails. } \\
\hline 8 & $11 / 2$ & & & & 2 & \multirow{2}{*}{\multicolumn{6}{|c|}{ Light wind. }} \\
\hline 9 & $\bar{I}$ & & & & 2 & & & & & & \\
\hline 10 & & \multirow{3}{*}{ 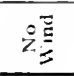 } & & & & \\
\hline II & & & & & & \multicolumn{6}{|c|}{$\begin{array}{l}\text { Clewed up mainsail and foresail. } \\
\text { Northwesterly swell. }\end{array}$} \\
\hline 12 & & & & & & \multicolumn{6}{|c|}{ Lowered staysails } \\
\hline $\bar{I}$ & $3 / 4$ & s & \multicolumn{2}{|c|}{ W S } & & \multicolumn{6}{|c|}{$\begin{array}{l}\text { Light wind; set foresail and mainsail, hoisted } \\
\text { topmast-staysail. }\end{array}$} \\
\hline 2 & 2 & SSE & & & & \multirow{2}{*}{\multicolumn{6}{|c|}{ Let out second reef in topsails. }} \\
\hline 3 & $41 / 2$ & & & & & & & & & & \\
\hline 4 & 4 & ESE & & & & \multicolumn{6}{|c|}{ Cloudy, rainy. } \\
\hline 5 & $5^{2}+$ & & & & & \multirow{2}{*}{\multicolumn{6}{|c|}{ Reef-topsail wind. }} \\
\hline 6 & 6 & $\mathrm{SE}$ & & & & & & & & & \\
\hline 7 & 73 & & & & & \multicolumn{6}{|c|}{ Hauled down topmast-staysails. } \\
\hline 8 & $7 \%$ & SSE & & & & \multicolumn{6}{|c|}{ Took second reef in topsails. } \\
\hline 9 & $3 \%$ & SSW & \multicolumn{2}{|c|}{ W' } & $\overline{34}$ & \multicolumn{6}{|c|}{ Drizzly, rainy. } \\
\hline 10 & $31 / 2$ & & & & 3 & \multicolumn{6}{|c|}{ Reef-topsail wind. } \\
\hline II & $31 / 2$ & & & & $3 / 4$ & \multicolumn{6}{|c|}{ Weather as before. } \\
\hline 12 & $3 \frac{3}{4}$ & $\mathrm{SW}$ & II & & & \multicolumn{6}{|c|}{ Hoisted fore-topmast-staysail. } \\
\hline & & & & & n 24 & hours & & & & m Vaua & \\
\hline & $\begin{array}{l}\text { ariati } \\
\text { of } \\
\text { ompa } \\
\text { rhum }\end{array}$ & $\begin{array}{l}\text { on } \\
\text { sss } \\
\mathrm{ab} E\end{array}$ & 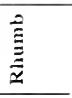 & 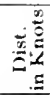 & $\stackrel{\Xi}{=}$ & 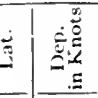 & 递总 & 元 & 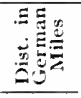 & $\stackrel{\stackrel{\Xi}{E}}{\Xi}$ & $\stackrel{\dot{\phi}}{\stackrel{\dot{\theta}}{0}}$ \\
\hline & $\begin{array}{l}\text { Dea } \\
\text { ckon }\end{array}$ & & ${ }^{\circ} \mathrm{W}$ & 60 & e & $\begin{array}{c}W \\
59.8 \\
\end{array}$ & $89.7^{\prime}$ & $48^{\circ} \mathrm{ro}$ & $25+3 \div 4$ & $\begin{array}{c}\text { E/S } \\
4^{\circ} 56^{\prime} \mathrm{S}\end{array}$ & $25^{\circ} 4 I^{\prime}$ \\
\hline
\end{tabular}


Actober I3, I74I. Afler Midday

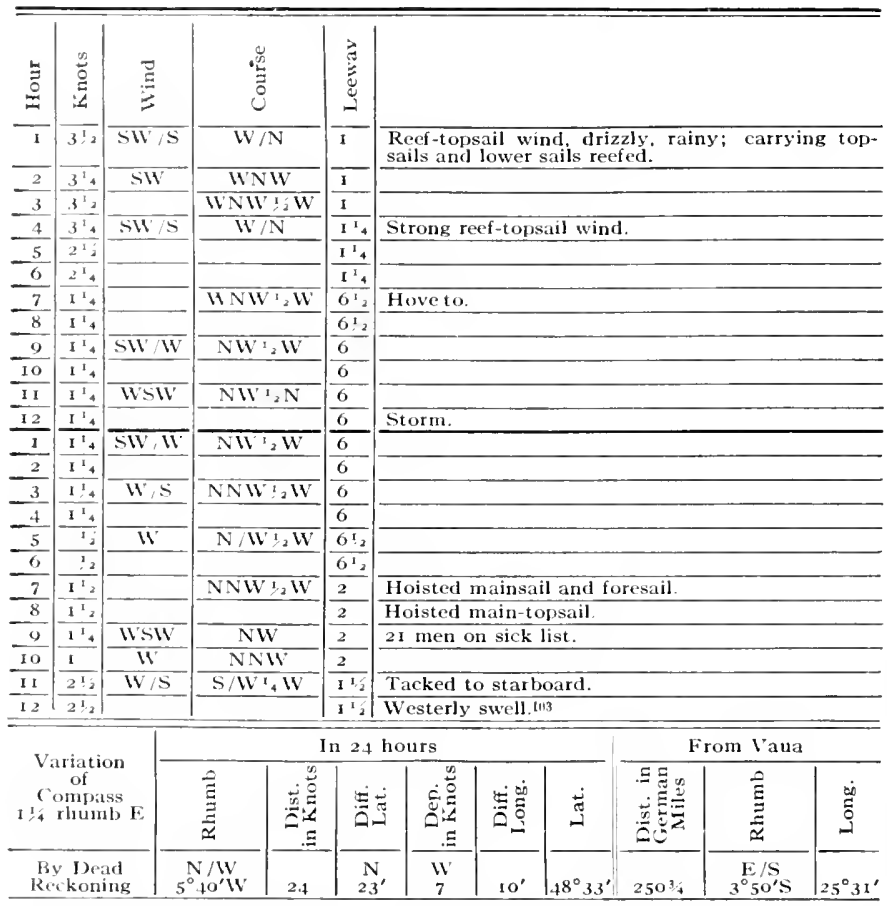

103 Khitrov's journal gives latitude by observation, $48^{\circ} 37^{\prime}$, used on the chart (Pl. I). 
$\Varangle$ October 14, 1741. Afler Midday

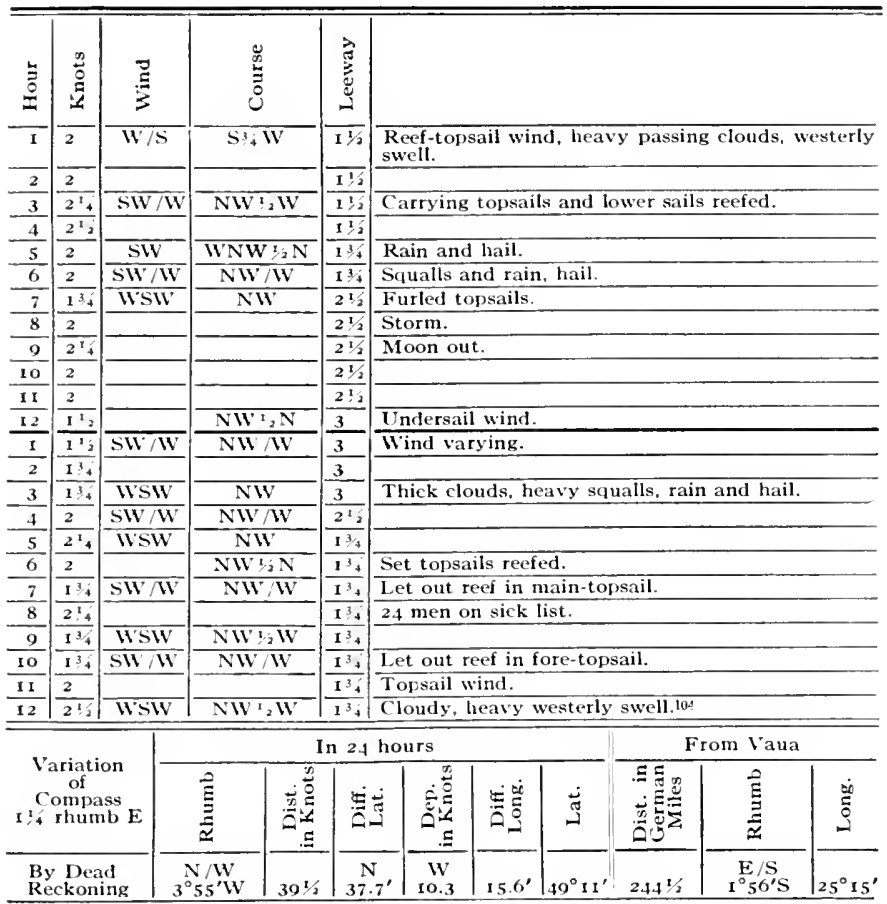

104 Khitrov's journal gives latitude by observation, $49^{\circ} 17^{\prime}$, used on the chart (Pl. I). 


\section{October 15, 1771. After Midday}

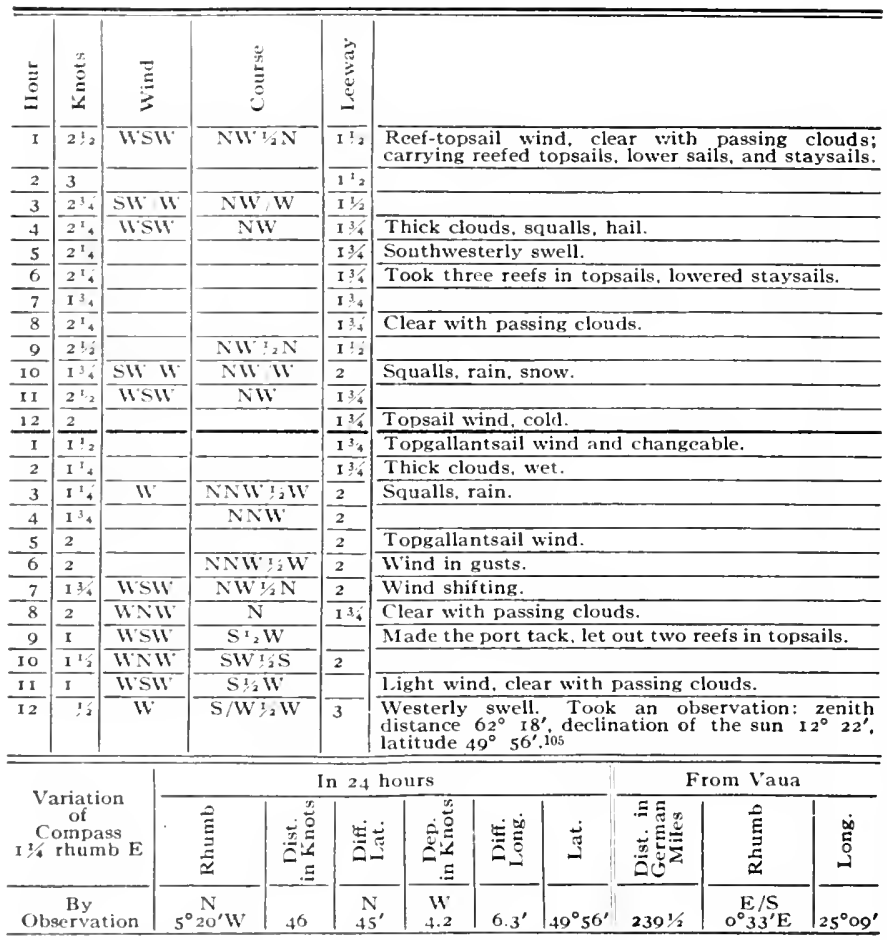

105 Khitrov's journal gives latitude by observation $49^{\circ} 58^{\prime}$. 
+ Oclober 16,1741 . Afler Midday

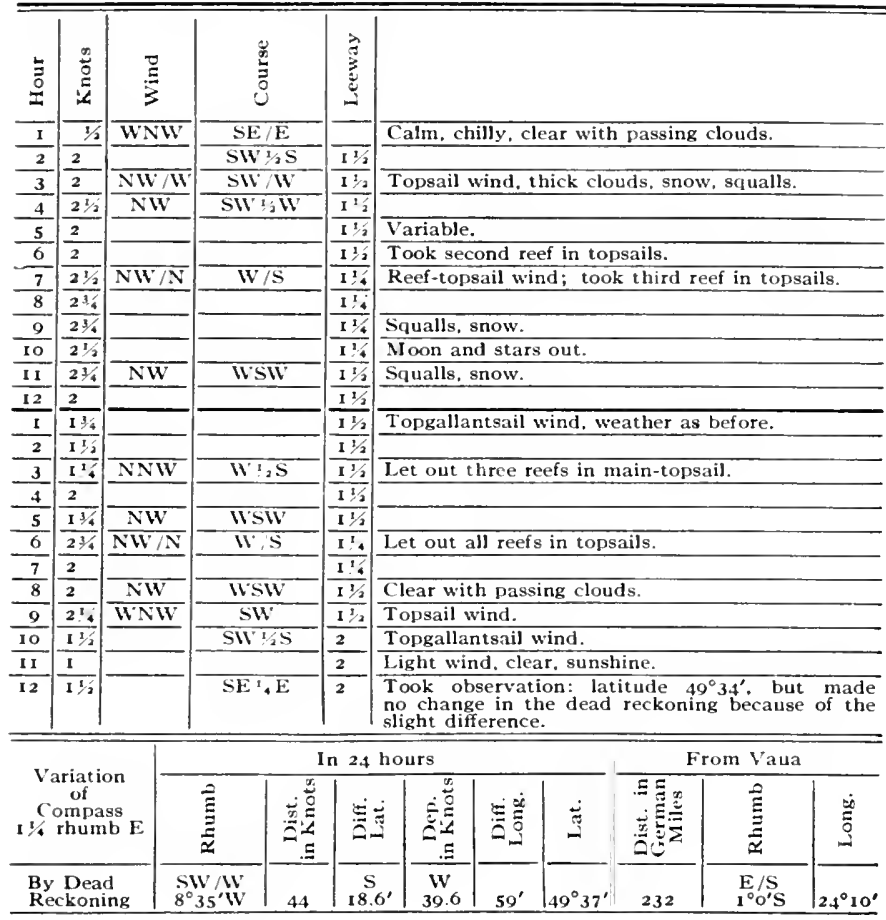


b) October 17, 17+1. After Midday

\begin{tabular}{|c|c|c|c|c|c|c|c|c|c|c|c|}
\hline 管 & $\stackrel{\infty}{\stackrel{0}{a}}$ & 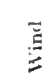 & \multicolumn{2}{|c|}{ 崖 } & 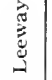 & & & & & & \\
\hline $\bar{E}$ & & \multirow{5}{*}{$\begin{array}{l}\vec{Z} \\
\stackrel{3}{3} \\
0 \\
z\end{array}$} & \multicolumn{2}{|r|}{ 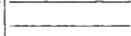 } & & \multicolumn{6}{|c|}{ Clear with passing clouds. } \\
\hline 2 & & & & & & & & & & & \\
\hline$\frac{3}{3}$ & & & & & & \multicolumn{6}{|c|}{$\begin{array}{l}\text { At times calm; repaired various damages caused } \\
\text { by the storm. }\end{array}$} \\
\hline 4 & 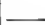 & & & & & \multirow{3}{*}{\multicolumn{6}{|c|}{ Topgallantsail wind; took second reef in topsails. }} \\
\hline 5 & & & & & & & & & & & \\
\hline 0 & 2 & $\mathrm{~s}$ & \multicolumn{2}{|c|}{$\mathrm{W} / \mathrm{S}$} & & & & & & & \\
\hline 7 & $3^{12}$ & S/ 1 & & & & \multicolumn{6}{|c|}{ 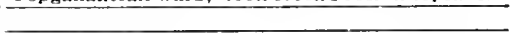 } \\
\hline 8 & 4 & & & & & \multicolumn{6}{|c|}{ Topsail wind. } \\
\hline 9 & 4 & & & & & \multirow{2}{*}{\multicolumn{6}{|c|}{$\begin{array}{l}\text { Drizzly. } \\
\text { Reef-topsail wind, thick clouds, wind freshening. }\end{array}$}} \\
\hline Io & 5 & & & & & & & & & & \\
\hline iI & $6^{1 / 2}$ & & & & & & & & & & \\
\hline 12 & 64 & & & & & \multicolumn{6}{|c|}{ Hauled down main-topmast-staysail. } \\
\hline $\mathrm{I}$ & $\overline{0^{12}}$ & & & & & \multirow{2}{*}{\multicolumn{6}{|c|}{$\begin{array}{l}\text { Cloudy, rainy, wind in gusts; hauled down fore- } \\
\text { topmast-staysail. topsails on the caps. }\end{array}$}} \\
\hline 2 & $6^{\prime 2}$ & & & & & & & & & & \\
\hline 3 & 614 & $\mathrm{SE}$ & & & & & & & & & \\
\hline 4 & $6 \cdot 4$ & & & & & \multirow{2}{*}{\multicolumn{6}{|c|}{ Gale, rain; took third reef in fore-topsail. }} \\
\hline 5 & $61^{2}$ & & & & & & & & & & \\
\hline 6 & $\overline{6^{1}} 4$ & & & & & \\
\hline 7 & $5^{\prime} 4$ & $\mathrm{E}$ & & & & \multicolumn{6}{|c|}{$\begin{array}{l}\text { Furled main-topsail, clewed up foresail because it } \\
\text { was torn in one place. }\end{array}$} \\
\hline 8 & 5 & & & & & \multirow{2}{*}{\multicolumn{6}{|c|}{ Strong reef-undersail wind. }} \\
\hline 9 & $4^{1} 4$ & & & & & & & & & & \\
\hline 10 & 4 & & & & & \multirow{2}{*}{\multicolumn{6}{|c|}{ Wind going down. }} \\
\hline 11 & $3^{3 / 4}$ & & & & & & & & & & \\
\hline 12 & $3^{2}+4$ & & & & & \multicolumn{6}{|c|}{$\begin{array}{l}\text { Let out reef in fore-topsail; reef-topsail wind, } \\
\text { drizzly, rainy. }\end{array}$} \\
\hline \multirow{2}{*}{\multicolumn{3}{|c|}{$\begin{array}{c}\text { Variation } \\
\text { of } \\
\text { Compass } \\
I+\text { rhumb E }\end{array}$}} & \multicolumn{9}{|c|}{ In 24 hours } \\
\hline & & & 葛 & 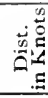 & 苾 & 逗总 & 电蒫 & 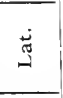 & 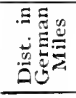 & है & $\stackrel{\infty}{\stackrel{0}{\circ}}$ \\
\hline & $\begin{array}{l}\text { De } \\
\text { eckot }\end{array}$ & & $\mathrm{W}_{4} \mathrm{~N}$ & 95 & $\begin{array}{r}N \\
4.7\end{array}$ & $\begin{array}{l}W \\
94.8\end{array}$ & $142^{\prime}$ & $49^{\circ}+2^{\prime}$ & 210 & $\underset{I^{\circ} 5^{\circ} / \mathrm{S}}{\mathrm{E} / \mathrm{S}}$ & $21^{\circ} 48^{\prime}$ \\
\hline
\end{tabular}


October I8, I74I. After Midday

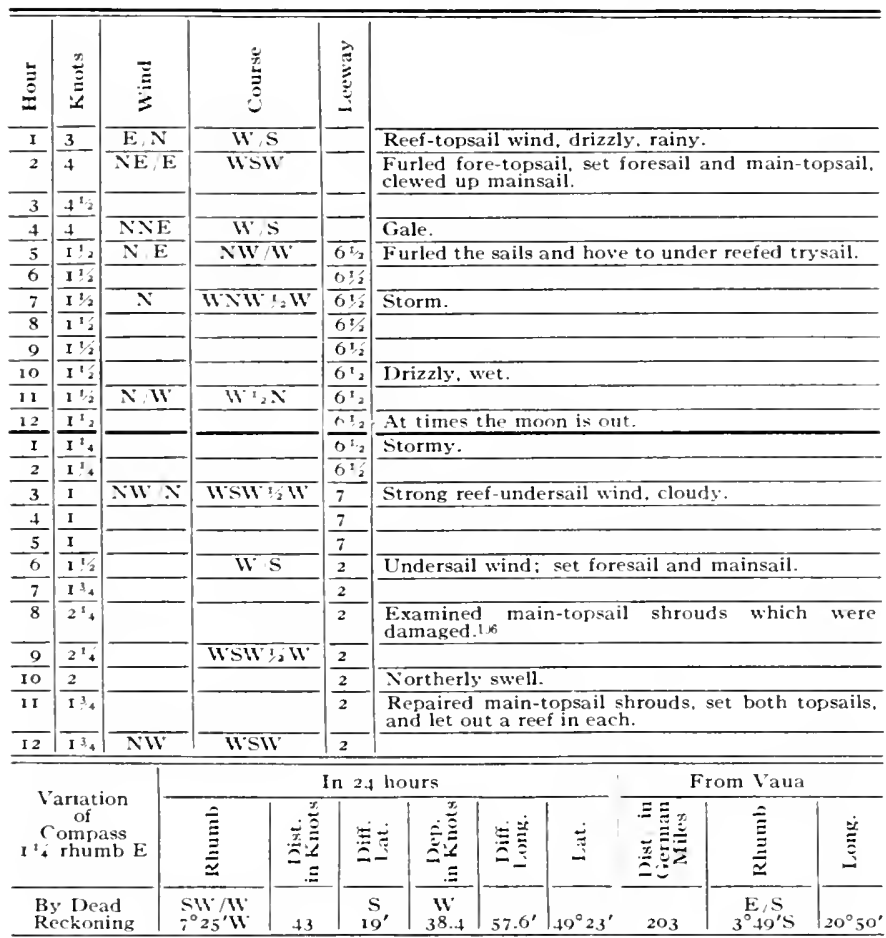

206 Khitrov's journal: "On sick list: Captain Commander and 32 men." 
(3) October I9, I7fI. After Midday

\begin{tabular}{|c|c|c|c|c|c|c|c|c|c|c|c|c|c|}
\hline$\stackrel{5}{3}$ & $\stackrel{3}{3}$ & $\stackrel{\Xi}{E}$ & & $\stackrel{\mathscr{E}}{\Xi}$ & & $\stackrel{2}{2}$ & & & & & & & \\
\hline I & $23_{4}$ & $\overline{N V}$ & & $\mathrm{SW} 34$ & & 1 & & $\begin{array}{l}\text { opsail u } \\
\text { psails ? }\end{array}$ & $\begin{array}{l}\text { ind, cle } \\
\text { nd lowe }\end{array}$ & $\begin{array}{l}\text { ar with } \\
\text { er sails }\end{array}$ & $\begin{array}{l}\text { passing } \\
\text { and sta }\end{array}$ & $\begin{array}{l}\text { clouds; c } \\
\text { sails. }\end{array}$ & rrying \\
\hline 2 & $2 \div$ & & & & & 1 & & & & & & & \\
\hline 3 & 23,4 & WN & & SW: & & $\mathbf{I}$ & & & & & & & \\
\hline 4 & 233 & $11 / 1$ & & $\mathrm{SW}$ & & 1 & & hick clo & uds, squ & ualls, we & et; haule & down st & ysails. \\
\hline 5 & $\sqrt{3 / 4}$ & & & SW & & $11 / 2$ & & & & & & & \\
\hline 6 & 234 & & & & & $11 / 2$ & & & & & & & \\
\hline 7 & $3 / 4$ & & & & & $11 / 2$ & & ight win & d. drizz & ly, wet. & & & \\
\hline 8 & $3 / 4$ & & & & & $11 / 2$ & & & & & & & \\
\hline 9 & $11 / 2$ & SII & & IVN & & 2 & & ith the & change & of wind & d we ma & the port & tack. \\
\hline 10 & 2 & SWV & & WI & & $11 / 2$ & & & & & & & \\
\hline II & $3 \times 4$ & SSV & & $W^{\prime} !$ & & $\mathrm{I}$ & & opsail y & ind, clo & udy. & & & \\
\hline 12 & $\overline{3^{1} 4}$ & SSI & & W & & & & & & & & & \\
\hline$I$ & $\overline{3^{34}}$ & 5,1 & & & & & & eef-tops & ail wind & & & & \\
\hline 2 & $3 \frac{3 / 4}{4}$ & & & & & & & rizzly. & ainy. & & & & \\
\hline 3 & 4 & $\mathrm{~S}$ & & & & & & & & & & & \\
\hline 4 & $4 \%$ & & & & & & & & & & & & \\
\hline 5 & 5 & & & & & & & rong re & f-topsa & il wind. & & & \\
\hline 6 & $51 / 2$ & & & & & & & eather & as befor & & & & \\
\hline 7 & 5 & & & & & & & $\begin{array}{l}y \text { the } w \\
\text { men o }\end{array}$ & $\begin{array}{l}\text { ill of } \mathrm{Gr} \\
\mathrm{a} \text { the sic }\end{array}$ & $\begin{array}{l}\text { od Alex. } \\
\text { ck list. }\end{array}$ & ei Kisele & died of & curvy; \\
\hline 8 & 5 & & & & & & & & & & & & \\
\hline 9 & 4 & & & & & & & ind goi & ng down & n; let o & ut reef $\mathrm{i}$ & topsails. & \\
\hline 10 & 4 & & & & & & & & & & & & \\
\hline 11 & 5 & & & & & & & ain. & & & & & \\
\hline 12 & $31 / 2$ & & & & & & & outherl & swell; & hauled & $\mathrm{up} \mathrm{topr}$ & ast stays & ils. \\
\hline \multirow{2}{*}{\multicolumn{3}{|c|}{$\begin{array}{c}\text { Variation } \\
\text { of } \\
\text { Compass } \\
\text { I t rhumb E }\end{array}$}} & \multicolumn{11}{|c|}{ In 2.1 hours } \\
\hline & & & & $\stackrel{\Xi}{\Xi}$ & $=$ & & 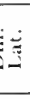 & 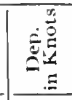 & $0 \frac{0}{0}$ & 藏 & 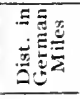 & E & $\stackrel{\dot{\varphi}}{\tilde{L}}$ \\
\hline \multicolumn{3}{|c|}{$\begin{array}{l}\text { By Dead } \\
\text { Reckoning }\end{array}$} & & $\begin{array}{l}W \\
44^{\prime} N\end{array}$ & 7 & & $\begin{array}{l}N \\
7^{\prime}\end{array}$ & $\begin{array}{r}\mathrm{W} \\
69.8 \\
\end{array}$ & $107.6^{\prime}$ & $49^{\circ} 30^{\prime \prime}$ & 186 & $\begin{array}{c}E / S \\
4^{\circ} 40^{\prime} S\end{array}$ & $19^{\circ} \mathrm{O2}^{\prime}$ \\
\hline
\end{tabular}


6 October 20, I74I. Afler Midday

\begin{tabular}{|c|c|c|c|c|c|c|c|c|c|c|c|c|}
\hline 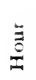 & 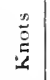 & $\vec{\Xi}$ & \multicolumn{2}{|c|}{ 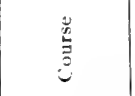 } & $\stackrel{\vec{y}}{3}$ & & & & & & & \\
\hline 1 & $\overline{21 / 2}$ & $s$ & \multicolumn{2}{|c|}{ W'/S } & $7 / 2$ & \multicolumn{7}{|c|}{$\begin{array}{l}\text { Topsail wind, drizzly, tainy; with the change of } \\
\text { wind we made the port tack. }\end{array}$} \\
\hline 2 & 3 & NIV & \multicolumn{2}{|c|}{ WSW } & $\mathbf{I}$ & & & & & & & \\
\hline 3 & $3 \frac{1 / 4}{4}$ & & \multirow{2}{*}{\multicolumn{2}{|c|}{ WSWt//2W }} & I & \multicolumn{7}{|c|}{ Undersail wind; lowered stay'sails. } \\
\hline 4 & 3 & & & & 1 & \\
\hline 5 & $2 \pi / 2$ & NW/W & \multicolumn{2}{|c|}{ SW/W } & $I^{2}+4$ & \multicolumn{7}{|c|}{ Furled topsails. } \\
\hline 6 & $\overline{1 \pm / 4}$ & & & & $I^{14}$ & \multicolumn{7}{|c|}{$\begin{array}{l}\text { Thick clouds, squalls, wet; furled foresail, hove to } \\
\text { under the mainsail and trysail. }\end{array}$} \\
\hline 7 & $1 \%$ & & & & & & & & & & & \\
\hline 8 & $1 \%$ & & & & 5 & \multicolumn{7}{|c|}{ Storm. } \\
\hline 9 & 19 & WNW & \multicolumn{2}{|c|}{ SW $1 / 2 \mathrm{~S}$} & 5 & \multicolumn{7}{|c|}{ Clear with passing showers. } \\
\hline 10 & $1 \mathrm{I} / 4$ & & & & 5 & \multirow{2}{*}{\multicolumn{7}{|c|}{ Thick clouds hail rain. }} \\
\hline II & $\mathrm{It}^{\mathrm{t}}$ & & & & 5 & & & & & \multicolumn{3}{|c|}{ Thick clouds, hail, rain. } \\
\hline 12 & $\mathrm{I}^{5}$ & & & & 5 & & & & & & & \\
\hline $\mathbf{I}$ & $I$ & & & & 5 & \multicolumn{7}{|c|}{ Wind going down. } \\
\hline 2 & $\mathrm{I}$ & & & & 5 & \\
\hline 3 & $I$ & $\mathrm{~W} / \mathrm{N}$ & \multirow{2}{*}{\multicolumn{2}{|c|}{$\frac{S S W: / 2 W}{S / W ! 2 W}$}} & 5 & & \multirow{2}{*}{\multicolumn{6}{|c|}{ Undersail wind. }} \\
\hline 4 & $I$ & WV & & & 5 & & & & & & & \\
\hline 5 & 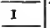 & & & & 5 & \multicolumn{7}{|c|}{ Heavy northerly swell. } \\
\hline 6 & $\mathrm{I}$ & $\mathrm{W} / \mathrm{S}$ & \multicolumn{2}{|c|}{$\mathrm{S} / 2 \mathrm{~W}$} & 5 & \multirow{2}{*}{\multicolumn{7}{|c|}{ Reef-topsail wind set foresail. }} \\
\hline 7 & $3 / 4$ & WSW & \multicolumn{2}{|c|}{ NW } & 4 & & & & & & & \\
\hline 8 & $\overline{134}$ & $\mathrm{SIV} / \mathrm{W}$ & \multicolumn{2}{|c|}{$\mathrm{NW} / \mathrm{W}$} & 2 & \multicolumn{7}{|c|}{$\begin{array}{l}\text { Set topsails reefed; Nikita Kharitonov died by } \\
\text { the will of God. }\end{array}$} \\
\hline 9 & $2 \frac{\pi}{2 / 2}$ & $\mathrm{~S} / \mathrm{W}$ & \multicolumn{2}{|c|}{$\mathrm{W} / \mathrm{N}$} & & \multirow{2}{*}{\multicolumn{7}{|c|}{$\begin{array}{l}\text { Clear with passing clouds; took three reefs in fore- } \\
\text { topsail; heavy squalls which obliged us to ease up } \\
\text { on the lanyards to the topsail shrouds; tacked to } \\
\text { port.107 }\end{array}$}} \\
\hline 10 & 4 & $\mathrm{~s}$ & \multicolumn{2}{|c|}{ W } & & & & & & & & \\
\hline II & $5 \frac{7 / 2}{4}$ & & & & & & & & & & & \\
\hline 12 & $31 / 2$ & SSW & & & 1 & & & & & & & \\
\hline & & & & & In 2. & h h & urs & & & & m Vaua & \\
\hline & $\begin{array}{l}\text { ariati } \\
\text { of } \\
\text { ompa } \\
\text { rhum }\end{array}$ & $\begin{array}{l}\mathrm{on} \\
\mathrm{ss} \\
\mathrm{b} \mathrm{E}\end{array}$ & 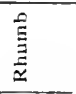 & 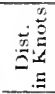 & 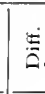 & & 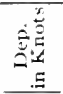 & 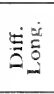 & 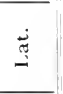 & 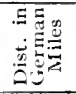 & $\frac{\Xi}{\Xi}$ & فํ. \\
\hline & Dea & & 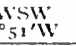 & 29.7 & ic & $3^{\prime}$ & $\begin{array}{l}\text { W } \\
28\end{array}$ & $12^{\prime}$ & $49^{\circ} 20^{\prime}$ & $180^{\prime \prime} z_{1}^{\prime}$ & $\underset{5^{\circ} 59^{\prime} S}{\mathrm{E} / \mathrm{S}}$ & $18^{\circ} 20^{\prime}$ \\
\hline
\end{tabular}

107 Khitrov's journal: "On sick list: Captain Commander, petty officers and men to the number of $32 . "$ 
$\Varangle$ Oclober 2I, I7tI. After Midday

\begin{tabular}{|c|c|c|c|c|c|c|c|c|c|c|c|c|}
\hline \multirow{2}{*}{$\frac{\stackrel{5}{3}}{1}$} & \multirow{2}{*}{ 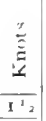 } & \multicolumn{2}{|l|}{ 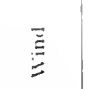 } & \multicolumn{2}{|c|}{$\begin{array}{l}3 \\
\vdots \\
5\end{array}$} & (2) & & & & & & \\
\hline & & \multicolumn{2}{|c|}{$\$ 11,8$} & \multicolumn{2}{|c|}{$\mathrm{WNW}_{2} \mathrm{WI}$} & 2 & \multicolumn{6}{|c|}{ Topgallantsail wind, wet, sunshine. } \\
\hline 2 & $I^{3} 4$ & \multirow{2}{*}{\multicolumn{2}{|c|}{$\mathrm{SWH}$}} & & & 2 & \\
\hline 3 & $\mathrm{I}^{\mathrm{I}} 4$ & & & \multicolumn{2}{|c|}{$\mathrm{NW}, \mathrm{W}$} & & \multicolumn{6}{|c|}{ Took three reefs in main-topsail. } \\
\hline 4 & $\sqrt{2^{1} 2}$ & \multicolumn{2}{|c|}{$\mathrm{WI}, \mathrm{N}$} & \multicolumn{2}{|c|}{$\mathrm{N} 1 / 2 \mathrm{~W}$} & I & \multicolumn{6}{|c|}{$\begin{array}{l}\text { Thick clouds, squalls, rain; made the starboard } \\
\text { tack. }\end{array}$} \\
\hline 5 & $22^{3}+$ & \multicolumn{2}{|c|}{ NW } & \multicolumn{2}{|c|}{ WSW } & $\overline{I^{1 / 2}}$ & & & & & & \\
\hline 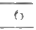 & $2^{2}+$ & & & & & $\mathrm{I}^{\mathrm{I}}$ & \multicolumn{6}{|c|}{ Stormy, wet. } \\
\hline 7 & $I^{3}+1$ & & & & & 3 & \multicolumn{6}{|c|}{ Furled topsails. } \\
\hline 8 & $\overline{\mathrm{I}_{2}}$ & & & & & 3 & \multicolumn{6}{|c|}{ Cloudy, rain, hail. } \\
\hline 9 & $\mathrm{I}^{1}+4$ & \multicolumn{2}{|c|}{$\mathrm{NW/W}$} & \multicolumn{2}{|c|}{$\mathrm{SW}^{\mathrm{t}} / \mathrm{W}$} & 5 & \multicolumn{6}{|c|}{$\begin{array}{l}\text { Gale; furled foresail, hove to under the mainsail } \\
\text { and trysail. }\end{array}$} \\
\hline 10 & $11^{1}$ & \multirow{2}{*}{\multicolumn{2}{|c|}{$11 \times 16$}} & \multicolumn{2}{|c|}{$S W+2 S$} & 5 & \multicolumn{6}{|l|}{ Stormy. } \\
\hline 11 & $I^{5} 4$ & & & & & 5 & \multicolumn{6}{|c|}{ Squalls, rain and hail. } \\
\hline 12 & $\mathrm{I}^{\mathrm{x}}$ & \multicolumn{2}{|c|}{$1 \mathrm{~N} N$} & SSIV & ${ }_{2} 11^{5}$ & 5 & Stars out & & & & & \\
\hline$I$ & $\overline{I^{1} 2}$ & 11 & & ss & & $3 \div 2$ & Reef-tops & if wit & $\mathrm{d} ;$ set $\mathrm{fo}$ & resail. & & \\
\hline 2 & $1^{1} 4$ & & & & & $3^{12}$ & Clear wit & passi & ng clond & & & \\
\hline 3 & 1 & W & & $\mathrm{S}_{2}$ & W & $3{ }^{1} 2$ & Came abo & at wit & the wit & $\mathrm{dd}$ and $\mathrm{n}$ & te the po & tack. \\
\hline 4 & $I^{3}+4$ & $\triangle 11$, & & $\mathrm{NW}$ & ${ }_{2} \mathrm{~W}$ & $2 \bar{i}$ & & & & & & \\
\hline 5 & $\mathrm{I}^{3} 4$ & 1151 & & $N$ & & $21 / 2$ & Set the to & osails & reefed. & & & \\
\hline 6 & $3^{1}+$ & 511 & II & $\mathrm{NW}$ & $1 \mathrm{~W}$ & $\mathrm{I}^{1}{ }_{2}$ & Hauled u & topn & ast-stay & sails. & & \\
\hline 7 & $2^{7}+$ & & & $\mathrm{NV}$ & ${ }_{4} \mathrm{IV}^{7}$ & $\mathrm{I}^{I_{12}}$ & Thick clo & $\mathrm{ds}, \mathrm{li}$ & il. snow & & & \\
\hline 8 & 23 & & & & & $\mathrm{I}^{1}{ }_{2}$ & Clear wit & passi & ng cloud & s, reef $-t$ & sail win & \\
\hline 9 & $2^{1}+$ & & & NW/I & $V: 2 \mathrm{VV}$ & $1 / 2$ & & & & & & \\
\hline 10 & $2 \div 2$ & & & $\mathrm{NW}$ & IV & $11 / 2$ & $\begin{array}{l}\text { Thick clo } \\
\text { ease up o } \\
\text { westerly }\end{array}$ & $\begin{array}{l}\text { ls, so } \\
\text { the } \\
\text { ell. }\end{array}$ & $\begin{array}{l}\text { alls; at } \\
\text { inyards }\end{array}$ & $\begin{array}{l}\text { times } \\
\text { to the }\end{array}$ & $\begin{array}{l}\text { were ob } \\
\text { pmast st }\end{array}$ & $\begin{array}{l}\text { ed to } \\
\text { ouds; } \\
\end{array}$ \\
\hline $1 \mathrm{I}$ & 2 & & & & & $\mathrm{I}^{1 / 2}$ & & & & & & \\
\hline 12 & $2: 2$ & & & $\mathrm{NW}$ & ${ }_{2} \mathrm{~W}$ & {$[1 / 2$} & $\begin{array}{l}\text { Took an } \\
\text { cause of } t \\
\text { ing it was }\end{array}$ & $\begin{array}{l}\text { obser } \\
\text { le sma } \\
\text { not ce }\end{array}$ & $\begin{array}{l}\text { ation: } \\
\text { ll differe } \\
\text { nsidered }\end{array}$ & $\begin{array}{l}\text { latitude } \\
\text { nce fro }\end{array}$ & $\begin{array}{l}9^{\circ} 27^{\prime}, \\
\text { he dead }\end{array}$ & $\begin{array}{l}\text { it be- } \\
\text { eckon- } \\
\end{array}$ \\
\hline & & & & & & n 24 & hours & & & & m Vaua & \\
\hline 8 & $\begin{array}{l}\text { driatic } \\
\text { of } \\
\text { onjas } \\
\text { rhum }\end{array}$ & $\begin{array}{l}\text { on } \\
1 \mathrm{ss} \\
\mathrm{ib} \mathrm{E}\end{array}$ & & $\stackrel{0}{\Xi}$ & 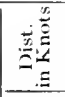 & 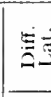 & 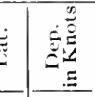 & $=\frac{0}{3}$ & 苞 & 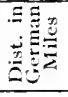 & $\stackrel{\vec{E}}{\Xi}$ & $\stackrel{\dot{x}}{\tilde{g}}$ \\
\hline $\begin{array}{l}\mathrm{I} 3 \mathrm{y} \\
\mathrm{K}\end{array}$ & $\begin{array}{l}\text { Deas } \\
\text { choni }\end{array}$ & & & $19^{\prime} \mathrm{W}$ & $16^{1 / 2}$ & $\mathrm{~N}$ & $\begin{array}{ll}\text { IV } \\
12.9\end{array}$ & $20^{\prime}$ & $1.40^{\circ} 30^{\prime}$ & $176^{3} 4$ & $\begin{array}{l}\mathrm{E} / \mathrm{S} \\
5^{\circ} 32^{\prime} \mathrm{S}\end{array}$ & $18^{\circ} 0^{\prime}$ \\
\hline
\end{tabular}




\section{October 22, 174I. After Midday}

\begin{tabular}{|c|c|c|c|c|c|c|c|c|c|c|c|c|c|}
\hline 岂 & 要 & $\underline{\Xi}$ & & 药 & & 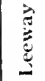 & & & & & & & \\
\hline I & $2 ! 4$ & WS & & NW: & & $\overline{11 / 2}$ & & $\begin{array}{l}\text { psail } \\
\text { uds. } \\
\text { fed. }\end{array}$ & $\begin{array}{l}\text { wind, } \\
\text { vail; 'ca }\end{array}$ & $\begin{array}{l}\text { lear wi } \\
\text { rrying }\end{array}$ & $\begin{array}{l}\text { ith pass } \\
\text { topsaits }\end{array}$ & $\begin{array}{l}\text { ig clouds. } \\
\text { and towe }\end{array}$ & $\begin{array}{l}\text { thick } \\
\text { r sails }\end{array}$ \\
\hline 2 & 2 & & & & & $I^{1}$ & & & & & & & \\
\hline 3 & 2 & IV & & $\mathrm{NN}$ & & $1 ! 2$ & & & & & & & \\
\hline 4 & $I^{3 / 4}$ & WN & & SV & & 2 & & ade $t h$ & starbc & ard tac & & & \\
\hline 5 & 134 & $\mathbf{W V}$ & & SSIV & $2 \mathrm{~V}$ & $\mathrm{I}^{3}+$ & & & & & & & \\
\hline 6 & 2 & & & & & $I^{3}+4$ & & psail , & ind. & & & & \\
\hline 7 & 2 & & & SSI & & $1 ! 2$ & & & & & & & \\
\hline 8 & $2 ! 4$ & & & & & $1 / 3$ & & & & & & & \\
\hline 9 & 2 & & & & & $I ! 2$ & & ick clc & uds, sc & uatls, al & nd wet. & & \\
\hline 70 & $21 / 2$ & & & & & $1_{12}$ & & dersaj & wind. & weather & r as befo & & \\
\hline$\overline{\text { II }}$ & $11 / 4$ & & & $\mathrm{~S} / \mathrm{W}$ & WW & 6 & & $\begin{array}{l}\text { rled t } \\
\text { in-stal }\end{array}$ & $\begin{array}{l}\text { psails, } \\
\text { sail an }\end{array}$ & $\begin{array}{l}\text { foresail } \\
\text { d trysai }\end{array}$ & il. mainsa & i, hove to & on the \\
\hline$\sqrt{12}$ & $1+4$ & & & & & 6 & & & & & & & \\
\hline I & $1^{1}$ & & & & & 6 & & & & & & & \\
\hline 2 & $1^{14}$ & II & & SSIV & $2 \mathrm{~W}$ & $5 \%$ & & the w & 11 of $\mathrm{Gr}$ & d died $t$ & he marir & Luka las & iakov. \\
\hline 3 & $1 ! \cdot$ & & & & & $5 \%$ & & & & & & & \\
\hline 4 & $I^{3}+4$ & & & & & $5 ! 2$ & & $\mathrm{rm}, \mathrm{st}$ & ualls. & tail. & & & \\
\hline 5 & 1 & $\mathrm{WN}$ & & sw & & 512 & & ry chi & & & & & \\
\hline 6 & $\mathrm{I}$ & & & & & $5 ! 2$ & & nd goi & ng dow & n a bit. & & & \\
\hline 7 & $\mathrm{I}$ & & & & & $\sqrt{5^{1 / 2}}$ & & & & & & & \\
\hline 8 & $I$ & & & & & $\overline{5^{1}}$ & & dersai & wind. & & & & \\
\hline 9 & $\overline{1 ! 3}$ & NW & & SW & & 2 & & fores & iil and & mainsai & & & \\
\hline 10 & 13 & & & & & 2 & & ar wit & h passi & $\mathrm{ng}$ cloud & & & \\
\hline II & $I \%$ & & & & & 2 & & topsa & ils reef & & & & \\
\hline$\sqrt{12}$ & $\sqrt{1 ! 4}$ & & & & & 2 & & $\begin{array}{l}\text { wrap } \\
\text { o the }\end{array}$ & $\begin{array}{l}\text { ped th } \\
\text { sea. }\end{array}$ & dead & marine & ad droppe & d him \\
\hline \multirow{2}{*}{\multicolumn{3}{|c|}{$\begin{array}{c}\text { Variation } \\
\text { of } \\
\text { Compass } \\
1 \text { thumb E }\end{array}$}} & \multicolumn{11}{|c|}{ In 24 hours } \\
\hline & & & & है & 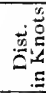 & & & 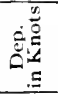 & 电题 & $\stackrel{\text { త }}{-1}$ & 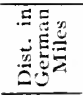 & 总 & $\stackrel{\infty}{5}$ \\
\hline \multicolumn{3}{|c|}{$\begin{array}{l}\text { By Dead } \\
\text { Reckoning }\end{array}$} & & $\begin{array}{l}S / W \\
\text { SII }\end{array}$ & 22 & & S, & $\begin{array}{l}W \\
7\end{array}$ & $10^{\prime}$ & $\left|49^{\circ} 09^{\prime}\right|$ & 17214 & $\begin{array}{c}\text { ESE } \\
4^{\circ} \mathrm{O}^{\prime} \mathrm{E}\end{array}$ & $117^{\circ} 50$ \\
\hline
\end{tabular}


October 23, $17+1$. After Midday

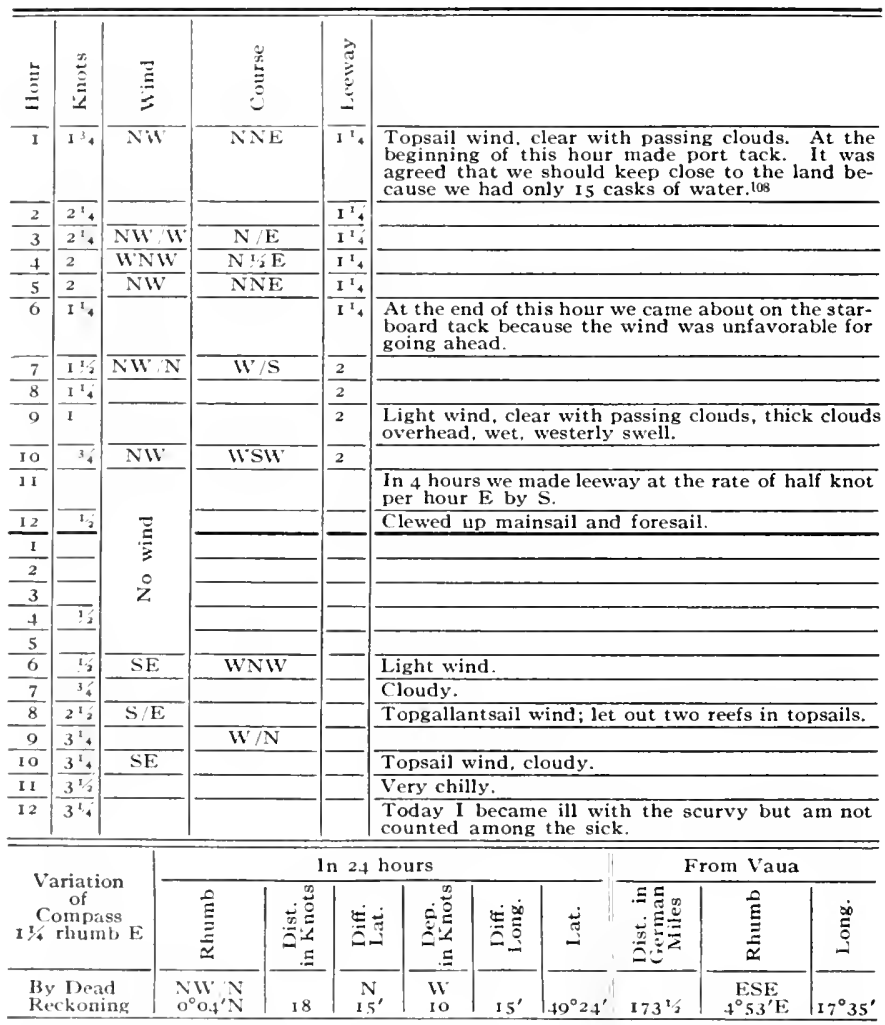

108 Khitrov's journal: "We and the petty officers had a consultation with the Captain Commander. The winds had continuously been contrary and of water we had only is casks. It was dangerous either to go on or to wait for a change of wind. and we therefore unanimously agreed to sail $\mathrm{N}$ in the hope of finding a place to take water, without which a great misfortune would overtake us." 


\section{I) October 24, 174I. After Midday}

\begin{tabular}{|c|c|c|c|c|c|c|c|c|c|c|c|}
\hline$\stackrel{5}{3}$ & $\underset{\stackrel{n}{g}}{\stackrel{2}{4}}$ & 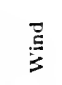 & \multicolumn{2}{|c|}{ 总 } & 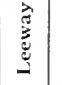 & & & & & & \\
\hline $\mathbf{I}$ & 4 & $\mathrm{SE}$ & \multicolumn{2}{|c|}{$W / N$} & & \multicolumn{6}{|c|}{$\begin{array}{l}\text { Topsail wind, chilly; carrying topsails, foresail. } \\
\text { mainsail reefed. }\end{array}$} \\
\hline 2 & 5 & & & & & & & & & & \\
\hline 3 & 4 & & & & & & & & & & \\
\hline 4 & 4 I/2 & $E / S$ & & & & \multicolumn{6}{|c|}{ Strong reef-topsail wind. } \\
\hline 5 & 5 & & & & & \multicolumn{6}{|c|}{$\begin{array}{l}\text { Took second and third reefs in fore-topsail; } \\
\text { heavy clouds, wet. }\end{array}$} \\
\hline 6 & $41 / 2$ & & & & & & & & & & \\
\hline 7 & $4^{3 / 2}$ & & \multicolumn{2}{|c|}{ IV } & & & & & & & \\
\hline 8 & $41 / 2$ & & 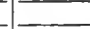 & & & \multicolumn{6}{|c|}{ Clewed up mainsail. } \\
\hline 9 & 5 & & & & & & & & & & \\
\hline IO & 434 & & & & & \multicolumn{6}{|c|}{ Rain, heavy passing clouds. } \\
\hline I 1 & $4 \%$ & & & & & & & & & & \\
\hline 12 & $+3 / 4$ & & & & & \multicolumn{6}{|l|}{ Snow. } \\
\hline I & 512 & & & & & \multicolumn{6}{|c|}{ Clewed up mainsail. } \\
\hline 2 & $51 / 3$ & & & & & \multirow{2}{*}{\multicolumn{6}{|c|}{ Undersail wind, drizzly, rain. }} \\
\hline 3. & 6 & $\mathrm{E}$ & & & & & & & & & \\
\hline 4 & 6 & & & & & & & & & & \\
\hline 5 & 6 & & & & & \multicolumn{6}{|c|}{ 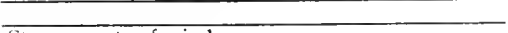 } \\
\hline 6 & $71 / 2$ & ENE & \multicolumn{2}{|c|}{ WI } & & \multicolumn{6}{|c|}{ Storm. gusts of wind. } \\
\hline 7 & 614 & & & & & \\
\hline 8 & $61 / 2$ & & & & & \multicolumn{6}{|c|}{ 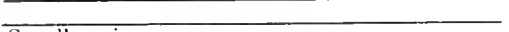 } \\
\hline 9 & 534 & $\mathrm{NE}$ & WN & & & \multicolumn{6}{|c|}{ Squalls, rain, snow. } \\
\hline 10 & 5 & & & & & \multicolumn{6}{|c|}{ Took three reefs in main-topsail. } \\
\hline 11 & $4 \%$ & & & & & \multicolumn{6}{|c|}{ Set mainsail. } \\
\hline 12 & 413 & & & & & \multicolumn{6}{|c|}{ Reef-topsail wind. weather the same. } \\
\hline \multirow{2}{*}{\multicolumn{3}{|c|}{$\begin{array}{c}\text { Variation } \\
\text { of } \\
\text { Compass } \\
1 \% \text { rhumb E }\end{array}$}} & \multicolumn{9}{|c|}{ In 24 hours } \\
\hline & & & $\stackrel{\vec{E}}{\underline{E}}$ & 总苔 & 胥 & $\stackrel{\stackrel{0}{5}}{\stackrel{5}{5}}$ & 氞递 & 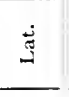 & 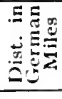 & $\stackrel{E}{\Xi}$ & 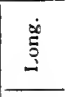 \\
\hline $\begin{array}{l}\mathrm{B} \\
\mathrm{R} \\
\end{array}$ & $\begin{array}{l}\text { Dea } \\
\text { eckon }\end{array}$ & & $\begin{array}{l}W V W \\
0^{\circ} 34^{\prime} W V\end{array}$ & 123 & $\begin{array}{r}N \\
46 \\
\end{array}$ & $\begin{array}{c}W \\
11+3 \\
\end{array}$ & $177^{\prime}$ & $50^{\circ} 10^{\prime}$ & $1+23 / 4$ & $\begin{array}{c}\mathrm{E} / \mathrm{S} \\
5^{\circ} 28^{\prime} \mathrm{S}\end{array}$ & $14^{\circ} 38^{\prime}$ \\
\hline
\end{tabular}


October 25, I74I. After Midday

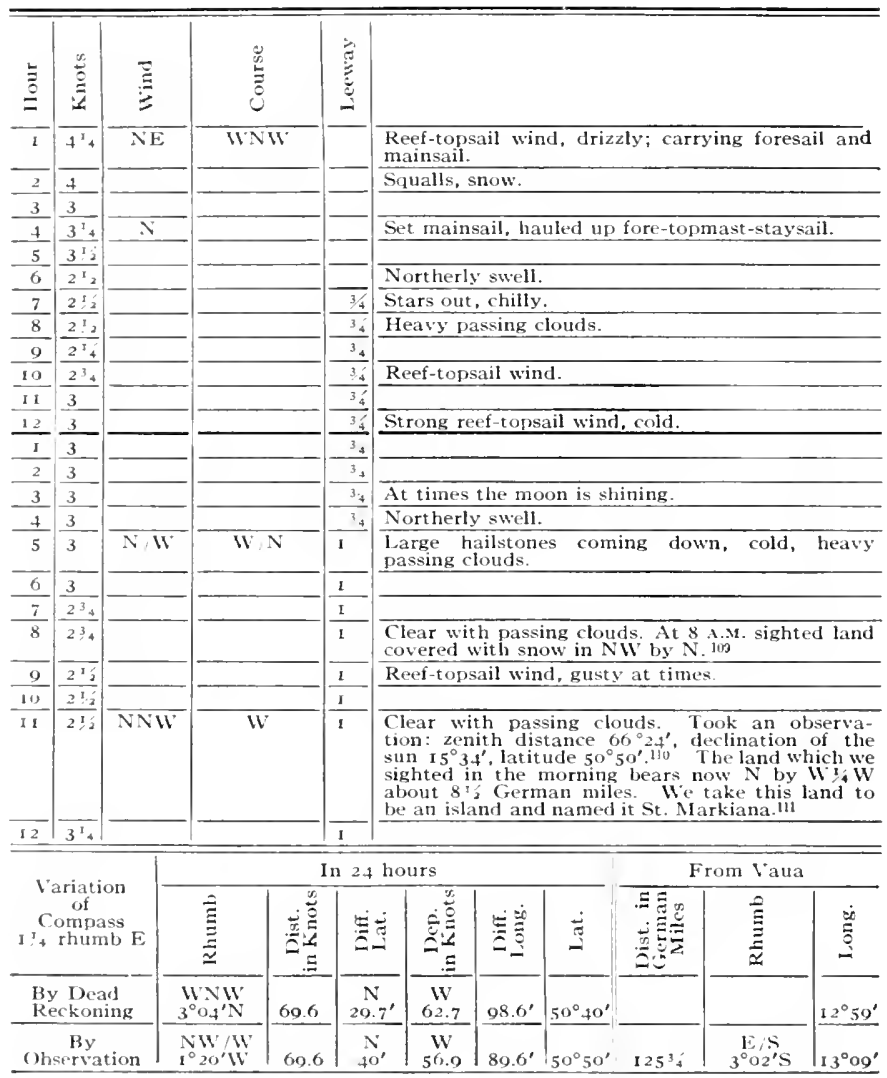

(Footnotes 100-1II on pp. 190-200.) 
(1) October 26, I74I. Afler Midday

\begin{tabular}{|c|c|c|c|c|c|c|c|c|c|c|}
\hline$\stackrel{\stackrel{2}{\Xi}}{\varrho}$ & 吾 & $\bar{\Xi}$ & \multicolumn{2}{|c|}{$\stackrel{\ddot{2}}{3}$} & | & & & & & \\
\hline 1 & $2+4$ & NNW & \multicolumn{2}{|c|}{ W' } & $\overline{1+4}$ & \multicolumn{5}{|c|}{$\begin{array}{l}\text { Reef-topsail wind, cloudy squalls, snow; carry- } \\
\text { ing staysails, topsails, and lower sails. }\end{array}$} \\
\hline 2 & 3 & & & & $\mathrm{I}^{1} 4$ & & & & & \\
\hline 3 & $2^{3 / 4}$ & & & & $I^{1} 4$ & & & & & \\
\hline 4 & 234 & & & & 13 & \multicolumn{5}{|c|}{ Northerly swell.11: } \\
\hline 5 & $2^{3}+4$ & \multirow[t]{2}{*}{$\mathrm{N} 1 \mathrm{~W}$} & \multicolumn{2}{|c|}{$W N$} & $11^{4}$ & \\
\hline 6 & $2: 2$ & & \multirow{2}{*}{\multicolumn{2}{|c|}{11}} & $1+4$ & & & & & \\
\hline 7 & 3 & N.NI & & & $I_{4}$ & \multicolumn{5}{|c|}{ Heavy passing clouds and snowstorm. } \\
\hline 8 & $22^{3} 4$ & & & & $\overline{I_{4}}$ & & & & & \\
\hline 9 & $22^{3}$ & & & & $1{ }^{i} 4$ & \multicolumn{5}{|c|}{ Clear with passing clouds. } \\
\hline Io & 27 & & & & $\overline{124}$ & \\
\hline 11 & $2+\frac{1}{2}$ & $\mathrm{NW}$ & \multicolumn{2}{|c|}{$\mathrm{II}+2 \mathrm{~N}$} & $\overline{I^{+2}}$ & & \multicolumn{2}{|c|}{ Wet. } & & \\
\hline 12 & 23 & & \multirow{2}{*}{\multicolumn{2}{|c|}{ WN }} & $\overline{\mathrm{I}^{\mathrm{E}}}$ & & & & & \\
\hline $\mathbf{I}$ & 234 & & & & $\overline{12}$ & & & & & \\
\hline 2 & $2 \div 4$ & & & & $1+2$ & \multicolumn{5}{|c|}{ Heavy passing clouds and rain. } \\
\hline 3. & 2 & & & & $1+\frac{1}{2}$ & & & & & \\
\hline 4 & $2 ! 4$ & & $W^{\prime 2}$ & & $\overline{13^{3}}$ & \multicolumn{5}{|c|}{ Heavy passing clouds, wind in gusts. } \\
\hline-5 & $2^{3} 2$ & $\mathrm{NNIV}$ & \multicolumn{2}{|c|}{11} & $1+2$ & \\
\hline 6 & $22_{4}$ & & & & $\overline{152}$ & \multicolumn{5}{|l|}{ Snow. } \\
\hline 7 & $2^{+} 4$ & & & & $\overline{I^{1}{ }_{2}}$ & \\
\hline 8 & $2{ }^{\mathrm{t}}$ & & & & $\overline{1: 2}$ & \multicolumn{5}{|c|}{$\begin{array}{l}\text { Reef-topsail wind, clear with passing clouds. } 30 \\
\text { on the sick list. }\end{array}$} \\
\hline 2 & $22^{3}$ & & & & $\overline{132}$ & & \\
\hline 10 & $2 \mathrm{~T}_{2}$ & 11 & 11 & & $\overline{I^{\prime} \prime_{2}}$ & \multicolumn{5}{|l|}{ Chilly. } \\
\hline $1 \mathrm{I}$ & $2{ }^{1} 2$ & & & & $11_{2}$ & \multicolumn{5}{|c|}{ Topsail wind, weather as before. } \\
\hline 12 & 2 & & & & $\overline{\mathrm{I}_{2} \mathrm{I}}$ & Topsail : & ind. & & & \\
\hline & & & & & n 24 & hours & & & om Vaua & \\
\hline C & $\begin{array}{l}\text { ariat } \\
\text { of } \\
\text { ompa } \\
\text { rhum }\end{array}$ & $\begin{array}{l}\text { on } \\
\text { iss } \\
\text { ab E }\end{array}$ & $\underline{\Xi}$ & 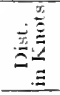 & 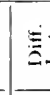 & 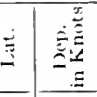 & 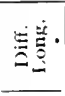 & 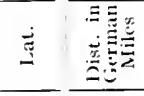 & $\underline{\Xi}$ & 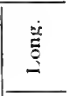 \\
\hline & Dea & & $\begin{array}{ll}W \\
25^{\circ} 6^{\prime}\end{array}$ & 58.6 & 2. & $\begin{array}{c}11 \\
58.2 \\
\end{array}$ & $92.6^{\prime}$ & $50^{\circ} 53^{\prime} \quad 111^{2}$ & $\begin{aligned} & \text { E/S } \\
& 4^{\circ} 29^{\prime} S\end{aligned}$ & $11^{\circ} 36^{\prime}$ \\
\hline
\end{tabular}

112 Khitrov's journal: "s o'clock in the afternoon sighted an island; southern point of it bore true N." [The "island" sighted was probably the southern end of Kiska Island, 1,200 to 1,500 feet high and with a radius of visibility of about -10 knots, and was not recognized as part of the same island of which it was the northern peak, t,050 feet higI and with a radius of visibility of about $70 \mathrm{knots}$, that had been sighted eight iours before. -EDIT. NOTE.]

\section{Footnotes to Log of October 25}

109 Khitrov's journal: "Saw land in NW by N, 8 miles distant, which we took for ar island.

110 Khitrov's journal: "The island we sighted is high, rocky, treeless, and covered with snow. Latitude $51^{\circ} 11^{\prime}$. . "This refers to the latitude of the ship at $8 \mathrm{~A}$. 1. , when the island was first sighted (see Waxel's report, p. 275); on the chart (PI. l) the October 25 noon position has been plotted accordingly, as this brings the subsequent landtalls into better agreement with the actual conditions than the observed latitude of $50^{\circ} 50^{\prime}$. which was probably a poor observation, and the recorded day"s run of 69.6 knots, whick probably underestimated the current and was too low.

111 Kiska lsland. [Sokolov (Zapiski Iydrogr. Depart. Vol. 9. St. Petersburg, 1851, pp. 30 $1-395)$ identifies St. Markiana Island as Amchitka and st. Sicphen Island, sighted on October 28 (here interpteted as Buldir), as Kiska. If the are of visibitity be plotted (Continuation of footnote on p. 2oo.) 
¿ October 27, I74I. After Midday

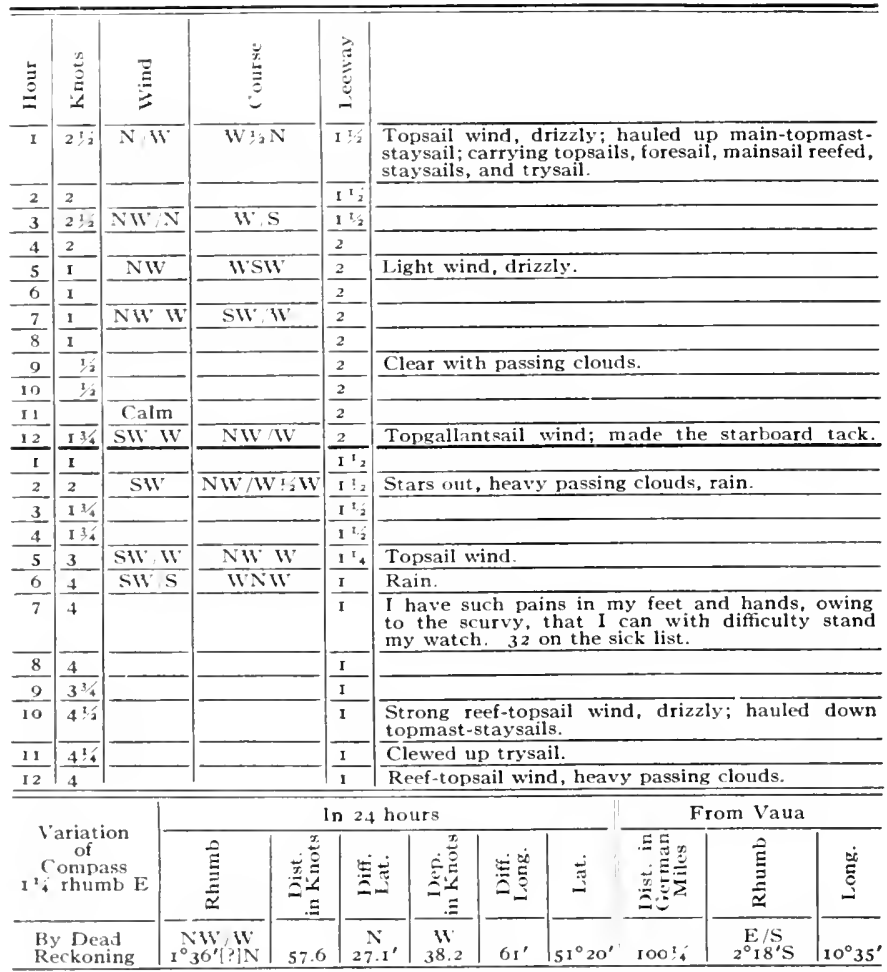

of the highest point of each of the two islands (Amchitka: height I,008 feet, visibility radius 32 knots: Kiska height 4,050 feet, visibility radius $70 \mathrm{knots}$ ) with reference to the probable course of the St. Peler as adjusted to the latitude of $5 I^{\circ} I^{\prime}$ and the bearing NW by $N$ at the time of sighting, it seems probable, however, that the island seen was Kiska and not Amchitka. This would also seem to be indicated by the characterization of the island as high. "Amchitka is ... low... The offshore navigator can not expect to see Amchitka at all as there are no commanding elevations" (United States (oast Pilot: Alaska. Part II, Ist edition, U.S. Coast and Geodetic Survey, Washington, I). (., 1916, p. 222),-EDIT. NOTE.I 


\section{$\Varangle$ October $28,17+1$. Afler Midday}

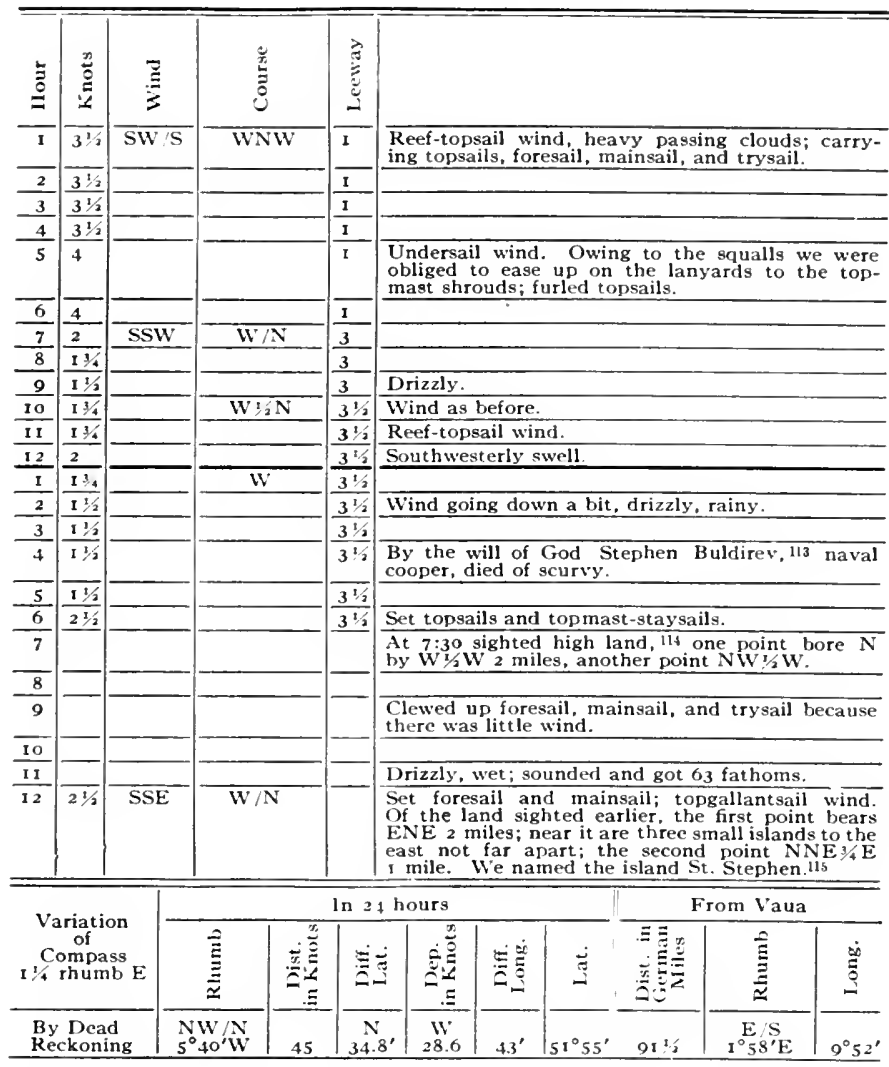

113 Another reading is Stepan Bogdriev. See, however, next footnote and entry under 12 p. m.

ili Buldir Island.

115 Khitrov's journal: "Land sighted proved to be an island extending WNW-ESE, the western point bearing NNE, the eastern ENE. From it were seen three small islands, distant $3 \frac{1}{2}$ or 4 miles. The longest seemed to be $4 \%$ German miles." 
2 October 20, 1741. After Midday

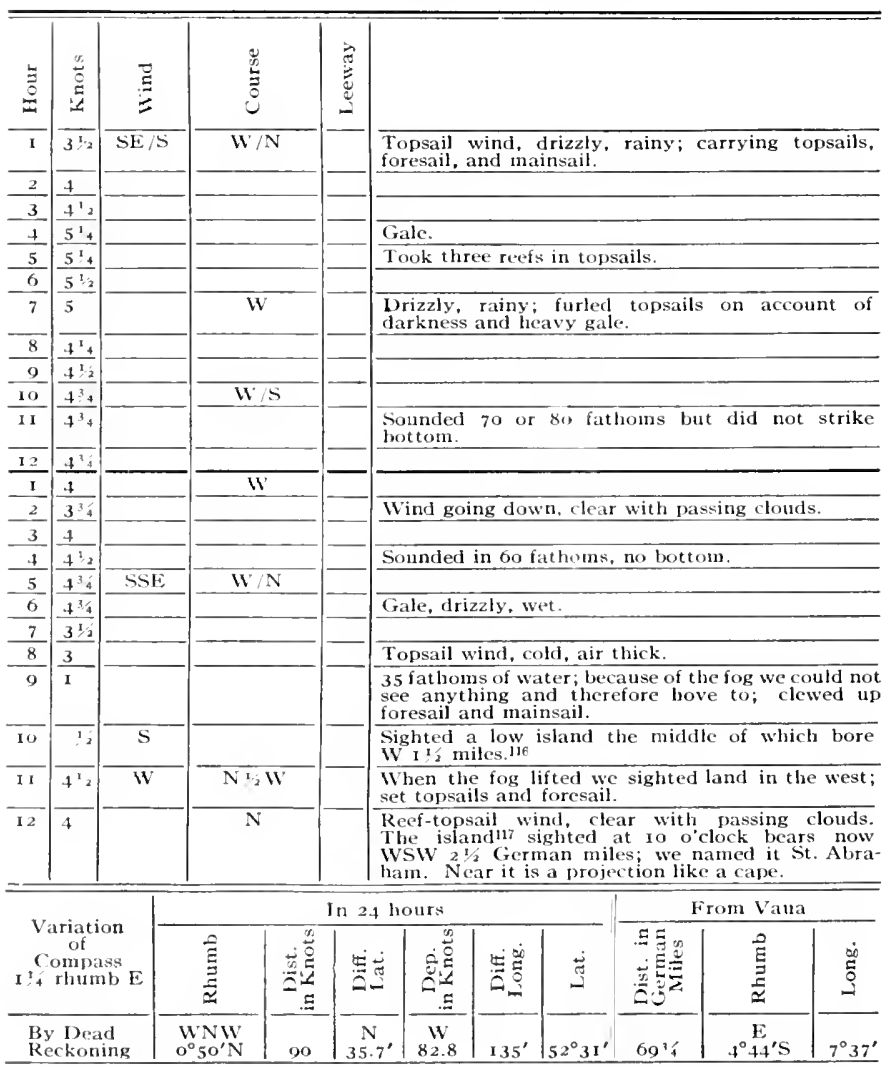

156 Khitrov"s journal: "Sighted an island; northern point $W$ by $N$, Southern point $W$ by $S 3^{1}$ miles. At noon the northern point of the island bore WSIV."

in Fisternmost of the Senichi lslands. 
Q October 30,17+1. Afler Midday

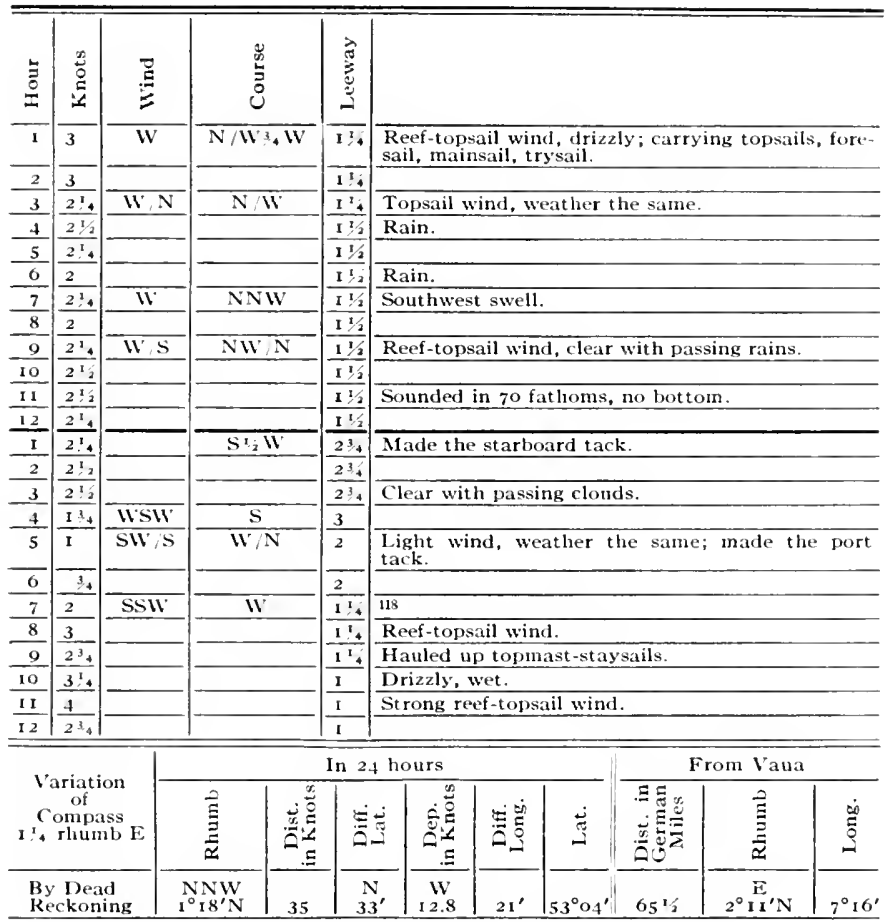

113 Khitrov's journal gives observed but discarded latitude $52^{\circ} 27^{\prime}$. 
h October $3 I, I 7+1$. After Midday

\begin{tabular}{|c|c|c|c|c|c|c|c|c|c|c|c|c|}
\hline$\stackrel{\Xi}{\Xi}$ & 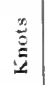 & 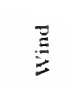 & \multicolumn{2}{|l|}{ 菤 } & בְ⿱ & & & & & & & \\
\hline $\bar{I}$ & $\overline{35}$ & S,W & \multicolumn{2}{|c|}{$\mathrm{W}^{3}{ }_{4} \mathrm{~S}$} & $11^{14}$ & \multicolumn{7}{|c|}{$\begin{array}{l}\text { Reei-topsail wind, drizzly, rain; carrying topsails, } \\
\text { foresail, trysail, and staysails. }\end{array}$} \\
\hline 2 & $3 \mathrm{x}_{2}$ & & \multirow{2}{*}{\multicolumn{2}{|c|}{$W N$}} & $1^{5}+$ & & & & & & & \\
\hline 3 & $22^{3}+$ & SW S & & & $I^{x_{4}}$ & & & & & & & \\
\hline 4 & 23 & & \multicolumn{2}{|c|}{ WNW } & $\overline{I^{1} \dot{2}}$ & \multicolumn{7}{|c|}{ Hauled down main-topmast-staysail. } \\
\hline 5 & $\overline{23.4}$ & & & & $\overline{I ! 3}$ & & & & & & & \\
\hline 6 & $\overline{23 / 4}$ & $\mathrm{SW} / \mathrm{W}$ & \multicolumn{2}{|c|}{ NW: SV } & $1 \mathrm{I/2}$ & \multirow{2}{*}{\multicolumn{7}{|c|}{ W'ind as before, heav'y passing clouds. }} \\
\hline 7 & $22^{32}$ & & & & $11 / 2$ & & & & & & & \\
\hline 8 & 25 & & \multicolumn{2}{|c|}{$N W$ ' } & $1+1 / 2$ & \multirow{2}{*}{\multicolumn{7}{|c|}{ Starsout }} \\
\hline 9 & $21_{1}$ & & & & $1 \div 2$ & \\
\hline 10 & $2 ! 2$ & & & & $1 \% / 2$ & & & & & & & \\
\hline$I I$ & 2 & & \multicolumn{2}{|c|}{$\mathrm{NW} W$} & $\overline{134}$ & \\
\hline 12 & $\overline{21_{4}}$ & & \multirow{3}{*}{\multicolumn{2}{|c|}{$N W W: 2 W$}} & $\overline{I^{3} 4}$ & \multicolumn{7}{|c|}{ Topsall wind. } \\
\hline $\mathrm{I}$ & $2^{1}+4$ & sil & & & $1: 2$ & \multicolumn{7}{|c|}{ Squally clouds; occasionally the stars are seen. } \\
\hline 2 & 234 & & & & $11 / 2$ & \multicolumn{7}{|c|}{ At $2: 30$ made the starboard tack. } \\
\hline 3 & $\overline{19}$ & SW: W & \multicolumn{2}{|c|}{$\mathrm{S} \mathrm{E}$} & 3 & \\
\hline 4 & 2 & & & & 3 & \multirow{2}{*}{\multicolumn{7}{|c|}{ Squally clouds, snow, cold. }} \\
\hline 5 & 216 & WSW & \multicolumn{2}{|l|}{$\mathrm{s}$} & 234 & & & & & & & \\
\hline 6 & 2 & & & & $23^{3} 4$ & \\
\hline 7 & 2 & & & & $23_{4}^{3}$ & \multirow{2}{*}{\multicolumn{7}{|c|}{$\begin{array}{l}\text { At } 8: 30 \text { came about with the wi nd and made the } \\
\text { port tack; unbent the foresail because it was no } \\
\text { longer sea worthy and bent on a new one. By the } \\
\text { will of God died the Yakutsk soldier Karp Pashen- } \\
\text { noi, and we lowered him into the sea. }\end{array}$}} \\
\hline 8 & 1 & & & & $23 / 4$ & & & & & & & \\
\hline 9 & $\overline{112}$ & SSW & \multicolumn{2}{|c|}{$\mathrm{WW}$} & $11_{4}^{13}$ & \\
\hline 10 & $2 \%$ & $\mathrm{~S}, \mathrm{WW}$ & \multicolumn{2}{|c|}{$W / N$} & 1 & \\
\hline II & 4 & & \multirow{2}{*}{\multicolumn{2}{|c|}{ W }} & $I$ & & & & & & & \\
\hline 12 & $3 / 2$ & $\mathrm{~s}$ & & & $3 / 2$ & \multicolumn{7}{|c|}{$\begin{array}{l}\text { Reef-topsail wind, snow, squally clouds; at times } \\
\text { the sun is seen. }\end{array}$} \\
\hline & & & & & $\ln 2$ & $\mathbf{h}$ & urs & & & & om Vaua & \\
\hline & $\begin{array}{l}\text { oriati } \\
\text { of } \\
\text { ompa } \\
\text { rhum }\end{array}$ & $\begin{array}{l}\text { Ion } \\
\text { ass } \\
\text { nb E }\end{array}$ & $\stackrel{\hat{\Xi}}{\underline{\Xi}}$ & $\frac{n}{0}$ & & 茪 & 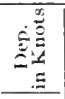 & 里菩 & $\stackrel{5}{S}$ & 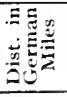 & 氖 & ஹٌ \\
\hline & $\begin{array}{l}\text { I Dea } \\
\text { eckon }\end{array}$ & & $\begin{array}{l}\text { NW/N } \\
1^{\circ} 23^{\prime},\end{array}$ & $37^{1}$ & & N. & $\begin{array}{c}1 \mathrm{~W} \\
21.6\end{array}$ & $36^{\prime}$ & $53^{\circ} 35^{\prime}$ & $60^{34}$ & $\begin{array}{c}\mathrm{E} / \mathrm{N} \\
1_{32^{\prime} \mathrm{E}} \\
\end{array}$ & $6^{\circ} 4^{\prime} 0^{\prime}$ \\
\hline
\end{tabular}


Fovember 1, I741. After Midday

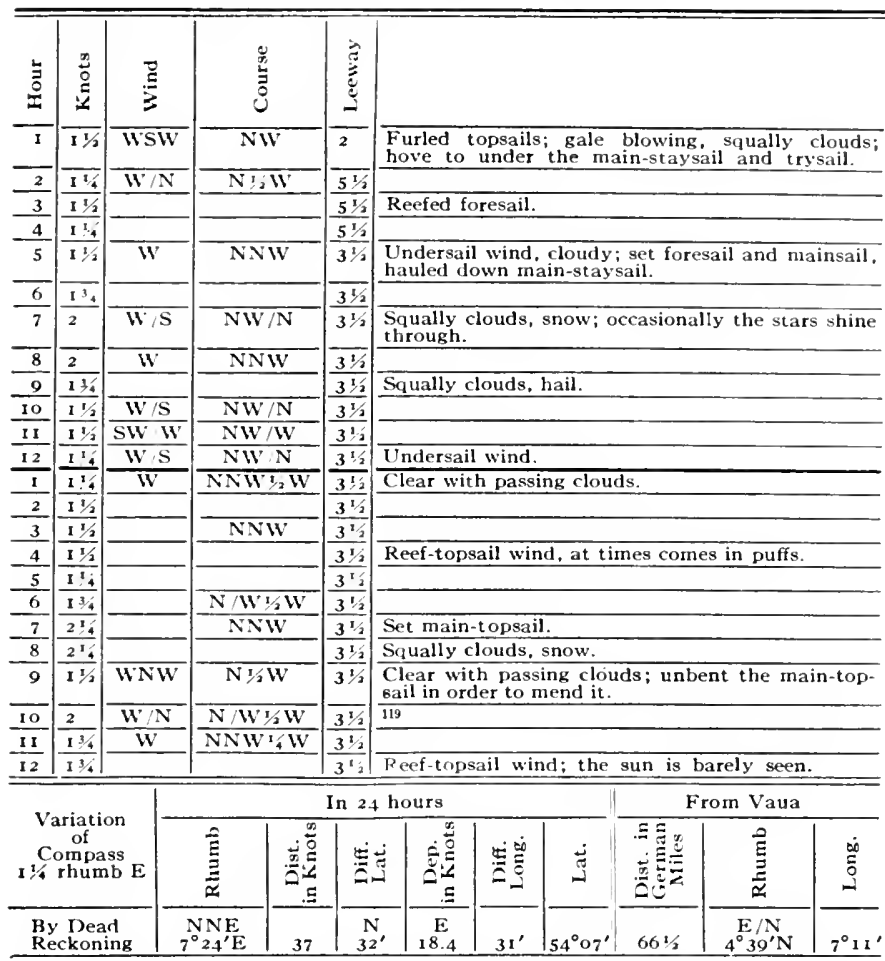

110 Khitrov's journal: "On the sick list: the Captain Commander and 36 men. With great difficulty the others manage to run the boat." 
(3) November 2, 174I. After Midday

\begin{tabular}{|c|c|c|c|c|c|c|c|c|c|c|c|}
\hline 点 & 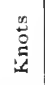 & 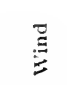 & 递 & & 苞 & & & & & & \\
\hline$I$ & $11 / 2$ & W & $\mathrm{NN}$ & & $\overline{3 ! 1}$ & Reef-tops & ail win & d, clear & with pa & sing clouds & \\
\hline 2 & $21 / 2$ & & & & 134 & $\begin{array}{l}\text { Set topsi } \\
\text { and trysa }\end{array}$ & $\begin{array}{l}\text { ils ree } \\
1 \text { in ad }\end{array}$ & $\begin{array}{l}\text { fed; ca } \\
\text { dition. }\end{array}$ & rrying & resail, ma & insail, \\
\hline 3 & $\begin{array}{l}2_{4}^{14} \\
\end{array}$ & $\mathrm{~W} / \mathrm{N}$ & $\mathrm{N} / \mathrm{W}$ & $2 \mathrm{WV}$ & $I 1 / 2$ & & & & & & \\
\hline 4 & $\overline{3^{12}}$ & & & & $11 / 2$ & & & & & & \\
\hline 5 & $1: 4$ & W & SS & & $1: 4$ & $\begin{array}{l}\text { Topgallat } \\
\text { tack. }\end{array}$ & tsail " & ind, cle & oudy; mi & de the sta & board \\
\hline 6 & $1^{3} 4$ & & & & $\begin{array}{r}3.4 \\
\end{array}$ & & & & & & \\
\hline 7 & $I^{5}$ & SW I & S & & 2 & & & & & & \\
\hline 8 & $I$ & SW & IV & & 2 & $\begin{array}{l}\text { Light win } \\
\end{array}$ & d. stan & sout; 1 & hade the & port tack. & \\
\hline 9 & $\mathrm{I}_{4}$ & SSE & IVS & & $1 / 2$ & & & & & & \\
\hline 10 & 214 & & & & $1 / 2$ & & & & & & \\
\hline II & 214 & & & & $1 / 2$ & Stars shin & ing. & & & & \\
\hline 12 & $2^{1 / 2}$ & & & & ${ }_{12}^{2}$ & Westerly & swell & & & & \\
\hline 1 & $21 / 2$ & $S E$ & WSW & $2 \mathrm{~W}$ & $\sqrt[3]{4}$ & Topsail $\mathrm{x}$ & ind. & & & & \\
\hline 2 & $23 \frac{1}{4}$ & & & & 34 & & & & & & \\
\hline 3 & $3 \%$ & SSE & WS & & & Reef-tops & ail win & d, chilly & drizzly & & \\
\hline 4 & 4 & & & & & & & & & & \\
\hline 5 & 4 & SE:S & SW & & & & & & & & \\
\hline 6 & 4 & & & & & $\begin{array}{l}\text { I am alt } \\
\text { stand my }\end{array}$ & $\begin{array}{l}\text { ogether } \\
\text { watch }\end{array}$ & $\begin{array}{l}\text { exhau } \\
\text { only be }\end{array}$ & $\begin{array}{l}\text { sted fro } \\
\text { cause of }\end{array}$ & $\begin{array}{l}\text { scurvy, } \\
\text { xtreme nec }\end{array}$ & $\begin{array}{l}\text { and I } \\
\text { essity. }\end{array}$ \\
\hline 7 & 4 & & & & & & & & & & \\
\hline 8 & 4 & & & & & & & & & & \\
\hline 9 & 4 & & & & & $\begin{array}{l}\text { Southerly } \\
\text { the naval }\end{array}$ & $\begin{array}{l}\text { swell. } \\
\text { carper }\end{array}$ & $\begin{array}{r}\text { By th } \\
\text { ater, die }\end{array}$ & $\begin{array}{l}\text { e will of } \\
\text { d. }\end{array}$ & rod Ivan 1 & etrov, \\
\hline 10 & $4^{12}$ & & & & & & & & & & \\
\hline 11 & 3 & & & & & $\begin{array}{l}\text { Strong u } \\
\text { the dead }\end{array}$ & $\begin{array}{l}\text { ndersai } \\
\text { body i }\end{array}$ & $\begin{array}{l}1 \text { wind } \\
\text { nto the }\end{array}$ & $\begin{array}{l}\text { furled } \\
\text { sea. }\end{array}$ & topsails; 1 & wered \\
\hline $\mathbf{1 2}$ & 3 & & & & & & & & $\ldots$ & & \\
\hline \multirow{2}{*}{\multicolumn{3}{|c|}{$\begin{array}{c}\text { Variation } \\
\text { of } \\
\text { Cornpass } \\
I^{1}+\text { rlumb E }\end{array}$}} & \multicolumn{6}{|c|}{ In 24 hours } & \multicolumn{3}{|c|}{ From Vaua } \\
\hline & & & 总 & 总造 & 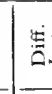 & 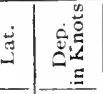 & 参高 & 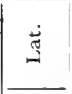 & 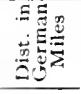 & 产 & 品 \\
\hline \multicolumn{3}{|c|}{$\begin{array}{l}\text { By Dead } \\
\text { Reckoning }\end{array}$} & $\begin{array}{l}11 / \mathrm{S} \\
8^{\circ} \mathrm{O} 7^{\prime} \mathrm{WV}\end{array}$ & 48.7 & 5 & \begin{tabular}{c|c} 
S & VV \\
$2^{\prime}$ & 8.4 \\
\end{tabular} & $82^{\prime}$ & $154^{\circ} \mathrm{O} 2^{\prime}$ & $54 \%$ & $\begin{array}{l}E N E \\
4^{\circ} 22^{\prime} \mathrm{E}\end{array}$ & $5^{\circ} 49^{\prime}$ \\
\hline
\end{tabular}


\& November 3, I741. After Midday

\begin{tabular}{|c|c|c|c|c|c|c|c|c|c|c|c|c|}
\hline$\stackrel{\Xi}{\Xi}$ & 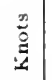 & $\frac{5}{3}$ & \multicolumn{2}{|c|}{ 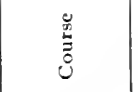 } & $\stackrel{\vec{E}}{\stackrel{\Xi}{\tilde{E}}}$ & & & & & & & \\
\hline $\mathbf{I}$ & $3 \%$ & SSE & \multicolumn{2}{|c|}{ SW $/ \mathrm{W}$} & I & \multicolumn{7}{|c|}{$\begin{array}{l}\text { Reef-undersail wind, squally clouds, rain; carry- } \\
\text { ing foresail, mainsail, and trysail. }\end{array}$} \\
\hline 2 & $\overline{3^{1}}$ & & & & $\mathrm{I}$ & & & & & & & \\
\hline 3 & 4 & SE & & & $66^{\mathrm{s}, 2}$ & \multicolumn{7}{|c|}{ Gale, cold, rain. } \\
\hline 4 & 4 & & & & $\overline{652}$ & \multirow{3}{*}{\multicolumn{7}{|c|}{$\begin{array}{l}\text { Furled foresail and mainsail; love to on the try- } \\
\text { sail because of the strong wind. } 1 \text { is dangerous } \\
\text { to go on our way esperially at niglit. }\end{array}$}} \\
\hline 5 & 4 & & & & $6 \%$ & & & & & & & \\
\hline 6 & $I$ & $\mathrm{E} / \mathrm{S}$ & \multicolumn{2}{|c|}{$\overline{\mathrm{S}}, \overline{\mathrm{E}}$} & $\overline{6 \div 2}$ & & & & & & & \\
\hline 7 & $\mathrm{I}$ & & \multirow{2}{*}{\multicolumn{2}{|c|}{ 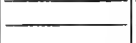 }} & $\overline{61 / 2}$ & \multicolumn{7}{|c|}{ to go on our way esperially at night. } \\
\hline 8 & $\mathrm{I}$ & & & & $61 / 2$ & \multirow{2}{*}{\multicolumn{7}{|c|}{ 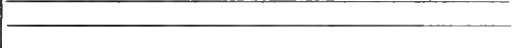 }} \\
\hline 9 & $I$ & & & & $61 / 2$ & & & & & & & \\
\hline 10 & I & & & & $6 \frac{1 / 2}{6}$ & \\
\hline II & $\mathrm{I}$ & $\mathrm{E}$ & \multicolumn{2}{|c|}{$\mathrm{S} \mathrm{E}$} & $\overline{61 \cdot 2}$ & \multicolumn{7}{|c|}{ W'ind going down, drizzly, rainy. } \\
\hline 12 & $I$ & & & & $\overline{6 ! 2}$ & \multicolumn{7}{|c|}{ 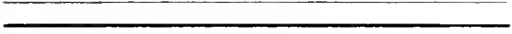 } \\
\hline $\mathbf{I}$ & $\mathbf{I}$ & & \multirow{2}{*}{\multicolumn{2}{|c|}{ SSE }} & $6 \div$ & \multirow{2}{*}{\multicolumn{7}{|c|}{ Rain. }} \\
\hline 2 & I & E N & & & & & & & & & & \\
\hline 3 & $\mathrm{I}$ & & & & \\
\hline 4 & $I$ & ENH & \multirow{2}{*}{\multicolumn{2}{|c|}{$\mathrm{SE}^{\mathrm{S}}{ }_{2} \mathrm{E}$}} & $\overline{6^{2}}$ & \multicolumn{7}{|c|}{ Undersail wind, clear with passing clouds. } \\
\hline 5 & I & & & & $\overline{6^{2}}$ & \multicolumn{7}{|c|}{ Drizzly, rainy. } \\
\hline 6 & I & & & $\overline{6,2}$ & \multirow{2}{*}{\multicolumn{7}{|c|}{ Set fore and main-topsatls. }} \\
\hline 7 & $3^{3}+4$ & & \multirow{2}{*}{\multicolumn{2}{|c|}{ WSW }} & & & & & & & & \\
\hline 8 & $3 \frac{1}{2}$ & & & & & \multicolumn{7}{|c|}{ Strong reef-topsail wind. } \\
\hline 9 & 4 & & & & & \\
\hline I0 & 4 & & & & & \multicolumn{5}{|c|}{ Clear with passing clouds. } & & \\
\hline II & $4+2$ & & & & & \multicolumn{7}{|c|}{ Undersail wind. } \\
\hline 12 & $\mid \overline{4 / 2}$ & & & & & \multicolumn{7}{|c|}{ Drizzly, heavy easterly swell. } \\
\hline \multirow{2}{*}{\multicolumn{3}{|c|}{$\begin{array}{l}\text { Variation } \\
\text { of } \\
\text { Compass } \\
\text { I rhumb E }\end{array}$}} & & & & 241 & ours & & & & m Vaua & \\
\hline & & & $\stackrel{0}{\Xi}$ & 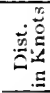 & & 每 & 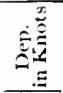 & 里音 & ตु่ & 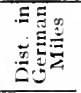 & $\hat{\vec{\Xi}}$ & 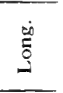 \\
\hline & $\begin{array}{l}\text { Dea } \\
\text { eckon }\end{array}$ & & $\begin{array}{l}\text { W/S } / \mathrm{S} \\
2^{\circ} 7^{\prime} \mathrm{S}\end{array}$ & $55^{1 / 2}$ & & $\mathrm{~s}_{1}$ & $\begin{array}{l}\text { W } \\
54\end{array}$ & $9 I^{\prime}$ & $53^{\circ} 49^{\prime}$ & $40^{3 / 4}$ & $\underset{2^{\circ} 50^{\prime} \mathrm{E}}{\mathrm{ENE}}$ & $4^{\circ} 18^{\prime}$ \\
\hline
\end{tabular}


† November 4, 1741. After Midday

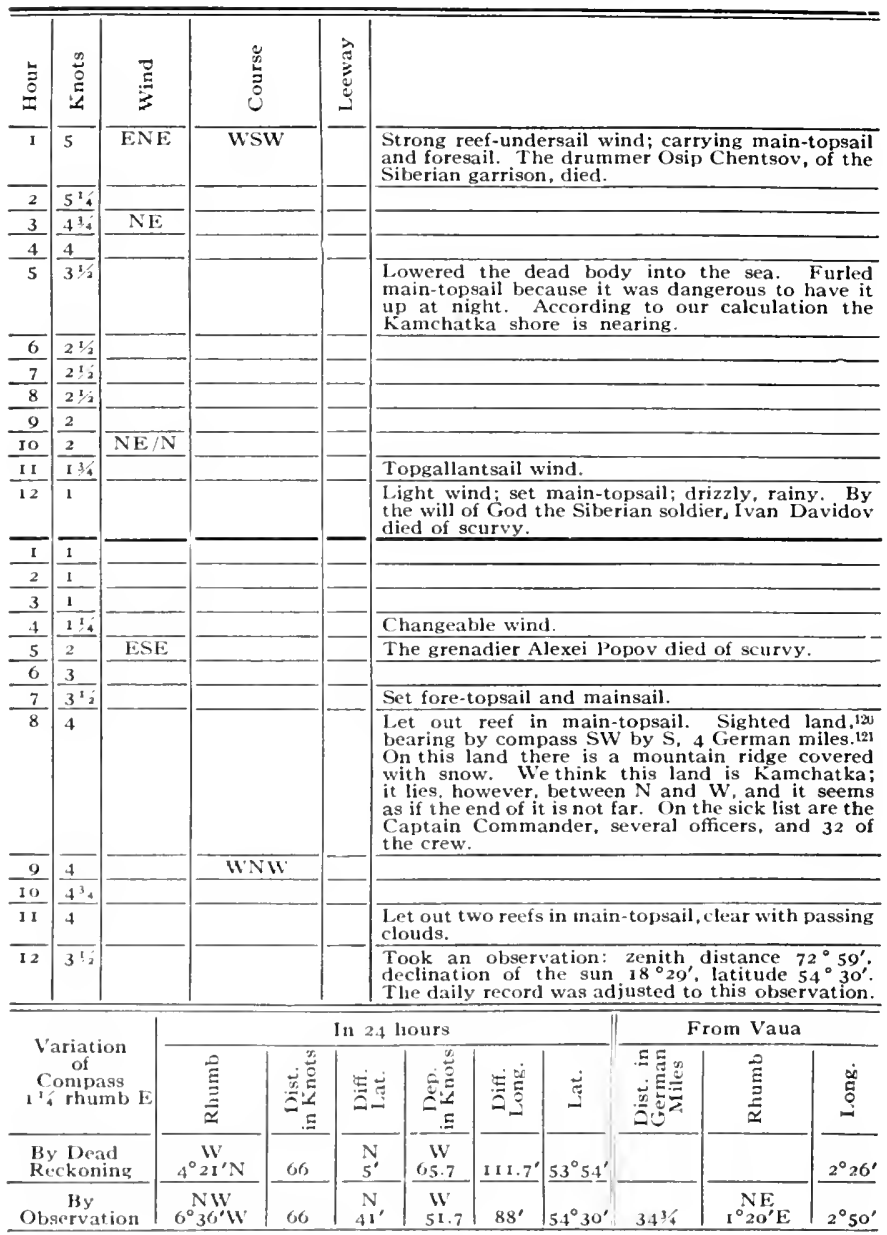

130 Copper Istand (Meini Island).

121 Khitrov's journal: "Siglted land with ligh snow-covered mountains SW by S 7 German miles." 


\section{2f November 5, 1741. After Midday}

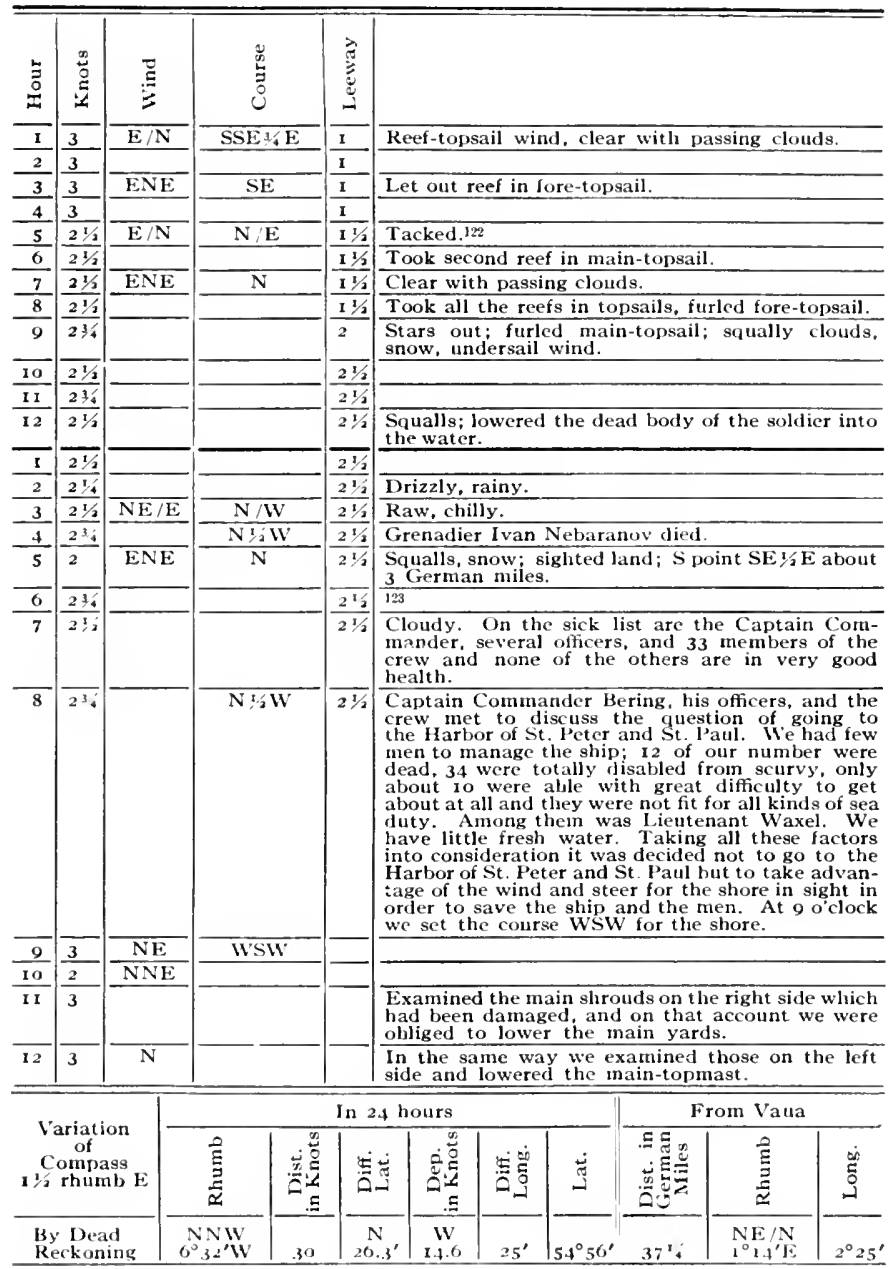

(Foornotes 122 and 523 at bottom of next page) 
ㅇ November 6, I741. After Midday

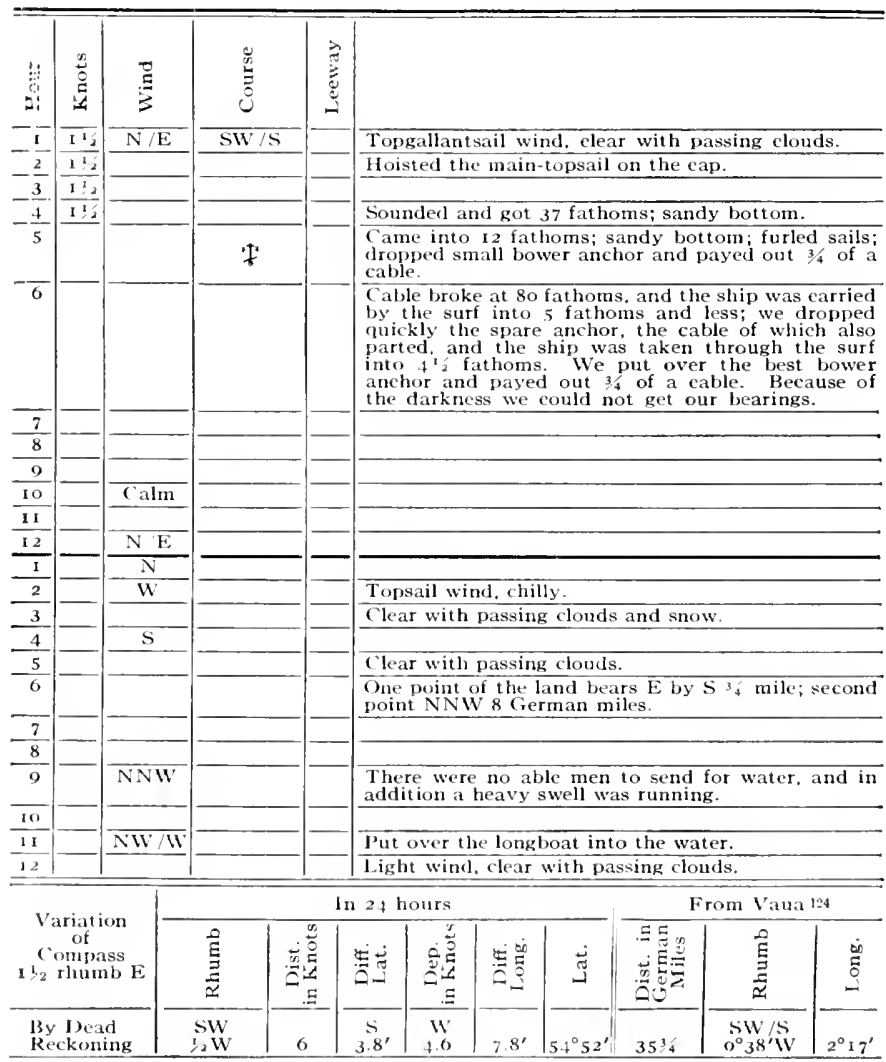

121 The rhumb here seems to be stated exceptionally as direction to Vaua from the slip; from Vaua it would be $\mathrm{NE} / \mathrm{N} 0^{\circ} 38^{\prime} \mathrm{E}$. The value for the difference in longitude between Vaua and the scene of the St. Peter's stranding on Bering Island, $2^{\circ} 17^{\prime}$, indicates the extent of the error in the reckoing of the ship's position. The true value is about $8^{\circ} 20^{\prime}$. On the general question of error in longitude, sec also Waxel's report, p. 276; Clirikov's report, p. 322 ; and footnote 22, p. 308 .

Footnotes to Log of Notember 5

122 Klitrov's journal: "Came ahout with the wind to get away from the shore. The S point of the sighted land bore SlE, E."

123 Khitrov's journal: "rhe Captain Commander, officers, and crew lad a meeting and deeided to make for the land aliead of us. W' rould not go on becarse we liatl no

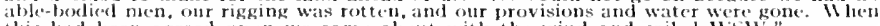
this had been agreed upon we came about with the wind and sailed WsW." 


\section{1) November 7, $17+1$. Afler Midday ${ }^{125}$}

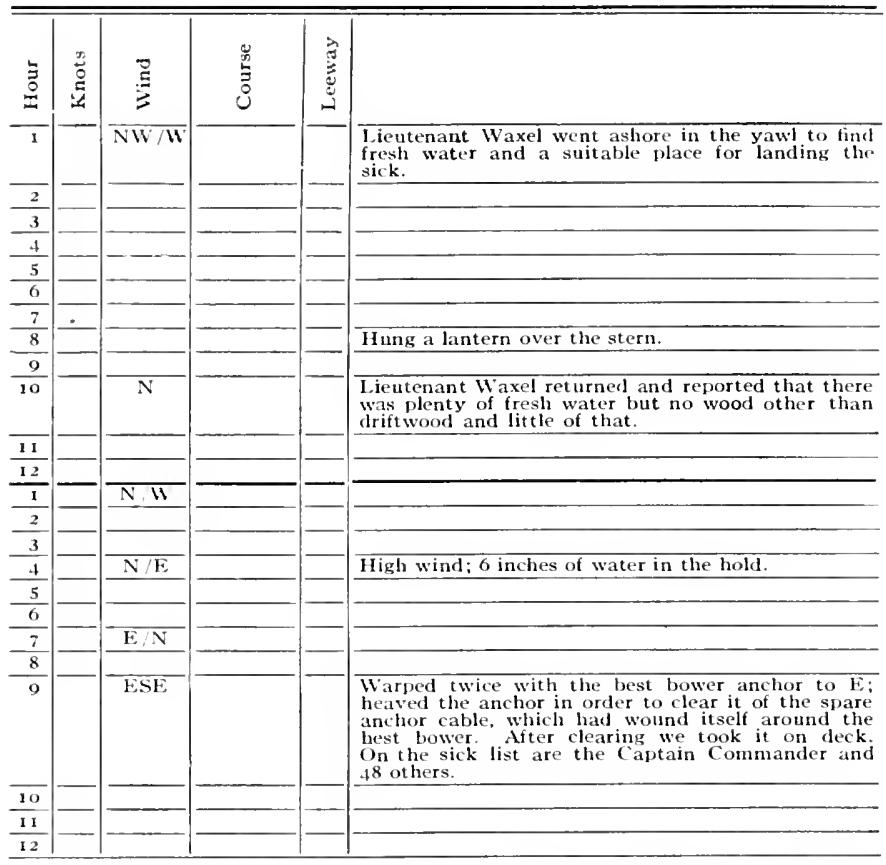

125 Khitrov's joumal. From November 7, 1741 to August 9, 1742, inclusive, Khitrov's journal is used. During this period Vushin's journal consists of a general aceount of happenings. These have, in the following, been inserted in their proper plares as foot notes to Khitrov's journal. 
November 8, 1741. After Midday

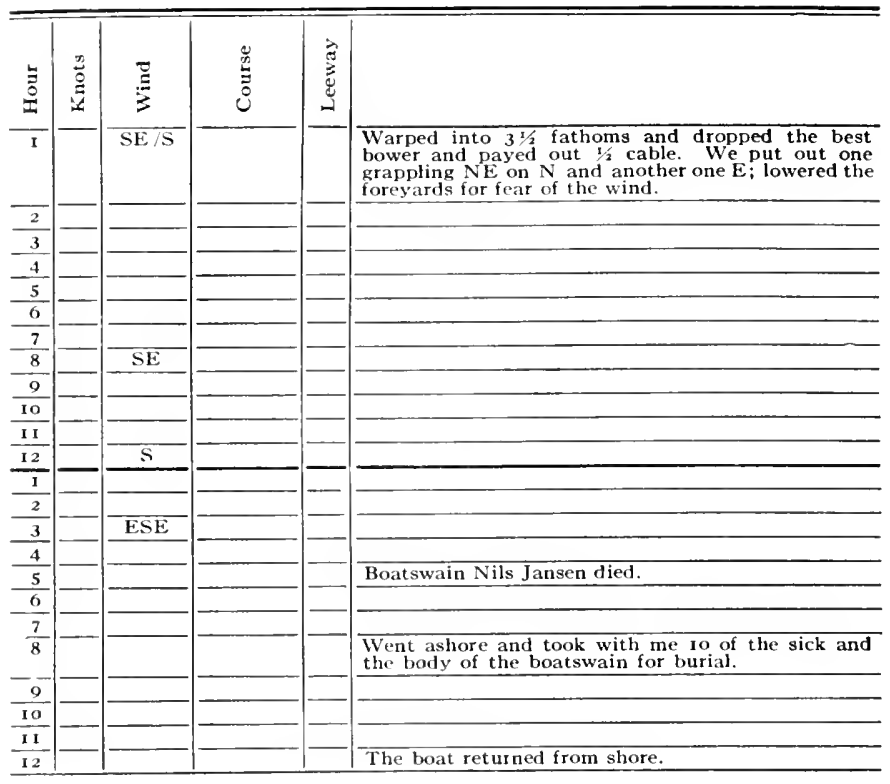


(3on'mber 9, 1741. After Midday

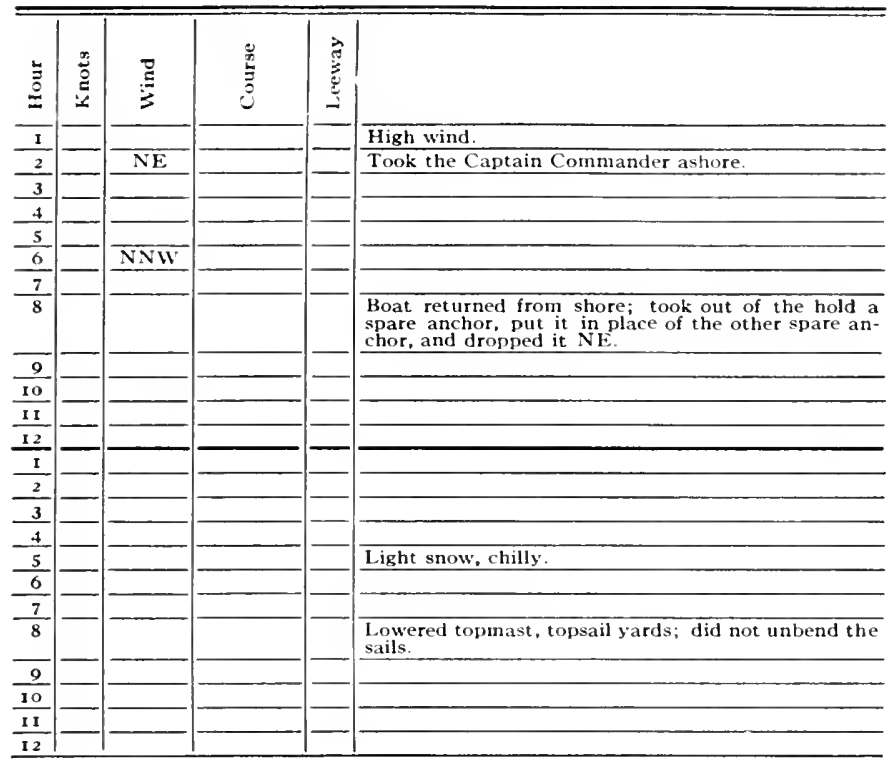


of November 10, 174I. After Midday

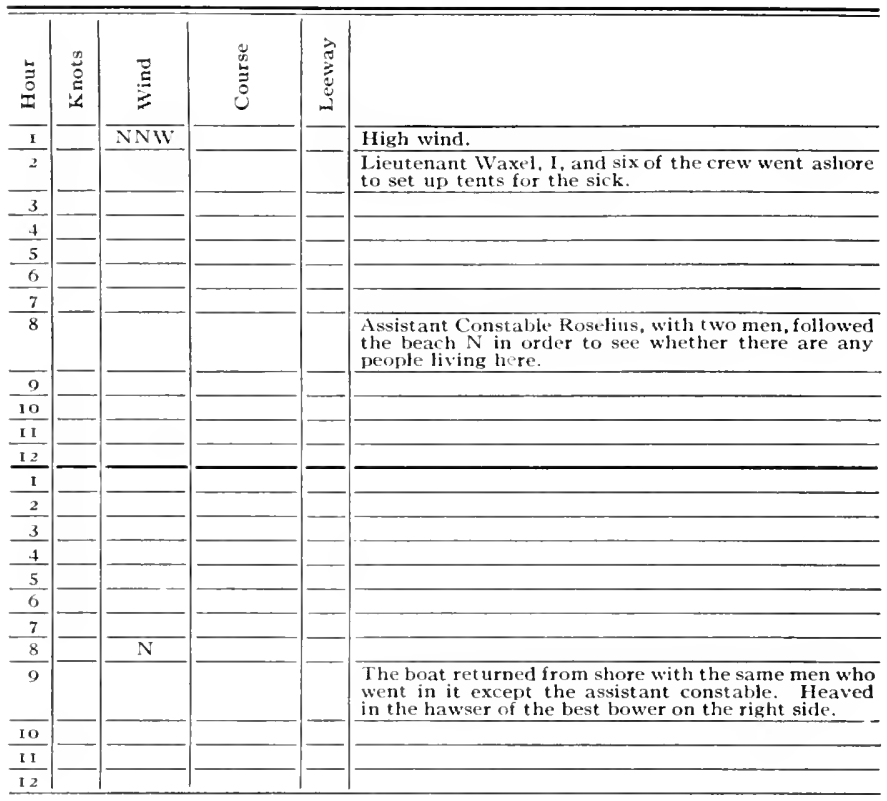


$\Varangle$ November $11,17+1$. After Midday

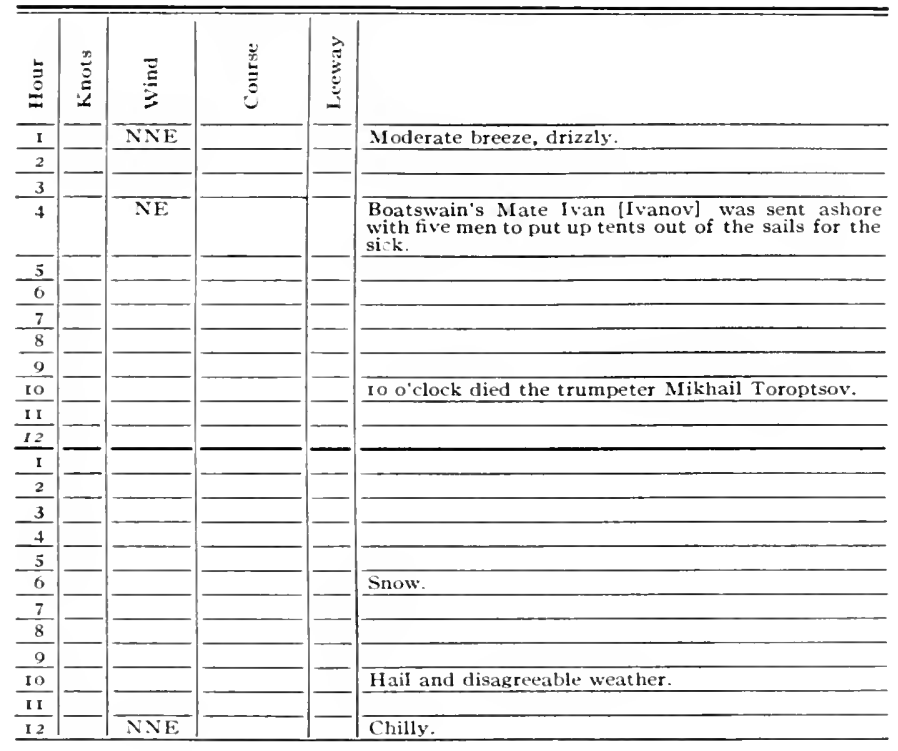


Z November 12, I74I. After Midday

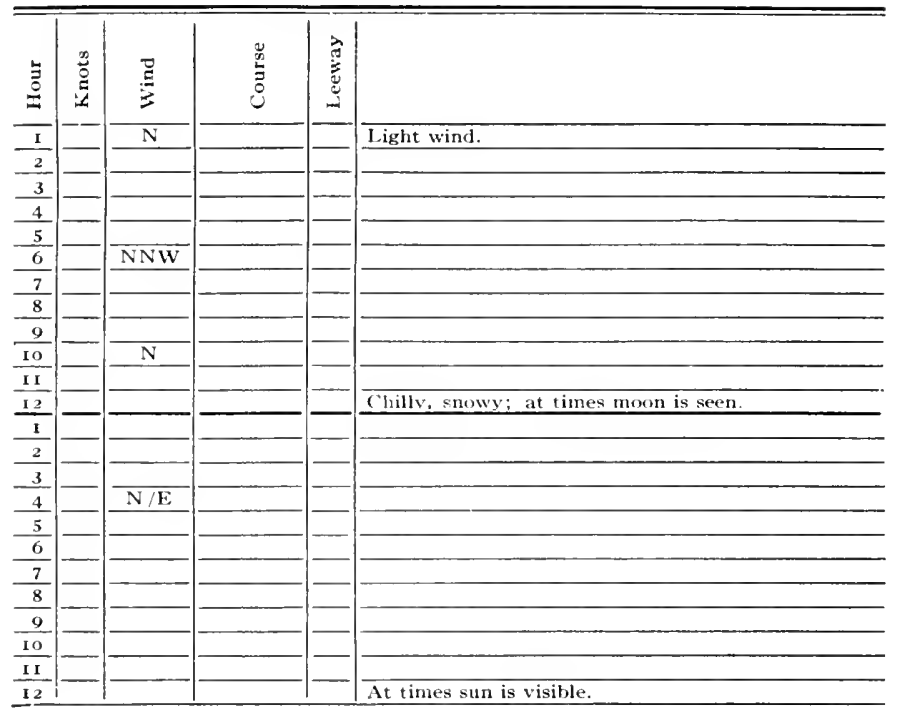


ㅇ November 13, 174I. Afler Midday

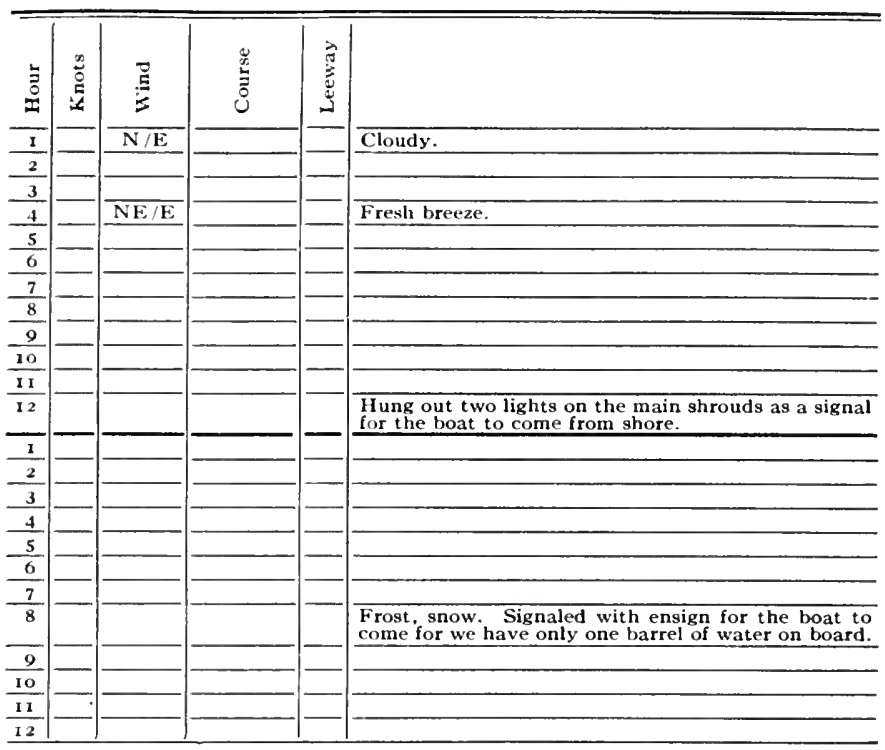


I) November 14, 174I. After Midday

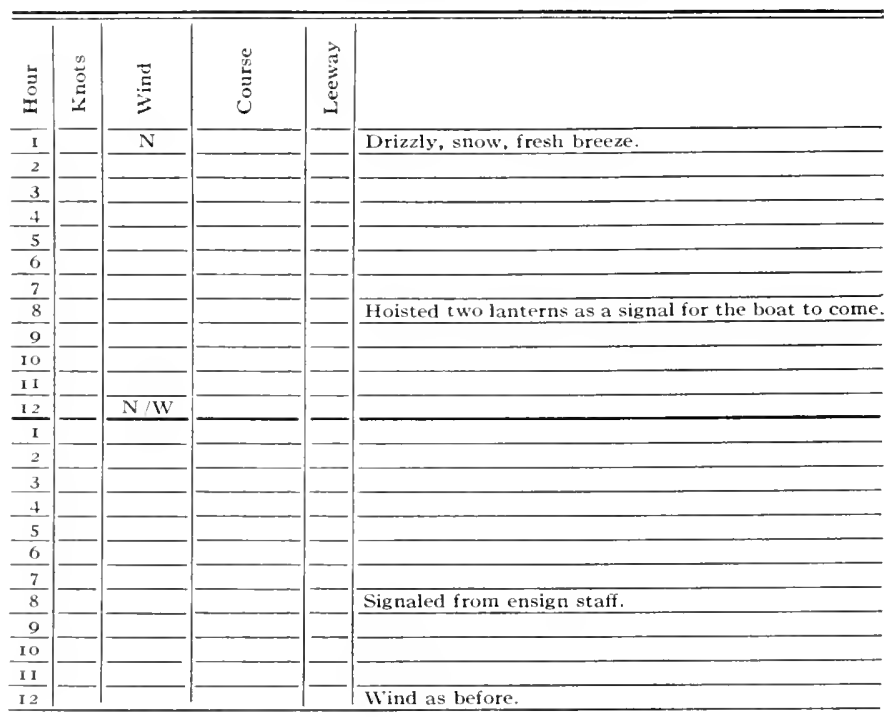


O November 15, 1741. After Midday

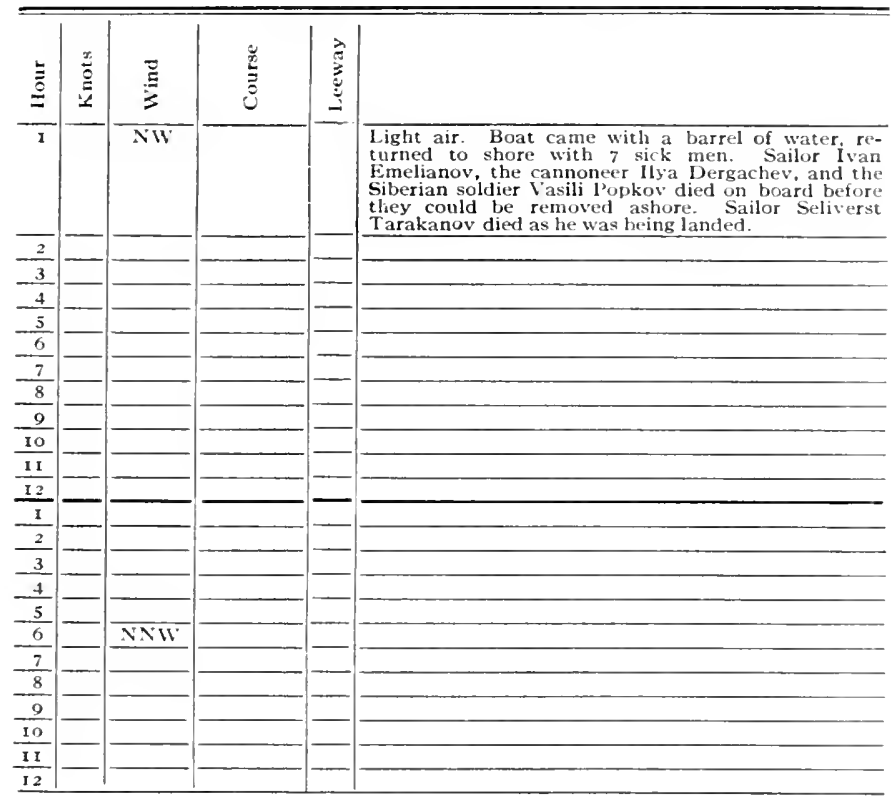


(3 November 16, 1741. After Midday

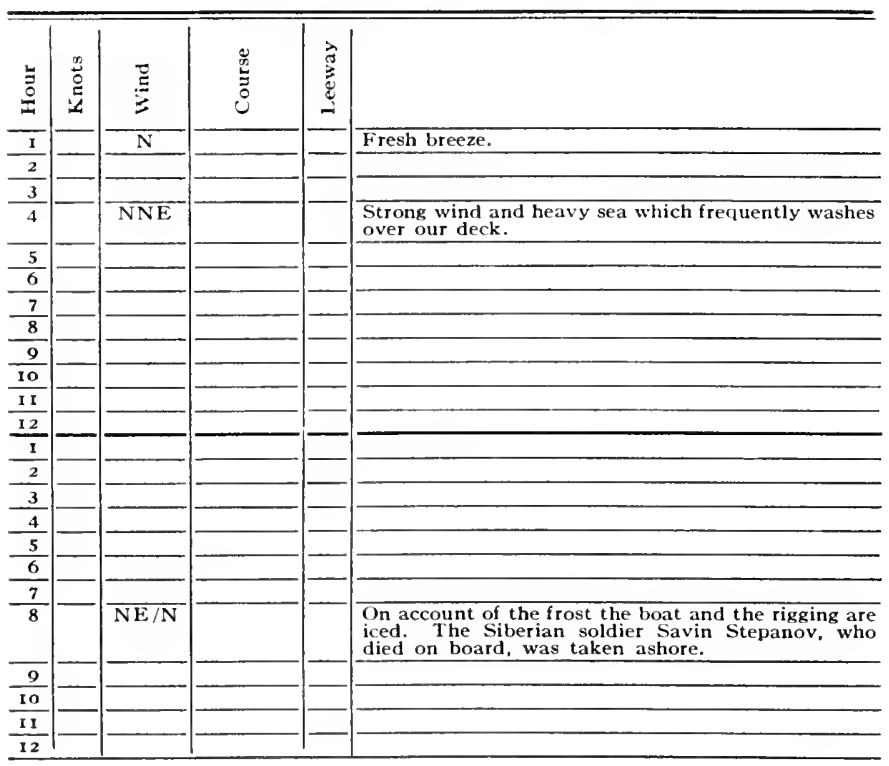




\section{o November 17,1741 . After Midday}

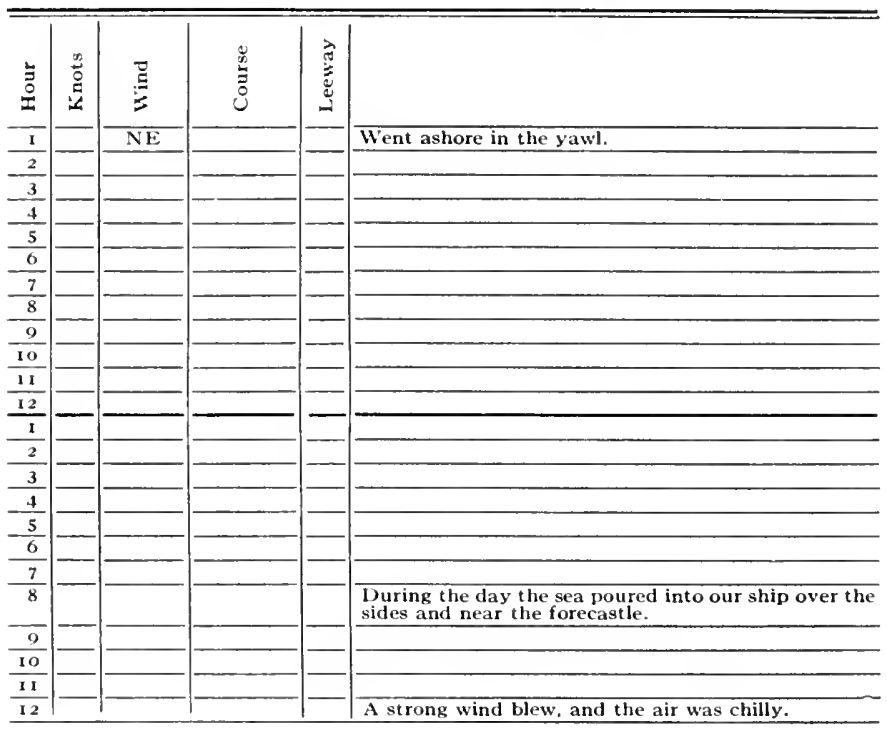


$\Varangle$ November $I S, I 7+I$. After Midday

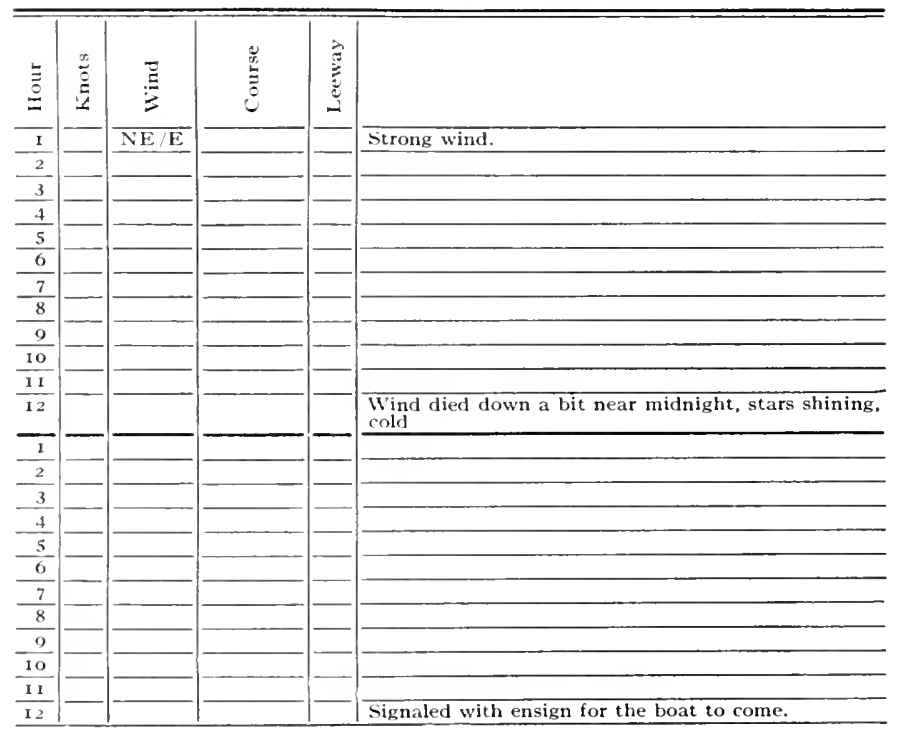


$2($ November 19, 1741. After Midday

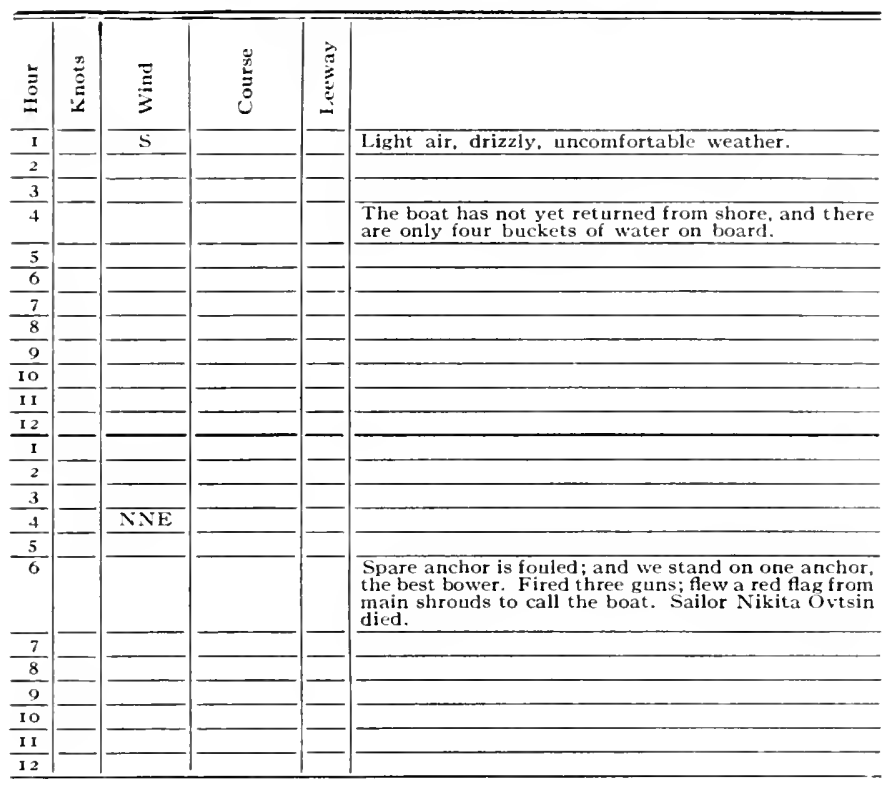


QNovember 20, 174I. After Midday

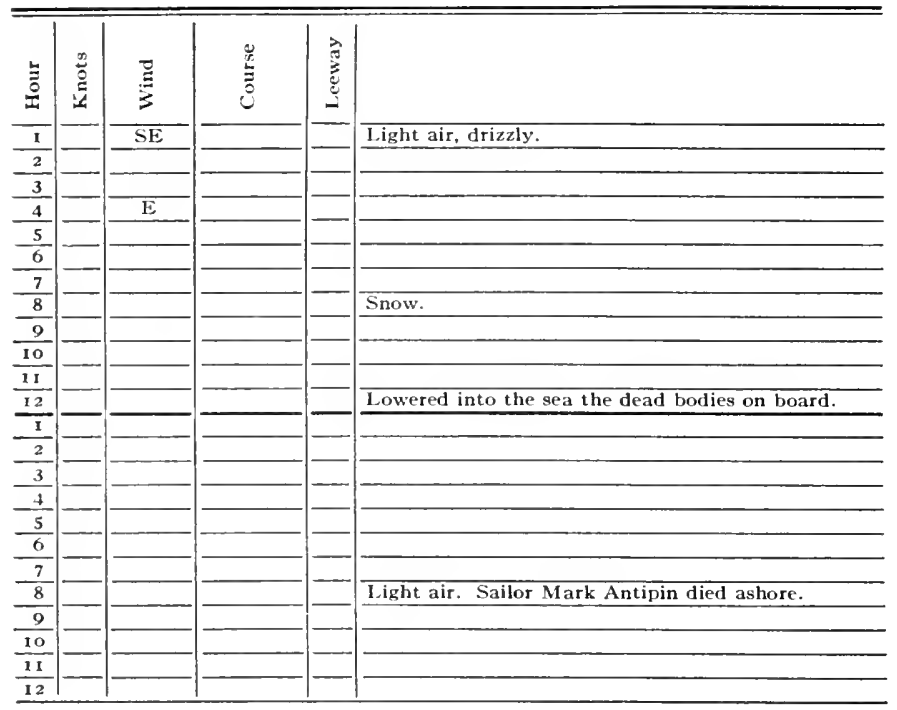


h November 2I, 174I. After Midday

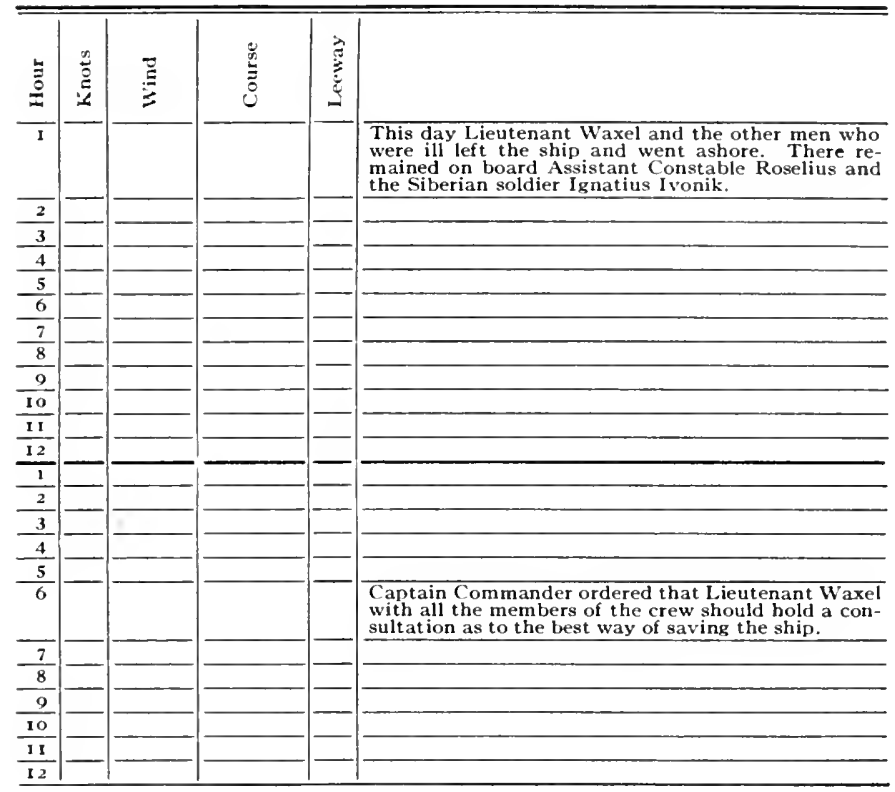


November 22, I7t1. After Midday

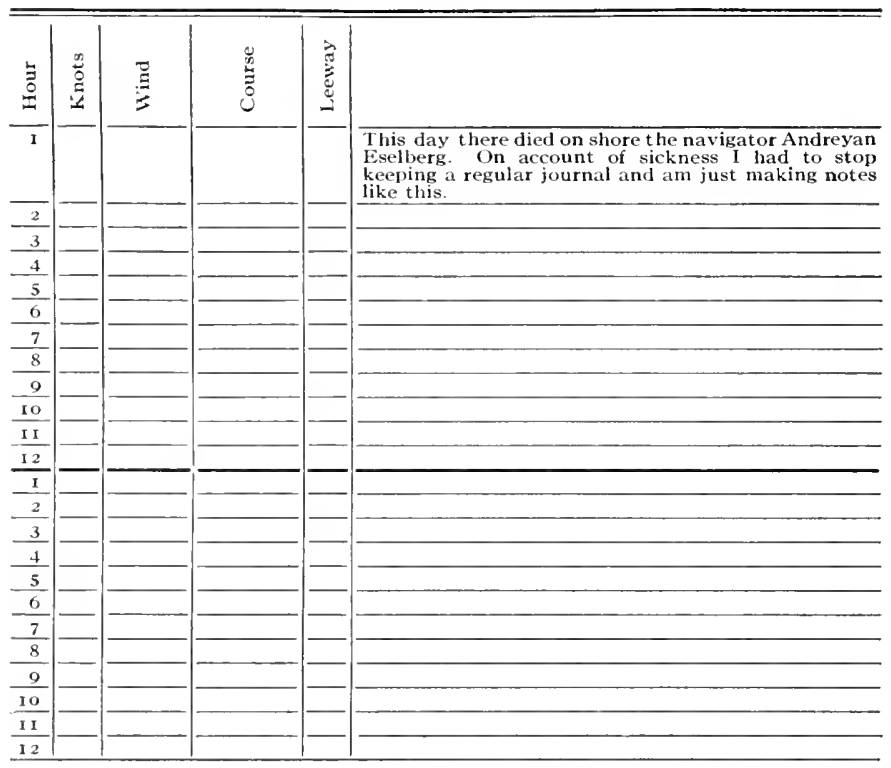


(1) Noiember 23, 174I. Afler. Midday

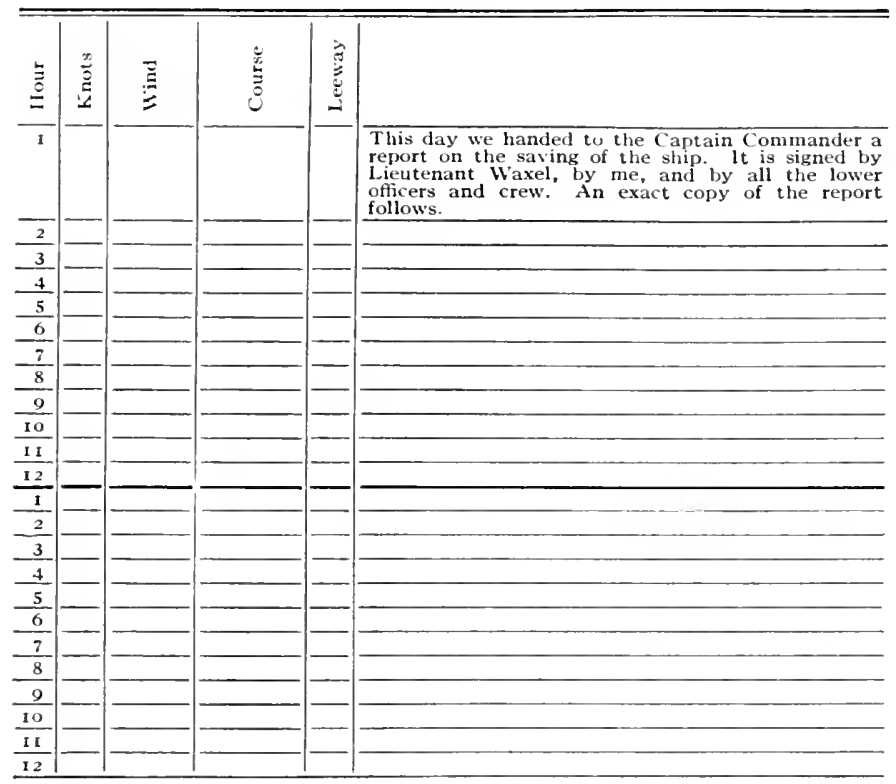




\author{
November 23, 1741 \\ REPORT ON SAVING THE SHIP
}

To His Highness Captain Commander Ivan Ivanovich Bering:

On November 21, I74I, your highness ordered me, the undersigned, to consult with the higher officers and the crew as to the best way of saving the St. Peter with the provisions and material on board and also as to a place where she might be hauled up for the winter. In accordance with your order I, Fleet Master Sofron Khitrov, all lower officers, and members of the crew met on November 22 to discuss these points.

r. The St. Peter has one anchor ont, the best bower, and its cable is not very dependable. We have no other anchors or grapplings aside from the 8-pood grappling. As you know, the ship lies out in the open sea and if a strong wind should come up from the east, southeast, west or northwest, one anchor would not hold. On the east, north, and west there are rocky reefs. If a strong wind should blow up from the south, or from between south and west. the ship would be driven out to sea.

2. We therefore agreed that at high tide the boat should be hauled up on the sandy beach facing it and made fast with hawsers. The water casks should not be emptied, the ballast should not be taken out, and the cargo should not be disturbed now; all these things can be done later in the winter, if nothing interferes. In order to save the provisions from the water in the ship, cables should be put under them.

We have no other suggestion to make, and we leave the matter for your highness' decision. Something must be done at once to save the vessel because of the winds, which may cause us to lose both the ship and supplies.

\title{
Lieutenant Sren WAXEL. Fleet Master Sofron KHItrov Assistant Navigator KHARLAM Yushin Assistant Skipper Nikita Khotyajntsov Assistant Constable Boris Roselius Boatswain's Mate AleXeI Ivanov
}

For the quartermaster, Luka Alexeiev, for all the crew, and for himself the sailor Timofei Anchiugov signed.

On the above written report on the saving of the St. Peter, I received, this $23 \mathrm{rd}$ day of November, an order from the Captain Commander to carry out the recommendations of the report at the very first opportunity. I am now waiting for favorable weather to do so.

$$
\text { Nonember 24, I74I }
$$

Strong wind, impossible to go aboard.

Nonember 25, $17+1$

Strong wind, impossible to go aboard. 


\section{November $26,1741^{126}$}

Today I am quite ill with scurvy and can barely stand on my feet. Nevertheless, this morning I went to the boatswain's mate, Alexei Ivanov, and [told him to go] to the Captain Commander and say that I am ready to go aboard the ship in order to lay it up if this can be done. The Captain Commander told Ivanov that in case the ship could not be hauled up we should at least bring ashore as much of the provisions as we could. When I walked down to the beach to go on board I was told by the above-mentioned Ivanov, who was on watch that day, that he had only five able-bodied men. In pushing out the yawl one of them, the soldier Elizar Zaikov, got wet and had to go back to the tent; and this left four men, namely the sailor Timofei Anchiugov, the soldier Grigori Izmailov, the Kamchatka servant I van Partinyagin, and the Chukchi and Koriak interpreter Alexei Lazukov. They were so weak that I realized that they could never weigh the best bower anchor. In addition, the wind was between north and west straight on the rocky reef which lay from the ship between south and east not more than $5^{\circ}$ fathoms away.

The shrouds and yards were also down. Although we might have enlisted a few more men, yet because of the reef and the wind it did not seem safe to try to lay up the ship as we had recommended in the report. When $I$ saw the impossibility of carrying out the task I went to Lieutenant Waxel and verbally explained the situation. He told me to report to the Captain Commander, which $I$ did at ten o'clock in the morning and gave him the reasons already mentioned. When I left him I went to see the sailor Dimitri Ovtsin who acted as the captain's adjutant, and told him to make a written statement of what has taken place and enumerate the reasons I gave.

November $27,17+1$

Today I was so ill that I could not leave the tent for any reason. In the same tent are Lieutenant Waxel and number of the crew.

126 Yushin's journal:

\section{VARIOUS Happenings in I 74 I After November 6}

This montl from the 6 th to the 22 nd we had at times fair wind and weather, but at other times the winds were so strong that it was impossible to come ashore. The sick were landed. and huts were made for them by digging holes in the ground and covering them over with sails. After the 22 nd we were all ashore and the $S t$. Peter was at anchor without a man on board, for there was no one well enough to stand watch or to unrig the ship; some of the men could not even move from their places and others were at the point of death.

According to our reckoning we were in Kamchatka, and therefore the assistant constable Roselius, with two men from Kamchatka, was sent north to examine the shore. Roselius returned in six days, after having walked more than so versts. because he and his med were so exhausted that they could not go farther. 


\section{November 28, I74I}

I was told this morning that during the northeast storm the anchor cable broke and the St. Peter was driven ashore on the spot where we had planned to lay her up.

\section{December $1, I 741$}

Captain Commander Bering sent the sailor Timofei Anchiugov with two men to examine the shore and to determine whether we are on the mainland or on some island. They were also to keep a lookout for forests.

\section{December 4,1741}

The calker Alexei Klementev died.

\section{December 8,1741}

5 A. M. Captain Commander Bering died, and Lieutenant Waxel succeeded to the command.

\section{December $9,174 I$}

Assistant Skipper Nikita Khotyaintsov died.

\section{December 17,1741}

Grenadier Ivan Tretyakov died.

\section{December $27,1741^{127}$}

Cannoneer Prokofei Efintsov died.

The sailor Timofei Anchiugov returned without bringing any information about the land [we are on].

$$
\text { January 2, } 1742
$$

The soldier Fedor Panov died.

\section{January 7,1742}

I I P. M. died the petty officer Ivan Lagunov.

127 Yushin's journal:

\section{THE MONTH OF DECEMBER, 1741}

By the will of God a strong NE wind broke the cable and drove the ship ashore near us. Its left side below the water line was badly damaged, the rudder was lost, and it suffered other injuries as well.

The sailor Anchiugov, with the two men from Kamchatka already mentioned, were orrered south to obtain information. He was gone about four weeks but did not leam anvthing definite. He silid that he thought that we were on an island. He could not follow the shore for any considerable distance because of the cliffs. He reported seeing many herds of sea otters. At the end of becember 1 began to recover somewhat from my illness. 
January 18,1742

\section{Statement on the Condition of the Ship}

Lieutenant Waxel, Master Khitrov, the lower officers and crew of the St. Peter met on this day to inspect the ship and determine whether it is fit for the continuation of our voyage. The condition of the ship was as follows:

I. The bottom, keel, sternpost and stem were all damaged, and the rudder was carried out to sea.

2. There was not a single anchor for the voyage, and there was no hope that we could recover one of those lost.

3. The rigging, shrouds, cables are not to be depended on; they snap and tear, and some are already torn.

4. It is impossible that the ship could be moved from its present position because it is already deeply buried in the sand.

5. Below the water line on the left side there is a crack crossway's.

Therefore, the ship is not fit for a continuation of our voyage further.

Lieutenant Svex WAXEL

Master Sofrow Khitrov

All the lower officers and crew of the St. Peter, except the sailor Dimitri Ovtsin, signed this.

\section{Counterstatement by the Sailor Dimitri Oytsin}

To His Highness Lieutenant Waxel:

You commanded that the crew should meet on January 22 to sign the report that the St. Peter is no longer fit for our voyage, and these were the reasons given.

I. The bottom, ked, sternpost, and stem were damaged, and the rudder was gone, and it is impossible to examine the inside because of the water in the hold.

2. We have not a single anchor, and there is no hope of finding one.

3. The rigging, shrouds, ropes are not to be depended on, for they break and in many places are already broken.

4. It is not probable that the ship can be moved from its place, since it is deeply buried in the sand.

5. Below the waterline there is a crack crossways. Therefore, the ship is unfit for further service.

On these points I should like to comment to your highness:

1. The sternpost and stem are not so badly injured that they cannot be repaired; although the false stem is gone, another can be made. As to the condition of the bottom, it can hardly be ascertained now owing to the snow and ice. Another rudder can be made, and timber for that purpose could probably be found.

2. We have not yet tried to recover the anchors, and it is not too much to expect that they may be found in summer. In the spring the winds are surely not as bad as in the fall, and the weather may become favorable so that we could get the ship in a position to repair it.

3. Some of the bad rigging could be repaired, other parts could be replaced from the stores; we have also spare ropes in case of need. If, of the four anchors, we find two, we should have enough for our purpose. 
4. Because of the snow and ice it is difficult to say whether the ship can be floated; if it can be floated, it can be repaired and used with the help of Almighty God.

5. At present it is difficult to say how badly damaged the bottom is; and, even if it were, it could be repaired.

These are my views, and these are the reasons why I refuse to sign the report to the effect that the ship was unfit for further service.

January 27, 1742 .

Sailor Dimitri OvtsiN

\section{January 29,1742}

\section{REBUTTAL}

Lieutenant Waxel, Fleet Master Khitrov, all the lower officers and members of the crew of the St. Peter met today to listen to the reading of the statement of Dimitri Ovtsin which he handed to Lieutenant Waxel on January 27 of this year. In this document Ovtsin said that he hoped that the St. Peter could be saved, that it could be floated, that the damage could be repaired, that is to say the stem and everything else, that a new rudder could be made out of timber to be found on the island, and that the anchors lost at sea near here could be recovered. After listening to his statement and reasons they were rejected by all who were present, because on January 8 they had examined the ship and found it unseaworthy. A report on this subject had been made and was signed by the officers and crew. Even if the ship were sound it could not be floated since we have no anchor, no timber, and not enough men for such work. Taking all this into consideration it was agreed (Ovtsin dissenting) that in Marcli the St. Peter should be broken up and out of the wreck some kind of small vessel should be made to take us to Kamchatka. In the meantime we should watch for an opportunity when the weather is favorable to empty ont the water from the hold and to inspect again the ship with a view of determining its seaworthiness.

\section{Lieutenant SVEN WAXEL Master Sof Ron KHITROV and all the officers and men ex- cept the Sailor Ovisin}

\section{February 23, 1742}

Assistant Navigator Yushin with four men was ordered to follow the shore to latitude $56^{\circ} 10^{\prime} \mathrm{N}$, which was the position of the mouth of the Kamchatka River. On the way he was to make a careful observation of the country.

\section{March 8, 1742}

Yushin and his party returned and reported that he had not gone farther than 70 versts from our camp because his advance was blocked by a rocky bluff which could not be passed even at low water. A fierce blizzard interfered also. Yushin said that when he was about 50 versts from camp he sighted land to the eastward, about ro miles away, which seemed like an island. ${ }^{127 a}$

ma See bracketed statement in third paragraph from bottom of p. 237 . 
March 10,1742

Lieutenant Waxel, Fleet Master Khitrov, all the lower officers, and the crew had another meeting to discuss Yushin's report. Since it told them nothing definite about the land, it was decided to send another party under the boatswain's mate Alexei Ivanov.

March I5, 17.42

Ivanov with four men was sent along the shore to explore the land.

March I9, $17 \$ 2$

Ivanov came back and reported that bluffs blocked his way to the westward.

March 22, 1742

Ivanov was sent out again with instructions to exert himself to the utmost to proceed farther and secure sone reliable information.

$$
\text { A pril 7, } 1742
$$

Ivanov and his party returned and reported that we were on an island because he had doubled the northern cape on the other side, which is about 150 versts from our camp.

\section{A pril 9,1742}

\section{Decision Made on Determination That Land Is an Island}

Lieutenant Waxel, Fleet Master Sofron Khitrov, Doctor Georg Steller, all the lower officers and crew of the St. Peter met to discuss the report of I vanov. He said that the land on which we live is an island because he had doubled the last northern cape on the western side and it was therefore evident that we were on an island. In order to reach Kamchatka it was decided to break up the St. Peler because it was not fit for further sea service, because it cannot be floated, and because of other reasons already mentioned, and make out of it some kind of small vessel.

It was agreed that during the period of the vessel's construction a number of our men under the leadership of Yushin should be detailed to hunt and to supply the camp with meat. Whatever they got should be divided equally among all according to the size of each group. From the stores of flour left after the April distribution, ro sacks should be set aside for the Kamchatka voyage. Until the voyage. the allowance should be reduced below that which had been allowed.

Lieutenant SVEN WAXEL

Fleet Master SAFron KHItrov and all the men

$$
\text { May } 2,1742
$$

Lieutenant Waxel, Fleet Master Khitrov, the lower officers, and some members of the crew looked about for a suitable place for building and launching a boat. Such a spot was found on the beach directly in front of the ship. 


\author{
May 6, 1742
}

With God's help we began the building of the ship by erecting the stem and the sternpost. It is to have a length of 36 feet, a 12 -foot beam, and depth of 5 feet 3 inches.

July $I, I 742$

I started along the beach toward $\mathrm{S}$ in order to examine the channel between us and the islands ${ }^{128}$ sighted and to see whether we could use it when we sailed from here. I was also anxious to learn whether there was some good harbor along the coast. Alexei I vanov went in the yawl to sce if he could find the anchors, but did not locate any except the 8-pood grappling.

July 9,1742

About 7 P. M. I returned with my party. We found a channel between the islands but no harbor. I should say that it is about 50 versts to the southern cape.

\title{
July 13,1742
}

Alexei Ivanov went again to look for the anchors but was unsuccessful. Weather clear and sunshiny. I received an order to make an inventory of all that we are to leave on the island.

\section{July 15,1742}

Light air from W, weather clear and sunshiny. When the ship was broken up Lieutenant Waxel, I, the lower officers, and those members of the crew who were engaged in the work inspected her and found that she was injured in twelve places.

$$
\text { July } 24,1742
$$

Yushin went in scarch of the anchors and did not find them.

Light air from SW, fine weather.

Midshipman Ivan Sint handed in his report of July 1, and with it was a list of the baggage and provisions each person had. The following is an exact copy:

128 Khitrov was under the impression that Copper Island was two islands. See bracketed statement in next-to-last paragraph on p. 237. 
List of Baggage of EAch Meiber of the Expedition

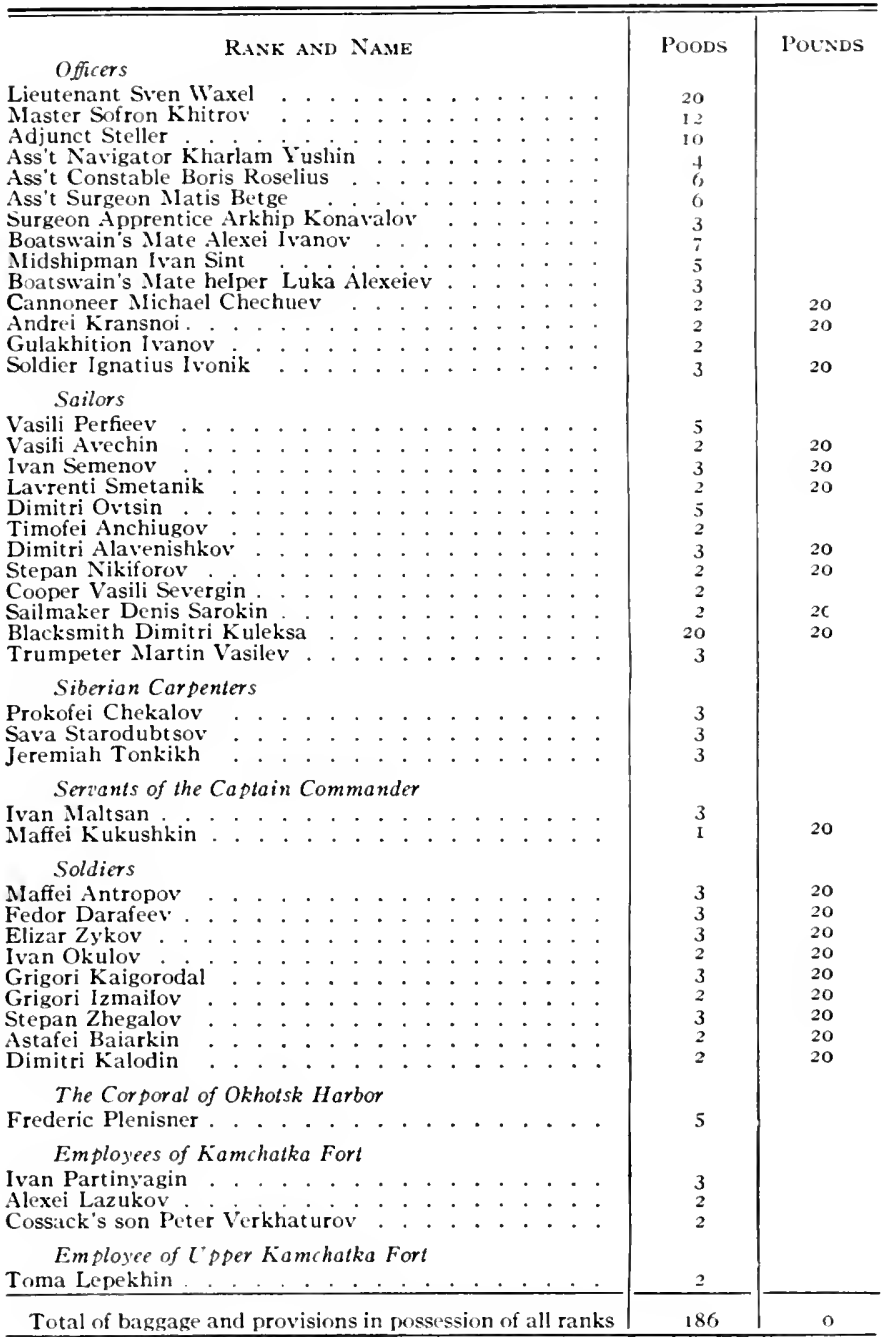




\author{
August I, 1742
}

\title{
Decision on Disposition of Supplies
}

Lieutenant Waxel, Fleet Master Khitrov, all lower officers and members of the crew of the St. Peter met. Our ship with God's help will soon be finished. The question came up what to do with all the supplies and materials on hand which we cannot take along for the following reasons.

I. To take all that material along would oblige us to leave behind half or more of the crew, and to leave these men on an unknown and uninhabited island is dangerous. There is little food to be lad here aside from what may be obtained by hunting sea animals, and even this source cannot be depended upon. Since our coming the game has been frightened away and driven off, and one may not in the future rely on hunting.

2. Of the materials and other supplies on hand only a small part could be taken along, but owing to the autumn storms of this region it would not be safe to overload our vessel. A large part of the supplies and materials is worthless and rotten, as may be seen from the inventory ${ }^{129}$ made out by Fleet Master Khitrov.

3. Although a small part of the above-mentioned material is good, yet it is not worth while to leave some one to watch it, because the island is uninhabited.

4. If we should leave a guard, we should have to come after him next year. There is no harbor here, nothing but rocks and reefs and the open sea, and there is great danger of wrecking the vessel.

5. Taking all these arguments into consideration, the undersigned have unanimously agreed to leave no one behind. Of the materials we should take iron enough to serve as ballast; of supplies, water, several casks of sea cow meat, and such other things as we need for the voyage.

All else should be left in the warehouses to be constructed.

\section{Lieutenant Sven Waxel, Fleet Master Sofron Khitrov, all the lower officers and crew}

\section{DESCRIPIION OF BERING ISLAND 130}

(A Description of the Island On Which We Lived From November, 1741, To August 13. 17.42, (to which are added] Certain Observations Which 1 Made.)

By agreement with Lieutenant Waxel I called the island "Bering Island" after the late Captain Commander Vitus Bering, who died and was buried there.

This island extended from latitude $54^{\circ}$ to $56^{\circ} \mathrm{N}$, that is from the southern cape, which we called Manati, that is to say Sea Cow. The island lies between NNW [and SSE?] and is about 130 versts long and from 7 to 10 versts wide. There are

129 The inventory exists but has not been included in the present work.

130 Yushin's journal:

VARIOUS HAPPENINGS IN I 742

On different days and montlis we secured information which led us to believe that we were on an island. This island is about eighteen German miles long and its axis lies NW and SE. It is wider in the north than in the south, averaging, from $N$ to $S$, is. Io, 8 , and 6 versts. It is impossible to go around some parts of the island on account of the clifts and rocky beaches, which 1 myself saw in many places. There are some bays around the island. It is not safe to approach the island nearer than about 5 versts. Across from our camp, or on the south side, there is a lake about I verst in circumference. and out of the lake a river flows into the sea. This river is about soo fathoms in length and 15 in width. I think that a small vessel, drawing not more than 5 feet of water. could enter this stream at high tide during the months of May and June. In the autumn 
no habitations on the island and no signs that there ever were any. It is a treeless region; there is no wood on it at all except in a few spots where a few willows grow, and they are so small that they are worthless for [fire] wood. There are mountain ridges, many volcanoes, and rocky bluffs; and therefore [it is] quite unsafe for ships to come near. The shore is everywhere rocky, some of the rocks extend a verst or more into the sea. In high water they are covered up, and in low water they are exposed. In those places where the shore is not rocky a heavy surf runs. There is no spot where a vessel drawing five or six feet of water may winter. We put ourselves to a great deal of trouble in looking for such a place-the boatswain's mate, Alexei Ivanov, was sent to the northern cape, and I went to the southern. There is only one spot, on the other [W] side of the island from our cape, where a vessel might go in. There is a lake over there which has an outlet to the sea, and in time of high water a ship might enter the lake and remain during the winter. But one cannot be sure even of this; for each year the old channel fills up, and a new channel is formed. Whether the entrance is always as [open?] as when we were there it is impossible to say.

When the west wind blew, it brought from Kamchatka to this island building timber which had been in use, [such as] smashed boats and Koriak deer sleds. When the east wind blew it brought from America big pine trees, oars, and weapons such as are used by the natives of America but not by those of Kamchatka.

When the weather was clear many of our men claimed that from the western side of our island they saw more than once snow-covered volcanoes to the westward. We assumed from our reckoning that they must be on Kamchatka but we could not be sure.

During the whole time that we were on this island we suffered great hardships. Our habitations were holes in the sand covered over with sails. We had to search the beach for driftwood, which we had to carry on our shoulders ro and i 2 versts. At that very time nearly all of us were very ill with scurvy which hung on so long that some of the men did not completely recover until spring, when green grass

the mouth of the river is covered with driftwood, and the surf plays there. The lake is deep enough, I think. I have sounded and got 3 feet [along the shore?], and at the mouth of the river the depth is 6 feet. On the western side of the island there is a breeding place for fur seals and sea lions. They came in the beginning of April, and soon their large numbers covered the beach. We used them for food when the sea otters became scarce in the spring.

By conmon consent we began to break up the $S t$. Peter in April, and in May we laid the keel of the new vessel, which measured 36 feet at the keel. Ten men were detailed for shipbuilding. the ot hers. petty officers and crew, broke up the old ship and brought the meat from the south side, a distance of about is versts over the hills. Each man carried about 2 poods, and it was hard work. An additional hasdship was the fact that we ran out of shoes. From the middle of June we began to hunt the sea cow. These are huge beasts, each giving from 50 to 60 poods of meat and even a greater amount of fat. They have two forefeet and [. ? ?

\section{POSITION OF BERING ISLAND}

In the north there is a cape NNW 8 German miles. From this cape there is a projection NW 5 miles. To the south of us there is a point ESE $3 / 2 \mathrm{E} 34$ of a mile. From this point the shore continues south 4 miles. From that point the shore extends NW to the northern point.

About Io German miles away, in the direction $N E$ by $N$ from Bering's Cross, there is an island which is not seen at all times. IThere is no island in the direction indicated. and what Yushin saw was prohally a cloud bank. Chirikov tells of sighting land on June 26-27. which, on further examination, proved to be a cloud bankl.

Another istand is seen $\mathrm{E}_{3 / 4} \mathrm{~N} 6$ German miles, a third island $\mathrm{E}$ by $\mathrm{S} 7$ German miles. These two islands extend $\mathrm{NE}$ and SW; they seem to be small but very high. IIn the direction indicated there is only one island, Copper lsland, which, when viewed from Bering Island, looks like two islands and is so noted on the early charts.

The latitude of Bering's Cross, when I took it on the 7 th day of July, was $55^{\circ} 05^{\prime} \mathrm{N}$. variation of the compass $13^{\circ} 58^{\prime}$, which I accepted as 1,4 rhumb E. 
appeared, which grass is known in Kamchatka as sweet grass and from which the government there manufactures a kind of wine. It tastes something like Russian borshch. ${ }^{131}$ We used this grass as well as other grasses for food. We had no provisions and had to secure our food with great trouble, and such as we found was bad and unfit for human beings. We were obliged to go along the beach 20 and 30 versts in the hope of killing a sea otter, sea lion, or hair seal. On seeing them we would steal up behind them as they were on the beach or on the rocks, when the tide was out, and kill them with clubs, and having killed them we tied them on ourselves with a load in front and a load behind. If for some reason we could not hunt these animals we were forced to find and eat dead ones, such as whales and sea cows, which had been cast up by the sea. During the winter we liunted the animals so hard that they became frightened, and by spring they were quite a distance from our camp. Just then large herds of fur seals came to breed on the other side of the island. They were so numerous on the western side that during the breeding season two or three men could barely get by them in safety, for at that period they are quite savage and attack people. Sometimes the men, in order to get to their destinations, had to cross over the mountain ridges, for they could not get around the beach on account of these animals. Their flesh was quite distasteful, and the longer we ate it the less we liked it. We then turned to lunting the sea cow and did it in the following manner. This animal does not stay far out at sea but keeps close to shore. Its back is above water, and with the flood tide it moves toward the shore to feed on the sea cabbage. As the tide goes out the sea cow goes along so as not to get stranded, being such a large animal. At the time when it was nearing the shore our men in the longboat would pull up close to it, and one of them, standing in the bow, hurled at it a large iron harpoon with sharp teeth. To the harpoon was made fast a cable from 4 to 5 inches in thickness, which cable was held by 20 men who were ashore. When the harpoon had penetrated the flesh and held, the men on shore pulled with all their might while the men in the boat went up close to the animal, cutting and stabbing with sharp iron weapons in order to weaken it and prevent it from breaking the rope, which sometimes happened. This cow is so powerful that it took all these men to hold her. Counting meat and fat, the sea cow gives about 200 poods. $^{132}$ From the time on when we began hunting the sea cow we were not in want. Of all the animals mentioned above the flesh of the cow is the best tasting. We brought with us to Kamchatka a considerable quantity of it salted.

At times the winds on the island are so strong that a person can with difficulty keep on his feet. We concluded that these winds are due to the high mountains and deep valleys. I may say that, owing to these $\mathrm{w}$ inds, the snow in the air, and the blizzards blowing off the mountains, we seldom saw a beautiful or clear day from December to March. From March on, during the spring and summer, there are heavy and continuous fogs and wet weather so that we seldom saw a pleasant day during this period. This kind of weather greatly interfered with the speed of our shipbuilding. In addition, the men were in such poor condition and so undisciplined that it was not safe to order them around, but everything had to be done by common consent. On this island there are no minerals or metals, for Adjunct Steller was sent purposely around the island without finding anything. There are no land animals on the island aside from the blue foxes. There are all kinds of land and sea birds.

131 A soup usually made out of beet root.

1327,200 lbs. 


$$
\text { August } 8,17+2
$$

All the men were preparing cables for launching the ship. After prayer we named the newly constructed vessel the hooker St. Peter and began to launch her. When she had slid down the ways 2 or 3 fathoms the boards of the platform gave way and she stopped.

$$
\text { August } 9,17+2 \text { [ciàil day] }
$$

With the help of God we got the hooker in to the water about 5 o'clock in the afternoon.

\section{The Log Book of the HoOker "St. Peter"}

$$
\text { August 10, 1742 [astronomical day] }{ }^{133}
$$

With God's help I begin this journal on the hooker St. Peter under the command of Lieutenant Waxel. I take as departure Bering's Cross which is in latitude $55^{\circ} 05^{\prime} \mathrm{N}$, variation of the compass $\mathrm{I} / 4$ rhumb easterly.

133 From this date until August 23 inclusive, Yushin's journal is used. Under August Io entry Khitrov's journal gives in addition: "Size of the hooker: length, 36 feet; beam, 12 feet; depth, from the deck to the keelson, $5 \%$ feet." 


\section{LOG BOOK OF THE HOOKER "ST. PETER"}

\section{of August IO, I742. After Midday}

\begin{tabular}{|c|c|c|c|c|c|}
\hline 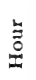 & 苞 & $\stackrel{\Xi}{Z}$ & $\begin{array}{l}\text { 品 } \\
\text { 苛 }\end{array}$ & 离 & \\
\hline I & & & & & Strong wind, heavy rain. \\
\hline 2 & & & & & \\
\hline 3 & & & & & Wind going down, light rain. \\
\hline 4 & & & & & Clear with passing clouds. \\
\hline 5 & & & & & 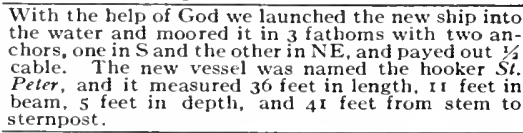 \\
\hline 6 & & & & & \\
\hline 7 & & & & & \\
\hline 8 & & & & & \\
\hline 9 & & & & & I am on watch. \\
\hline Jo & & & & & Wind comes in puffs. \\
\hline II & & & & & \\
\hline 12 & - & & & & Light wind. \\
\hline I & - & & & & Cloudy, stars out. \\
\hline 2 & & & & & \\
\hline 3 & & & & & Chilly. \\
\hline 4 & & & & & $\begin{array}{l}\text { The hooker is drawing } 2 \text { feet } 10 \text { inches of water, and } \\
\text { at the stern } 3 \text { feet. }\end{array}$ \\
\hline 5 & & & & & \\
\hline 6 & & & & Z & \\
\hline 7 & - & & & & \\
\hline 8 & — & & & & \\
\hline 9 & 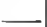 & & & & Shears were brought on board for setting the masts. \\
\hline 10 & & & & & Masts and gear were brought. \\
\hline II & 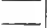 & & & 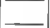 & Rudder was put in place. \\
\hline 12 & & & & & $\begin{array}{l}\text { Fixed the shears for the masts and made ready to } \\
\text { set them in place. }\end{array}$ \\
\hline
\end{tabular}


$\Varangle$ August 11, 1742. After Midday

\begin{tabular}{|c|c|c|c|c|c|}
\hline$\stackrel{\underline{\Xi}}{\stackrel{2}{0}}$ & $\underset{\stackrel{0}{0}}{\stackrel{0}{0}}$ & 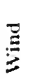 & 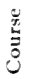 & $\stackrel{\vec{z}}{\stackrel{\Xi}{Z}}$ & \\
\hline I & & & & & Clear. \\
\hline 2 & & & & & Took the shears ashore. \\
\hline 3 & & & & & \\
\hline 4 & & & & & Since noon we set and rigged the masts and yards. \\
\hline 5 & & & & & \\
\hline 6 & & & & & \\
\hline 7 & & & & & $\begin{array}{l}\text { Assistant Constable Roselius relieves me on watch } \\
\text { duty. }\end{array}$ \\
\hline$\overline{8}$ & & & & & \\
\hline 9 & & & & & Clear with passing clouds. \\
\hline to & & & & & Stars out. \\
\hline II & & & & & Light wind and unsteady. \\
\hline 12 & & & & & \\
\hline$I$ & & & & & \\
\hline 2 & & $\mathrm{~S}$ & & & As above. \\
\hline 3 & & & & & \\
\hline 4 & & & & & Cloudy. \\
\hline 5 & & & & & \\
\hline 6 & & & & & Sunshiny. \\
\hline 7 & & & & & The main yard was brought on board and rigged. \\
\hline 8 & & & & & \\
\hline 9 & & & & & $\begin{array}{l}\text { The bowsprit and topsail yard were brought from } \\
\text { shore, the bowsprit was shipped in place. }\end{array}$ \\
\hline 10 & & & & & Clear with passing clouds. \\
\hline II & & & & & $\begin{array}{l}\text { By noon we had stowed away Io barrels of water, } 5 \\
\text { barrels salt meat. I I sacks of flour, some full others } \\
\text { only partly full. }\end{array}$ \\
\hline 12 & & & & & \\
\hline
\end{tabular}




\section{LOG BOOK OF THE HOOKER "ST. PETER"}

2 August 12, 1772. After Midday

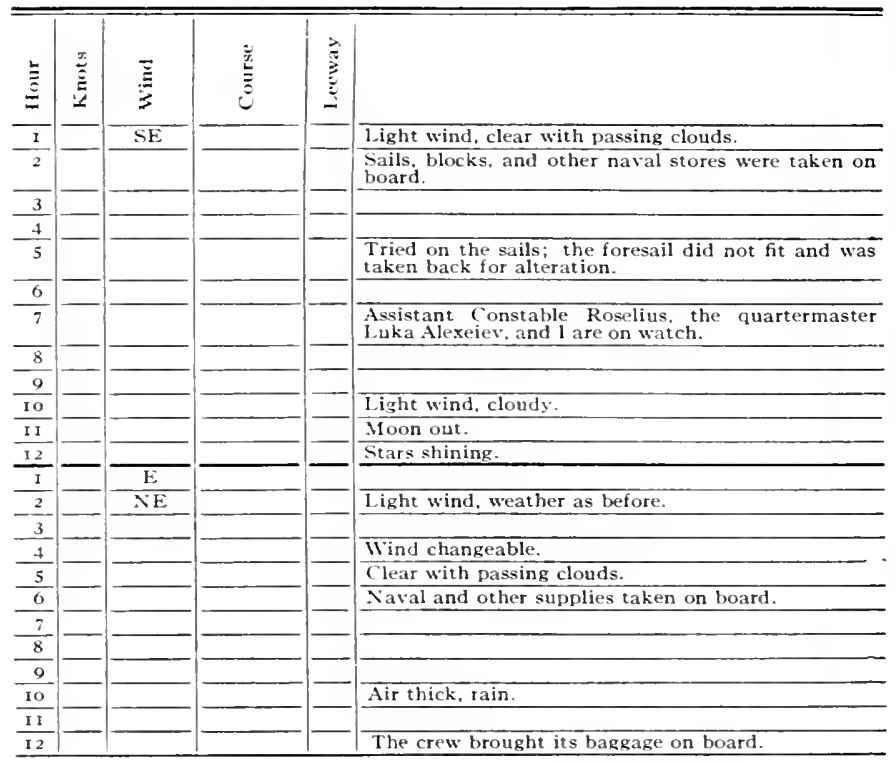


I August 13, 1742. After Midday

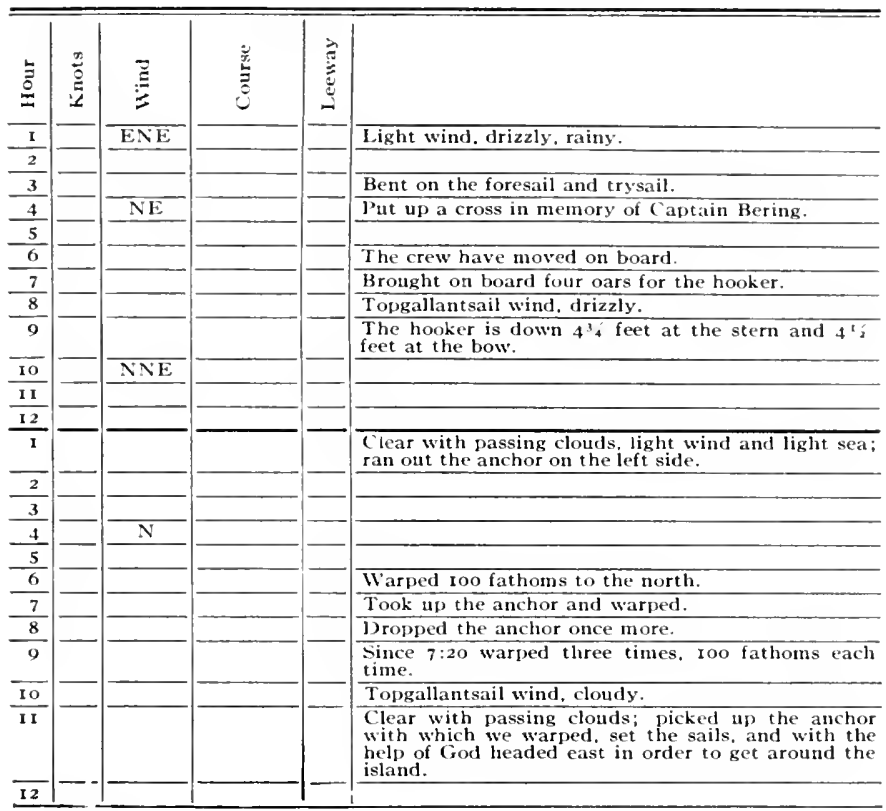




\section{$2+4$ LOG BOOK OF THE HOOKER "ST. PETER"}

\section{h August 14, 1742. Afler Midday}

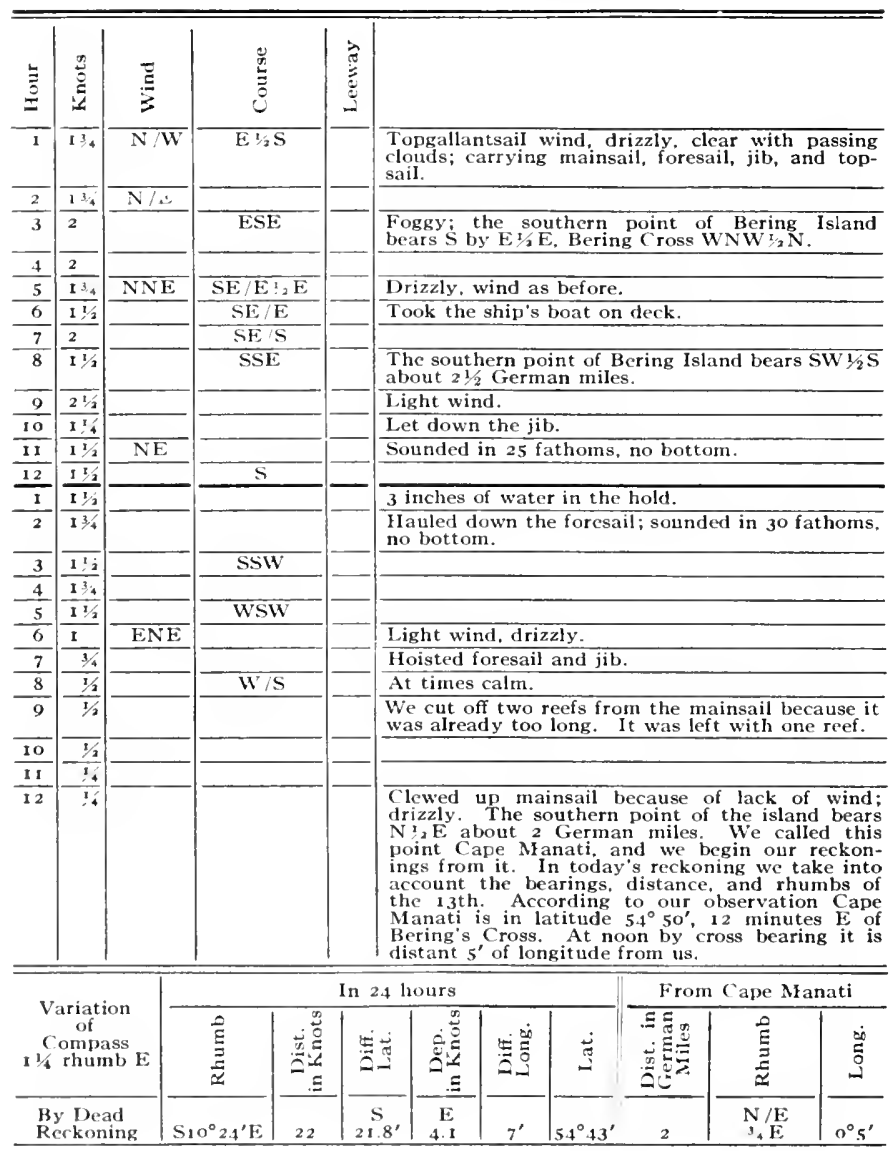


August 15,1772. After Midday

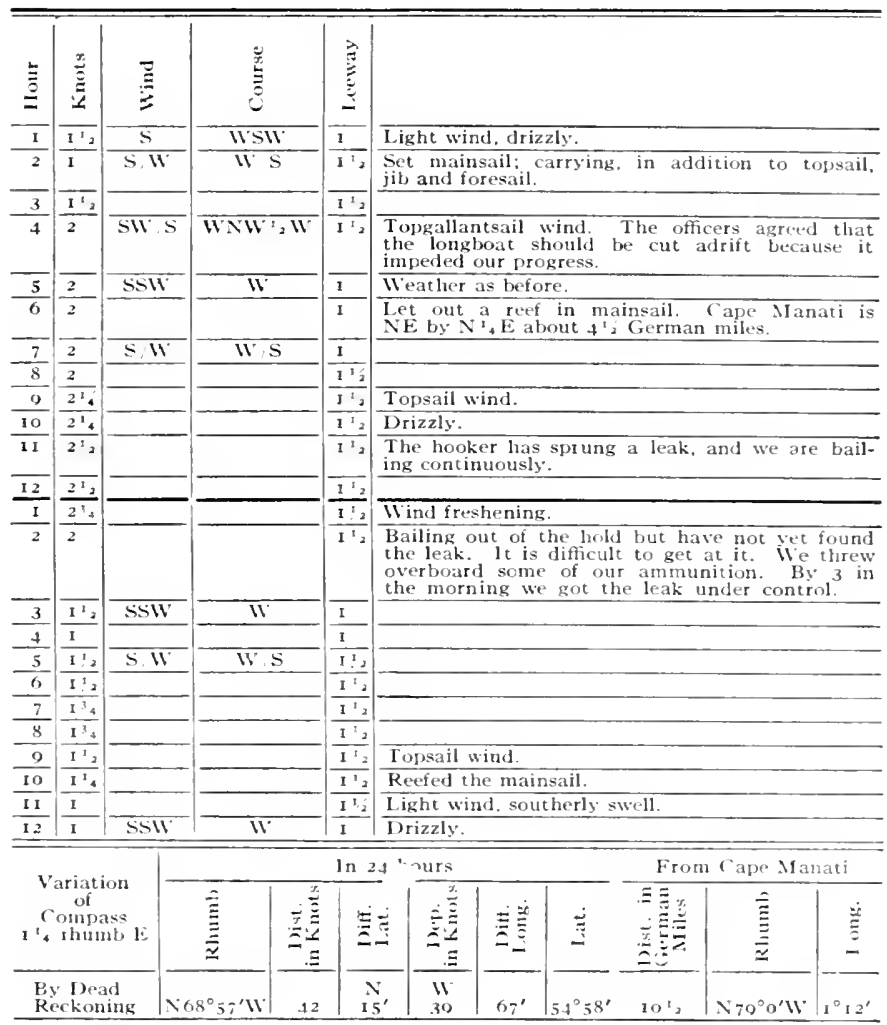


(1) August I6, 1742. After Midday

\begin{tabular}{|c|c|c|c|c|c|c|c|c|c|c|c|c|c|}
\hline \multirow{2}{*}{$\frac{\stackrel{2}{3}}{1}$} & \multirow{2}{*}{$\frac{\stackrel{\infty}{0}}{34}$} & \multicolumn{2}{|c|}{$\frac{\bar{E}}{3}$} & \multicolumn{2}{|l|}{ 苋 } & \multicolumn{2}{|c|}{ 㤹 } & & & & & & \\
\hline & & \multicolumn{2}{|c|}{ SW } & \multicolumn{2}{|c|}{ WNW } & \multirow{4}{*}{2} & \multicolumn{7}{|c|}{$\begin{array}{l}\text { Light wind, drizzly, wet; carrying mainsail, fore- } \\
\text { sail, jib, and topsail. }\end{array}$} \\
\hline 2 & $1 / 2$ & & \multirow{2}{*}{\multicolumn{2}{|c|}{ NW }} & & & & & & & & \\
\hline 3 & \multirow{5}{*}{$\left|\begin{array}{ll}1 & \\
1 / 2\end{array}\right|$} & \multicolumn{2}{|c|}{ WSW } & & & & & & & & & & \\
\hline 4 & & \multirow{4}{*}{\multicolumn{2}{|c|}{ 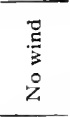 }} & & & & \multicolumn{7}{|c|}{$\begin{array}{l}\text { Heavy swell from south at the rate of } 1 / 2 \text { a knot } \\
\text { in } 4 \text { hours. }\end{array}$} \\
\hline 5 & & & & \multicolumn{2}{|c|}{ NNW } & \multirow{3}{*}{3} & \multicolumn{7}{|c|}{ Clear with passing clouds. } \\
\hline 6 & & & & & & & & & & & & & \\
\hline 7 & & & & & & & & ggy, w & & & & & \\
\hline 8 & $1 / 2$ & \multicolumn{2}{|c|}{ WSW } & $\mathrm{NW}$ & & & \multicolumn{7}{|c|}{ Changeable wind. } \\
\hline 9 & \multirow{4}{*}{$1 / 2$} & \multirow{4}{*}{\multicolumn{2}{|c|}{$\begin{array}{l}\bar{E} \\
\ddot{3} \\
\dot{Z} \\
\end{array}$}} & & & \multirow{4}{*}{3} & & vell at & le rat & of $1 / 21$ & not in 4 & hours. & \\
\hline Io & & & & & & & & & & & & & \\
\hline I I & & & & & & & & ars out & & & & & \\
\hline 12 & & & & & & & & & & & & & \\
\hline$I$ & $x_{2}$ & St & $\overline{S E}$ & WSW & & & & ght wi & driz & zly. & & & \\
\hline 2 & $1 / 2$ & & & & & & & gg, wet & clear & with pas & $\operatorname{sing~} \mathrm{clc}$ & uds. & \\
\hline 3 & $\mathbf{I}$ & & & & & & & & & & & & \\
\hline 4 & $11 / 2$ & & & & & & & & & & & & \\
\hline 5 & $11^{12}$ & & & & & & & psail y & nd. & & & & \\
\hline 6 & $2 \div$ & & & & & & & t out & reef ir & mains & il and $t$ & opsail. & \\
\hline 7 & 23.4 & & & & & & & pgalla & sail & ind, at & nospher & e thick. & \\
\hline 8 & 214 & & & & & & & & & & & & \\
\hline 9 & $21 / 2$ & $\mathrm{SE}$ & $/ \mathrm{S}$ & SW /V & & & & ear wit & passi & ng clou & & & \\
\hline 10 & $21 / 4$ & & & & & & & & & & & & \\
\hline II & 2 & & & & & & & ually $c$ & uds. & & & & \\
\hline & $21 / 2$ & & & & & & & & & & & & \\
\hline & & & & & & & 4 & lours & & & Fror & n Cape Man & ati \\
\hline $\begin{array}{r}\mathrm{C} \\
\mathrm{C} \\
\mathrm{I}\end{array}$ & $\begin{array}{l}\text { ariation } \\
\text { of } \\
\text { onjpass } \\
\text { rhumb }\end{array}$ & & & $\frac{\hat{\Xi}}{\vec{\Xi}}$ & 兽造 & & & 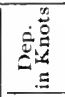 & 象茪 & 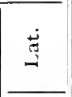 & 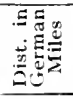 & $\stackrel{\stackrel{\varrho}{\Xi}}{\stackrel{\Xi}{\Xi}}$ & 泀 \\
\hline $\mathrm{By}$ & $\begin{array}{l}\text { Dead } \\
\text { ckonin }\end{array}$ & & 583 & ${ }^{\circ} 24^{\prime} \mathrm{II}$ & 22 & & $\mathrm{~S}$ & $\begin{array}{c}\text { W } \\
21.6\end{array}$ & $37^{\prime}$ & $54^{\circ} 56^{\prime}$ & 1534 & $\left|\mathrm{~N} 84^{\circ} 33^{\prime} \mathrm{W}\right|$ & $1^{\circ} 49^{\prime}$ \\
\hline
\end{tabular}


\& August 17, 1742. After Midday

\begin{tabular}{|c|c|c|c|c|c|c|c|c|c|c|c|c|c|}
\hline \multirow{2}{*}{$\frac{\stackrel{5}{3}}{1}$} & \multirow{2}{*}{ 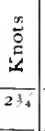 } & \multicolumn{2}{|l|}{ 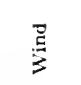 } & \multicolumn{2}{|l|}{ 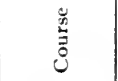 } & 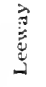 & & & & & & & \\
\hline & & \multicolumn{2}{|c|}{$\mathrm{S} / \mathrm{E}$} & \multicolumn{2}{|c|}{$\mathrm{SW} / \mathrm{W}$} & \multirow{6}{*}{ I } & \multicolumn{7}{|c|}{$\begin{array}{l}\text { Topsail wind drizzly, rainy; carrsing foresail, } \\
\text { mainsail, jib, and topsail. }\end{array}$} \\
\hline 2 & $22^{s_{4}}$ & \multirow{2}{*}{\multicolumn{2}{|c|}{$\mathrm{s}$}} & & & & & & & & & & \\
\hline 3. & 3 & & & \multicolumn{2}{|c|}{ IISW } & & \\
\hline 4 & 3 & \multirow{2}{*}{\multicolumn{2}{|c|}{-}} & & & & \multirow{2}{*}{\multicolumn{7}{|c|}{ Topgallantsail wind, southerly swell. }} \\
\hline 5 & $21 / 2$ & & & & & & \multirow{2}{*}{\multicolumn{7}{|c|}{ Topgallantsail wind, southerly swell. }} \\
\hline 6 & 2 & \multirow{2}{*}{\multicolumn{2}{|c|}{ SE S }} & & & & & & & & & & \\
\hline 7 & 2 & & & & & & \multicolumn{7}{|c|}{ Weather as before. } \\
\hline 8 & $25 / 2$ & \multirow{2}{*}{\multicolumn{2}{|c|}{$\mathrm{SSE}$}} & & & & & & & & & & \\
\hline 9 & $\overline{3^{3}+4}$ & & & \multicolumn{2}{|c|}{ SIV/W } & & \multicolumn{7}{|c|}{ Reef-topsail wind, foggy, rainy. } \\
\hline 10 & 3 & \multicolumn{2}{|c|}{ 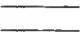 } & \multirow{2}{*}{\multicolumn{2}{|c|}{ 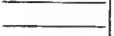 }} & & \\
\hline 11 & $22^{3+}$ & \multirow{2}{*}{\multicolumn{2}{|c|}{ 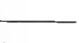 }} & \multirow{2}{*}{\multicolumn{2}{|c|}{ 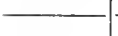 }} & & \multicolumn{7}{|c|}{ Lowered topsail. } \\
\hline 12 & $\overline{21_{2}}$ & & & & & & \multirow{2}{*}{\multicolumn{7}{|c|}{ Reef-topsail wind, rain. }} \\
\hline 1 & $\overline{2+2}$ & & & & & & & & & & & \\
\hline$\frac{2}{2}$ & $22^{34}$ & & & & & & & & & & & & \\
\hline 3 & $22_{4}$ & $\mathrm{SE}$ & & SW & & & & dersail & wind. & & & & \\
\hline 4 & $33_{i}^{\prime}$ & E & & & & & & uled ds & wn jit & and fo & esail. & & \\
\hline 5 & $44^{1 / 4}$ & & & & & & & & & & & & \\
\hline 6 & $4 ! 2$ & $\mathrm{ESE}$ & & & & & & ef-unds & rsail w & ind. & & & \\
\hline 7 & $4 \cdot 4$ & & & & & & & nd in $\mathrm{g}$ & usts. & eavy ra & & & \\
\hline 8 & $31_{2}$ & & & & & & $F_{0}$ & & & & & & \\
\hline 9 & $33^{3}$ & & & & & & & ar wit & passí & ig clous & & & \\
\hline 10 & 3 & $\mathrm{SE} / 1$ & & SSW & & & & $\begin{array}{l}\text { ick fo } \\
\text { hy wa }\end{array}$ & nid & $\begin{array}{l}\text { hted } \\
\text { Wh abou }\end{array}$ & $\begin{array}{l}\text { igh sno } \\
t 3 \text { Germ }\end{array}$ & $\begin{array}{l}\text { ow-covered } \\
\text { lan miles aw }\end{array}$ & $\begin{array}{ll}\text { land } \\
\text { ay. } 134 \\
\end{array}$ \\
\hline 11 & $22^{3}$ & & & & & 1 & & nd goil & ig dow & a bit. & weather & as hefore. & \\
\hline 12 & $2 ! 2$ & & & & & & & & & & & & \\
\hline & & & & & & & $\mathrm{h}$ & ours & & & From & Cape Man & ati \\
\hline & $\begin{array}{l}\text { of } \\
\text { of } \\
\text { aumb }\end{array}$ & & & $\stackrel{\vec{\Xi}}{\underline{\underline{\Xi}}}$ & 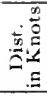 & & & 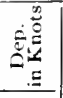 & 题 & 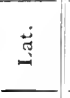 & 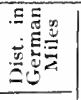 & 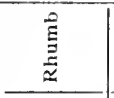 & 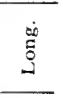 \\
\hline & $\begin{array}{l}\text { Dea } \\
\text { ckoni }\end{array}$ & & Se & $5^{\circ} 23^{\prime} \mathrm{W}$ & $7 \mathrm{I}$ & & & $\begin{array}{c}W \\
6.4 .6\end{array}$ & $\mathrm{III}^{\prime}$ & $54^{\circ} 27^{\prime}$ & $32 \frac{3 \%}{4}$ & $\mathrm{~S}_{79^{\circ}}{ }_{4} 6^{\prime} \mathrm{WV}$ & $3^{\circ}{ }^{4} 0^{\prime}$ \\
\hline
\end{tabular}

134 Khitrov's journal: "At 9:30 we sighted, through the mist, land in the Wr from which rose a high, snow-covered volcano, which we identified as in Kamchatka, near Cape Kronotski. We steered away from the land." Cape Kronotski is shown on Pl. I. 


\section{LOG BOOK OF THE HOOKER "ST. PETER"}

$\Varangle$ August $18,17+2$. Afier Midday

\begin{tabular}{|c|c|c|c|c|c|c|c|c|c|c|c|}
\hline \multirow{2}{*}{$\frac{\stackrel{y}{a}}{1}$} & \multirow{2}{*}{ 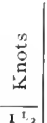 } & $\stackrel{\Xi}{\Xi}$ & 总 & \multicolumn{2}{|c|}{ 密 } & & & & & & \\
\hline & & $\mathrm{E} / \mathrm{S}$ & $s$ & & & \multicolumn{6}{|c|}{ Strong undersail wind, rain, sunshine. } \\
\hline$\frac{2}{2}$ & 2 & \multirow{2}{*}{ NE } & & & & \multirow{2}{*}{\multicolumn{6}{|c|}{ Wind going down; set topsail hoisted jib }} \\
\hline 3 & 2 & & & & & & & & & & \\
\hline 4 & $1 ! 2$ & 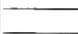 & & & & \multicolumn{6}{|c|}{ Heavy southerly swell. } \\
\hline 5 & 4 & II & & & & \multicolumn{6}{|c|}{ Clear with passing clouds. } \\
\hline 6 & $\overline{3^{2}}$ & W S & & & & \multirow{2}{*}{\multicolumn{6}{|c|}{ Topgallantsail wind, chilly. }} \\
\hline 7 & $1 \div 2$ & WSW & $\mathrm{Si}_{2} \mathrm{E}$ & \multirow{3}{*}{\multicolumn{2}{|c|}{$1 / 2$}} & \multirow{2}{*}{\multicolumn{6}{|c|}{$\begin{array}{l}\text { Topgallantsail wind, chilly. } \\
\text { Sighted a high volcano } \mathrm{W} \text { by } \mathrm{N} \text {. another volcano } \\
\text { W by S, some distance farther to the north } \\
\text { a point } \mathrm{N} \text { r E } 5 \text { miles. }\end{array}$}} \\
\hline 8 & $\mathbf{I}$ & & & & & & & & & & \\
\hline 9 & $2 ! / 2$ & $\mathrm{SW} / \mathrm{W}$ & $\mathrm{S} / \mathrm{E}$ & & & \multicolumn{6}{|c|}{ Topsail wind. } \\
\hline Io & $\begin{array}{l}2^{3 / 4} \\
\end{array}$ & & & & \multicolumn{6}{|c|}{ Lowered topsail. } \\
\hline 11 & 3 & \multirow[b]{2}{*}{$\mathrm{SW}$} & & & & \multirow{2}{*}{\multicolumn{6}{|c|}{ Undersail wind, stars out. }} \\
\hline 12 & $22^{3} 4$ & & $\mathrm{SSE}$ & & & & & & & & \\
\hline $\mathrm{I}$ & 4 & & & & & \multicolumn{6}{|c|}{ Heavy clouds, squalls; tacked to port. } \\
\hline 2 & 2 & IV & NNW & & & & & & & & \\
\hline 3 & $1 \div 2$ & & & & & & & & & & \\
\hline 4 & $\mathrm{I}$ & & & & & Stars out. & chilly & & & & \\
\hline 5 & $\overline{1} 1 / 2$ & $\mathrm{~W} / \mathrm{s}$ & NW/I & & & & & & & & \\
\hline 6 & $I^{1 / 2}$ & & & & 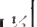 & Topgallan & sail $n$ & ind. & & & \\
\hline 7 & $\overline{14}$ & & & & & Sunshiny. & & & & & \\
\hline 8 & $\bar{I}$ & & & & & Light win & & & & & \\
\hline 9 & $\mathrm{I}^{\mathrm{I}_{2}}$ & & & & & Set topsai & reefe & & & & \\
\hline 10 & 2 & & & 1 & & Topsail w & nd. & & & & \\
\hline II & $\overline{2 ! 4}$ & WSW & $\mathrm{NW}$ & & & Clear wit! & passi & ig cloud & & & \\
\hline 12 & $\overline{2 ! 4}$ & & & & I & $\begin{array}{l}\text { Took obs } \\
\text { lination of } \\
\text { voleano s } \\
\text { it is }{ }_{4}\end{array}$ & $\begin{array}{l}\text { ervati } \\
\text { the } \\
\text { ghted } \\
\text { rerma }\end{array}$ & $\begin{array}{l}\text { n: } \\
\text { un } 9^{\circ} \\
\text { is } \mathrm{l}^{\circ} \\
\text { miles. } \\
\text {. }\end{array}$ & $\begin{array}{l}\text { dista } \\
\text { y, latitu }\end{array}$ & $\begin{array}{r}44^{\circ}+4 \\
54^{\circ} 14^{\prime} \\
\text { point n }\end{array}$ & $\begin{array}{l}\text { dec- } \\
\text { High } \\
\text { h of } \\
\end{array}$ \\
\hline & & & & & & $2+$ hours & & & Fron & Cape $\mathbf{M}$ & \\
\hline & $\begin{array}{l}\text { of } \\
\text { omps } \\
\text { hum }\end{array}$ & & $\underline{\underline{E}}$ & 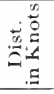 & & 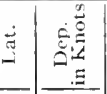 & 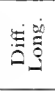 & ذ્ّ & 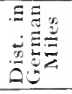 & 蔦 & 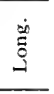 \\
\hline & $\begin{array}{l}\text { Dez } \\
\text { ckon }\end{array}$ & ing & 135 & 135 & 13 & \begin{tabular}{l|l} 
& $W$ \\
0, & 0.3 \\
\end{tabular} & $18^{\prime}$ & $54^{\circ} 13^{\prime}$ & $33 ! 4$ & $\begin{array}{l}\text { WV/S } \\
4^{\circ} 55^{\prime} \mathrm{S}\end{array}$ & $3^{\circ} 40^{\prime}$ \\
\hline
\end{tabular}

13.5 Khitrov's journal: Rhumb), SW $/ \mathrm{S} 0^{\circ} 5 \mathrm{I}^{\prime}$ S [i.e. S $32^{\circ} 54^{\prime} \mathrm{W}$ ]; Dist. in Knots, 20. 
2 August 19. 1742. After Midday

\begin{tabular}{|c|c|c|c|c|c|c|c|c|c|c|c|c|}
\hline 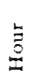 & 䓵 & 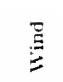 & \multicolumn{2}{|l|}{ 蒫 } & 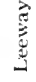 & & & & & & & \\
\hline 1 & 213 & WsW & \multicolumn{2}{|l|}{ NW } & \multirow{6}{*}{1} & \multirow{2}{*}{\multicolumn{7}{|c|}{$\begin{array}{l}\text { Topsail wind, clear with passing clouds; carry- } \\
\text { ing all sails; topsial and mainsail are reefect. } \\
\text { Southerly swell. }\end{array}$}} \\
\hline 2 & $22_{2}$ & & & & & & & & & & & \\
\hline 3 & $\frac{22^{12}}{2}$ & & \multirow{2}{*}{\multicolumn{2}{|c|}{$\ldots$}} & & \\
\hline 4 & $11^{3}$ & & & & & \multirow{2}{*}{\multicolumn{7}{|c|}{ Topgallantsail wind, cloudy. }} \\
\hline 5 & $1 \frac{1}{2}$ & & \multicolumn{2}{|l|}{ 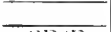 } & & & & & & & & \\
\hline 6 & $11 / 4$ & $\overline{S / W}$ & \multicolumn{2}{|l|}{$\mathrm{SE} E$} & & \multicolumn{7}{|c|}{$\begin{array}{l}\text { Came within }{ }^{3}+\text { mile of shore, tacked to star- } \\
\text { board. }\end{array}$} \\
\hline 7 & 2,4 & & \multirow{2}{*}{\multicolumn{2}{|c|}{ SE,E, E }} & \multirow{4}{*}{ I } & & & & & & & \\
\hline 8 & $22^{3} 4$ & & & & & \multicolumn{7}{|c|}{ Topsail wind. } \\
\hline 9 & 4 & & \multicolumn{2}{|l|}{$\mathrm{SE} \mathrm{E}$} & & \multicolumn{7}{|c|}{ Strong recf-topsail wind; lowered topsail. } \\
\hline 10 & $3+2$ & & & & \\
\hline 11 & 3 & $\mathrm{~s}$ & & & \multirow{5}{*}{$I: 2$} & & & & & & & \\
\hline 12 & $2 ! 4$ & $\mathrm{SE}$ & \multicolumn{2}{|l|}{ ES } & & & \multicolumn{6}{|c|}{ Undersail wind. heavy rain. } \\
\hline$I$ & $2+2$ & & & & & \multicolumn{7}{|c|}{ Recf-topsail wind, drizzly, rainy, chilly. } \\
\hline 2 & 2 & 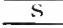 & \multicolumn{2}{|l|}{ ESLE } & & & & & & & & \\
\hline 3 & 2 & & & & & \multicolumn{7}{|c|}{ Hauled down the jib. } \\
\hline 4 & $1^{3}+4$ & SSW & \multicolumn{2}{|l|}{$\mathrm{SE}$} & \multirow{3}{*}{2} & \multicolumn{7}{|c|}{ Topsail wind. } \\
\hline 5 & 1 & & & & & & & & & & & \\
\hline 6 & $1^{1 / 2}$ & $11 / \mathrm{s}$ & \multicolumn{2}{|c|}{$\mathrm{NW} N$} & & \multicolumn{7}{|c|}{ Tacked to port. } \\
\hline 7 & 32 & SW & SSE & & & \multirow{2}{*}{\multicolumn{7}{|c|}{$\begin{array}{l}\text { Tacked to starboard. } \\
\text { Set topsail and jib. }\end{array}$}} \\
\hline 8 & $\bar{T}_{2}$ & & & & 3 & & & & & & & \\
\hline 9 & I & WSII & s & & , & & opgallax & tsiil & ind. $c$ & ar with & passing c & utls. \\
\hline 10 & $\begin{array}{ll}3 i \\
\end{array}$ & & & & & & & & & & & \\
\hline II & $1 / 2$ & $8 \bar{\Xi}$ & & & & & $\begin{array}{l}\text { Cather } \\
\text { le rate. }\end{array}$ & as be & 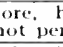 & $\begin{array}{l}\text { avy so } \\
\text { hour di }\end{array}$ & $\begin{array}{l}\text { therly s } \\
\text { ing } 2 \text { ho }\end{array}$ & $\begin{array}{ll}11 \\
s .\end{array}$ \\
\hline 12 & & $7 \equiv$ & $\mathrm{N}$ & & & & $\begin{array}{l}\text { he nort } \\
\text { ile. }\end{array}$ & hern & oint 1 & ars Ni & 3214 & rman \\
\hline & & & & & In & 1 & ours & & & Fron & Cape $\mathrm{N}_{\text {: }}$ & \\
\hline & 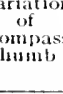 & & $\underline{\bar{\Xi}}$ & 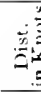 & & & 总 & 穷蓄 & $\stackrel{5}{-5}$ & 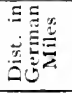 & $\underset{\underline{E}}{\underline{\underline{E}}}$ & $\stackrel{0}{5}$ \\
\hline & $\begin{array}{l}\text { Dead } \\
\text { ckonit }\end{array}$ & $g$ & $1^{\circ} 44^{\prime} \mathrm{E}$ & 23. & & 3 & $\begin{array}{c}E \\
23.4\end{array}$ & $30.8^{\prime}$ & $54^{\circ} 10^{\prime}$ & 28 & $\begin{array}{l}\text { W'SW } \\
I^{\circ} 33^{\prime} W \mathrm{~W}\end{array}$ & $3^{\circ}$ \\
\hline
\end{tabular}




\section{$25^{\circ}$ LOG BOOK OF THE HOOKER "ST. PETER"}

\section{August 20, 1742. After Midday}

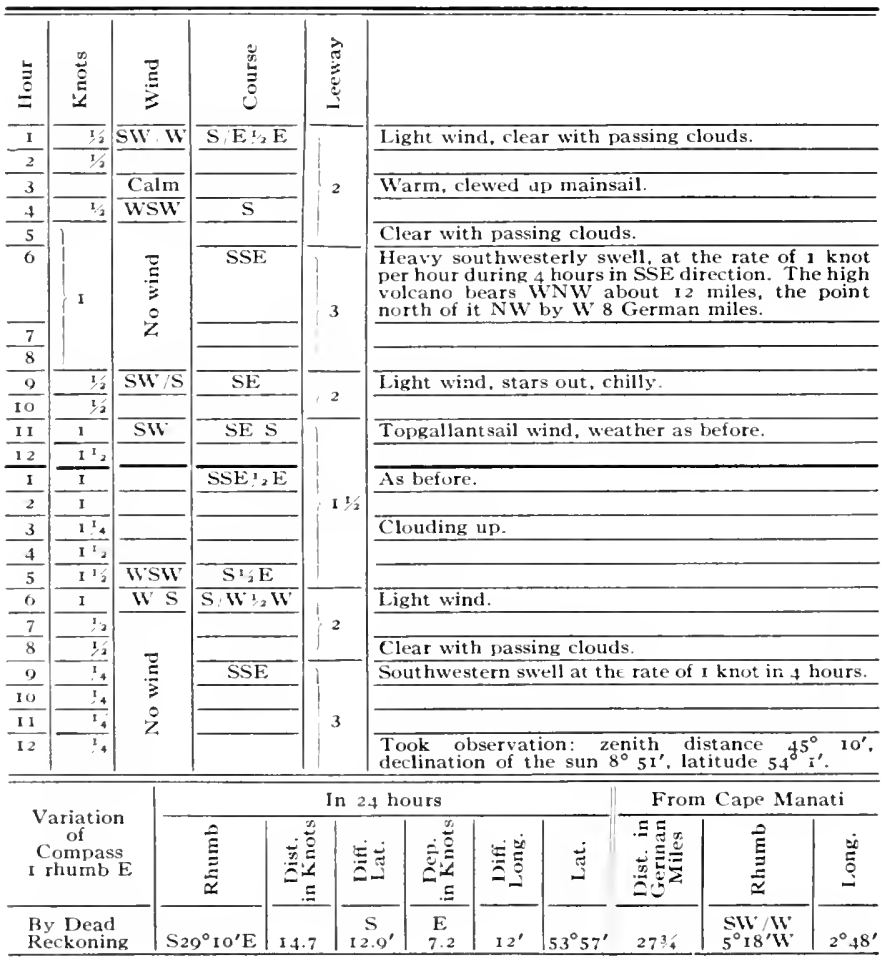




\section{b August 21, 1742. After Midday}

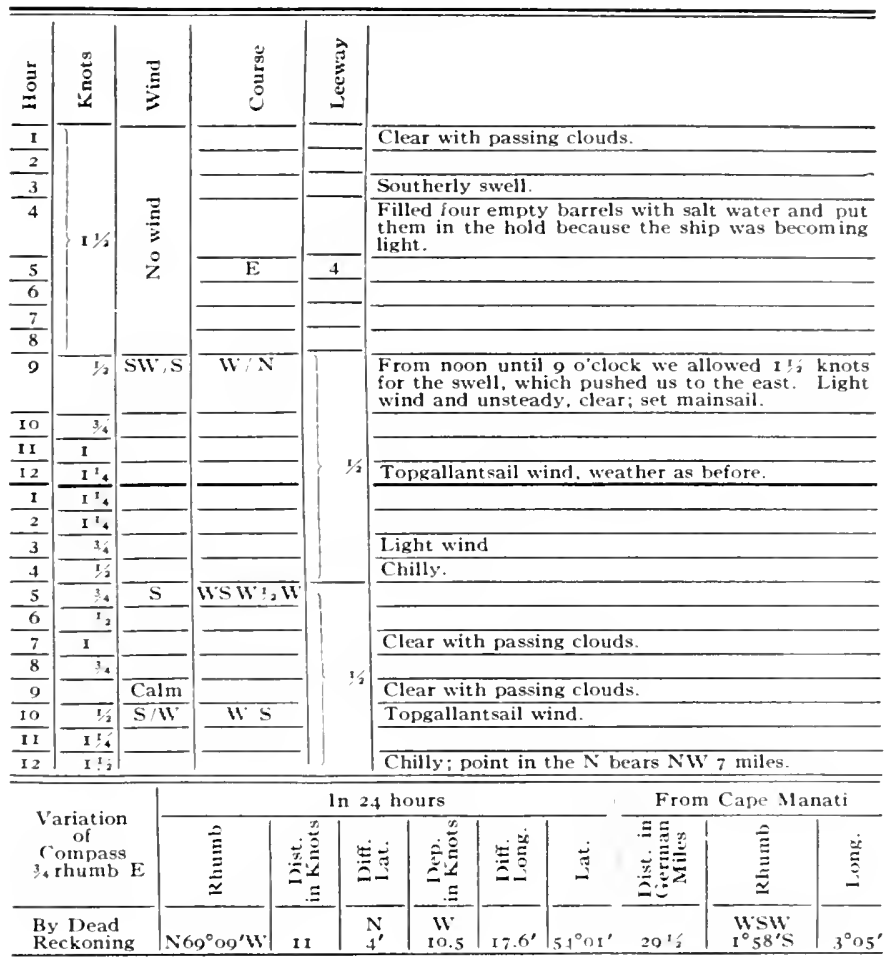


August 22, I742. After Midday

\begin{tabular}{|c|c|c|c|c|c|c|c|c|c|c|c|}
\hline 岂 & 葍 & 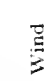 & & $\stackrel{\stackrel{\mathscr{m}}{\sharp}}{\stackrel{\Xi}{\Xi}}$ & & $\stackrel{E}{E}$ & & & & & \\
\hline $\mathrm{I}$ & $\overline{\mathrm{T}^{2}}$ & S & & WS & & & Topgalla & ts sail & ind, drizzly; car & ying the for & esails. \\
\hline 2 & I & & & & & 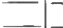 & & & & & \\
\hline 3 & $3 \frac{34}{3}$ & & & & & & & & & & \\
\hline 4 & & Cal & & & & & Light rai & & & & \\
\hline 5 & $\begin{array}{ll}312 \\
\end{array}$ & $\mathbf{E}$ & & Sil & & & light wi & d, we & ather as before. & & \\
\hline 6 & $\frac{\mathrm{I}^{1}}{2}$ & & & & & & Tongalla & itsail & vind. & & \\
\hline 7 & $\overline{2 \overline{3}}$ & & & & & & Reefed t. & psail, & lowered jib and & oresai.. & \\
\hline 8 & 3 & & & $\mathrm{SW}$ & & & & & & & \\
\hline 9 & $4^{34}$ & & & & & & Topsail y & ind, $d$ & rizzly, heavy rai & & \\
\hline 10 & 4 & & & & & & & & & & \\
\hline II & 5 & & & & & & Undersai & wind & lowered topsail & & \\
\hline $\mathrm{I} 2$ & $4^{3}$ & & & & & & Simally. & & & & \\
\hline $\bar{I}$ & 4 & $\overline{\mathrm{NN}}$ & & $\sin$ & 211 & & & & & & \\
\hline 2 & $44^{12}$ & & & & & & Keet-und & rsail & vind, chilly, hea & y rain. & \\
\hline 3 & $44^{12}$ & & & & & 7 & Took a $\mathrm{r}$ & ef in & nainsail. & & \\
\hline 4 & $4^{3}+$ & & & & & & Frale, rai & & & & \\
\hline 5 & $4^{1} 4$ & & & WS & & & & & & & \\
\hline 6 & $33^{3}$ & & & & & & $\begin{array}{l}\text { Wind go } \\
\text { clouds. }\end{array}$ & ng do & wn, air thick, $c$ & ear with $p$ & $\overline{\text { assing }}$ \\
\hline 7 & $\overline{3 i 2}$ & & & & & & Let out $r$ & ef in & mainsail. & & \\
\hline$\frac{8}{8}$ & $23^{3}$ & & & & & & Topsail y & ind, $n$ & ortheasterly swel & 1. light rain & \\
\hline$\frac{9}{9}$ & $23_{4}$ & $\mathrm{~N}$ & & & & -7 & Clear wit & 1 pass & ing clouds. & & \\
\hline 10 & 3 & & & & & & Set topsa & & & & \\
\hline$I 1$ & $\overline{I^{34}}$ & & & & & & & & & & \\
\hline 12 & 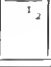 & & & & & & $\begin{array}{l}\text { Light wit } \\
\text { zenith } \\
8^{\circ} 08^{\prime}, 1 \mathrm{da} \\
\end{array}$ & $\begin{array}{l}\text { d, sun } \\
\text { stance } \\
\text { itude }\end{array}$ & $\begin{array}{l}4 x^{\circ}, \text { ajr clear } \\
45^{\circ} 0^{\prime} \text {, declin } \\
53^{\circ} 08^{\prime} \text {. }\end{array}$ & $\begin{array}{l}\text { Ook obsery } \\
\text { ation of } t 1\end{array}$ & $\begin{array}{l}\text { ation: } \\
\text { e sun }\end{array}$ \\
\hline \multirow{2}{*}{\multicolumn{3}{|c|}{$\begin{array}{c}\text { Variation } \\
\text { of } \\
\text { Compass } \\
3 \text { rhumb } \mathrm{E}\end{array}$}} & \multicolumn{9}{|c|}{ From Cape Manati } \\
\hline & & & & $\stackrel{\stackrel{0}{\Xi}}{\Xi}$ & 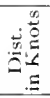 & 跣 & - & 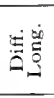 & 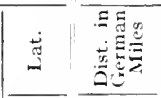 & 芑 & 这 \\
\hline \multicolumn{3}{|c|}{$\begin{array}{l}\text { By Dearl } \\
\text { Reckoning }\end{array}$} & $s_{5}$ & $3^{\circ} 30^{\prime} \mathrm{W}$ & 66 & 39.8 & $\begin{array}{r}W \\
52.0 \\
\end{array}$ & $80^{\prime}$ & $53^{\circ} 21^{\prime}$ & & $4^{\circ} 36^{\prime}$ \\
\hline \multicolumn{3}{|c|}{ By } & $s_{3}$ & 8,4615 & 6,6 & s. & $\underset{.1 .3}{W}$ & $71.7^{\prime}$ & $53^{\circ} 08^{\prime} \quad 45^{\prime} z$ & $\mathrm{~S}_{55^{\circ} 59^{\prime} \mathrm{W}}$ & $4^{\circ} \mathrm{I} 7^{\prime}$ \\
\hline
\end{tabular}


(S August 23, I742. Afler Midday

\begin{tabular}{|c|c|c|c|c|c|c|c|c|c|c|c|c|c|}
\hline \multirow{3}{*}{$\frac{\frac{5}{5}}{\frac{1}{2}}$} & $\frac{n}{0}$ & \multicolumn{2}{|c|}{$\Xi$} & \multicolumn{2}{|l|}{ 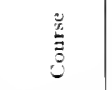 } & 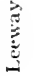 & & & & & & & \\
\hline & & \multicolumn{2}{|c|}{$\overline{\mathrm{NE}, \mathrm{E}}$} & \multicolumn{2}{|l|}{ sW } & & \multicolumn{7}{|c|}{ Liglit wind, clear with parsing clonds. } \\
\hline & & \multirow{4}{*}{\multicolumn{2}{|c|}{ 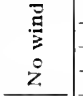 }} & & & \multirow{2}{*}{-} & \multirow{2}{*}{\multicolumn{7}{|c|}{ Heary northeasterly sweth. }} \\
\hline 3 & \multirow{3}{*}{$1 / 2$} & & & & & & & & & & & & \\
\hline-4 & & & & & & & & wed $u$ & main & & & & \\
\hline-5 & & & & & & & & owed f & r the & well $s_{2}$ & knot in & 3 hours. & \\
\hline 6 & $t_{2}$ & \multicolumn{2}{|c|}{ SW } & SSE & & \multirow{7}{*}{ I } & \multicolumn{7}{|c|}{ 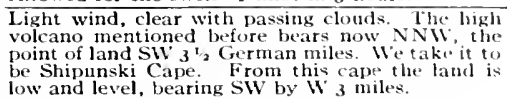 } \\
\hline 7 & 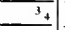 & & & & & & \multicolumn{7}{|c|}{ Lowered topsail, hoisted jib. } \\
\hline 8 & $13_{2}$ & & & & & & \multicolumn{7}{|c|}{ Topgallantsail wind, stars out. } \\
\hline 9 & $2 \frac{2 \%}{4}$ & & & & & & \multicolumn{7}{|c|}{ Chilly. } \\
\hline 10 & 2312 & & & & & & \multicolumn{7}{|c|}{ Topsail wind, weather as before. } \\
\hline 11 & 23,2 & & & & & & & & & & & & \\
\hline 12 & 2 & & & & & & \multicolumn{7}{|c|}{ At the end of the hour tacked to port. } \\
\hline 1 & $21 / 2$ & & \multicolumn{2}{|l|}{ IINII } & \multirow{5}{*}{$\mathbf{I}$} & & & & & & & \\
\hline 2 & $22_{4}$ & & & & & & & & & & & & \\
\hline 3 & $1^{3}+4$ & \multirow{2}{*}{\multicolumn{2}{|c|}{$=$}} & & & & \multicolumn{7}{|c|}{ Topgallant sail wind, stars out. } \\
\hline 4 & $\mathrm{I}^{\mathrm{T}_{3}}$ & & & & & & \multicolumn{7}{|c|}{ Set topsail. } \\
\hline 5 & $(1+1$ & \multicolumn{2}{|c|}{ - } & & & & \multicolumn{7}{|c|}{ Light wind. } \\
\hline 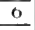 & $1+2$ & SW & $\bar{s}$ & W N & & & & shini & & & & & \\
\hline 7 & I & & & & & $1 / 2$ & & & & & & & \\
\hline 8 & 1 & $\mathrm{Cal}$ & & & & & & ondy. & & & & & \\
\hline 9 & & Si & & W.NW & & & & $y$ ligh & wind & nd uns & eady. & & \\
\hline 10 & 12 & & & & & & & ar wit & passi & g cloud & & & \\
\hline II & 1 & & & & & & & & & & & & \\
\hline 12 & $2 / 2$ & & & & & & & 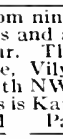 & $\begin{array}{l}\text { o'clo } \\
\text { dvane } \\
\text { ecape } \\
\text { uchen } \\
4 \text { Ger } \\
\text { ichat } \\
\text { ul is a }\end{array}$ & $\begin{array}{l}\text { unt il } \\
\text { d at } t h \\
\text { or poin } \\
\text { vole } \\
\text { ban nil } \\
\text { and th } \\
\text { ant } 12 \\
\text { ont } 12\end{array}$ & $\begin{array}{l}\text { loon we } \\
\text { rate of } \\
\text { bears } \\
\text { no W's } \\
\text { s. It is } \\
\text { at the } \mathrm{H} \\
\text { german }\end{array}$ & $\begin{array}{l}\text { worked wi } \\
\text { : of a } \mathrm{kn} \\
\mathbf{1}, 2 \mathrm{~S}, \mathrm{G} \\
\text { the cape } \\
\text { luite eviden } \\
\text { arbor of st. } \\
\text { miles to the }\end{array}$ & $\begin{array}{l}\text { th the } \\
\text { ot per } \\
\text { erman } \\
\text { to the } \\
\text { that } \\
\text { l'eter } \\
\text { west. }\end{array}$ \\
\hline & & & & & & $\ln 2$ & $4 \mathrm{~h}$ & urs & & & From & Cape Man & lati \\
\hline & $\begin{array}{l}\text { ariatior } \\
\text { of } \\
\text { ompas } \\
\text { rhumb }\end{array}$ & & & 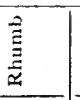 & 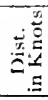 & & & 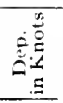 & 参 & $\ddot{\square}$ & 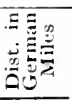 & 写 & 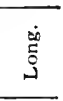 \\
\hline & $\begin{array}{l}\text { Dead } \\
\text { ckonin }\end{array}$ & & S 50 & $\circ^{\circ} 2 I^{\prime} W$ & 6.2 & & & $\begin{array}{l}\text { WV } \\
5.4\end{array}$ & $g^{\prime}$ & $53^{\circ} \circ 5^{\prime}$ & $463 / 4$ & $\mathrm{~S}_{5} 6^{\circ} \mathrm{O} 8^{\prime} \mathrm{WW}$ & $4^{\circ} 26^{\prime}$ \\
\hline
\end{tabular}




\section{LOG BOOK OF THE HOOKER "ST. PETER"}

\section{of August 24, 1742. After Midday ${ }^{136}$}

\begin{tabular}{|c|c|c|c|c|c|c|c|c|c|c|c|c|c|}
\hline$\stackrel{\text { o }}{0}$ & 营 & \multicolumn{2}{|c|}{$\stackrel{\frac{2}{3}}{3}$} & \multicolumn{2}{|l|}{ 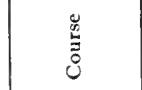 } & है & & & & & & & \\
\hline $\mathbf{I}$ & $\because 4$ & \multicolumn{2}{|c|}{$\mathrm{SW} / \mathrm{W}$} & \multicolumn{2}{|c|}{ NW $/ W$} & $\mathrm{I}$ & \multicolumn{7}{|c|}{ Light wind; carrying all sails. } \\
\hline 2 & $3 / 4$ & & & & & 1 & & & & & & & \\
\hline 3 & $\overline{1}$ & \multirow{2}{*}{\multicolumn{2}{|c|}{ Calm }} & & & 1 & \\
\hline 4 & $1 / 4$ & & & \multicolumn{2}{|c|}{ SW } & & \multicolumn{7}{|c|}{ Rowed. } \\
\hline 5 & $9 / 4$ & & \multirow{2}{*}{\multicolumn{2}{|c|}{ SSE }} & & \multicolumn{7}{|c|}{ Rowed. } \\
\hline 6 & 8 & \multicolumn{2}{|c|}{ SW } & & & I & \multicolumn{7}{|c|}{$\begin{array}{l}\text { Light wi nd: let out mainsail and foresail. Shipun- } \\
\text { ski Cape W by S } \mathrm{S} \text {, from it a projection WSW } 2 \\
\text { miles. Clewed up mainsail, lowered topsail. }\end{array}$} \\
\hline 7 & $13 / 2$ & \multicolumn{2}{|c|}{ NNW } & \multicolumn{2}{|c|}{$\mathrm{SW} / \mathrm{S}$} & & & & & & & & \\
\hline 8 & & \multicolumn{2}{|c|}{ Calm } & & & & & & & & & & \\
\hline \multicolumn{14}{|l|}{9} \\
\hline Io & $8 / 2$ & \multicolumn{2}{|c|}{ WSW } & \multicolumn{2}{|l|}{$\mathrm{S}$} & 1 & \multicolumn{7}{|c|}{ Set mainsail. } \\
\hline I I & 1 & & & & \\
\hline 12 & & \multicolumn{2}{|c|}{$3 / 2$} & & & \\
\hline I & $3 / 4$ & & & & & I & & & & & & & \\
\hline 2 & $1 \%$ & W & & SSV & & $1 / 2$ & & isted $t$ & psail. & & & & \\
\hline 3 & $13 / 2$ & & & & & $3 / 2$ & & & & & & & \\
\hline 4 & $3 / 4$ & & & & & $2 / 2$ & & & & & & & \\
\hline 5 & I & W/ & & SW & & $3 / 4$ & & & & & & & \\
\hline 6 & $\mathbf{I}$ & & & & & $3 / 4$ & & nshine & & & & & \\
\hline 7 & $\sqrt[3 / 4]{4}$ & & & & & $3 / 4$ & & & & & & & \\
\hline 8 & $1 / 2$ & & & & & $3 / 4$ & & & & & & & \\
\hline 9 & & Cal & & & & & & wed u & mair & sail. & & & \\
\hline 10 & & & & & & & & nshine & pleas & nt weat & her. & & \\
\hline I I & & & & & & & & & & & & & \\
\hline 12 & & & & & & & & $\begin{array}{l}\text { ipunsk } \\
\text { lcano }\end{array}$ & $\begin{array}{l}\text { Cape } \\
V \text { by }\end{array}$ & $\begin{array}{l}\mathrm{NW} 1 / 2 \mathrm{~W} \\
\mathrm{~N}_{12} \mathrm{~N}\end{array}$ & ( 3 miles & distant, $\mathrm{H}$ & urning \\
\hline & & & & & & In 2. & tho & urs & & & Fron & Cape $\mathrm{Ma}$ & nati \\
\hline $3 / 4$ & $\begin{array}{l}\text { ariat } \\
\text { of } \\
\text { ompa } \\
\text { rlium }\end{array}$ & $\begin{array}{l}\text { on } \\
\text { ass } \mathrm{E}\end{array}$ & & $\frac{0}{\Xi}$ & 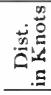 & & 苟 & 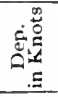 & 㝳苔 & $\stackrel{\ddot{3}}{\leftrightarrows}$ & 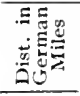 & 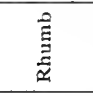 & $\stackrel{\infty}{\dot{1}}$ \\
\hline & $\begin{array}{l}\text { y De } \\
\text { eckon } \\
\text { chitr }\end{array}$ & $\begin{array}{l}\operatorname{ad} \\
\text { ing } \\
\text { av) }\end{array}$ & & 专W/S & 9.2 & & s, & $\begin{array}{l}W \\
6\end{array}$ & IO ${ }^{\prime}$ & $53^{\circ} 13^{\prime}$ & $501 / 2$ & $\underset{5^{\circ} \mathrm{I} 0^{\prime} \mathrm{W}}{\mathrm{SW}}$ & $5^{\circ} \mathrm{o3}{ }^{\prime}$ \\
\hline & $\begin{array}{l}\text { y } \\
\text { cekon } \\
\text { Yush }\end{array}$ & $\begin{array}{l}\text { ad } \\
\text { ing } \\
\text { in) }\end{array}$ & $\mathrm{S} 2$ & $4^{\circ} 24^{\prime} \mathrm{W}$ & 12 & & $\begin{array}{l}S \\
.8\end{array}$ & $\begin{array}{r}\text { IV } \\
4.9\end{array}$ & $8^{\prime}$ & $52^{\circ} 54^{\prime}$ & $?$ & $?$ & {$\left[4^{\circ} 34^{\prime}\right]$} \\
\hline
\end{tabular}

136 Khitrov's journal is used from now on to the end because Yushin's is in part illegible. Vushin's 24 hour summaries are retained, however, because they are based on the observed position of August 22, while Khitrov's seen based on that day's position by dead reckoning. 
$\Varangle$ August 25, 1742. After Midday

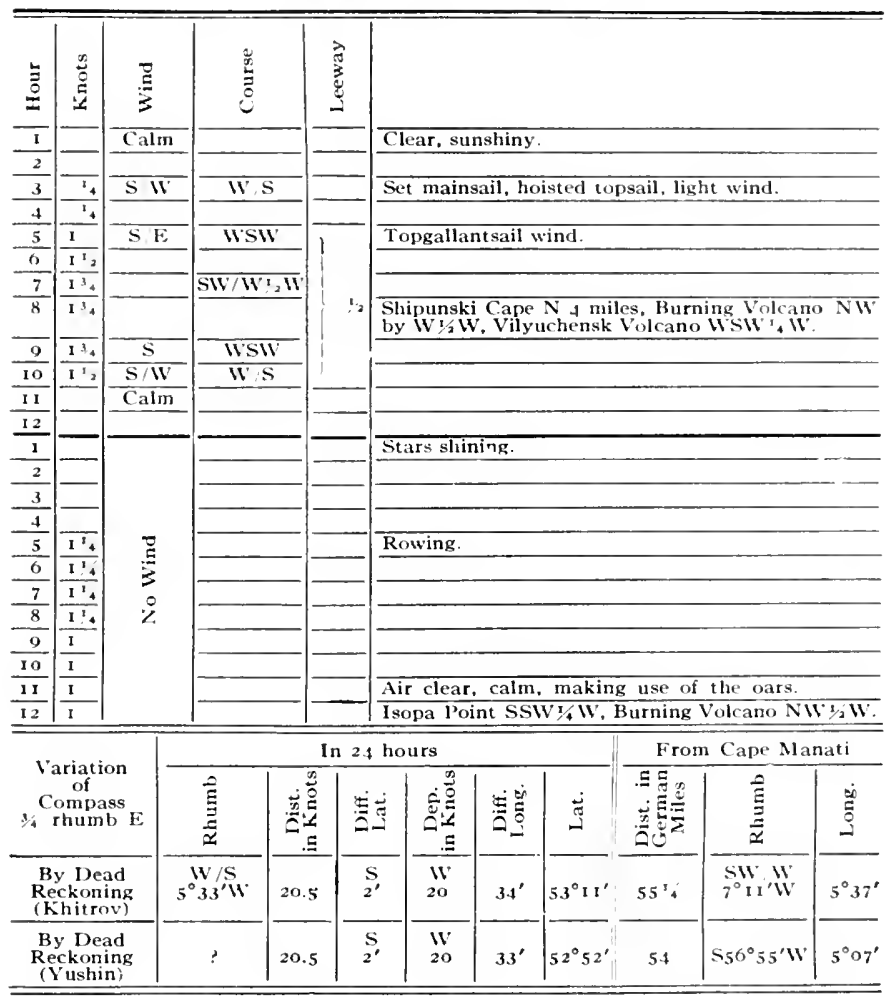




\section{August 26, I772. After Midday}

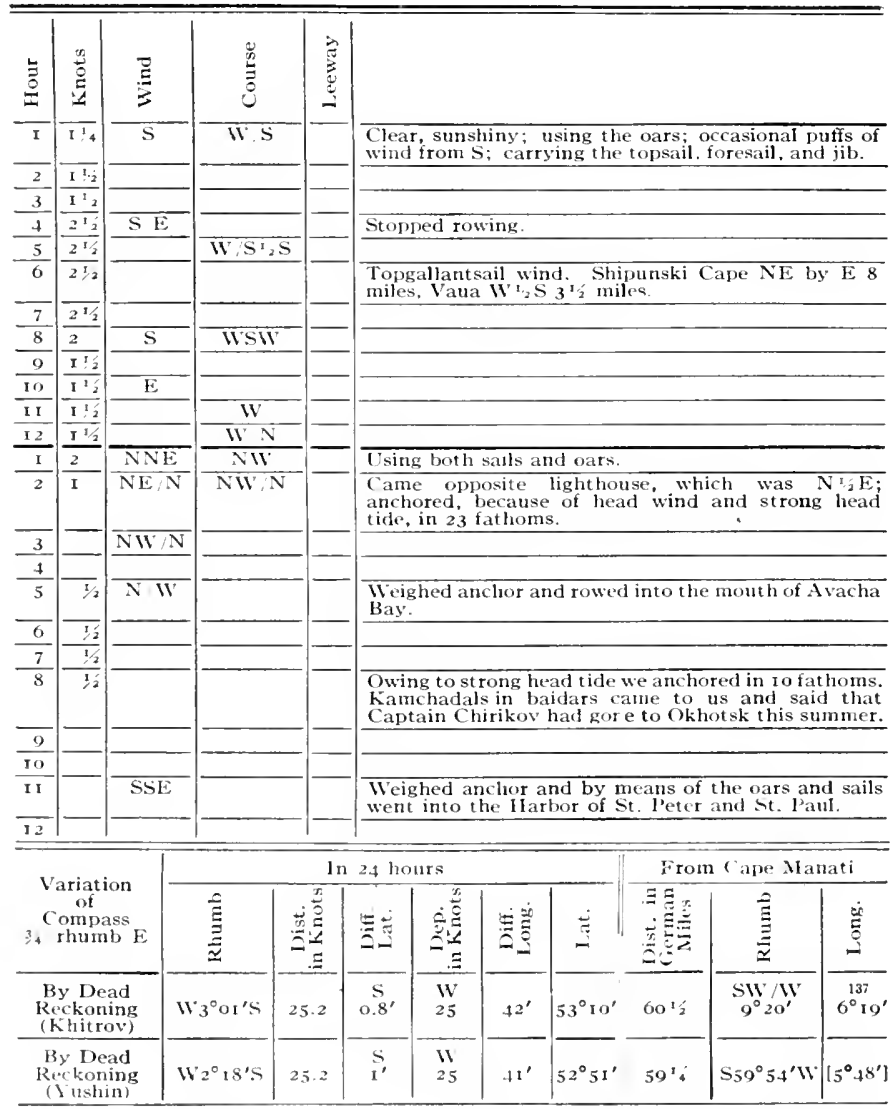

137 Fvon with the fresh start in reckoning 1 rom lering Island, this value is she $r$ about $\mathrm{I}^{2} \mathrm{~s}^{\circ}$ of the trHe longitude difference between Cape Manati and l'etropavlovsk. See foutnote i 24 , above. 
I August 27, 1742. After Midday

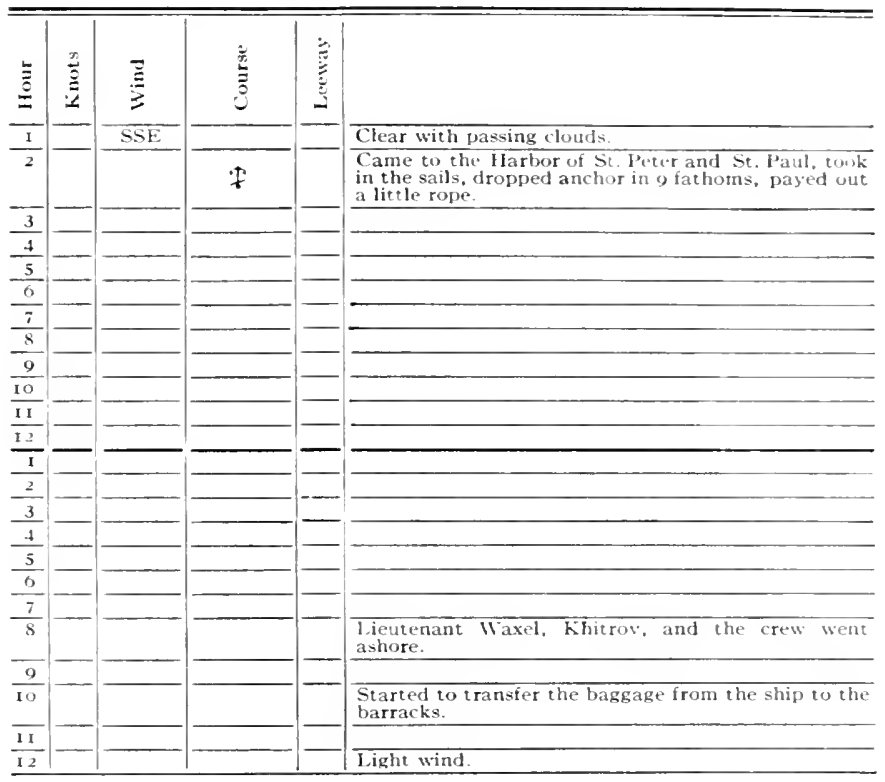




\section{LOG BOOK OF THE HOOKER "ST. PETER"}

\section{h) A ugust 28, 1742. After Midday}

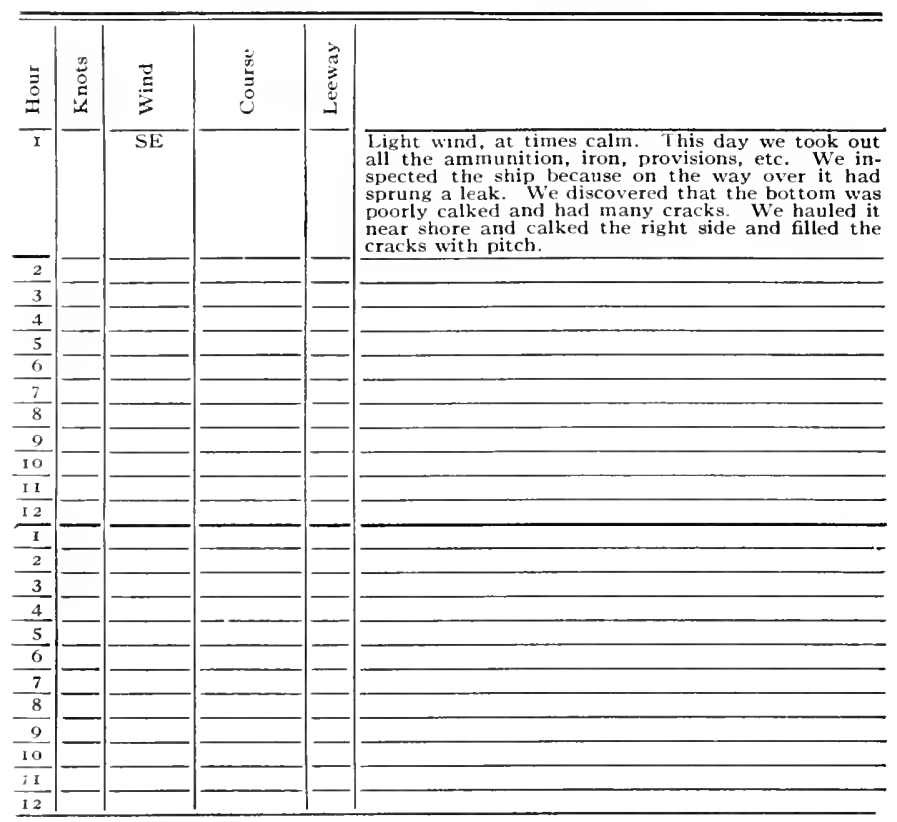


August 29, 1742. After Midday

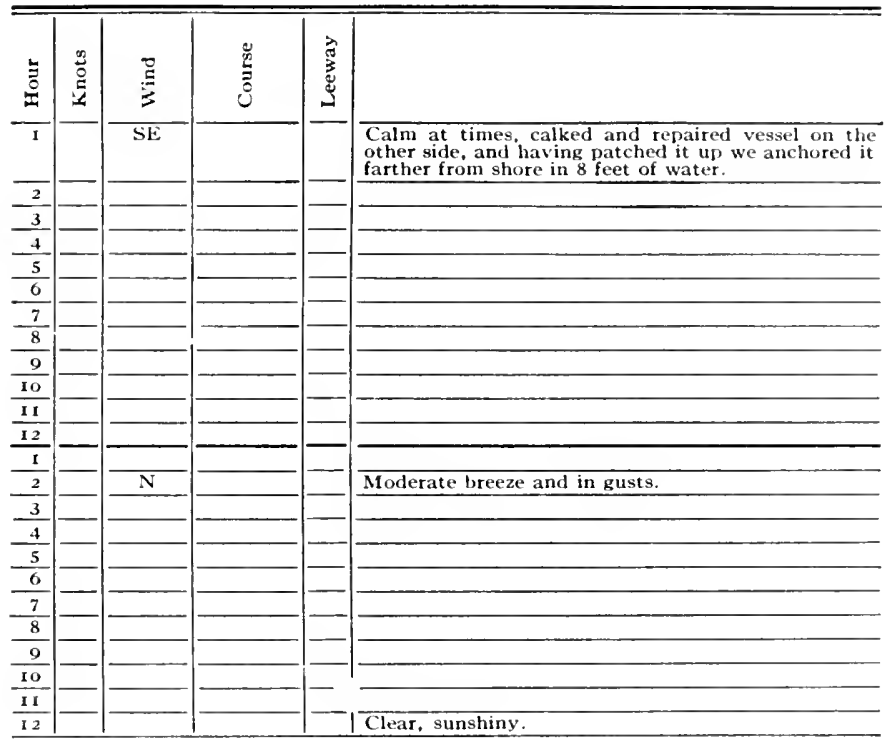




\section{LOG BOOK OF THE HOOKER "ST. PETER"}

(1) August 30, 1742. After Midday

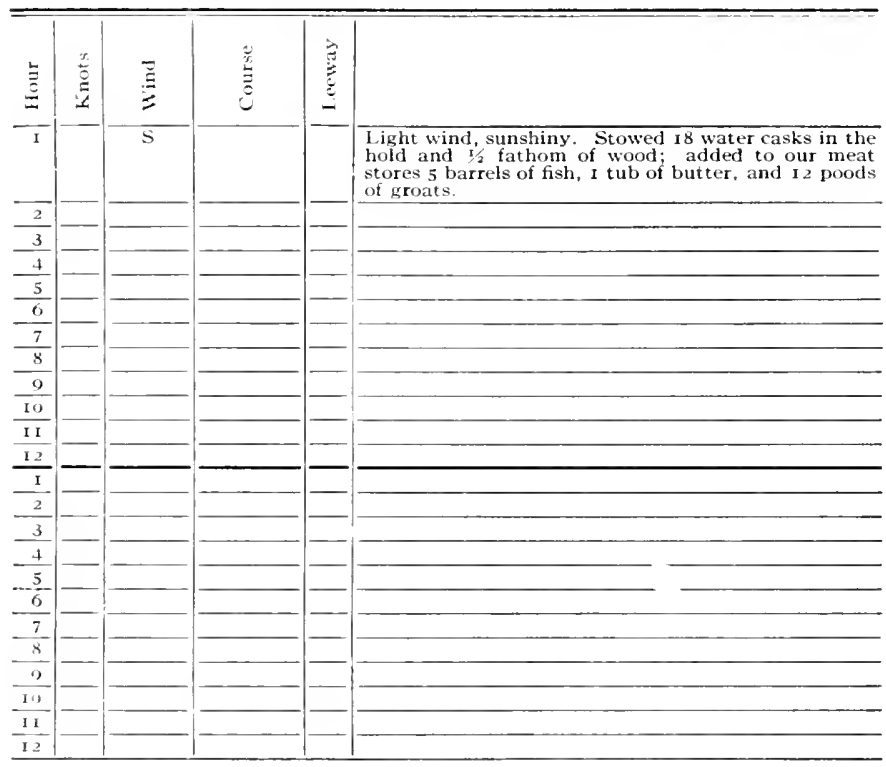


1. Alugust $31,17+2$. Afler Midday

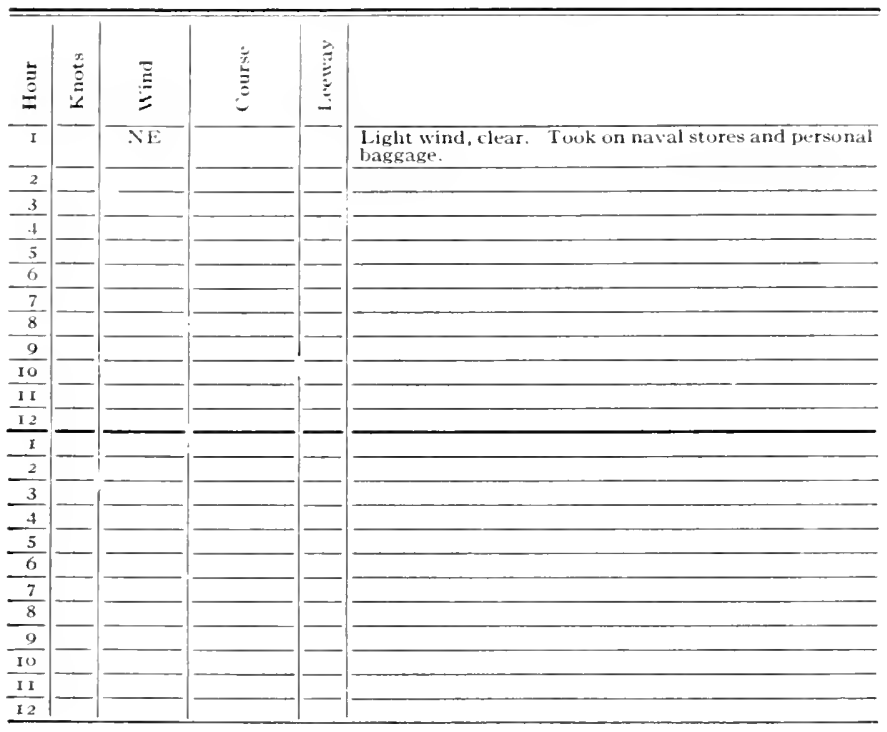


ఫ September 1,1742 . After Midday

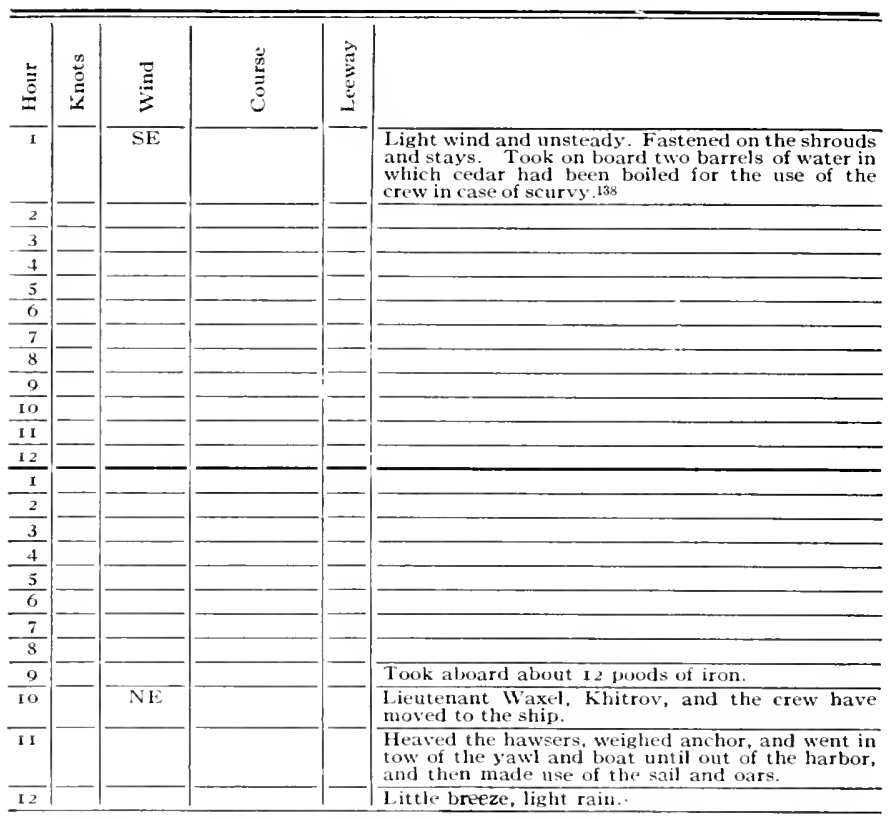

134 The cedar in question is, according to Dr. Stejneger, the Pinus cembra, the prostrate nut pine. Krasheninnikov (History of Kamcliatka. Russian edit., St. Petersburg, I 755, Vol. 2, pp. 192-193) say's: "This tree differs from the regular cedar in that it is smaller and does not grow straight but creeps along the ground, and for that reason it is called slanels. It is a valuable remedy for scurvy."

Cook refers to it: "One of these [cedars] grows upon the coast. sort we made our essence for beer, and found it excellent for the purpose. . We were told by the old Toion [chief | at St. Peter and St. Paul that Beering, during the time lie lay in that harbour, first taught them the use of the decoction of these pines and that it had froved a most excellent remedy for the scurvy." (James Cook: A royage to the I'acific Ocean, Lundon, 1784 . Vol. 3. pp. 332-333.) 
2f September 2, 1742, After Midday

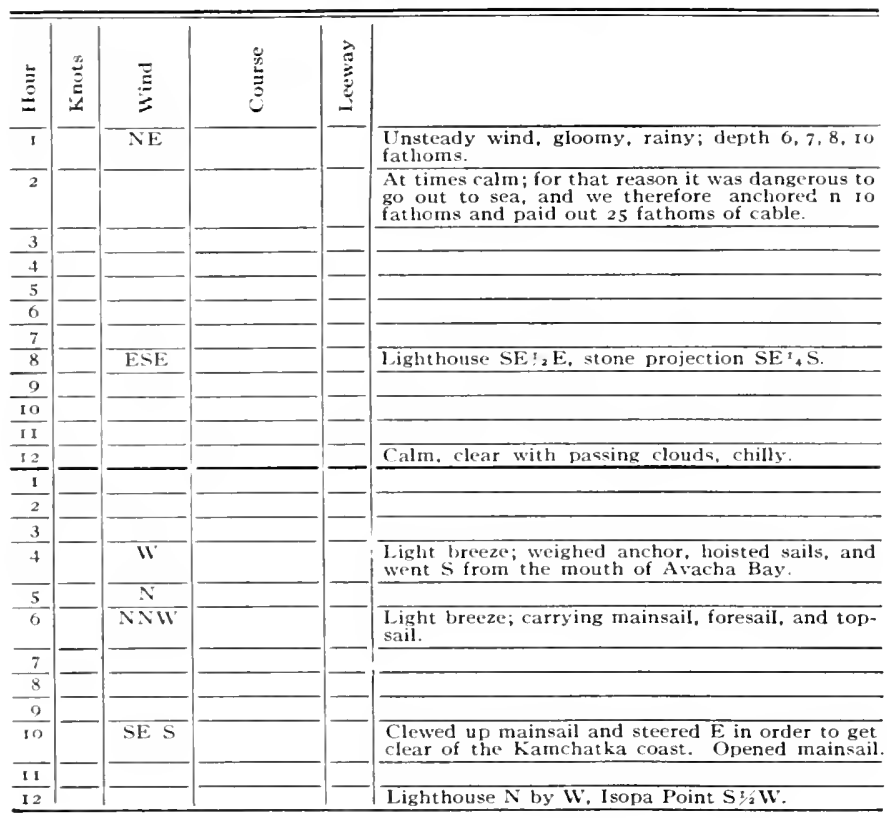




\section{LOG BOOK OF THE HOOKER "ST. PETER"}

Q September 3, I742. After Midday

\begin{tabular}{|c|c|c|c|c|c|}
\hline $\begin{array}{l}\stackrel{\Xi}{\Xi} \\
\Xi\end{array}$ & $\underset{3}{\stackrel{n}{0}}$ & 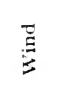 & 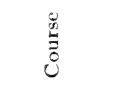 & $\hat{\tilde{E}}$ & \\
\hline I & & $\mathrm{s}$ & ESE & & light wind, clear \\
\hline$\frac{2}{2}$ & & & & & Light breeze. \\
\hline$\frac{3}{3}$ & & & & & \\
\hline 4 & & $S W$ & $\mathrm{SE} / \overline{\mathrm{E}} / 2 \mathrm{E}$ & & $\begin{array}{l}\text { Vaua } \mathrm{NW}_{2} \mathrm{Wl}_{3} \text { miles, Isopa SSW, Shipunski } \\
\text { Cape NE: }{ }_{2} \mathrm{~N} \text {. }\end{array}$ \\
\hline 5 & & s & ESE & & \\
\hline$\frac{6}{6}$ & & SW & $\overline{\mathrm{SE} / \mathrm{E}}$ & & Wind freshening, chilly; took a reef in topsail. \\
\hline 7 & & & & & \\
\hline 8 & & & & & $\begin{array}{l}\text { Topgallantsail breeze; came about with the wind } \\
\text { and sailed W. }\end{array}$ \\
\hline 9 & & SSIV & W & & \\
\hline 10 & & & & & \\
\hline II & & & & & \\
\hline 12 & & & & & Very little wind. \\
\hline $\bar{I}$ & & & & & \\
\hline 2 & & & & & Tacked to starboard. \\
\hline 3 & & Calm & & & \\
\hline 4 & & & & & Clewed up mainsail. \\
\hline 5 & & & & & \\
\hline 6 & & S.W & SF, E & & Hoisted mainsail; rain falling. \\
\hline 7 & 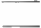 & & $\mathrm{SE}+\mathrm{E}$ & & Topgallantsail wind. \\
\hline 8 & & & & & Lowered topsail. \\
\hline 9) & & & W N & & $\begin{array}{l}\text { Tieutenant Waxel and Navigator Y ushin agreed to } \\
\text { return to Avacha, because the wind was contrary } \\
\text { and no headway was heing made by tacking and } \\
\text { because the vessel was leaking hadly. } \\
\end{array}$ \\
\hline In) & & & WNW & & \\
\hline II & & & & & Sailed into Avacha Bay. \\
\hline 12 & & & $\mathrm{NW}+\mathrm{W}_{2}$ & & Vaua lighthouse NiW by $\mathrm{N}$ i ${ }_{1}$, German mile. \\
\hline
\end{tabular}


1) September f, 1742. After Midday

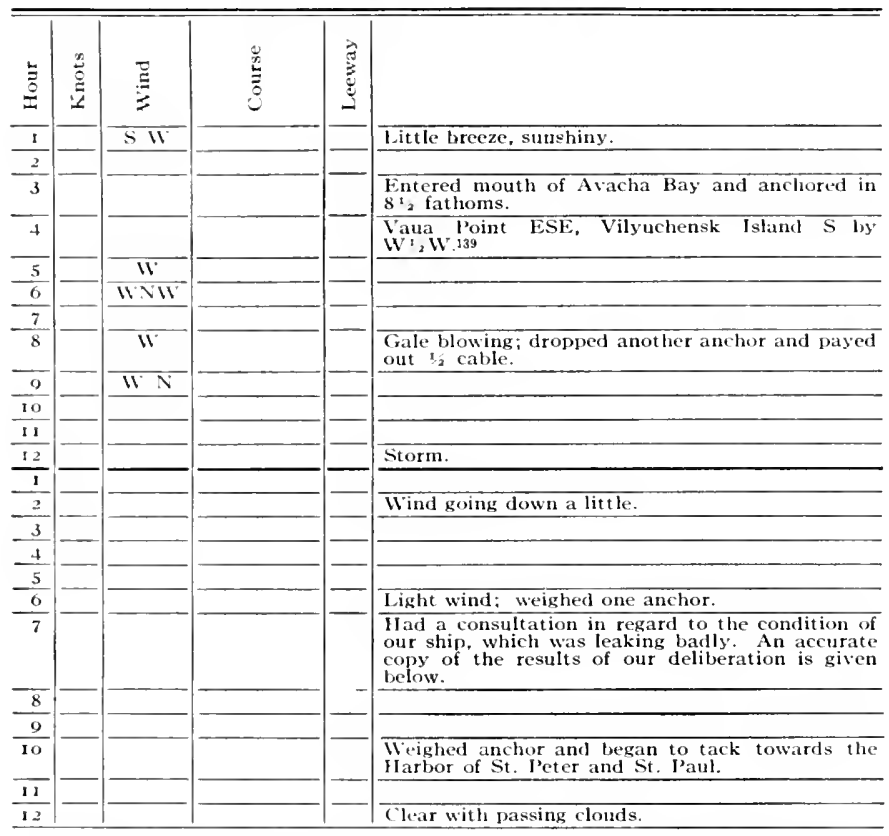

139 "Vilyuchensk" Island has disappeared from the maps. It is probably the same as "Starichkov" lsland of later maps. Stelle: "Beschreibung von dem Lande Kantschatka," p. 18) refers to it: "Diesem Haven [Avacha] zur Rechten ist eine kleine steinerne Insel ... Diese wird Wiliutschisky ostrow genennet." 


\title{
266 LOG BOOK OF THE HOOKER "ST. PETER"
}

\author{
Seplember 4,1742 \\ Decision Reached at the Consultation on September 4, 1742
}

Lieutenant Waxel, Fleet Master Khitrov, and all the lower officers on board on the voyage from $A$ vacha to Okhotsk came to the following decision:

I. We tried to repair the hooker St. Peter so that it would not leak as much as it had on the way from Bering Island to Avacha, and when we were at Avacha we had hoped that this had been done.

2. On September I, after we had [made out a report?] of the activities of our company, based on the original journal and chart, we were ready to go on our way. We went as far as eight miles from the bay but could not make much headway owing to the strong head wind which prevented us from sailing on our course, which was south and between south and west. At the same time our ship sprung a leak as before, forcing us to bail all the time and in the end to turn back to the aforementioned bay.

3. We knew, from former experiences, that after the middle of this month the strong north winds would begin to blow and that our vessel could not weather them and could never make Okhotsk in time [to escape them?]. We had time to go as far as the mouth of the Bolshaya River and might have done so, but we knew that at the Bolsheretsk Post there were not provisions enough foro ur men. Having taken all these reasons into consideration we decided to return to Avacha Bay.

\author{
Lieutenant Sven WAXEL \\ Master SOFRON KHITROV \\ Assistant Navigator KHARLAM YUSHIN \\ For the Boatswain Alexer Ivanov \\ Boatswain's mate GRIGORI TRUBESHIN \\ Midshipman IVAN SiNT
}




\section{FINAL RETURN TO PETROPAVLOVSK}

Seplember 5, 1742. After Midday

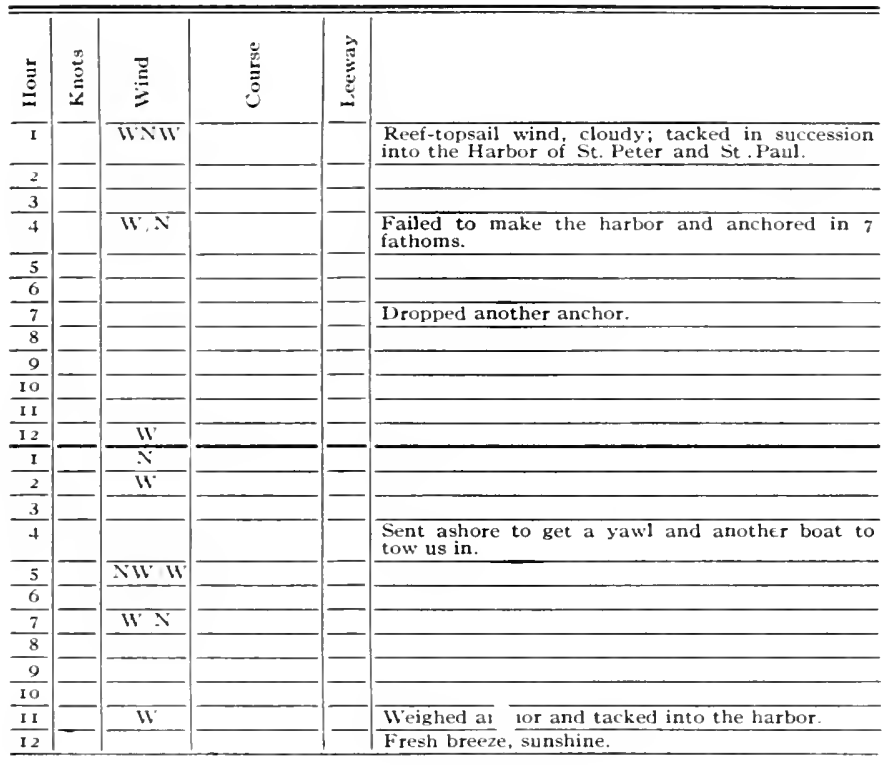


268 LOG BOOK OF THE HOOKER "ST. PETER"

(S September 6, I742. After Midday

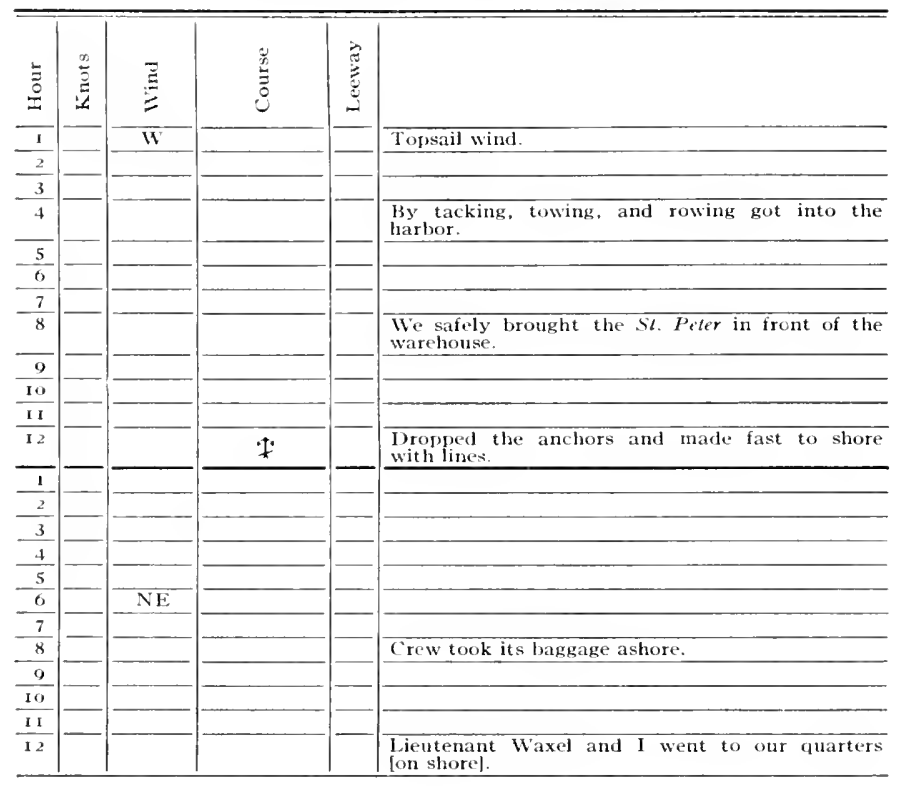


\& September $7, I 7+2$. Afler Midlay 110

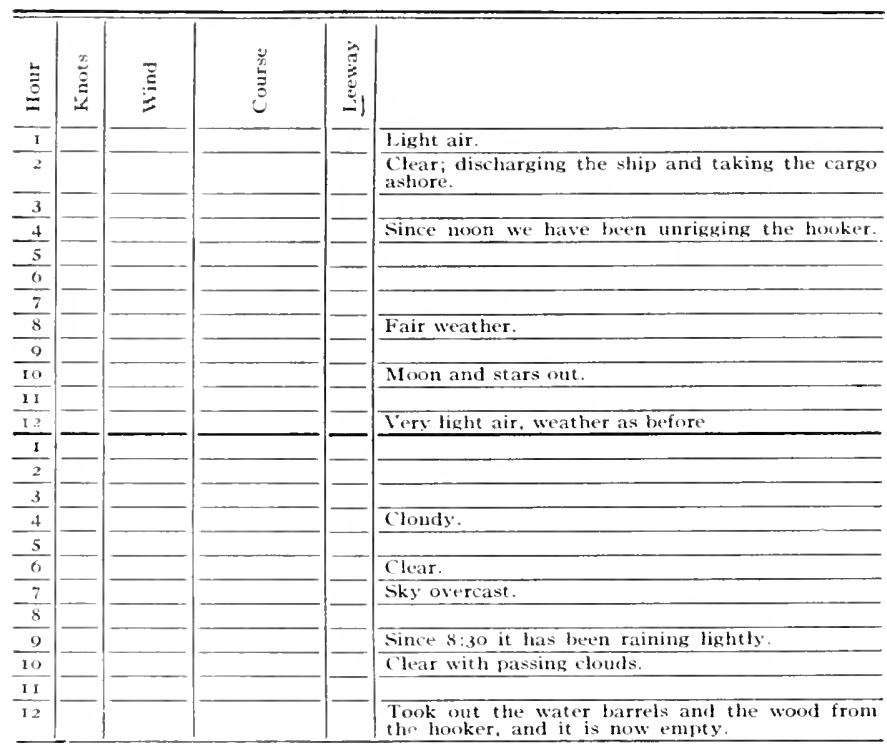

1to Y'ushin's journal. 


\section{CHAPTER VI}

\section{LIEUTENANT WAXEL'S REPORT ON THE VOYAGE OF THE ST. PETER}

Although the log book of the St. Peter gives an intimate, dayby-day insight into the happenings of the voyage, and is therefore a valuable historical document, its stereotyped form and routine information of ten obscure the human interest of the story. This element is supplied by the simple and straightforward narrative of Lieutenant Sven Waxel's report, which he, as officer in command after Bering's death, forwarded to the Admiralty College from Petropavlorsk by special messenger in November, 1742. A translation from the original ${ }^{1}$ in the Russian archives follows.

\section{To the Imperial Adiriralty College, a Most HUMBLE REPORT}

Last May, I 74I, the late Captain Commander Bering called a council, in which participated Captain Chirikov, Professor de la Croyère, the officers, and navigators, to decide on the first course to sail after leaving the Harbor of St. Peter and St. Paul [Petropavlovsk] in order to discover the Terra Juan de Gama which is marked on the map of the abovenamed Professor de la Croyère and which extends northerly to the 47th parallel. It was agreed to steer, after going out of Avacha Bay, SE by $E$, true compass, to the 46 th degree in order to see whether there is any land on that parallel and if such be discovered to coast alongside of it between north and east or north and west, depending on the lay of the land. If, lrowever, no land is seen on that parallel, it was concluded to sail from there on an $\mathrm{E}$ by $\mathrm{N}$ course until land is found, and when found to follow it in a northerly direction for the purpose of observation as long as time will permit so as to return during the last days of Septem-

1 Archives of the Ministry of Marine, Petrograd: Papers of the Admiralty College, 17.42-17.46, No. 2, pp. 233 ff. On the top of the first page is written "Received September 4, 1743. from the hands of Boatswain Alexei Ivanov, who was sent here for that purjose." The report was passed on to the Senate two day's later, and to the Cabinet on September 17. 
ber to the Harbor of St. Peter and St. Paul, where we would pass the winter. All having agreed to this plan, Captain Commander Bering on the St. Peter, accompanied by Captain Chirikoy on the St. Paul, sailed out of Avacha Bay on June 4, I 74I, and on getting out to sea kept the course agreed upon, that is SE by E, and a little closer at times.

The wind was fair, and we kept together with Captain Chirikov until June 19, when there was a strong easterly wind which compelled us and him to take in sail and heave to. The St. Paul was seen to the northwest of us until about eleven $0^{\prime}$ clock at night, being then in latitude $49^{\circ} 52^{\prime}$ $\mathrm{N}$ and from Vaua $17^{\circ} 49^{\prime}$, but after that time we lost sight of her With the coming of day we set out to find her on the course on which we saw her during the night, and in this neighborhood so far as the weather allowed we spent 43 hours but without success. Then Captain Commander Bering, his officers agreeing, decided to pursue the course originally agreed upon between $\mathrm{S}$ and $\mathrm{E}$, and we kept within that quarter of the compass on various rhumbs, according to the wind, to $45^{\circ} 13^{\prime}$ and to the east of Vaua $16^{\circ} 23^{\prime}$, and about 200 German miles without finding any land. The Captain Commander then changed the course to $\mathrm{E}$ by $\mathrm{N}$, the same which lad been agreed upon in council, and we sailed on it to latitude $48^{\circ} 38^{\prime}$ and longitude from Vaua $36^{\circ}$, but even at this distance no land was seen. The Captain Commander, his officers agreeing, decided to keep a little more to the north in the hope of soon discovering some land. Between $\mathrm{N}$ and $\mathrm{E}$ we sailed until July I6, on which day we saw land [Mt. St. Elias] in the northwest. We steered for it and on July 20 anchored alongside one of the islands, which we named St. Elias [Kayak].

This island is situated in latitude $59^{\circ} 40^{\prime} \mathrm{N} ;^{2}$ longitude from Vaua $48^{\circ} 50^{\prime}$, and on the course $\mathrm{E}$ by $\mathrm{N}$ from Vaua $4173 / 4$ German miles. That same day the Captain Commander sent Fleet Master Khitrov in the longboat to look about among the other islands in sight for a good anchoring place where we might run for shelter in case of need. Khitrov returned during the day and made a verbal report to the Captain to the effect that he found anchorage between the mainland and the island in the roadstead, where he got 3 to $3 \frac{1}{2}$ fathoms of water. He also reported that on that island he came across a hut built of hewn boards and that there were indications that people occupied the hut shortly before our coming. He brought to show us a wooden basket, a shovel, also a stone which had on it many marks of copper. The Adjunct Steller, who went in the small boat to the island of St. Elias, found there an earthen hut in which were dried fish, cured that very summer; we saw also human footprints on the sand and a fireplace. It was evident that on seeing

${ }^{2}$ Yushin gives $59^{\circ} 49^{\prime}$, Khitrov $59^{\circ} 39^{\prime}$. The correct latitude of the southern point (Cape St. Elias) is $59^{\circ} 48^{\prime}$, of the northern point $60^{\circ} 0^{\prime}$, and of the St. Peter's anchorage $59^{\circ} 56^{\prime \prime}$. 
us the people ran into the roods to hide or that their homes are on the mainland and they visit this island to catch fish and hunt sea animals. In order to show his good will to the native people the Captain Commander sent to the liut a number of presents- $16 \frac{1}{2}$ arshins of green-colored goods, 2 iron knives, 20 Chinese strings of beads, 2 iron [smoking] pipes, all of which were left there. We could not tell whether on the mainland there was any good timber or not, but we did see the huge mountains and volcanoes covered with snow. On the island there is plenty of small timber, namely fir, larch, and other trees, which are not fit for building and not even for repairing a ship, for we looked for material for topyards but did not find it.

When we had provided ourselves with water from the island of St. Elias, the Captain Commander, mainly because it was dangerous to lie out in the open sea, departed July $2 \mathrm{I}$, and we steered between S and W, following the lay of the land, for the purpose of observation. In this way we sailed on various courses and came to latitude $56^{\circ} 54^{\prime}$ by July 26 . Here we sighted that same coast [Sitkalidak Island] in the direction NE by $\mathrm{N}$ from us, about seven miles distant. On taking soundings we had 3.5 to 40 fathoms of water. It was quite unsafe to keep near the land because of the sand banks, continuous heavy fogs, and violent winds; on that account, as well as our ignorance of the nature of the coast, which had dangers for us, we steered different courses in order to draw away from it more and more-all of which is clearly noted down in our log book. This maneuvering we kept up until August 2. During that night as the fog lifted, we saw quite close to us an island [Chirikov Island], the water being 18,17 , and 16 fathoms deep. On that account, as well as the darkness and the fog, we let go the anchor in 18 fathoms. We named this island Tumannoi [foggy]. In the morning we sailed away.

The mainland was from us in a NNV direction and not a great distance away. August 4 we sighted five islands [Semidi], and from this time on we kept farther and farther out to sea. For the course we were following the winds were quite contrary. By August Io we were in latitude $53^{\circ}$ I $8^{\prime} \mathrm{N}$ and from Vaua a little less than 400 German miles; and taking all this into consideration, also the report submitted by the assistant surgeon, to the effect that 2 I of our company were down with scurvy and that, if obliged to remain out at sea during the autumn weather, they would be altogether unfit for service, the Captain Commander with his higher and lower officers decided that if the winds permitted it we should from this day on keep on the $53 \mathrm{rd}$ parallel in order to see whether or not American land is to be found on that parallel, although the last we saw of it was on the 55th. We attenuted to put our decision into execution but, until August 27, were lindered by the severe headwinds from the west, being then still more than 300 German niles fron Vaua and on the 53rd parallel of latitude. It was quite clear to the Captain Commander that 
if these violent head winds held on we should not advance very fast. At this time we had only 25 barrels of water, and to attempt with no more on hand to reach the Harbor of St. Peter and St. Paul, assuming even that headwinds would no longer trouble us, would be very dangerous and might lead to a great misfortune.

Taking these possibilities into consideration it was agreed on this 27 th day of August to go in search of water on the land which according to our calculation was about 60 miles away. On the 20 th we saw many islands [Shumagin], towards which we steered closer and closer, having $55,50,45$, and I 5 fathoms of water, with an even, sloping, sandy bottom. When we had come near enough we lowered the small yawl and sent the Assistant Navigator Yushin to find anchorage among the islands. While he was gone we anchored in 24 fathoms. When he returned and said that he could not find good anchorage we weighed anchor, put over the longboat, and went in its tow into the bay among the islands, where we had 25 and I 5 fathoms of water. Near one of the islands we ancliored and sent the navigator ashore to look for water, and, on returning, he reported to the Captain Commander that he found plenty of it. The mainland was seen behind these islands about 12 miles away. During the night we saw fire on one of the islands to the NNE, about a mile and a half away from us.

The following morning, August 30, Fleet Master Khitrov was sent in the small yawl to the place where fire had been seen, with instructions that, if he found people, he should treat them kindly; and with that in view he was given a number of things to present to them. In the meantime a strong wind was blowing, accompanied by squalls, so that it was not safe to remain longer near the island, as we were wholly unprotected. We were therefore obliged to seek shelter, though it was slight, among the islands, where we anchored. On September 2 we sent the longboat to Khitrov because, owing to the violent wind, he could not get off shore in the small yawl. The next day, September 3 , he came safely aboard but without the small yawl, which he had to leave behind owing to the strong wind and heavy surf. He made a report to the Captain Commander that he was at the place where fire had been seen, that he found the fireplace but did not meet any people. This same day we weighed anchor and tacked among the islands, going around the first island to the island [Bird Island] which was to the east of it, about two miles distant, and there anchoring in 15 fathoms. Here we remained, September 4. About noon of the 5 th we heard a loud noise and saw two men, who sat in two baidarkas rowing towards our ship. When they came within $5^{\circ}$ fathoms of us they stopped and called to $t$ s in their tongue, which our interpreters of the Chukchi and Koriak languages conld not make out. Our interpreters hailed them in Koriak and in Chukchi, and it was evident that they could not understand, because 
they pointed to their ears, waved their hands to us, and motioned to the shore. After this, one of the two approached much closer to our ship but would not come alongside. At the order of the Captain Commander we threw to him, tied on a board, several arshins of red (Chinese) silk, mirrors, iron pipes for smoking Chinese tobacco (called shar), and several small copper bells. He seemed to receive these gif ts with pleasure, and in return he threw to us two thin sticks planed smooth, to one of which were tied birds' feathers and to the other a bird's claw with the feathers on. which feathers we identified as that of the hawk. When we had accepted the presents the Americans pulled away for the shore, at the same time shouting to us in a loud voice and waving their hands toward the beach.

The Captain Commander ordered that the longboat should be lowered; in this I was sent to the Americans. I took with me an interpreter who understood the Chukchi and Koriak tongues, several armed men, also presents and Russian liquor. When I came near the place of the Americans I anchored because, owing to the surf, the strong wind, and submerged rocks, it was impossible to land. I allowed the interpreter and several men of my party to wade ashore. At the same time I offered the Americans presents, which they would not accept. To one of them I lianded a cup of liquor, which he took and drank but immediately spat it out. In the meantime the interpreter who went ashore was led to their camp, where he was offered whale blubber, which he accepted. When he wished to go back to the boat the Americans detained him and would not allow it; just how fast they held him it was difficult to say. In order to free him I was obliged to command the soldiers to fire in the air. When the guns were fired they all fell on the ground, and this gave the interpreter the chance to come aboard. The Americans made a dash for the boat, seized the painter, and started to liaul the boat ashore. I feared that it would be smashed on the rocks and therefore shouted to cut the painter and slip the cable. With all my men I returned safe on board and made a verbal report to the Captain Commander. At night the Americans built a big fire on the beach. That night there was an unusually strong wind, and we were compelled to strike the main and fore yards and wait for daylight. As soon as the 5 th of September dawned we weighed anclior and tacked near the island with the intention of proceeding on our course, but the wind was contrary and the tide against us. We went back again to the island and anchored in 7 fathoms. While we were at anchor there rowed to us from the aforementioned island seven baidarkas, two of which came right up to the ship. It was evident that they had never before seen a gun, for when they came to us they were not in the least afraid. We gave them at this time an iron kettle and a few needles. They gave to us as presents two hats made of bark on which were fastened ivory images in the form of a luman 
being." These images are being sent together with this humble report to the honorable Admiralty College, but the hats as well as the sticks were lost at the time of our misfortune.

All these islands are uninhabited and treeless. It is evident that the Americans come in baidarkas from the mainland to the islands to hunt sea animals and to catch fish. As to the way the Americans make their baidarkas from seal hide, it may be seen from the detailed drawing which I made on the map which is sent to the Admiralty College. Having taken on water we got under sail September 6 and continued on our course. We called that island Shumagin. We intended to go straight to Avacha Bay, but contrary winds from the west as well as violent winds hindered us and caused us much suffering. Frequently we had to heave to in order to save ourselves. On September 24 we came to latitude $5 \mathrm{I}^{\circ} \mathrm{N}$ where we sighted several islands [Adak, Atka] and, behind them on the WNW, the American mainland [Great Sitkin]. At this place there blew an indescribable gale from the west which continued until October I3. During the whole time we had to heave to and were carried to the eastward about 80 German miles. In order that the Admiralty College may form some idea of the storm it should be pointed out that the old navigators said that they had seldom seen anything like it. We were saved from it with great difficulty. A number of the men were down with scurvy, some died of it, and those who survived were too weak to work. Although, on account of the hard labor and the continuous inclement weather, we were at the end of our strength, yet with the help of God we made every effort to reach the Harbor of St. Peter and St. Paul. On October 25 , being then in latitude $5 \mathrm{I}^{\circ}$ and some minutes, we sighted a high, rocky, and treeless island in NW by N, which we named St. Markiana [Kiska Island]. On the 28 th we saw another island to the NNIV, about three miles distant, and in its neighborhood three small islands. As far as we could make out they were all bare and treeless. This island we named St. Stephen [Buldir]. The following day it was foggy, and on taking soundings and getting only 35 fathoms of water we hove to and did not take any chances. When it cleared a bit we noticed an island to the west which we named St. Abraham [one of the Semichi]. We then hoisted the sails and went on our way. It became very difficult to run the ship because, in addition to those who died, 40 were ill and those who were still about were very feeble. We continued under these conditions

These so-called hats were eye shades which the hunters used when out at sea. Cook gives the following description of them: "All of them [Aleuts of Unalaska] have a kind of oval snouted cap, made of wood, with a rim to admit the head. These caps are dyed with green and other colors; and round the upper part of the rim are stuck the long bristles of some sea animal [sea lion] on which are strung glass beads; and on the front is a small image or two made of bone." (James Cook: A Voyage to the Pacific Ocean, London, 1784. Vol. 2, p. 51.) See Fig. 12, lower. 


\section{REPORT ON VOYAGE OF THE "ST. PETER"}

until November 4, on which day we sighted land [Copper Island] which we looked upon as a godsend because it was beyond our strength to keep any longer at sea. By observation this land is in latitude $54^{\circ}$, but by dead reckoning we thought we were in latitude $53^{\circ}$ and in longitude some I 40 minutes from Vaua. From this reckoning we hoped that the land in sight might be Kamchatka, some little distance north of Shipunski Cape. Our faulty reckoning was due to the fact that we had for a long time (as may be seen from our log book) thick weather and no sunshine to set us right.

We and our men were greatly weakened from the severe attack of the scurvy and at the end of our strength and in great danger, for it may be said at that time the ship was without control. Of our men there were only eight who, with great pain, could look after themselves, and of these three, one of them being the Captain Commander's man, could come on deck. All the others were sick unto death. We had only six barrels of water on board. As to provisions we had no sea biscuit or such like and only a little flour, butter, and meat. On top of this the shrouds on the mainsail above the catharpings on the right side were all torn, and therefore we could not carry any sail on the mainmast and, as was noted above, there was no one to do any repairing. At the particular time of our misfortune the wind was contrary for getting around the cape seen by us. Therefore, on November 5, realizing that we were in a bad state to remain longer at sea and that it was dangerous to wait for fair winds because our men were giving out so fast that we feared we should be left without any help whatever and the ship would be lost with all on board. the Captain Commander called together for consultation all the higher and lower officers and those of the men who were still able to drag themselves to the cabin. The nembers of the crew announced that because of their sickness and feebleness they were no longer able to do their work at sea. Taking this into account the Captain Commander and the higher and lower officers agreed to look for an anchoring place where we might winter in order to save ourselves and not be entirely destroyed by such a dreadful disease. All the men agreed to this, and we sailed before the wind towards the land and drew near it about five o'clock in the afternoon and anchored with the small bower in 12 fathoms of water and paid ont alout three-fourths of a cable. It hroke about 80 fathoms from the end, and we were carried by the surf 5 fathoms, when we let go another anchor with the same result, and we were carried by the surf over [the reef] closer to the shore into $4 / 2$ fathoms, whore we let fall the best bower and paid out three-fourths of a cable. By this time we lad 49 of the crew on the sick list. On November 6 we lowered the longboat and moored the ship, but only after great difficulty since we were so shorthanderl. After this week, with the help of those men who, although with some cffort, were still able to be on their feet, we did our best to transport 
the sick ashore and to put up for them tents made out of sails. When these were up we took the sick Captain Commander ashore on November 8; Fleet Master Khitrov, who was also ill, on the $15 \mathrm{th}$; and many of the crew, not a few of whom died as they were being transported. On the 2 Ist I, too, was so ill that I had to be taken off the ship because I was almost helpless, and there was no one to bring water on board, and no one to look after the ship.

At this time the Captain Commander sent Constable Roselius with two other men with instructions to follow the shore in a northerly direction to determine whether the land on which we were was part of Kamchatka or some island; and, if Kamchatka, Roselius was to continue on his way until he cane to an inhabited place and there carry out such orders as the Captain Commander gave him. Roselius was so weak that he did not go farther than 30 miles from our camp and returned without news. On November 2 r, the Captain Commander ordered me, with the higher and lower officers and members of the crew, to submit a report on the best way to save the ship. This was done, and the report was submitted on the $23 \mathrm{rd}$, in which we recommended that the ship should be hauled up on the sandy beach near our camp and that the cargo should not be unloaded so that in case of a strong offshore wind and the breaking of the cables the ship would not be carried out to sea. The Captain Commander accepted the report and that same day, the $23 \mathrm{rd}$, ordered Master Khitrov to put it into execution at the very first opportunity. On the 25 th Khitrov started to go on board and, when he came to where the longboat was hauled up, was told by the boatswain. Alexei Ivanov, who was on watch, that there were only five able-bodied men. In dragging the longboat into the water one of the men got drenched to the skin and had to give up, which left four men who were completely exhausted. When Master Khitrov realized that with this handful of men he could not weigh the best bower anchor, that the wind at the time was blowing NNW straight on the rocky reef, which was about 150 fathoms in SSE dircetion from where the ship was at that time, and that the topmasts and yards were down, and even if there had been enough men it would be dangerous to move the ship in such weather, owing to the above-mentioned reef, he became convinced that he could not carry out the task assigned to him. He then came to me and explained the situation, and 1 advised him to make his report to the Captain Commander, which he dicl. From the $25^{\text {th }}$ to the 28 th it blew hard so that it was not possible to go on board to carry out the above-mentioned order. In addition to the other troubles Fleet Master Khitrov was quite ill with scurvy and unable to be about and was put in the same tent with the rest of us. (n) the night of November 28 a violent storm from NE broke the cable and drove the ship on the sandy beach on which we had planned to lay her up. On December I the Captain Commander sent the sailor 
Anchiugov with two other men along the shore to the south to explore and to learn whether the land on which we were was the mainland or some island. They came back on December 27 without any definite information.

On December 8, I 74I, by the will of God, Captain Commander Bering died of scurvy from which he had been suffering for four months, and we buried him on the island where we lived. I succeeded him in command. and, with the assistance of Fleet Master Khitrov, every effort was made to find out where we were; but, owing to bad weather and other serious obstacles, it was not before the month of April that we knew anything certain. As soon as we were sure that we were on an island, we made a general inspection of the St. Peter to determine whether she was in good condition for going to Kamchatka and whether it was possible to get her into the water. Our examination showed that the vessel was so badly injured that she could not be repaired, that we had nothing to repair her with, and that we had not the facilities for getting her off since she was buried in something like seven feet of sand above the keel. We took council together as to how to get away from the island, and we found no other means (since the island was bare and treeless) than to break up the ship and make out of her for our voyage to Kamchatka as large a vessel as the timber would allow. To this plan all agreed, and we went to work in April and continued operations until May 5. On the following day (May 6), with the help of God, we commenced building the new vessel, the keel of which was 36 feet, beam I 2, and depth 5 feet 3 inches. We worked as fast as we could, making use of every favorable moment.

The island on which we wintered extends from $54^{\circ}$ to $56^{\circ}$ north latitude, and from the southern cape, which we named Cape Manati, that is to say Sea Cow, it stretches from NNIV to SSE. It is about I3o versts long and about ro wide. There are no habitations on it and no signs that people ever lived there. There are no trees of any kind except in some places a few small willows. On the island are high mountains and volcanoes. In many places there are rocky bluffs, making it extremely difficult for seagoing vessels to approach, for on the whole island there are very few places where the beach is not full of rocks, stretching out into the sea for a verst or more, which are submerged at high tide and exposed at low tide. Where the beach is not rocky the surf runs high, and therefore it is quite dangerous to anchor in the roadstead. For ships that draw five or six feet of water there is no place for wintering, for we looked into the question of a harbor and sent for that purpose Fleet Master Khitrov to the south and boatswain Alexei Ivanov to the north, but they could not find one. When the wind blew from the west it brought us from Kamchatka pieces of timber that had been used in the building of huts, planks that had been used for fastening together rafts of logs, and broken deer sleds such as are used by the wandering Koriaks. 
Many times when the weather was clear some of our men saw, from the west side of the island, what seemed to be snow-covered volcanoes, but on account of the great distance we could not be sure about that, but our reckoning on the 1742 voyage leaves no room for doubt that the volcanoes in question were those of the Kamchatka coast. From the American side there floated over to us big pine trees, arrows, and paddles such as are not found in Kamchatka. While on the island we lived very poorly because our dwellings were holes made in the sand covered over with sail. The gathering of wood was particularly difficult, for we were obliged to search for it along the beach and carry it on our backs as far as 10 or 12 versts. While we were there we and nearly all the men in our command suffered greatly from scurvy and for such a long time that some did not fully recover before spring when there were found some greens which could be used for food. During the whole winter our food, because we had no other provision, was, we might say, very poor, difficult to obtain, and not fit to eat. We had to hunt along the beach sometimes as far as 20 and 30 versts in the hopes of killing a sea animal, sea otter, sea lion, or hair seal, which is commonly called tulen. Having killed the beast we had to carry it on our backs the same long distance back to our camp. If we could not find live animals to kill we had to content ourselves with dead ones that were washed ashore, such as sea cows and whales. By spring the game was frightened away, and we had to live on the fur seals which came here at that time to breed. Their meat was especially nauseating; and, as it became more so as time went on, we took to hunting sea cows, huge animals, weighing no less than two hundred poods. One may form an idea of the appearance of these animals-fur seal, sea lion, and sea cow-from the sketches of them made on the map which I send to the Admiralty College. ${ }^{4}$ From this time on we lived on sea cow meat, which is better than that of the other animals aforementioned.

On the island where we lived there were violent winds and even blizzards during the winter. It may be said that between December and March there was seldom a fair day. From March and during the spring and summer months there was almost continuous fog and dampness and little pleasant weather.

The delay in the construction of our vessel was due in part to the disagreeable atmospheric conditions just mentioned and in part to the poor health of the men and the great distance from the source of authorit $y$, which made it unsafe to hold them strictly to their work, and nothing could be done without the consent of all concerned. Adjunct Steller. who was with us, prospected the island for metals and minerals but found none. When with God's help the new ship was finished, I, with Fleet Master Khitroy and all the men, had a meeting to discuss what we should

\footnotetext{
4 Reproduced by W. H. Dall as PI. 70. Appendix 19. L. S. Coast and Geodelic Survey Rept. for 1890 .
} 
do with all the material on hand. We agreed to take with us a certain amount of irou for ballast, such provisions as we needed, a supply of fresh water, several barrels of salted sea cow meat, and all the crew with their baggage and to sail for Kamchatka. All the other material and supplies, the greater part of which was worthless, according to the testimony of the Fleet Master and the petty officers, it was decicied to leave behind in the warehouse which we built for that purpose. Owing to the fall weather and our ignorance of the seaworthiness of our craft we did not think it wise to overload it. A list of all the material, good and bad, left behind is sent to the Admiralty College.

On August io we launched our newly constructed ship which we named the hooker St. Peter, and we rigged it on August 13. Having taken on board all the men to the number of 46 , we started, with God's help, on our way to Kamchatka. On the night of August 15, while out at sea, the vessel, owing to her poor construction, sprang a big leak so that there was soon about two feet of water in the hold. We were in great danger, and to lighten the ship we threw out of the hold cammon balls and shot. By means of buckets and pumps we got rid of some of the water. When the leak had been somewhat attended to we proceeded on our course. On August 20 we sailed safely into the Harbor of the Holy Apostles St. Peter and St. Paul. There we heard that Captain Chirikov and his company lad left this same summer for Okhotsk. We repaired the hooker St. Peter and set sail on September I with the intention of following bim to Okhotsk, but after being out at sea for five days another big leak was discovered. In addition to other troubles the wind was contrary, so that we decided, all agreeing, to return for the winter to the harbor so that we might not suffer misfortune from such a leak in such an unreliable vessel and so late a season.

We are now in Kamchatka, where we plan to remain until the coming April, when we will repair the hooker and go to Okhotsk. If Captain Chirikov slould still be there, I and my company will place ourselves at his orders; if he should be gone, we will proceed to Yakutsk, where I will try to carry out the instructions of the Admiralty College which I shall there receive; if there are no orders on hand. I will wait until they come.

I take this occasion to report to the Admiralty College that from the time we left the Harbor of St. Peter and St. Paul in the year I 7fI, and during the time we were out at sea and on the island, there died of scurvy at different times, officers and ochers, 31 men. The names of these persons and the dates of their death are sent on a special list.

Article 9 of the instructions which the Arlmiralty College gave to Captain Commander Buring reads that when the expedition shall have come to an end there should be forwarded to the Admiralty College the log book and the chart that shall lave been made by the offeers who were on the voyage. I and Fleet Master Khitrov fully meant to do that [make 
the chart] but we were in such trouble and so ill (all of which I have reported above) that we have not as yet been able to accomplish it. But the log book and a sketch map giving the outline of the American coast (which we wished to name, atter the manner of other European discoverers, "New Russia" but did not feel empowered to do so without orders of the Admiralty (College), as well as sketch maps of the islands of St. Elias, Shumagin, and the other neighboring islands are being sont to the Admiralty College this 15 th day of November, 1742 , by a special messenger, one of my men, the boatswain Alexei Ivanov. This man was a tower of strength when we were in trouble. The Captain Commander made him boatswain, and he fulfilled the duties of his position in an able manner. For greater salety in going through the country of the natives Ivanov is accompanied by Ivan Okulov, one of our soldiers, from a Tobolsk regiment. They are ordered to go by way of the Anadyr Fost and to lose no time.

That which I have reported above about our hardships and suffering I humbly pray that the Admiralty College may receive.

Harbor of the Holy Apostles

Lieutenant SVEX WAXEL

St. Peter and St. Paul

Nonember 15,1742

\section{ENCLOSURE}

List of the Officers and Mex of the "St. Peter," Which Salled from the Harbor of St. Peter axo St. Pall 1N 17it. Who by the Will of God DIEO OF SCLRVY EITHER AT SEA OR ON THE ISLAND WHeRE WE WINTERED

The name of each person and the date when he died are noted below; and the island where we wintered, and which has been described above, we named Bering's Island.

Sailor, 2nd rank

Marine grenadier

Marine soldier

Kamchat ka soldier

Marine soldier

Admiralty cooper

Soldier of the Siberian garrison

Admiralty carpenter

Drummer of the Siberian garrison

Marine grenadier

Soldier of the Siberian garrison

Marine grenadier

Boatswain

Trumpeter

Sailor, Ist rank

Camnoneer, 2nd rank

Cadet of the Yakutsk regiment

Sailor, 2nd rank

Soldier of the lakutsk regiment.
Nikita Shumagin

Andrei Tretyakov

Alexei Kiselev

Nikita Kharitonov

Luka Zaviakov

Stepan Bogdriev

Karp Pashennoi

Ivan Petrov

Osip Clientsov

Alexei Popov

Ivan Davidov

Ivan Nebaranov

Nils Jansen

Mikliael Toroptsov

Ivan Enelianov

Ilya Dergachev

Vasili Popkov

Seliverst Tarakanov

Savin Stepanov
Alig. 31. 1741

Sept. 24, 1741

Oct. I9, 17t1

Oct. 20, 17t1

Oct. 22, 1741

Oct. 28, r7t

Oct. 31, 1741

Nov. 2, 1741

Nov. 4. I7tI

Nov. 4.17.41

Nov, 4, 17t1

Nov. 5.1741

Nov. $8,17+1$

Nov. 11, 1741

Nov. 15, 17 71

Nov. 15, 1741

Nov. 15, 1741

Nov. 15, 1741

Nov. I6, 1741 


\section{REPORT ON VOYAGE OF THE "ST. PETER"}

Sailor, Ist rank

Navigator

Admiralty calker

Underskipper

Marine grenadier

Cannoneer, and rank

Marine soldier

Ensign

Personal servants of the officers, two men

Mark Antipin

Semen Artemev

Andreyan Eselberg

Alexei Klementev

Nikita Khotyaintsov

Ivan Tretyakov

Prokofei Efintsov

Fedor Panov

Ivan Lagunov

Altogether, with the Captain Commander, 3 I men.6

\section{Lieutenant Sven Waxel}

${ }^{5} \mathrm{His}$ death not mentioned in the $\log$ book.

${ }^{6}$ Waxel left out the name of the sailor Nikita Ovtsin, who died on November 19 (see log book).
Nov. 20, 174I

Nov. 22, I74I

Nov, 22, I74I

Dec. $4,174 \mathrm{I}$

Dec. 9, $174 \mathrm{I}$

Dec. 17,1741

Dec. $27,174 \mathrm{I}$

Jan. $\quad 2,1742$

Jan. 8,1742

(see log book). 


\section{CHAPTER VII}

\section{THE JOURNAL OF CHIRIKOV'S VESSEL, THE "ST. PAUL"}

The second of the two vessels that started on June 4, I74I, from Petropavlovsk on that eventful voyage was the St. Paul, Captain Alexei Chirikov in command. The first-hand records consist of a log book, a daily journal of the voyage, and Captain Chirikov's report to the Admiralty College in St. Petersburg. Of the originals ${ }^{1}$ in the Russian archives, a translation of the journal is given in this chapter and of Captain Chirikov's report in the next chapter. It has not been deemed necessary to publish the log book, as, other than routine navigational information, it contains nothing that is not reported even more fully, in the journal and report. The log book has, however, been used in plotting the track of the St. Paul (see p. 330, foot note I).

\section{Journal Kept on the Ship "St. Paul" in Command of Captain Alexei Chirikov at the Time It Made the Voyage East From the Kamchatka Harbor of the Holy a postles \\ Peter and Paul to the Western Coast of AMERICA IN THE YEAR I74I}

The reckoning begins from the landmark called Vaua at the mouth of Avacha Bay, situated in latitude $52^{\circ} 53^{\prime}$ north, longitude (according to the observation taken recently by Professor of Astronomy Delisle de la Croyère) I $27^{\circ} 55^{\prime}$ from the meridian of St. Petersburg and $175^{\circ} 55^{\prime}$ from the Peak of Teneriffe Island. For our reckoning we take the meridian which runs through Vaua as the first meridian. We reckon in knots, of which there are sixty to the degree. After June 4 we marked on the chart our daily position in latitude and longitude, the rlumb, and the distance from Vaua.

${ }^{1}$ Log book: Archives of the Hydrographic Section of the Ministry of Marine, Petrograd: 1741-43. Nos. 645-648. Another log book, under 1742, No. 1706. Journal: Archives of the Ministry of Marine, Petrograd: Papers of the Admiralty College, i743, No. i6.

Report: Archives of the Ministry of Marine, Petrograd: Papers of Captain Commander Bering, No. 44. 
June 4. $17+1$

From Vaua we sailed on the rhumb $\mathrm{S}_{5} 0^{\circ} \mathrm{E}$, distance 8.5 knots.

June 5, I 741

From Vaua rhumb $\mathrm{S} 68^{\circ} \mathrm{E}$.

Saw the St. Peter, Captain Commander Bering in command, on the rhumb W by $S, 5$ knots distant. We fired a gun as a signal for the Captain Commander to come closer to us since the wind was such that we could not go to him. From us it seemed that the coursc sailed was more southerly than agreed upon. It had been decided by all the officers and by Professor Delisle de la Croyère to sail SE by E true compass. That same hour the Captain Commander acknowledged our signal by firing a gun and stood straight for us.

Although Juan de Gama land is an island, consequently not a part of the American continent, yet we decided on the course we did because our instructions called for an examination of the islands lying on the way to America. It was agreed in case we failed to find land in latitude $46^{\circ} \mathrm{N}$, to sail steadily $\mathrm{E}$ by $\mathrm{N}$ until we met with success. If on either of the courses SE by $\mathrm{E}$ or $\mathrm{E}$ by $\mathrm{N}$ we discovered land it was our plan to coast along it from east to north or north to west, depending on its position, but not to follow it up if it extended between south and east. In that case we were to leave it and go east until we came across [other] land and keep this in sight while likewise going norther$1 y$, to latitude $65^{\circ}$ or at least as far as, with the help of God, the time would permit. If we reached latitude $65^{\circ}$ in good time we planned to sail due west to the Chukchi country and in this way determine the distance between America and Asia and when that was accomplished to return to this harbor. If on the above-mentioned rhumb we should run into hearl winds then we agreed to keep as close to the rhumb as possible until, with God's help, we discovered land, which we would examine according to the instructions of the Captain Commander. In planning the voyage we had to keep in mind that we must return to this larbor towards the end of September. Although we discussed the plan suggested by the Adniralty College, before we left, of sailing (because the distance was supposed to be short) across from the Chukchi land to America, yet we did not do so because it was too early.

\section{June 6, 174 I}

latitule by observation $52^{\circ} \mathrm{oz}^{\prime} \mathrm{N}$; from Vaua. longitude $\mathrm{I}^{\circ} 57^{\prime} 3^{\prime \prime} \mathrm{E}$, rhumb $\$ 54^{\circ} 30^{\prime} \mathrm{E}$, distance 87.9 knots. ${ }^{2}$

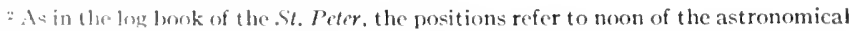
day. For the difference between the civil and the astronomical date, see footune. (). D. 50. In the journal of the St. P'aul, however, the rhumb and distance 
At $1: 30$ in the afternoon the Captain Commander, in answer to our signal mentioned above, came close to our ship. Through the speaking trumpet we told him that we signaled because he was on the rhumb IV by S, 5 knots distant, wind SW by $\mathrm{W}$, and that we ought to keep closer together. If we failed to do so we might become separated in foggy weather and spend valuable time in looking for one another, for we were on the course agreed upon and the wind fair.

The same day the Captain Commander signaled for one of our lieutenants. Captain Chirikov requested that, unless it was an urgent matter. the communication should be made througl the speaking trumpet, for to send a lieutenant necessitated the lowering of a boat and the loss of time. It was replied (from the St. Peter) that if we had any communication of importance to make to the Captain Commander we should send a lieutenant; if we had not we need not send him, and that this was all they wished to tell us. After this the signal was taken down.

\section{June 7,1741}

Latitude $50^{\circ} 55^{\prime}$; from Vaua, longitude $4^{\circ} 26^{\prime} 7^{\prime \prime}$, rhumb $\mathrm{S}_{5} 4^{\circ} 2 \mathrm{I}^{\prime} \mathrm{E}$, distance 201.4 knots.

\section{June 8, I7+I}

Latitude $49^{\circ} 5^{\prime}$; from Vaua, longitude $6^{\circ} 49^{\prime}$, rhumb $\mathrm{S}_{5} 4^{\circ} 4 \mathrm{I}^{\prime} \mathrm{E}$, distance 313 knots.

At the fourth hour in the afternoon the Captain Commander signaled for our ship to take the lead.

\section{June $9,174 I$}

Latitude by observation $48^{\circ}+2^{\prime}$; from Vaua, longitude $9^{\circ} 09^{\prime} 6^{\prime \prime}$, rhumb $\mathrm{S}_{5} 4^{\circ} \mathrm{O}^{\prime} \mathrm{E}$, distance 428.4 knots.

\section{June IO, I74I}

Latitude by observation $47^{\circ} 45^{\prime}$; from Vaua, longitude $10^{\circ} 53^{\prime} 9^{\prime \prime}$, rhumb $\mathrm{S}_{5} 3^{\circ} 33^{\prime} \mathrm{E}$, distance 5 I 8 knots.

At the fifth hour the St. Peter came near us, and we told them that at night we would heave to in order to take soundings and when we did that we would hang a lantern at the ensign staff and that they should keep a lookout for it and not run into us.

from Vaua are reckoned differently from what they are in the log of the St. Peter. The rhumb is expressed as direction within a quadrant instead of in points of the compass, as there (footnote 2, p. 37); the distance, in knots, or nautical miles (bo to a mean degree of latitude), instead of in German miles (15 to a degree). Comparing the entries for June 6 and reducing them to a common term. we get: (1) rhumb: St. Peter, SE by E $3^{\circ} 30^{\prime} \mathrm{E}$, or, in azimuth, $120^{\circ} 15^{\prime}$; St. Paul, S5. $34^{\circ} \mathrm{E}$, or $125^{\circ} 30^{\prime} ;$ (2) distance, St. Peter, $25^{\prime}$ German miles, or ros knots; St. Paul, 87.9 knots. For the track of the St. Paul Pl. I should be consulted throughout. 


\section{June II, I74I}

Latitude $46^{\circ} 58^{\prime}$; from Vaua, longitude $2^{\circ} 38^{\prime} 8^{\prime \prime}$, rhumb $\mathrm{S}_{5}{ }^{\circ} 56^{\prime} \mathrm{E}$, 603 knots.

At the sixth hour Captain Commander signaled to wait for him and to do that we hove to. At 6:30 we talked over with him the latitude, the longitude, and the distance from Vaua. Proceeded on our course. It was understood that if in the nighttime we took soundings and got bottom we should fire a gun and bring to.

\section{June I2, I74I}

Latitude by observation $46^{\circ} \mathrm{O} 5^{\prime}$; from Vaua, longitude $13^{\circ} 35^{\prime} 5^{\prime \prime}$, rhumb $\mathrm{S}_{5} 2^{\circ} 20^{\prime} \mathrm{E}$, distance 667.7 knots.

\section{June I3, 1741}

Latitude $46^{\circ} \mathrm{O} 3^{\prime}$; from Vaua, longitude $14^{\circ} \mathrm{o}^{\prime} \mathrm{I}^{\prime \prime}$, rhumb $\mathrm{S}_{53^{\circ}} \mathrm{oz}{ }^{\prime} \mathrm{E}$. distance 682 knots.

At 3:30 in the afternoon we hove to while the St. Peter came up to us. Lieutenant Waxel, through the speaking trumpet, announced that according to the decision reached by the Captain Commander, the officers, and the Professor of Astronomy, Delisle de la Croyère, it was time to change the rhumb and to sail $\mathrm{E}$ by $\mathrm{N}$ by true compass. From our ship it was replied that Captain Chirikov and his officers agreed to this, since the observations made at noon [recorded under June 12 ] gave our position as latitude $46^{\circ} \mathrm{o5}^{\prime} \mathrm{N}$, rhumb from Vaua $\mathrm{S}_{52}{ }^{\circ} 2 \mathrm{o}^{\prime} \mathrm{E}$. The conversation ended by all agreeing to sail $\mathrm{E}$ by $\mathrm{N}$ true compass. That same hour we looked from the crosstrees for land between $S$ and $W$, also $S$ and $E$, but saw no land. We filled our sails and went on the rhumb agreed upon,

\section{June 14, 174 I}

Latitude $46^{\circ}$ I $8^{\prime}$; from Vaua, longitude $14^{\circ} \mathrm{Io}^{\prime} 7^{\prime \prime}$, rhumb $54^{\circ} 20^{\prime} \mathrm{E}$, distance $677 \mathrm{knots}$

\section{June 15, 174I}

latitude $47^{\circ} 41^{\prime}$; from Vaua, longitude $14^{\circ} 23^{\prime} 4^{\prime \prime}$, rhumb $\mathrm{S} 60^{\circ} 26^{\prime} \mathrm{E}$, distance 632.3 knots.

\section{June 16,1741}

Latitude $47^{\circ} 55^{\prime}$; from Vaua, longitude $14^{\circ} 39^{\prime} I^{\prime \prime}$, rhumb S6I $58^{\prime} \mathrm{E}$, distance $634 . \mathrm{I}$ knots.

At the beginning of the fourth hour in the afternoon we came about and went on a port tack in order to get closer to the Captain Commander, who was from us SE by $\mathrm{S}$, about $31 / 2$ (?) knots away. About the middle 
of the hour we shook out the first reef in the fore-topsail; at the end of the hour we came about on a starboard tack.

At the end of the fif th hour we came up with the St. Peter. We lowered the staysail, clewed up the foresail, and inquired through the speaking trumpet how far north we should go in case this east wind held on and whether a signal would be given us when it was time to put about. In reply we were told that no changes would be made without a signal. At the beginning of the eighth hour the Captain Commander came about and so did we.

\section{June I7, I74I}

Latitude $48^{\circ} 12^{\prime}$; from Vaua, longitude $15^{\circ} 49^{\prime} 5^{\prime \prime}$, rhumb S6 $65^{\circ} \mathrm{O}^{\prime} \mathrm{E}$, distance 665 knots.

\section{June 18,1741}

Latitude $48^{\circ} 53^{\prime}$; from Vaua, longitude $17^{\circ} 30^{\prime} 05^{\prime \prime}$, rhumb $\mathrm{S}_{7} 0^{\circ} 04^{\prime} \mathrm{E}$, distance 70.4 knots.

About the middle of the fif th hour in the morning a gun was fired from the St. Peter as a signal for us to come up. We brought to, since we were to the leeward; and in the meantime the St. Peter drew close to us, and Master Khitrov, speaking through the trumpet, informed us that we were now near $49^{\circ} o^{\prime} \mathrm{N}$, and that the place on the 46th parallel which lies on the agreed course from Vaua is now $\mathrm{S} / 2 \mathrm{~W}$ from us; consequently we should now come about on another tack. I replied that we should [not?] come about with the wind as it stands because we are now sailing NE1/2E true compass, which course is the same as $\mathrm{E}$ by $\mathrm{N}$ from the 46 th parallel, a difference of $21 / 2$ rhumbs. If we came about on another tack we should be sailing SSW $1 / 2 \mathrm{~W}$, a difference of II $1 / 2$ rhumbs, which would lead us far away from our main course. Therefore I suggested that we proceed on the course we are on, making use of the north and west winds, until we had come at least as far as the 53rd parallel. No reply was made to this. We filled our sails and went our way.

\section{June 19,1741}

Latitude $49^{\circ} 3 \mathrm{I}^{\prime}$; from Vaua, longitude $18^{\circ} 36^{\prime} 2^{\prime \prime}$, rhumb $\mathrm{S} 73^{\circ} 53^{\prime} \mathrm{E}$, distance 727.7 knots.

\section{June 20, I74I}

Latitude $48^{\circ} 49^{\prime}$; from Vaua, longitude $17^{\circ} 41^{\prime} 7^{\prime \prime}$, rhumb $669^{\circ} 58^{\prime} \mathrm{E}$, distance 712 knots.

At the third hour in the morning the St. Peter was 10 knots distant; at the fifth hour she began to disappear from sight.

The spot where we last saw the ship was to the north, latitude $48^{\circ} 59^{\prime}$, longitude from Vaua $18^{\circ} \mathrm{O}^{\prime}$, distance Io knots. 


\section{June 2I, I74I}

I atitude by observation $48^{\circ} 27^{\prime}$; from Vaua, longitude $17^{\circ} 29^{\prime}$, rhumb S6 $68^{\circ} \mathrm{IO}^{\prime} \mathrm{E}$, distance $7 \mathrm{I} 6 \mathrm{knots}$.

At the beginning of the ninth hour wind moderated; hoisted mainsail and foresail and proceeded on our course; lowered main-staysail. The signal code which the Captain Commander gave us said that in case we parted we should look for one another at the place where we last saw one another. I gave the order to steer as near as possible for that spot, but this was difficult as it was NNE from us and the wind was dead aliead.

\section{June 22, I74I}

Latitude $48^{\circ} 06^{\prime}$; from Vaua, longitude $\mathrm{I} 8^{\circ} 37^{\prime} 8^{\prime \prime}$, rhumb S6 $7^{\circ} 59^{\prime} \mathrm{E}$, distance 765 knots.

\section{June 23, I74I}

Latitude $48^{\circ} \mathrm{I}^{\prime}$; from Vaua, longitude $20^{\circ} 20^{\prime} 3^{\prime \prime}$, rhumb S70 I $5^{\prime} \mathrm{E}$, distance 823 knots.

At the fifth hour in the morning we gave up looking for the St. Peter and with the assent of all the officers of the St. Paul we went on our way.

\section{June $24,17+1$}

Latitude by observation $48^{\circ} 03^{\prime}$; from Vaua, longitude $23^{\circ} \mathrm{I} 4^{\prime}$, rhumb

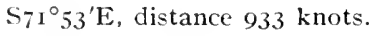

\section{June $25,174 I$}

Latitude $48^{\circ} 22^{\prime}$; from Vaua, longitude $26^{\circ} 24^{\prime} 2^{\prime \prime}$, rhumb $\mathrm{S} 74^{\circ} 54^{\prime} \mathrm{E}$, distance $\mathrm{I}, \mathrm{o}^{\circ} \mathrm{o}$ knots.

\section{Iune 26, I74I}

Latitude by observation $48^{\circ}$ I I' $^{\prime}$; from Vaua, longitude $29^{\circ} \mathrm{I} 4^{\prime}$, rhumb $\mathrm{S} 75^{\circ} 48^{\prime} \mathrm{E}$, distance $\mathrm{I}, \mathrm{I} 49$ knots.

At the beginning of the twelfth hour in the morning we thought we sighted mountains between $\mathrm{N}$ and $\mathrm{NE}$, and to make sure we steered $\mathrm{NE}$ by compass.

$$
\text { June } 27,174 I
$$

Latitude $48^{\circ} 34^{\prime}$; from Vaua, longitude $30^{\circ} 52^{\prime} 4^{\prime \prime}$, rhumb $\mathrm{S} 77^{\circ} 32^{\prime} \mathrm{E}$, distance, I,200 knots.

At the end of the fourth hour in the afternoon we discovered that the supposed mountains were clouds and therefore went back to the regular course.

\section{June $28,17+1$}

Latitude $48^{\circ} 59^{\prime}$; from Vaua, longitude $33^{\circ} \mathrm{o}^{\prime} 5^{\prime \prime}$, rhumb $\mathrm{S} 79^{\circ} 24^{\prime} \mathrm{E}$, distance 1,269 knots. 


\section{June 29, I7.4I}

Latitude $49^{\circ} 22^{\prime}$ I $^{\prime \prime}$; from Vaua, longitude $34^{\circ} 58^{\prime} \mathrm{I}^{\prime \prime}$, rhumb $\mathrm{S} 80^{\circ} 53^{\prime} \mathrm{E}$, distance 1,332 knots.

$$
\text { June } 30,17+71
$$

Latitude $49^{\circ} 47^{\prime}$; from Vaua, longitude $37^{\circ} 06^{\prime} 8^{\prime \prime}$, rhumb S82 $24^{\prime} \mathrm{E}$, distance 1,407 knots.

$$
J u l y I, 17+11
$$

Latitude $50^{\circ} 27^{\prime}$; from Vaua, longitude $40^{\circ} 3 \mathbf{1}^{\prime}$, rhumb $584^{\circ} 28^{\prime} \mathrm{E}$, distance $1,51+$ knots.

$$
\text { July } 2,17+1
$$

Latitude $50^{\circ} 48^{\prime}$; from Vaua, longitude $42^{\circ} \mathrm{o}^{\prime} 4^{\prime \prime}$, rhumb $\mathrm{S} 85^{\circ} 24^{\prime} \mathrm{E}$, distance 1,559 knots.

$$
\text { July } 3,174 I
$$

Latitude $50^{\circ} 50^{\prime}$; from Vaua, longitude $42^{\circ} 06^{\prime}$, rhumb $\mathrm{S} 85^{\circ} 29^{\prime} \mathrm{E}$, distance 1,562 knots.

$$
J u l y+17+1
$$

Latitude $51^{\circ} \mathrm{O}^{\prime}$; from Vaua, longitude $42^{\circ} 40^{\prime}$, rhumb S86 OI'E, distance 1,584 knots.

$$
\text { July } 5,1741
$$

Latitude $5 \mathrm{I}^{\circ} 32^{\prime}$; from V'aua, longitude $45^{\circ}$ I $2^{\prime} 8^{\prime \prime}$, rhumb $887^{\circ} 13^{\prime} \mathrm{E}$, distance 1,668 knots.

$$
\text { July } 6, I 7.7 I
$$

Latitude $5 \mathrm{I}^{\circ} 58^{\prime}$; from Vaua, longitude $47^{\circ} 33^{\prime} \mathrm{I}^{\prime \prime}$, rhumb $\mathrm{S} 88^{\circ}$ I $2^{\prime} \mathrm{E}$, distance $1,75 \mathrm{I}$ knots.

\section{July $7,17+1$}

Latitude $52^{\circ} 10^{\prime}$; from Vaua, longitude $48^{\circ} 36^{\prime}$, rhumb $588^{\circ} 37^{\prime} \mathrm{E}$, distance $1,773 \mathrm{knots}$.

At the eighth hour in the evening we noticed considerable green-looking vegetation afloat, which we thought was grass, and therefore we brought to in order to take soundings. No bottom at roo fathoms.

Examined the vegetation and learned that it was not (sea) grass but a species found in thick water resembling a sea nettle which is washed ashore in large quantities.

$$
\text { July } 8,17+1
$$

Latitude $52^{\circ} 32^{\prime}$; from Vaua, longitude $48^{\circ} 45^{\prime}$, rlumb $\$ 89^{\circ} \mathrm{I} g^{\prime} \mathrm{T}$, distance 1,772 knots.

$$
\text { July } 9,17.4 I
$$

Latitude $52^{\circ} 49^{\prime}$; from Vaua, longitude $49^{\circ} 08^{\prime}$, rhumb $\$ 89^{\circ} 50^{\prime} \mathrm{E}$, distance 1,782 knots. 
July IO, 1741

Latitude $53^{\circ} \mathrm{IO}^{\prime}$; from Vaua, longitude $5 \mathrm{I}^{\circ} \mathrm{I}^{\prime}$, rhumb $\mathrm{N} 89^{\circ} 28^{\prime} \mathrm{E}$, distance $\mathrm{I}, 852$ knots.

$$
\text { July II, I74I }
$$

Latitude $53^{\circ} 2 \mathrm{I}^{\prime}$; from Vaua, longitude $52^{\circ} \mathrm{o4}^{\prime}$, rhumb $\mathrm{N} 89^{\circ} \mathrm{og} 9^{\prime} \mathrm{E}$, distance $\mathbf{I}, 874$ knots.

$$
\text { July } 12,1741
$$

Latitude by observation $54^{\circ} 27^{\prime}$; from Vaua, longitude $55^{\circ} \mathrm{I} 6^{\prime}$, rhumb $\wedge 87^{\circ} 15^{\prime} \mathrm{E}$, distance 1,959 knots.

Saw a shore duck today.

$$
\text { July } 13,174 I
$$

Latitude by observation $54^{\circ} 4 \mathrm{I}^{\prime}$; from Vaua, longitude $58^{\circ} 03^{\prime} 5^{\prime \prime}$, rlumb N86 $59^{\prime} \mathrm{E}$, distance 2,053 knots.

Saw a shore duck, a gull, and two old floating trees.

$$
\text { July } 14,1741
$$

Latitude $55^{\circ} 09^{\prime} 6^{\prime \prime}$; from Vaua, longitude $60^{\circ} 41^{\prime}$, rhumb N86 $21^{\prime} \mathrm{E}$, distance 2,145 knots.

At the beginning of the first hour in the morning we noticed near the ship three wide and long strips of very white water. We brought to and took soundings, but no bottom at $\mathrm{s} o 0$ fathoms. We drifted into the first strip, which was about half a verst long and 200 fathoms wide, and later into the other strips, which were smaller. The color of the water was different, possibly owing to the movement of a school of fish; but we do not know for certain the cause of it. As the night was dark and foggy we were afraid of running ashore and therefore during the second and third hours we sailed by the wind on the course $\mathrm{W}$ by $\mathrm{N}$ by the compass.

At the third hour in the morning we saw a large number of shore ducks, gulls, a whale, porpoises, and three medium-sized pieces of driftwood that had been in the water for some time.

$$
\text { July } 15,174 I
$$

Latitude $55^{\circ} 36^{\prime} 3^{\prime \prime}$; from Vaua, longitude $61^{\circ} 51^{\prime}$, rhumb $N 85^{\circ} 42^{\prime} \mathrm{E}$, distance 2,178 knots.

At the beginning of the fourth hour in the morning [i.e., 3 A. M.] we sighted land, took its bearings, and went by the wind on a left tack. Shook out reef in fore-topsail. At the ninth hour took soundings and at 60 fathoms got bottom of gray sand. The parts of the shore which we saw today are quite mountainous. We took a number of soundings within three to five knots from shore and got nowhere less than 60 fathoms, bottom gray 


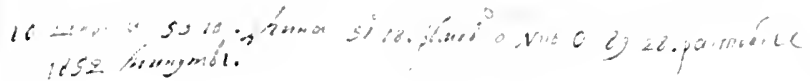

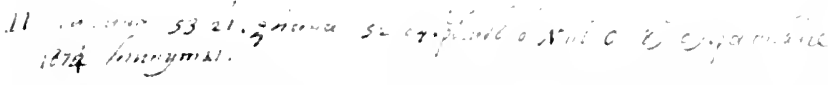

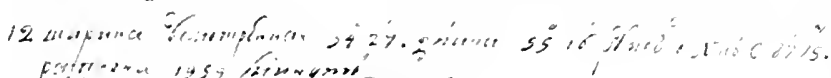

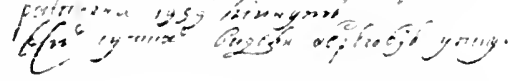

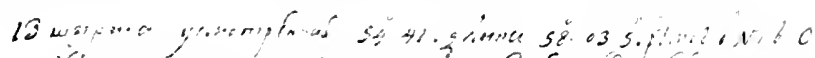
if

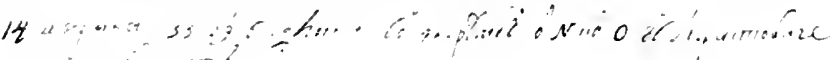

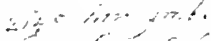

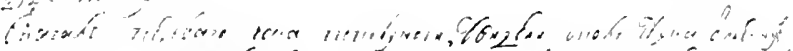

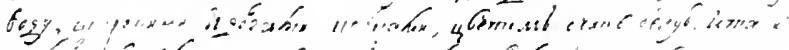

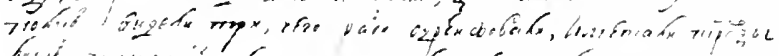

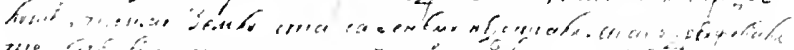

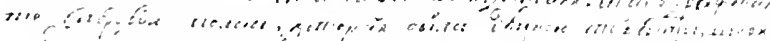

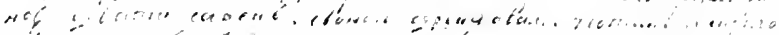

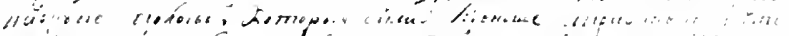

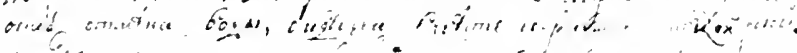

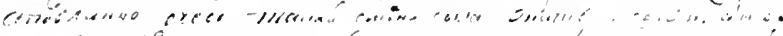

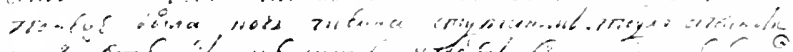

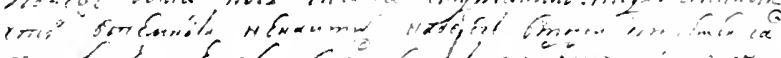

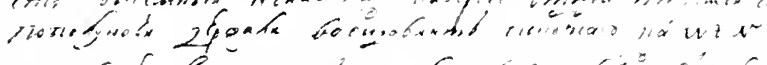

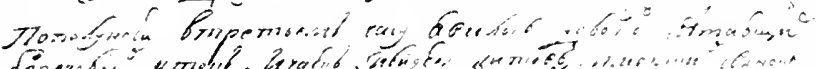

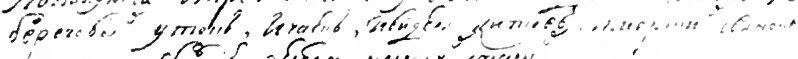

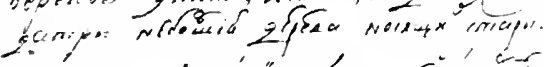

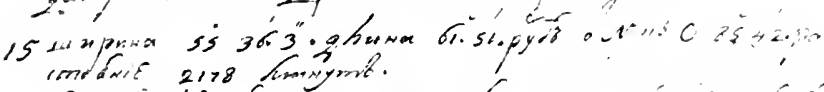

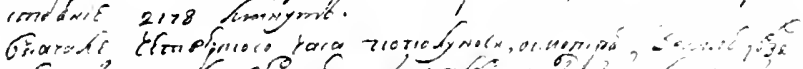

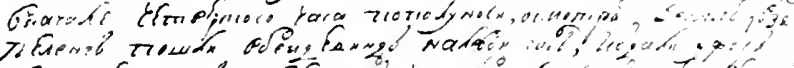

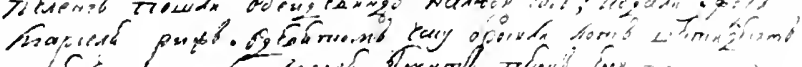

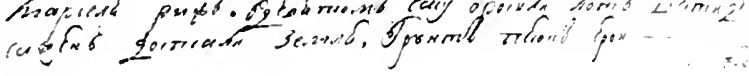

FIf. I3-Tacsimile of a page of the journal of the St. Paul: entries of July 10-It and first part of July $15,17+1$, the last recording the discovery of land (for text, see orposite page). 

sand and in places small rocks. Many different kinds of ducks tlew past our ship.

At two in the morning we distinguished some very high mountains and, as the light at the time was not very good, we brouglit to. An hour later the land stood out much better and we could make out trees. This must be America, judging by the latitude and the longitude, for we were at that lour in latitude $55^{\circ} 2 \mathrm{I}^{\prime} \mathrm{N}$ and longitude from Vaua $6 \mathrm{I}^{\circ} 55^{\prime}$. From this place we took the following bearings according to the corrected compass: in the soutl the land seemed to end in $\mathrm{S}_{3} 6^{\circ}{ }_{19^{\prime}} \mathrm{E}$, distant about 30 knots; the shore nearest to us had three projections in $\mathrm{N}_{72}{ }^{\circ} 4 \mathrm{I}^{\prime} \mathrm{E}$; the cape ${ }^{3}$ ending in the north bore $\mathrm{N}_{19}{ }^{\circ}+1^{\prime} \mathrm{E}$. From the third hour proceeded $\mathrm{N} 3 / 4 \mathrm{~W} 8$ knots. At 5:30 in the morning the three projections were on the rhumb, $\mathrm{S}_{70^{\circ}} 19^{\prime} \mathrm{E}$; the cape which seemed to end in the north was on the rhumb

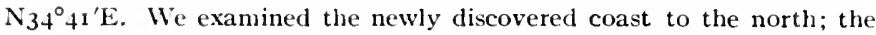
low cape was on the rhumb $\mathrm{N} 39^{\circ} 22^{\prime} \mathrm{E}$; at 8:30 in the morning the coast seemed to end in $\mathrm{N}_{3} 3^{\circ} \mathrm{o}$ 'W. ${ }^{4}$

\section{July $16,174 I$}

Latitude by observation $56^{\circ} 15^{\prime}$; from Vaua, longitude $60^{\circ} 57^{\prime} 2^{\prime \prime}$. rhumb $\mathrm{N} 84^{\circ} 48^{\prime} \mathrm{E}$, distance 2,140 knots.

At the fourth hour in the afternoon we lowered a boat and sent boatswain Trubitsin and eight men to the bay sighted an hour and a half ago. When the reached there he had orders to take soundings and to examine it to see whether it offered good anchorage and protection from the winds. He was told to keep an eye on our ensign flagstaff and to listen for guns signaling for him to return to the ship. At 6:30 in the afternoon we saw our boat near the shore; we came about so as to get closer to it and hove to.

At the beginning of the eighth hour the boat came back and we took it in tow and stood off. Trubitsin reported that he took soundings within 60 fathoms of the cape and got 40 fathoms and that the bay was unprotected from south and west winds. He said also that he saw large fir, spruce, and pine trees on the beach, many sea lions on the rocks, but no sign of liuman beings or their habitations. At the seventh hour in the morning there was an endless flight of large flocks of birds past the ship, the same species that are found in Kamchatka and known as aru and urili [murres and cormorants].

At noon we were from seven to three knots from shore, depth of water

3 Cape Addington. (Identifications in this and the next chapter by E. P. B.)

4 Coronation lsland.- - The lack of chronological sequence in the entry under July 15 may be due to the fact that the first paragraph is a general summary of the events of the day, while the second paragraph takes them up in detail. To this may also be due the se'ming discrepancy in the hour when land was first sighted. At $2 \mathrm{~A}$. M. the mountain tops were first seen; at $3 \mathrm{~A}$. M. the coast itself became visible.-EDit. NOTE.] 
from 60 to 62 fathoms. Observed many ducks and gulls of different species, also sea animals-whales, sea lions, and walrus.

At the ninth hour in the morning the cape to which Trubitsin was sent

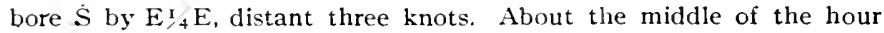
[ninth] we sighted on the rhumb E by $\mathrm{S} / 4 \mathrm{E}$, distant six knots, three small islet $s^{5}$ close to one another and right up against the shore or very close to it, for we could not see clearly because of the fog. At end of the tenth hour land ${ }^{6}$ loomed up through the fog on the rhumb $\mathrm{N}$ by $\mathrm{E} 3 / 4 \mathrm{E}$ or almost ENE, but the fog came down again and hid it. We took soundings, no bottom. Proceeded on our course.

At noon ${ }^{7}$ we caught sight through the fog of a very mountainous land on the rhumb NI/4E, distant not more than three knots, which stretched from $\mathrm{NWW}_{/ 4} \mathrm{~W}$ to $\mathrm{SE} / 4 \mathrm{E}$. We sailed parallel to it, keeping it on the east from three to five knots.

$$
\text { July } I 7,1741
$$

Latitude by observation $57^{\circ} 39^{\prime}$; from Vaua, longitude $58^{\circ} 54^{\prime} 2$ ", rhumb $\$ 82^{\circ}+3^{\prime} \mathrm{E}$, distance 2,059 knots.

About the middle of the sixth hour in the afternoon the fog began to clear and we caught sight of the coast parallel to which we were running. At the seventh hour we took soundings, depth 75 fathoms. At the tenth hour in the morning lowered the boat for the purpose of sending it ashore. From the first to the ninth hour in the afternoon we went parallel to the shore, having it to the east of us about three or four knots distant. The land was full of mountains, some covered with snow and all with trees. At the eighth hour in the evening the coast seemed to end ${ }^{8}$ in $\mathrm{N} 3 / 4 \mathrm{E}$, distant 8 knots; and in its place appeared low land with sea cliffs, with the high mountains receding in the background. At the ninth hour in the morning the coast parallel to which we were sailing ended ${ }^{9}$ in $\mathrm{NNW}_{1 / 2} \mathrm{~W}$, distant 30 knots. Behind us there was a capelo in $\mathrm{SE}$ by $\mathrm{S} / 4 \mathrm{E}$, distant 45 knots.

At noon ${ }^{11}$ the cape which we saw at the ninth hour in NNWI/4 [sic], distant 30 knots, is now on that same rhumb, distant 19 knots.

$$
\text { July IS, } 1741
$$

At $3: 30$ in the afternoon we went as close to the shore as we dared. We sent the boat ashore in charge of Fleet Master Dementiev who had with him ten armed men. He took with him a hand compass, a small lead, two empty water casks, a grapnel, and a cable. He had written

SHazy Islands.

${ }^{6}$ Cape Ommaney.

'Close to Puftin Point, just north of Cape Ommaney.

t The south shores of Sitka Sound.

9 Cape Cross.

${ }^{10}$ Cape Edgecumbe.

"At noon the St. Paul was off Cape Edward. 
orders; and among other things he was told to make for the opening "? which seemed to $u$ s a bay and to take its bearings. The position of the bay and other details relating to it are recorded in their places so that it can be more easily identified.

At the eighth hour in the afternoon we sighted a low shore in $\mathrm{N}$ by E $3 / 4 \mathrm{E}$, distant 7 knots, which stretched to SE and SE by E. The bearings of the place into which the boat was sent we did not see.

At the beginning of the fifth hour in the afternoon the second mountain, behind which is another broad mountain covered with snow, is in $N \mathrm{E} 3 / 4 \mathrm{E}$; the bay into which the boat was orkered bears $N N E 3 / 4 \mathrm{E}$, distant 5 knots. There is still wnother mountain with a sharp peak, somewhat crooked on the right side; and, when you look at it, it is in ENE ${ }_{4} \mathrm{E}$; opposite, or a little south of it, is a small island or a piece of rather low land covered with medium-sized trees. North of this island and opposite the bay were seen in places projections of bare rocks.

At the end of the day we saw high snow-covered mountains extending to the northward and ending in NV by N.

According to the reckoning at $4: 30$ in the afternoon, taking into consideration the bearing of the bay, we set down the position of the bay into which the boat was sent as latitude $57^{\circ} 23^{\prime} \mathrm{N}$, longitude from Vaua $59^{\circ} 36^{\prime}$, distance 2,059 knots, rhumb N82 ${ }^{\circ} 28^{\prime} \mathrm{E}$; by correction the latitude is $57^{\circ} 50^{\prime}$, longitude $58^{\circ} 54^{\prime}$.

From this place we again started a new reckoning, because for some time after July 17 we beat up and down the bay so as not to get too far from it; but the strong winds and tides carried us out 20,30 , and more knots.

\section{July $19,174 I$}

At the third hour in the afternoon took the bearings cf a round, forested volcano; below it was a low, thick, dark green forest on a white cliff in $\mathrm{NE} / 4 \mathrm{E}$; the white cliff was distant about 6 knots. Another volcano to the left of it and smaller was in NE by $\mathrm{N} / 4 \mathrm{E}$, some distance from shore; a high bare cape covered with a clump of high trees in $\operatorname{SSE} 1 / 4 \mathrm{E}$, distant I 2 knots.

At the middle of the fifth hour in the afternoon we saw a mountain in $\mathrm{NE}$ by $\mathrm{N}_{4}^{1} \mathrm{E}$, distant 30 knots, another in NNE $3 / 4 \mathrm{E}$, distant 32 knots; after this we saw no more land to the north beyond the high mountain which is in $\mathrm{N}^{2}+\mathrm{V}$, distant 30 knots; then the mountain was $\mathrm{N}$ by $\mathrm{WV} 3 / 4 \mathrm{~W}$, distant fo knots. From this hour we observed lower ground through the cape in $\mathrm{E}$ by $\mathrm{S}_{4} \mathrm{E}$, opposite which we supposed our boat had gone into the bay.

12 The St. Paul was off Lisianski Strait. 
At the eighth hour in the afternoon we saw a high bare cape which at the third hour bore $\mathrm{SSE} / 4 \mathrm{E}$, distant $\mathrm{I} 2$ knots; now it bears $\mathrm{SE}$ by $\mathrm{S} 1 / 4 \mathrm{E}$, 15 knots.

At the fourth hour in the morning we saw that the small far-away cape which at the eighth hour bore $\mathrm{SE}$ by $\mathrm{S} / \mathrm{E}$ now bears $\mathrm{SE} 1 / 4 \mathrm{E}$, distant 25 knots; the mountain which at the fifth hour bore $\mathrm{N}$ by E1/4 E, 24 knots, is now NE3/4E, 12 knots. Now the mountains extend N1/4 W.

\section{July $20,174 I$}

During the twenty-four hours the wind shifted about; it was foggy, cloudy, rainy. The heavy wind and rain carried us away from the place where the boat had gone and from which it had not yet returned.

$$
\text { July } 2 I, I 74 I
$$

Boat has not yet returned. We worked hard to come closer to the place to which it had gone.

$$
\text { July 22, } 1741
$$

No boat. We went near the place into which it had been ordered.

$$
\text { July 23, } 174 I
$$

From about the first hour in the afternoon we kept parallel to the coast and, according to our opinion, close to the place where the boat went; but owing to the heavy fog we could not identify the landmarks.

At the second hour we thought we were opposite the exact spot where the boat was, and to summon it we fired two guns. Depth of water 7o, 75 , and 80 fathoms; bottom of sand and large rocks.

At the fourth hour the fog lifted a little and we noticed snoke on the beach $\mathrm{E}$ by $\mathrm{N} / 4 \mathrm{E}, 5$ knots, and we supposed that it was made by our men because during all the time that we followed the shore we saw no human habitations, no boats, and no fires. Took soundings and got 80 fathoms.

At the seventh hour wind very light, and the air clear from fog, and the landmarks of the place into which the boat was sent stood out. The fire burned in the very place into which the boat went, and we took it for granted that it was kept up by our men, and we fired seven times at intervals as a signal for them to come out; but no boat appeared, although the weather was fair. We noticed, however, that after we signallect the fire on the beach grew bigger.

At the tenth hour in the evening we hung out a lantern at the ensign staff so that the boat might see us if it came out.

$M$ idnight. The fire on the beach bore ENE $/ 4 \mathrm{E}$.

At the ninth hour in the morning no fire on shore; half hour later a little smoke in $\mathrm{ENE} 3 \frac{3}{4}$, and then it again became invisible. 
At the eleventh hour a walrus swam past the ship. At the middle of the twelfth hour lowered the small boat for the purpose of sending it ashore to help the men in the large boat.

Noon. The place into which the boat was sent bore ENE1/2E, 9 bnots.

No boat, although we sailed close up to the place into which it went.

$$
\text { July 24, I74I }
$$

The weather was fair enough for a considerable length of time for the boat to come out; since it did not appear we concluded that it must be damaged and could not. All the officers, higher and lower, were of the opinion (which they put in writing) that it was necessary to find out what was the matter with the boat and for that purpose to send, while the sea was quiet. the small boat with a carpenter and a calker with the necessary tools to repair the boat, as well as provisions, since those ashore had only enough for a week. Boatswain Sidor Savelev volunteered to take the mechanics ashore, and the sailor Sidor Fadiev, who expressed a desire to go along, was detailed to accompany them. The carpenter's name was Nariazhev Polkovnikov and the calker's was Gorin. They left at one o'clock in the afternoon, and we, having a light wind, followed them to shore. The boatswain had orders that as soon as he landed he should build a fire to notify us of his arrival and to signal the condition of the men and the boat. He was ordered to leave ashore the carpenter and the calker to repair the boat, but he himself was to return without delay to the ship with Dementiev and as many others as he could accommodate. We sailed up quite close to the land, on which a heavy sea was running, so that we had difficulty in getting away, for the sails made little headway against the sea. WVe saw the boatswain near the shore in the sixth hour of the afternoon; but he made no signals and, although the weather was fair, did not come back.

In the fifth hour we brought to in order to wait for the two boats; we watehed for signals but in vain; we took soundings and got 65 fathoms and a bottom of big rocks. For two or three versts from shore the water was 70,65 , and 60 fathoms, and the bottom bad. Many rocks were seen both under and above water on which the surf was playing. For these reasons we could not anchor.

At the seventh hour we came about and moved away from shore because we had no signals from the boatswain and the men.

At the ninth hour we steered for the shore to watch for signals. Half an hour later we were so close to shore that we could see the rocks and the surf playing on them, but no signals. Because it was night we decided to go a little farther from land. The place where the two boats went bore NE3/4 E. 3 knots. We fired a gun as a summons to the men ashore; at the time there was hardly any wind, the ship was making almost no 
headway, and those ashore had the weather in their favor for coming out. As we fired from our cannon we thought we saw some one on shore shooting a gun, but we heard no noise. We observed also a fire on the beach as if in answer to us. We fired another gun.

At the ninth hour in the evening we observed fire on the beach. Took soundings, 66 fathoms; very little wind. We hung out two lanterns, one at the ensign staff and the other at the gaff. The fire on the beach appeared and disappeared by turns.

At the first hour in the afternoon we saw the fire.

At the third hour we fired guns to summon the boats.

At the fourth hour we fired guns.

At the tenth, eleventh, and twelfth hours we tacked close to the place into which the boats had gone. Our position is latitude $57^{\circ} 5 \mathrm{I}^{\prime} \mathrm{N}$.

$$
\text { July } 25, I 7+1
$$

Latitude by observation $57^{\circ} 50^{\prime} 6^{\prime \prime}$; from l'aua, longitude $57^{\circ} 10^{\prime} 2^{\prime \prime}$, rhumb $N 82^{\circ} 35^{\prime} \mathrm{E}$, distance 2,007 knots.

First hour. We noticed two boats rowing from the bay into which our boats had gone; one of them was large, and the other small. We naturally thought they were our boats, and we stood towards them. There was very little wind stirring, and I ordered that the sails be taken in and the shrouds tightened[?]. When the small boat drew close to us we became aware that it was not our boat, for it had a sharp bow, and that those in it did not row with oars but paddled. The boat did not, however, come near enough so that we could see the faces of those in it. All that we did see was that it contained four persons, one at the stern and the others at the paddle. One of them had on clothes of red material. Being that far away they stood up and shouted twice "Agai, Agai," waved their hands, and turned back to shore. I commanded my men to wave white kerchiefs and to invite those in the boat to come to our ship. Many of my men did that but it did no good; the boat proceeded on its way to shore. We could not pursue them because in the first place we had no wind, in the second place the small boat went very fast, and the large one had stopped a considerable distance from us. They continued to pull away and finally disappeared in the bay from which they had come. We then became convinced that some misfortune had happened to our men, ${ }^{12 a}$ for it was the eighth day since the Fleet Master lad left; during that period there was plenty of fine weather for returning, and we had sailed quite close to the place, and yet he did not appear. Since the boatswain had gone we had not been away from the place, the weather had been fair, and if a misfortune had not overtaken them they would have returned. The fact that the Americans did not dare to approach our ship leads us to believe that they have either killed or detained our men. We reto a see note on p. 311 . 
mained close to the place until evening, hoping that our boats would come out. Towards evening, however, we had to keep away from shore because of the danger.

At the fourth hour in the afternoon we came about and steered for shore where le observed a fire, also two boats which came out and, after keeping close to land, put back and disappeared. This is the last of boats and signals that we saw, except on the spot where we formerly observed fire we now had smoke.

Ninth lour in the afternoon. Because of the night we took a reef in the topsails. There were neither signals nor fires on shore. We hung out a lantern on the ensign staff so that, if, against all hope, our boats should come, they could find us.

This day we began again our reckoning. The bay into which the boats were sent is in latitude $57^{\circ} 50^{\prime} \mathrm{N}$, longitude $58^{\circ} 54^{\prime}$.

\section{July $26,17+1$}

Latitude by observation $58^{\circ} 21^{\prime} 5^{\prime \prime}$; from Yaua, longitude $57^{\circ} 7^{\prime}$. rhumb $\mathrm{N} 80^{\circ}+2^{\prime} \mathrm{E}$, distance $\mathrm{I}, 954$ knots.

At the fourth hour in the afternoon we saw large quantities of grass, the kind that grows near the beach.

At noon we saw high, snow-covered mountains ${ }^{13}$ on the land ending in the north, bearing $\mathrm{N}^{3} / 4 \mathrm{E}$, to knots.

\section{July $27,174 I$}

Latitude by observation $58^{\circ} 37^{\prime} 3^{\prime \prime}$; from Vaua, longitude $54^{\circ} \mathrm{II}^{\prime} 4^{\prime \prime}$, rhumb $\times 180^{\circ} 23^{\prime} \mathrm{E}$, distance 1,862 knots.

At the first hour in the afternoon Captain Chirikov, Lieutenants Chikhachev and V'range, Navigators Michacl Plautin and Elagin came to the following decision, which they put in writing: Owing to the fact that there were no small boats by the help of which the shore could be examined and water brought aboard (of which there were only 45 casks left) it would be better to go straight to the Harbor of St. Peter and St. Paul, in Avacha Bay in eastern Kamchatka.

Ninth hour. Wind strong; cloudy, rainy. Ordered the crew to catch the rain water from the sails for drinking purposes, for even before this the men made use of it without being obliged to do so, and no harm came to them. Considering the distance we were from port the amount of water on hand was far too little, and therefore I commanded that it should be rationed out to the crew.

$$
\text { July } 28,17+I
$$

Latitude by observation $58^{\circ} 54^{\prime}$; from Vaua, longitude $52^{\circ} 57^{\prime}$, rhumb $\mathrm{N}_{7} 8^{\circ}+7^{\prime} \mathrm{E}$, distance $\mathrm{I}, 815$ knots.

${ }^{13}$ Mt. Fairweather. 
July 29, I74I

Latitude $59^{\circ} 05^{\prime} 6^{\prime \prime}$; from Vaua, longitude $51^{\circ} 38^{\prime} 4^{\prime \prime}$, rhumb $N_{77^{\circ}} 52^{\prime} \mathrm{E}$, distance 1,773 knots.

During the day we saw many shore ducks and gulls, which indicated that there is land not far from us to the north; but owing to the fog and the gloomy weather it could not be seen.

$$
\text { July } 30,1741
$$

Latitude $58^{\circ} 25^{\prime}$; from Vaua, longitude $47^{\circ} 3^{\prime}$, rhumb $\mathrm{N}_{7} 8^{\circ} 19^{\prime} \mathrm{E}$, distance 1,640 knots.

Saw one shore duck.

July $3 I, I 74 I$

Latitude $58^{\circ} 38^{\prime} 7^{\prime \prime}$; from Vaua, longitude $45^{\circ} 52^{\prime}$, rhumb $N 77^{\circ} 24^{\prime} \mathrm{E}$, distance $I, 587$ knots.

During the day we saw many shore ducks and gulls, a little sea cabbage, water of greenish color-all of which go to show that land is not far from us to the north. The reason we did not see it was due either to the atmosphere or to the lowness of the shore.

\section{A ugust I, I74I}

Latitude by observation $58^{\circ} 46^{\prime}$; from Vaua, longitude $45^{\circ} 21^{\prime}$, rhumb $\mathrm{N} 76^{\circ} 58^{\prime} \mathrm{E}$, distance 1,565 knots.

At the fifth hour sighted land in N by W, 30 knots; it ended NNW about 18 knots. A walrus dived near the ship.

At noon land ended ${ }^{14}$ in WNW $1 / 2 \mathrm{~W}, 50$ knots; northern ending $\mathrm{N}$ by $\mathrm{E} 1 / 2 \mathrm{E}$, about 30 knots; middle of it $\mathrm{NNW} 1 / 2 \mathrm{~W}, 20$ knots. There were high, snow-covered mountains on the land. During the day flocks of shore ducks and gulls were seen on the wing.

$$
\text { Augusı 2, I741 }
$$

Latitude $58^{\circ} 24^{\prime}$; from Vaua, longitude $45^{\circ} 12^{\prime}$, rhumb $N_{77^{\circ}} 47^{\prime} \mathrm{E}$, distance $\mathrm{r}, 564$ knots.

At noon the land in the west seemed to end in $\mathrm{W}, 65 \mathrm{knots}$, with high, snow-covered mountains. ${ }^{15}$ Saw flying ducks and gulls.

\section{A ugust 3, I74I}

Latitude by observation $57^{\circ} 37^{\prime}$; from Vaua, longitude $44^{\circ} 49^{\prime}$, rhumb N79 $29^{\prime} \mathrm{E}$, distance $1,556 \mathrm{knots}$.

During the day many flocks of gulls flew over us, also red-billed ducks and another species with white bellies and white under wings. Sandpipers (kuliki) came on board, sea animals swam alongside of us.

14 Cape Elizabeth, the southwestern end of Kenai Peninsula.

${ }^{15}$ Probably northern end of Afognak Island, with Mt. Douglas back of it on the mainland. 
A ugust 4, I74I

Latitude $57^{\circ} 10^{\prime}$; from Vaua, longitude $45^{\circ} \mathrm{o}^{\prime}$, thumb $\mathrm{N} 80^{\circ} 34^{\prime} \mathrm{E}$, distance 1,575 knots.

Saw many whales.

August 5, I74I

Latitude $56^{\circ} 49^{\prime} 4^{\prime \prime}$; from Vaua, longitude $43^{\circ} 41^{\prime}$, rhumb N8 $\mathrm{r}^{\circ} \mathrm{O} 5^{\prime} \mathbf{E}$, distance 1,525 knots.

Numerous flocks of birds were in the air, the color of the water was not altogether like the sea, and therefore we concluded that land must be near, hidden by the fog.

August 6, I74I

Latitude $56^{\circ} 32^{\prime} 4^{\prime \prime}$; from Vaua, longitude $44^{\circ} \mathrm{I}^{\prime}$, rhumb N8 $\mathbf{1}^{\circ} 5 \mathrm{I}^{\prime} \mathrm{E}$, distance 1,542 knots.

Did not see land.

August 7, I74I

Latitude $56^{\circ} 3 \mathrm{I}^{\prime} 4^{\prime \prime}$; from Vaua, longitude $44^{\circ} 20^{\prime}$, rhumb N8 $\mathrm{I}^{\circ} 55^{\prime} \mathrm{E}$, distance 1,533 knots.

August 8, 174I

Latitude $55^{\circ} 57^{\prime} 7^{\prime \prime}$; from Vaua, longitude $44^{\circ} 32^{\prime}$, rhumb $\mathrm{N} 83^{\circ} 13^{\prime} \mathrm{E}$, distance 1,564 knots.

August 9, I74I

Latitude $55^{\circ} \mathrm{o}^{\prime} 7^{\prime \prime}$; from Vaua, longitude $44^{\circ} \mathrm{o} 3^{\prime}$, rhumb N85 $15^{\prime} \mathrm{E}$, distance $\mathrm{I}, 554^{\circ}$ knots.

$$
\text { August 10, I74I }
$$

Latitude by observation $54^{\circ} \mathrm{or}^{\prime}$; from Vaua, longitude $42^{\circ} 2 \mathrm{I}^{\prime}$, rhumb $\mathrm{N} 87^{\circ} 20^{\prime} \mathrm{E}$, distance $\mathrm{I}, 513$ knots.

\section{A ugust II, 1741}

Latitude $53^{\circ} 20^{\prime}$; from Vaua, longitude $41^{\circ} 25^{\prime}$, rhumb N88 $58^{\prime} \mathrm{E}$, distance 1,492 knots.

Saw many whales near the ship and in the distance.

A ugust 12, 1741

Latitude $53^{\circ} \mathrm{o} 8^{\prime}$; from Vaua, longitude $40^{\circ} 52^{\prime}$, rhumb from $\mathrm{N} 89^{\circ} 25^{\prime} \mathrm{E}$, distance $\mathrm{I}, 475$ knots.

Saw many whales near the ship and in the distance.

A ugust 13, 174I

Iatitude $53^{\circ} \mathrm{I}^{\prime}$; from Vaua, longitude $40^{\circ} 23^{\prime}$, rhumb $N 89^{\circ} 06^{\prime} \mathrm{E}$, distance $1,45^{6}$ knots. 
August If, I7. I

Latitude $52^{\circ} 32^{\prime} 4^{\prime \prime}$; from Vaua, longitude $39^{\circ} 30^{\prime}$, rhumb $S 89^{\circ} 1 \mathbf{I}^{\prime} \mathrm{E}$, distance 1,436 knots.

$$
\text { A ugust 15, I7 } 1 \text { I }
$$

Latitude $52^{\circ} 24^{\prime}$; from Vaua, longitude $38^{\circ} 37^{\prime}$, rhumb $\mathrm{S} 88^{\circ} 49^{\prime} \mathrm{E}$, distance $I, 418$ knots.

Saw one shore duck-the kind that iives on the rocks.

August 16, 174I

Latitude $52^{\circ} 31^{\prime}$; from Vaua, longitude $38^{\circ} 43^{\prime}$, rhumb $\mathrm{S} 89^{\circ} \mathrm{O} 6^{\prime} \mathrm{E}$, distance 1,408 knots.

Saw three shore gulls, which looked like sea terns, and one fur seal that dived near the ship.

\section{August I7, 1741}

Latitude $53^{\circ} 10^{\prime}$; from Vaua, longitude $37^{\circ} 56^{\prime}$, rhumb $N 89^{\circ} I 7^{\prime} \mathrm{E}$, distance 1,369 knots.

Saw one large shore duck.

\section{August 18,1741}

Latitude by observation $52^{\circ} 30^{\prime}$; from Vaua, longitude $37^{\circ} 32^{\prime} 4^{\prime \prime}$, rhumb $\mathrm{S} 89^{\circ} \mathrm{O} 2^{\prime} \mathrm{E}$, distance $\mathrm{I}, 363$ knots.

\section{August I9, I74I}

Latitude $52^{\circ}$ I $5^{\prime}$; from Vana, longitude $36^{\circ} 39^{\prime} \mathrm{I}^{\prime \prime}$, rhumb S88 $22^{\prime} \mathrm{E}$. distance 1,337 knots.

$$
\text { August 20, I7+I }
$$

Latitude $52^{\circ}$ or'; from Vaua, longitude $35^{\circ} 43^{\prime}$, rhumb $587^{\circ} 43^{\prime} \mathrm{E}$, listance 1,306 knots.

Saw one shore duck and one fur seal which dived near the ship.

A ugust 21, 1741

Latitude $51^{\circ} 35^{\prime}$; from Vaua, longitude $34^{\circ} 50^{\prime}$, rhumb $\mathrm{S} 86^{\circ} 3 \mathrm{I}^{\prime} \mathrm{E}$, distance 1,284 knots.

Saw two shore gulls.

$$
\text { August 22, } 1741
$$

Latitude $52^{\circ} 16^{\prime}$, from Vaua, longitude $34^{\circ} 42^{\prime}$, rhumb $\$ 88^{\circ} 20^{\prime} \mathrm{E}$, distance 1,266 knots.

Saw one large shore gull and a large root of sea cabbage. 
August 23, 174t

Latitude $52^{\circ} 15^{\prime}$; from Vaua, longitude $34^{\circ} 23^{\prime}$, rhumb $S 88^{\circ} 16^{\prime} \mathrm{E}$, distance 1,254 knots.

Saw a shore duck and floating grass.

August 24, I7+I

I.atitude $52^{\circ} 23^{\prime} 6^{\prime \prime}$; from Vaua, longitude $33^{\circ} 42^{\prime}$, rhumb $\mathrm{S} 89^{\circ} 2 \mathrm{I}^{\prime} \mathrm{E}$, distance 1,228 knots.

Saw floating green grass-the kind that grows near shore.

\section{August 25, 1741}

Latitude $52^{\circ} \mathrm{o}^{\prime}$; from Vaua, longitude $34^{\circ} 20^{\prime}$, rhumb $\mathrm{S} 87^{\circ} 35^{\prime} \mathrm{E}$, distance 1,256 kilots.

Saw plenty of floating grass near the ship.

$$
\text { August 26, I74I }
$$

latiturle $5 \mathrm{r}^{\circ} 5^{8^{\prime}}$; from Vaua, longitude $34^{\circ} 26^{\prime}$, rhumb $\mathrm{S} 87^{\circ} 30^{\prime} \mathrm{E}$, distance 1,260 knots.

$$
4 \text { ugust } 27,1741
$$

Latitude $52^{\circ} \mathrm{O} 3^{\prime}$; fron Vaua, longitude $34^{\circ} 33^{\prime} 2^{\prime \prime}$, rhumb S87 $45^{\prime} \mathrm{E}$, distance 1,263 knots.

$$
\text { August 28, I74I }
$$

Latitude $51^{\circ} 48^{\prime}$; from Vaua, longitude $34^{\circ} 15^{\prime}$, rhumb $887^{\circ} 02^{\prime} \mathrm{E}$, distance 1,256 knots.

Saw about ten white shore gulls.

$$
\text { August } 29,1741
$$

Latitude $52^{\circ} 05^{\prime} 9^{\prime \prime}$; fron laua, longitude $33^{\circ} 55^{\circ}$, rhumb $\mathrm{S} 87^{\circ} 5 \mathrm{O}^{\prime} \mathrm{E}$, clistance $I, 239$ knots.

$$
\text { August } 30,174 t
$$

Latitude by observation $52^{\circ} 21^{\prime}$; from Vaua, longitude $33^{\circ} 52^{\prime}$, rhumb $\mathrm{S} 88^{\circ} 3 \mathrm{I}^{\prime} \mathrm{E}$, distance 1,233 knots.

\section{A ugusi 3I, I74I}

Latitude $52^{\circ} 36^{\prime}$; from V'aua, longitude $31^{\circ} 18^{\prime}$, rhumb $\mathrm{S} 89^{\circ} \mathrm{og} 9^{\prime} \mathrm{E}$, distance 1,138 knots.

An old, somewhat dried-up tree floated past us; we saw also one redbilled duct.

\section{September $I, I 74 I$}

Iatitude $52^{\circ} 52^{\prime}$; from Vaua, longitude $26^{\circ} 39^{\prime}$, rhumb E, distance 965.3 knots. 
September 2, 17.1I

Latitude $52^{\circ} 47^{\prime} 5^{\prime \prime}$; from Vaua, longitude $23^{\circ} 44^{\prime}$, rhumb S- E, distance 860.3 knots.

Seplember 3, I74I

Latitude $52^{\circ} 21^{\prime} 5^{\prime \prime}$; from Vaua, longitude $23^{\circ} 18^{\prime}$, rhumb $\mathrm{S} 87^{\circ} 53^{\prime} \mathrm{E}$, distance 849 knots.

September 4, I74I

Latitude by observation $52^{\circ} 23^{\prime}$; from Vaua, longitude $2 \mathrm{I}^{\circ} \mathrm{IO}^{\prime}$, rhumb $\mathrm{S} 88^{\circ} 19^{\prime} \mathrm{E}$, distance 770 knots.

At the beginning of the seventh hour in the morning we sighted land ahead of us and a little to the left, but owing to the mist we could not observe it carefully. At noon the land ${ }^{16}$ which we sighted at the seventh hour appears now NE by N, 40 knots, N by E⿺辶/ $\mathrm{E}, 30$ knots; mountains (not very high) in NNW, 25 knots; high land. on which there are several high peaks, in NNIV3/4W, 25 knots; a large round, snow-covered volcano NIV by $\mathrm{N} 3 / 4 \mathrm{WW}$; the land ends $\mathrm{W}$ by $\mathrm{N} 3 / 4 \mathrm{WV}$, distant about 50 knots. Sailed past a dead whale on which were many sea birds called fulmars. ${ }^{17}$

\section{Seplember 5, 1741}

Latitude by observation $51^{\circ} 53^{\prime}$; from Vaua, longitude $19^{\circ} 55^{\prime}$, rhumb $\$ 85^{\circ} 22^{\prime} \mathrm{E}$, distance 730.4 knots.

\section{September 6, I74I}

Latitude $51^{\circ} 29^{\prime}$; from Vaua, longitude $18^{\circ} 58^{\prime}$, rhumb $\mathrm{S} 83^{\circ} \mathrm{O} 8^{\prime} \mathrm{E}$, distance 702.5 knots.

\section{September 7, 17.41}

Latitude $51^{\circ} 23^{\prime}$; from Vaua, longitude $16^{\circ} 29^{\prime}$, rhumb S81 $34^{\prime} \mathrm{E}$, distance 613.7 knots.

\section{Scptember $8,17+1$}

Latitude $51^{\circ} 36^{\prime}$; from Vaua, longitude $12^{\circ} 20^{\prime}$, rhumb $\mathrm{S} 80^{\circ} 21^{\prime} \mathrm{E}$, distance 459.4 knots.

From six in the morning until noon we saw floating sea cabbage, also many shore ducks and gulls flying.

\section{September 9, I74I}

Jatitude by observation $51^{\circ} 40^{\prime}$; from Vaua, longitude $11^{\circ} 28^{\prime} 5^{\prime \prime}$, rhumb $\$ 79^{\circ} 39^{\prime} \mathrm{F}$, distance 429 knots.

At the ninth hour in the evening raid out 75 fathoms of cable. Took soundings, 25 fathouns; bottom fine gray sand. Heard the breaking of

16 Islands of Four Mountains.

17 Procellaria gtacialis gtupischa Stejneger 
the surf on the beach to the SW, but owing to the mist we could not see the land.

At the ninth hour in the morning the fog lifted a bit, and we saw the shore $^{18}$ about 200 fathoms away. High, snow-covered mountains and tall green grass were seen, but no trees. In places cliffs came down to the very sea, and close to the shore were many rocks above and below the water. We caught sight of two men walking through the grass across the hills from north to south. They were close to a high hill out of which ran a small stream. It was evident that they had caught sight of us and came a lit tle nearer so as to have a good look at our ship. We called to them in Russian and in Kamchadal that they should come to us. At the end of the hour we heard people shouting to us from the shore in the direction SSW, but we could not see them and owing to the surf could not make out their words. We replied, through the speaking trumpet and without it, asking them to come out to us.

At the tenth hour in the morning seven small boats, one man in each, were seen rowing towards us. Each of these boats was about is feet long. 3 feet wide, the bow very sharp, the stern somewhat rounded and blunt, and the whole covered with hair seal and sea lion skins. The deck was roundish and, like the sides, was sewed with some kind of skins except one spot between the center and the stern, where there was a round hole in which the man sat. He was dressed in a kind of shirt which covered his head and his arms and was made from the intestines of a whale or some other animal. There was something outside the hole that resembled leather breeching and which tied around the man. Some of these breechings were not tied, and we could see that there were rocks in the boats. They have light double paddles, made of birch wood, with which they paddle on both sides; and, as far as we could make out, the men were quite fearless in the water. They were not deterred by any kind of waves or seas and went through the water at a rapid rate. When they came within 50 fathoms of the ship they began to shout, turning first to one side and then to the other, not in the manner as if they wished to speak but as the Yakuts and Tungus do in their incantations. From this act we concluded that the people before us were doing the same thing and were praying that no harm might come to them from us. Whether this is the true explanation of their behavior or not I cannot tell. After they had carried on like this about seven or eight minutes they began to converse with one another in the usual tone of voice. While they were doing this we looked pleasant, bowed to them, and waved our hands as an invitation for them to come nearer our ship; but they did not quite dare to do so. They made a gesture with their hands as if drawing a bow, which showed that they were afraid we might

ss Adak Island. 
attack them. To the best of our ability we tried to convince them that they had nothing to fear from us. We pressed our hands to our hearts as a sign that we would receive them in a friendly manner. To convince them still more I threw them a Chinese cup as a mark of friendship. One of the men picked it up and after examining it made a gesture as if to indicate that he had no use for it. He was about to throw it back to the ship when we bowed and motioned for him to keep it, but he threw it into the water. I ordered that two pieces of damask should be tied to a board and thrown into the water near the ship. They picked it up and after handling it for a little while threw it away. I then gave the order to bring up the different things we had to give as presents-small boxes, small bells, needles, Chinese tobacco, pipes-and, holding them up, I invited them to come near. During this time I had few men on deck but had them concealed below with their guns loaded in case of danger. In spite of our efforts we could not convince them of our good will if they came close to the ship. It was only after we made them understand that we were out of water and had nothing to drink and that we looked to them for help that one of them dared approach us. We gave him Chinese tobacco and a pipe which he took and placed on the deck of his boat. When the others saw this they, too, became bold and paddled up. We distributed among them boxes, bells, needles, all of which they received rather indifferently as if not knowing what to do with them. They did not even know that needles would sink and did not make any effort to keep them out of the water, for among the presents we gave them some of the needles fell into the water, and instead of saving them they merely watched them go down. Among them we noticed several who raised one hand to their mouth and with the other hand made a quick motion as if cutting something near the mouth. This gave us the idea that they wanted knives, because tlee Kamchadals and the other peoples of this region when they eat meat or anything of that kind cut it at the mouth. I ordered that a knife should be given them, and when they saw it they were overjoyed and seized it from one another and with great eagerness begged for more. We then invited as many of them as would to come aboard so that we might show them friendly attentions and persuade some of them to accompany us, as the instructions of the Captain Commander required. Not being able to understand one another, we not only failed to persuade them to go with us but even to coax one of them to come on board so that we might show them how friendly we really were. We gave them a small barrel in which to bring us water from the shore. They understood what we meant, but they would not take the barrel and showed us that they had bladders for that purpose. Three of them paddled towards the beach and returned with water. When they came alongside one of them held up a bladder and indieated that he wished to have a knife in 


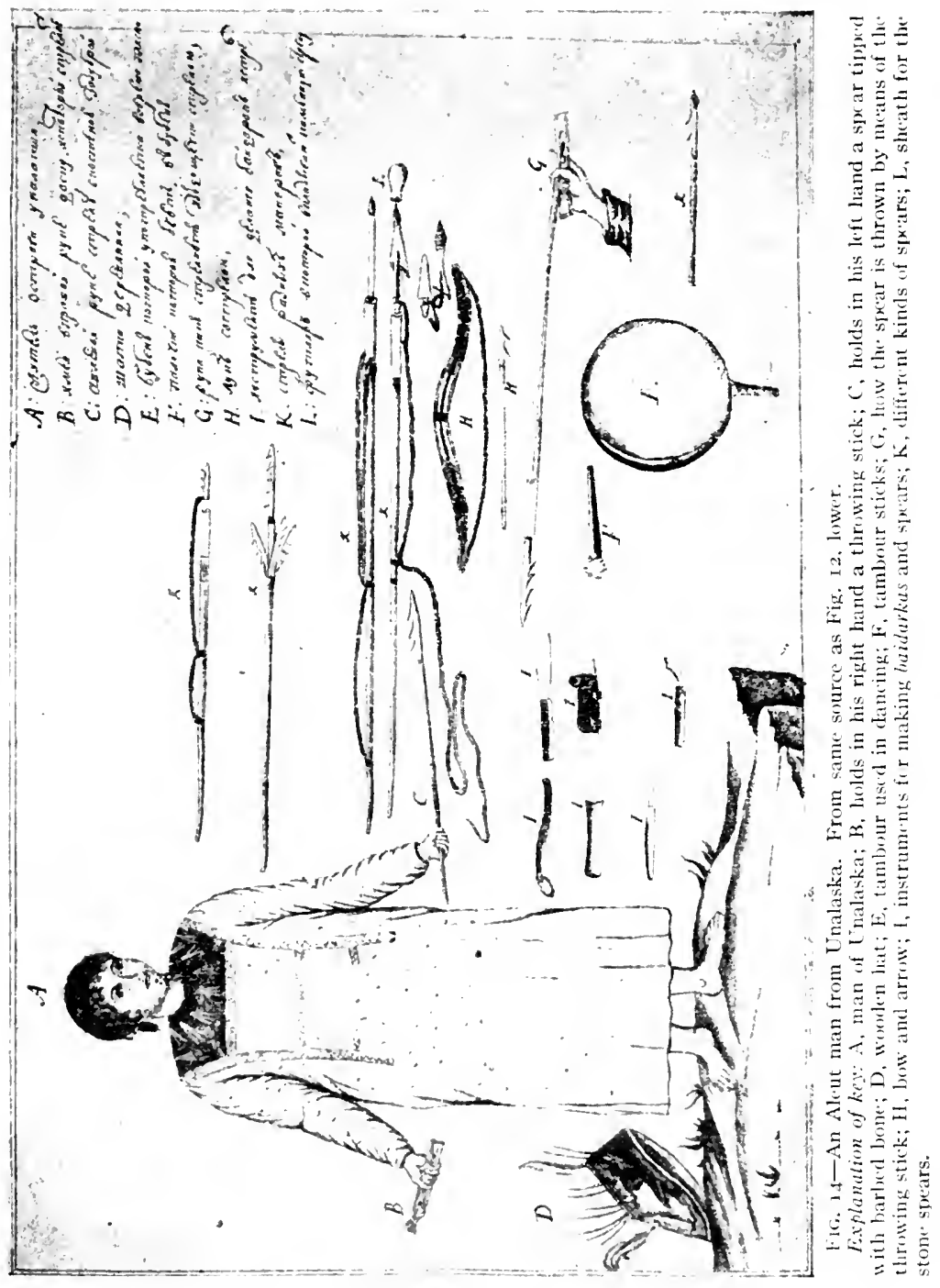




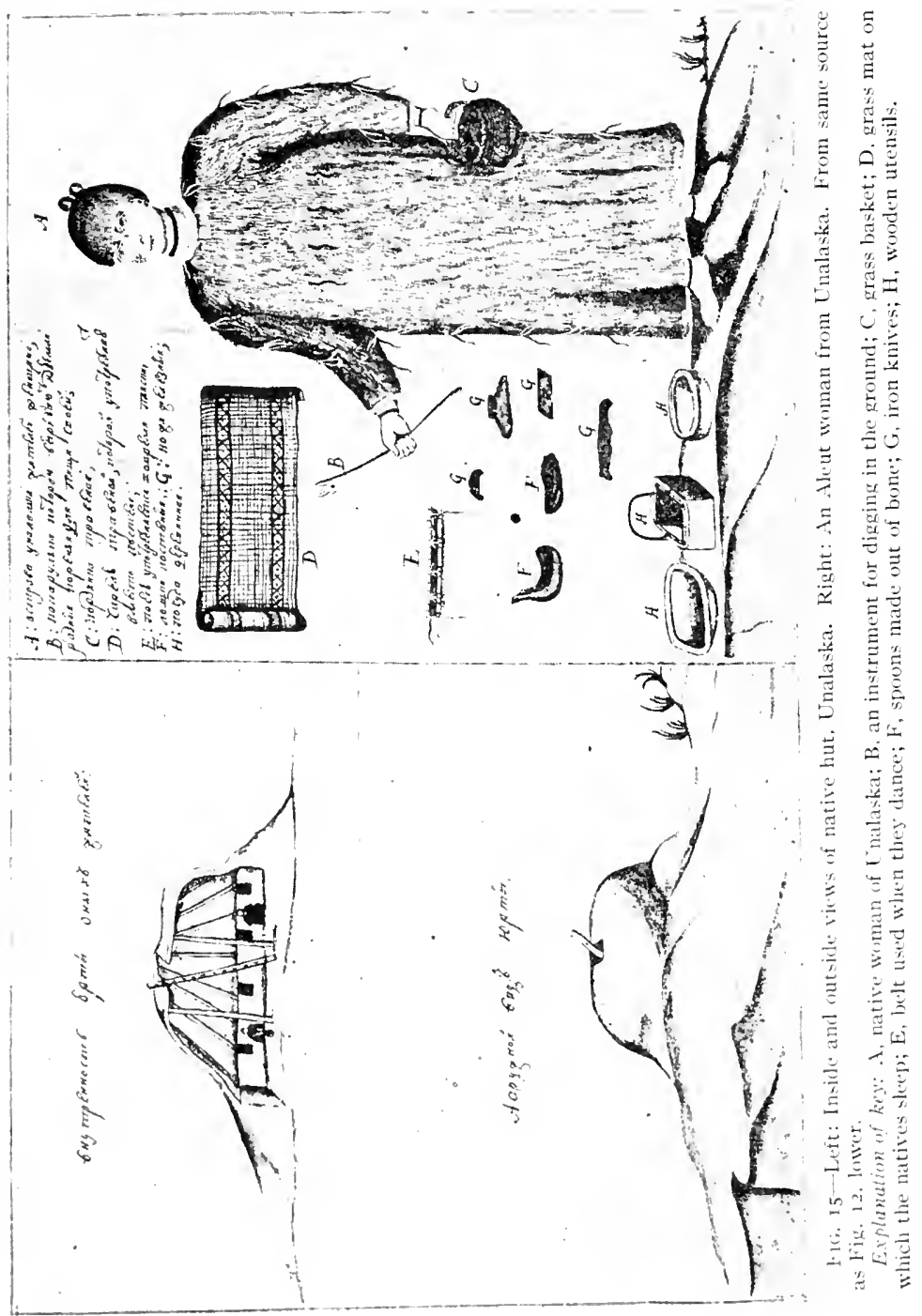


payment. This was given him; but, instead of handing over the bladder, he passed it to the second man, who also demanded a knife. When he got it he passed the bladder to the third man, who equally insisted on a knife. This act, as well as some other things they did, proves that their conscience is not highly developed.

These men seem to be of fair size and resemble the Tatars. They are pale and seemingly healthy. Almost all of them were beardless, whether naturally so or because they had plucked out the hair it is hard to tell; only two or three of then had anything like beards. They stuff roots into their noses which causes them to bleed. Some of these roots they were eating and gave us a few for the same purpose. In order to determine what these roots are we brought them along. In returm for their gift we made them a present of sea biscuits. They brought us some kind of mineral wrapjed in sea weeds. Aside from arrows, of which we secured four, there was nothing on board their boats. They wear on their heads a kind of hat made of thin birch boards, which are decorated with various colors and feathers. Some of these dippers (hats) had in the top small ivory statues. We secured one of these dippers by giving in trade a dull axe, which they received gladly. As a mark of friendship we offered them a copper kettle, which they kept for a little while and then handed back.

When they had been near the ship for a considerable time they returned to the shore. In the afternoon we were visited by fourteen of these boats, one man in each, among whom were a number who had been with us bewre noon. When they neared the ship they made the same kind of noise as before. Although they spent three or four hours alongside and we talked a great deal, yet we do not know anything they said, and we could not persuale one of them to come aboard. They would have remained near us even longer if I had not ordered that signs should be made to them to go ashore. I did this because the wind began to rise, making it possible for us to get out of the bay into which we had unintentionally entered. After much difficulty and the loss of an anchor we finally, with God's help, got out.

\section{September IO, $17+1$}

Latitude $51^{\circ} 12^{\prime}$; from Vaua, longitude $I^{\circ} 54^{\prime} 6^{\prime \prime}$, rhumb $577^{\circ} \mathrm{O}^{\prime} \mathrm{E}$, distance 45 knots.

At the sixth hour in the afternoon there was a very light offshore breeze, but we could see from the way the clouds were being driven eastward that beyond the mountains there was a west wind.

By the eighth hour the wind freshened a little. We began to heave in the cable and had already 35 fathoms aboard when all of a sudden a squall from the mountains struck us. The anchor began to drag. We hoisted the topsails and foresail. The tide was setting towards the north. 
forcing us on the land, which was about 300 fathoms to the east. Fearing that we might be driven ashore and dashed on the submerged rocks farther to the west we cut the cable ( 35 fathoms of which we had already heaved in) of the spare small bower anchor at the hawsehole, crowded on all sails and stood out to sea in a SE direction, and after great difficulty and great danger succeeded.

September II, I74I

Latitude $50^{\circ} 52^{\prime}$; from Vaua, longitude $I_{1}^{\circ} 45^{\prime} 8^{\prime \prime}$, rhumb $\mathrm{S}_{74^{\circ}} 28^{\prime} \mathrm{E}$, distance 451.8 knots.

September $12,174 I$

Latitude by observation $5 \mathrm{I}^{\circ} \mathrm{I} 2^{\prime}$; from Vaua, longitude $10^{\circ} 52^{\prime} 3^{\prime \prime}$, rhumb $\mathrm{S}_{75^{\circ}} 59^{\prime} \mathrm{E}$, distance $4 \mathrm{I}+$ knots.

\section{September I3, I74I}

Latitude $51^{\circ} \mathrm{oI}^{\prime}$; from Vaua, longiture $10^{\circ} 05^{\prime} 9^{\prime \prime}$, rhumb $\mathrm{S}_{73}{ }^{\circ} 18^{\prime} \mathrm{E}$, distance 389.7 knots.

Saw a considerable quantity of floating weeds near the ship.

September 14,1741

Latitude $50^{\circ} 3 \mathrm{I}^{\prime}$; from Vaua, longitude $8^{\circ} 19^{\prime} \mathrm{o}^{\prime \prime}$, rhumb $\mathrm{S} 65^{\circ} 22^{\prime} \mathrm{E}$, distance $3+\mathrm{I} .5$ knots.

Saw a shore cormorant on the wing.

September 15,1741

Latitude $5 \mathrm{I}^{\circ}$ oI'; from Vaua, longitude $6^{\circ} 27^{\prime} 9^{\prime \prime}$, rhumb $664^{\circ} 53^{\prime} \mathrm{E}$, distance 264 knots.

September 16,1741

Latitude $51^{\circ} 54^{\prime}$; from Vaua, longitude $5^{\circ} 26^{\prime} 5^{\prime \prime}$, rhumb $\mathrm{S}_{73}{ }^{\circ} 30^{\prime} \mathrm{E}$, distance 207.7 knots.

Captain Chirikov, Lieutenant Chikhachev, and the members of the crew are very ill, owing to the lack of water and the long and hard sea voyage. They are no longer able to work. One of the strongest, the sailmaker Michael Usachev, died of scurvy, and we lowered the body into the sea. All during the day we saw sea cabbage and floating grass, the kind that grows near the shore; the color of the water was green, unlike the color of sea water.

September 17,1741

Iatitude $51^{\circ} 30^{\prime}$; from Vaua, longitude $5^{\circ} \mathrm{I}^{\prime} 9^{\prime \prime}$, rhumb $666^{\circ} 56^{\prime} \mathrm{E}$, distance 209.5 knots.

This day we saw the same kind of sea cabbage, grass, and water as the diy before. 
September 18,1741

Latitude by observation $52^{\circ} 33^{\prime}$; from Vaua, longitude $4^{\circ} 44^{\prime} 8^{\prime \prime}$, rhumb $\$ 83^{\circ} 20^{\prime} \mathrm{E}$, distance 172.5 knots.

Saw the same kind of sea cabbage, grass, and green water.

\section{September 19, 1741}

Latitude $52^{\circ} 37^{\prime}$; from Vaua, longitude $4^{\circ} 36^{\prime} 8^{\prime \prime}$, rhumb S $83^{\circ} 12^{\prime} \mathrm{E}$, distance 167.5 knots.

\section{September 20, $174 I$}

Latitucle $52^{\circ} 36^{\prime}$; from Vaua, longitude $4^{\circ} 17^{\prime}$, rhumb S8 $3^{\circ} 46^{\prime} \mathrm{E}$, distance 156.6 knots.

\section{September 21,1741}

Latitude $52^{\circ} 35^{\prime}$; from Vaua, longitude $3^{\circ} 29^{\prime} 5^{\prime \prime}$, rhumb S8 $\mathrm{I}^{\circ} 51^{\prime} \mathrm{E}$, distance 127 knots.

At the eighth hour we saw a small fish near the ship, took soundings, and got 60 fathoms. No land in sight, but for the sake of safety we put about.

At the tenth hour in the morning we saw land straight ahead of us, which did not look like Kamchatka. We came about on a ight tack and went parallel to the shoreon the east, distant about 4 knots. At noon the land was still in sight, extending from WSW, 8 knots, ${ }^{19}$ to $\mathrm{NW} 1 / 2 \mathrm{~W}, 6$ knots. Until $12: 30$ the southern half of the land seemed level, and the northern half mountainous; but the mountains were neither high nor snow-covered. A small island ${ }^{20}$ loomed up in $\mathrm{N}$ by $\mathrm{W}_{1 / 2} \mathrm{~W}, 10$ knots. In a bay situated between the above-mentioned land in NW1/2W and NE by $N$ high, snow-covered mountains were seen. ${ }^{21}$

\section{September 22, I74I}

Latitude $52^{\circ} 07^{\prime}$; from Vaua, longitude $2^{\circ} 34^{\prime} 3^{\prime \prime}$, rhumb S6 $3^{\circ} 54^{\prime} \mathrm{E}$, distance 104.5 knots.

At the sixth hour in the afternoon the cape which at noon bore WSW, 8 knots, bears now NE by N3/4E, 12 knots, and the other cape, the western one, bears $\mathrm{N}$ by $\mathrm{W}, 6$ knots; between them the shore is low and level; the low, snowless mountains, which at noon seemed to stretch from south to north, and the high, snow-covered mountains in the bay bear $\mathrm{NW}$ by $\mathrm{W} / 2 \mathrm{~W}, 15 \mathrm{knots}$; and the land which we sighted yesterday in $\mathrm{N}$ by $\mathrm{W}$ is now $\mathrm{N}$, distant about 15 knots.

Today we saw two otters asleep in the water.

19 This was the southeastern point of Agattu Island.

so One of the Semichi Islands.

21 The high land of Attu. 
September 23, 1741

Latitude $5 \mathrm{I}^{\circ} 48^{\prime}$; from Vaua, longitude $3^{\circ}$ I $^{\prime} 4^{\prime \prime}$, rhumb $\mathrm{S}_{54}{ }^{\circ} 5^{\prime} \mathrm{E}$, distance I 12.9 knots.

September 24, I74I

Latitude $5 \mathrm{I}^{\circ} 5 \mathrm{O}^{\prime}$; from Vaua, longitude $\mathrm{I}^{\circ} 59^{\prime} \mathrm{I}^{\prime \prime}$, rhumb $449^{\circ} \mathrm{O} 6^{\prime} \mathrm{E}$, distance 96.2 knots.

\section{September 25, 1741}

Iatitude $51^{\circ} 27^{\prime}$; from Vaua, longitude $I^{\circ} \mathrm{o}_{3}^{\prime} \mathrm{I}^{\prime \prime} \mathrm{E}$, rhumb $\mathrm{S}_{3} \mathrm{I}^{\circ} \mathrm{O}^{\prime} \mathrm{E}$, distance I 00.5 knots.

\section{September 26, I74I}

Latitude $52^{\circ}$ I $6^{\prime}$; from Vaua, longitude $0^{\circ} 6^{\prime} \mathrm{I} 3^{\prime \prime} \mathrm{W},{ }^{22}$ rhumb $\mathrm{S} 5^{\circ} 45^{\prime} \mathrm{W}$, distance 46.8 knots.

Assistant Constable Osip Kachikov died of scurvy and we lowered the body into the sea. Captain Chirikov, Lieutenants Chikhachev and Plautin, and six of the crew are very ill with scurvy; all the others are so weak from long sickness, hard work, and lack of water that they can barely come on deck and do their work. We have not more than seven barrels of water on board.

\section{September 27,1741}

Latitude $5 \mathrm{I}^{\circ} 44^{\prime}$; from Vaua, longitude $0^{\circ} 38^{\prime} 27^{\prime \prime} \mathrm{E}$, rhumb $\mathrm{Sr} 8^{\circ} 44^{\prime} \mathrm{E}$, distance 72.9 knots.

Captain Chirikov, Lieutenants Chikhachev and Plautin, Professor of Astronomy Delisle de la Croyère, and twelve members of the crew are very sick with scurvy; and the others are weak and get about with great difficulty. We have only six small barrels of water, and the daily need is five cups per person. We no longer cook kasha. ${ }^{23}$

22 All the longitudes ought, of course, to be $\mathrm{E}$, as the whole voyage lay to the eastward of Vaua. That their calculations from now on gave $W$ longitudes (the $\mathrm{E}$ positions of September 27 and 28 were temporary setbacks bcause of head winds) made the officers of the St. Paul aware of the error in the ship's position: the total error in longitude amounted to $11^{\circ} 39^{\prime}$ (see footnote 14, p. 322). The distances, rhumbs, and longitudes become intelligible when referred to the assumed position of Vaua, which was about in $170^{\circ} 30^{\prime} \mathrm{E}$ of Greenwich (and $52^{\circ} 53^{\prime} \mathrm{N}$ ).

In his report (Chapter VIII, p. 322) Chirikov discusses this matter of error in longitude and its cause. He correctly ascribes it to lack of a known point at the farther end of his journey wherewith to check his obscrvations. He also correctly infers that the currents are partially responsible; he made attempts to determine this element but was not able to. It is precisely in the adjustment to known positions through the identification of landfalls and in the allowance made for currents that lies the value of such modern reconstructions of Bering and Chirikov's tracks ats is afforded by Pl. I. It is interesting to compare this chart with the reproduction of the original chart of the navigators accompanying Sokolov's memoir in Zapiski II yilrogr. Itepart. Vol. 9, St. l'etersburg, I 85 I (copied on Lauridsen's "Vitus Bering," 1889, Map 111.)-EnT. Note.

${ }^{23}$ Buckwheat mush. 
September $28,17+1$

Latitude $5 \mathrm{I}^{\circ} 17^{\prime}$; from Vaua, longitude $0^{\circ} 5^{0^{\prime}} 37^{\prime \prime} \mathrm{E}$, rhumb $\mathrm{S}_{1} 7^{\circ} 52^{\prime} \mathrm{E}$, distance IoI knots.

\section{Seplember 29, $17+1$}

Latitude $52^{\circ} 11^{\prime}$; from Vaua, longitude $0^{\circ} 21^{\prime} 53^{\prime \prime} \mathrm{W}$, rhumb $\operatorname{Si}_{1} 7^{\circ} 8^{\prime} \mathrm{W}$, distance 44 knots.

\section{September 30,1741}

l.atitude $5 \mathrm{I}^{\circ} 55^{\prime}$; from Vaua, Iongitude $\mathrm{I}^{\circ} \mathrm{o} 2^{\prime} 53^{\prime \prime} \mathrm{W}$, rhumb S3 $3^{\circ} \mathrm{I} 9^{\prime} \mathrm{W}$, distance 69.4 knots.

At the ninth hour in the morning we had a heavy storm with squalls, hail, and snow. We furled the mainsail, lowered the main yards and fore yards, and hove to under the mizzen. The waves threw up on deck a small fish cailed stickleback (khakhalcha), which is not more than $21 / 2$ inches long.

\section{October I. $17+1$}

Latitude $51^{\circ} 28^{\prime}$; from Vaua, longitude $0^{\circ} 32^{\prime} 33^{\prime \prime}$ (?) $\mathrm{W}$, rhumb $\mathrm{S}_{1} 3^{\circ} 22^{\prime} \mathrm{W}$, distance 87.4 knots.

Heavy sea running; rain, snow, and cold.

$$
\text { October 2, } 17+1
$$

Latitude $5 \mathrm{I}^{\circ} 46^{\prime}$; from Vaua, longitude $2^{\circ} 19^{\prime} 6^{\prime \prime} \mathrm{W}$, rhumb $\mathrm{S}_{5} \mathrm{I}^{\circ} 5 \mathrm{O}^{\prime} \mathrm{W}$, distance 108.4 knots.

\section{October 3, I74I}

Latitude $51^{\circ} 58^{\prime}$; from Vaua, longitude $3^{\circ} 48^{\prime} \mathrm{W}$, rhumb S6 $68^{\circ} 34^{\prime} \mathrm{W}$, distance 150.5 knots.

\section{October $4,174 I$}

Latitude by observation $52^{\circ} 56^{\prime}$; from Vaua, longitude $5^{\circ} 31^{\prime} 4^{\prime \prime} \mathrm{W}$ rhumb $\mathrm{N} 89^{\circ} \mathrm{O} 8^{\prime} \mathrm{W}$, distance $199.9^{\prime}$ knots.

At the second hour in the afternoon we had a heavy rain, and the crew had a chance to satisfy their thirst and to catch seven buckets of water in addition.

\section{October 5, I74I}

Latitude $52^{\circ} 33^{\prime}$; from Vaua, longitude $6^{\circ} 48^{\prime} 4^{\prime \prime} \mathrm{W}$, rhumb $S 85^{\circ} 23^{\prime} \mathrm{W}$, distance 248.4 knots.

At the ninth hour in the morning topsail wind, heavy snow, and very cold. The men were so weak that they suffered much at their work.

\section{October 6, I74I}

Latitude $52^{\circ} 29^{\prime}$; from Vaua, longitude $6^{\circ} 4 \mathrm{I}^{\prime} 7^{\circ} \mathrm{W}$, rhumb S8 $84^{\circ} 22^{\prime} \mathrm{W}$, distance 244.5 knots. 


\section{Oclober 7, 1741}

Latitude $53^{\circ} \mathrm{I} 2^{\prime}$; from Vaua, longitude $9^{\circ} 32^{\prime} \mathrm{W}$, rhumb $\mathrm{N} 86^{\circ} 54^{\prime} \mathrm{W}$, distance 352 knots.

At the fifth hour in the afternoon Vasili Nizhegorodok of Yakutsk, the servant of Lieutenant Plautin, died of scurvy, and we lowered the body into the sea. At end of the tenth hour Lieutenant Chikhachev died of scurvy.

\section{October $8,17+1$}

Latitude $52^{\circ} 37^{\prime}$; from Vaua, longitude $11^{\circ} 04^{\prime} I^{\prime \prime} \mathrm{W}$, rlumb $585^{\circ} \mathrm{o} 7^{\prime} \mathrm{W}$, distance 399.4 knots.

Navigator Vrange and Lieutenant Michael Plautin died of scurvy in the third hour of the afternoon. At the seventh hour in the morning we sighted land-high, snow-covered mountains. According to our opinion and according to the position of the land it must be the shore running north from Isopa to Vaua.24 Owing, however, to the mist we could not see very distinctly.

At noon we were certain that the land in sight was Shipunski Cape bearing NNE3/4E; Avacha, or Burning, Volcano bore $\mathrm{NW}$ by $\mathrm{N} 3 / 4 \mathrm{~W}$, Vilyuchensk Volcano $\mathrm{W}$ by N3/4 $\mathrm{W}$; the land ended to the S and SW by IV; Vaua could not be seen owing to the mist.

\section{October 9, I74I}

Latitude $52^{\circ} 57^{\prime}$; from Vaua, longitude i ${ }^{\circ} 39^{\prime} 4^{\prime \prime} \mathrm{W}$, rhumb N89 $27^{\prime} \mathrm{W}$, distance 422 knots.

At the sixth hour we sighted Vaua; but, owing to the head wind and the coming on of night, we could not enter the bay and therefore put out to sea. At noon the wind was very light, and the fog cleared a bit, and Vaua stood out, bearing according to compass $W$ by $N$, distant about io knots.

\section{October 10,1741}

At the fifth hour in the afternoon the wind from $\mathrm{N}$ increased a little, and we sailed WNW into the mouth of the bay.

At the eighth hour noticed a light on Vaua lighthouse.

At the ninth hour we safely passed Vaua and entered the mouth of Avacha Bay, where we anchored in seven fathoms.

At the seventh lour we fired five guns as a signal for small boats to come out.

At the ninth hour Ensign Levashev came out and told us that Captain Commander Bering on the $S t$. Peter had not yet returned, that the galliot Okhotsk, in command of Assistant Navigator Andrei Sheganov, had

if It was not the coast south of Vaua but north of it that was sighted, as was soon realized, as the first sentence of the next paragraph shows. 
come from Okhotsk bringing 1,500 poods of provisions, that Captain Spanberg with five ships had reached Bolsheretsk from Okhotsk on his way to Japan.

Professor of Astronomy Delisle de la Croyère died of scurvy at ten o'clock.

At noon Captain Chirikov was taken ashore in a very sick condition.

October 12,1741

Brought the ship around into the Harbor of St. Peter and St. Paul for the winter and began to discharge cargo.

\section{Note oN tHE LOSS OF CHIRIKOV'S MEN}

The loss of Chirikov's men is the most dramatic incident of the voyage of the St. Paul. The question naturally arises, What was theit fate? Has any account of this episode been handed down in the traditions of the Indian tribes of the region? This question was submitted to a number of authorities on the ethnology of the Pacific Coast. None of them had heard of any such tradition. A mong previous investigators. Professor George Davidson to judge by a foot note on v. 20 of his "The Tracks and Landfalls of Bering and Chiriko on the Northwest Coast of America," San Francisco, rgor, made an attempt in 1901 to ascertain the traditions of the Tlingits through the medium of two members of the Alaska Commercial Company. It is not known that any information was secured.

One of the ethnologists consulted, Lieutenant George T. Emmons, whose study of a gimilar first contaet between a Pacific Coast tribe and white men (The Meeting between La Pérouse and the Tlingit, Imer. Anthropologist. Vol. 13, 1911 , pp. 294-298), together with his long labors among the Tlingits, makes him peculiarly. well equipped to deal with this question, has likewise, during thirty ' 'ears' investigation among the coast tribes, never been able to learn anything of the loss of Chirikov's men. However, in the communication that he kindly sends, he suggests that Chirikov's two boats may have been swamped in the strong tidal rips that occur at the mouths of such narrow fiord arms as Lisianski Strait, especially if they entered with the strength of a flood tide. That this might easily happen to small boats is evidenced by the fact that today even powerful steamers, as he statcs, enter Peril Strait, a similar passage somewhat farther sonth, only at slack water. The assumption that the boats were swamped and their occunants drowned would seem to be borne out by the fact that two native canoes put off from shore and approached the $S t$. Paul. If the Russians had landed and if, in spite of Chirikov's admonitions to his men, there bad been a fight, the natives, after this first experience of firearms, even if they had overpowered the landing parties, would certainly not have exposed themselves to the greater risk of facing the main body of the Russians. The fact that they approached the ship would rather prove their innocence. Also, the similarity of their call of "agai," as reported by Chirikov, to the Tlingit "agou," which means "come here," would seem to imply friendly intentions. In most later instances the first meetings of natives of this coast with Europeans, before the Russians commenced to appropriate their hunting grounds, says lieutenant Emmons in conclusion. Were friendly; indeed, the Tlingits were rather ready to trade.

The only suggestion of a tradition among the coast tribes that might shed light on the loss of Chirikov's men is contained in a recently published book, "The Story of Sitka. The Historic Outpost of the Northwest Coast. The Chief Factory of the Russian American Company." by C. L. Andrews, Seattle, 1922. The relevant passages (pp. 9-10) follow. The author assumes the locality of the tragedy to be Sitka Sound.

"Nearly two centuries have passed since the Russian seamen landed and no word has come from them. For mote than seventy years the Russian Government sought for some sign of their fate.* Tales were told of a colony of Russians existing on the coast, but each upon investigation proved lut a tumor.

"There is a dim tradition among the Sitkas of men being lured ashore in the long ago. They say that Chief Annahootz, the predecessor of the chief of that name who was a firm friend of the whites at Sitka in 1878 . was the leading actor in the tragedy. Annahootz dressed himself in the skin of a bear and played along the beach. So skillfully did he simulate the sinuous motions of the animal that the Russians in the excitement of the chase plunged into the woods in pursuit and there the savage warriors killed them to a man, leaving none to tell the story. The disappearance of Chirikof's men has remained one of the many unsolved mysteries of the Nothlland, and their fate will never be known to a certainty."-EDIT. NOTE.

* "January 2oth, 1820, a letter written by the Directory at St. Petersburg to Chief Manager Muravief at Sitka enclosing instructions previously given to Hagemeister, instructing him to find the descendants of Chirikof's lost men, urging that it must be done and expressing surprise that it had been neglected thus long. (Russian American [Company's] Archives, IState Department, Washington, D. C..] Correspondence, Vol. 2, No. 108)." 


\section{CHAPTER VIII}

\section{CHIRIKOV'S REPOR'T ON THE IOYAGE OF THE "ST. PAUL"}

Captain Chirikov's report on the voyage of the St. Paul, together with a supplementary report in which he expresses his readiness to continue the explorations, with their respective enclosures, translated from the originals ${ }^{1}$ in the Russian archives, reads as follows.

\section{To the Imperial Admiralty College, A RePort}

On May 4, I 74I, Captain Commander Bering, I, Captain Chirikov, Lieutenant Chikhachev, Navigator Vrange, Fleet Lieutenants Sven Waxel and Plautin, Professor of Astronomy Louis Delisle de la Croyère, Flect Master Sofron Khitrov, Acting Fleet Master Avraam Dementiev, and Navigators Andreyan Eselberg and Ivan Elagin met to hear the written instructions given to Captain Commander Bering by the Imperial Admiralty College, especially Articles 9, I0, and I 7, and Article 6 of the general order of the ruling Senate, ${ }^{2}$ which was with the said instructions. After reading them we all agreed that, according to the instructions, to find the Amcrican coast we should sail at first, after leaving this harbor, SE by $\mathrm{E}$ by true compass and keep on this rhumb to the 46 th parallel of north latitude unless we sooner found land, for, on the map of Professor Delisle de la Croyère, Juan de Gama Land was located on the 47th parallel of latitude and right on our course. We believed that it was a part of Anerica because, on the general charts, land is indicated all the way from California to Juan de Gama Land, and this indication is also on the map of Professor Delisle de la Croyère.

Although Juan de Gama Land is represented as an island, consequently not a part of the American continent, yet we decided on the course we lid because our instructions called for an examination of the islands lying on the way to America. It was agreed that in case land was not found in the latitude just mentioned we should sail steadily $\mathrm{E}$ by $\mathrm{N}$

1 Archives of the Ministry of Marine, Petrograd: Papers of Captain Commander Bering, No. 4.4.

2 The instructions of the Admiralty College to Bering are not given in the present work, but the orders of the Senate are. Article 6 will be found in Chapter IV, p. 30 . 
until we met with success. If land was discovered either on the course $\mathrm{SE}$ by $\mathrm{E}$ or $\mathrm{E}$ by $\mathrm{N}$, we determined to coast alongside of it from east to north or north to west, depending on its position, but not to follow it up if it stretched between south and east. In the latter case, we were to leave it and sail east until we sighted [other] land and when found to keep it in view likewise while going northerly, to the $65^{\text {th }}$ parallel or at least as far as, God willing, time would permit. If we reached the $65^{\text {th }}$ parallel in good season we planned to sail due west to the Chukchi country and determine the distance between America and Asia and when we had done that to steer for this port. If head winds should prevent us from keeping on the above-mentioned rhumb, we agreed to keep as close to it as possible until with God's help we discovered land and examined it in accordance with the instructions of the Captain Commander. In planning the voyage we had to keep in mind that we were to be back in this harbor towards the end of September. Although we discussed the suggestion of the Admiralty College, made before we left St. Petersburg, to go first to the Chukchi country and from there towards America (for the distance must be short), we did not act upon it because the season was too early and there was too much ice in the neighborhood of the Chukchi land, which is situated near latitude $65^{\circ} \mathrm{N}$.

Therefore we concluded to follow the course first mentioned, and on May 29 we sailed out of the Harbor of St. Peter and St. Paul [Petropavlovsk] and anchored in the roadstead of Avacha Bay and there remained, on account of head winds, until June 4 , when the wind died down and we put out to sea and kept the course agreed upon for Juan de Gama Land. By June 12 we had come as far as the 46 th parallel without finding the said land, and it became quite evident that it did not exist. since we had sailed over the region where it was supposed to be. On June 13 we changed the course in order to find America and sailed $\mathrm{E}$ by $\mathrm{N}$, or as near to it as the winds permitted. On June 20 because of the continuous fogs, which are common in this region, and the stormy winds (which obliged us to heave to under the mizzensail) the Captain Commander and I became separated. During this bad weather I searched for him as long a time as he allowed for such a purpose but without success. From this time on I did not again see his ship and was, therefore, compelled to continue the voyage alone on the course laid out.

On July 15 (having, from the mouth of Avacha Bay, where we had put up a lighthouse named Vaua, come east $61^{\circ} 51^{\prime}$ of longitude, according to our reckoning, distance on the rhumb $\mathrm{E}$ by $N 6^{\circ} 57^{\prime} \mathrm{E}, 2,178$ knots or Italian miles, or 3,793 Russian versts, counting $1041 / 2$ versts to a degree -in returning we made $73^{\circ} 30^{\prime}$ of longitude, distance on the rhumb E by $\mathrm{N} 7^{\circ} 38^{\prime}$ E, 2.589 knots, or 4,509 Russian versts-) we discovered land [Cape Addington] in latitude $55^{\circ} 36^{\prime}$ north [noon position]. This land was without doubt the American coast, because, according to the map 
of the Nuremberg geographer Johann Baptist Homann and others, we were not far from parts of America that are well known. To illustrate this point more clearly we have joined (on the chart which is being sent to the Admiralty College) our discoveries with the American coast as it appears on the map of Homann and Professor Delisle de la Croyère, namely the northern part of California, the mouth of the Moozemleck River, ${ }^{3}$ a portion of the interior of the eastern part of Hudson Strait. The longitude of Kamchatka, as given on the chart, is referred to Teneriffe Island and St. Petersburg as calculated by Professor Delisle de la Croyère.

When we stood near the land on the above-noted date we looked about for anchorage in order to make our observations as we were instructed. We approached within three versts of the shore and even nearer in some places, sounding as we went along, but found no good anchorage, for the depth was about 70 fathoms or more. The coast is irregular and mountainous; these mountains had a fine growth of timber and in places were covered with snow. In the journal and on the general chart may be seen the lay of the land, for we paralleled it. Not finding a good anchorage $I$ sent the quartermaster Grigori Trubitsin with eight men in the longboat into a bay to ascertain whether it could be entered, the kind of anchorage, and the depth of the water. He did as he was told and returned in lour hours and made a written report to the effect that he had been within 60 fathoms of the shore opposite the bay, where he found 40 fathoms of water and gravelly, sandy bottom. There was shelter from north and east winds, but no protection from west and south winds. It offered anchorage for only a small boat. The width of the bay from the cape to the shore is son awhere from 4 to 5 versts. In going by the cape we noticed on the rocks many sea lions. On the mountains were large trees of fir and pine. Human habitation we did not see. On July 16 , at eight $0^{\prime}$ clock in the evening, we took the longboat aboard, because there was no good place to anchor; and, for more safety, we steered away from shore for the night. About five in the morning, the wind being fair, we came about and sailed north on the sane rhumb on which we last saw land [Cape Ommaney] in the north the night before. At ten o'clock in the norning we came within a half verst of it. At the time it was quite foggy, and in order not to get too close we paralleled it, keeping between north and west. It was my intention to make a careful survey of a part of the American coast, but my plans were ruined by the misfortune of July 18 . On that day we were in the 58 th degree, and I noticed that the mountains had more snow on them than those we had passed. Evidently we were going into colder country, where it is more difficult to make observations than in warmer. With this idea in mind l ordered, my officers

${ }^{3}$ ()11 Homann's map (1712?) this river falls into the Gulf of California. 
agreeing, the acting fleet master Arraam Dementiev with ten armed men to take the yawl and examine the bay. It was then in the fourth hour. I followed them with the ship, with the purpose of anchoring when 1 was near the bay into which they entered. Much to our disappointment we found no place to anchor; everywhere the shore is broken up, and the mountains come right down to the water's edge; and, as is usual in such cases, the water is deep, which on sounding we found to be true, as may be seen in the officers' journals.

We approached within two versts of the bay, sounding as we went along, but got nowhere less than 65 fathoms. Almost everywhere the bottom was gravelly, and in many places rocks were seen above and below water. For these reasons we did not anchor but hove to and tacked in front of the bay, the bearings of which we took. Before he set out I handed Dementiev a copy of the instructions which had been given to us to display in public. He read it over several times. I gave him also the following order which was signed by me:

You are put in command of the longboat and ten armed men, one copper cannon and two rockets; and you are to go ashore and do the following things.

(1) When you come near enough to the shore, make a landing if possible; if not. come back to the ship and for our information fire two guns. (2) If with God's help you get ashore, look about for human beings; if you find them, be gentle with them and present them with a few small presents with which the ensign Choglokov will provide you, namely a copper and an iron kettle, two hundred beads, three packages of Chinese tobacco, one piece of nankeen, one piece of damask, five rattles [?], and a paper of needles. From me you will receive ten-ruble pieces which you may distribute among the inhabitants as you think best. Among other questions ask them, in case the Koriak interpreter who goes with you can enter into conversation with them (for it is not likely that any other language but his will be of any help), what kind of land this is and under what government they are; and ask some of them to come aboard our ship. (3) See whether there is a safe place for a ship to come in and anchor for a time, take soundings, and make a sketch map of the harbor, even if it is only rough. (4) Note the kind of trees and grasses on shore. (5) Examine the rocks and the soil to see whether they contain precious minerals; in order to help you a piece of silver ore is given you to take along and if you find something like it bring it aboard. (6) Ask the natives in what direction the land extends, whether it has any rivers that flow into the sea, and where they are; and obtain such other information asyou can. (7) If the inhabitants should act in an unfriendly manner and make it unsafe to remain, return to the ship as quickly as you can; but do them no harm nor allow your men to do so. (8) Make every effort to carry out quickly the above instructions so that you may return to the ship the same day or at least not later than the next day. If thick weather should set in, making it impossible to see the ship, you had better not come out. If stormy weather should come on, delay your departure and, with that in view, take with you provisions enough to last your company a week. (9) As soon as you land signal to us with a rocket and when you embark let off another one. While ashore keep up a big fire, especially at night, if you think 
We are likely to see the flame, or the smoke in the day time. (10) When you are within a verst of the shore begin sounding to ascertain the depth and the kind of bottom. (II) Fill with fresh water the two barrels which are being sent with you. In all things conduct yourself as a true and good servant of Her Imperial Majesty.

We had no signal of any kind from him. We saw them approach the shore, and that is all. Hoping that he would come out, we kept under sail for five days and as near the bay as we dared.

At first the weather was such that the longboat could have come without any trouble; later we had heavy rains, fog, and strong winds which carried us from the said bay a distance of about 30 knots. On July 23 we returned and went up quite close to the bay and there saw a fire which we thought was made by our men. During all the time that we had followed the coast we had seen no fire, no buildings, no boats, nor any other signs of human beings and therefore supposed that the country was uninhabited. When we observed the flame we fired a gun at intervals as a signal to the boat to come out; but no boat came, atthough the weather was fair for that purpose and we sailed quite close to the shorc. As we fired the blaze on the beach grew bigger.

On July 2.4 we concluded that it was quite probable that the boat was damaged and could not come out. With this idea in mind all the officers consulted and decided (in writing) to send ashore the small boat with the carpenter, the calker, and the necessary tools to repair the longboat. Boatswain Sidor Savelev volunteered to accompany them, and the sailor Fadiev was asked to go and help row because he had expressed a desire to do so. These are the instructions (a copy of which is enclosed ${ }^{4}$ ) which were given to the boatswain. On reaching shore and finding the boat in need of repairs he was to leave the carpenter and calker; and he, with Dementiev and three or four of the men, was to return to the ship without delay. When he departed the weather was very still; we followed him quite close to slore and saw him approach it. According to our time it was exactly six o'clock in the afternoon. The signals which had been agreed upon he failed to make and at the expected time did not return. The weather was fair. Next day, July 25, at one o'clock in the afternoon we sighted coming ont of the bay into which we had sent our men two boats, one snall and the other larger, and we concluded that they must be our boats returning. We went to meet them; as we drew near we noticed that they were not our boats, because their bows were sharp and the men did not row as we do but paddled. They did not come near cnough for us to make ont their faces; all we saw was that there were four men in one boat; one man stood at the stern and three paddled. One of them wore something red. We saw them stand up, motion with

- See below, 0. 323. 
their hands, and heard them call twice, "Agai, Agai"; and then they turned about and paddled for the shore. I ordered white kerchiefs to be waved as an invitation for them to board our ship. This was done by a number of our men, but it did no good; the people in the boats paid no attention, proceeded shoreward, and entered the bay out of which they had come. We could not follow them because in the first place there was little wind and in the second place the small boat was fast and the larger one had not come very near us. We became convinced that some misfortune had happened to our men. ${ }^{46}$ Dementiev had been gone eight days, and during that time the weather was fair for returning and we stood close by. From the time that the boatswain left us we had hardly moved, and the weather was quiet. Surely something must have happened to them; otherwise they would have come. The action of the natives, their fear to come close to us, made us suspect that they had either killed our men or held them. We stood near the place the rest of the day; in the evening we kept offshore a bit but had a lantern at the stern so that in case our men came out they would see us.

The next morning at eleven we came about and coasted along the shore between north and west. By the end of July 26, we had come to latitude $5^{\circ} 2 \mathrm{I}^{\prime} \mathrm{N}$ and longitude from Vaua, according to our reckoning as we went along, $54^{\circ} \mathrm{Ir}^{\prime} .^{5}$ At this place and on July 27 we discussed whether we should still keep up the search. We had no small boat and therefore no means of sending a party ashore or bringing water and provisions on board. According to our reckoning in coming over we were nearly 2,000 knots from the Harbor of St. Peter and St. Paul, and we had no idea what kind of wind we should have. We had 45 casks of water, hardly enough for such a distance; we did not know whether they were full or partly empty (later it proved that we were seven short). Having taken all these circumstances into consideration, we-that is, I, Lieutenant Chikhachev, Navigator Vrange, Fleet Lieutenant Plautin, and Navigator Ivan Elagin-agreed not to continue on our course [not to follow the coast] but to start back at once for the Harbor of St. Peter and St. Paul. I enclose an accurate copy of our decision. ${ }^{6}$ At the time we realized that it was too soon for going back and, had it not been for our misfortunes, we should not have done so. Altogether we saw about 400 versts of the [American] coast.

Having decided, we put about and sailed for this harbor. On the way we had contrary winds from northwest and southwest almost continuously. We unexpectedly ran into some land [Adak Island] which was on our course and nearly lost the ship and all on board. We saw whales, sea lions, walruses, porpoises, birds, many white-backed ducks,

${ }^{5}$ According to the journal (Chapter VII, p. 297) the latitude is that of July 26 but the longitude that of July 27 .

${ }^{6}$ See below, p. 324.

sa See note on p. 311 . 
also another kind with a red crooked bill, and many different kinds of gulls. All along the coast the mountains are high, the shore steep, and the water deep.

The mountains along the shore which we first sighted were covered with growths of good-sized trees; some of the mountains had snow on them, increasing in quantity the farther north we went. From the place we turned back we could see in N3/4 E very high snow-covered mountains [Mt. Fairweather], far higher than any in Kamchatka. On August I, at five in the afternoon, we sighted land in NW $1 / 2$ W [SW end of Kenai Peninsula], about 30 knots distant, and towards the end of the day we took bearings of it. On this land there was a chain of snow-covered mountains, and we were of the opinion that this was a continuation of the land from which we had turned back a few days before, because in the interval we saw birds, gulls, ducks, also floating grass which grows along the shore. These we did not see on our outward voyage, when we were a considerable distance from land, except during two or three days when we ran into a little floating grass and observed one bird a day (not counting gray gulls and another kind of small bird that looks like the kestrel of the steppes, ${ }^{7}$ which we always saw far out). On account of this we kept a little south of the regular course and the fair wind. Beginning with July 31 the color of the water was different from what it is far out at sea, but we saw no land on that day, probably because of the fog. On August I and 2 we caught sight of it and at the end of the day took its bearings. On August 4 and 5 we took soundings and got bottom between 43 and 90 fathoms. Many birds flew about, and the color of the water indicated that we were near land; but we did not see it. During the night of August 6 we came to a bank where the water was only 30 fathoms and the bottom sandy and rocky, and we were therefore obliged to come about and go on an easterly course. The land which we sighted on August I is marked on the chart alongside of the date, and our daily positions are also indicated there. The winds were contrary, and the fresh water was decreasing.

On Angust I I consulted with the officers and with their consent gave the order that the crew should have cooked kasha ${ }^{8}$ once a day for two days and twice on the third day, and of drinking water no more than is necessary to quench the thirst. As to the officers, they were to have one cooked meal a day. When it rained the crew set buckets and other

7 According to Pallas, pustolga is the Russian name for the kestrel (Falco tinnun(ulus), which is common enough on the Russian steppes but does not occur in eastern Asia or in Naska. The bird seen by Chirikov must therefore be another species of small hawk or falcon, possibly Circus hudsonius, or more likely Falco columbarius, the $t y$ pical form of which has been recorded from the Prince William Sound region by (irinnell (Lniv' of California P'ubls, in Zool., Vol. 5, I910, P. 387) while the dark form (Falco C. suckliyi) prevails between Sitka and northern ('ahifornia. (L. Stejneger.) $8 \mathrm{~A}$ buckwheat mush. 
vessels to catch the water from the sails; and, although it was bitterish and tasted of tar, yet the men drank it gladly and said that it was good for the health and that the tar bitterness eured them of seurvy. In order that the men might not become too weak from lack of sufficient food I ordered that on those days when they had kasha just once they should be given (this did not apply to the officers and their servants) a eup of wine in addition to the usual allowance. As the head winds continued and the supply of water ran short without any hope of our getting more, the distance from the harbor being still considerable, I ordered that the crew should have kasha only every other day. The men took it in the right spirit, and on the days when they had no kasha they lived on biscuit and butter. On those days when additional food was allowed they had salt meat cooked in sea water. After September It the crew had cooked kasha but once a week, and on the other six days they lived on cold food. It was understood that if any one wished to use his allowance of drinking water for cooking his biscuit he night do so, but this would be the only warm food he would receive. These privations began to tell; many of the men came down with scurvy, and both officers and crew did their work under great difficulties. Some of the men were so feeble that they could not even come on deck. I began to fear that the worst might happen and therefore ordered that after September if the members of the crew should have daily two cups of wine and the petty officers one above the usual allowance.

About seven in the morning of September 4 we sighted land [1slands of Four Mountains], and at the end of the day we took the bearings of it. The reckoning on our returning voyage gave latitude $52^{\circ} 23^{\prime} \mathrm{N}$, longitude from Vaua $32^{\circ} 49^{\prime}$, distance on the rhumb E by $\mathrm{S} 9^{\circ} 48^{\prime} \mathrm{E}$, I, 186 knots, ${ }^{9}$ or $2,065^{1} / 2$ Russian versts. The land was full of mountains, the highest of which was covered with snow. At eight o'clock we came about to get away from the land and sailed on a course between $\mathrm{S}$ and $\mathrm{W}$ so that after we got by it we could continue on our regular course. On Septembcr 8 we had many indications of the nearness of land, namely birds and floating grass, and on that account between seven in the evening and seven in the morning we kept one or two points more to the south than usual, and after that we sailed on the regular course for a short time. At nine o'clock the fog set in and the signs of land were still plentiful, and I was again obliged to sail one point south of our regular course, or west by true compass. At the end of the seventh hour in the evening the wind, by God's merey, suddenly moderated. We took soundings and found bottom at 50 fathoms.

While we made ready to anchor the sea carried us into 30 fathoms and sandy bottom, and there we let go the small bower anchor and

9 These are the corrected tongitude and distance. The journal for September 4 (see p. 302 ) gives: longitude, $21^{\circ}{ }^{\circ} 0^{\prime}$, distance 770 knots. 
paid out a half cable. At the bow there was 28 fathoms and at the stern 24. We leard the breaking of the surf on the rocks, but the heavy log hid everything from sight. Towards nine in the morning, when it cleared a bit, we saw land to the west [Adak Island], about 200 fathoms away. The high mountains on it were bare of trees but covered with grass. The beach was irregular, the eastern part seemed lower and about 3 oo fathoms away. Near the shore, on both sides, there were many rocks above and below the water, and we could see the surf breaking over them. To the north no land was seen.

As we examined the shore we eaught sight of two men walking along the beach. We shouted to them in Russian and in the Kamchadal language to come aboard; a little later we heard human voices calling to us, but the breaking of the surf made so much noise that we could not make out what was said. Through the speaking trumpet and without it we again invited them to come. At ten o'clock seven men in seven small skin boats came near us and, after looking on for some time, went back to shore. In the afternoon (September 9) fourteen of these skin boats, one man in each, paddled up to our ship, and from them we secured one of the hats they wore (which was made of birch wood) and four arrows. They also gave us, wrapped up in seaweeds, some kind of mineral which I think is antimony (or stibnite), which I have sent to Bolsheretsk Post to be assayed by the assayer Gardebol; but up to this time I have not heard from him. They also gave us the roots of a grass with which they stuff their noses, and a few of these roots we brought with us for exhibition. As to how they came, their kind of boats, their dealings with us, and other such matters are written up in the journal.

At the eighth hour in the evening of September ro, the wind began to blow from the west, and, trusting to God's help, we attempted to get away from where we stood before it was too late. We started to heave in but while doing this drifted easterly to within 300 fathoms of the shore and wele in danger of being blown on it. I feared also that there might be submerged rocks on the west. I, therefore, ordered to cut the cable ( $3+$ fathoms of which was still out) at the hawse hole, to put on all sail, and to go SE. This was done witl God's help, but it was a narrow escape, for a strong wind blew off the mountains and from all directions.

The place where we were at anchor is in latitude $5^{\circ} 40^{\prime} \mathrm{N}$, distant from Vaua, reckoning from our returning point on the rhumb $\mathrm{E}$ by $\mathrm{S} 6^{\circ} 2 \mathrm{o}^{\prime} \mathrm{E}$, 852 knots, ${ }^{10}$ or 1,484 Russian versts. After we cleared the land we proceeded to sail a little more westerly than our laid-out course, but the liead winds greatly hindered us.

On September 21 , at eight in the evening, we noticed a small fish close to the ship. We took soundings and got 60 fathoms. We let the ship drift

${ }^{20}$ This is the corrected distance. The journal for September 9 (p. 302) gives $429 \mathrm{knots}$. 
and took more soundings and got the same depth with sandy bottom. There was no land in sight, probably because it was hidden by the darkness and fog; but we took no chances and stood out easterly for two hours and then hove to until three in the morming. when we proceeded on our course. At the end of the ninth hour in the morning, land [Agattu Island] was sighted on the rlumb W by N, but I knew that it was not yet Kamchatka. In the tenth hour we came about in order to clear it in the south, for the wind was favorable and we were keeping parallel with the shore between south and west. At the end of the day we took the bearings of the land, which was latitude $52^{\circ} 35^{\prime} \mathrm{N}$, distant from Vaua $95^{6}$ Russian versts, "reckoning from the returning point. The shore seemed broken up; here and there were mountains of no great size, but in the distance were high snow-covered mountains; but on the lower mountains and on the level there was no snow. At different times we noticed near the land four otters, the kind that live near Kamchatka, but which we had found nowhere else. We had the land in view until six in the morning of September 22. I am of the opinion that the land which we sighted on September 4,9 , and $2 \mathrm{I}$ is one and the same body, and it is probably due to the fog that we did not always see it. We had enough evidence of it in the shape of shore birds, different kinds of animals that keep close to land, and floating grasses. As we sailed south these signs either decreased or disappeared altogether. On our outward voyage, when we kept much farther south than on the inward, we saw hardly any of the above indications, aside from sea animals and a small amount of fairly broken-up sea grass and that rarely. I am inclined to think that all the land which we saw is one body because from August 6, when it disappeared from view, until September 4 , when we caught sight of it once more, there were signs that it was near. Another good bit of evidence to substantiate my theory is this: If the people we met with on September 9 were on an island it goes to show that the mainland is near, for how could they have come here otherwise? If it is not an island then it is the mainland itself. I cannot say for certain which it is.

By the end of September all members of the crew were down with scurvy; many of them could not stand their watch, and those that came on deck did so because it was necessary and not because they had the strength. Between September 16 and the time when we returned to the harbor six men died. Their names, as well as those who were left on the American coast, are recorded in the register. ${ }^{12}$ On the night of October 7 , at ten o'clock. Lieutenant Chikhachev died; on the next day Navigator Vrange and Fleet Master Michael Plautin passed away. Chikhachev could not stand his watch for three weeks before he died, and Plautin

"Equivalent to 549 knots. This would be the corrected distance. The journal for September 21 ( p. 307 ) gives 127 knots.

12 Not published in the present work. 
for two weeks. I was so ill with scurvy and so weak that I expected death any moment. From September $2 \mathrm{I}$ to the time that we reached port I was not able to come on deck. The ship was navigated by Ivan Elagin, who, although very ill, yet would not give in and because of dire necessity remained on deck almost continuously. I gave him such help as I could, for, thanks to God, my mind did not leave me. I worked out the courses from the $\log$ book and told him what to do. When, according to our reckoning on the outward voyage, we should have sighted Kamchatka but failed to do so, I ordered him to steer west close to the parallel of this harbor. Elagin has good judgment, and, if it had not been for him and the strength which God gave him, some great misfortune would have happened to the ship. Because of his great merits, good judgment, soberness, as well as for numerous difficult services, I promoted him to the rank of flect lieutenant, for he not only did the work of that office but ran the whole ship.

By the will of God we caught sight of Kamchatka at seven in the morning of October 8, and two days later, nine in the evening, we sailed into Avacha Bay and anchored. At the time we had but two barrels of water, which we had boiled out of sea water. At ten o'clock in the morning of October ro Professor Delisle de la Croyère died. With God's help we entered the Harbor of the Holy Apostles Peter and Paul on October I 2. At the time of this writing the Captain Commander has not yet arrived, and it is not known whether on account of some misfortune he has been obliged to winter somewhere. We had enough provisions on board both ships to last a half year in case of necessity.

Enclosed is a list of the crew (with their names) who are with the Captain Commander. ${ }^{13}$ I am submitting to the Admiralty College the journal of our voyage and a Mercator chart. According to our reckoning the number of degrees of longitude between Kamcluatka and America differs in going and coming, ${ }^{14}$ owing to the fact that during all the time that we were at sea we reckoned from only one base, the meridian of Vaua, and had no other base or way to correct by. The outward voyage and the land discovered on it are marked on the chart in black ink; and the return voyage, with the land sighted and its extension, in red ink. During such a long voyage and period of time it is impossible even for the best navigators to escape errors altogether. If a more accurate map is desired, the professors of the Academy can make it from the reckonings going and coming or in some other way from the log, journal, and chart. To what extent the error in longitude is due to the currents I cannot tell. In going we tried twice to determine the current but failed. Along the American coast there was a tidal current running up and down parallel

13 Not published in the present work.

14 Going, $61^{\circ} 51^{\prime}$; coming, $73^{\circ} 30^{\prime}$ (see Chirikov's report, above, p. 313; also Chapter VII, footnote 22, p. 308). 
to the shore. In returning we had no small boat and could not make a study of the tide.

It took six weeks to go from Kamchatka to America; these weeks were in the months of June and July (by the middle of June we were in latitude $46^{\circ} \mathrm{N}$ ), and yet it was as cold as our middle autumn. We had almost continuous logs; only occasionally about the middle of the day did the sun show itself. During these six weeks we had only three really fair days. The same kind of weather prevailed along the American coast, for while we were there the weather was not fair. In August and September, when we returned, we had many more clear days with the usual strong autumu winds. In my opinion the best time to navigate these seas is August and half of September and not June and July.

The instructions of the Admiralty College require that this report be sent in care of an officer who took part in the expedition. Unfortunately this is not possible, as the officers are dead; and therefore I am sending it in care of the marine Semeon Splavshikov, who will follow the winter overland route. This route as far as Yakutsk is not very safe because of the unsubdued natives living between Kamchatka and the Kolyma Post, who sometimes kill, plunder, or detain Russian travelers. If I were not to send it, I should be blamed for delay. For fear that these papers may not reach their destination $I$ intend, as soon as navigation to Okhotsk opens in the spring, to send to the Admiralty College exact copies of the report, journal, chart, list of the crew with their names, by a special messenger, the midshipman Yurlov. He will also take along the things we secured from the natives of the newly discovered land and hand them to the Admiralty College. I beg to add that in this journal the day of the month begins at noon as is customary in all naval journals.

To the Admiralty College from its humble servant.

December 7, 1741

\section{Captain Alexel Chirikov}

\section{ENClosures}

\section{INSTRUCTIONS TO BoAtswain Sidor SAVEleV}

On the igth of this month Acting Fleet Master and Navigator Dementiev went ashore and has not been heard of since, and it is possible some harm has come to him. We are now near the spot where he was sent, and I order you to go in the small boat to the place where he went. Take with you a carpenter and a calker and such tools as are needed for repairing the boat. When you come close to the shore, if you see no hostile natives or see Dementiev or some of his men, make a landing. If the boat is lamaged and can be repaired, set the carpenter and some of Dementiev's men to work; but do you come back at once and bring with you Dementiev and as many of his men as are not needed to do the repair work. If the boat is beyond repairs bring aboard as many men as your boat will hold, and 
for the others another trip will be made. If you find his party and boat in good condition, signal that fact to us by making two fires-the smoke of which we could see in day time and the flames at night. If the boat can be repaired, build three fires; it it cannot be, make four some little distance apart. But you are to come aboard with Dementiev and as many others as the boat will accommodate without overloading. Should you start back in the evening or at night, lay out the fires as best you can.

July $23,174 I$

Captain ChIRIKov

\section{Decision to Return}

On July 26, 1741, Captain Chirikov and the undersigned officers decided, owing to the loss of the two ship's boats with Dementiev and fourteen men, not to tollow out the original plan but to start for Kamchatka that very day. There were no more small boats on board, which made it impossible to examine the shore or to take on fiesh water. We had only 45 barrels of water, and this is a very small amount considering the long distance to Avacha, which is nearly 2,000 knots. We were not even sure that some of the water had not leaked out of the casks. We were equally in divubt as to the kind of winds we might have. Because of this shortage of water it was agreed not to examine the coast but to sail for Kamchatka so as not to suffer some great misfortune.

\section{Captain Alexel Chirikov Lieutenants $\left\{\begin{array}{l}\text { IvaN Chikhachev } \\ \text { Michael PlaUtin }\end{array}\right.$ Navigator IVAN ELAGIN}

\section{To the Admiralty College, A [Supplementary] Report}

Captain Commander Bering's instructions required us to examine the land which according to rumor lies opposite the Chukchi country, some distance to the east, also the last land we saw on our voyage, 956 versts from here [Agattu]. It is my humble duty as well as my great desire to secure full information about both of these lands. Of the company that was put under my command, that is to say the lieutenant, two navigators, constable, boatswain, and crew, some, by the will of God, have died, others were lost on the American coast (their names I have sent with my first report to the Admiralty College). At the present time I have only one man on board for navigating the ship, keeping the log, and mapping the coast, and that is the navigator Elagin. Although on the list of officers there is the name of the assistant navigator Yurlov (who was not taken along on the last expedition), yet he does not know his business. (It would seem that he was taken from a warelouse, where he acted as watchman, and put to study navigation, being then of an age when he could learn neither the theory nor the practice of it; and after a time. because of length of service, he was given the rank and pay of assistant navigator.) As to myself, I am quite unfit for sea duty. The scurvy is sleep in my system, and this it is difficult to shake off because of the 
heavy atmosphere and especially because of the poor and insufficient food. Twice on the return voyage I nearly died of the disease, the last time it was October 26. Now, by God's mercy, I am just able to sit up; my feet are drawn up and full of spots, and the teeth are loose in the gums. The drugs on hand are so old that they are worthless. A similar state of ill health exists anong the erew; some of them are not fit for sea service. From the enclosed lists ${ }^{15}$ may be seen how many officers and men we still have, how many were left ashore, how many we should have according to the regulations of the Admiralty College, and how nuany we need. The ship's rigging is in bad condition, and $\mathbf{I}$ attach the report ${ }^{16}$ made by the lower officers on that subject. We cannot replace it because we liave no other rigging. Taking all these things into consideration-my illness, shortage in men, food, material-it is impossible to put to sea for the purpose of making a detailed investigation of the above-mentioned lands, namely the one we discovered and the one opposite the Clukchi country. I am going to ask help from Captain Spanberg (who is now at Bolsheretsk with five ships). If he himself cannot take part in the expedition, he may be willing to give us a navigator or an assistant navigator and provisions, a list of which I have made out and sent to him. If I should receive assistance, even if only enough to repair my rigging, and if $I$ am not too ill I will do my duty and go to sea. If help should not come the best thing to do would be to take the men on board and go to Okhotsk or lakutsk and wait there for orders. It would perhaps be better to go on to Yakutsk so as not to use up the provisions at Okhotsk, where they could be used later for the expedition.

Should it be the wish of her Imperial Majesty for us to explore the newly discovered land as well as that part which is supposed to lie opposite the Chukchi country and (according to the instructions to Captain Commander Bering) to bring the inlabitants under Her Imperial Majesty's subjection, it would be necessary to increase the number of men on the ship to the full complement as laid down by the Admiralty College. How many men and of what rank they should be is indicated on the enclosed list. ${ }^{17}$ We need also new rigging, spare parts, and a number of other things. If I were at Yakutsk to hurry the material along it would reach here more quickly than by my waiting for it in these harbors. If I should receive no aid, I, with the assent of my officers, will do the best that we can for the interests of Her Imperial Majesty and will report to the Admiralty College.

According to the instructions of the Admiralty College we were required to bring to St. Petersburg a few inhabitants of the newly discovered land, or of land we might discover in the future. We could not

\footnotetext{
15 See p. 327.

16 Not published here.

17 See p. 320.
} 
persuade them to come, and to force them against their will without special instructions was dangerous. Not having any of these people with me and not knowing their language I can say little about them. It is not likely that they will come on board willingly, and I do not suppose Her Imperial Majesty would lave us use force. For that purpose a larger crew is necessary than we have had so far.

To the Admiralty College from its humble servant.

December $9,17+1$

\section{Captain AlexeI Chirikov}

\section{Enclosures}

List of the Complement of Men on My Ship, According to the Regulation of the Admiralty College, the Number on Hand (Besides Thuse Who Are Unfit for Sea Service), the Number Needed, and Their Rank

$$
\text { Rank }
$$

Captain

Lieutenant

Navigator

Assistant navigators

Surgeon

Assistant surgeon

Surgeon's apprentices

Boatswain

Boatswain's mates

Quartermasters

Purser

Assistant constable

Cannoneers

Copyist

Assistant storekeeper

Sailors

Calkers

Sailmakers

Carpenters

Coopers

Corporal

Soldiers

Drummer

Monk

Total

\section{Complement \\ Present}

I

\begin{tabular}{|c|c|}
\hline 1 & I \\
\hline I & \\
\hline I & I \\
\hline 2 & \\
\hline I & \\
\hline I & I \\
\hline 2 & I \\
\hline I & \\
\hline 2 & 2 \\
\hline 2 & I \\
\hline I & I \\
\hline I & \\
\hline 6 & 4 \\
\hline I & 1 \\
\hline I & I \\
\hline 12 & 8 \\
\hline 3 & \\
\hline 3 & 2 \\
\hline 3 & 3 \\
\hline 3 & 2 \\
\hline I & \\
\hline 24 & 24 \\
\hline I & I \\
\hline I & \\
\hline 75 & 54 \\
\hline
\end{tabular}

In addition we need a boatswain's mate in place of Evdokim Fedorov. 
List of Admilty MeN, Artillerists, and Siberians of Vapious Ranks Who Are Registered 1N the Harbor of the Holy Apostles St. Peter and St. PaCl. Some of these men are on land and others absent

\begin{tabular}{|c|c|c|c|c|c|c|c|c|}
\hline \multirow{2}{*}{\multicolumn{2}{|c|}{ Rank }} & \multirow{2}{*}{ Registered } & \multicolumn{4}{|c|}{$.1 b \operatorname{sent}$} & \multirow{2}{*}{ Absent } & \multirow{2}{*}{ Present } \\
\hline & & & (a) & (b) & (c) & (d) & & \\
\hline \multirow{12}{*}{\multicolumn{2}{|c|}{$\begin{array}{l}\text { Captain } \\
\text { Ensigns } \\
\text { Assistant surgeon } \\
\text { Surgeon's apprentice } \\
\text { Navigator } \\
\text { Assistant navigator } \\
\text { Assistant storekeeper } \\
\text { Midshipman } \\
\text { Clerk } \\
\text { Copyist } \\
\text { Boatswain's mates } \\
\text { Registrars } \\
\text { Cannoneer corporal }\end{array}$}} & I & & & & & & I \\
\hline & & 2 & & & & & & 2 \\
\hline & & I & & & & & & I \\
\hline & & & & & & & & I \\
\hline & & I & & & & & & I \\
\hline & & 1 & & & & & & I \\
\hline & & $\begin{array}{l}\mathrm{I} \\
\mathrm{I}\end{array}$ & & & & & & $\begin{array}{l}1 \\
1\end{array}$ \\
\hline & & I & & & & & & I \\
\hline & & I & & & & & & I \\
\hline & & 2 & & & & & & 2 \\
\hline & & 2 & & & & & & 2 \\
\hline & & $\begin{array}{l}1 \\
2\end{array}$ & & & & & & $\begin{array}{l}1 \\
2\end{array}$ \\
\hline \multirow{3}{*}{$\begin{array}{l}\text { Cannoneers } \\
\text { Quartermaster } \\
\text { Sailors }\end{array}$} & 2nd class & I & & & & & & I \\
\hline & & 1 & & & & & & 1 \\
\hline & $\begin{array}{l}\text { Ist class } \\
\text { 2nd class }\end{array}$ & 3 & & & & & & 3 \\
\hline \multirow{2}{*}{$\begin{array}{l}\text { Ranked as } \\
\text { sailors }\end{array}$} & Siberian car- & 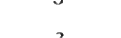 & & & & & & 3 \\
\hline & $\begin{array}{l}\text { penters } \\
\text { Workman }\end{array}$ & $\begin{array}{l}2 \\
1\end{array}$ & & & & & & $\frac{2}{1}$ \\
\hline \multirow{2}{*}{\multicolumn{2}{|c|}{$\begin{array}{l}\text { Assistant corporal } \\
\text { Soldiers }\end{array}$}} & 1 & & & & & & I \\
\hline & & 2 & I & & & & I & 1 \\
\hline & $\begin{array}{l}\text { Carpenters } \\
\text { Turner }\end{array}$ & 4 & & & & & & $\begin{array}{l}4 \\
1\end{array}$ \\
\hline & Blacksmiths & & & & & & & \\
\hline & $\begin{array}{l}\text { Foreman } \\
\text { Smiths }\end{array}$ & $\begin{array}{l}1 \\
2\end{array}$ & & & & & & I \\
\hline \multirow{5}{*}{ Admiralty } & Cooper & 1 & & & & & & $\begin{array}{l}2 \\
1\end{array}$ \\
\hline & $\begin{array}{l}\text { Assistant } \\
\text { cooper and }\end{array}$ & & & & & & & \\
\hline & $\begin{array}{l}\text { cooper and } \\
\text { Siberian }\end{array}$ & & & & & & & \\
\hline & carpenter & I & & & & & & I \\
\hline & Calkers & 2 & & & & & & 2 \\
\hline \multirow{2}{*}{$\begin{array}{l}\text { Secretaries } \\
\text { Siberian }\end{array}$} & Sallmakers & $\begin{array}{l}3 \\
2\end{array}$ & & & & & & $\begin{array}{l}3 \\
2\end{array}$ \\
\hline & Drummer & I & & & & & & I \\
\hline garrison & Soldiers & I0 & & & 1 & & 1 & I 8 \\
\hline \multirow{3}{*}{\multicolumn{2}{|c|}{$\begin{array}{l}\text { From lakutsk regiment } \\
\text { Siberian carpenters } \\
\text { Employees of Kamchatka posts }\end{array}$}} & 8 & & I & & & I & 7 \\
\hline & & 4 & & & I & & I & 3 \\
\hline & & 2 & & & & 2 & 2 & \\
\hline \multicolumn{2}{|l|}{ Total } & 83 & I & I & 2 & 2 & 6 & 77 \\
\hline
\end{tabular}

Captain CHiRikov

(a) Sent to St. Petersburg with a report.

(b) Sent to buy deer.

(c) Guard the deer herds at Bolsheretsk.

(d) In Lower Kamchatka Post. 


\section{CHAP'TER IX}

\section{THE END OF THF, EXPEDITION}

Bering's second expedition came officially to an end in 1749 , although very little in the line of exploration and discovery was done after 1742. Counting from the time when Peter the Great signed the instructions for the first expedition, twenty-five years had been spent in the attempt to solve the mysteries of the North Pacific and the Arctic. Nany of them were solved. It was proved that a northeast passage was impracticable; that Novaya Zemlya was not a peninsula; that the Asiatic coast extended much farther to the eastward than was supposed; that Terra de Ieso, Company Land, and Gama Land, as pictured by the cartographers, did not exist; that Japan was an island; and that the American coast ran in a northwesterly direction from Cape Blanco. In brief, it made clear all the points which were in doubt at the time of Peter's last visit to Paris.

Unfortunately, the one question, the important question, the raison d'être of these undertakings, whether Asia and America were united, was not answered,owing to the fact that the explorers along the Arctic coast were unable to go from the Kolyma to the Anadyr. The same questions were asked after the second expedition as at the end of the first expedition; and they continued to be asked until another Russian, Baron Ferdinand Wrangell, answered them during the winters of 1821 and I823, when he walked along the Arctic shore from the Kolyma to Kolyuchin Island, a place that had been charted by Billings, of the Russian Navy, in I79I. So, after all, Russia did solve that problem, and to her belongs the credit.

It may be of some interest to the reader to know what became of the officers who survived the hard royage. Chirikor passed the winter of $1741-1742$ at Petroparlovsk, and in the spring he 
sailed on the St. Paul to the eastward in the hope of finding Bering and locating the American coast opposite East Cape. He failed in both of his objects largely because of the poor health of his men, the poor equipment, and the unseaworthiness of his vessel. He lid, however, reach the islands of Attu and Atka, the first being a new discovery, the second probably a resighting of a11 island seen by Bering on September 25, 1741. During the summer of $17+2$ he sailed for Okhotsk, where he left the St. Paul, and from there he proceeded inland. He remained in Siberia as the head of the expedition until 1745 , when he was asked to come to the capital and was promoted to the rank of captain commander; but he died in 1748 as a result of a disease contracted on the voyage. Waxel left Kamchatka in 1743 for Okhotsk, and from there he went to Yakutsk and Yeniseisk, where he found Chirikov. When the latter was called to St. Petersburg, llaxel assumed command until he was summoned home. There he was made captain of the first rank, and when he died his widow was granted a pension of 2,000 rubles.

Khitroy was made rear-admiral in 1753 . Ortsin in 1757 was in command of the Poltar'a, holding at the time the rank of oberster Kriegskommisar.

All others who took part in these expeditions were advanced one grade in rank, dating from July I5, I744, and their wages were paid accordingly. The Senate requested the Admiralty College to reward them as it should seem best.

When Bering's death became known at Kamchatka his private property was sold at auction and the proceeds, about I, ooo rubles, were sent to his family. Whatever wages were due him at the time of his death were also paid over to his wife and children. In addition the Senate voted him a reward of 5,000 rubles. 


\title{
NOTE TO ACCONPANY THE CHART OF THE VOYAGE OF BERING AND CHIRIKOV FROM KAMCHATKA TO THE ALASKAN COAST AND RETURN, 1741
}

\author{
By the late \\ ELLSWORTH P. BERTHOLF \\ Captain-Commandant U. S. Coast Guard (retired)
}

The tracks of both vessels, as laid down on the accompanying chart (Pl. I), show the approximate noon positions throughout the voyage and indicate the several portions of the coast and the islands sighted or examined by the navigators. ${ }^{2}$

\section{The Jonnt Voyage of the "St. Peter" and the "St. Paul"}

The brigs St. Peter and St. Pautl, commanded respectively by Captain Commander Bering and Captain Chirikov, sailed out of Avacha Bay, Kamchatka, on June 4, I74I, and entered on their eventful voyage of discovery. Bering's instructions from the Admiralty College required that he should steer SE by E, true, until he reached latitude $46^{\circ}$ in order to locate Juande Gama Land, which, according to the map of the astronomer Louis Delisle de la Croyère, extended to the 47 th parallel of north latitude. If no land was sighted on that course he was to steer $\mathrm{E}$ by $\mathrm{N}$, true, until he came to the American continent. The wind being fair, the ships were able to make good the true course, SE by E, and found themselves on June 12 in latitude $46^{\circ}$. No land being

1 The following material (translated by Professor F. A. Golder) was made use of in constructing the chart. In connection with the St. Peter: (a) Yushin's log book; (b) Khitrov's tog book; (c) the report of Lieutenant Waxel, who succeeded to the command after Bering's death; and (d) the journal of the naturalist Steller (published in Vol. II of the present work). In connection with the St. Paul: (a) the log book, (b) the daily journal, and (c) the report of Captain Chirikov. [Captain Bertholf, shortly before his sudden death in November, I92r, was able to revise a proof of the chart. As it now stands it represents his final judgment.-EDit. Note.]

2 In the course of several tours of duty in Alaskan waters, as executive and commanding officer in the U.S. Revenue Cutter Service and the U. S. Coast Guard, the writer became familiar with the coast and islands of that territory, and this knowledge has been of great help to him in identifying the land sighted by Bering and Chirikov. 
sighted and no bottom being found at 100 fathoms, Bering, on

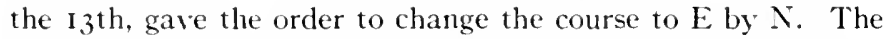
wind was now unfarorable, but the ressels continued in company until the 2oth, when stormy weather overtook them and they became separated during the night. Although up to this time the ressels had exchanged signals almost daily and had frequently spoken to each other and compared positions, nevertheless the noon positions recorded in the log books of the two vessels do not always agree even on those days when they both obtained observations of the sun. On June ro both ressels took observations within sight of each other, but the St. Peter's log records the latitude as $47^{\circ} 29^{\prime}$, while the St. Paul's log gives the latitude as $47^{\circ} 45^{\prime}$. In plotting the noon position of the ressels while in company we have therefore averaged the positions recorded in the two log books.

On June 20 the wind was strong from the eastward with a heavy sea running, and both vessels were under short sail. The St. Peter's $\log$ for that day records that at 10 P.s. the St. Paul bore XW distant 2 miles, and that at I A.M. the latter was no longer within sight, in consequence of which the St. Peter hove to. The $\log$ of the St. Paul for June 20 records that at 3 A.M. the St. Peter was Io miles distant, and at 5 A.M. she began to disappear from sight, whereupon the St. Paul hove to, and both vessels, being hove to on the port tack, drifted to the southward and westward. The following day the weather moderated, and in accordance with Bering's previous instructions each vessel began to search for the other in the direction in which the other was last seen. Bering believed the St. Paul to be northwest of him and began his search in that direction. Chirikor, however, reckoned the St. Peter to be north-northeast of him and endeavored to search in that direction, but as the wind was from that same quarter the St. Paul made little progress.

\section{The Separate Voyalie of the "St. Peter"}

Bering searched for the St. Paul until the 22nd and then resumed the voyage; but, after taking council with his officers, he 
decided to shape again his course to the southward as far as latitude $46^{\circ}$ in order to make another attempt to locate the elusive Gama Land. Noon of the $25^{\text {th }}$ found the ship in latitude $45^{\circ} 16^{\prime}$, with clear weather and no land in sight. This finally convinced Bering that the map of Delisle de la Croyère was incorrect and that Juan de Gama Land did not exist, and consequently he changed the course to $\mathrm{E}$ by $\mathrm{N}$, true. He had fair winds and made good progress, but no land being sighted by July 6 he began to keep more to the northward. On July I4, being fearful of the water supply, a council of the officers was called, and it was decided to steer more towards the north, in the hope of reaching some land where the empty water casks might be refilled.

\section{DISCOVERY OF LAND}

At noon July 16 (the end of the log book day ${ }^{3}$ ), the ship was in latitude $58^{\circ} 17^{\prime}$ by observation. Thirty minutes later land was sighted, high mountains covered with snow, and at I P.M., July 17 , a particularly high snow-covered mountain bore $\mathrm{N}$ by II. This was Mt. St. Elias, Bering's first glimpse of the American continent, at a distance of about 120 nautical miles. The longitude according to the reckoning was $151^{\circ} 26^{\prime} \mathrm{IV}$, but this was more than $9^{\circ}$ in error, the correct approximate longitude of the St. Peter at noon July 16 having been $142^{\circ} \mathrm{Io}^{\prime} \mathrm{IV}$. These early navigators were sailing unknown seas and had no means of knowing that during the greater part of their voyage a current set them constantly to the eastward. The errors in reckoning the ship's position were due in small part to crude methods and lack of instruments, and in a larger part to the unknown currents. In plotting the positions of Bering and Chirikov we have endeavored to make due allowance for these currents.

\footnotetext{
${ }^{3}$ While the civil day begins at midnight and ends the following midnight, the astronomical day is reckoned from noon to noon. The log books of the St. Peter and the St. Paul were kept according to the astronomical date, and the log date of July 16 begins at noon July 15 and ends at noon July 16 , The civil date coincides with the log date during the hours of the forenoon, but during the hours of the afternoon the log date is one day in advance of the civil date. The St. Peter's log lowk records that land was sighted July 17 at 12.30 P.M., or 30 minutes after that day began; the civil date was July 16, I2.30 P.M. (Russian ealendar).
} 
In approaching land the vessel made slow progress against the light head winds prevailing, and on the 18 th foggy weather obliged Bering to proceed with caution. No land was seen that day, although there were many indications that the shore was close aboard. At 8 P.M. on the $19^{\text {th }}$ the fog lifted, and the St. Peter fourid herself in the bight to the eastward of Kayak Island, with the southern point of the island and its detached Pinnacle Rock in plain view bearing SW by $\mathrm{W}$. During the night the ship was worked around the point, and on the morning of the 2 oth the St. Peter dropped her anchor on the west side of Kayak Island about midway of its length. Bering named the island St. Elias, and the southern point Cape St. Elias. The two boats were put overboard, and men were sent ashore to fill the empty casks. Khitroy sounded out the passage beeween Kayak and Wingham Islands to find sheltered anchorage in case of need; he also landed and examined Wingham Island.

\section{SKIRTING TIIE ALASKAN COAST}

The next morning at daybreak, the wind being fair, Bering, taking into consideration the lateness of the season and the signs of scurvy among the crew, gave orders to weigh anchor and begin the return voyage. After leaving Kayak Island early in the morning of July 21, the St. Peter made Cape Hinchinbrook, opened out the passage into Prince Villiam Sound, and then hauled to the southward along Montague Island. The officers took bearings of the Mooded Islands, and at noon of the 22 nd the ship was just below Cape Cleare and about 25 miles distant. The weather being thick no land was seen on the 23 rd, 24 th, and $25^{\text {th }}$, although on the $24^{\text {th }}$ the course was changed more to the westward in order to draw near to land. At 4 A.M. on July 26 they suddenly made high land bearing NE by $\mathrm{N}$ distant about 8 miles and got bottom at 35 fathoms. This was Black Point, the southern point of Sitkalidak Island. ${ }^{4}$ After sighting Black Point, the St. Peter stood about due south until noon, covering a

- It has been stated that Bering named the point Cape St. Hermogenes, but no mention of this is made in any of the ship's records or in Steller's diary. 
distance of some 32 miles, and then hauled more to the westward until noon of the 27 th. Frequent soundings were taken and the water shoaled to 35 fathoms. The weather being thick, no land was seen after leaving Black Point, but at noon of the 27 th Bering decided that they were getting too near the land and hauled to the southward and eastward to work offshore until the weather cleared. $^{5}$

From noon of the 27 th until noon of the 3 Ist the St. Peter stood off shore in a general southeasterly direction. On the 3 Ist the weather cleared, and shortly before noon the course was changed to the northward and westward in order to get in touch with the land. Towards evening of August 2, a heavy fog set in, and the ressel was again headed offshore as the water had shoaled to 30 fathoms. At I A.M. the fog temporarily cleared and disclosed land close aboard bearing SE by $S$. The log records that a sounding shoaled 20 fathoms, whereupon the ship was put about and anchored in 18 fathoms to await daylight. Steller records this incident in his diary as follows: "About one o'clock in the night it was discovered on heaving the lead that the boat was in 4

${ }^{5} \mathrm{Mr}$. George Davidson in his "The Tracks and Landfalls of Bering and Chirikof, on the Northwest Coast of America," San Francisco, Igor, concluded that the St. Peter, after leaving Black Point, passed through Douglas Channel between the Trinity Islands and the southern end of Kodiak Island. There is nothing in the original records to support that conclusion, while there is much evidence to the contrary. After sighting Black Point at 4 A.M., July 26, the St. Peler made 32 miles due south up to noon and was then some 25 miles to the eastward of Douglas Channel. From this point the vessel turned to the westward, and if she did indeed pass through this channel she must have done so between noon and 9 P.M. because at that hour the course was changed to WSW, true. From noon to I P.M. the St. Peler made SW by S, true; from I to 2 P.M., SW $l_{2} W$, true; from 2 to 3 P.M.. W by $S$, true; and from 3 to 9 EP.M.. W $12 S$, true, covering from noon until 9 P.M. a distance of 29 miles according to the log. All this time soundings were taken frequently, but no depth less than 35 fathoms is recorded. As the water in Douglas Channel shoals to 7 fathoms, it is clear the ship could not have reached the narrowest part of the channel by 9 P.M. From 9 P.M. to I I P.M. the course was IVSW, true; from I 1 P.M. until daylight the course was generally SW, true; and at midnight they sounded in 40 fathoms. If the exact position of the St. Peter at noon on the $26 \mathrm{th}$ was such that the courses steered between noon and 9 P.M. carried her in the direction of Douglas Channel, then at 9 P.M. she must have been in the immediate vicinity of the eastern entrance to the channel, because the soundings show she had not passed through the channel up to 9 P.M. Being in that position at 9 P.M., the course steered after that hour would have put the ship ashore on the Trinity Islands.

By plotting on the modern chart the hourly runs of the St. Peter from 4 A.M., July 26 , until noou of July 27 , making due allowance for the tides and currents and 
fathoms of water, but it was otherwise reported to the captain. Gradually the ship worked out of this into 18 and 20 fathoms, where anchor was dropped to wait for daylight."

This was Chirikov Island, and at daylight the bearings showed the ship was anchored about $1 / 2$ miles off the northern end. They named this island Tumannoi (foggy). Towards evening of the 3 rd the anchor was raised, and the St. Peter stood to the northward and westward. At 3 P.M. of August $4 \mathrm{Mt}$. Chiginigak was sighted, and at 8 P.I. the officers made out the mainland from Cape Providence to Chignik Bay, with Sutwik Island in plain riew. The ressel was now working to the southward; but the wind had fallen to light variable airs, and she made little progress during the night. In the morning the wind freshened from the eastward; and at noon August 4 , according to the bearings, the St. Peter was 5 miles to the eastward of Anowik Island of the Semidi group. The largest and most northerly island of the group must have been hidden by the fog, as both the log and IIaxel's report state that only five islands were seen. Owing to the fog, unsteady winds, and numerous islands, Bering decided to work off-

noting at the proper intervals the soundings recorded in the log, it at once becomes apparent that the vessel passed to the southward of the Trinity Islands and at noon of the 27 th was about 15 miles south of Tugidak Island. Mr. Davidson states that at night the vessel found herself in shoal water, tossed by heavy current rips. This would indeed have been the case had she passed through Douglas Channel with the SE storm prevailing, but there is no entry in the log indieating any unusual conditions. At 7 and 8 P.s. the $\log$ records depths of 35 and 40 fathoms, and at 9 P.... is the following entry: "Sounded, but no bottom. We are in danger of running on sand banks and therefore cannot keep close to land, hence have kept off a few points." At that hour the course was changed to WSW, true; and at midnight, having found bottom at 40 fathoms, the vessel was hauled off to SW, true. Waxel makes no mention of unusually shoal water at this time, and Steller, who was an exceptionally keen observer and painstaking recorder of events. makes only the following comment in his diary covering the $26 \mathrm{th}$ and $27 \mathrm{th}$ : "These gentlemen (the navigators) thought it necessary alway's to hug the coast, instead of which it might have been better after sailing a hundred versts to try and go north one or two degrees. Their navigation and a small storm drove us during the night of July. 27 on a bank 50 fathoms under water, that stretehed out from the shore into the sea, but the land itself, to our great fortune, was so distant as not to be seen." Steller apparently seized every opportunity to criticize Bering and the navigators. On more than one occasion he recites that shoal water and other unusual events were kept from the knowledge of the Captain and suppressed from the log, and it is fair to assume that if the St. Peter had passed through Douglas Channel on the stormy night of the $27 \mathrm{th}$. Steller would not have failed to note the unusual conditions and record them in his diary. 
shore and, after sighting the Semidi Islands, stood to the southward until August Io when he concluded to work to the westward along the 53rd parallel of latitude. But he now experienced a succession of head winds and storms and made so little progress on the homeward voyage that on the 27 th he again became troubled over the water supply and called a council of the officers. There were but 25 casks of fresh water remaining in the hold, and if the westerly winds continued for any length of time they could not hope to reach Avacha Bay before the water supply was exhausted. According to the reckoning land was about 240 miles $^{6}$ distant, and it was agreed "for safety's sake, to go nearer the land with a view to finding good anchorage where we might take on waterenough to last until our return so that in case of head winds we should not suffer extremely:"

\section{LANDING ON THE SHUMAGIN ISLANDS}

Accordingly, on August 27, the ship was headed to the northward; on the 29 th, shortly after daylight, a group of islands came into view, and on the afternoon of the 3 oth the St. Peter anchored between Near Island and Nagai Island of the Shumagin group. The following morning the task of filling the empty water casks was begun, and Khitrov was sent ashore to examine the land in the vicinity of a fire.which had been seen the night before. ${ }^{7}$ Steller went ashore with the water party to pursue his studies, the results of which he has minutely recorded in his diary.

On September I the work of filling the water casks was completed, and the sick men were brought on board in the longboat; but the wind had freshened and kicked up a sea, and Khitrov, who had landed in the small boat and extended his explorations some distance along the shores of Nagai Island, was unable to return to the ship. Towards noon of the 2 nd the weather moder-

6 "60 German miles."

"There were now ten of the ship's company down with the scurvy, and these men were sent ashore for rest and fresh air; but one of the sick seamen, Nikita Shumagin, died just after he was landed. He was buried on shore, and in his memory Bering named the island Shumagin. But the name has come down to us as applied to this entire group, and the particular island which Bering named Shumagin is now known as Nagai Istand. 
ated somewhat, and the longboat was sent ashore for Khitrov and his party, but, owing to the fog and squally weather, the party did not return until the forenoon of the 3 rd. Khitrov reported finding the place where the fire had been and many signs of human beings but had seen no people.

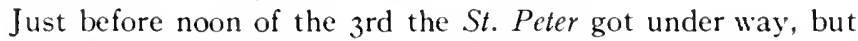
the wind fell to light airs from the SIV, and, being unable to work out to sea, Bering anchored off the north end of Bird Island on

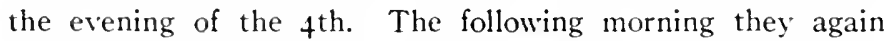
attempted to get to sea; but the wind was baffling and the current against them, and in the afternoon of the $5^{\text {th }}$ Bering again anchored close to Bird Island. Scarcely had the anchor been dropped when human voices were heard from the shore; soon two baidarkas, or skin boats, with one man in each, approached the St. Peter, and Bering and his ship's company had their first glimpse of the people who inhabited the land they had discovered. The longboat was lowered, and a party went ashore to distribute presents and interview the natives. The account of this incident, as given in the $\log$ and in Steller's diary, will be found very interesting.

On the afternoon of the 6 th another attempt was made to get to sea; but it was unsuccessful, and the St. Peter was obliged to return to the anchorage off Bird Island. The following morning the St. Peter sailed around the northern end of the island, passed out to sea through Otter Strait between Bird and Chernabura Islands, and by noon was clear of the land, standing to the southward. This course was held until the 8 th, when Bering again turned to the westward along the $53 \mathrm{rd}$ parallel. The wind still held from the westward and the progress towards Avacha was necessarily slow.

\section{LANDFALL OF ADAK AND ATKA ISLANDS}

On September 22 an observation of the sun gave their latitude as $50^{\circ} 27^{\prime}$, and the ressel was headed more to the northward to work towards the 53 rd parallel. On the $24^{\text {th }}$ they got another sight of the sun at noon and found themselves in latitude $5 \mathrm{I}^{\circ}$ 
30'. A few hours later. 3 P.M. of September 25 , land was signted; one point of land bore $\mathrm{IV}$ by $\mathrm{S}$, another point bore ENE, and a high snow-covered mountain, which they concluded was on the mainland, bore NIV by $W 1 / 2 W$. They sounded and got no bottom, but nevertheless the ship was put about and pointed off shore. According to our adjustments for errors in reckoning and currents, the St. Peter was in longitude $175^{\circ} 23^{\prime} \mathrm{W}$, and the snow-covered mountain was the 5,000-foot peak on Great Sitkin Island. The land seen to the westward was the south shore of Adak Island or one of the small islands close by, and to the eastward they saw the shores of Atka Island. No name was given in the $\log$ to the land sighted on the 25 th.

\section{BESET BY STORMS AND SCURVY}

A series of westerly gales now set in which continued with varying violence, driving the ship to the eastward, until October 11. On September 30 a particularly violent gale broke upon the little craft and rendered her all but helpless for two days. Steller records that "We had never experienced the like of it before, and it is hard to even imagine it. We expected to be smashed to pieces any minute; we could neither stand, sit, nor lie down. No one stood his watch, and the storm drove the ship where it willed. Half of our crew were sick and feeble, and the other half were on their feet only because they had to be, but on account of the storm and the violent motion of boat were ont of their heads."

On October I I the weather moderated and a noon observation of the sun was obtained. The St. Peter was in latitude $48^{\circ} 15^{\prime}$, longitude $168^{\circ} 25^{\prime} \mathrm{W}$, having been driven some $35^{\circ}$ miles southeast from the landfall of September 25. On October 12 they made fair progress to the westward, but another storm forced them to the northward until the 15 th, when the wind again permitted working to the westward. The scurvy was now in full swing; 32 of the ship's company were ill, several had died, and deaths were occurring almost daily. Bering himself was affected and, although he had found some temporary relief in the antiscorbutic plants gathered by Steller on the Shumagin Islands, he gradually 
grew worse. On October I 8 he was unable to leave his bed and from that date was carried on the sick tist.

\section{LANDFALLS OF KISKA AND BULDIR ISLANDS}

On October 2.3 the course was changed more to the northward in order to regain the 5.3 rd parallel; and at $8 \mathrm{~A} . \mathrm{M}$. on the $25^{\text {th }}$ a high, rocky, and treeless island was sighted in the northwest which IVaxel in his report states they named St. Markiana. This was Kiska Island, the approximate position of the St. Peter being latitude $5 \mathrm{I}^{\circ} \mathrm{I} \mathrm{I}^{\prime}$, longitude $\mathrm{I} 78^{\circ} 23^{\prime} \mathrm{E}$.

Passing to the northward and westward beyond Kiska Island the St. Peter encountered the heary tide rips frequently met with in that vicinity, and at daylight on October 28 was close to Buldir Island. Steller records in his diary that "it was quite evident we were in a strait because the waves, even in stormy weather, were not so high." Early in the morning of the 28th signs of land were observed; and, the weather being foggy, the vessel was put under short sail. Shortly after daylight the fog cleared and disclosed a small island directly in the path of the vessel not more than 3 miles distant. The log records that early in the morning one of the ship's company, Stephen Buldirev, died, and his body was lowered into the sea after land was sighted. Waxel states in his report that they named the island St. Stephen; the modern name is Buldir.

\section{LANDING ON BERING ISLAND}

The wind was now fair, and the St. Peter steered between IV and IV NVV, true, until Io A.M. the following morning, October 29, when another island was sighted which they named St. Abraham. This was the eastern of the Semichi Islands. The St. Peter passed to the northward of these islands and the island of Attu, and on the morning of November 4 the eastern island of the Commander group was sighted. At first it was believed the land was Kamchatka, but after rounding the southern point they realized their error. By this time conditions on board were pitiful. Twelve of the crew had already died, and of the remaining 65 Waxel reports 
that there were only eight who, with great pain, could help themselves, and only three of these were able to be on deck. Bering called into consultation such of the officers and men as were able to drag themselves into the cabin; and, being convinced they would not be able to handle the ship in the event of a storm, it was decided immediately to seek an anchorage where they might winter on shore and rid themselves of the dreadful disease that had gripped the ship's company.

The St. Peter was therefore worked over to Bering Island, and on November 6 a small anchor was dropped in 12 fathoms of water. The cable parted, and another small anchor was let go; but this cable parted also, and by the time the heavy anchor could be got overboard to hold the ship the fresh easterly breeze had carried her into $4 \frac{1}{2}$ fathoms, close to the shore. The longboat was lowered, and the task of getting the sick men ashore was begun; but with the very few men who were able to move about this was not completed until the $5_{5}$ th, and meanwhile the scurvy had claimed seven more victims.

A narrow section of sandy beach was discovered near the camp, and it was decided to haul the St. Peter ashore at this place, where they might secure her against the winter storms. It was found, however, that there was no sufficient strength left in the crew even to raise the anchor, and the ship remained where they left her until November 28, when a heavy gale drove her ashore and wrecked her on the very beach where they had planned to lay her up.

Bering was very ill when he was carried ashore on November 8 and realized that he could not last many days; but he retained his mind and power of speech and continued to direct affairs to the end. At 2 o'clock in the morning of December 8, I74I, he passed away, and his comrades reverently interred his remains on the island that bears his name and marked the spot with a cross.

With the fresh water and fresh meat which they were able to procure on shore the health of the men rapidly improved, and by Christmas most of them were on their feet again. As they 
regained their strength Waxel, who had succeeded to the command, sent out exploring parties, which reported that they were on an island and that the Kamchatka coast was not far distant.

\section{RETURN TO PETROPAVLOVSK}

In the spring they began to break up the St. Peter to build a small craft to carry them to the mainland. On August io the boat was launched, and preparations were made to leave the island. This boat was 36 feet long, with a beam of 12 feet and a depth of 5/4 feet, sloop-rigged with a bowsprit. They named her the St. Peter and in this small craft Waxel loaded his 45 men together with food, water, and baggage, and set sail from Bering Island on August 13. On the 15th the hooker began to leak badly; but the carpenter managed to patch up the hole, and they kept on. On August 27 they sailed into the harbor of Petropavlovsk. Of the 77 officers and men who left on the St. Peter fourteen months before only 45 returned, and these had suffered shipwreck and untold hardships; but their misfortunes did not end with their safe return, for they soon learned that they had been given up as lost and that the personal property they had left behind had been appropriated by the inhabitants of Avacha! Truly the lot of those early explorers was not altogether a happy one.

\section{The Separate Voyage of the "St. Paul"}

When the weather moderated on the morning of June $2 \mathrm{I}$, Chirikov reckoned that the St. Peter was NNE from him and began his search in that direction; but the wind was ahead, and little progress could be made. On the 23rd he gave up the search and resumed the $\mathrm{E}$ by $\mathrm{N}$ course agreed upon. On July I, no land having been sighted, Chirikov steered more to the northward. Both vessels had been favored with fair winds since they parted company; but, as Bering had stood south to the $45^{\text {th }}$ parallel before resuming the easterly course, the St. Paul was considerably in advance of the St. Peter, and Chirikov's noon position on July I was within 30 miles of Bering's noon position on July 8. 


\section{DISCOYERY OF LAND}

On July i4 large numbers of shore ducks and gulls indicated that land was not far distant, and at daybreak on the $15^{\text {th }}$ the land to the northward of Dixon's Entrance was sighted. At 3 A.M. Capes Addington and Bartolome were in plain sight; but, the wind being very light, it was not until noon that the St. Paul could be worked in under the shores of Cape Addington. In the afternoon of the 16 th a boat was sent to examine the bay between the two capes; but, finding the anchorage unprotected from the west and south winds, Chirikor stood offshore when the breeze freshened at sundown. At daylight the course was changed to the northward, and the St. Paul passed outside the Hazy Islands, which were sighted at nine o'clock in the forenoon. An hour later the highland of Cape Ommaney loomed up through the mist, and at noon, July i6, the St. Paul was under the hills of Puffin Point. Chirikov now paralleled the coast until 9 P.M. of the I 7 th, keeping from three to four miles from the shore, and then hauled offshore until I I P.M. when he hove to until daylight. From noon until 8 P.M. of the $I 7$ th the ressel logged a distance of 36 knots; adding to this a favorable current of about one knot per hour would place the St. Paul at 8 P.M. abreast that part of the coast where the land falls away to form the southern shores of Sitka Sound, and the log records that at 8 P.M. the "coast seemed to end in $\mathrm{N} 3 / 4 \mathrm{E}$, distant 8 knots, and in its place appeared low land with sea cliffs, with the high mountains receding in the background." An hour later the St. Paul's course was changed to $\mathrm{WNW} / 4 \mathrm{~W}$, true, for two hours. At I I P.M. she was hove to, and at daylight the course parallel to the coast was resumed. All this time the current was setting to the northward along the coast so that the St. Paul passed Sitka Sound in the night and was well north of Cape Edgecumbe at daylight. Indeed the $\log$ records at $9 \mathrm{~A} . \mathrm{M}$. on the $\mathrm{I}_{\mathrm{th}} \mathrm{h}$ a cape to the SE, which could be no other than Cape Edgecumbe. At the same time another point of land (Cape Cross) loomed up about NNW; Chirikov's observation on the 17 th gave the noon latitude as $57^{\circ} 39^{\prime}$; the bearings at noon as given in the log and the courses and distances 
run since the previous noon, augmented by allowance for current, all coincide in fixing the position of the St. Paul at noon, July I 7 , as abreast Cape Edward, and about ten miles off shore. At 3:30 o'clock in the afternoon of July 18 the longboat was lowered and Fleet Master Dementiev with ten armed men was sent to examine the shore. Comprehensive instructions were given him, which are fully set forth in Chirikov's report, and Dementiev was to signal with a rocket as soon as he landed.

\section{LOSS OF TWO LANDING PARTIES}

In his report Chirikor states "we had no signal of any kind from him. We saw them approach the shore, and that is all." For five days the St. Paul kept as near the bay as possible, waiting for the boat to return. At first the weather was such that the longboat could have come off without trouble, but later they had strong winds that at times carried the ship a distance of 30 miles. On July 23 the St. Paul returned and upon approaching the bay saw a fire which they thought had been made by Dementiev, because all the time they had followed the coast they had seen no fire, buildings, boats, nor any other signs of human beings and supposed the coast was uninhabited. Upon noting the fire a gun was fired at intervals as a signal, and the St. Paul was sailed close to the shore. As the gun was fired the fire on shore appeared to grow brighter, but there was no sign of the boat. On July 24 Chirikov concluded the longboat had been damaged and was unable to come off, and it was decided to send the small boat ashore with tools for repairing the longboat. Accordingly, boatswain Savelev was sent in the small boat with a carpenter, a calker, and a seaman.

Chirikor reports that when Savelev departed "the weather was very still; we followed him quite close to the shore and saw him approach it. According to our time it was exactly six o'clock in the afternoon. The signals which had been agreed upon he failed to make and at the expected time did not return." We can well imagine the anxiety with which Chirikov and his ship's 
company watched and waited that night and the following day, for their last boat had gone and this left them no means of reaching the shore; but at one o'clock in the afternoon (July 25) two boats were seen coming out of the bay which the St. Paul's boats had entered. One of the boats was larger than the other, and at first it was thought the ship's boats were returning; but they soon saw their error, for the small boat approached rapidly and the four men in it used paddles instead of oars. This boat stopped at some distance from the ship; the four occupants stood up and shouted twice "Agai, Agai," waved their hands, and turned back to the larger boat, which had stopped at a still further distance from the St. Paul. White kerchiefs were waved, and every effort was made to induce the boats to come closer, but to no avail; and the two boats rapidly returned to the bay from which they had come. The actions of the natives and their fear to come close to the ship convinced Chirikov that his men had either been killed or held captive, and the ship's company began to realize the full extent of the disaster that had overtaken them. They had lost I 5 of their shipmates and their only two boats; they could not follow the native boats in the St. Paul and were helpless to avenge or release their comrades, since they had no means of reaching the shore. The remainder of the afternoon the St. Paul kept close to the mouth of the bay but at sundown headed offshore for the night and in the morning coasted along the shore to the northward. At noon of the 26 th, being in latitude $58^{\circ} 2 \mathbf{I}^{\prime}$, Chirikov called a council of the officers, and it was decided that as the remaining water supply was barely sufficient for the return voyage and they had no boats with which to obtain a fresh supply or even to examine the shore, it was the part of wisdom to sail at once for Kamchatka.

\section{IDENTIFICATION OF PLACE WIERE CIIIRIKOV'S MEN WERE LOST}

At this point it is of interest to discuss the location of the bay where the St. Paul lost her two boats and i 5 of her crew, as there appears to have been some doubt on this point. The position of 
the St. Paul at noon on July $\mathbf{1} 7$, checked by her observed latitude, the bearings of the land given in the log, and the traverse table from the previous noon, was latitude $57^{\circ} 39^{\prime}$, longitude $136^{\circ} 34^{\prime} \mathrm{W}$, about io miles SIV by IV from Cape Edward. Three hours and a half later (3:30 P.M., July I 8 ) the longboat was sent ashore, and in that time the St. Paul had made good 8 miles N, true, which placed her about 5 miles WSW, true, from the entrance to Lisianski Strait. On the $24^{\text {th }}$ when the small boat was sent ashore the weather was clear, and Chirikov took a noon observation close to the mouth of the bay and found the latitude to be $57^{\circ} 50^{\prime}$. This is the correct latitude of Lisianski Strait. On other occasions where it has been possible to check Chirikov's observed latitude with the definitely known position of the ship, his noon sights have proved to be very good, and there is no reason to doubt the general accuracy of his observation on this occasion. Mr. Davidson states ${ }^{8}$ that Chirikov designated the place as "a great bay in latitude $57^{\circ} 15^{\prime}$." Chirikov in his report simply refers to a bay with nothing to indicate particularly whether it was large or small. The log book which was kept by Chirikov records the latitude as $57^{\circ} 50^{\prime}$ and gives the impression that the bay was considered small. The entry in the log for July 18 recites that "At 3:30 stood for the land as near as we dared and sent ashore in the boat the Fleet Master Dementiev and ten armed men. . . . He carried a written order which, among other things, told him to make for the opening, which seemed to us a bay, and take its bearings."

Another entry on the same day recites that "According to the reckoning at 4.30 in the afternoon, taking also in to consideration the position of the bay into which Dementiev was sent, we set down the position of the said bay as $57^{\circ} 23^{\prime} \mathrm{N}$, longitude from Vaua $59^{\circ} 36^{\prime}$. . . . By correction the latitude is $57^{\circ} 50^{\prime}$, longitude $58^{\circ} 54^{\prime}$. The true latitude we got by observation on the 24 th."

Chirikov's observed latitude, the traverse table, the bearings taken during the several days the St. Paul remained in the vicinity, and the references in the log all point with certainty to

8 P. 19 of work cited in footnote 5. D. 334. 
Lisianski Strait as the place where the boats of the St. Paul met with disaster.

\section{BEGINNING OF HONEWARD VOYAGE}

In accordance with the decision of the conncil the homeward voyage was begun. At noon of the $26 \mathrm{th}$ Mt. Fairweather was sighted at a distance of 40 miles, and on the morning of July 27 they made out Ocean Cape where the land falls away to form Yakutat Bay. On August I the Kenai Peninsula came into view, and Chirikov hauled to the southward. At noon Cape Elizabeth was 50 miles distant. At noon of August 2, although the St. Paul. was keeping well offshore, the northern end of Afognak Island was sighted with Mt. Douglas in the distance, and on the $3^{\text {rd }}$ they caught a glimpse of the high land in the ricinity of Cape Chiniak. Chirikor now worked to the southward and westward until the parallel of $53^{\circ}$ was reached on the 12 th, when he again hanled to the westward; but a spell of contrary winds and bad weather set in, and between that date and the 30 th the St. Paul advanced but 100 miles to the westward. On August $3 \mathrm{I}$ the wind was fair, and good progress was made until September 4, when Umnak Island and the Islands of Four Mountains were sighted. At noon the St. Paul was about 25 miles to the southward of the islands, the weather was clear, and as the land seemed to extend to the southward and westward Chirikor stood in that direction for two days before continuing west. On September 8 there were many indications that the ship was again approaching

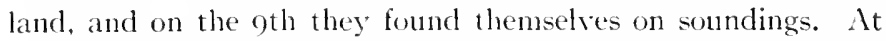
sundown it was calm with a dense fog; a cast of the lead showing only 30 fathoms of water, Chirikov wisely brought his ship to anchor. During the night they conld hear the surf breaking on the beach, and the lifting of the fog the following morning revealed a rocky shore scarcely a quarter of a mile away. The St. Paul had anchored in one of the smalt bights on the south shore of Adak Island, probably the eastermmost one. During the night the tide was running to the westward, and but for Chirikov's prudence the night before the St. Paul would have drifted upon the rocks in the fog. 


\section{LANDFALLS IN THE ALELTIAN ISLANDS}

Soon after the fog cleared two men were seen on the beach, and in a short timeseven baidarkas, each carryingone man, approached the ship. Gifts were distributed by Chirikov, but the natives could not be persuaded to come on board. In the afternoon (September Io) I4 of these skin boats came out to the ship; but none of the natives would come on board, and Chirikor had no boats in which to send a party ashore. The log for September 9 has an interesting account of this meeting. Late in the afternoon of September Io the wind freshened, heavy squalls came down the hills. the St. Paul began to drag towards the rocks which lined the shore, and Chirikov was obliged to cut the cable and put hastily to sea. For the next four days, until September If. Chirikov steered to the southward of west to keep clear of the land, but on the latter date, being in latitude $50^{\circ} 3 \mathrm{I}^{\prime}$ with no signs of land, the course was changed to the northward of west so as to work up to the 53rd parallel. This course took the St. Panl within 20 miles of Kiska Island on the I6th, but the weather was overcast and no land was seen.

In the latter part of August. Chirikov found it necessary to put the crew on short allowance of both food and water, and he reports that the crew had cooked kasha (a buckwheat mush) but once a week and on the other six days lived on cold food, with an allowance of water just sufficient to quench the thirst. These privations soon sapped the vitality of the men, and on September I6 the log records the death of one man from scurvy and the fact that many of the men were very ill. Chirikov among the number.

At sundown of September 2 I a cast of the lead indicated 60 fathoms, and Chirikov, with his customary prudence, gave orders to shorten sail and heave to for the night. In the morning land was sighted, and at noon the St. Paul was close under the eastern shores of Agattu Island. The atmosphere was clear, and they made out the Semichi Islands and the peaks of Attu in the distance. $\mathrm{By}$ this time all but one of the officers and most of the crew were down with scurvy, and only a few were able to drag themselves on deck to work the ship. Chirikov himself was so 
ill that after September $2 \mathrm{I}$ he never left the cabin until he was carried ashore when they made port. The only officer not confined to his bed was Ivan Elagin, who although affected by the scurvy would not give up and kept the deck almost continuously. Chirikov, lying in his bed, worked out the sights and the reckoning and directed Elagin what courses to steer; when according to their reckoning they had run up their longitude and no land was sighted Chirikov gave directions to keep to the westward close to the 53rd parallel. On October 7 Lieutenant Chikhachev died, and the next day both Lieutenant Plautin and Navigator Vrange passed away.

\section{RETURN TO PETROPAVLOVSK}

On October 8 they were gladdened by the sight of land, at noon they recognized the familiar outlines of Cape Shipunski, and two days later the St. Paul anchored in Avacha Bay. Professor de la Croyère died just as the anchor was dropped. Chirikov was very low, but he was immediately taken ashore, where he rallied sufficiently to write his report and resume direction of affairs. He never fully regained his health. Thusended the eventful voyage of the St. Paul. Of her 76 officers and men who sailed from Avacha Harbor June 4 but 54 returned, and all of these were suffering from the scurvy.

The voyages of Bering and Chirikov are events of great importance. These two navigators crossed the Pacific and discovered the northwestern coast of the American continent at a tremendous cost and in the face of untold difficulties; and a seaman reads the details of their struggle with increasing respect and admiration for the men who achieved so great an object with tools so inadequate for the purpose. 


$$
\mid
$$




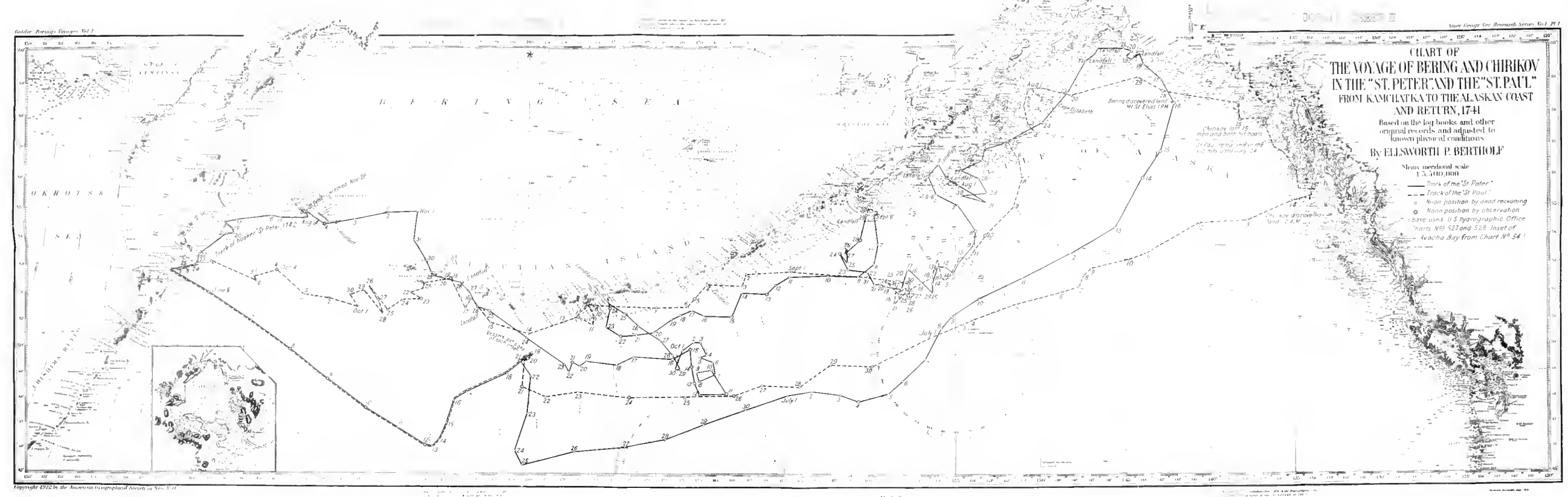




\section{BIBLIOGRAPHICAI, NOTE}

\section{Manuscript Sources}

Golder's "Guide to Materials for American History in Russian Archives" (Carnegie Instn. Publ. No. 239, Washington, D. C., 1917) lists all the unpublished documents on this subject that are to be found in Petrograd and Moscow.

In the Archives of the Ministry of Marine at Petrograd there are classified under the heading "Captain Commander Bering" I I I bundles of documents, some of which contain between 2,000 and 3,000 pages. In addition there are many other papers in the archives relating to this subject catalogued under different heads, such as "Admiralty College," "Count Apraksin," and various other names. These are by no means all the documents. The Hydrographic Section of the Ministry of Marine, the Academy of Sciences, the Archives of the State, the Ministry of War, and most of the other archives, both at Petrograd and Moscow, have manuscript material in this field.

This vast amount of material is, however, out of proportion to its importance. Nany of the III bundles associated with the name of Bering have nothing whatever to do with him, his work, or his period, but deal nearly altogether with purely Siberian affairs of a much later time. In the remaining bundles which do concern the Bering expeditions, much of the material contained is worthless because of duplication. Each undertaking went through the hands of several administrative bodies and bureaus, all of which made copies and comments; by the time a decision was reached a large number of papers had accumulated that were merely repetitions of the same subject. To give concrete illustrations: The trouble between Spanberg and one of his lieutenants fills many bundles of documents-the same charge (a very petty one) being repeated again and again. The petition of 
Bering's family, after his death, that the pay due him should be handed over to them makes two or more bundles of affidavits, copies of certificates, comments of various bureaus, etc. The good and the worthless material is all tied up together.

In the archives of the Ministry of Marine at Paris there is a collection of valuable papers as yet unpublished. These are letters, copies of journals, charts, reports of conversation, newspaper clippings, and other such material collected by the members of the Delisle family-all of which throw interesting side lights on the period and the men. The papers which are of special importance for this study are those gathered by Joseph Nicolas Delisle during his twenty-one years' (17261747) residence at the Russian capital as an officer of the Acadeny. Those of his papers which have recently come to light in the Bibliothèque Nationale have been discussed by Isnard.

The principal materials for the present work are the original $\log$ books and other naval papers of the navigators. They have all been preserved except the journal of the St. Peter, which was lost at the time of the wreck of the ship. The documents dealing with Bering's second expedition have never been published, not even in Russia, and have been used only once before-by Sokolov for his study. But even Soliolov was ignorant of the existence of some of the material. On his return from Petrograd in 1917 the author prided himself on having examined all the documents, but it seems that he was mistaken. His attention has recently been called to a reference by Eugen Büchner ("Die Abbildungen der Nordischen Seekuh," St. Petersburg, I 89 I, p. I) to "eine handsschriftliche Beschreibung der zweiten Bering'schen Expedition . . . . die den Schiffs-Capitain Swen Waxell . . . zum Verfasser hat," which is deposited in the Emperor's private library in Tsarskoe Selo. Whether this is merely a copy of the document here published or something different it is difficult to say. It cannot be greatly different since the same man wrote both; however, that is an open question for the time being. 
When the officers returned to Kamchatka they had several copies made of their papers and forwarded them to the Admiralty College. These copies occasionally differ from one another, as might be expected, because of the copyists; but it is possible to check up the errors. Many of the expressions then used are now archaic and can be found only in old Russian and Dutch dictionaries.

\section{Published Sources}

For the first two decades of the eighteenth century the printed material is found in the "Pamyatniki Sibirskoi Istorii." The "Polnoe Sobranie Zakonov Rossiiskoi Imperii" contains the instructions to the officers in Siberia and the navigators. Bering's report to the Empress on his first voyage is printed without comment in Zapiski Voenno-Topograficheskago Depo. Berkh in his "Pervoe Morskoe Puteshestvie Rossiyan" edited the log book of the first voyage which was kept by the midshipman, Peter Chaplin. Vakhtin in "Russkiye Truzheniki Morya" printed additional documents on this voyage. Gvozdev's report and other material bearing on the sighting of the American coast in 1732 may be found in Zapiski Hydrograficheskago Departamenta. Steller's "Reise von Kamtschatka nach Amerika" is the only original document of importance dealing with the second sea voyage which has up to this time appeared in print.

Mïller, Gmelin, and Krasheninnikov were members of Bering's second expedition though they did not go to sea with him. Their writings may be classed partly as original and partly as contemporary documents, depending altogether on the topics which they discuss. About the middle of the nineteenth century a friendly controversy arose between Karl Ernst von Baer, a German scholar residing at St. Petersburg, and Lieutenant Sokolov of the Russian Navy. In 1848 and 1849 Baer wrote a series of newspaper articles on Peter the Great's contribution to the advancement of geographical knowledge. These papers were translated into Russian and published in the Proceedings (Zapiski) of the Geographical Society in 1849 and 1850 . As soon as they ap- 
peared Sokolov replied to them, heading his papers "Bering and Chirikov." His contention was that Chirikov did not receive all thecredit he merited for the part he took in the Bering expeditions. Baer took notice of the attack and defended his position in the St. Petersburger Zeitung (Nos. I I4, I I5, 1 I6). The controversy had this good in it: it stimulated a study of the original documents, and the results of these researches appeared in the Zapiski Hydrograficheskago Departamenta and brought out many points unknown before. In I 872 Baer, then eighty years of age, completed his monograph and summarized the points of the controversy in an admirable way. Towards the end of the nineteenth century Lauridsen, a Dane, took up the cudgels in behalf of Bering.

\section{Secondary Materials}

Mezhov's "Bibliographia Sibirica" is the best published work on the subject but is neither complete nor wholly reliable. A hitherto unpublished card bibliography of Alaska which has been prepared by Judge James Wickersham of Juneau is much better than Mezhov's for Alaska.

The secondary material is not altogether satisfactory. It is almost two hundred years since Bering received his commission to undertake his first voyage, and during that long period only six men have been sufficiently interested in the subject to give it careful consideration, either in whole or in part. These men are Müller, Coxe, Sokolov, Baer, Bancroft, and Lauridsen. One of these is a Russian, and the two Germans were in the service of Russia at the time of their writing. The most important book in this field is, after all, the third volume of Müller's "Sammlung Russischer Geschichte," published in $175^{8}$. Soon after its appearance this work was translated into Russian, English, and French. Although since that time much paper and ink have been used up in telling this story, yet very little that is new has been added to our knowledge of the subject. Both Russian and non-Russian scholars have preferred to follow Müller's version than to consult the originals. Müller's work, although very valuable, should not be used as a source, but along with the sources. Müller was too 
much a part of the period to see it in its true proportions. His judgment of the men of his time is not critical; he usually rates their deeds at a higher value than they deserve. His lenient attitude may be partly explained by the fact that many of the men of whom he was writing, or their friends, were yet alive, and it was wise not to say anything which might offend.

"Peter's des Grossen Verdienste um die Erweiterung der geographischen Kenntnisse," the monograph by Karl Ernst von Baer referred to above, gives an excellent summary of the Bering voyages. It is scholarly, readable, and fair in its judgments. Lauridsen's "Vitus Bering" has brought to light many interesting facts. Bancroft's "History of Alaska" is of much value and may be used with profit in connection with the sources. Dall's "Critical Review of Bering's First Expedition" is the first really critical discussion in English of the first expedition. There are many helpful papers in the Morskoi Sbornik. Fischer's "Sibirische Geschichte," Slovtsov's "Istoricheskoe Obozryenie Sibiri," and Pallas' "Neue Nordische Beiträge" have much important material. The journals of Cook, Lapérouse, Krusenstern, and other navigators in the North Pacific Ocean help in understanding the difficulties and problems which confronted the Russian sailors of the seventeenth and eighteenth centuries.

In conclusion it should be said that nearly all of the manuscript material used in this work was photostated and is now deposited in the Library of Congress at Mashington, D. C., and the library of the University of Washington at Seattle. As far as printed material is concerned, the Yudin Collection of the Library of Congress is richer for the study of this subject than any library in Western Europe and, according to the statements of certain Russian scholars, even than the Imperial Library at Petrograd.

\section{Biblography}

In the following list of references the author's original entries have been editorially amplified, classified, and commented upon. This amplification includes the addition of a number of 
entries, especially the papers published during the first half of the nineteenth century by Russian students of the subject. The author has not had occasion to see proofs of the bibliography. The titles are grouped by topics in the natural order of the development of the theme. Within each group the titles are arranged logically, beginning with the more general ones, or chronologically. Comment is of ten added to characterize the title's relation to the Bering expeditions. Russian titles are transliterated and translated.

\section{History of Siberia}

MEZHOV, V. I. [on title page in quasi-French transliteration, MÉJOw]. Bibliographia sibirica: Bibliographie des livres et articles de journaux russes et étrangers concernant la Sibérie. 4 vols. in three. St. Petersburg, I 89I-92. [Vol. 2 deals with geography and voyages.]

Akty istoricheskie (Historical documents). Collected and published by the Archeographical Commission. Vols. 1-5, for 1334-1 700. St. Petersburg, I84I-43. Separate index, 1843.

Dopolneniya $\mathrm{k}$ aktam istoricheskim (Supplementary historical documents). Collected and published by the Archeographical Commission. Vols. I-12, for II 50-1700. St. Petersburg, 1846-72. Separate index to Vols. I-IO, 1875 .

Pamyatniki Sibirskoi istorii xviii vyeka (Memoirs for Siberian history of the eighteenth century). Edited for the Archeographical Commission by A. I. Timofeev. 2 vols. (Vol. I, I 700-1713; Vol. 2, 1713-1724). St. Petersburg, I $882-85$.

FISCHER, J. E. Sibirische Geschichte von der Entdekkung Sibiriens bis auf die Eroberung dieses Landes durch die russischen Waffen. 2 vols. Acad. of Sci., St. Petersburg, I 768 . [Russian edition was published in 1774 Fischer was one of the scientists who were members of Bering's second expedition.]

ANDRYevich, V. K. Istoriya Sibiri (History of Siberia). 2 vols. St. Petersburg, 1889 .

Slovtsov, P. A. Istoricheskoe obozryenie Sibiri (Historical survey of Siberia). 2 vols. in one. St. Petersburg, 1886.

KUZNETSOV, E. V. Sibirskii lyetopisets. Lyetopis kontsa xvii i nachala xviii stolyetii, vedennaya $\mathrm{v}$ Tobolskye (Annals of Siberia. Annals of the seventeenth and beginning of the eighteenth century, recorded in Tobolsk). Tobolsk, I892.

[TIrov, A. A.] Sibir v xvii vyekye. Sbornik starinnykh Russkikh statei o Sibiri i prilezhashchikh $\mathrm{k}$ ner zemlyakh. S prilozheniem snimka so 
starinnoi karty Sibiri. (Siberia in the seventeenth century. A collection of old Russian publications on Siberia and its borderlands, accompanied by a reproduction of an old map of Siberia.) Edited by G. Iudin. Moscow, I 890 .

Trrov, A. A. Tyumen $v$ xvii stolyetii (Tyumen in the seventeenth century). Published by A. I. Chukmalsina, Moscow, I903.

Sukachev, V. P., edit. Pervoe stolyetic Irkutska (First century of Irkutsk). St. Petersburg, 1902.

Remezov, S. Chertezhnaya kniga Sibiri (Map book of Siberia). Archeographical Commission, St. Petersburg, I882. IA modern reproduction of an atlas of Siberia and the accompanying notes made at the request of the tsar by Remezov towards the end of the seventeenth century. The important general map of Siberia in this atlas is reproduced in A. E. Nordenskiöld's Periplus, Stockholm, I897, Pl. 37.]

Cahen, Gaston. Les cartes de la Sibérie au xviiie siècle: Essai de bibliographie critique. Paris, I9I I.

Popov, M. Pervaya morskaya expeditsiya $k$ ustyu ryeki Obi (First sea expedition to the mouth of the Ob). St. Petersburg, I907.

\section{Geographical Knouledge of the North Pacific Prior to Bering's Expeditions}

Nachod, Oskar. Ein unentdecktes Goldland: Ein Beitrag zur Geschichte der Entdeckungen im nördlichen Grossen Ocean. Separate from Mitt. der Deutschen Gesell. für Natur und Völkerkunde Ostasiens. [Tokyo, I9oo.] [Deals with the voyages of the Spaniard Vizcaino from Mexico in $161 \mathrm{I}$ and 1612 in search of the rumored gold islands east of Japan.]

HeEres, J. E., edit. Abel Janszoon Tasman's journal of his discovery of Van Diemens Land and New Zealand in I642, with documents relating to his exploration of Australia in 1644. Amsterdam, I 898. [Contains a good brief account of Tasman and Quast's voyage in 1639 in search of the rumored gold islands, on which they reached $42^{\circ} \mathrm{N}$. 600 Dutch miles east of Japan and discovered the Bonin Islands.]

Leupe, P. A., edit. Reize van Maarten Gerritsz[oon] Vries in $\mathbf{I 6} 43$ naar het noorden en oosten van Japan, volgens het journaal gehouden door C. J. Coen, op het schip Castricum. Published under the auspices of the Koninklijk Instituut voor Taal-, Land-, en Volkenkunde van Nederlandsch Indië. Amsterdam, 1858. [First publication of the journal of Vries's voyage of I643.]

Telekr, Paul. Atlas zur Geschichte der Kartographie der Japanischen Inseln, nebst dem holländischen Journal der Reise Mathys Quasts und A. J. Tasmans zur Entdeckung der Goldinseln im Osten von Japan 
i. d. J. I639 und dessen deutscher Übersetzung. Budapest, I9o9. [Contains the complete journal of Quast on Tasman and Quast's voyage of 1639 in search of the rumored gold islands east of Japan; also an account of Vries's voyage of 1643.]

Witsen, NicolaAs. Noord en oost Tartaryen, behelzende eene beschryving van verscheidene Tartersche en nabuurige gewesten, in de noorder en oostelykste deelen van Aziën en Europa. 2 vols. 2nd edit., Amsterdam, I785. [First edition, I692.]

Histoire [i. e. Mémoires] de l'Académie des Sciences [de Paris]. Vols. for I714, I 720, I725, I 729. [These volumes contain papers by Guillaume and Joseph Delisle. The volume for $\mathrm{I} 720$ has one by Guillaume Delisle, for instance (Détermination géographique de la situation et de l'étendue des différentes parties de ia terre), in which he excellently sums up the geographical knowledge of the North Pacific at the time. In this he advances the opinion that Terra de lezo is a part of the continent of Asia and Japan a peninsula of Yezo. From 1699 to 1789 the title of the Mémoires was Histoire.]

VAUGONDY, ROBERT DE. Observations critiques sur les nouvelles découvertes de l'Amiral Fuentes. Paris, 1753. [Bartolomé de Fuentes was a Spanish admiral who made a voyage in the North Pacific in I640 to discover a northwest passage. See also the paper by J. N. Delisle listed on p. $360-36 \mathrm{r}$.

KAEMPFER, ENGELBERT. The history of Japan, giving an account of the antient and present state and government of that empire. Written in High Dutch by E. Kaempfer ... and translated from his original manuscript, never before printed, by J. G. Scheuchzer ... 2 vols. London, 1728. [One of the accompanying maps shows Kamchatka and Terra de Yezo as the same land.]

Strahlenberg, P. J. von. An historico-geographical description of the north and eastern parts of Europe and Asia, but more particularly of Russia, Siberia, and Great Tartary ... Written originally in High German by Mr. Philip John von Strahlenberg ... London, 1738. Original edition, Stockholm, 1730. [Strahlenberg was a Swedish officer who was made prisoner by the Russians at the Battle of Pultava. During his captivity in Siberia, where he enjoyed much freedom of movement, he made the observations on which this book is based. The important map of northern Eurasia accompanying the original edition is reproduced in facsimile in A. E. Nordenskiöld's Periplus, Stockholm, 1897, Pl. 38.]

BERNARD, J. F., edit. Recueil de voyages au nord, contenant divers mémoires très utiles au commerce et à la navigation. Io vols. Amsterdam, I 725-38. [Vol. 4, published I 732, contains account of report by Jesuits in 1566 of body of land north of Nippon called Yezo.] Charlevoix, [P. F. X.] DE. Histoire et description générale du Japon. 
2 vols. Paris, I736. [One of the accompanying maps, by Bellin, "Ingénieur au Dépôt des Cartes de la Marine" (facsimile reproduction as P1. I5 (I) in Teleki's atlas, cited above on p. 355), shows Kamchatka as called Terra de Yezo by the Japanese.]

Ruge, Sophus. Fretum Anian. Dresden, 1873.

\section{Minor Expeditions: Shestakov and Grozdev}

SGibNEv, A. S. Materialy dlya istorii Kamchatki, etc. (Materials for the history of Kamchatka: Expedition of Shestakov). Morskoi Sbornik, Vol. Ioo, Section 2, St. Petersburg, 1869.

Gvozdev, Mikhael Spiridonovich. Report of his voyage of 1732. Archives of the Ministry of Marine, Petrograd: Papers of Count Chernishev, 1762-68, No. 367, pp. 46-49. [Manuscript. For published version, see the present work, pp. 22-24.]

Plolonskir], A. [S.]. Pokhod geodezista Mikhaila Gvozdeva, etc. (Journey of the surveyor Mikhail Gvozdev to Bering Strait in 1732). Morskoi Sbornik, Vol. 4, pp. 389-402, St. Petersburg, I85o. [Discusses Gvozdev's voyage mainly on the basis of a preliminary first report made by him and Skurikhin, another member of the expedition, in I 74I.]

Pervyi pokhod Russkikh k Amerikye 1732 goda (First voyage of the Russians to America in 1732). Zapiski Hydrograficheskago Departamenta, Vol. 9, pp. 78-107, St. Petersburg, 1851. [Publishes (pp. 88I 03) Gvozdev's second report, made to Spanberg in 1743, which is fuller than the first preliminary report of $174 \mathrm{r}$.]

Delisle, Joseph Nicolas Navigation et découvertes faites par les Russes dans la Mer Orientale entre les deux voiages du Capitaine Beerings vers les années I731 et I732: Nouvelles connoissances sur les terres orientales données par Mr. Fcodor Ivanitch Soimonof le I Mars, 1738. Delisle MSS., No. XXV, I6 and I6K, Archives du Dépôt des Cartes et Plans de la Marine, Paris. [Note, with map, on Gvozdev's voyage. Published, with translation, in F. A. Golder: Russian Expansion in the Pacific, I64I-1850, Cleveland, O., I914, Appendix E. pp. 298-301; map on p. I54.J

Bering's Expedilions: Accounts of Participantsor Persons Direclly Concerned (a) Manuscript

(See also entries in F. A. Golder: Guide to Materials for American History in Russian Archives, Carnegie Instn. Publ. No. 239. Washington, D. C., 1917, especially pp. I16-135)

Chaplin, Peter. Journal and log book of Bering's first expedition. Archives of the Hydrographic Section of the Ministry of Marine, 
Petrograd: Nautical Journals, 1725-1731, No. 641. [For abridged published version, see book by Berkh, I 832, listed below on p. 363.]

BERING, Vitus. Report on his first expedition. Archives of the General Staff, Petrograd: Section X, No. 566. [For published version, see reference listed below on p. 359 and translation of the report in the present work. pp. 9-20.]

Itshin, Kharlam. Log book of the St. Peter. Archives of the Hydrographic Section of the Ministry of Marine, Petrograd: Nautical Journals, 1741, 1742, Nos. 642, 643, 644. [Photostat copies in the Library of Congress, Washington, D. C., and the library of the University of Washington, Seattle, Wash.]

Khitrov, Sofron. Log book of the St. Peter. Archives of the Imperial Academy of Science, Petrograd: MS. No. 120, 32: 16: 19. [Photostat copy in the library of the University of Washington. Seattle, Wash.]

Khitrov, Sofron. Log book of the St. Peter [another copy]. Archives of State. Petrograd, XXIV, No. 9. [Photostat copy in Library of Congress, Washington, D. C.]

Waxel. Sien. Report on voyage of the St. Peter. Archives of the Ministry of Marine, Petrograd: Papers of the Admiralty College, Bundle No. 2. [Photostat copies in the Library of Congress, Washington, D. C., and the library of the University of Washington, Seattle, Wash.]

Log book of the St. Paul. Archives of the Hydrographic Section of the Ministry of Marine, Petrograd: Nautical Journals, I 741-1743. Nos. $645-648$.

Journal of the St. Paul. Archives of the Ministry of Marine, Petrograd: Papers of the Admiralty College, Bundle No. I6.

Chirikov, Alexei. Report on the voyage of the St. Paul. Archives of the Ministry of Marine, Petrograd: Papers of Captain Commander Bering, Bundle No. 44. [Photostat copies of all three St. Paul documents are in the Library of Congress, Washington. D. C., and the library of the University of Washington, Seattle, Wash.]

Delisle Manuscripts. Archives du Dépôt des Cartes et Plans de la Marine, Paris. [See under Delisle, J. N., above, p. 357, and below, this page, and, under Isnard, p. 361.]

\section{(b) Published}

Makov, L. N., edit. Razskazy Nartova o Petrye Velikom (Nartov's narratives about Peter tle Great). Acad. of Sci., St. Petersburg. 1891. [A. K. Nartov (1683-I756) was almost in constant attendance on the Emperor during his last days, and records his own words concerning his plans for Bering's first expedition.]

Lelisle, Josepit Nicolas. Explication de la carte de la Mer Orientale dressée pour montrer le plus court chemin de l'Asie à l'Amérique. 
Lue à l'Académie [des Sciences de St. Pétersbourg] l'an I 732. Delisle MSS., No. XXV, I4, Archives du Dépôt des Cartes et Plans de la Marine, Paris. [Memoir explaining the map (Delisle MSS.. XXVI, 3B) which was given to Bering on his second expedition; De Gama and Company Lands were indicated on it. Original memoir, with translation, published in F. A. Golder: Russian Expansion in the Pacific, I64I-I850, Cleveland, O., I9I4. Appendix F, pp. 302-3 I3.]

Donesenie Flota Kapitana Beringa ob ekspeditsii ego k vostochnym beregam Sibiri (Report of Fleet Captain Bering on his expedition to the eastern coast of Siberia). Zapiski Voenno-Topograficheskago Depo, Vol. Io, pp. 69-75 (report) and 77-79 (tables), St. Petersburg, 1847. [For a translation from the original manuscript, see the present work, pp. 9-20.]

Müller, Gerhard Friedrich. Nachrichten von Seereisen und zur See gemachten Entdeckungen, die von Russland aus längs den Küsten des Eismeeres und auf dem östlichen Weltmeere gegen Japan und Amerika geschehen sind, zur Erläuterung einer bei der Academe der Wissenschaften vertertigten Landkarte. In series: Sammlung Russischer Geschichte (9 vols., Acad. of Sci., St. Petersburg, I 732I 764). Vol. 3, Parts I-3, St. Petersburg, 1758. [This is the most important account by a contemporary. Müller was one of the scientists who were members of Bering's second expedition. He did not go to sea with him, however.]

MÜller, Gerhard Friedrich. Voyages from Asia to America for completing the discoveries of the north west coast of America. To which is prefixed a summary of the voyages made by the Russians on the Frozen Sea in search of a north east passage. Serving as an explanation of a map of the Russian discoveries, published by the Academy of Sciences at Petersburgh. Translated from the High Dutch of S. [i. e. G.] Muller . . By Thomas Jefferys . . London, I761. [Translation of the author's German work.]

Müller, Gerhard Friedrich. Voyages et découvertes faites par les Russes le long des côtes de la Mer Glaciale et sur l'Océan Oriental, tant vers le Japon que vers l'Amérique. Ouvrage traduit de l'allemand de Mr. G. P. [sic] Miller, par C. G. F. Dumas. 2 vols. Amsterdam, 1766. [Translation of the author's German work. The accompanying map, which shows the results of Bering's expeditions as well as the tracks of his vessels, is reproduced in facsimile in A. E. Nordenskiöld's Periplus, Stockholm, I 897 , Fig. 42 on p. 98.]

Steller, Georg Wilheli. Beschreibung von dem Lande Kamtschatka, dessen Einwohnern, deren Sitten, Nahmen. Lebensart und verschiedenen Gewohnheiten, hrsg. von J. B. S. . . . Frankfort and Leipzig, I774.

STELLER, GEORG WiLHELM. Reise von Kamtschatka nach Amerika 
mit dem Commandeur-Capitän Bering. Ein Pendant zu dessen Beschreibung von Kamtschatka. St. Petersburg, I793. Also in P. S. Pallas: Neue Nordische Beiträge, Vol. 5, pp. I 29-236; Vol. 6, pp. I-26, Leipzig, I 795. [Translated in Vol. 2 of the present work.]

GMELIN, JohANN GEORG. Reise durch Sibirien, von dem Jahr I733 bis 1743. (In series: Sammlung Neuer und Merkwürdiger Reisen zu Wasser und zu Lande, Parts 4-7). 4 vols. in two. Göttingen, I75152. [Gmelin was one of the scientists who were members of Bering's second expedition. He took part only in the land expedition, and did not go to sea with him.]

Gmelin, Johann Georg. Voyage au Kamtschatka par la Sibérie. In A. F. Prévost: Histoire générale des voyages, Vol. 24, pp. 94499. The Hague, I779. [Translation of the author's German work.]

[Krasheninnikov, Stepan PeTrovich.] The history of Kamtschatka and the Kurilski Islands, with the countries adjacent; illustrated with maps and cuts. Published at Petersbourg in the Russian language, by order of Her Imperial Majesty, and translated into English by James Grieve, M.D. London, I764. [Krasheninnikov was one of the scientists who were members of Bering's second expedition. He did not go to sea with him, however. An abridged translation from the Russian original, which was published in 1755 . A complete translation of the original appeared in French at Amsterdam in $177^{\circ}$. A second Russian edition was pubished in $\mathbf{3} 786$.

Pallas, P. S. Nachrichten von den russischen Entdcckungen zwischen Asia und Europa. Büsching's Magazin für die Neue Historie und Geographie, Vol. I6, pp. 235-286. Halle, I782.

Delisle, Joseph Nicolas. Explieation de la carte des nouvelles découvertes au nord de la Mer du Sud. Paris, 1752. Accompanied by a map: Carte des nouvelles découvertes au nord de la Mer du Sud, tant à l'est de la Sibérie et du Kamtchatka, qu'à l'ouest de la Nouvelle France, dressće sur les mémoires de Mr. Del' Isle . . par Philippe Buache (facsimile reproduction as Pl. I4 (1) in Teleki's atlas, cited above on p. 355). [Contains a paper read by J. N. Delisle after his return from his twenty-one years' stay in St. Petersburg (1 726-1 747) before the Paris Academy of Sciences on April 8, 1750, entitled "Nouvelles découvertes au nord de la Mer du Sud." In this paper Delisle said that Bering was wrecked on an island and had not been in America. while Chirikov and Delisle de la Croyère had touched on the American coast. This statement was probably made in good faith because Delisle did not know the true facts of the voyage of the St. Peter, many of the original journals and charts still being in Siberia and the Admiralty College probably not yet having given out accurate information.]

Delisle, Joseph Nicolas. Nouvelles cartes des découvertes de l'amiral 
de Fonte et autres navigateurs . . dans les mers septentrionales, avec leur explication. Paris, 1753. [Accompanied by a map on which the discoveries of Bering and Chirikov, whose tracks are shown, are correlated with the alleged discoveries along the northwestern coast of North America of the Spanish navigator Bartolomé de Fuentes in 1640. A facsimile of this map accompanies A. IV. Greely's paper, listed on p. 367, as P1. 21, Nall. Geogr. Magaz., Vol. 3, 1891.]

1sNard, Albert. Joseph-Nicolas Delisle: Sa biographie et sa collection de cartes géographiques à la Bibliothèque Nationale. Bull. Section de Géogr., Comité des Travaux Hist. et Sci., Vol. 3o, 1915, pr. 34-164. Minist. de 1'Instruction Publ. et des Beaux-Arts, Paris. LOf the collection of manuscripts and maps brought back to Paris by. Delisle from his twenty-one years' stay in St. Petersburg (1 726-1 747) one part was deposited in the archives of the Dépôt des Cartes et Plans de la Marine. Reference is made to these above, on p. 358. The remaining part was deposited in the Bibliothèque Royale. These, long missing, have recently been found. They are now in the Section Géographique of the Bibliothèque Nationale and are described and listed in the present paper. The collection contains first-hand copies of maps of Bering's route on his first expedition from Tobolsk to Bering Strait (Nos. I65, I66, I66 bis, 167).]

Omont, H. Lettres de J.-N. Delisle alı Comte de Maurepas et à l'Abbé Bignon sur ses travaux géographiques en Russie (1726-1730). Bull. Section de Géogr.. Comité des Traz'aux Hist. et Sci., Vol. 32, 1917, pp. I30-I64. Minist. de 1'Instruction Publ. et des Beaux-Arts, Paris. [Seven letters, of which five from Delisle to the then Minister of Marine and to the librarian of the Bibliothèque Royale, informing them of his activities during the first years of his sojourn in Russia. Letters III and VII, on pp. I $41-147$ and I60-I62, deal at first hand with Bering's first expedition and the state of geographical knowledge of the North Pacific prior to that expedition, including von Strahlenberg's studies (see above, p. 356). "C'est du capitaine Beerings luy-mêtne, qui est icy de retour depuis quelques mois, que j'ay appris ce que je viens d'en marquer à Votre Grandeur" (Letter VIl, dated June 25,1730 ).]

Froidevaux, Henri. Les études géographiques de Joseph-Nicolas Delisle sur l'Empire Russe. La Géographie. Vol. 33, I920, pp. $219-228$. Paris. [An abstract of the papers by Isnard and Omont listed above.]

Buache, Philippe. Considćrations géographiques et physiques sur les nouvelles découvertes au nord de la grande mer appelée vulgairement la Mer du Sud, avec des cartes qui y sont relatives. Paris, r 753 . [Buache, who was "Premier Géographe du Roy" and in close touch with J. N. Delisle, in this memorr discusses the geography of the North Pacific mainly as established by Bering's expeditions. The 
memoir is accompanied by 13 maps, of which two are reproduced in facsimile as Figs. Io and I I on pp. I 40 and I $4 \mathrm{I}$ of Teleki's atlas, cited above on P. 355.]

[Waxel, Sven](?). Lettre d'un officier de la marine russienne à un Seigneur de la Cour concernant la carte des nouvelles découvertes au nord de la Mer du Sud, et la mémoire qui y sert d'explication, publiée par M. de l'Isle à Paris en I752. Traduit de l'original russe. Berlin, I753. [In this anonymous pamphlet the origin and inception of the Bering expeditions are discussed and exception is taken to J. N. Delisle's statement (see entry on p. 360) that Bering had not reached America. From internal evidence A. W. Greely (p. 222 of paper listed on p. 367) concludes that Sren Waxel was the author. Long quotations from the pamphlet are there given in translation. A careless English translation appeared in London in 1754.]

\section{Near-Contemporary Accounts of Bering's Expeditions, and Compendiums}

Du Halde, Jean Baptisie. Description géographique, historique, chronologique, politique, et physique de l'empire de la Chine et de la Tartarie chinoise ... 4 vols. The Hague, 1736. [Vol. 4, p. 562 , contains Bering's report on his first voyage. In the atlas to accompany Du Halde's work (J. B. B. d'Anville's Nouvel atlas de la Chine, de la Tartarie chinoise et du Thibet, The Hague, I737) is reproduced Bering's original map of his first voyage. This map, which he made in Moscow in $173 \mathbf{I}$, was presented by the Russian government to the King of Poland, who gave it to the Jesuit father Du Halde. It is from a copy of this map for use in Sweden that our Fig. 5 is reproduced.]

Weber, - Das neuveränderte Russland. At least 3 vols. Frankfort and Leipzig, I 744. [Weber, who knew and associated with Bering. in this account follows verbatim a report of Bering's first expedition which appeared in the Copenhagen periodical Nye Tidende immediately after Bering's return in March, I730, and which Lauridsen (work cited on p. 366) ascribes to Bering himself.]

HARrIS, JoHN, edit. Navigantium atque itinerantium bibliotheca. Or, a complete collection of voyages and travels. Consisting of above six hundred of the most authentic writers . . Originally published . . by John Harris . . 2 vols. London, 174t-1748. [Vol. 2, pp. IOI 8-1022, contains an account, by John Campbell, based on Bering's report, of his first expedition and, facing $p$. IOI 6 , a reproduction of his map.]

Chappe d'Auteroche, Jean. Voyage en Sibérie, fait par ordre du roi en I 76I; contenant les mours, les usages des Russes, et l'état actual 
de cette puissance; la description géographique et le nivellement de la route de Paris à Tobolsk. 2 vols. in three, and atlas. Paris, I 768. [Vol. 2 contains a translation of Krasheninnikov's history of Kamchatka.]

[SCHERER, J. B.] (?) Neue Nachrichten von denen neuentdekten Insuln in der See zwischen Asien und Amerika; aus mitgetheilten Urkunden und Auszügen verfasset von J. L. S.—. Hamburg and Leipzig, I 776. [Attributed to J. B. Scherer, who wrote the introduction to and edited Steller's "Beschreibung von dem Lande Kamtschatka," I 774.]

Coxe, Willian. Account of the Russian discoveries between Asia and America. To which are added the conquest of Siberia and the history of the transactions and commerce between Russia and China. With Supplement: A comparative view of the Russian discoveries with those nade by Captains Cook and Clerke. $3 \mathrm{~d}$ edit., London, 1787. [Important book. The author lived in Russia during the second half of the eighteenth century. In his account of the Russian voyages up to and including the Bering period, he follows Müller closely. Earlier edition, I 780.]

Hallager, Morten, comp. Udförlige og troeværdige efterretninger om de fra Rusland af langs med kysterne af Iishavet til söes giorte opdagelser; tilligemed de i russiske tieneste værende danske söeofficerers, commandeur-capitain Vitus Berings og capitain Morten Spangbergs söe-reiser, foretagne $\mathrm{i}$ aarene $I 728,1729,1738$, I $74 \mathrm{I}$ til I 743, paa det östlige ocean fra Kamtschatka af til Japon og Amerika; samt en beskrivelse over de siden den tid $i$ dette hav fundne öer .. uddragne af ovenmeldte söefareres dagböger og af Statsraad Müllers, Adjunctus Stellers, Prof. de l'Isle's, Dr. Pallas's beretninger og Skrifter. . . Copenhagen, I 784 .

Forster, JohAN REINHOLd. History of the voyages and discoveries made in the North. Translated from the German. London, I 786 ,

BCRNEY, JAMES. A chronological history of the discoveries in the South Sea or Pacific Ocean . . 5 vols. London, I803-1 7 .

BURNEY, JANIES. A chronological history of north-eastern voyages of discovery; and of the early eastern navigations of the Russians. London, I819.

\section{Modern Discussions of Bering's Expeditions}

Berkh, Vasili N. Pervoe morskoe puteshestvie Rossiyan, predprinyatoe dlya ryesheniya geograficheskoi zadachi, Soedinyaetsya li Aziya s Amerikoyu? i sovershennoe v I 727,28 i 29 godakh pod nachalstrom Flota Kapitana I-go ranga Vitusa Beringa (First sea voyage of the 
Russians undertaken for settling this geographical problem, Are Asia and America united? and performed in $1727-1729$, under the command of Captain of the first rank Vitus Bering). Imp. Acad. of Sci. Press, St. Petersburg, 1823. [The leading discussion of the first expedition, based on a study of the archives. It includes an abridged version of the log book kept by midshipman Peter Chaplin and a map showing the route of the sea voyage, reproduced in Fig. 6 of the present work.]

Polonskir, A. S. Pervaya Kamchatskaya Ekspeditsiya Beringa, 1 725-29 goda (First Kamchatka Expedition of Bering, 1725-29). Zapiski Hydrograficheskago Departamenta, Vol. 8, pp. 535-556. St. Petersburg, 1850. [Contains Spanberg's and Chirikov's arguments as to proceeding farther after expedition had reached $67^{\circ} 18^{\prime} \mathrm{N}$. See the present work, p. 19.l

P[olonskir], A. [S.]. Pervaya Kamchatskaya Ekspeditsiya Beringa, 1 725-29 goda (First Kamchatka Expedition of Bering, 1725-29). Otechestveniya Zapiski, I851, No. 3 .

Naznachenie pervoi Beringovoi ekspeditsii (Destination of Bering's first expedition). Zapiski Hydrograficheskago Departamenta, Vol. 9, pp. 642-644, St. Petersburg, 1851 .

Berkh, Vasili N. Puteshestvie Kap. Komandora Beringa i Kap. Chirikova, etc. (Voyage of Capt. Commander Bering and Capt. Chirikov to the northwest coast of America: Second expedition). Blagonamyerennyi Zhurnal, Vol. 2, 1818, No. 6(?), St. Petersburg.

Baer, Karl Ernst von. Zaslugi Petra Velikago po chasti rasprostraneniya geograficheskikh poznanii o Rossii $\mathrm{i}$ pogranichnikh s neyu zemlyakh Azii (The merits of Peter the Great in the extension of geographical knowledge about Russia and her Asiatic frontier regions). Zapiski Russkago Geograficheskago Obshchestia, Vol. 3, 1849, pp. 217253; Vol. 4, 1850, pp. 260-283. [Read at the meeting of Jan. 14, 1848, of the Russian Geographical Society. This was the paper which led to the friendly controversy between von Baer and Sokolov as to the relative merits of Bering and Chirikov which is reflected in the entries that follow.]

Sokolov, Aleksandr. Bering i Chirikov. Syeerernaya Pchela, 1849 , No. 98 (May 5), pp. 391-392; No. 99 (May 6), pp. 395-396, St. Petersburg. [In reply to von Baer's article in Zapiski Russ. Geogr. Obshch., Vols. 3 and 4, 1849-50. Also published separately, 13 pp., St. Petersburg, I 8.49.]

Baer, Karl Ernst von. [Bering und Tschirikow](?) St. Petersburger Zeitung, 1849, Nos. 114, 115, 116. [Reply to Sokolov's article in Syevernaya Pchela, 8449 , Nos. 98 and 99.]

Bafr, Kari. ERNSt von. Bering i Chirikov. Russkii Invalid ili Voenniya Vyedomosti, 1849, Nos. 121, 122, 123, St. Petersburg. [Russian 
translation of von Baer's article in St. Petersburger Zeitung, 1849. Nos. I $14-1$ 16.]

Sokolov, Aleksandr. [Reply to Mr. Baer of the Academy of Sciences.] Syevernaya Pchela, 1849, No. 133, St. Petersburg. [Reply to von Baer's translated article in Russkii Invalid, 1849. Nos. 121-1 23.]

Sokolov, Aleksindr. Syevernaya ekspeditsiya, 1733-43 goda (The northern expeditions, I 733-43). Zapiski Hydrograficheskago Departamenta, Vol. 9, pp. I90-469, St. Petersburg, 1851. [The leading discussion of the second expedition, based on a study of the archives. It contains a map on Mercator's projection, equatorial scale I : 17,500,000, showing Bering's and Chirikov's tracks according to their own calculations, and Khitrov's map of Kayak Island, both reproduced by Lauridsen in his "Vitus Bering," cited on p. 366.]

Sokolov, Aleksandr. Primyechaniya, etc. (Remarks on the "Account of the Northern Expeditions, 1733-43.' published in Vol. 9 of the Journ. of the Hydrographic Department). Morskoi Sbornik, Vol. 25. No. I3, Section 4, pP. 103-105. St. Petersburg, 1856.

Astronomicheskie $\mathrm{i}$ geodezicheskie instrumenty Delil-de-la Kroera, vtoroi Kamchatskoi ekspeditsii (Astronomical and geodetic instruments used by Delisle de la Croyère on the second Kamchatka expedition). Zapiski Hydrograficheskago Departamenta, Vol. 7, pp. 537-542, St. Petersburg, 1849 .

Berki, VAsili N. Biograficheskoe svyedenie o Kapitan-Komandorye Vitusye Beringye (Biographical sketch of Capt. Commander Vitus Bering). Syevernyi Arkhiv, Vol. 6, No. 8, St. Petersburg, 1823.

BERKH, VASILI N. Zhizneopisaniya pervykh rossiiskikh admiralov, etc. (Biographies of the first Russian admirals, or an attempt at the history of the Russian Navy). 4 vols., St. Petersburg, 1831-36.

BaER, Karl ERnst vos. Peter's des Grossen Verdienste um die Erweiterung der geographischen Kenntnisse. (In series: Beiträge zur Kenntnis des russischen Reiches, Vol. I6.) St. Petersburg, 1872.

StejNeger, Leonhard. Contributions to the history of the Commander Islands, No. I: Notes on the natural history, including descriptions of new cetaceans. Proc. U. S. Natl. Museum, Vol. 6, 1883. pp. 58-89.

STEJNEGER, LeONIARD. Contributions to the history of the Commander Islands, No. 2: Investigations relating to the date of the extermination of Steller's sea-cow. Proc. U. S. Natl. Museum, Vol. 7, I 884 , pp. I $81-189$.

Stejneger, Leonhard. Fra det yderste Osten. Naturen, Vol. 8, 1884 , PD. 65-69. Christiania.

Stejneger, Leonhard. Eine Umsegelung der Bering's-Insel, Herbst 1882. Deutsche Geogr. Blätter, Vol. 3, I 885, pp. 225-273. Bremen.

[DAHi.GREN, E. W.] Berings karta öf ver Sibirien. Ymer, Vol. 4, I884, 
pp. 93-94. IOn copies in Sweden of Bering's map of his first expedition.]

NoRdenskiöld, A. E. Den första på verkliga iakttagelser grundade karta öf ver norra Asien. Ymer, Vol. 7, 1887, pp. I33-144. Stockholm.

BANCrofT, H. H. Alaska, I730-1885. (In his "History of the Pacific States of North America," San Francisco, I 882-9o, Vol. 28.) San Francisco, 1886. [Bering's voyages are treated in Chapters 3-5. There is a valuable but undifferentiated list of "Authorities quoted," pp. xxiii-xxxviii.]

Lauridsen, Peter. Vitus Jonassen Bering. Geografisk Tidskrift, Vol. 6,1862, pp. 89-98. Copcnhagen. [A study preliminary to Lauridsen's major work, listed in the next entry. This paper discusses the first expedition only.]

Lauridsen, Peter. Vitus I. Bering og de russiske opdagelsesrejser fra $1725-43$. Copenhagen, I 885 . [The Danish original of the next entry, which see. Reviewed by A. E. Nordenskiöld in Journ. Amer. Geogr. Soc., Vol. I 7, I 885 , pp. 285-298.]

Lauridsen, Peter. Vitus Bering, the discoverer of Bering Strait. Revised by the author, and translated from the Danish by Julius E. Olson. . . With an introduction to the American edition by Frederick Schwatka. . . Chicago, 1889. [An a pologia of Bering. In spite of this bias it is valuable as one of the first VVest European discussions of the subject taking the Russian sources into consideration. The author had access to the Russian archives in $\mathbf{1 8 8 3}$, but even with the assistance received from Admiral Wessalgo, then director of the Hydrographic Section of the Ministry of Marine, and others, the handicap of incomplete familiarity with Russian could not be entirely overcome. Lauridsen reproduces (map III) in quasi-facsimile from Sokolov, Zapiski Hydrogr. Dept., Vol. 9, combined, the tracks of the St. Peter and the St. Paul from the original charts of Waxel and Khitrov and of Chirikov. But, as the accumulated error in longitude of the ship's position in each case is not prorated but is thrown into one day, the two tracks are not properly related to each other. However, the map is of importance as representing the navigators' own conception of their tracks. Lauridsen also reproduces (map IV), from the same publication, Khitrov's map of Kayak Island from his journal.]

VAkitis, V. Russkiye truzlieniki morya. Pervaya morskaya ekspeditsiya Beringa, etc. (Russian self-sacrificing navigators. Bering's first expedition, etc.). St. Petersburg, 1890 . [Publislies documents on the first voyage.]

DALI. IV. H. Alaska and its resources. Boston, 1870. [Pp. 297-30I refer to Bering's voyages.]

DAL., WV. H. Notes on an original chart of Bering's expedition of $1725^{-}$ $173^{\circ}$, and on an original manuscript chart of his sccond expedition; 
together with a summary of a journal of the first expedition, kept by Peter Chaplin, and now first rendered into English from Bergh's Russian version. Appendix No. 19, U. S. Coast and Geodetic Survey Rept. for 1890 , pp. 759-774. Washington, D. C., I891. WValuable because it makes easily accessible Chaplin's journal of Bering's first expedition. The translation is from the work by Berkh listed above on p. 363. Of the accompanying facsimile of the map of the first expedition the eastern section is reproduced in our Fig. 5. The likewise accompanying map of the second expedition is a facsimile of a manuscript chart by Sven Waxel showing the discoveries of that expedition.]

DALL, W. H. A critical review of Bering's first expedition, 1725-30, together with a translation of his original report upon it. Natl. Geogr. Mag., Vol. 2, I 89o, pp. I I I-I69. [The first modern discussion in English of Bering's first expedition based on a critical consideration of the sources available at the time of publication.]

Greely, A. W. The cartography and observations of Bering's first voyage. Natl. Geogr. Mlag., Vol. 3, I891-92, pp. 205-23o. [Important critical remarks on some of the sources, based on copies in the author's possession.]

Scнort, C. A. On the magnetic observations of Bering during his first expedition. Appendix No. 5, U. S. Coast and Geodetic Survey Rept. for 1801 . Washington, D. C., I892. Reprinted from U.S. Coast and Geodetic Survey Bull. 20.

Davidson, George. The tracks and landfalls of Bering and Chirikof on the northwest coast of America from the point of their separation in latitude $49^{\circ} \mathrm{Io}^{\prime}$, longitude $176^{\circ} 4 \mathrm{o}^{\prime}$ west, to their return to the same meridian, June, July, August, September, October, I $74 \mathrm{I}$. [San Francisco], I gor. Also published in Trans, and Proc. Geogr. Soc. of the Pacific. Ser. 2, Vol. 1. [Accompanied by an important map showing the tracks of the St. Peter and St. Paul, east of the point of their separation, adjusted to the actual outline of the Alaskan coast. The sources used for plotting the ship's positions are not indicated in the text.]

Golder, F. A. Russian expansion on the Pacific, 1641-1850: An account of the earliest and later expeditions made by the Russians along the Pacific coast of Asia and North America, including some related expeditions to the Arctic regions. Cleveland, I9I4. [Bering's voyages are dealt with in Chapters VI and VIII. Terra de Yezo in Chapter V, and Gvozdev's voyage in Chapter VII.]

I.titer Voyages to the Same Region Which Shed Light on the Conditions Under Which Bering's Expeditions Were Made

Cook. JAmes. A voyage to the Pacific Ocean. Undertaken, by the 
command of His Majesty, for making discoveries in the Northern hemisphere, to determine the position and extent of the west side of North America; its distance from Asia; and the practicability of a northern passage to Europe. Performed under the direction of Captains Cook, Clerke, and Gore, in His Majesty's ships the Resolution and Discovery, in the years $1776, I 777,1778, I 779$, and 1780 . Vol. I and II written by Captain James Cook, F.R.S. Vol. III by Captain James King, 1...... and F.R.S. Published by order of the Lords Commissioners of the Admiralty. 3 vols. and atlas. London, I 784 .

Dixon, George. A voyage round the world; but more particularly to the north-west coast of America; performed in 1785, I 786, I 787 , and $\mathrm{I} 788$, in the King George and Queen Charlotte, Captains Portlock and Dixon. . . London, 1789 .

Shelekhov, GrigoriI. Grigori Schelechof russischen Kaufmanns erste und zweyte Reise von Ochotsk in Siberien durch den östlichen Ocean nach den Küsten von Amerika in den Jahren 1783 bis 1789 . Nebst umständlicher Beschreibung der von ihm neuentdeckten Inseln Küktak, Afagnak und mehrerer andrer, zu welchen selbst der berühmte Cap. Cook nicht gekommen und die sich der russischen Herrschaft unterworfen haben. Aus dem Russischen übersetzt von J. Z. Logan. St. Petersburg, I793.

Lapérouse, Jean Françors de Galaup, Comte de. Voyage de La Pérouse autour du monde, publié conformément au décrit du 22 avril I 79r, et rédigé par M. L. A. Millet-Mureau. 4 vols. and atlas. Paris, I 797 .

SAUER, MARTIN. An account of a geographical and astronomical expedition to the northern parts of Russia, for ascertaining the degrees of latitude and longitude of the mouth of the river Kovima; of the whole coast of the Tshutski, to East Cape; and of the islands in the eastern ocean, stretching to the American coast. Performed . . . by Commodore Joseph Billings, in the years 1785, etc., to I794. The whole narrated from the original papers. London, iso2. [Reference, p. 194, to a native met with on Billings' expedition who remembered Bering's landing on Kayak Island.]

Sarycinev, Gavrin. Account of a voyage of discovery to the northeast of Siberia, the Frozen Ocean, and the north-east sea. 2 vols. in one. Translated from the Russian. London, I 806-07. [Gives an account of the meeting, on Billings' expedition, of a native who remembered Bering's landing on Kayak Island.]

VAncouver, George. A voyage of discovery to the North Pacific Ocean, and round the world, in which the coast of north-west America has been carefully examined and accurately surveyed. Undertaken by His Majesty's command, principally with a view to ascertain 
the existence of any navigable communication between the North Pacific and North Atlantic oceans; and performed in the years 1790 , 1791, I792, 1793, I794, and 1795, in the Discovery, sloop of war, and armed tender Chatham, under the command of Captain George Vancouver . . 3 vols. and atlas. London, I 798.

Broughton, W. R. A voyage of discovery to the North Pacific Ocean, in which the coast of Asia, from the lat. of $35^{\circ}$ north to the lat. of $52^{\circ}$ north, the island of Insu (commonly known under the name of the land of Jesso, ) the north, south, and cast coasts of Japan, the Lieuchieux and the adjacent isles, as well as the coast of Corea, have been examined and surveyed. Performed in His Majesty's sloop Providence and her tender, in the years 1795. I796, I797, 1798. London, I 804 .

KRUSEnstern, A. J. von. Voyage round the world, in the years I 803 . I 804 , I 805 , and I 806 , by order of His Imperial Miajesty Alexander the First, on board the ships Nadeshda and Neva, under the command of Captain A. J. von Krusenstern. . . Translated from the original German, by Hoppner. 2 vols. in one. London, 1813.

LANGSDORFF, G. H. voN. Voyages and travels in various parts of the world, during the years I803, 1804, 1805, I806, and 1807. 2 vols. London, 1813-14. [Translation of the author's "Bemerkungen auf einer Reise um die Welt," Frankfort, I812.]

Kotzenue, Otto von. A voyage of discovery, into the South Sea and Beering's Straits, for the purpose of exploring a north-east passage, undertaken in the years $1815-1818$, at the expense of His Highness . . Count Romanzoff, in the ship Rurick, under the command of the lieutenant in the Russian imperial navy, Otto von Kotzebue .. 3 vols. London, I 821 .

Choris, Louis. Voyage pittoresque autour du monde, avec des portraits de sauvages d'Amérique, d'Asie, d'Afrique, et des îles du Grand Océan. Paris, 1822. [Choris accompanied Kotzebuc's expedition as artist.]

Wrangkll, Fredinand von. Narrative of an expedition to the Polar Sea, in the years 1820 , I 821 , I 822 , and I 823 . Edited by Major Edward Sabine. . . . London, 1840. [Translated from G. Engelhardt's German translation of the then unpublished Russian manuscript.]

Beechey, F. W. Narrative of a voyage to the Pacific and Beering's Strait, to co-operate with the polar expeditions, performed in $\mathrm{His}$ Majesty's ship Blossom, under the command of Captain F. W. Beechey . . in the years $1825,26,27,28,2$ vols. London, 1831 .

Dobeli., Peter. Travels in Kamtchatka and Siberia; with a narrative of a residence in China. 2 vols. London, 1830.

MiddendorfF, A. T. von. Reise in den äussersten Norden und Osten Sibiriens während der Jahre I 843 und 1844 , mit allerhöchster Genehmi- 
gung auf Veranstaltung der $k$. Akademie der Wissenschaften zu St. Petersburg ausgefuhrt und in Verbindung mit vielen Gelehrten herausgegeben. 4 vols. in seven, and atlas. Acad. of Sci., St. Petersburg, $1847-1875$.

Kittlitz, F. H. von. Denkwürdigkeiten einer Reise nach dem russischen Amerika, nach Mikronesien und durch Kamtschatka. 2 vols. Gotha, 1858 .

NordenskiöLd, A. E. The voyage of the Vega round Asia and Europe; with a historical review of previous journey's along the north coast of the Old World. Translated [from the Swedish] by Alexander Leslie. 2 vols. London, I88I. [Chapter I3 contains references to Bering's voyages.]

Gulllemard, F. H. H. The cruise of the Marchesa to Kamschatka \& New Guinea. IVith notices of Formosa, Liu-Kiu, and various islands of the Malay archipelago. 2nd edit. London, I889.

Burney, Janes. Memoir on the geography of the north-eastern part of Asia. London, I 818 . [Paper read before a seientific body in London in which the author, who had been with Cook in Bering Strait, insisted that it was not conclusively proved that the Old World and the New World were two distinet continents.]

Venianinov, IVAN (Innokentii, metropolitan of Moscow). Zapiski ob ostrovakh Unalashkinskago otdyela. Izdano izhdiveniem RossiiskoAmerikanskoi Kompanii. (Notes on the islands of the Unalaska district. Published at the expense of the Russian-American Company.) 3 vols. in two. St. Petersburg, I 840 .

HolmberG, H. J. Etlınographische Skizzen über die Völker des russischen Amerika. 2 vols. Helsingfors, I $855-[63]$. [Vol. I reprinted from Vol. 4 of the Actu of Finska Vetenskaps-Societeten; Vol. 2 is a separate (pp. [35]-IOI) from Vol. 7 of the Acta.]

Dall, IV. II., and George Gibis. Tribes of the extreme Northwest, by W. H. Dall. Tribes of western Washington and northwestern Oregon, by George Gibbs. Contributions to North American Ethnology. Vol. I. Bur. of Ethnol., Washington, D. C. 1877.

Sluinis, N. V. Okhotsko-Kamehatkii krai (Okhotsk-Kamchatka region). Vol. I. St. Petersburg, 1900. [Description of the natural history of the region, with map.]

Rlabl'sinski, li. l'. Kamehatskaya ekspeditsiya. Moscow, I9I2. [Account of a scientific expedition to Kanchatka financed by the author, on whicl Waldenar Jochelson, known for his work on the Jesup North Pacific Expedition, was in charge of ethnology.] 


\section{Official Documents, and Bibliographies}

Polnoe sobranie zakonov Rossiiskoi Imperii (Complete collection of the laws of the Russian Empire). Vols. 3, 4, 7, 8. Imperial Printing Press, St. Petersburg, ca. I830.

Obshchii morskoi spisok (General navy list). I3 vols. and I vol. of index. St. Petersburg, I885-1907. [Useful in following the careers of the officers who took part in Bering's voyages.]

Opisanie dyyl arkhiva Morskago Ministerstva s poloviny xvii do nachala xix stolyctiya (Description of the papers in the archives of the Ministry of Marine from the middle of the seventeenth to the beginning of the nineteentl century). Io vols. Published by direction of the Ministry of Marine, St. Petersburg, 1877-1906.

DALl, W. II., and MARCus BAKER. Partial list of charts, maps, and publications relating to Alaska and the adjacent region, from Puget Sound and Hakodadi to the Arctic Ocean, between the Rocky and the Stanovoi Mountains, in: Pacific Coast Pilot: Coasts and Islands of Alaska, Second Series, pp. 163-374, U. S. Coast and Geodetic Survey, Washington, D.C., I 879. [Invaluable bibliography. Strong on Russian sources; Russian titles are given in Cyrillic. The contents of periodical series are analyzed. Maps are listed on pp. 167-223, text publications on pp. 225-374.].

Golder, F. A. Guide to materials for American history in Russian archives. Carnegie Instn. Publ. No. 239. Washington, D. C., 1917. The documents relating to the Bering expeditions are mainly in the archives of the Ministry of Marine (listed on Pp. I19-130), of the Hydrographic Section of that Ministry (pp. 130-136), and of the General Staff (PD. II6-II9).] 
. 
This book is DUE on the last date stamped below

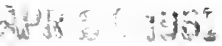

iut $2 \cdots 480$ 
$\frac{y}{y r}$ Gb |||||||||||||||||||||||||||||||

L $\begin{array}{llll}009 & 529 & 355 & 1\end{array}$ 
